\title{
Finit element solution of Poisson Equation over Polygonal Domains using a novel auto mesh generation technique and an explicit integration scheme for linear convex quadrilaterals of cubic order Serendipity and Lagrange families
}

\author{
H. T. Rathod ${ }^{a^{*}}$, Md.Shafiqul. Islam ${ }^{b}$, Bharath Rathod ${ }^{c}$, K. Sugantha Devi ${ }^{d}$ \\ a Department of Mathematics, Central College Campus, Bangalore University, \\ Bangalore -560001, Karnataka State, India. \\ ${ }^{\mathbf{b}}$ Department of Applied Mathematics, University of Dhaka,Dhaka-1000, \\ Bangladesh \\ ${ }^{c}$ Xavier Institute of Management and Entrepreneurship, Hosur Road, \\ Electronic City Phase II, Bangalore, Karnataka 560034, Karnataka State, India. \\ ${ }^{\mathrm{d}}$ Department of Mathematics, Dr. T. Thimmaiah Institute of Technology, Oorgam Post, \\ Kolar Gold Field, Kolar District, Karnataka state, Pin- 563120, India.
}

\begin{abstract}
:
This paper presents an explicit integration scheme to compute the stiffness matrix of twelve node and sixteen node linear convex quadrilateral finite elements of Serendipity and Lagrange families using an explicit integration scheme and discretisation of polygonal domain by such finite elements using a novel auto mesh generation technique, In finite element analysis, the boundary value problems governed by second order linear partial differential equations, the element stiffness matrices are expressed as integrals of the product of global derivatives over the linear convex quadrilateral region. These matrices can be shown to depend on the material properties matrices and the matrix of integrals with integrands as rational functions with polynomial numerator and the linear denominator $(4+\xi+\eta)$ in the bivariates $\xi$ and $\eta$ over a 2 -square $(-1 \leq \xi, \eta \leq 1)$ with the nodes on the boundary and in the interior of this simple domain. The finite elements up to cubic order have nodes only on the boundary for Serendipity family and the finite elements with boundary as well as some interior nodes belong to the Lagrange family. The first order element is the bilinear convex quadrilateral finite element which is an exception and it belongs to both the families. We have for the present ,the cubic order finite elements which havee 12 boundary nodes at the nodal coordinates $\{(-1,-1),(1,-1),(1,1),(-$ $1,1),(-1 / 3,-1),(1 / 3,-1),(1,-1 / 3),(1,1 / 3),(1 / 3,1),(-1 / 3,1),(-1,1 / 3),(-1,-1 / 3)\}$ and the four interoior nodal coordinates at the points $(-$ $1 / 3,-1 / 3),(1 / 3,-1 / 3),(1 / 3,1 / 3),(-1 / 3,1 / 3)\}$ in the local parametric space $(\xi, \eta)$. In this paper, we have computed the integrals of local derivative products with linear denominator $(4+\xi+\eta)$ in exact forms using the symbolic mathematics capabilities of MATLAB. The proposed explicit finite element integration scheme can be then applied to solve boundary value problems in continuum mechanics over convex polygonal domains. We have also developed a novel auto mesh generation technique of all 12-node and 16-node linear(straight edge) convex quadrilaterals for a polygonal domain $\Omega \subset \mathcal{R}^{2}$ which provides the nodal coordinates and the element connectivity. We have used the explicit integration scheme and this novel auto mesh generation technique to solve the Poisson equation $-\nabla^{2} \mathrm{u}=f$, where $\mathrm{u}$ is an unknown physical variable and $f$ is a known smooth function in $\Omega \subset \mathcal{R}^{2}$ with Dirichlet boundary conditions over the convex polygonal domain.
\end{abstract}

Key words: Explicit Integration, Finite Element Method,cubic order 2-D finite elements of Serendipity and Lagrange families, Matlab Symbolic Mathematics, All Quadrilateral Mesh Generation Technique,Poisson Equation,Dirichlet Boundary Conditions ,Polygonal Domain, Gauss Legendre Quadrature Rules.

\section{Introduction :}

In recent years, the finite element method (FEM) has emerged as a powerful tool for the approximate solution of differential equations governing diverse physical phenomena. Today, finite element analysis is an integral and major component in many fields of engineering design and manufacturing. Its use in industry and research is extensive, and indeed without it many practical problem in science, engineering and emerging technologies such as nanotechnology, biotechnology, aerospace, chemical. etc would be incapable of solution [1,2,3]. In FEM, various integrals are to be determined numerically in the evaluation of stiffness matrix, mass matrix, body force vector, etc. The algebraic integration needed to derive explicit finite element relations for second 
order continuum mechanics problems generally defies our analytic skill and in most cases, it appears to be a prohibitive task. Hence, from a practical point of view, numerical integration scheme is not only necessary but very important as well. Among various numerical integration schemes, Gauss Legendre quadrature, which can evaluate exactly the $(2 \mathrm{n}-1)^{\text {th }}$ degree polynomial with ' $n$ ' Gaussian integration points, is mostly used in view of the accuracy and efficiency of calculation. However, the integrands of global derivative products in stiffness matrix computations of practical applications are not always simple polynomials but rational expressions which the Gaussian quadrature cannot evaluate exactly [7-15]. The integration points have to be increased in order to improve the integration accuracy but it is also desirable to make these evaluations by using as few Gaussian points as possible, from the point of view of the computational efficiency. Thus it is an important task to strike a proper balance between accuracy and economy in computation. Therefore analytical integration is essential to generate a smaller error as well as to save the computational costs of Gaussian quadrature commonly applied for science, engineering and technical problems. In explicit integration of stiffness matrix, complications arise from two main sources, firstly the large number of integrations that need to be performed and secondly, in methods which use isoparametric or equivalently the subparametric finite elements, the presence of determinant of the Jacobian matrix ( we refer this as Jacobian here after ) in the denominator of the element matrix integrands. This problem is considered in the recent work [16] for the four node linear convex quadrilateral which proposes a new discretisation method and use of pre computed universal numeric arrays which do not depend on element size and shape. In this method a linear polygon is discretized into a set of linear triangles and then each of these triangles is further discretised into three linear four node convex quadrilateral elements by joining the centroid to the mid-point of sides. These quadrilateral elements are then mapped into 2-squares $(-1 \leq \xi, \eta \leq 1)$ in the natural space $(\xi, \eta)$ to obtain the same expression of the Jacobian, namely $\mathrm{c}(4+\xi+\eta)$ where $\mathrm{c}$ is some appropriate constant which depends on the geometric data for the triangle. We can always devise finite elements with higher order interpolation or shape functions by placing more nodes over these quadrilateral regions.

Many important problems in engineering,science and applied mathematics are formulated by appropriate differential equations with some boundary conditions imposed on the desired unknown function or the set of functions. There exists a large literature which demonstrates numerical accuracy of the finite element method to deal with such issues [1]. Clough seems to be the first who introduced the fnite elements to standard computational procedures [2]. A further historical development and present day concepts of finite element analysis are widely described in references [1, 3]. In this paper the well-known Laplace and Poisson equations will be examined by means of the finite element method applied to an appropriate 'mesh'. The class of physical situations in which we meet these equations is really broad. Let's recall such problems like heat conduction, seepage through porous media, irrotational flow of ideal fluids, distribution of electrical or magnetic potential, torsion of prismatic shafts, lubrication of pad bearings and others [4]. Therefore, in physics and engineering arises a need of some computational methods that allow us to solve accurately such a large variety of physical situations. The considered method completes the above-mentioned task. Particularly, it refers to a standard discrete pattern allowing to find an approximate solution to continuum problem. At the beginning, the continuum domain is discretized by dividing it into a finite number of elements for which properties must be determined from an analysis of the physical problem (e. g. as a result of experiments). These studies on a particular problem allow us to construct the so called the stiffness matrix for each element that, for instance, in elasticity comprising material properties like stress strain relationships [2,5]. Then the corresponding nodal loads associated with elements must be found. The construction of accurate elements constitutes the subject of a mesh generation recipe proposed by the author within the presented article. In many realistic situations, mesh generation is a time consuming and error prone process because of various levels of geometrical complexity. Over the years, there were developed both semi automatic and fully automatic mesh generators obtained, respectively, by using the mapping methods or, on the contrary, algorithms based on the Delaunay triangulation method [6], the advancing front method [7] and tree methods [8]. It is worth mentioning that the first attempt to create fully automatic mesh generator capable to produce valid finite element meshes over arbitrary domains has been made by Zienkiewicz and Phillips [9].

In the present paper, we propose a similar discretisation method for linear polygon in Cartesian two space (x,y). This discrtisation is carried in two steps, We first discretise the linear polygon into a set of linear triangles in the Cartesian space (x,y) and these linear triangles are then mapped into a standard triangle in a local space $(\mathrm{u}, \mathrm{v})$. We further discretise the standard triangles into three linear quadrilaterals by joining the centroid to the midpoints of triangles in $(\mathrm{u}, \mathrm{v})$ space which are finally mapped into 2square in the local $(\xi, \eta)$ space. We then establish a derivative product relation between the linear convex quadrilaterals in the Cartesian space, $(\mathrm{x}, \mathrm{y})$ which are interior to an arbitrary triangle and the linear quadrilaterals in the local space $(\mathrm{u}, \mathrm{v})$ interior to the standard triangle. In this procedure, all computations in the local space $(\mathrm{u}, \mathrm{v})$ for product of global derivative integrals are free from geometric properties and hence they are pure numbers. We then propose a numerical scheme to integrate the products of global derivatives. We have shown that the matrix product of global derivative integrals is expressible as matrix triple product comprising of geometric properties matrices and the product of local derivative integrals matrix. We have obtained explicit integration of the product of local derivatives which is now possible by use of symbolic integration commands available in leading mathematical softwares MATLAB, MAPLE, MATHEMATIKA etc. In this paper, we have used the MATLAB symbolic mathematics to compute the integrals of the products of local derivatives in $(\mathrm{u}, \mathrm{v})$ space .The proposed explicit integration scheme is shown as a useful technique in the formation of element stiffness matrices for second order boundary value problems governed by partial differential equations[27-33].

This paper presents an explicit integration scheme to compute the stiffness matrices of linear convex quadrilateral elements belonging to cubic order twelve node Serendipity and sixteen node Lagrange families using the symbolic mathematics and discretisation of polygonal domain by such finite elements using a novel auto mesh generation technique, In finite element analysis, the boundary value problems governed by second order linear partial differential equations, the element stiffness matrices are expressed as integrals of the product of global derivatives over the linear convex quadrilateral region. These matrices can be shown to depend on the material properties and the matrix of integrals with integrands as rational functions with polynomial numerator and the linear denominator $(4+\xi+\eta)$ in the bivariates $\xi$ and $\eta$ over a 2 -square $(-1 \leq \xi$, $\eta \leq 1)$ with nodes at the boundary points $\{(-1,-1),(1,-1),(1,1),(-1,1),(-1 / 3,-1),(1 / 3,-1),(1,-1 / 3),(1,1 / 3),(1 / 3,1),(-1 / 3,1),(-1,1 / 3),(-1,-1 / 3)\}$ and the 
four interoior nodal coordinates at the points $(-1 / 3,-1 / 3),(1 / 3,-1 / 3),(1 / 3,1 / 3),(-1 / 3,1 / 3)\}$ in the local parametric space $(\xi, \eta)$. In this paper, we have computed these integrals in exact forms using the symbolic mathematics capabilities of MATLAB. The proposed explicit finite element integration scheme can be applied to solve boundary value problems in continuum mechanics over convex polygonal domains. We have also developed a novel auto mesh generation technique of all 12- node and 16-node linear convex quadrilaterals for a polygonal domain $\Omega \subset \mathcal{R}^{2}$ which provides the nodal coordinates and element connectivity. We have used the explicit integration scheme and this novel auto mesh generation technique to solve the Poisson equation $-\nabla^{2} \mathrm{u}=$ $f$, where $\mathrm{u}$ is a unknown physical variable and $f$ is a known smooth function in $\Omega \subset \mathcal{R}^{2}$ with given Dirichlet boundary conditions over convex polygonal domains. We need a small amount of numerical integration to complete the solution of the Poisson boundary value problem when $f$ is a known smooth function other than a constant.

\section{POISSON EQUATION}

\subsection{Statement of the Problem}

The Poisson equation

$$
-\nabla^{2} \mathrm{u}=f
$$

is the simplest and

most famous elliptic partial differential equations. The source (or load) function is given on some two or three dimensional domain $\Omega \subset \mathcal{R}^{2}$ or $\mathcal{R}^{3}$. A solution u satisfying (1.1) will also satisfy boundary conditions on the boundary $\partial \Omega$ of $\Omega$;for example $\alpha u+\beta \frac{\partial \mathrm{u}}{\partial \mathrm{n}}=\mathrm{g} \quad$ on $\quad \partial \Omega$

where $\partial \mathrm{u} / \partial \mathrm{n}$ denotes directional derivative in the direction normal to the boundary $\partial \Omega$ (conveniently pointing outwards) and $\alpha$ and $\beta$ are constants, although variable coefficients are also possible.The combination of (1.1) and (1.2) together is referred to as boundary value problem. If the constant $\beta$ in (1.2) is zero,then the boundary condition is known as the Dirichlet type, and the boundary value problem is referred as the Dirichlet problem for the Poisson equation. Alternatively, if the constant $\alpha$ in (1.2) is zero,then we correspondingly have a Neumann boundary value problem. A third possibility is that Dirichlet conditions hold on part of the boundary $\partial \Omega_{\mathrm{D}}$ and Neumann conditions(or indeed mixed conditions where $\alpha$ and $\beta$ are both nonzero) hold on remainder $\partial \Omega \backslash \partial \Omega_{\mathrm{D}}$. The case $\alpha=0, \beta=1$ in (1.2) demands special attention.First, since $\mathrm{u}=$ constant satisfies the homogeneous problem with $f=0, g=0$, it is clear that a solution to a Neumann problem can only be unique up to an additive constant.Second,integrating (1.1) over $\Omega$ using Gauss's theorem gives

$-\int_{\partial \Omega} \frac{\partial \mathrm{u}}{\partial \mathrm{n}}=-\int_{\Omega} \nabla^{2} \mathrm{u}=\int_{\Omega} f$

thus a necessary condition for the existence of a solution to the Neumann problem is that the source and boundary data satisfy the compatibility condition:

$$
\int_{\partial \Omega} g+\int_{\Omega} f=0
$$

2.2 Weak Formulation of the Poisson Boundary Value Problem

A sufficiently smooth function u satisfying both eqns(1) and (2) is known as classical solution to the Poisson boundary value problem. For a Dirichlet problem, $\mathrm{u}$ is a classical solution only if it has continuousecond derivatives in $\Omega$ (i.e. $\mathrm{u}$ is $C^{2}(\Omega)$ and is continuous up to the boundary i.e.u is in $C^{0}(\bar{\Omega})$ ). In case of nonsmooth domains or discontinuous source functions,the function $\mathrm{u}$ satisfying eqns(1) and (2) may not be smooth (or regular) enough to be regarded as classical solution. For problems which arise from, perfectly reasonable mathematical models an alternative description of the boundary value problem is required. Since this alternative description is less restrictive in terms of admissible data it is called weak formulation.

To derive a weak formulation of a Poisson problem,we require that for an appropriate set of test functions $v$,

$\int_{\Omega}\left(\nabla^{2} u+f\right) v=0$

This formulation exists provided that the integrals are well defined. If $u$ is a classical solution then it must also satisfy eqn (5). If $v$ is sufficiently smooth however,then the smoothness required of $u$ can be reduced by using the derivative of a product rule and the divergence theorem

$$
\begin{aligned}
-\int_{\Omega} v \nabla^{2} u & =\int_{\Omega} \nabla u \cdot \nabla v-\int_{\Omega} \nabla \cdot(v \nabla u) \\
& =\int_{\Omega} \nabla u \cdot \nabla v-\int_{\partial \Omega} v \frac{\partial \mathrm{u}}{\partial \mathrm{n}},
\end{aligned}
$$

so that

$\int_{\Omega} \nabla u \cdot \nabla v=\int_{\Omega} v f+\int_{\partial \Omega} v \frac{\partial \mathrm{u}}{\partial \mathrm{n}}$

The point here is that the problem posed byeqn(6) may have a solution $u$ called a weak solution,that is not smooth enough to be a classical solution. If a classical solution does exist then eqn(6)is equivalent to eqns (1) and (2) and the weak solution is classical. The case of Neumann problem $(\alpha=0, \beta=1)$ in eqn(2) is particularly straight forward. Substituting from eqn(2) into eqn(6)gives us the following formulation: find $u$ defined on $\Omega$ such that 
$\int_{\Omega} \nabla u \cdot \nabla v=\int_{\Omega} v f+\int_{\partial \Omega} v g$

for all suitable test functions $v$.

\subsection{Finite Elements for Poisson's Equation with Dirichlet conditions: Implementation and Review} Of Theory

\subsubsection{Weak Form}

Given Poisson Equation:

$-\Delta u(\mathrm{x})=f(\mathrm{x})$ for all $\mathrm{x} \in \Omega$

.........................(7a)

$u=g(x)$ on $\partial \Omega$

We have already obtained in eqn $(6)$ with $(\alpha=1, \beta=0)$ the weak form of the equation by multiplying both sides by a test function $v$ (i.e a function which is infinitely differentiable and has compact support,integrating over the domain $\Omega$ and performing integration by parts or by application of Divergence(GREEN) theorem. The result is

$\int_{\Omega} \nabla u \cdot \nabla v d x=\int_{\Omega} v f d x$

..............................(7c)

$u=g(x)$ on $\partial \Omega$

For all test functions $v$

2.3.2 Finite Elements

To find an approximation to the solution $u$, we choose a finite dimensional space $V_{h}$ and ask that eqn(7a-b) is satisfied only for $v$ in $V_{h}$ rather than for all test functions $v$. Then we look for a function $u_{h} \in V_{h}$ which satisfies

$\int_{\Omega} \nabla u_{h} \cdot \nabla v d x=\int_{\Omega} v f d x \quad$ for all $\quad v \in V_{h}$

$u_{h}$ is called the finite element solution and functions in $V_{h}$ are called finite elements.

Note that it is also common for the triangles or quadrilaterals in the mesh to be called elements.

If a basis for $V_{h}$ is $\left\{\varphi_{j}\right\}_{j=1}^{j=N}$ then we can write $u_{h}=\sum_{j=1}^{\mathrm{j}=\mathrm{N}} \alpha_{\mathrm{j}} \varphi_{\mathrm{j}}$. Substituting this in eqn(8) and choosing $v$ to be a basis function $\varphi_{i}$ gives the following set of equations

$\sum_{j=1}^{N} \alpha_{j} \int_{\Omega} \nabla \varphi_{i} \cdot \nabla \varphi_{j} d x=\int_{\Omega} f \varphi_{i} d x \quad, \mathrm{i}=1,2,3, \ldots ., \mathrm{N}$

This is really a linear system of the form

$K u=f$

Where, $u=\left(\alpha_{1}, \alpha_{2}, \alpha_{3}, \ldots \ldots \ldots \alpha_{N}\right)^{T}$ and

$$
\begin{aligned}
& K_{i, j}=\int_{\Omega} \nabla \varphi_{i} \cdot \nabla \varphi_{j} d x \\
& f_{i}=\int_{\Omega} f \varphi_{i} d x
\end{aligned}
$$

and $K$ is called stiffness matrix because the linear system looks like Hookes law if $f$ represents forces and $u$ represents displacemewnts.

In general, $\Omega=\sum_{e=1}^{N_{e}} \Omega^{e}$, where $N_{e}$ is the number of elements discritised in the domain $\Omega$. In two dimensions the mesh elements are triangles or quadrilaterals. The choice of finite element spaces are usually piecewise polynomials.

2.3.3 Overview on the implementation of Finite Element Method

Once we have choosen the finite element space (and the element type),then we can implement the finite element method.The implementation is divided into three steps:

1. Mesh Generation:how does one perform a triangulation or quadrangulation of the domain $\Omega$ ?

2. Assembling the Stiffness Matrix:how does one compute the entries in the stiffness matrix in an efficient way?

3. Solving the linear System:What kind of methodse suited for solving the linear system?

In this paper,we present new approach to mesh generation [ ] and explicit computations for the entries in the stiffness matrix [ ] which is vital in Assembling the Stiffness Matrix,since we believe that the methods of solving linear system are well researched and standardised.

We shall first take up the derivations regarding the topic on Assembling the Stiffness Matrix. The Mesh Generation topic will be discussed immediately there after.

\subsubsection{Assembling the Stiffness Matrix}

In order to assemble the stiffness matrix, we need to compute integrals of the form(see eqn(11) in section (2.3.2)

$$
K_{i, j}=\int_{\Omega} \nabla \varphi_{i} \cdot \nabla \varphi_{j} d x
$$


The most obvious way to assemble the stiffness matrix is to compute the integrals $K_{i, j}$ for the nodal pairs $\mathrm{i}$ and $\mathrm{j}$; this is a node oriented computation and we need to know the common support of basis functions $\varphi_{i}$ and $\varphi_{j}$. This means we need to know which elements contain both $\mathrm{i}$ and $\mathrm{j}$.The mesh generator provides us with the information regarding the nodes on a particular element so we would need to do some extra processing to find the elements that contain a particular node.This is an issue which is very complicated.Hence, in practice assembling is focussed on elements rather than on nodes. We note that on a particular element,the basis functions have a simple expression and the elements themselves are very simple domains like triangles and quadrilaterals. It is very easy to make a change of variables for integrals over triangles and quadrilaterals to standard triangles and squares. In the element oriented computation,we rewrite or interpret the integral in eqn(11) as

$K_{i, j}=\sum_{\Omega^{e} \varepsilon \Omega_{h}^{e}} K_{i, j}^{e}=$

where

$K_{i, j}^{e}$

$$
=\int_{\Omega} e \nabla \varphi_{i} \cdot \nabla \varphi_{j} d x
$$

and $\Omega_{h}^{e}$ is the set of (mesh) elements in $\Omega$ contributing to $K_{i, j}$ and $\Omega=\sum_{e=1}^{N_{e}} \Omega^{e}, \Omega^{e}$ is an element contained in the set $\Omega_{h}^{e}$ .This says us that we can compute $K_{i, j}$ by computing the integrals over each element $\Omega^{e}$ and then summing up over all elements $\Omega_{h}^{e}$.

Notice that the integrals

$K_{i, j}^{e}=\int_{\Omega^{e}} \nabla \varphi_{i} \cdot \nabla \varphi_{j} d x$ look like the entries $K_{i, j}=\int_{\Omega} \nabla \varphi_{i} \cdot \nabla \varphi_{j} d x=\sum_{\Omega^{e}} \Omega_{h}^{e} \quad \int_{\Omega^{e}} \nabla \varphi_{i} \cdot \nabla \varphi_{j} d x$ except the domain of integration is an element $\Omega^{e}$. So,we only need to save all entries of $K^{e}=\left[K_{i, j}^{e}\right]$ which corresponds to nodes on $\Omega^{e}$. Then if $\Omega^{\mathrm{e}}$ has $\mathrm{d}$ nodes, we can think of $K^{e}$ as a dxd matrix. In view of the above,the procedure for computing the stiffness maerix is done on an element by element basis.

We must also compute the integrals

$$
f_{i}=\int_{\Omega} f \varphi_{i} d x=\sum_{e=1}^{N_{e}} f_{i}^{e}
$$

where

$f_{i}^{e}$

Now further assume that on an element $\Omega^{\mathrm{e}}, u_{h}=\mathrm{u}^{\mathrm{e}}=\sum_{\mathrm{j}=1}^{\mathrm{j}=\mathrm{d}} \mathrm{u}_{\mathrm{j}}^{\mathrm{e}} \varphi_{\mathrm{j}}$

From eqn(9) and eqns(12a-d) it follows that $K u=f$ is equivalent to

Where

$$
\sum_{e=1}^{N_{e}} K^{e} \mathrm{u}^{\mathrm{e}}=\sum_{e=1}^{N_{e}} f^{e}
$$

$\mathrm{u}^{\mathrm{e}}=\left(\mathrm{u}_{1}^{\mathrm{e}}, \mathrm{u}_{2}^{\mathrm{e}}, \mathrm{u}_{3}^{\mathrm{e}}, \ldots \ldots \ldots \ldots \mathrm{u}_{\mathrm{d}}^{\mathrm{e}}\right)^{T}, f^{\mathrm{e}}=\left(f_{1}^{\mathrm{e}}, f_{2}^{\mathrm{e}}, f_{3}^{\mathrm{e}}, \ldots \ldots \ldots \ldots f_{\mathrm{d}}^{\mathrm{e}}\right)^{T}$

d referes to number of nodes per element, $N_{e}$ referes to the total number of elements in the domain $\Omega$

\subsubsection{Computing the Integrals $K_{i, j}^{e}$ and $f_{i}^{e}$}

In order to compute the local/element stiffness matrices, we need to compute the integrals $K_{i, j}^{e}=\int_{\Omega^{e}} \nabla \varphi_{i} \cdot \nabla \varphi_{j} d x$ .These integrals are computed by making a change of variables to a reference element. We now outline a brief procedure for element oriented computation

(1)For each element $\Omega^{e}$, compute it's local stiffness matrix $K^{e}$. This requires computing the integrals $K_{i, j}^{e}=\int_{\Omega} \nabla \nabla \varphi_{i} \cdot \nabla \varphi_{j} d \mathrm{x}$ which we compute by transforming to a reference element. In two dimensions $\Omega^{e}$ is an arbitrary linear triangle and each triangle will be further discritised three convex quadrilaterals $Q_{3 e-2}, Q_{3 e-1}$ and $Q_{3 e}$ Each triangle will be transformed to the corresponding reference elements:the standard triangle(a right isosceles triangle) and further Each triangle will be transformed to the corresponding reference elements:the standard triangle(a right isosceles triangle) and further Each triangle will be transformed to the corresponding reference elements:the standard triangle(a right isosceles triangle) and further each quadrilateral will be transformed into a standard square(1-square or a 2-square). Since in two dimensional space $\mathrm{x}=(\mathrm{x}, \mathrm{y})$ the explicit form of $K_{i, j}^{e}=$ $\int_{\Omega^{e}} \nabla \varphi_{i} \cdot \nabla \varphi_{j} d \mathrm{x}$ is given by

$K_{i, j}^{e}=\int_{\Omega^{e}} \nabla \varphi_{i} \cdot \nabla \varphi_{j} d \mathrm{x}$

$\left.=\int_{\Omega} e \frac{\partial \varphi_{i}}{\partial x} \frac{\partial \varphi_{j}}{\partial x}+\frac{\partial \varphi_{i}}{\partial y} \frac{\partial \varphi_{j}}{\partial y}\right\} d x d y=\sum_{e=1}^{N_{e}} \sum_{n=0}^{2} \int_{Q_{E}}\left\{\frac{\partial \varphi_{i}}{\partial x} \frac{\partial \varphi_{j}}{\partial x}+\frac{\partial \varphi_{i}}{\partial y} \frac{\partial \varphi_{j}}{\partial y}\right\} d x d y$

$=\sum_{e=1}^{N_{e}} \sum_{n=0}^{2} S_{i, j}^{E}$

.....................(12g)

Where $S_{i, j}^{E}=\int_{Q_{E}}\left\{\frac{\partial \varphi_{i}}{\partial x} \frac{\partial \varphi_{j}}{\partial x}+\frac{\partial \varphi_{i}}{\partial y} \frac{\partial \varphi_{j}}{\partial y}\right\} d x d y$ and $\mathrm{E}=3 \mathrm{e}+\mathrm{n}-2, \mathrm{e}=1,2, \ldots N_{e}$ andn $=0,1,2$

and hence we must be careful about the derivatives when we perform the change of variables. These bring extra factors involving the affine transformations (when $\Omega^{e}$ is an arbitrary linear triangle) and bilinear transformations(when $\Omega^{e}$ is an arbitrary linear convex quadrilateral)

$f_{i}^{e}=\int_{\Omega^{e}} f \varphi_{i}$ dxdy can be computed in a straight forward manner if $f$ is a simple function otherwise we have to apply numerical integration 
(2) For each element $\Omega^{e}$, first compute the local stiffness matrices $S^{E}=\left[S_{i, j}^{E}\right]$ and then add contribution of $K^{e}=S^{3 e-2}$ $+S^{3 e-1}+S^{3 e}$, to the global stiffness matrix $\mathrm{K}$. We repeat this procedure for all elements i.e for e $=1,2, \ldots \ldots ., N_{e}$; where $N_{e}$ is the number of elements $\Omega^{e}$ which are discritised in the domain $\Omega$, in fact we have $\Omega=\sum_{e=1}^{N_{e}} \Omega^{e}=\sum_{e=1}^{N_{e}} \sum_{n=0}^{2} Q_{E}, \mathrm{E}=3 \mathrm{e}+\mathrm{n}-2$

\subsection{Finite Element Types}

\subsubsection{Linear Convex Quadrilateral Elements :}

Let us first consider an arbitrary four noded linear convex quadrilateral in the global (Cartesian) coordinate system (u, v) as in Fig 1a, which mapped into a 2 -square in the local(natural) parametric coordinate $(\xi, \eta)$ as in Fig $1 \mathrm{~b}$.

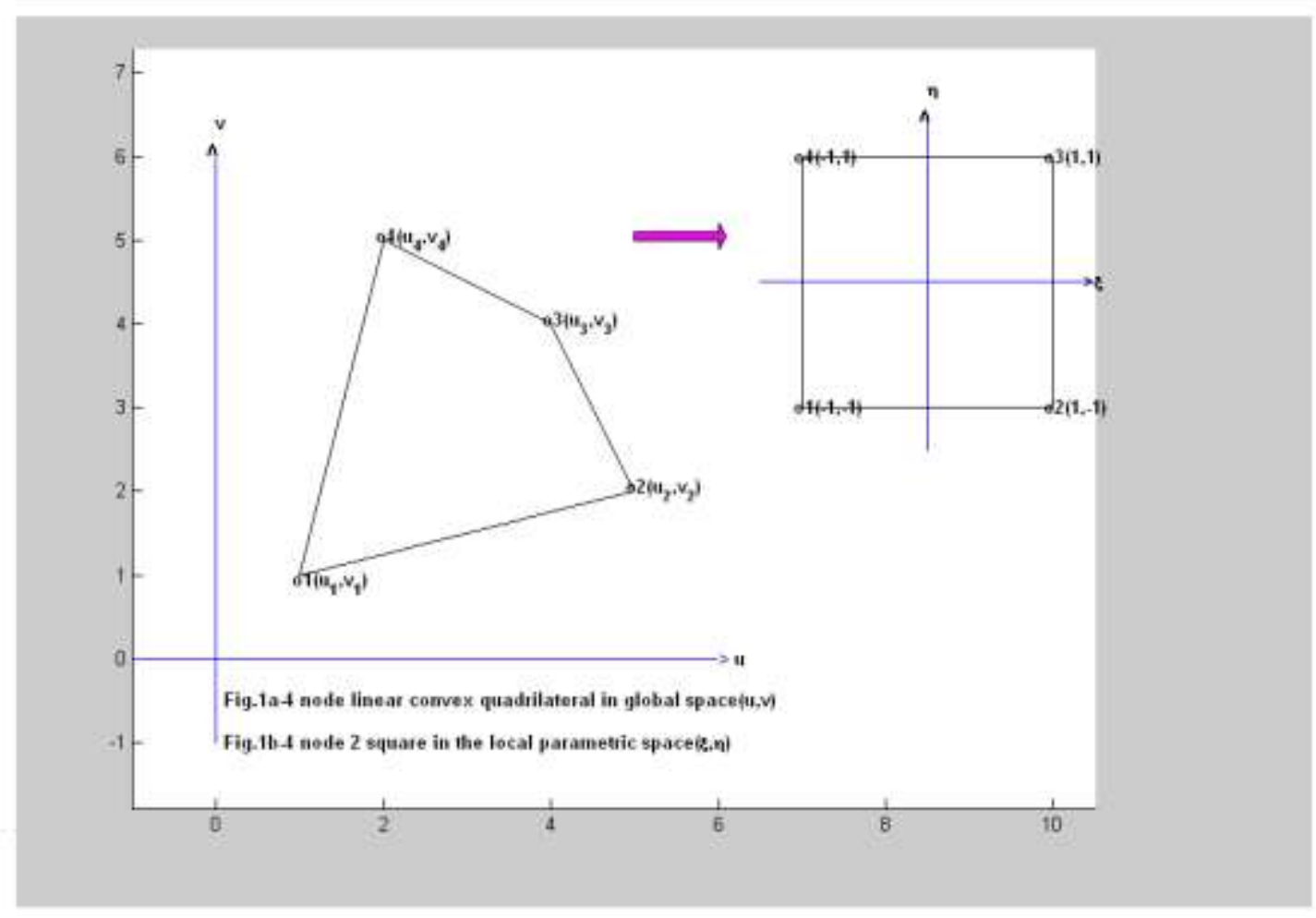

$\left(\begin{array}{l}\mathrm{u} \\ \mathrm{v}\end{array}\right)=\sum_{\mathrm{k}=1}^{4}\left(\begin{array}{l}\mathrm{u}_{\mathrm{k}} \\ \mathrm{v}_{\mathrm{k}}\end{array}\right) \mathrm{M}_{\mathrm{k}}(\xi, \eta)$

Where $\left(\mathrm{u}_{\mathrm{k}}, \mathrm{v}_{\mathrm{k}}\right),(\mathrm{k}=1,2,3,4)$ are the vertices of the original arbitrary linear convex quadrilateral in (u, v) plane and $\mathrm{M}_{\mathrm{k}}(\xi, \eta)$ denote the well known bilinear basis functions [1-3] in the local parametric space $(\xi, \eta)$ and they are given by

$$
\mathrm{M}_{\mathrm{k}}(\xi, \eta)=\frac{1}{4}\left(1+\xi \xi_{\mathrm{k}}\right)\left(1+\eta \eta_{\mathrm{k}}\right), \quad \mathrm{k}=1,2,3,4
$$

Where $\left\{\left(\xi_{\mathrm{k}}, \eta_{\mathrm{k}}\right), \mathrm{k}=1,2,3,4\right\}=\{(-1,-1),(1,-1),(1,1),(-1,1)\}$

describes a geometric transformation over a linear convex quadrilateral element from the original global space into the local parametric space.

\subsubsection{Isoparametric Transformation :}

For the isoparametric coordinate transformation over the linear convex quadrilateral element as shown in Fig 1, we select the field variables, say $\phi, \psi$, etc governing the physical problem as

$\left(\begin{array}{c}\phi \\ \psi\end{array}\right)=\sum_{\mathrm{k}=1}^{4}\left(\begin{array}{c}\phi_{\mathrm{k}} \\ \psi_{\mathrm{k}}\end{array}\right) \mathrm{N}_{\mathrm{k}}^{\mathrm{e}}(\xi, \eta)$ 
Where $\phi_{\mathrm{k}}, \Psi_{\mathrm{k}}$ refer to unknowns at node $\mathrm{k}$ and the shape functions $\mathrm{N}_{\mathrm{k}}{ }^{\mathrm{e}}=\mathrm{M}_{\mathrm{k}}$, and $\mathrm{M}_{\mathrm{k}}$ are defined as in Eqn.(14a-b)

We have considered the application of explicit stiffness matrix integration scheme and automesh generation technique to find FEM solution of Poisson equation boundary value problems over polygonal domains using linear convex quadrilateral elements under isoparametric transformations[ ].

\subsubsection{Subparmetric Transformation :}

For the subparametric transformation over the nde - noded element we define the field variables $\phi, \psi$ (say) governing the physical problem as

$\left(\begin{array}{c}\phi \\ \psi\end{array}\right)=\sum_{\mathrm{k}=1}^{\mathrm{nde}}\left(\begin{array}{c}\phi_{\mathrm{k}}^{\mathrm{e}} \\ \psi_{\mathrm{k}}^{\mathrm{e}}\end{array}\right) \mathrm{N}_{\mathrm{k}}^{\mathrm{e}}(\xi, \eta)$

Where $\phi_{\mathrm{k}}, \psi_{\mathrm{k}}$ refer to unknowns at node $\mathrm{k}$ and nde $>4$

In our recent paper, the explicit finite element integration scheme is presented by using the isoparametric transformation over the 4 node linear convex quadrilateral element for which we set nde $=4$

In the present paper, we consider the subparametric transformation for a linear convex quadrilateral element for which nde $=12$, a 12 noded 2 square of cubic Serendipity type and nde=16, a 16-node 2-square of cubic Lagrange type.

\section{Cubic order Linear Convex Quadrilateral Elements :}

In this section, we give a brief description of the 12- node cubic Serendipity quadrilateral element under subparametric transformation as shown in Fig 1c, Fig 1d; and the 16- node cubic Lagrange quadrilateral element under subparametric transformation as shown in Fig 1e, Fig 1f.

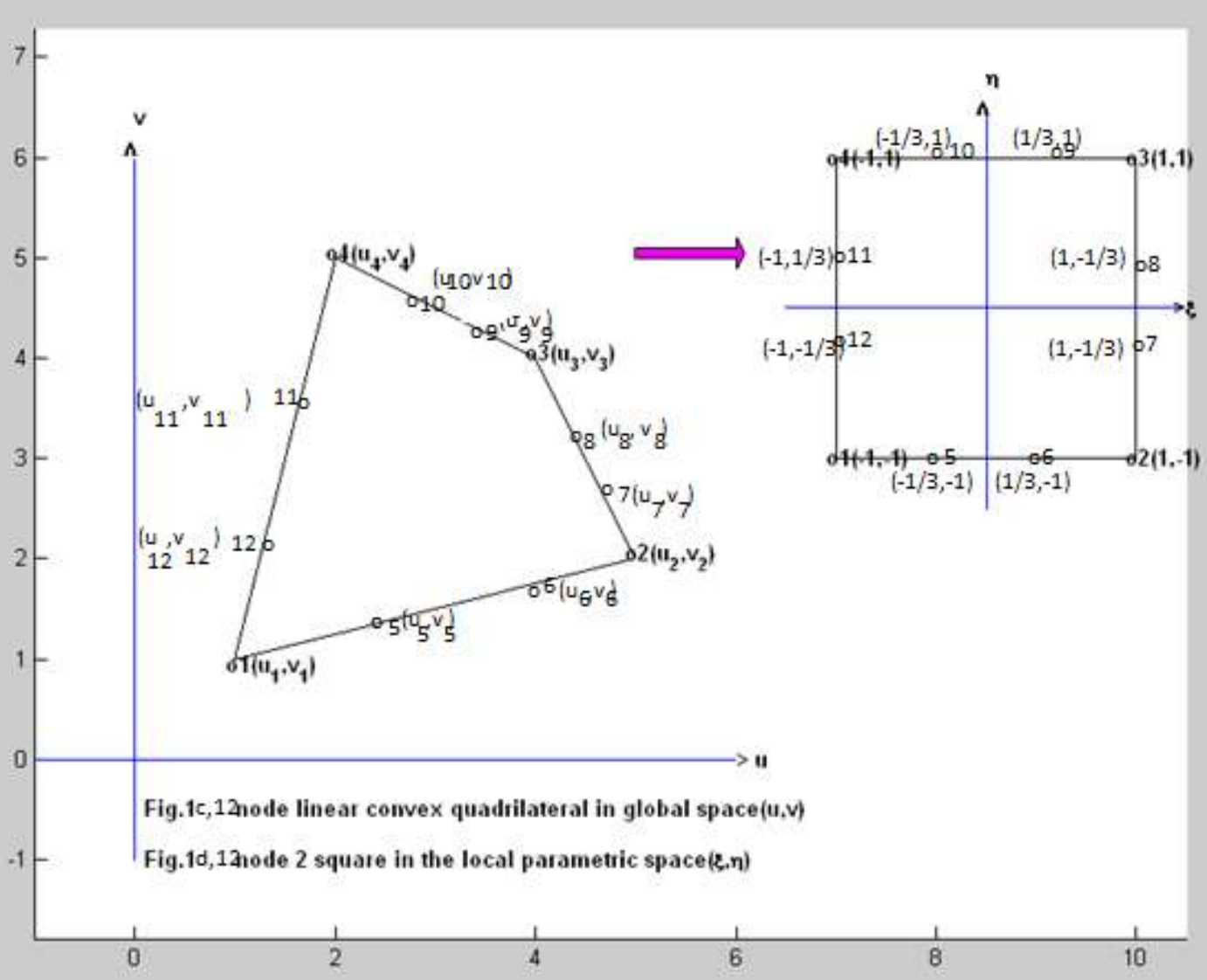




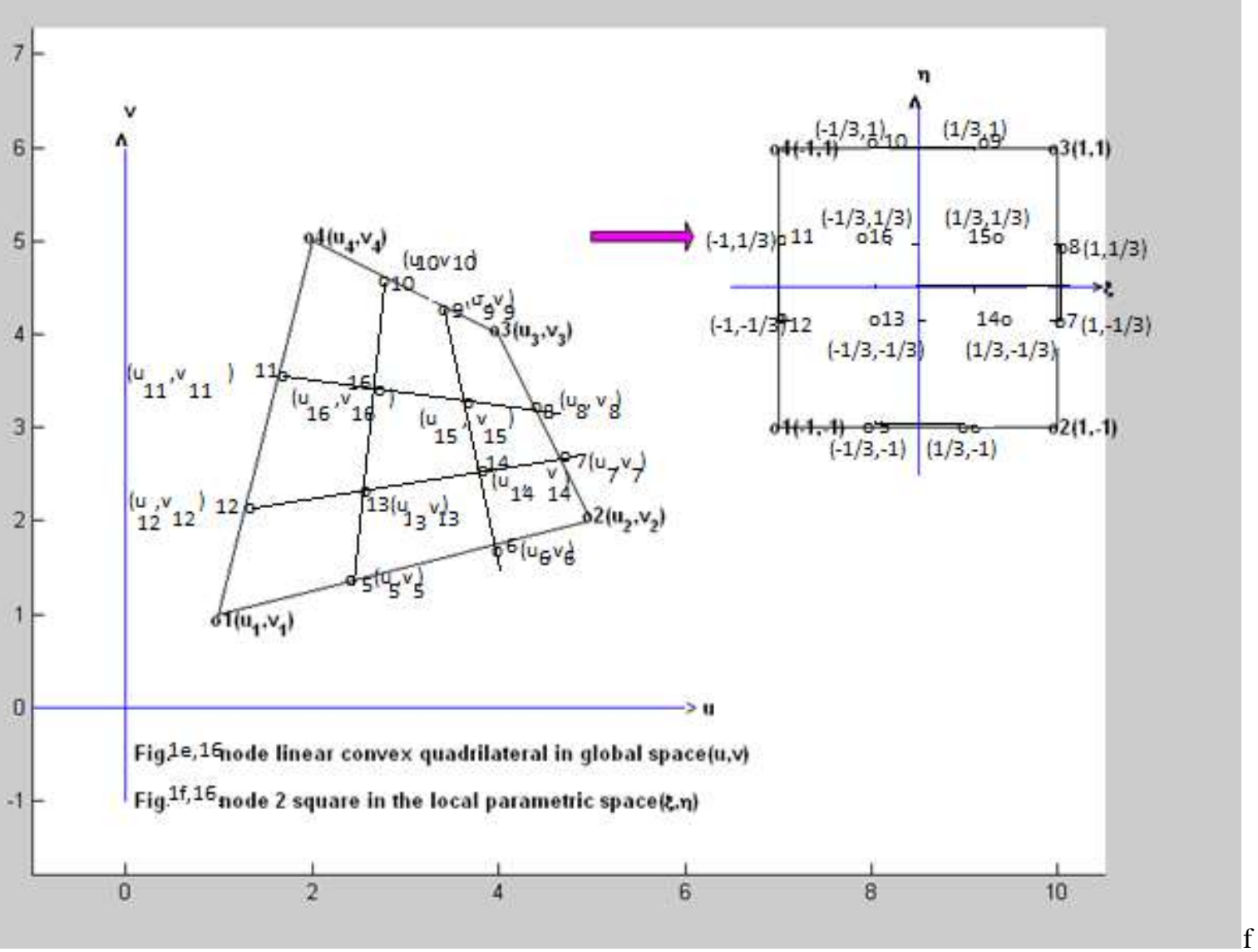

We use the transform of Eqns.(13-14) to define the element geometry i.e.

$\left(\begin{array}{c}\mathrm{u}(\xi, \eta) \\ \mathrm{v}(\xi, \eta)\end{array}\right)=\left(\begin{array}{l}\mathrm{u} \\ \mathrm{v}\end{array}\right)=\sum_{\mathrm{k}=1}^{4}\left(\begin{array}{c}\mathrm{u}_{\mathrm{k}} \\ \mathrm{v}_{\mathrm{k}}\end{array}\right) \mathrm{M}_{\mathrm{k}}(\xi, \eta)$

Where $\mathrm{M}_{\mathrm{k}}(\xi, \eta)=\frac{1}{4}\left(1+\xi \xi_{\mathrm{k}}\right)\left(1+\eta \eta_{\mathrm{k}}\right), \quad(\mathrm{k}=1,2,3,4)$

(14a)

With $\left.\left(\mathrm{u}\left(\xi_{\mathrm{k}}, \eta_{\mathrm{k}}\right), \mathrm{v}\left(\xi_{\mathrm{k}}, \eta_{\mathrm{k}}\right)\right), \quad \mathrm{k}=1,2,3,4\right)$ are the vertices of the linear convex quadrilateral in global $(\mathrm{u}, \mathrm{v})$ space .

$\left\{\left(\xi_{\mathrm{k}}, \eta_{\mathrm{k}}\right), \mathrm{k}=1,2,3,4\right\}=\{(-1,-1),(1,-1),(1,1),(-1,1)\}$

$(14 b)$

Using the transformation of Eqns.(13-14) and from Fig 1c, Fig 1d; we see that there is a one to one correspondence between $\left(\left(\xi_{\mathrm{k}}, \eta_{\mathrm{k}}\right), \mathrm{k}=1(1) 12\right)$ and $\left(\left(\mathrm{u}_{\mathrm{k}}, \mathrm{v}_{\mathrm{k}}\right)=\left(\mathrm{u}\left(\xi_{\mathrm{k}}, \eta_{\mathrm{k}}\right), \mathrm{v}\left(\xi_{\mathrm{k}}, \eta_{\mathrm{k}}\right)\right), \mathrm{k}=1(1) 12\right)$ for cubic Serendipity element.

Using the transformation of Eqns.(13-14) and from Fig 1e, Fig 1f ; we see that there is a one to one correspondence between $\left(\left(\xi_{\mathrm{k}}, \eta_{\mathrm{k}}\right), \mathrm{k}=1(1) 16\right)$ and $\left(\left(\mathrm{u}_{\mathrm{k}}, \mathrm{v}_{\mathrm{k}}\right)=\left(\mathrm{u}\left(\xi_{\mathrm{k}}, \eta_{\mathrm{k}}\right), \mathrm{v}\left(\xi_{\mathrm{k}}, \eta_{\mathrm{k}}\right)\right), \mathrm{k}=1(1) 16\right)$ for cubic Lagrange element.

Where, the coordinates of the boundary nodes are :

$\left(\left(\xi_{\mathrm{k}}, \eta_{\mathrm{k}}\right), \mathrm{k}=1(1) 12\right)=\{(-1,-1),(1,-1),(1,1),(-1,1),(-1 / 3,-1),(1 / 3,-1),(1,-1 / 3),(1,1 / 3),(1 / 3,1),(-1 /$ $3,1),(-1,1 / 3),(-1,-1 / 3)\}$ $-(14 c)$

and coordinates of the interior nodes are:

$\left(\left(\xi_{\mathrm{k}}, \eta_{\mathrm{k}}\right), \mathrm{k}=13,14,15,16\right)=\{(-1 / 3,-1 / 3),(1 / 3,-1 / 3),(1 / 3,1 / 3),(-1 / 3,1 / 3)\}$

$(14 \mathrm{~d})$

where 


$$
\begin{aligned}
& \left(u_{5}, v_{5}\right)=\left(\left(2 u_{1}+u_{2}\right) / 3,\left(2 v_{1}+v_{2}\right) / 3\right), \\
& \left(u_{6}, v_{6}\right)=\left(\left(u_{1}+2 u_{2}\right) / 3,\left(v_{1}+2 v_{2}\right) / 3\right), \\
& \left(u_{7}, v_{7}\right)=\left(\left(2 u_{2}+u_{3}\right) / 3,\left(2 v_{2}+v_{3}\right) / 3\right), \\
& \left(u_{8}, v_{8}\right)=\left(\left(u_{2}+2 u_{3}\right) / 3,\left(v_{2}+2 v_{3}\right) / 3\right), \\
& \left(u_{9}, v_{9}\right)=\left(\left(2 u_{3}+u_{4}\right) / 3,\left(2 v_{3}+v_{4}\right) / 3\right), \\
& \left(u_{10}, v_{10}\right)=\left(\left(u_{3}+2 u_{4}\right) / 3,\left(v_{3}+2 v_{4}\right) / 3\right), \\
& \left(u_{11}, v_{11}\right)=\left(\left(u_{1}+2 u_{4}\right) / 3,\left(v_{1}+2 v_{4}\right) / 3\right) \\
& \left(u_{12}, v_{12}\right)=\left(\left(2 u_{1}+u_{4}\right) / 3,\left(2 v_{1}+v_{4}\right) / 3\right), \\
& \left(u_{13}, v_{13}\right)==\left(\left(4 u_{1}+2 u_{2}+u_{3}+2 u_{4}\right) / 9,\left(4 v_{1}+2 v_{2}+v_{3}+2 v_{4}\right) / 9\right), \\
& \left(u_{14}, v_{14}\right)==\left(\left(2 u_{1}+4 u_{2}+2 u_{3}+u_{4}\right) / 9,\left(2 v_{1}+4 v_{2}+2 v_{3}+v_{4}\right) / 9\right), \\
& \left(u_{15}, v_{15}\right)==\left(\left(u_{1}+2 u_{2}+4 u_{3}+2 u_{4}\right) / 9,\left(v_{1}+2 v_{2}+4 v_{3}+2 v_{4}\right) / 9\right), \\
& \left(u_{16}, v_{16}\right)==\left(\left(2 u_{1}+u_{2}+2 u_{3}+4 u_{4}\right) / 9,\left(2 v_{1}+v_{2}+2 v_{3}+4 v_{4}\right) / 9\right),
\end{aligned}
$$

We then define the variation of physical variables $\phi^{\mathrm{e}}, \psi^{\mathrm{e}}$ (say) over 12- node cubic Serendipity element of Fig 1c , 1d and 16node cubic Lagrange element of Fig 1e , 1f by Eqn.(16) with nde = 12,16

$\left(\begin{array}{c}\phi^{\mathrm{e}} \\ \psi^{\mathrm{e}}\end{array}\right)=\sum_{\mathrm{k}=1}^{\mathrm{nde}} \mathrm{N}_{\mathrm{k}}^{\mathrm{e}}(\xi, \eta)\left(\begin{array}{c}\phi_{\mathrm{k}}^{\mathrm{e}} \\ \psi_{\mathrm{k}}^{\mathrm{e}}\end{array}\right)$

Where $\phi_{\mathrm{k}}{ }^{\mathrm{e}}, \psi_{\mathrm{k}}{ }^{\mathrm{e}}$ are the nodal values at node $\mathrm{k}$

The shape functions $\mathrm{N}_{\mathrm{i}}{ }^{\mathrm{e}}$ of the 12- node cubic Serendipity element shown in Fig 1c, Fig 1d are given by

$\mathrm{N}_{1}{ }^{\mathrm{e}}(\xi, \eta)=\left((1-\xi)(1-\eta)\left(-10+9\left(\xi^{2}+\eta^{2}\right)\right)\right) / 32 ;$

$\mathrm{N}_{2}{ }^{\text {e }}(\xi, \eta)=\left((1+\xi)(1-\eta)\left(-10+9\left(\xi^{2}+\eta^{2}\right)\right)\right) / 32$;

$\mathrm{N}_{3}{ }^{\text {e }}(\xi, \eta)=\left((1+\xi)(1+\eta)\left(-10+9\left(\xi^{2}+\eta^{2}\right)\right)\right) / 32$;

$\mathrm{N}_{4}{ }^{\mathrm{e}}(\xi, \eta)=\left((1-\xi)(1+\eta)\left(-10+9\left(\xi^{2}+\eta^{2}\right)\right)\right) / 32$;

$\mathrm{N}_{5}{ }^{\mathrm{e}}(\xi, \eta)=(9 / 32)\left((1-\eta)\left(1-\xi^{2}\right)(1-3 \xi)\right)$;

$\mathrm{N}_{6}{ }^{\mathrm{e}}(\xi, \eta)=(9 / 32)\left((1-\eta)\left(1-\xi^{2}\right)(1+3 \xi)\right)$;

$\mathrm{N}_{7}{ }^{\mathrm{e}}(\xi, \eta)=(9 / 32)\left((1+\xi)\left(1-\eta^{2}\right)(1-3 \eta)\right)$

$\mathrm{N}_{8}{ }^{\mathrm{e}}(\xi, \eta)=(9 / 32)\left((1+\xi)\left(1-\eta^{2}\right)(1+3 \eta)\right)$;

$\mathrm{N}_{9}{ }^{\mathrm{e}}(\xi, \eta)=(9 / 32)\left((1+\eta)\left(1-\xi^{2}\right)(1+3 \xi)\right)$;

$\mathrm{N}_{10} \mathrm{e}(\xi, \eta)=(9 / 32)\left((1+\eta)\left(1-\xi^{2}\right)(1-3 \xi)\right)$;

$\mathrm{N}_{11}{ }^{\mathrm{e}}(\xi, \eta)=(9 / 32)\left((1-\xi)\left(1-\eta^{2}\right)(1+3 \eta)\right)$;

$\mathrm{N}_{12}$ e $(\xi, \eta)=(9 / 32)\left((1-\xi)\left(1-\eta^{2}\right)(1-3 \eta)\right)$;

and we may check that

$\mathrm{N}_{\mathrm{k}}{ }^{\mathrm{e}}\left(\xi_{\mathrm{k}}, \eta_{\mathrm{k}}\right)=1, \mathrm{~N}_{\mathrm{k}}{ }^{\mathrm{e}}\left(\xi_{\mathrm{j}}, \eta_{\mathrm{j}}\right)=0$, when $\mathrm{j} \neq k$

$\left(\left(\xi_{\mathrm{k}}, \eta_{\mathrm{k}}\right), \mathrm{k}=1(1) 12\right)=\{(-1,-1),(1,-1),(1,1),(-1,1),(-1 / 3,-1),(1 / 3,-1),(1,-1 / 3),(1,1 / 3),(1 / 3,1),(-1 /$

$3,1),(-1,1 / 3),(-1,-1 / 3)\}$ $(17 \mathrm{~b})$

The shape functions $\mathrm{N}_{\mathrm{i}}{ }^{\mathrm{e}}$ of the 16- node cubic Serendipity element shown in Fig 1e, Fig 1f are given by

$\mathrm{N}_{1}{ }^{\mathrm{e}}(\xi, \eta)=\left(-9 / 16 \xi^{3}+9 / 16 \xi^{2}+1 / 16 \xi-1 / 16\right)\left(-9 / 16 \eta^{3}+9 / 16 \eta^{2}+1 / 16 \eta-1 / 16\right)$

$\mathrm{N}_{2}{ }^{\mathrm{e}}(\xi, \eta)=\left(9 / 16 \xi^{3}+9 / 16 \xi^{2}-1 / 16 \xi-1 / 16\right)\left(-9 / 16 \eta^{3}+9 / 16 \eta^{2}+1 / 16 \eta-1 / 16\right)$;

$\mathrm{N}_{3}{ }^{\mathrm{e}}(\xi, \eta)=\left(9 / 16 \xi^{3}+9 / 16 \xi^{2}-1 / 16 \xi-1 / 16\right)\left(9 / 16 \eta^{3}+9 / 16 \eta^{2}-1 / 16 \eta-1 / 16\right) ;$

$\mathrm{N}_{4}{ }^{\text {e }}(\xi, \eta)=\left(-9 / 16 \xi^{3}+9 / 16 \xi^{2}+1 / 16 \xi-1 / 16\right)\left(9 / 16 \eta^{3}+9 / 16 \eta^{2}-1 / 16 \eta-1 / 16\right)$

$\mathrm{N}_{5}{ }^{\mathrm{e}}(\xi, \eta)=\left(27 / 16 \xi^{3}-9 / 16 \xi^{2}-27 / 16 \xi+9 / 16\right)\left(-9 / 16 \eta^{3}+9 / 16 \eta^{2}+1 / 16 \eta-1 / 16\right)$;

$\mathrm{N}_{6}{ }^{\mathrm{e}}(\xi, \eta)=\left(-27 / 16 \xi^{3}-9 / 16 \xi^{2}+27 / 16 \xi+9 / 16\right)\left(-9 / 16 \eta^{3}+9 / 16 \eta^{2}+1 / 16 \eta-1 / 16\right)$; 
$\mathrm{N}_{7}{ }^{\mathrm{e}}(\xi, \eta)=\left(9 / 16 \xi^{3}+9 / 16 \xi^{2}-1 / 16 \xi-1 / 16\right)\left(27 / 16 \eta^{3}-9 / 16 \eta^{2}-27 / 16 \eta+9 / 16\right) ;$

$\mathrm{N}_{8}{ }^{\mathrm{e}}(\xi, \eta)=\left(9 / 16 \xi^{3}+9 / 16 \xi^{2}-1 / 16 \xi-1 / 16\right)\left(-27 / 16 \eta^{3}-9 / 16 \eta^{2}+27 / 16 \eta+9 / 16\right) ;$

$\mathrm{N}_{9}{ }^{\mathrm{e}}(\xi, \eta)=\left(-27 / 16 \xi^{3}-9 / 16 \xi^{2}+27 / 16 \xi+9 / 16\right)\left(9 / 16 \eta^{3}+9 / 16 \eta^{2}-1 / 16 \eta-1 / 16\right) ;$

$\mathrm{N}_{10}{ }^{\mathrm{e}}(\xi, \eta)=\left(27 / 16 \xi^{3}-9 / 16 \xi^{2}-27 / 16 \xi+9 / 16\right)\left(9 / 16 \eta^{3}+9 / 16 \eta^{2}-1 / 16 \eta-1 / 16\right) ;$

$\mathrm{N}_{11}{ }^{\mathrm{e}}(\xi, \eta)=\left(-9 / 16 \xi^{3}+9 / 16 \xi^{2}+1 / 16 \xi-1 / 16\right)\left(-27 / 16 \eta^{3}-9 / 16 \eta^{2}+27 / 16 \eta+9 / 16\right)$;

$\mathrm{N}_{12}{ }^{\text {e }}(\xi, \eta)=\left(-9 / 16 \xi^{3}+9 / 16 \xi^{2}+1 / 16 \xi-1 / 16\right)\left(27 / 16 \eta^{3}-9 / 16 \eta^{2}-27 / 16 \eta+9 / 16\right)$;

$\mathrm{N}_{13}$ e $(\xi, \eta)=\left(27 / 16 \xi^{3}-9 / 16 \xi^{2}-27 / 16 \xi+9 / 16\right)\left(27 / 16 \eta^{3}-9 / 16 \eta^{2}-27 / 16 \eta+9 / 16\right) ;$

$\mathrm{N}_{14}{ }^{\text {e }}(\xi, \eta)=\left(-27 / 16 \xi^{3}-9 / 16 \xi^{2}+27 / 16 \xi+9 / 16\right)\left(27 / 16 \eta^{3}-9 / 16 \eta^{2}-27 / 16 \eta+9 / 16\right)$;

$\mathrm{N}_{15}{ }^{\mathrm{e}}(\xi, \eta)=\left(-27 / 16 \xi^{3}-9 / 16 \xi^{2}+27 / 16 \xi+9 / 16\right)\left(-27 / 16 \eta^{3}-9 / 16 \eta^{2}+27 / 16 \eta+9 / 16\right)$;

$\mathrm{N}_{16}{ }^{\mathrm{e}}(\xi, \eta)=\left(27 / 16 \xi^{3}-9 / 16 \xi^{2}-27 / 16 \xi+9 / 16\right)\left(-27 / 16 \eta^{3}-9 / 16 \eta^{2}+27 / 16 \eta+9 / 16\right)$;

and we may check that

$\mathrm{N}_{\mathrm{k}}{ }^{\mathrm{e}}\left(\xi_{\mathrm{k}}, \eta_{\mathrm{k}}\right)=1, \mathrm{~N}_{\mathrm{k}}{ }^{\mathrm{e}}\left(\xi_{\mathrm{j}}, \eta_{\mathrm{j}}\right)=0$, when $\mathrm{j} \neq k, \mathrm{k}=1(1) 16$

$\left(\left(\xi_{\mathrm{k}}, \eta_{\mathrm{k}}\right), \mathrm{k}=1(1) 12\right)=\{(-1,-1),(1,-1),(1,1),(-1,1),(-1 / 3,-1),(1 / 3,-1),(1,-1 / 3),(1,1 / 3),(1 / 3,1),(-1 /$

$3,1),(-1,1 / 3),(-1,-1 / 3)\}$ and $(17 b)$

$\left(\left(\xi_{\mathrm{k}}, \eta_{\mathrm{k}}\right), \mathrm{k}=13,14,15,16\right)=\{(-1 / 3,-1 / 3),(1 / 3,-1 / 3),(1 / 3,1 / 3),(-1 / 3,1 / 3)\}$

$--(17 \mathrm{c})$

\section{Explicit Form of the Jacobian and Global Derivatives :}

\subsection{Jacobian}

Let us consider an arbitrary linear convex quadrilateral in the global Cartesian space (u, v) as in Fig 1a, c which is mapped into a 8- node 2- square in the local parametric space $(\xi, \eta)$ as in Fig $1 \mathrm{~b}, \mathrm{~d}$

From the Eq.(1) and Eq.(2), we have

$\frac{\partial \mathrm{u}}{\partial \xi}=\sum_{\mathrm{k}=1}^{4} \mathrm{u}_{\mathrm{k}} \frac{\partial \mathrm{M}_{\mathrm{k}}}{\partial \xi}=\frac{1}{4}\left[\left(-\mathrm{u}_{1}+\mathrm{u}_{2}+\mathrm{u}_{3}-\mathrm{u}_{4}\right)+\left(\mathrm{u}_{1}-\mathrm{u}_{2}+\mathrm{u}_{3}-\mathrm{u}_{4}\right) \eta\right]$

$\frac{\partial \mathrm{u}}{\partial \eta}=\sum_{\mathrm{k}=1}^{4} \mathrm{u}_{\mathrm{k}} \frac{\partial \mathrm{M}_{\mathrm{k}}}{\partial \eta}=\frac{1}{4}\left[\left(-\mathrm{u}_{1}-\mathrm{u}_{2}+\mathrm{u}_{3}+\mathrm{u}_{4}\right)+\left(\mathrm{u}_{1}-\mathrm{u}_{2}+\mathrm{u}_{3}-\mathrm{u}_{4}\right) \xi\right]$

$\frac{\partial \mathrm{v}}{\partial \xi}=\frac{1}{4}\left[\left(-\mathrm{v}_{1}+\mathrm{v}_{2}+\mathrm{v}_{3}-\mathrm{v}_{4}\right)+\left(\mathrm{v}_{1}-\mathrm{v}_{2}+\mathrm{v}_{3}-\mathrm{v}_{4}\right) \eta\right]$

$\frac{\partial \mathrm{v}}{\partial \eta}=\frac{1}{4}\left[\left(-\mathrm{v}_{1}-\mathrm{v}_{2}+\mathrm{v}_{3}+\mathrm{v}_{4}\right)+\left(\mathrm{v}_{1}-\mathrm{v}_{2}+\mathrm{v}_{3}-\mathrm{v}_{4}\right) \xi\right]$

Hence the Jacobian, J can be expressed as [1, 2, 3]

$\mathrm{J}=\frac{\partial(\mathrm{u}, \mathrm{v})}{\partial(\xi, \eta)}=\frac{\partial \mathrm{u}}{\partial \xi} \frac{\partial \mathrm{v}}{\partial \eta}-\frac{\partial \mathrm{u}}{\partial \eta} \frac{\partial \mathrm{v}}{\partial \xi}=\alpha+\beta \xi+\gamma \eta$

Where

$\alpha=\frac{1}{8}\left[\left(u_{4}-u_{2}\right)\left(v_{1}-v_{3}\right)+\left(u_{3}-u_{1}\right)\left(v_{4}-v_{2}\right)\right]$

$\beta=\frac{1}{8}\left[\left(u_{4}-u_{3}\right)\left(v_{2}-v_{1}\right)+\left(u_{1}-u_{2}\right)\left(v_{4}-v_{3}\right)\right]$

$\gamma=\frac{1}{8}\left[\left(\mathrm{u}_{4}-\mathrm{u}_{1}\right)\left(\mathrm{v}_{2}-\mathrm{v}_{3}\right)+\left(\mathrm{u}_{3}-\mathrm{u}_{2}\right)\left(\mathrm{v}_{4}-\mathrm{v}_{1}\right)\right]$

\subsection{Global Derivatives:}

If $\mathrm{N}_{\mathrm{i}}^{\mathrm{e}}$ denotes the basis functions of node $\mathrm{i}$ of any order of the element $\mathrm{e}$, then the chain rule of differentiation from Eq.(1) we can write the global derivative as in

$[1,2,3]$ 
$\left(\begin{array}{c}\frac{\partial \mathrm{N}_{\mathrm{i}}^{\mathrm{e}}}{\partial \mathrm{u}} \\ \frac{\partial \mathrm{N}_{\mathrm{i}}^{\mathrm{e}}}{\partial \mathrm{v}}\end{array}\right)=\frac{1}{\mathrm{~J}}\left[\begin{array}{cr}\frac{\partial \mathrm{v}}{\partial \eta} & -\frac{\partial \mathrm{v}}{\partial \xi} \\ -\frac{\partial \mathrm{u}}{\partial \eta} & \frac{\partial \mathrm{u}}{\partial \xi}\end{array}\right]\left[\begin{array}{c}\frac{\partial \mathrm{N}_{\mathrm{i}}^{\mathrm{e}}}{\partial \xi} \\ \frac{\partial \mathrm{N}_{\mathrm{i}}^{\mathrm{e}}}{\partial \eta}\end{array}\right]$

Where $\frac{\partial u}{\partial \xi}, \frac{\partial u}{\partial \eta}, \frac{\partial v}{\partial \xi}$ and $\frac{\partial v}{\partial \eta}$ are defined as in Eqs.(18a)-(18d) while J is defined in Eq.(19a-b), $(\mathrm{i}, \mathrm{j}=1,2,3,-----$, nde $)$, nde $=$ the number of nodes per element. We may recall that the explicit integration for linear convex quadrilateral with nde $=4$ is already presented by the authors in their recent paper [18]. We take nde $=12,16$ for the present study.

\section{Discretisation of an Arbitrary Triangle :}

A linear convex polygon in the physical plane (x, y) can be always discretised into a finite number of linear triangles. However, we would like to study a particular discretization of these triangles further into linear convex quadrilaterals. This is stated in the following Lemma [26].

We first consider an arbitrary triangle $\triangle \mathrm{PQR}$ in the Cartesian space $(x, y)$ with vertices $P\left(x_{p}, y_{p}\right), Q\left(x_{q}, y_{q}\right)$, and $R\left(x_{r}, y_{r}\right)$, Let $Z\left(\left(x_{p}+x_{q}+x_{r}\right) / 3,\left(y_{p}+y_{q}+y_{r}\right) / 3\right)$ be its centroid and also let $S, T, U$ be the midpoints of sides $P Q, Q R$, and $R P$ respectively Now by joining the centroid $Z$ to the midpoints $S, T, U$ by straight lines, we divided the triangle $\Delta \mathrm{PQR}$ into three special quadrilaterals $\mathrm{Q}_{1}, \mathrm{Q}_{2}$, and $\mathrm{Q}_{3}$ (say) which are spanned by vertices $\langle\mathrm{Z}, \mathrm{U}, \mathrm{P}, \mathrm{S}\rangle,\langle\mathrm{Z}, \mathrm{S}, \mathrm{Q}, \mathrm{T}\rangle$, and $\langle\mathrm{Z}, \mathrm{T}, \mathrm{R}, \mathrm{U}\rangle$ respectively. This is shown in Fig. 2.0 a

We next consider the standard triangle $\triangle \mathrm{ABC}$ in the Cartesian space $(\mathrm{u}, \mathrm{v})$ with vertices, centroid and midpoints $\mathrm{A}(1,0), \mathrm{B}(0,1)$, $\mathrm{C}(0,0), \mathrm{G}\left(\frac{1}{3}, \frac{1}{3}\right), \mathrm{D}\left(\frac{1}{2}, 1 / 2\right), \mathrm{E}(0,1 / 2)$ and $\mathrm{F}\left(\frac{1}{2}, 0\right)$. We now divide the $\Delta \mathrm{ABC}$ into three special quadrilaterals $\widehat{Q_{1}}, \widehat{Q_{2}}$ and $\widehat{Q_{3}}$ (say) which are spanned by vertices $\langle\mathrm{G}, \mathrm{E}, \mathrm{C}, \mathrm{F}\rangle,\langle\mathrm{G}, \mathrm{F}, \mathrm{A}, \mathrm{D}\rangle$ and $\langle\mathrm{G}, \mathrm{D}, \mathrm{B}, \mathrm{E}\rangle$ respectively. This is shown in Fig. $2.0 \mathrm{~b}$

We apply linear transformations to map an arbitrary triangle into a triangle of our choice. In this section, we use the well known transformation which maps an arbitrary triangle into a standard triangle (a right isosceles triangle). We assume the special discretization scheme of the previous section for the following developments

5.1 Lemma 1. There exists a unique linear transformation to map the special quadrilaterals $Q_{i}$ in to $\widehat{Q_{l}} \quad(i=1,2,3)$ satisfying the conditions

(i) $\sum_{i=1}^{3} Q_{i}=\triangle P Q R$, the arbitrary triangle in the $(\mathrm{x}, \mathrm{y})$ space.

(ii) $\sum_{i=1}^{3} \widehat{Q}_{l}=\triangle A B C$, the standard triangle (right isosceles triangle ) in the (u, v) space , then the Jacobian $\mathrm{J}^{\mathrm{e}}$ for each element $\mathrm{Q}_{\mathrm{e}},(\mathrm{e}=1,2,3)$ is given by

$\mathrm{J}=\mathrm{J}^{\mathrm{e}}=\frac{1}{48} \Delta \operatorname{pqr}(4+\xi+\eta), \quad \mathrm{e}=1,2,3$

Where $\Delta$ pqr is the area of the triangle $\Delta \mathrm{PQR}$

$2 \Delta p q r=\left|\begin{array}{lll}1 & x_{p} & y_{p} \\ 1 & x_{q} & y_{q} \\ 1 & x_{r} & y_{r}\end{array}\right|=\left[\left(x_{p}-x_{r}\right)\left(y_{q}-y_{r}\right)-\left(x_{q}-x_{r}\right)\left(y_{p}-y_{r}\right)\right]$ 


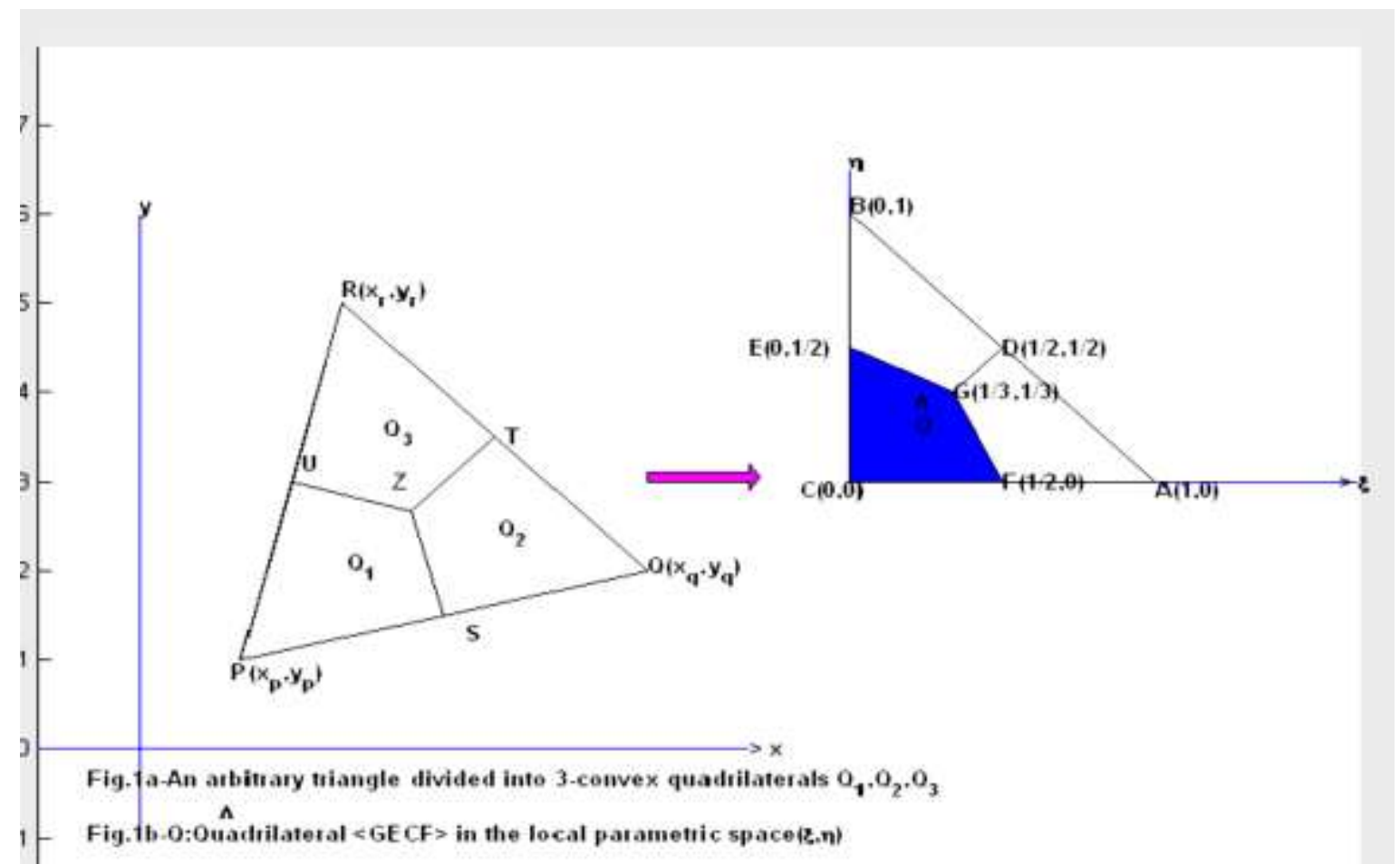

Fig 2.0

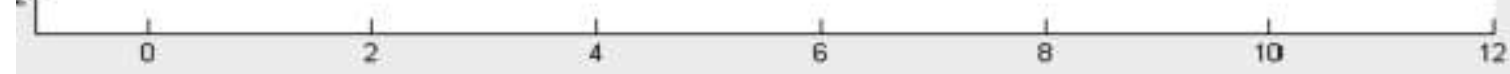

Proof: We shall now refer to Fig 2.0 a, b for the following developments and consider the following linear transformation between $(\mathrm{x}, \mathrm{y})$ and $(\mathrm{u}, \mathrm{v})$ spaces.

$\left(\begin{array}{l}x \\ y\end{array}\right)=\left(\begin{array}{l}x_{p} \\ y_{p}\end{array}\right) w+\left(\begin{array}{l}x_{q} \\ y_{q}\end{array}\right) u+\left(\begin{array}{l}x_{r} \\ y_{r}\end{array}\right) v, w=1-u-v$,

We can verify that the above transformation of eqn. Above maps the arbitrary triangle $\triangle P Q R$ into the standard triangle $\triangle A B C$. The points $\mathrm{P}, \mathrm{Q}, \mathrm{R}, \mathrm{S}, \mathrm{T}, \mathrm{U}$ and $\mathrm{Z}$ are respectively mapped into the points $\mathrm{A}, \mathrm{B}, \mathrm{C}, \mathrm{D}, \mathrm{E}, \mathrm{F}$ and $\mathrm{G}$ respectively. The quadrilaterals $Q_{i}$ are mapped into quadrilaterals $\widehat{Q}_{l}$. This proves the existence of the required transformation.

5.2 Lemma 2. There exists three linear transformations to map the quadrilaterals $Q_{i}(i=1,2,3)$ of $\triangle P Q R$ into a unique triangle $\hat{Q}=Q_{i}$ (say) of the standard triangle $\triangle A B C$ which satisfy the following conditions

(i) $\sum_{i=1}^{3} Q_{i}=\triangle P Q R$, the arbitrary triangle in the $(\mathrm{x}, \mathrm{y})$ space.

(ii) $\sum_{i=1}^{3} \widehat{Q_{l}}=\triangle A B C$, the standard triangle (right isosceles ) in the $(u, v)$ space

Proof: We again refer to Fig $1 \mathrm{a}, 1 \mathrm{~b}$ and consider the following linear transformations between $(\mathrm{x}, \mathrm{y})$ and $(u, v)$ spaces.

$\left(\begin{array}{l}x^{(1)} \\ y^{(1)}\end{array}\right)=\left(\begin{array}{l}x_{p} \\ y_{p}\end{array}\right) w+\left(\begin{array}{l}x_{q} \\ y_{q}\end{array}\right) u+\left(\begin{array}{l}x_{r} \\ y_{r}\end{array}\right) v, w=1-u-v$

$\left(\begin{array}{l}x^{(2)} \\ y^{(2)}\end{array}\right)=\left(\begin{array}{l}x_{q} \\ y_{q}\end{array}\right) w+\left(\begin{array}{l}x_{r} \\ y_{r}\end{array}\right) u+\left(\begin{array}{l}x_{p} \\ y_{p}\end{array}\right) v, w=1-u-v$

$\left(\begin{array}{l}x^{(3)} \\ y^{(3)}\end{array}\right)=\left(\begin{array}{l}x_{r} \\ y_{r}\end{array}\right) w+\left(\begin{array}{l}x_{p} \\ y_{p}\end{array}\right) u+\left(\begin{array}{l}x_{q} \\ y_{q}\end{array}\right) v, w=1-u-v$

It is quite clear that each of the above transformations map the arbitrary triangle $\triangle P Q R$ into $\triangle A B C$. We may further note the following 
(i) The transformation of eqn.(2) maps the vertices $\mathrm{P}, \mathrm{Q}, \mathrm{R}$ in $(\mathrm{x}, \mathrm{y})$ space into vertices $\mathrm{C}(0,0), \mathrm{A}(1,0), \mathrm{B}(0,1)$ in $(\mathrm{u}, \mathrm{v})$ space

(ii) The transformation of eqn.(3) maps the vertices $\mathrm{Q}, \mathrm{R}, \mathrm{P}$ in $(\mathrm{x}, \mathrm{y})$ space into vertices $\mathrm{C}(0,0), \mathrm{A}(1,0), \mathrm{B}(0,1)$ in $(\mathrm{u}, \mathrm{v})$ space

(iii) The transformation of eqn.(4) maps the vertices $\mathrm{R}, \mathrm{P}, \mathrm{Q}$ in $(\mathrm{x}, \mathrm{y})$ space into vertices $\mathrm{C}(0,0), \mathrm{A}(1,0), \mathrm{B}(0,1)$ in $(\mathrm{u}, \mathrm{v})$ space

We can now verify that the transformation of eqn.(2) maps the quadrilateral $\mathrm{Q}_{1}$ spanning the vertices $<\mathrm{Z}, \mathrm{U}, \mathrm{P}, \mathrm{S}>$ in $(\mathrm{x}, \mathrm{y})$ space into the quadrilateral $\hat{Q}=Q_{1}$ spanning the vertices $\langle\mathrm{G}, \mathrm{E}, \mathrm{C}, \mathrm{F}>$ in the $(\mathrm{u}, \mathrm{v})$ space. In a similar manner, we find that using the transformation of eqn.(3), the quadrilateral $Q_{2}$ spanned by vertices $<\mathrm{Z}, \mathrm{S}, \mathrm{Q}, \mathrm{T}>$ in (x, y) space is mapped into the quadrilateral $\widehat{Q}=\widehat{Q_{1}}$ spanned by the vertices $<\mathrm{G}, \mathrm{E}, \mathrm{C}, \mathrm{F}>$ in the (u, v) space. Finally on using the linear transformation of eqn.(4), the quadrilateral $Q_{3}$ spanned by vertices $<\mathrm{Z}, \mathrm{T}, \mathrm{R}, \mathrm{U}>$ in $(\mathrm{x}, \mathrm{y})$ space is mapped into the quadrilateral $\hat{Q}=Q_{1}$ spanning the vertices $<$ $\mathrm{G}, \mathrm{E}, \mathrm{C}, \mathrm{F}>$ in the (u, v) space. This completes the proof of Lemma 2.

We may note here that the linear transformations $\left(x^{(1)}, y^{(1)}\right)^{T}$ in eqn.(23) and $(x, y)^{T}$ in eqn.(22) are identical. We wish to say in advance that the application of the above lemmas will be of immense help in the development of this paper.

5.3 Lemma 3. Let $\triangle \mathrm{ABC}$ be an arbitrary triangle with the vertices $\mathrm{A}(1,0), \mathrm{B}(0,1)$ and $\mathrm{C}(0,0)$ and 1 et $\mathrm{D}(1 / 2,1 / 2), \mathrm{E}(0,1 / 2)$ and $\mathrm{F}(1 / 2,0)$ be midpoints of sides $\mathrm{AB}, \mathrm{BC}$ and $\mathrm{CA}$ respectively and also let $\mathrm{G}(1 / 3,1 / 3)$ be its centroid. Then the Jacobian of the three special quadrilaterals $\widehat{Q_{e}}(e=1,2,3)$ viz. $\langle\mathrm{G}, \mathrm{E}, \mathrm{C}, \mathrm{F}\rangle,\langle\mathrm{G}, \mathrm{F}, \mathrm{A}, \mathrm{D}\rangle$ and $\langle\mathrm{G}, \mathrm{D}, \mathrm{B}, \mathrm{E}\rangle$ have the same expression given by

$\widehat{J}=\frac{\partial(\mathrm{u}, \mathrm{v})}{\partial(\xi, \eta)}=\widehat{J}^{\mathrm{e}}=\frac{1}{96}(4+\xi+\eta), \quad(e=1,2,3)$

Proof: We can immediately verify that eqn.(10a) is true by substituting the nodal values of $\widehat{Q_{e}}$ in eqn.(9a-b). The general result for a special quadrilateral $Q_{e}(e=1,2,3)$ follows by direct substitution of geometric coordinates of the vertices in eqns.(9a - b) or by chain rule of partial differentiation and use of eqn.(1):

$J=J^{e}=\frac{\partial(\mathrm{x}, \mathrm{y})}{\partial(\xi, \eta)}=\frac{\partial(\mathrm{x}, \mathrm{y})}{\partial(\mathrm{u}, \mathrm{v})} \frac{\partial(\mathrm{u}, \mathrm{v})}{\partial(\xi, \eta)}=\left(2 \Delta_{p q r}\right) \frac{1}{96}(4+\xi+\eta)=\frac{\Delta_{p q r}}{48}(4+\xi+\eta)$

We have shown in the foregoing Lemma that an arbitrary linear triangle can be discretised into three linear convex quadrilaterals. Further, each of these quadrilaterals in xy plane can be mapped into a unique linear convex quadrilateral spanned by the vertices $\mathrm{G}(1 / 3,1 / 3), \mathrm{E}(0,1 / 2), \mathrm{C}(0,0), \mathrm{F}(1 / 2,0)$, using a proper linear transformation as given Eqn.(23) - (25).

We note that the quadrilaterals can be converted into 12-node cubic Serendipity and 16-node cubic Lagrange element by placing additional nodes on the boundary and interior of the quadrilaterals $Q_{i}(i=1,2,3)$ in $(\mathrm{x}, \mathrm{y})$ space,$\widehat{Q}_{\imath}(i=1.2 .3)$ in $(\mathrm{u}, \mathrm{v})$ space and the 2 -square in $(\xi, \eta)$ space

\section{Integration over a Triangular Region :}

\subsection{Composite Integration}

We shall now establish a composite integration formula for an arbitrary triangular region $\Delta$ PQR shown in Fig 2a or Fig $3 a$. Let $\phi(\mathrm{x}, \mathrm{y})$ be an arbitrary and smooth function defined over the region $\Delta \mathrm{PQR}$. We now consider

$\mathrm{II}_{\Delta \mathrm{PQR}}=\iint_{\Delta \mathrm{PQR}} \phi(\mathrm{x}, \mathrm{y}) \mathrm{dxdy}=\sum_{\mathrm{e}=1}^{3} \iint_{\mathrm{Q}_{\mathrm{e}}} \phi(\mathrm{x}, \mathrm{y}) \mathrm{dxdy}$

$=\iint_{\widehat{Q}} \sum_{\mathrm{e}=1}^{3}\left[\phi\left(\mathrm{x}^{(\mathrm{e})}(\mathrm{u}, \mathrm{v}), \mathrm{y}^{(\mathrm{e})}(\mathrm{u}, \mathrm{v})\right) \frac{\partial\left(\mathrm{x}^{(\mathrm{e})}(\mathrm{u}, \mathrm{v}), \mathrm{y}^{(\mathrm{e})}(\mathrm{u}, \mathrm{v})\right)}{\partial(\mathrm{u}, \mathrm{v})}\right] \mathrm{dudv}$

$=\left(2 \Delta_{\mathrm{pqr}}\right) \iint_{\widehat{\mathrm{Q}}}\left\{\sum_{\mathrm{e}=1}^{3}\left[\phi\left(\mathrm{x}^{(\mathrm{e})}(\mathrm{u}, \mathrm{v}), \mathrm{y}^{(\mathrm{e})}(\mathrm{u}, \mathrm{v})\right)\right]\right\} \mathrm{dudv}$ 
Where $\left.\left(x^{(e)}(u, v), y^{(e)}(u, v)\right), e=1,2,3\right)$ are the linear transformations of Eqs.(23)-(25) and $\widehat{Q}$ is the linear convex 4-node quadrilateral GECF spanning the vertices $\mathrm{G}(1 / 3,1 / 3), \mathrm{E}(0,1 / 2), \mathrm{C}(0,0), \mathrm{F}(1 / 2,0)$ and $\Delta_{\mathrm{pqr}}$ is the area of triangle $\Delta \mathrm{PQR}$, Now, we further use the bilinear transformation of Eqns.(1)-(2) in Eqn.(15) and obtain.

$$
\mathrm{II}_{\Delta \mathrm{PQR}}=\left(2 \Delta_{\mathrm{pqr}}\right) \int_{-1}^{1} \int_{-1}^{1}\left\{\sum_{\mathrm{e}=1}^{3}\left[\phi\left(\mathrm{x}^{(\mathrm{e})}(\mathrm{u}, \mathrm{v}), \mathrm{y}^{(\mathrm{e})}(\mathrm{u}, \mathrm{v})\right) \frac{\partial(\mathrm{u}, \mathrm{v})}{\partial(\xi, \eta)}\right\} \mathrm{d} \xi \mathrm{d} \eta\right.
$$

In Eq.(16) we have used the bilinear transformation given in Eqns.(13)- (14)

$$
\begin{aligned}
& u=u(\xi, \eta)=\frac{1}{3} M_{1}(\xi, \eta)+\frac{1}{2} M_{4}(\xi, \eta) \\
& v=v(\xi, \eta)=\frac{1}{3} M_{1}(\xi, \eta)+\frac{1}{2} M_{2}(\xi, \eta)
\end{aligned}
$$

to map the arbitrary linear convex 4 noded quadrilateral into a 2 - square in integral of Eqn.(28) simplifies to the following.

$\mathrm{II}_{\Delta \mathrm{PQR}}=\left(2 \Delta_{\mathrm{pqr}}\right) \int_{-1}^{1} \int_{-1}^{1}\left[\sum_{\mathrm{e}=1}^{3}\left(\frac{4+\xi+\eta}{96}\right) \phi\left(\mathrm{x}^{(\mathrm{e})}(\mathrm{u}, \mathrm{v}), \mathrm{y}^{(\mathrm{e})}(\mathrm{u}, \mathrm{v})\right)\right] \mathrm{d} \xi \mathrm{d} \eta$

We can evaluate Eqn.(30) either analytically or numerically depending on the form of the integrand.

Using Numerical Integration, we have from Eqn.(30)

$\mathrm{II}_{\Delta \mathrm{PQR}}=2 \Delta_{\mathrm{pqr}} \sum_{\mathrm{i}=1}^{\mathrm{N}} \sum_{\mathrm{j}=1}^{\mathrm{N}}\left(\frac{\mathrm{w}_{\mathrm{i}}^{(\mathrm{N})} \mathrm{w}_{\mathrm{j}}^{(\mathrm{N})}\left(4+\xi_{\mathrm{i}}^{(\mathrm{N})}+\eta_{\mathrm{j}}^{(\mathrm{N})}\right)}{96}\right) \sum_{\mathrm{e}=1}^{3} \phi\left(\mathrm{x}^{(\mathrm{e})}\left(\mathrm{u}_{\mathrm{i}, \mathrm{j}}^{(\mathrm{N})}, \mathrm{v}_{\mathrm{i}, \mathrm{j}}^{(\mathrm{N})}\right), \mathrm{y}^{(\mathrm{e})}\left(\mathrm{u}_{\mathrm{i}, \mathrm{j}}^{(\mathrm{N})}, \mathrm{v}_{\mathrm{i}, \mathrm{j}}^{(\mathrm{N})}\right)\right)$

Where from Eqn.(29), we write

$u_{i, j}^{(N)}=u\left(\xi_{i}^{(N)}, \eta_{j}^{(N)}\right)$

$\mathrm{v}_{\mathrm{i}, \mathrm{j}}^{(\mathrm{N})}=\mathrm{v}\left(\xi_{\mathrm{i}}^{(\mathrm{N})}, \eta_{\mathrm{j}}^{(\mathrm{N})}\right)$

and $\left(\mathrm{W}_{\mathrm{i}}^{(\mathrm{N})}, \xi_{\mathrm{i}}^{(\mathrm{N})}\right),\left(\mathrm{W}_{\mathrm{j}}^{(\mathrm{N})}, \xi_{\mathrm{j}}^{(\mathrm{N})}\right)$ are the weight coefficients and sampling points along $\xi, \eta$ directions of the $\mathrm{N}^{\text {th }}$ order Gauss Legendre quadrature rules. We could also use Gauss Labatto quadrature rules as well to evaluate the integral of Eqn.(18).

The above composite rule is applied to numerical Integration over polygonal domains using convex quadrangulation and Gauss Legendre Quadrature Rules[27].

In the next section 6.2, we shall apply the above derivations and compute the integral of eqn.(26) by assuming the integrand $\phi(x$, $\mathrm{y})$ as the product of global derivatives, which are not explicit function of global variates (x, y)

6.2 Global Derivative Integrals :

If $\mathrm{N}_{\mathrm{i}}^{(\mathrm{e})}(\mathrm{i}=1(1) 9)$ denotes the basis functions for node $\mathrm{i}$ of a linear convex 9- node linear convex quadrilateral element $\mathrm{e}$, then by use of chain rule of partial differentiation

$\left(\begin{array}{l}\frac{\partial N_{i}^{\mathrm{e}}}{\partial \mathrm{x}} \\ \frac{\partial \mathrm{N}_{\mathrm{i}}^{\mathrm{e}}}{\partial \mathrm{y}}\end{array}\right)=\left[\begin{array}{ll}\frac{\partial \mathrm{u}}{\partial \mathrm{x}} & \frac{\partial \mathrm{v}}{\partial \mathrm{x}} \\ \frac{\partial \mathrm{u}}{\partial \mathrm{y}} & \frac{\partial \mathrm{u}}{\partial \mathrm{y}}\end{array}\right]\left[\begin{array}{c}\frac{\partial \mathrm{N}_{\mathrm{i}}^{\mathrm{e}}}{\partial \mathrm{u}} \\ \frac{\partial \mathrm{N}_{\mathrm{i}}^{\mathrm{e}}}{\partial \mathrm{v}}\end{array}\right]$

We note that to transform 8- node linear convex quadrilateral $\mathrm{Q}_{\mathrm{e}}(\mathrm{e}=1,2,3)$ of $\Delta \mathrm{PQR}$ in Cartesian space $(\mathrm{x}, \mathrm{y})$ into $\widehat{\mathrm{Q}}$, the 8- node linear convex quadrilateral spanned by vertices $(1 / 3,1 / 3),(1 / 6,5 / 12),(0,1 / 2),(0,1 / 4),(0,0),(1 / 4,0),(1 / 2,0)$ and $(5 / 12,1 / 6)$ in uvplane.

We must now use the earlier transformations.

$\left(\begin{array}{l}x^{1} \\ y^{1}\end{array}\right)=\left(\begin{array}{l}x_{p} \\ y_{p}\end{array}\right)+\left(\begin{array}{l}x_{q}-x_{p} \\ y_{q}-y_{p}\end{array}\right) u+\left(\begin{array}{l}x_{r}-x_{p} \\ y_{r}-y_{p}\end{array}\right) v$ for $Q_{1}$ in $\Delta P Q R$ 
$\left(\begin{array}{l}x^{2} \\ y^{2}\end{array}\right)=\left(\begin{array}{l}x_{q} \\ y_{q}\end{array}\right)+\left(\begin{array}{l}x_{r}-x_{q} \\ y_{r}-y_{q}\end{array}\right) u+\left(\begin{array}{c}x_{p}-x_{q} \\ y_{p}-y_{q}\end{array}\right) v$ for $Q_{2}$ in $\Delta P Q R$

$\left(\begin{array}{l}x^{3} \\ y^{3}\end{array}\right)=\left(\begin{array}{l}x_{r} \\ y_{r}\end{array}\right)+\left(\begin{array}{l}x_{p}-x_{r} \\ y_{p}-y_{r}\end{array}\right) u+\left(\begin{array}{l}x_{q}-x_{r} \\ y_{q}-y_{r}\end{array}\right) v$ for $Q_{3}$ in $\Delta P Q R$

And we note that the above transformations viz Eqns.(23)-(25) are of the form

$\left(\begin{array}{l}x \\ y\end{array}\right)=\left(\begin{array}{l}x_{c} \\ y_{c}\end{array}\right)+\left(\begin{array}{l}x_{a}-x_{c} \\ y_{a}-y_{c}\end{array}\right) u+\left(\begin{array}{l}x_{b}-x_{c} \\ y_{b}-y_{c}\end{array}\right) v$

which can map an arbitrary triangle $\triangle \mathrm{ABC}, \mathrm{A}\left(\mathrm{x}_{\mathrm{a}}, \mathrm{y}_{\mathrm{a}}\right), \mathrm{B}\left(\mathrm{x}_{\mathrm{b}}, \mathrm{y}_{\mathrm{b}}\right), \mathrm{C}\left(\mathrm{x}_{\mathrm{c}}, \mathrm{y}_{\mathrm{c}}\right)$ in $\mathrm{xy}-$ plane into a right isosceles triangle in the uv plane

Hence, we have from Eqn.(34)

$$
\left(\begin{array}{l}
u \\
v
\end{array}\right)=\left(\begin{array}{ll}
\left(x_{a}-x_{c}\right) & \left(x_{b}-x_{c}\right) \\
\left(y_{a}-y_{c}\right) & \left(y_{b}-y_{c}\right)
\end{array}\right)^{-1}\left(\begin{array}{l}
x-x_{c} \\
y-y_{c}
\end{array}\right)
$$

This gives

$\mathrm{u}=\left(\propto_{\mathrm{a}}+\beta_{\mathrm{a}} \mathrm{x}+\gamma_{\mathrm{a}} \mathrm{y}\right) /\left(2 \Delta_{\mathrm{abc}}\right)$

$\mathrm{v}=\left(\propto_{\mathrm{b}}+\beta_{\mathrm{b}} \mathrm{x}+\gamma_{\mathrm{b}} \mathrm{y}\right) /\left(2 \Delta_{\mathrm{abc}}\right)$

where

$\propto_{\mathrm{a}}=\left(\mathrm{x}_{\mathrm{b}} \mathrm{y}_{\mathrm{c}}-\mathrm{x}_{\mathrm{c}} \mathrm{y}_{\mathrm{b}}\right), \quad \propto_{\mathrm{b}}=\left(\mathrm{x}_{\mathrm{c}} \mathrm{y}_{\mathrm{a}}-\mathrm{x}_{\mathrm{a}} \mathrm{y}_{\mathrm{c}}\right)$,

$\beta_{\mathrm{a}}=\left(\mathrm{y}_{\mathrm{b}}-\mathrm{y}_{\mathrm{c}}\right), \quad \beta_{\mathrm{b}}=\left(\mathrm{y}_{\mathrm{c}}-\mathrm{y}_{\mathrm{a}}\right)$,

$\gamma_{\mathrm{a}}=\left(\mathrm{x}_{\mathrm{c}}-\mathrm{x}_{\mathrm{b}}\right), \quad \gamma_{\mathrm{b}}=\left(\mathrm{x}_{\mathrm{a}}-\mathrm{x}_{\mathrm{c}}\right)$,

and

$\frac{\partial(\mathrm{x}, \mathrm{y})}{\partial(\mathrm{u}, \mathrm{v})}=2 \Delta_{\mathrm{abc}}=\left|\begin{array}{lll}1 & \mathrm{x}_{\mathrm{a}} & \mathrm{y}_{\mathrm{a}} \\ 1 & \mathrm{x}_{\mathrm{b}} & \mathrm{y}_{\mathrm{b}} \\ 1 & \mathrm{x}_{\mathrm{c}} & \mathrm{y}_{\mathrm{c}}\end{array}\right|=2 *$ area of the triangle $\Delta \mathrm{ABC}$

$$
=\left(\gamma_{\mathrm{b}} \beta_{\mathrm{a}}-\gamma_{\mathrm{a}} \beta_{\mathrm{b}}\right)
$$

From Eqn.(33) and Eqn.(36), we obtain

$\left(\begin{array}{c}\frac{\partial \mathrm{N}_{\mathrm{i}}^{\mathrm{e}}}{\partial \mathrm{x}} \\ \frac{\partial \mathrm{N}_{\mathrm{i}}^{\mathrm{e}}}{\partial \mathrm{y}}\end{array}\right)=\left(\begin{array}{ll}\beta_{\mathrm{a}}^{*} & \beta_{\mathrm{b}}^{*} \\ \gamma_{\mathrm{a}}^{*} & \gamma_{\mathrm{b}}^{*}\end{array}\right)\left(\begin{array}{c}\frac{\partial \mathrm{N}_{\mathrm{i}}^{\mathrm{e}}}{\partial \mathrm{u}} \\ \frac{\partial \mathrm{N}_{\mathrm{i}}^{\mathrm{e}}}{\partial \mathrm{v}}\end{array}\right)$

where $\quad \beta_{\mathrm{a}}{ }^{*}=\frac{\beta_{\mathrm{a}}}{\left(2 \Delta_{\mathrm{abc}}\right)} \quad, \quad \beta_{\mathrm{b}}{ }^{*}=\frac{\beta_{\mathrm{b}}}{\left(2 \Delta_{\mathrm{abc}}\right)}$

$$
\gamma_{\mathrm{a}}^{*}=\frac{\gamma_{\mathrm{a}}}{\left(2 \Delta_{\mathrm{abc}}\right)} \quad, \quad \gamma_{\mathrm{b}}{ }^{*}=\frac{\gamma_{\mathrm{b}}}{\left(2 \Delta_{\mathrm{abc}}\right)}
$$

Letting,

$$
\mathrm{D}_{\mathrm{x}, \mathrm{y}}^{\mathrm{i}, \mathrm{e}}=\left(\begin{array}{c}
\frac{\partial \mathrm{N}_{\mathrm{i}}^{\mathrm{e}}}{\partial \mathrm{x}} \\
\frac{\partial \mathrm{N}_{\mathrm{i}}^{\mathrm{e}}}{\partial \mathrm{y}}
\end{array}\right), \mathrm{P}=\left(\begin{array}{cc}
\beta_{\mathrm{a}}{ }^{*} & \beta_{\mathrm{b}}{ }^{*} \\
\gamma_{\mathrm{a}}{ }^{*} & \gamma_{\mathrm{b}}{ }^{*}
\end{array}\right), \quad \mathrm{D}_{\mathrm{u}, \mathrm{v}}^{\mathrm{i}, \mathrm{e}}=\left(\begin{array}{c}
\frac{\partial \mathrm{N}_{\mathrm{i}}^{\mathrm{e}}}{\partial \mathrm{u}} \\
\frac{\partial \mathrm{N}_{\mathrm{i}}^{\mathrm{e}}}{\partial \mathrm{v}}
\end{array}\right)
$$

We obtain from Eqn.(38) and Eqn.(39)

$D_{x, y}^{i, e}=P D_{u, v}^{i, e}$

Hence from Eqn.(39) and Eqn.(40) 


$$
\begin{aligned}
& G_{x, y}^{i, j, e}=\left(\begin{array}{c}
\frac{\partial N_{i}^{e}}{\partial x} \\
\frac{\partial N_{i}^{e}}{\partial y}
\end{array}\right)\left(\frac{\partial N_{j}^{e}}{\partial x} \frac{\partial N_{j}^{e}}{\partial y}\right)=\left(D_{x, y}^{i, e}\right)\left(D_{x, y}^{j, e}\right)^{T} \\
& =\left(\begin{array}{cc}
\frac{\partial N_{i}^{e}}{\partial x} \frac{\partial N_{j}^{e}}{\partial x} & \frac{\partial N_{i}^{e}}{\partial x} \frac{\partial N_{j}^{e}}{\partial y} \\
\frac{\partial N_{i}^{e}}{\partial y} \frac{\partial N_{j}^{e}}{\partial x} & \frac{\partial N_{i}^{e}}{\partial y} \frac{\partial N_{j}^{e}}{\partial y}
\end{array}\right) \\
& \mathrm{G}_{\mathrm{u}, \mathrm{v}}^{\mathrm{i}, \mathrm{j}, \mathrm{e}}=\left(\begin{array}{c}
\frac{\partial \mathrm{N}_{\mathrm{i}}^{\mathrm{e}}}{\partial \mathrm{u}} \\
\frac{\partial \mathrm{N}_{\mathrm{i}}^{\mathrm{e}}}{\partial \mathrm{v}}
\end{array}\right)\left(\frac{\partial \mathrm{N}_{\mathrm{j}}^{\mathrm{e}}}{\partial \mathrm{u}} \frac{\partial \mathrm{N}_{\mathrm{j}}^{\mathrm{e}}}{\partial \mathrm{v}}\right)=\left(\mathrm{D}_{\mathrm{u}, \mathrm{v}}^{\mathrm{i}, \mathrm{e}}\right)\left(\mathrm{D}_{\mathrm{u}, \mathrm{v}}^{\mathrm{j}, \mathrm{e}}\right)^{\mathrm{T}} \\
& =\left(\begin{array}{ll}
\frac{\partial N_{i}^{e}}{\partial u} \frac{\partial N_{j}^{e}}{\partial u} & \frac{\partial N_{i}^{e}}{\partial u} \frac{\partial N_{j}^{e}}{\partial v} \\
\frac{\partial N_{i}^{e}}{\partial v} \frac{\partial N_{j}^{e}}{\partial u} & \frac{\partial N_{i}^{e}}{\partial v} \frac{\partial N_{j}^{e}}{\partial v}
\end{array}\right)
\end{aligned}
$$

We have now from Eqn.(40) and Eqn.(41a- b)

$$
\begin{aligned}
G_{\mathrm{x}, \mathrm{y}}^{\mathrm{i}, \mathrm{j} e} & =\left(P D_{\mathrm{u}, \mathrm{v}}^{\mathrm{i}, \mathrm{e}}\right)\left(D_{\mathrm{u}, \mathrm{v}}^{\mathrm{j}, \mathrm{e}} \mathrm{P}\right)^{\mathrm{T}} \\
& =P\left(D_{\mathrm{u}, \mathrm{v}}^{\mathrm{i}, \mathrm{e}}\right)\left(D_{\mathrm{u}, \mathrm{v}}^{\mathrm{j}, \mathrm{e}}\right)^{\mathrm{T}} \mathrm{P}^{\mathrm{T}} \\
& =P G_{\mathrm{u}, \mathrm{v}}^{\mathrm{i}, \mathrm{j}, \mathrm{e}} \mathrm{P}^{\mathrm{T}}
\end{aligned}
$$

We now define the submatrices of global derivative integrals in $(x, y)$ and $(\mathrm{u}, \mathrm{v})$ space associated with the nodes $\mathrm{i}$ and $\mathrm{j}(\mathrm{i}, \mathrm{j}=$ $1,2,3,4,5,6,7,8,9)$ as

$S^{i, j, e}=\iint_{Q_{e}} G_{x, y}^{i, j, e} d x d y$,

$$
\mathrm{K}^{\mathrm{i}, \mathrm{j}, \mathrm{e}}=\iint_{\widehat{\mathrm{Q}}} \mathrm{G}_{\mathrm{u}, \mathrm{v}}^{\mathrm{i}, \mathrm{j}, \mathrm{e}} \mathrm{du} \mathrm{dv}
$$

where, we have already defined the 8- node linear convex quadrilaterals $Q_{e}(e=1,2,3)$ in $(x, y)$ space and $\widehat{Q}$ in $(u, v)$ space in Fig 3a- 3b. From Eqns.(41)-(43), we obtain the following relations connecting the submatrices $\mathrm{S}^{\mathrm{i}, \mathrm{j}, \mathrm{e}}$ and $\mathrm{K}^{\mathrm{i}, \mathrm{j}, \mathrm{e}}$

We now obtain the submatrices $S^{i, j, e}$ and $K^{i, j, e}$ in an explicit form from Eqs.(41a)- (41b)

$$
\begin{aligned}
& S^{i, j, e}=\iint_{Q_{e}} G_{x, y}^{i, j, e} d x d y=\left(\begin{array}{cc}
\iint_{Q_{e}} \frac{\partial N_{i}^{e}}{\partial x} \frac{\partial N_{j}^{e}}{\partial x} d x d y & \iint_{Q_{e}} \frac{\partial N_{i}^{e}}{\partial x} \frac{\partial N_{j}^{e}}{\partial y} d x d y \\
\iint_{Q_{e}} \frac{\partial N_{i}^{e}}{\partial y} \frac{\partial N_{j}^{e}}{\partial x} d x d y & \iint_{Q_{e}} \frac{\partial N_{i}^{e}}{\partial y} \frac{\partial N_{j}^{e}}{\partial y} d x d y
\end{array}\right) \\
& =\left(\begin{array}{cc}
\mathrm{S}_{2 \mathrm{i}-1,2 \mathrm{j}-1}^{\mathrm{e}} & \mathrm{S}_{2 \mathrm{i}-1,2 \mathrm{j}}^{\mathrm{e}} \\
\mathrm{S}_{2 \mathrm{i}, 2 \mathrm{j}-1}^{\mathrm{e}} & \mathrm{S}_{2 \mathrm{i}, 2 \mathrm{j}}^{\mathrm{e}}
\end{array}\right) \quad \text { (say) }
\end{aligned}
$$

and in similar manner

$$
\begin{aligned}
& K^{i, j, e}=\iint_{\widehat{Q}} G_{u, v}^{i, j, e} d u d v=\left(\begin{array}{cc}
\iint_{\widehat{Q}} \frac{\partial N_{i}^{e}}{\partial u} \frac{\partial N_{j}^{e}}{\partial u} d u d v & \iint_{\widehat{Q}} \frac{\partial N_{i}^{e}}{\partial u} \frac{\partial N_{j}^{e}}{\partial v} d u d v \\
\iint_{\widehat{Q}} \frac{\partial N_{i}^{e}}{\partial v} \frac{\partial N_{j}^{e}}{\partial u} d u d v & \iint_{\widehat{Q}} \frac{\partial N_{i}^{e}}{\partial v} \frac{\partial N_{j}^{e}}{\partial v} d u d v
\end{array}\right) \\
& =\left(\begin{array}{cc}
\mathrm{K}_{2 \mathrm{i}-1,2 \mathrm{j}-1}^{\mathrm{e}} & \mathrm{K}_{2 \mathrm{i}-1,2 \mathrm{j}}^{\mathrm{e}} \\
\mathrm{K}_{2 \mathrm{i}, 2 \mathrm{j}-1}^{\mathrm{e}} & \mathrm{K}_{2 \mathrm{i}, 2 \mathrm{j}}^{\mathrm{e}}
\end{array}\right) \quad \text { (say) }
\end{aligned}
$$

We have now from the above Eqns.(41)-(45)

$S^{i, j, e}=\iint_{Q_{e}} G_{x, y}^{i, j, e} d x d y=\iint_{\widehat{Q}}\left(P G_{u, v}^{i, j, e} P^{T}\right) \frac{\partial(x, y)}{\partial(u, v)} d u d v$ 


$$
\begin{gathered}
=2 \Delta_{\mathrm{abc}} \iint_{\widehat{Q}}\left(P \mathrm{G}_{\mathrm{u}, \mathrm{v}}^{\mathrm{i}, \mathrm{j}, \mathrm{e}} \mathrm{P}^{\mathrm{T}}\right) d u d v \\
=2 \Delta_{\mathrm{abc}} \mathrm{P}\left(\iint_{\widehat{Q}} \mathrm{G}_{\mathrm{u}, \mathrm{v}}^{\mathrm{i}, \mathrm{j}, \mathrm{e}} \mathrm{du} d v\right) \mathrm{P}^{\mathrm{T}} \\
=2 \Delta_{\mathrm{abc}} \mathrm{P}\left(\mathrm{K}^{\mathrm{i} \mathrm{j}, \mathrm{e}}\right) \mathrm{P}^{\mathrm{T}} \quad,(\mathrm{i}, \mathrm{j}=1,2,3,4,5,6,7,8,9)
\end{gathered}
$$

We can thus obtain the global derivative integrals in the physical space or Cartesian space $(\mathrm{x}, \mathrm{y})$ by using the matrix triple product established in Eqn.(46).

We note that $\widehat{Q}$ is the 8 - node linear convex quadrilateral in $(u, v)$ space spanned by the vertices $(1 / 3,1 / 3),(1 / 6,5 / 12),(0,1 / 2),(0$, $1 / 4),(0,0),(1 / 4,0),(1 / 2,0),(5 / 12,1 / 6)$ and $(5 / 24,5 / 24)$ in uv- plane hence from Eqn. $(45)$

$$
\begin{aligned}
K^{\mathrm{i}, \mathrm{j}, \mathrm{e}} & =\iint_{\widehat{Q}} G_{\mathrm{u}, \mathrm{v}}^{\mathrm{i}, \mathrm{e}} \mathrm{du} d v \\
& =\int_{-1}^{1} \int_{-1}^{1} G_{\mathrm{u}, \mathrm{v}}^{\mathrm{i}, \mathrm{j}, \mathrm{e}} \frac{\partial(\mathrm{u}, \mathrm{v})}{\partial(\xi, \eta)} \mathrm{d} \xi \mathrm{d} \eta
\end{aligned}
$$

We now refer to section 6.1 of this paper, in this section, we have derived the necessary relations to integrate Eq.(47). As in Eqns.(27)-(28), we use the transformation of Eqn.(29) to map the 9- node quadrilateral $\widehat{Q}$ to the 9- node 2 -square $-1 \leq \xi, \eta \leq 1$ Using Eqn.(29) in Eqn.(48), we obtain

$\mathrm{K}^{\mathrm{i}, \mathrm{j}, \mathrm{e}}=\iint_{\widehat{Q}} G_{\mathrm{u}, \mathrm{v}}^{\mathrm{i}, \mathrm{j}, \mathrm{e}}\left(\frac{4+\xi+\eta}{96}\right) \mathrm{d} \xi \mathrm{d} \eta$

Thus, we have from Eq.(46)

$\mathrm{S}^{\mathrm{i}, \mathrm{j}, \mathrm{e}}=\left(2 \Delta_{\mathrm{abc}}\right) \mathrm{P}\left(\mathrm{K}^{\mathrm{i}, \mathrm{j}, \mathrm{e}}\right) \mathrm{P}^{\mathrm{T}}$

Where $\mathrm{K}^{\mathrm{i}, \mathrm{j}, \mathrm{e}}$ is given in Eqn.(49)

In Eqn. (50), $2 \Delta_{\mathrm{abc}}=2 *$ area of the triangle spanning vertices $\mathrm{A}\left(\mathrm{x}_{\mathrm{a}}, \mathrm{y}_{\mathrm{a}}\right), \mathrm{B}\left(\mathrm{x}_{\mathrm{b}}, \mathrm{y}_{\mathrm{b}}\right), \mathrm{C}\left(\mathrm{x}_{\mathrm{c}}, \mathrm{y}_{\mathrm{c}}\right)$ is a scalar.

The matrices $\mathrm{P}, \mathrm{P}^{\mathrm{T}}$ depend purely on the nodel coordinates $\left(\mathrm{x}_{\mathrm{a}}, \mathrm{y}_{\mathrm{a}}\right),\left(\mathrm{x}_{\mathrm{b}}, \mathrm{y}_{\mathrm{b}}\right),\left(\mathrm{x}_{\mathrm{c}}, \mathrm{y}_{\mathrm{c}}\right)$ the matrix $\mathrm{K}^{\mathrm{i}, \mathrm{j}, \mathrm{e}}$ can be explicity computed by the relations obtained in section $2-6$. We find that $\mathrm{K}^{\mathrm{i}, \mathrm{j}, \mathrm{e}}$ is a $(2 \mathrm{X} 2)$ matrix of integrals whose integrands are rational functions with polynomial numerator and the linear denominator $(4+\xi+\eta)$.

Hence these integrals can be explicity computed. The explicit values of these integrals are expressible in terms of logarithmic constants. We have used symbolic mathematics software of MATLAB to compute the explicit values and their conversion to any number of digits can be obtained by using variable precision arithmetic (vpa) command. The matrix $\mathrm{K}^{\mathrm{e}}$ as noted in Eqn.(45) is of order 8-node convex quadrilateral element.

We have computed $\mathrm{K}^{\mathrm{e}}$ for the four node element $\mathrm{n}_{\mathrm{de}}=4$ in our resent paper [18]. In the present paper, we have computed $\mathrm{K}^{\mathrm{e}}$ for the 8- node linear convex quadrilateral $\widehat{\mathrm{Q}}$ in $\mathrm{uv}$ - space. This is listed in Table 1A and Table 1B.

We may note that In order to compute the local/element stiffness matrices for the Poisson Boundary Value problem, we need to compute the integrals Eqns(12a-b)

$K_{i, j}^{e}=\int_{\Omega^{e}} \nabla \varphi_{i} \cdot \nabla \varphi_{j} d x=\int_{\Omega^{e}}\left\{\frac{\partial \varphi_{i}}{\partial x} \frac{\partial \varphi_{j}}{\partial x}+\frac{\partial \varphi_{i}}{\partial y} \frac{\partial \varphi_{j}}{\partial y}\right\} d x d y$

from the above derivations, we can rewrite $K_{i, j}^{e}$ in the notations of this sections by taking $\varphi_{i}=\mathbf{N}_{i}$ and $\varphi_{j}=N_{j}$ and $\Omega^{e}=Q_{e}$ so that

$K_{i, j}^{e}=\int_{Q_{e}} \nabla \mathrm{N}_{i} \cdot \nabla N_{j} d \mathrm{x}=\int_{Q_{e}}\left\{\frac{\partial N_{i}}{\partial x} \frac{\partial N_{j}}{\partial x}+\frac{\partial N_{i}}{\partial y} \frac{\partial N_{j}}{\partial y}\right\} d x d y=\mathrm{S}_{2 \mathrm{i}-1,2 \mathrm{j}-1}^{\mathrm{e}}+\mathrm{S}_{2 \mathrm{i}, 2 \mathrm{j}}^{\mathrm{e}}$ 
6.3 Computation of $K_{i, j}^{e}$

The explicit integration scheme explained above compute four derivative product integrals as given in eqn(44) and they are necessary to compute the stiffness matrix entries of plane stress/plane strain problems in elasticity and sevral other applications in continuum mechanics.But this computation requires matrix triple product as given in eqn (50).Since,we only need the sum of two of these integrals viz : $S_{2 i-1,2 j-1}^{e}+S_{2 i, 2 j}^{e}$. We now present an efficient method to compute this sum by using matrix product.

Let $F_{p . q}^{i, j}=\frac{\partial N_{i}}{\partial p} \frac{\partial N_{j}}{\partial q}, I_{p . q}^{i, j}=\int_{Q_{e}} F_{p . q}^{i, j} d p d q$, then we have from eqns(44-45):

$$
\begin{aligned}
& S^{i, j, e}=\iint_{Q_{e}} G_{x, y}^{i, j, e} \quad d x d y=\left(\begin{array}{cc}
\iint_{Q_{e}} \frac{\partial N_{i}^{e}}{\partial x} \frac{\partial N_{j}^{e}}{\partial x} d x d y & \iint_{Q_{e}} \frac{\partial N_{i}^{e}}{\partial x} \frac{\partial N_{j}^{e}}{\partial y} d x d y \\
\iint_{Q_{e}} \frac{\partial N_{i}^{e}}{\partial y} \frac{\partial N_{j}^{e}}{\partial x} d x d y & \iint_{Q_{e}} \frac{\partial N_{i}^{e}}{\partial y} \frac{\partial N_{j}^{e}}{\partial y} d x d y
\end{array}\right) \\
& =\left(\begin{array}{cc}
S_{2 i-1,2 j-1}^{e} & S_{2 i-1,2 j}^{e} \\
S_{2 i, 2 j-1}^{e} & S_{2 i, 2 j}^{e}
\end{array}\right) \quad(s a y) \\
& =\left(\begin{array}{ll}
I_{x, x}^{i, j} & I_{x, y}^{i, j} \\
I_{y, x}^{i, j} & I_{y, y}^{i, j}
\end{array}\right)
\end{aligned}
$$

$$
\begin{aligned}
& K^{i, j, e}=\iint_{\widehat{Q}} G_{u, v}^{i, j, e} \quad d u d v=\left(\begin{array}{cc}
\iint_{\widehat{Q}} \frac{\partial N_{i}^{e}}{\partial u} \frac{\partial N_{j}^{e}}{\partial u} d u d v & \iint_{\widehat{Q}} \frac{\partial N_{i}^{e}}{\partial u} \frac{\partial N_{j}^{e}}{\partial v} d u d v \\
\iint_{\widehat{Q}} \frac{\partial N_{i}^{e}}{\partial v} \frac{\partial N_{j}^{e}}{\partial u} d u d v & \iint_{\widehat{Q}} \frac{\partial N_{i}^{e}}{\partial v} \frac{\partial N_{j}^{e}}{\partial v} d u d v
\end{array}\right) \\
& =\left(\begin{array}{ll}
\mathbf{K}_{2 \mathbf{i}-1,2 \mathbf{j}-1}^{\mathrm{e}} & \mathbf{K}_{2 \mathbf{i}-1,2 \mathbf{j}}^{\mathrm{e}} \\
\mathbf{K}_{2 \mathbf{i}, 2 \mathbf{j}-1}^{\mathrm{e}} & \mathbf{K}_{2 \mathbf{i}, 2 \mathbf{j}}^{\mathrm{e}}
\end{array}\right) \quad \text { (say) } \\
& =\left(\begin{array}{ll}
I_{u, u}^{i, j} & I_{u, v}^{i, j} \\
I_{v, u}^{i, j} & I_{v, v}^{i, j}
\end{array}\right)
\end{aligned}
$$

Let $P=\left(\begin{array}{ll}P_{11} & P_{12} \\ P_{21} & P_{22}\end{array}\right), P^{T}=\left(\begin{array}{ll}P_{11} & P_{21} \\ P_{12} & P_{22}\end{array}\right)$

From eqns( 44$),(46)$ and (52a-b)

$$
\begin{aligned}
& S^{i, j, e}=\iint_{Q_{e}} G_{x, y}^{i, j, e} d x d y=2 \Delta_{a b c} P\left(\iint_{\widehat{Q}} G_{u, v}^{i, j, e} d u d v\right) P^{T} \\
& =2 \Delta_{\text {abc }}\left(\begin{array}{ll}
P_{11} & P_{12} \\
P_{21} & P_{22}
\end{array}\right)\left(\begin{array}{cc}
I_{u, u}^{i, j} & I_{u, v}^{i, j} \\
I_{v, u}^{i, j} & I_{v, v}^{i, j}
\end{array}\right)\left(\begin{array}{ll}
P_{11} & P_{21} \\
P_{12} & P_{22}
\end{array}\right)
\end{aligned}
$$

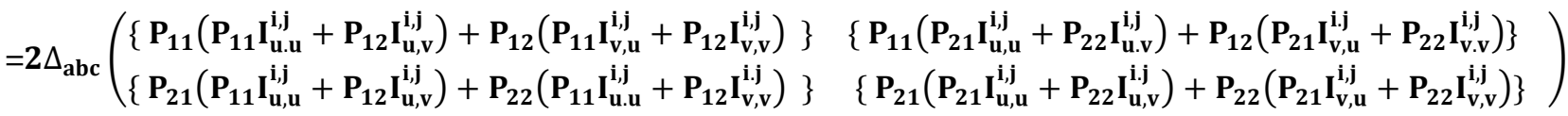

From eqn(51a-b) and eqn(46), we find

$\operatorname{trace}\left(S^{\mathrm{i}, \mathrm{j}, \mathrm{e}}\right)=\operatorname{trace}\left(\iint_{Q_{\mathrm{e}}} G_{\mathrm{x}, \mathrm{y}}^{\mathrm{i}, \mathrm{j}, \mathrm{e}} \mathrm{dxdy}\right)=\left(S_{2 \mathrm{i}-1,2 \mathrm{j}-1}^{\mathrm{e}}+S_{2 \mathrm{i}, 2 \mathrm{j}}^{\mathrm{e}}\right)=K_{i, j}^{e}=\int_{Q_{e}} \nabla \mathrm{N}_{i} \cdot \nabla N_{j} d \mathrm{x}=\int_{Q_{e}}\left\{\frac{\partial N_{i}}{\partial x} \frac{\partial N_{j}}{\partial x}+\frac{\partial N_{i}}{\partial y} \frac{\partial N_{j}}{\partial y}\right\} d x d y$

$$
=\left(P_{11}^{2}+P_{21}^{2}\right) I_{u, u}^{i, j}+\left(P_{11} P_{12}+P_{21} P_{22}\right)\left(I_{u, v}^{i, j}+I_{v, u}^{i, j}\right)+\left(P_{12}^{2}+P_{22}^{2}\right) I_{v, v}^{i, j}
$$

W e can obtain the above integraql $\int_{Q_{e}}\left\{\frac{\partial N_{i}}{\partial x} \frac{\partial N_{j}}{\partial x}+\frac{\partial N_{i}}{\partial y} \frac{\partial N_{j}}{\partial y}\right\} d x d y$ by use of matrix operations which doesnot need the computation matrix triple product.This procedure is presented below 
From eqn (44b) and eqn(45),let us do the following:

$$
\left(\begin{array}{ll}
P^{T} P
\end{array}\right) \cdot *\left(\begin{array}{ll}
I_{u, u}^{i, j} & I_{u, v}^{i, j} \\
I_{v, u}^{i, j} & I_{v, v}^{i, j}
\end{array}\right)=\left[\begin{array}{cc}
\left(P_{11}^{2}+P_{21}^{2}\right) I_{u, u}^{i, j} & \left(P_{11} P_{12}+P_{21} P_{22}\right) I_{u, v}^{i, j} \\
\left(P_{11} P_{12}+P_{22} P_{21}\right) I_{v, u}^{i, j} & \left(P_{12}^{2}+P_{22}^{2}\right) I_{v, v}^{i, j}
\end{array}\right]
$$

We observe from eqn(56) that sum of all the entries gives us the value of the integral i.e

$\int_{Q_{e}}\left\{\frac{\partial N_{i}}{\partial x} \frac{\partial N_{j}}{\partial x}+\frac{\partial N_{i}}{\partial y} \frac{\partial N_{j}}{\partial y}\right\} d x d y=\operatorname{sum}\left(\operatorname{sum}\left(\left(P^{T} P\right) \cdot *\left(\begin{array}{cc}I_{u, u}^{i, j} & I_{u, v}^{i, j} \\ I_{v, u}^{i, j} & I_{v, v}^{i, j}\end{array}\right)\right)\right)$

Where,sum is a Matlab function.We note that $S=\operatorname{sum}(X)$ gives the sum of the elements of vector $X$. If $X$ is a matrix then $S$ is a row vector with the sum over each column.It is clear that $\operatorname{sum}(\operatorname{sum}(X))$ gives the sum of all the entries in a matrix $X$

6.4 Computing of Force Vector Integrals $\int_{\Omega^{e}} f \varphi_{i} \mathrm{dxdy}$

We shall now propose numerical integration for the complicated integrands in the force vector integrals over the domain $\Omega^{e}$ which is an arbitrary linear triangle and $\phi(x, y)=f \varphi_{i}$.We also refer to the section 2 for the theory necessary to derive the composite numerical integration formla

We shall now establish a composite integration formula for an arbitrary linear triangular region $\Delta$ PQR shown in Fig $2 a$ or Fig 3a. We have for an arbitrary smooth function $\phi(x, y)$

II $_{\triangle \mathrm{PQR}}=\iint_{\Delta \mathrm{PQR}} \phi(\mathrm{x}, \mathrm{y}) \mathbf{d x d y}=\sum_{\mathrm{e}=1}^{3} \iint_{\mathrm{Q}_{\mathrm{e}}} \phi(\mathrm{x}, \mathrm{y}) \mathbf{d x d y}$

---------- (58)

$=\iint_{\widehat{Q}} \sum_{\mathbf{e}=1}^{3}\left[\phi\left(\mathbf{x}^{(\mathbf{e})}(\mathbf{u}, \mathbf{v}), \mathbf{y}^{(\mathbf{e})}(\mathbf{u}, \mathbf{v})\right) \frac{\partial\left(\mathbf{x}^{(e)}(\mathbf{u}, \mathbf{v}), \mathbf{y}^{(e)}(\mathbf{u}, \mathbf{v})\right)}{\partial(\mathbf{u}, \mathbf{v})}\right] \mathbf{d u d v}$

$=\left(2 \Delta_{\mathrm{pqr}}\right) \iint_{\widehat{Q}}\left\{\sum_{\mathrm{e}=1}^{3}\left[\phi\left(\mathbf{x}^{(e)}(\mathbf{u}, \mathbf{v}), \mathbf{y}^{(e)}(\mathbf{u}, \mathbf{v})\right)\right]\right\} \mathbf{d u d v}$

Where $\left.\left(x^{(e)}(u, v), y^{(e)}(u, v)\right), e=1,2,3\right)$ are the transformations of Eqs.(8)-(10) and $\widehat{Q}$ is the quadrilateral in uv- plane spanned by vertices $\mathrm{G}(1 / 3,1 / 3), \mathrm{E}(0,1 / 2), \mathrm{C}(0,0)$ and $\mathrm{F}(1 / 2,0)$, and $\Delta_{\mathrm{pqr}}$ is the area of triangle $\Delta$ PQR, Now using the transformations defined in Eqs.(1)-(2) we obtain

$\mathbf{I I}_{\triangle \mathrm{PQR}}=\left(2 \Delta_{\mathbf{p q r}}\right) \iint_{\widehat{Q}}\left\{\sum_{\mathbf{e}=1}^{3}\left[\boldsymbol{\phi}\left(\mathbf{x}^{(\mathbf{e})}(\mathbf{u}, \mathbf{v}), \mathbf{y}^{(\mathbf{e})}(\mathbf{u}, \mathbf{v})\right) \frac{\partial(\mathbf{u}, \mathbf{v})}{\partial(\xi, \eta)}\right\} \mathbf{d} \xi \mathbf{d} \boldsymbol{\eta}\right.$

In Eq.(14) we have used the transformation

$\mathbf{u}(\xi, \eta)=\frac{1}{3} \mathbf{N}_{1}(\xi, \eta)+\frac{1}{2} \mathbf{N}_{4}(\xi, \eta)$

$\mathbf{v}(\xi, \eta)=\frac{1}{3} \mathbf{N}_{1}(\xi, \eta)+\frac{1}{2} \mathbf{N}_{2}(\xi, \eta)$

(61)

to map the quadrilateral $\widehat{Q}$ into a 2 -square in $\xi \eta$ - plane.

We can now obtain from Eqs.(13)-(14)

II $_{\triangle \mathrm{PQR}}=\left(2 \Delta_{\mathrm{pqr}}\right) \int_{-1}^{1} \int_{-1}^{1}\left[\sum_{\mathrm{e}=1}^{3}\left(\frac{4+\xi+\eta}{96}\right) \phi\left(x^{(e)}(u, v), y^{(e)}(u, v)\right)\right] \mathbf{d} \xi \mathbf{d} \eta$

We can evaluate Eq.(16) either analytically or numerically depending on the form of the integrand.

Using Numerical Integration ;

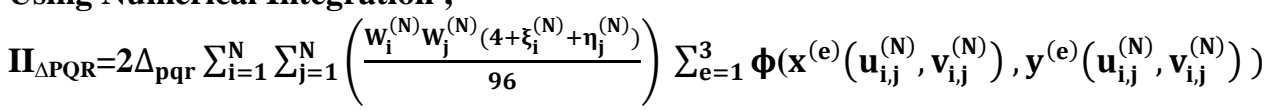

Where, 
$\mathbf{u}_{\mathbf{i}, \mathbf{j}}^{(\mathrm{N})}=\mathbf{u}\left(\boldsymbol{\xi}_{\mathbf{i}}^{(\mathrm{N})}, \boldsymbol{\eta}_{\mathbf{j}}^{(\mathrm{N})}\right)$ and $\mathbf{v}_{\mathbf{i}, \mathbf{j}}^{(\mathrm{N})}=\mathbf{v}\left(\boldsymbol{\xi}_{\mathbf{i}}^{(\mathrm{N})}, \boldsymbol{\eta}_{\mathbf{j}}^{(\mathrm{N})}\right)$

and $\left(W_{i}^{(N)}, \xi_{i}^{(N)}\right),\left(W_{j}^{(N)}, \xi_{j}^{(N)}\right)$ are the weight coefficients and sampling points of $N^{\text {th }}$ order Gauss Legendre Quadrature rules.

The above composite rule is applied to numerical Integration over polygonal domains using convex quadrangulation and Gauss Legendre Quadrature Rules[27].

The above method will help in integrating $\int_{\Omega^{e}} f \varphi_{i} \mathrm{dxdy}$, when the intgrand $f \varphi_{i}$ is complicated

\section{A NEW APPROACH TO MESH GENERATION}

The first step in implimenting finite element method isto generate a mesh.In a recent work the author and his co-workers have proposed a new approach to mesh generation which can discretise a convex polygon into an all quadrilateral mesh.This will be presented next.This new approach to mesh generation meets the necessary requirements of regularity on the shape of elements. There are two types of them which usually suffice in finite element computations.The first is called shape regularity. It says that the ratio of the diameter of the element to the radius of the inner circle must be less than some constant. For triangles,the diameter of the triangle is related to the smallest circle which contain the triangle.The inner circle refers to the largest circle which fits inside the triangle. Shape regularity focuses on the shape of individual triangles and doesnot refer to how the shapes of different elements relate to each other. So some elements can be large wshile others might be very small. There is a second type of requirement on the shape of elements. This requirement says that ratio of the maximum diameter of elements to the radius of the inner circle of an element must be less than some constant .If a mesh satisfies this requirement,it is called quasiuniform.This requirement is more important when we perform refinements. We must note that a mesh generation gives us the nodes on a particular element as well as the coordinates of the nodes. We now give an account of this novel mesh generation technique with an aim to use it further in the solution of Poisson problem. Stated in eqn(7a-b).

In our recent papers [ - ], the explicit finite element integration scheme is presented by using the isoparametric transformation over the 4 node, 8 node and 9 node linear convex quadrilateral elements which is applied to torison of a square shaft, on considering symmetry of the problem domain, mesh generation for 1/8 of the cross section which is a triangle was discritised into an all quadrilateral mesh. In this paper we consider applications to Poisson boundary values for nonconstant functions over polygonal domains

\subsection{An automatic indirect quadrilateral mesh generator}

A wide range of problems in applied science and engineering can be simulated by partial derivative equations(PDE).In the last few decade,one of the most relevant techniques to solve is the Finite Element Method(FEM).It is well known that a good quality mesh is required in order to obtain an accurate solution.Hence the construction of a mesh is on e of the most important steps.

In the next few sections, we present a novel mesh generation scheme of all quadrilateral elements for convex polygonal domains. This scheme converts the elements in background triangular mesh into quadrilaterals through the operation of splitting. We first decompose the convex polygon into simple subregions in the shape of triangles. These simple subregions are then triangulated to generate a fine mesh of triangles. We propose then an automatic triangular to quadrilateral conversion scheme in which each isolated triangle is split into three quadrilaterals according to the usual scheme, adding three vertices in the middle of edges and a vertex at the barrycentre of the triangular element. Further, to preserve the mesh conformity a similar procedure is also applied to every triangle of the domain and this fully discretizes the given convex polygonal domain into all quadrilaterals, thus propogating uniform refinement. In section 4.2, we present a scheme to discretize the arbitrary and standard triangles into a fine mesh of six node triangular elements. In section 4.3, we explain the procedure to split these triangles into quadrilaterals. In section 4.4,we have presented a method of piecing together of all triangular subregions and eventually creating a all quadrilateral mesh for the given convex polygonal domain. In section 4.5,we present several examples to illustrate the simplicity and efficiency of the proposed mesh generation method for standard and arbitrary triangles,rectangles and convex polygonal domains.

\subsection{Division of an Arbitrary Triangle}

We can map an arbitrary triangle with vertices $\left(\left(x_{i}, y_{i}\right), i=1,2,3\right)$ into a right isosceles triangle in the (u,v) space as shown in Fig. 4a, b. The necessary transformation is given by the equations.

$x=x_{1}+\left(x_{2}-x_{1}\right) u+\left(x_{3}-x_{1}\right) v$ 
$y=y_{1}+\left(y_{2}-y_{1}\right) u+\left(y_{3}-y_{1}\right) v$

The mapping of eqn.(1) describes a unique relation between the coordinate systems. This is illustrated by using the area coordinates and division of each side into three equal parts in Fig. 5a Fig. 5b. It is clear that all the coordinates of this division can be determined by knowing the coordinates $\left(\left(x_{i}, y_{i}\right), i=1,2,3\right)$ of the vertices for the arbitrary triangle. In general, it is well known that by making ' $n$ ' equal divisions on all sides and the concept of area coordinates, we can divide an arbitrary triangle into $n^{2}$ smaller triangles having the same area which equals $\Delta / n^{2}$ where $\Delta$ is the area of a linear arbitrary triangle with vertices $\left(\left(x_{i}, y_{i}\right), i=1,2,3\right)$ in the Cartesian space.

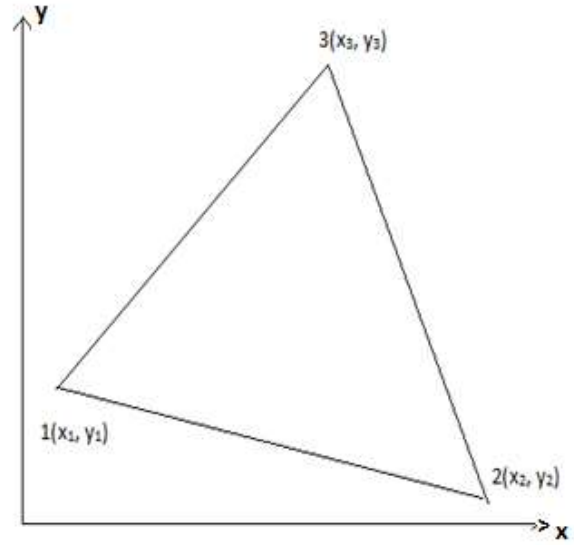

4.a

Fig. 4a An Arbitrary Linear Triangle in the (x, y) space space

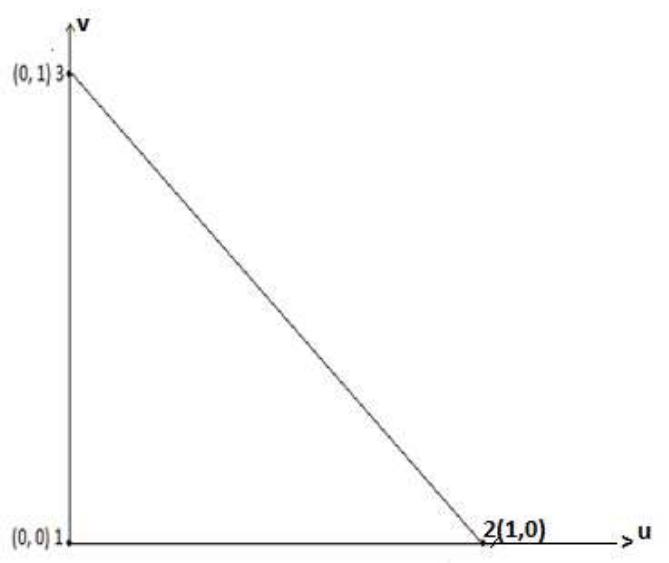

$4 \mathrm{~b}$

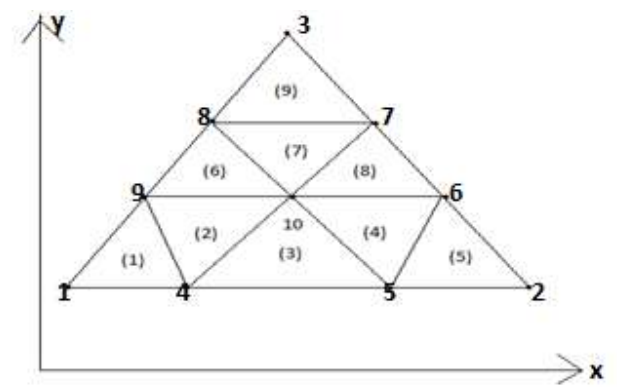

5 a

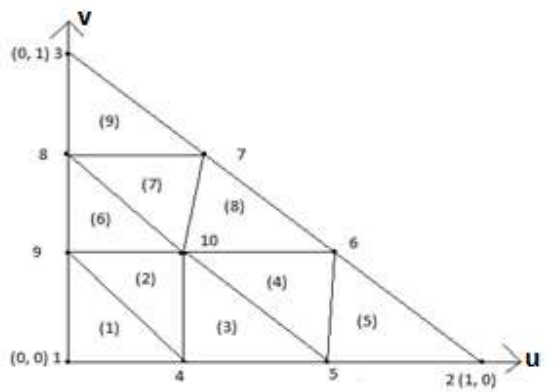

$5 \mathrm{~b}$

Fig. 5a Division of an arbitrary triangle into Nine triangles in Cartesian space

Fig. $5 \mathrm{~b}$ Division of a right isosceles triangle into Nine right isosceles triangles in ( $u, v)$ space 


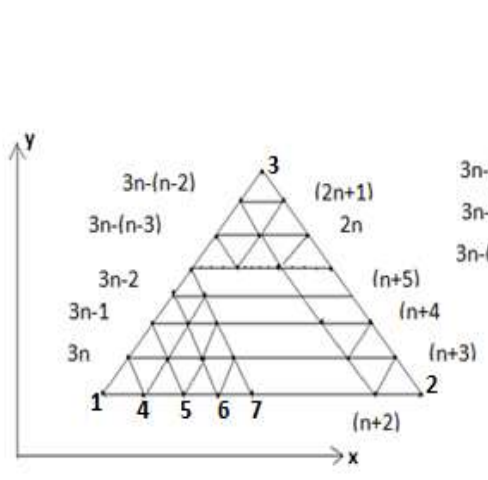

6a

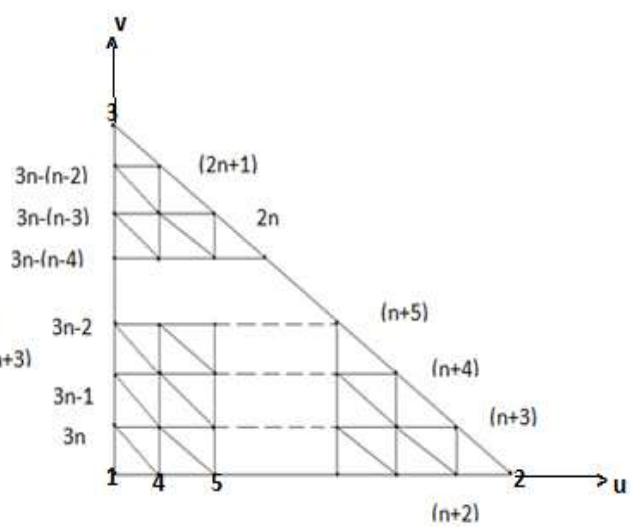

Fig.6a Division of an arbitrary triangle into $n^{2}$ triangle in Cartesian space $(\mathrm{x}, \mathrm{y})$, where each side is divided into $\mathrm{n}$ divisions of equal length

Fig. 6b Division of a right isosceles triangle into $n^{2}$ right isosceles triangle in $(\mathrm{u}, \mathrm{v})$ space, where each side is divided into $\mathrm{n}$ divisions of equal length

We have shown the division of an arbitrary triangle in Fig. 6a, Fig. 6b, We divided each side of the triangles (either in Cartesian space or natural space) into $n$ equal parts and draw lines parallel to the sides of the triangles. This creates (n+1) $(n+2)$ nodes. These nodes are numbered from triangle base line $l_{12}$ (letting $l_{i j}$ as the line joining the vertex $\left(x_{i}, y_{i}\right)$ and $\left(x_{j}, y_{j}\right)$ ) along the line $v=0$ and upwards up to the line $v=1$. The nodes $1,2,3$ are numbered anticlockwise and then nodes $4,5,-----,(n+2)$ are along line $v=0$ and the nodes $(n+3),(n+4),-----, 2 n,(2 n+1)$ are numbered along the line $l_{23}$ i.e. $u+v=1$ and then the node $(2 n+2),(2 n+3),------, 3 n$ are numbered along the line $u=0$. Then the interior nodes are numbered in increasing order from left to right along the line $v=\frac{1}{n}, \frac{2}{n},---, \frac{n-1}{n}$ bounded on the right by the line $u+v=1$. Thus the entire triangle is covered by $(n+1)(n+2) / 2$ nodes. This is shown in the $\underline{r} r$ matrix of size_(n+1) $\times$ $(n+1)$, only nonzero entries of this matrix refer to the nodes of the triangles

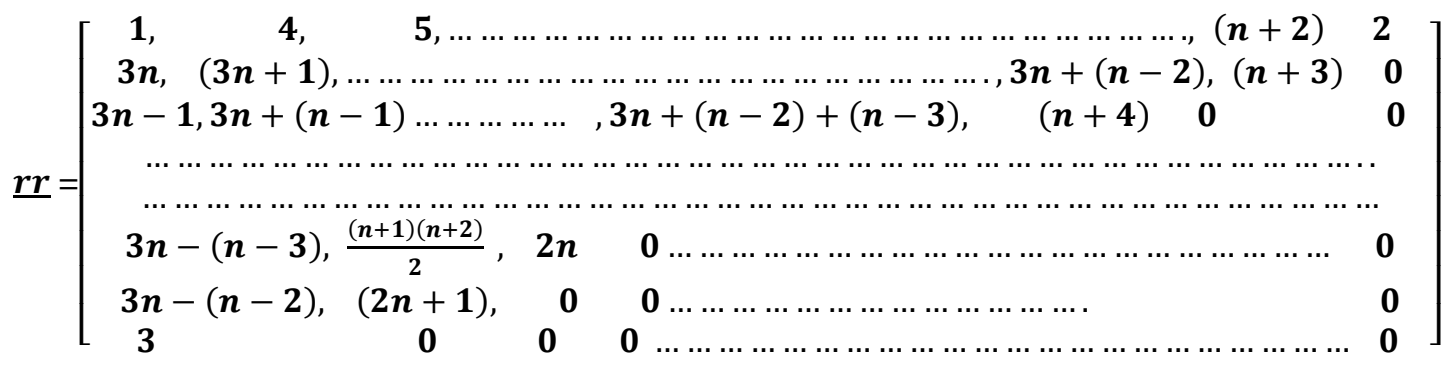

\subsection{Quadrangulation of an Arbitrary Triangle}

We now consider the quadrangulation of an arbitrary triangle. We first divide the arbitrary triangle into a number of equal size six node triangles. Let us define $l_{i j}$ as the line joining the points $\left(x_{i}, y_{i}\right)$ and $\left(x_{j}, y_{j}\right)$ in the Cartesian space $(x, y)$. Then the arbitrary triangle with vertices at $\left(\left(x_{i}, y_{i}\right), i=1,2,3\right)$ is bounded by three lines $l_{12}, l_{23}$, and $l_{31}$. By 
dividing the sides $l_{12}, l_{23}, l_{31}$ into $n=2 m$ divisions $(\mathrm{m}$, an integer $)$ creates $m^{2}$ six node triangular divisions. Then by joining the centroid of these six node triangles to the midpoints of their sides, we obtain three quadrilaterals for each of these triangle. We have illustrated this process for the two and four divisions of $l_{12}, l_{23}$, and $l_{31}$ sides of the arbitrary and standard triangles in Figs. 4 and 5

Two Divisions of Each side of an Arbitrary Triangle

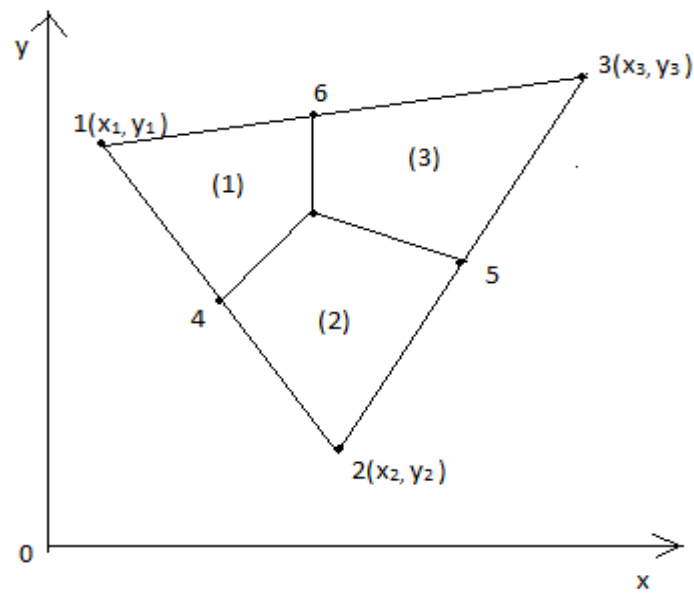

7(a)

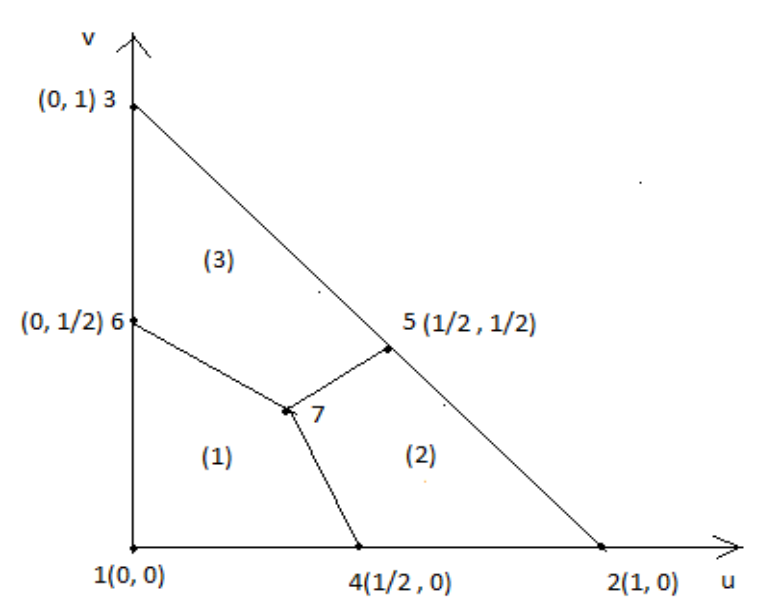

7(b)

Fig 7(a). Division of an arbitrary triangle into three quadrilaterals

Fig 7(b). Division of a standard triangle into three quadrilaterals

Four Divisions of Each side of an Arbitrary Triangle

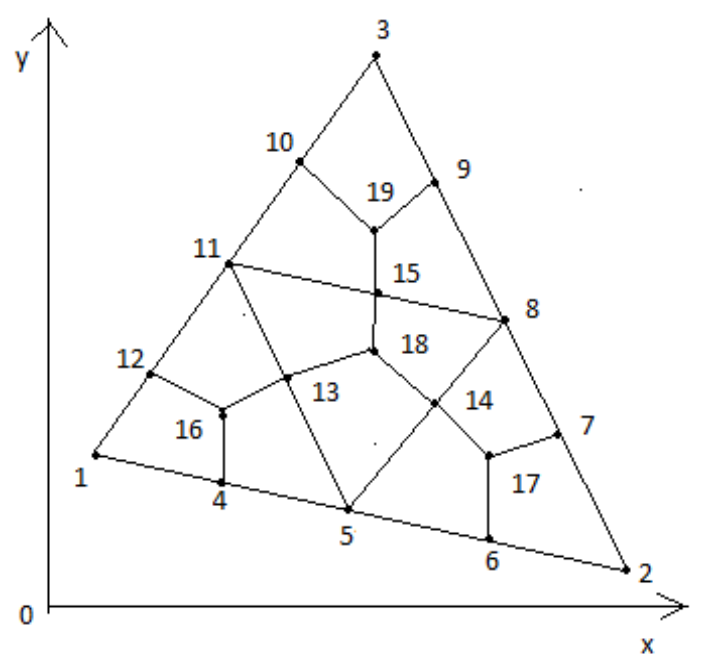

$8 \mathbf{a}$

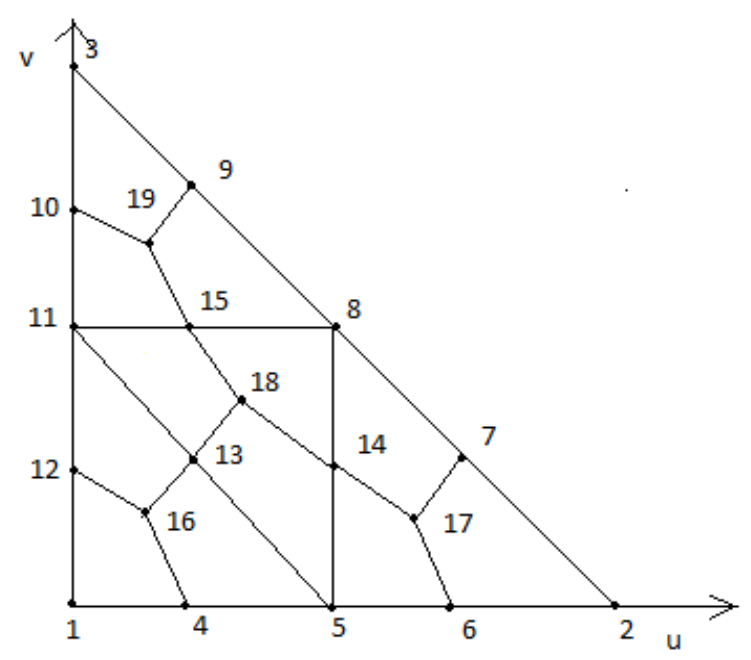

$\mathbf{8 b}$

Fig 8a. Division of an arbitrary triangle into 4 six node triangles

Fig $8 b$. Division of a standard triangle into 4 right isosceles triangle

In general, we note that to divide an arbitrary triangle into equal size six node triangle, we must divide each side of the triangle into an even number of divisions and locate points in the interior of triangle at equal spacing. We also do similar divisions and locations of interior points for the standard triangle. Thus $\mathbf{n}$ (even ) divisions creates $(\mathbf{n} / \mathbf{2})^{2}$ six node triangles in both the spaces. If the entries of the sub matrix $\underline{\operatorname{rr}}(i ; i+2, j ; j+2)$ are nonzero then two six node triangles can be 
formed. If $\underline{r r}(i+1, j+2)=\underline{r r}(i+2, j+1 ; j+2)=0$ then one six node triangle can be formed. If the sub matrices $\underline{r}(i ; i+2, j ; j+2)$ is a $(3 \times 3)$ zero matrix , we cannot form the six node triangles. We now explain the creation of the six node triangles using the $\underline{\boldsymbol{r} \boldsymbol{r}}$ matrix_of eqn.( ). We can form six node triangles by using node points of three consecutive rows and columns of $\underline{r} \boldsymbol{r}$ matrix. This procedure is depicted in Fig. 9 for three consecutive rows $i, i+1$, $i+2$ and three consecutive columns $j, j+1, j+2$ of the $\underline{r r}$ sub matrix

Formation of six node triangles using sub matrix $\underline{r} \boldsymbol{r}$

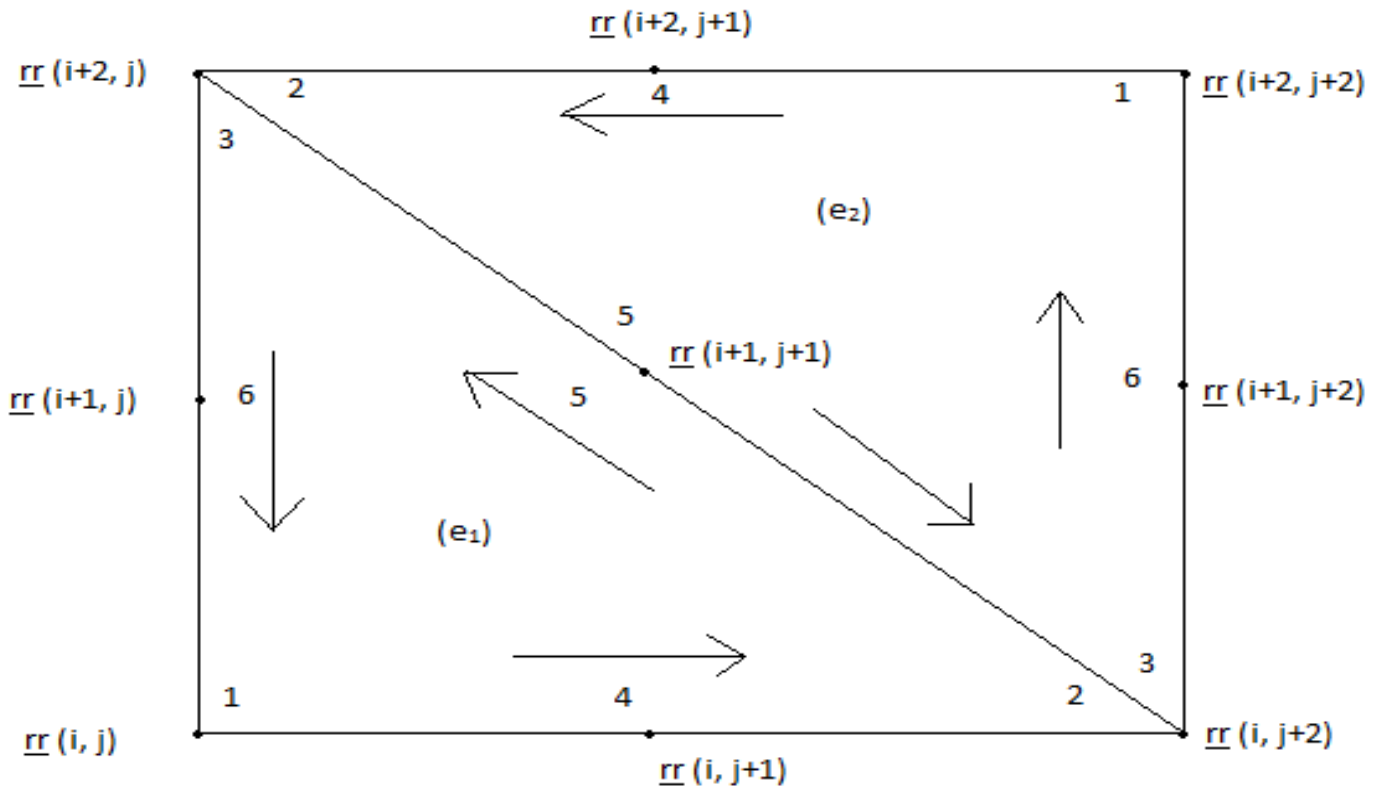

Fig. 9 Six node triangle formation for non zero sub matrix $\underline{r}$

If the sub matrix ( $(\underline{r r}(k, l), k=i, i+1, i+2), l=j, j+1, j+2)$ is nonzero, then we can construct two six node triangles. The element nodal connectivity is then given by

$\left(\mathrm{e}_{1}\right)<\underline{r r}(i, j), \underline{r r}(i, i+2), \underline{r r}(i+2, j), \underline{r r}(i, j+1), \underline{r r}(i+1, j+1), \underline{r r}(i+1, j)>$

$\left.\left(\mathrm{e}_{2}\right)<\underline{r r}(i+2, j+2), \underline{r r}(i+2, j), \underline{r r}(i, j+2), \underline{r r}(i+2, j+1), \underline{r r}(i+1, j+1), \underline{r r} i+1, j+2\right)>$

If the elements of sub matrix ( $(\underline{r} \boldsymbol{r}(k, l), k=i, i+1, i+2), l=j, j+1, j+2)$ are nonzero, then as standard earlier, we can construct two six node triangles. We can create three quadrilaterals in each of these six node triangles. The nodal connectivity for the 3 quadrilaterals created in $\left(e_{1}\right)$ are given as

$Q_{3 n_{1}-2}<c_{1}, \underline{r r}(i+1, j), \underline{r r}(i, j), \underline{r r}(i, j+1)>$

$Q_{3 n_{1}-1}<c_{1}, \underline{r r}(i, j+1), \underline{r r}(i, j+2), \underline{r r}(i+1, j+1)>$

$\begin{array}{lllllllllll}Q_{3 n_{1}} & < & c_{1} & , & \underline{r} & (i+1, j+1), & \underline{r} & (i+2, j), & \underline{r r} & (i+1, j) & >\end{array}$

and the nodal connectivity for the 3 quadrilaterals created in $\left(e_{2}\right)$ are given as

$Q_{3 n_{2}-2}<c_{2}, \underline{r r}(i+1, j+2), \underline{r r}(i+2, j+2), \underline{r r}(i+2, j+1)>$

$Q_{3 n_{2}-1}<c_{2}, \underline{r r}(i+2, j+1), \underline{r r}(i+2, j), \underline{r r}(i+1, j+1)>$

$Q_{3 n_{1}}<c_{2}, \underline{r r}(i+1, j+1), \underline{r r}(i, j+2), \underline{r r}(i+1, j+2)>$

---- (61) 
We can generate polygonal meshes by piecing together triangular with straight sides. Subsection (called LOOPs). The user specifies the shape of these LooPs by designating six coordinates of each LOOP

As an example, consider the geometry shown in Fig. 8(a). This is a a square region which is simply chosen for illustration. We divide this region into four LOOPs as shown in Fig.8(d). These LOOPs 1,2,3 and 4 are triangles each with three sides. After the LOOPs are defined, the number of elements for each LOOP is selected to produce the mesh shown in Fig. 8(c).The complete mesh is shown in Fig.8(b)

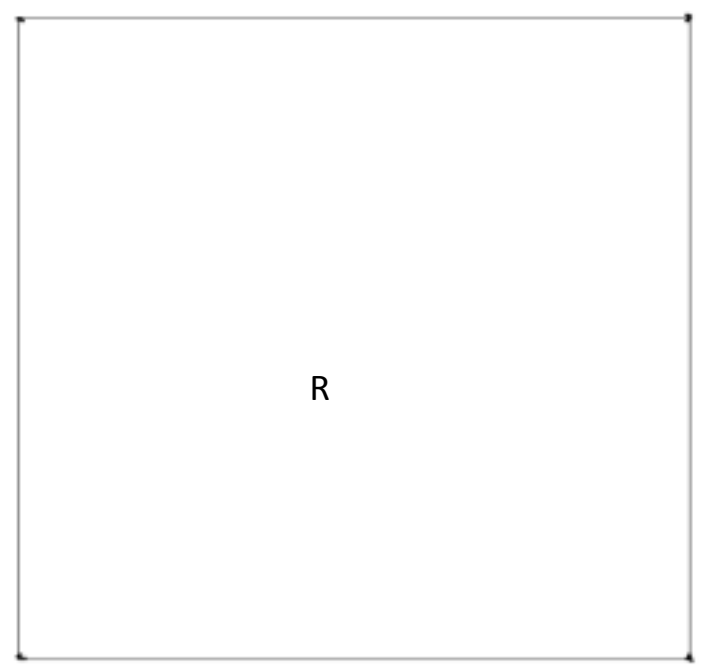

$10 \mathbf{a}$

(i)Fig.10a: Region $R$ to be analyzed

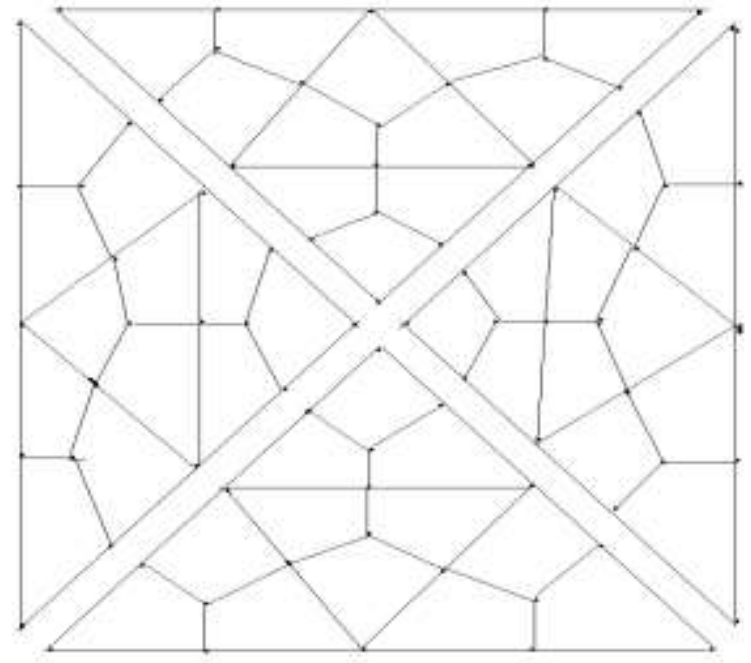

$10 \mathrm{c}$

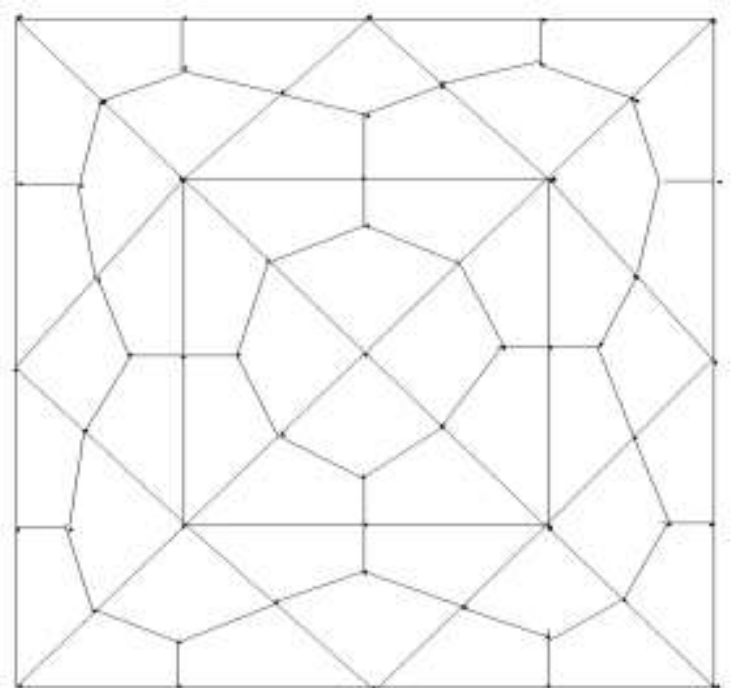

10b

(ii) Fig.10b: Example of completed mesh

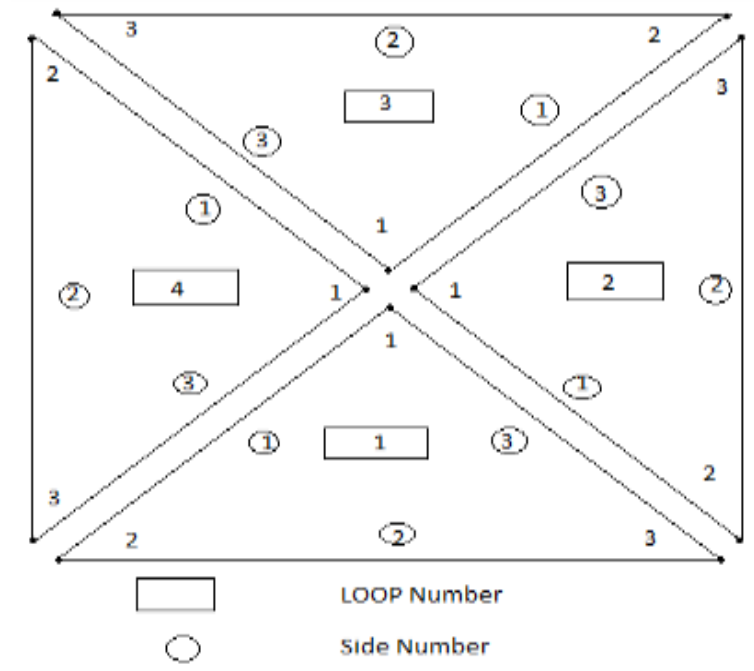

10d

(iii)Fig.10c:Exploded view showing four loops (iv)Fig.10d:Example of a loop and side numbering scheme

How to define the LOOP geometry, specify the number of elements and piece together the LOOPs will now be explained

Joining LOOPs : A complete mesh is formed by piecing together LOOPs. This piecing is done sequentially thus, the first LOOP formed is the foundation LOOP, with subsequent LOOPs joined either to it or to other LOOPs that have already been defined. As each LOOP is defined, the user must specify for each of the three sides of the current LOOP. 
In the present mesh generation code, we aim to create a convex polygon. This requires a simple procedure. We join side 3 of LOOP 1 to side 1 of LOOP 2, side 3 of LOOP 2 will joined to side 1 of LOOP 3, side 3 of LOOP 3 will be joined to side 1 of LOOP 4. Finally side 3 of LOOP 4 will be joined to side 1 of LOOP 1.

When joining two LOOPs, it is essential that the two sides to be joined have the same number of divisions. Thus the number of divisions remains the same for all the LOOPs. We note that the sides of LOOP $(i)$ and side of LOOP $(i+1)$ share the same node numbers. But we have to reverse the sequencing of node numbers of side 3 and assign them as node numbers for side 1 of $\operatorname{LOOP}(i+1)$. This will be required for allowing the anticlockwise numbering for element connectivity.

The auto mesh generation technique discritises a polygonal domain into all four node special quadrilateral elements. We can convert these into 12-node Serendipity and 16-node Lagrange special quadrilateral elements by adding two nodes at the trisectional points on each side and also at the interior of four node special quadrilateral elements. We have written codes to carry this conversion schemes in the programs of all four node special quadrilaterals proposed in[27-32 ].We include here some meshes of all 12-node and 16-node special quadrilaterals at initial stagtes of mesh generation which is self explainatory.

Example 1: right isosceles triangle

$\mathrm{x}=([0 ; 1 / 2 ; 1 / 2])$

$\mathrm{y}=\left(\left[\begin{array}{ll}0 & 0 ; 1 / 2]\end{array}\right)\right.$

We use this mesh to solve torsion of a square cross section, due to symmetry considerations mesh generation over the above domain is sufficient.This is a case of Poisson equation with constant right hand side(=-2). Our main aim here is to compute torsional constant and Prandtl stress function values for the given domain.

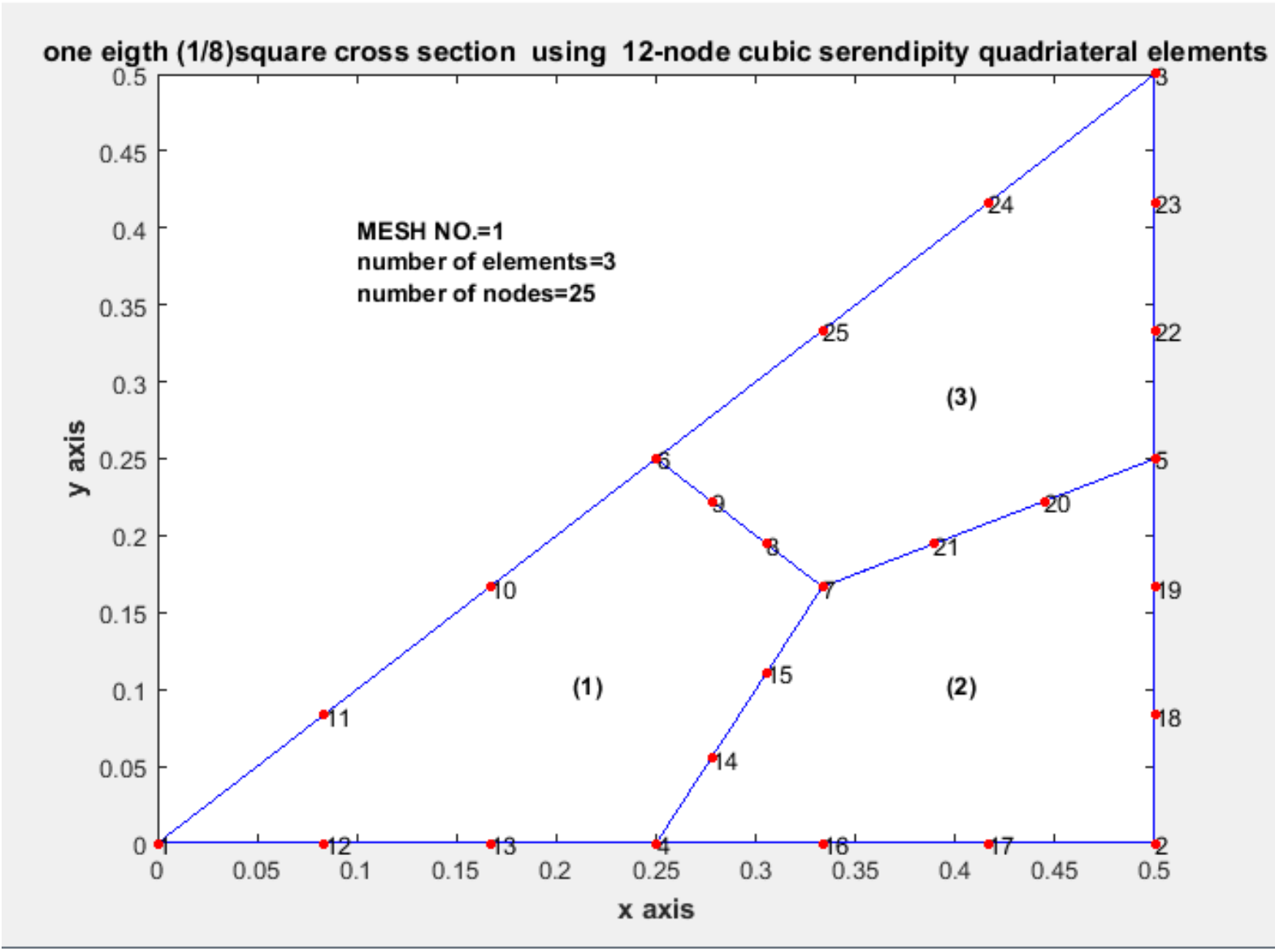

Fig.11a Initial mesh of right isosceles triangle(12-node quadrilaterals) 


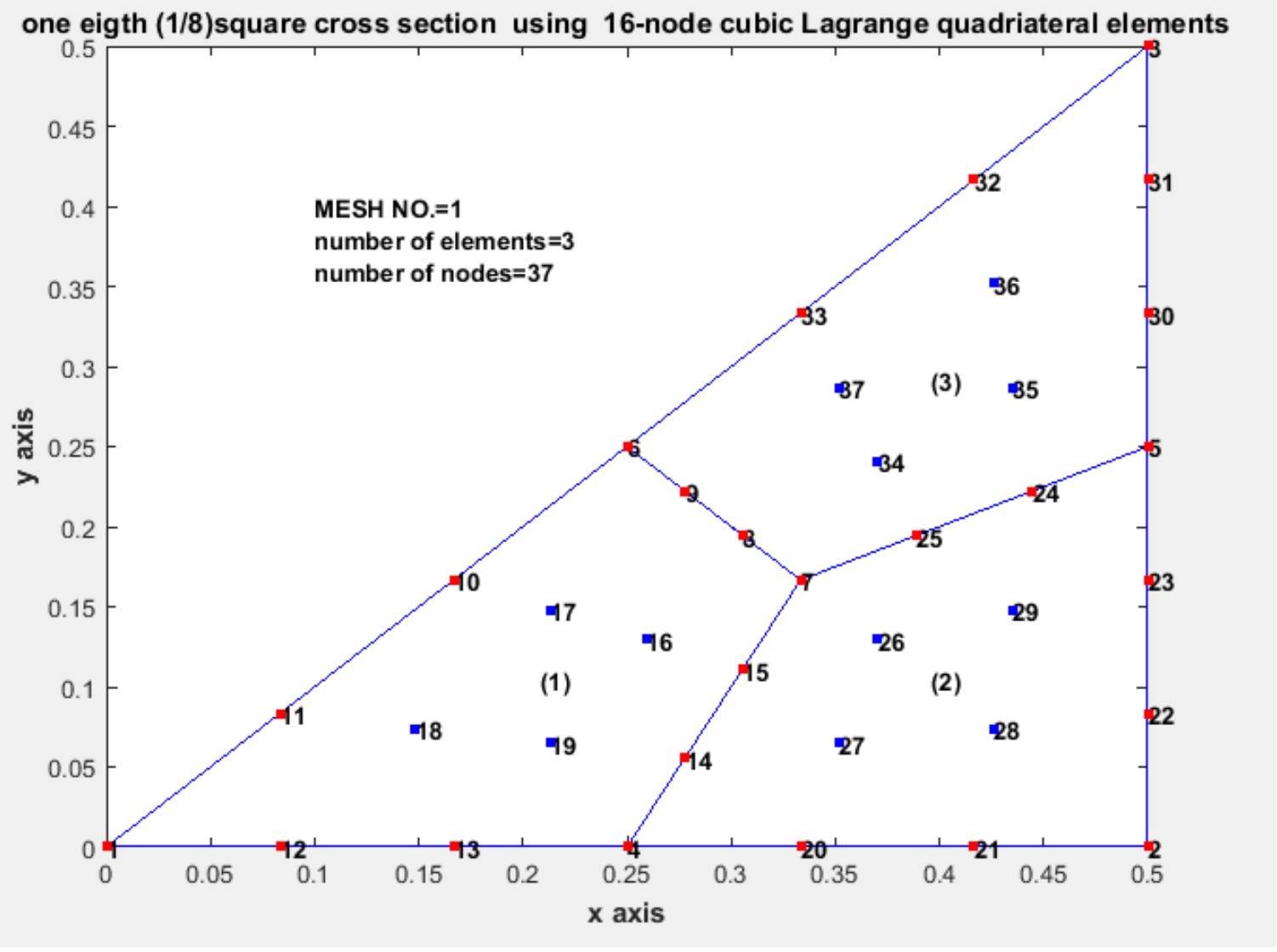

Fig.11b Initial mesh of right isosceles triangle(16-node quadrilaterals)

Example 2: equilateral triangle,each side $=2 * \operatorname{sqrt}(3)$

$\mathrm{x}=([-\sqrt{3} ; \sqrt{3} ; 0])$

$\mathrm{y}=([-1 ;-1 ; 2])$

We use this mesh to solve torsion of an arbitrary triangular cross section. This is a case of Poisson equation with constant right hand side(=-1). Our aims are to compute torsional constant and contour lines of Prandtl stress function 
equilateral triangular cross section using 12-node cubic serendipity quadriateral elements

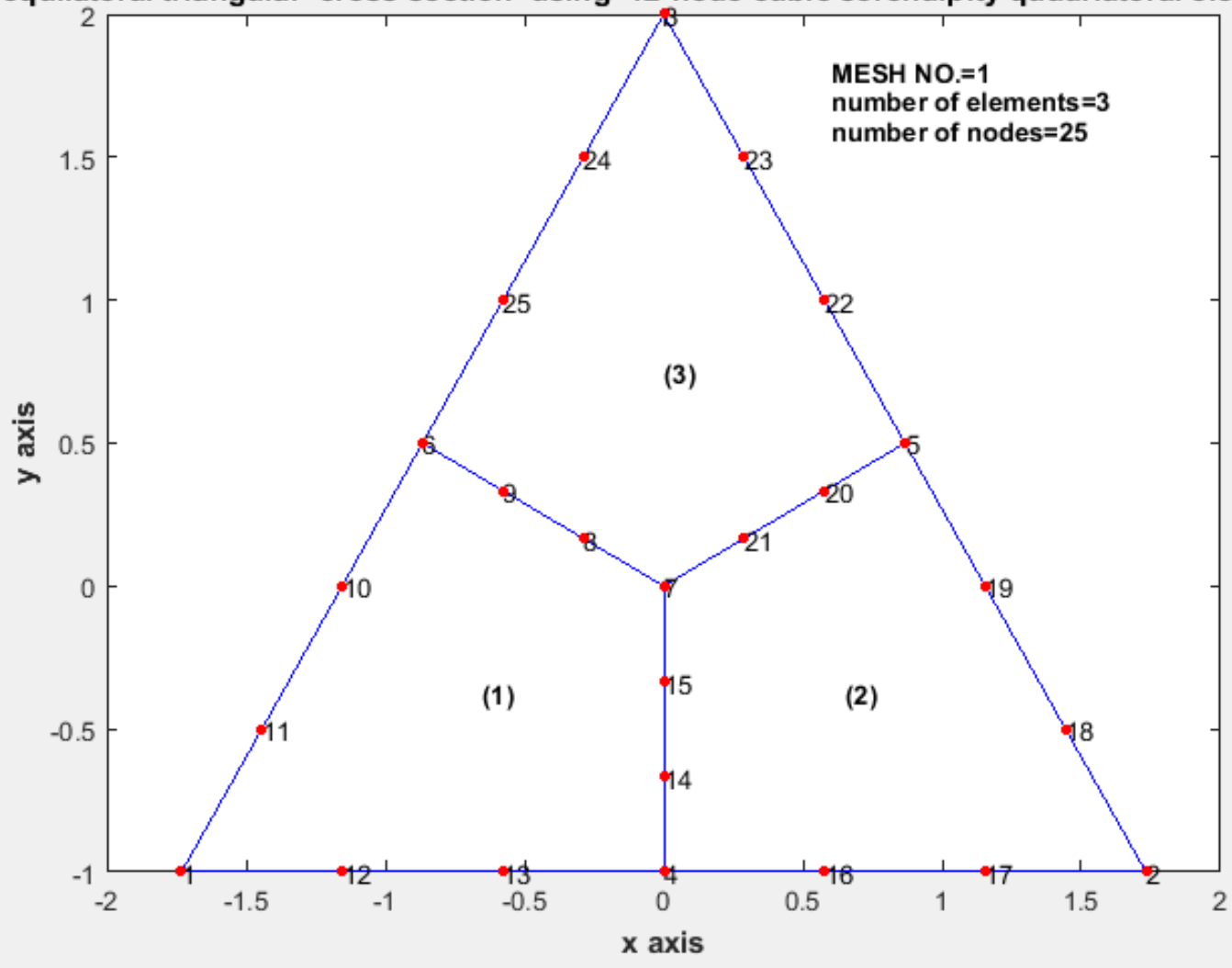

Fig.12a Initial mesh of equilateral triangle,each side $=2 * \operatorname{sqrt}(3)$ (12-node quadrilaterals)

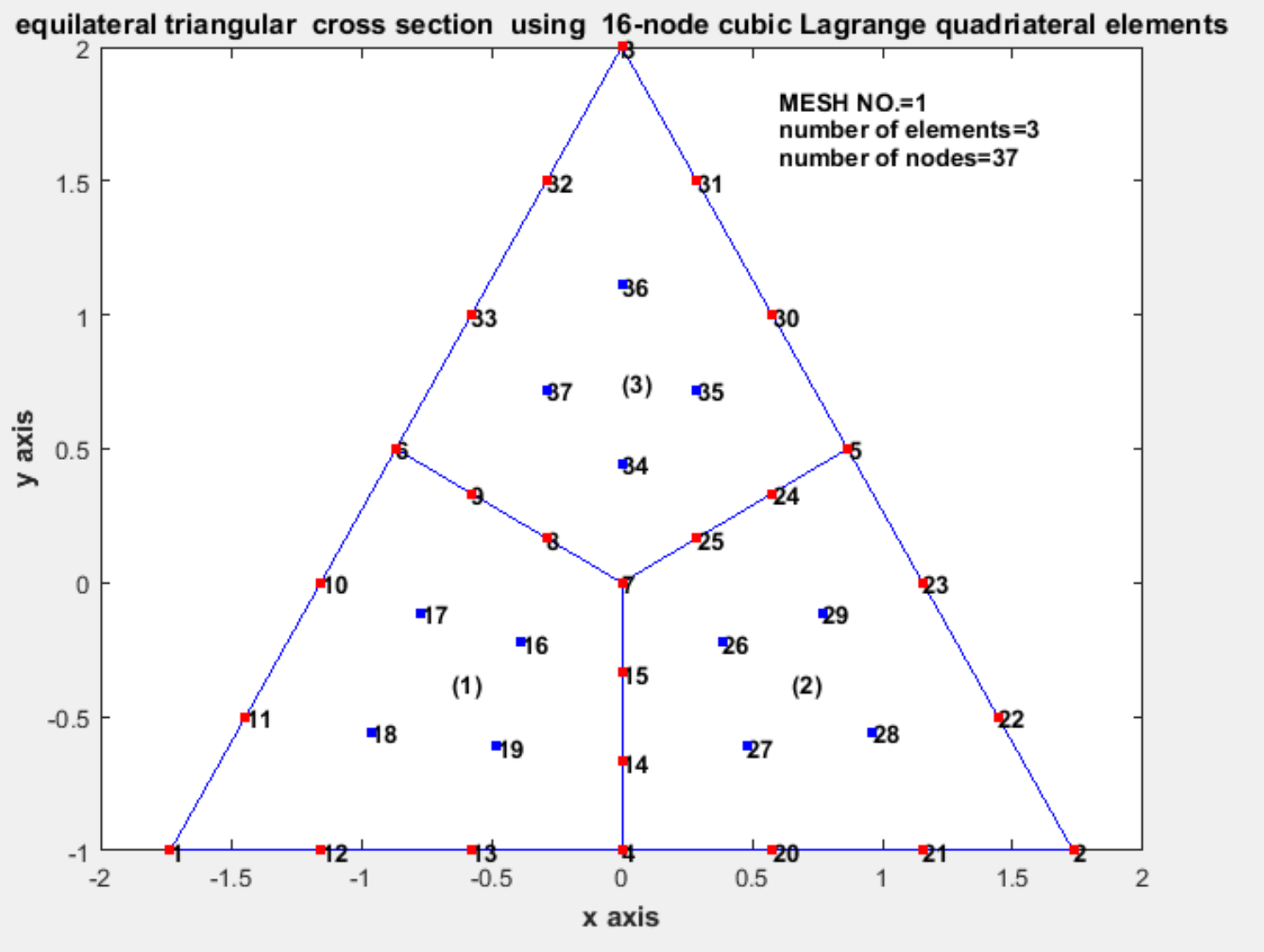

Fig.12b Initial mesh of equilateral triangle,each side $=2 * \operatorname{sqrt}(3)$ (16-node quadrilaterals)

Example 3 :a square domain with eight triangles(9-boundary nodes) 
$\mathrm{x}=([1 / 2 ; 1 / 2 ; 1 ; 1 ; 1 ; 1 / 2 ; 0 ; 0 ; 0]) \%$ FOR UNIT SQUARE

$\mathrm{y}=([1 / 2 ; 0 ; 0 ; 1 / 2 ; 1 ; 1 ; 1 ; 1 / 2 ; 0]) \%$ FOR UNIT SQUARE

We use this mesh to solve torsion of a square cross section. We would like to draw contour lines of Prandtl stress function over the entire domain. This is a case of Poisson equation with constant right hand $\operatorname{side}(=-2)$

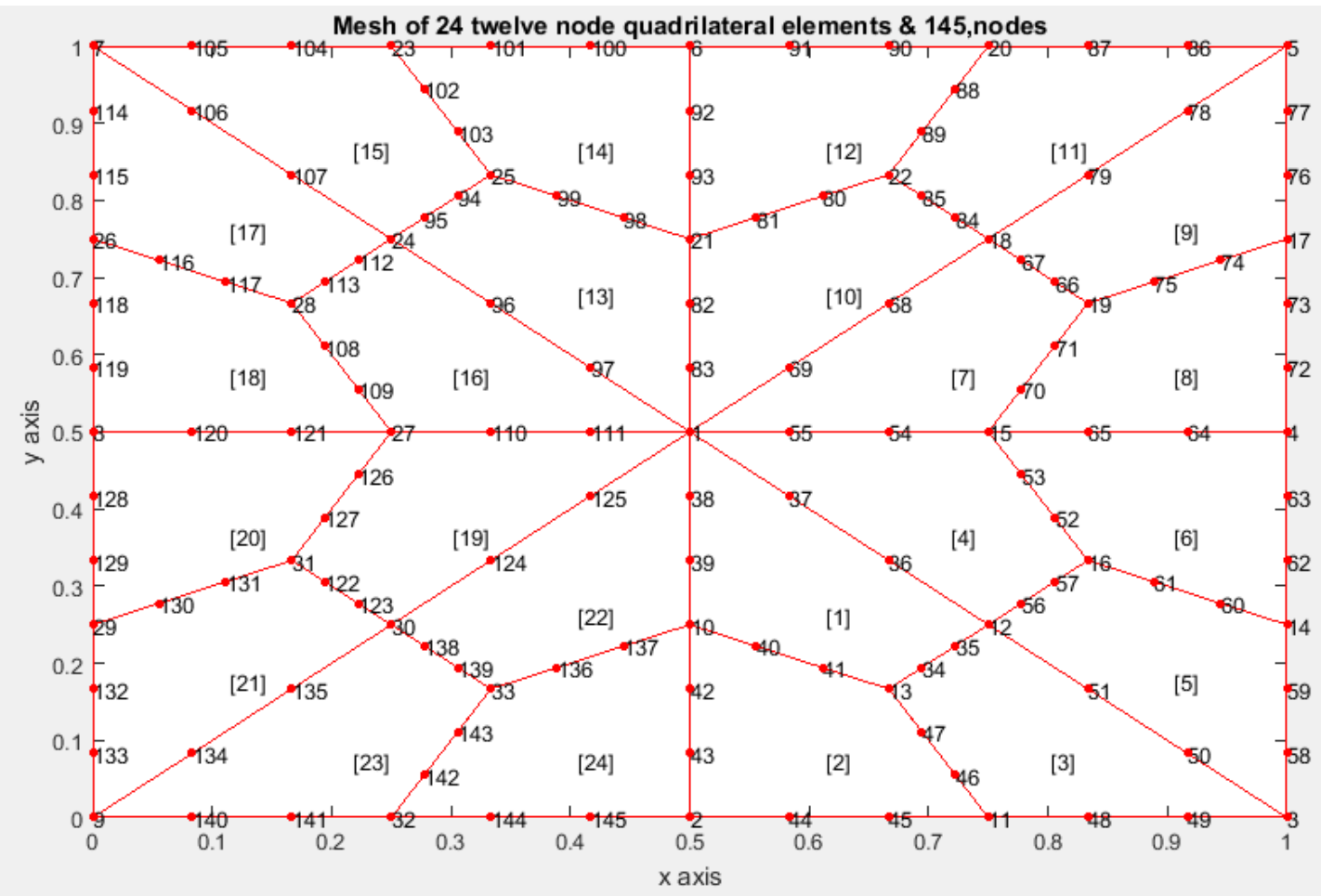

Fig.13a Initial Mesh for a square domain with eight triangles-12 noded quadrilaterals

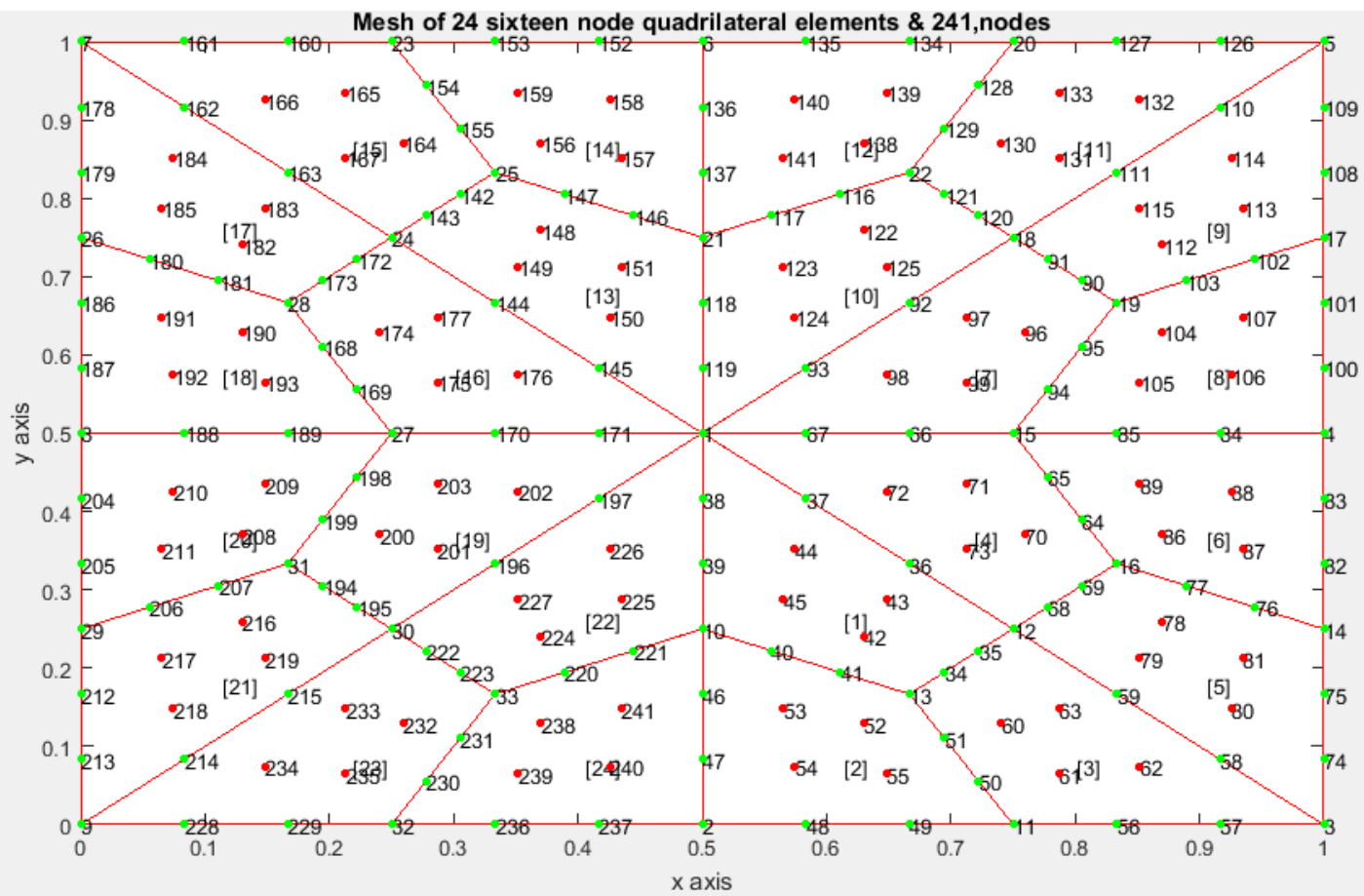

Fig.13b Initial Mesh for a square domain with eight triangles-16 noded quadrilaterals

Fig.13b Initial Mesh for a square domain with eight triangles(9-boundary nodes)

Example 4:pentagonal domain with seven triangles(8-boundary nodes) 
$\mathrm{x}=([1 / 2 ; 1 / 2 ; 1 ; 1 ; 1 / 2 ; 0 ; 0 ; 0]) \%$ for MOIN EXAMPLE

$\mathrm{y}=([1 / 2 ; 0 ; 0 ; 1 / 2 ; 1 ; 1 ; 1 / 2 ; 0]) \%$ for MOIN EXAMPLE

We use this mesh to solve Poisson equation with a nonconstant smooth function on right hand side, with a known analytical solution

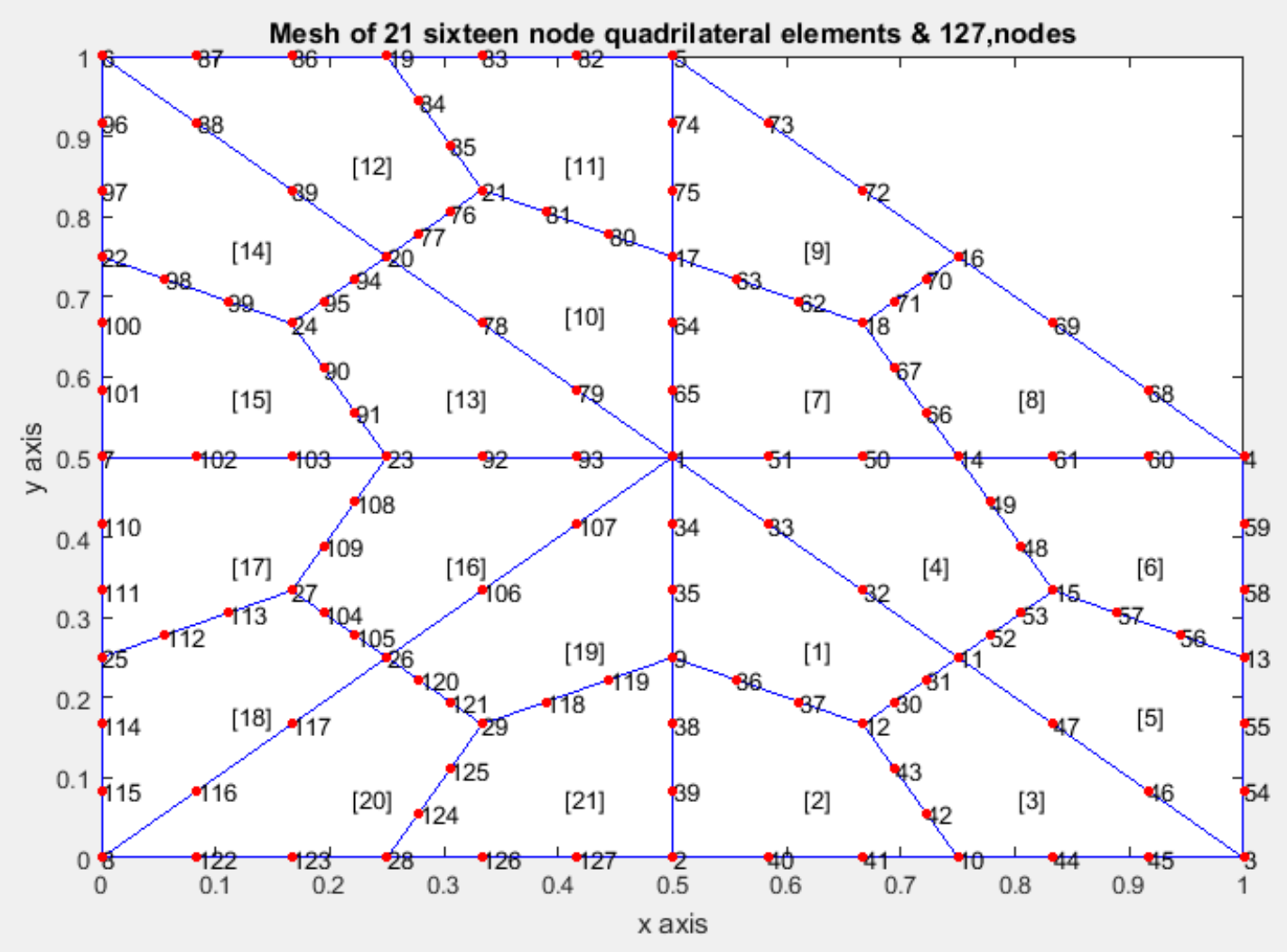

Fig 14a:Initial Mesh for a pentagonal domain seven triangles-12 noded quadrilaterals

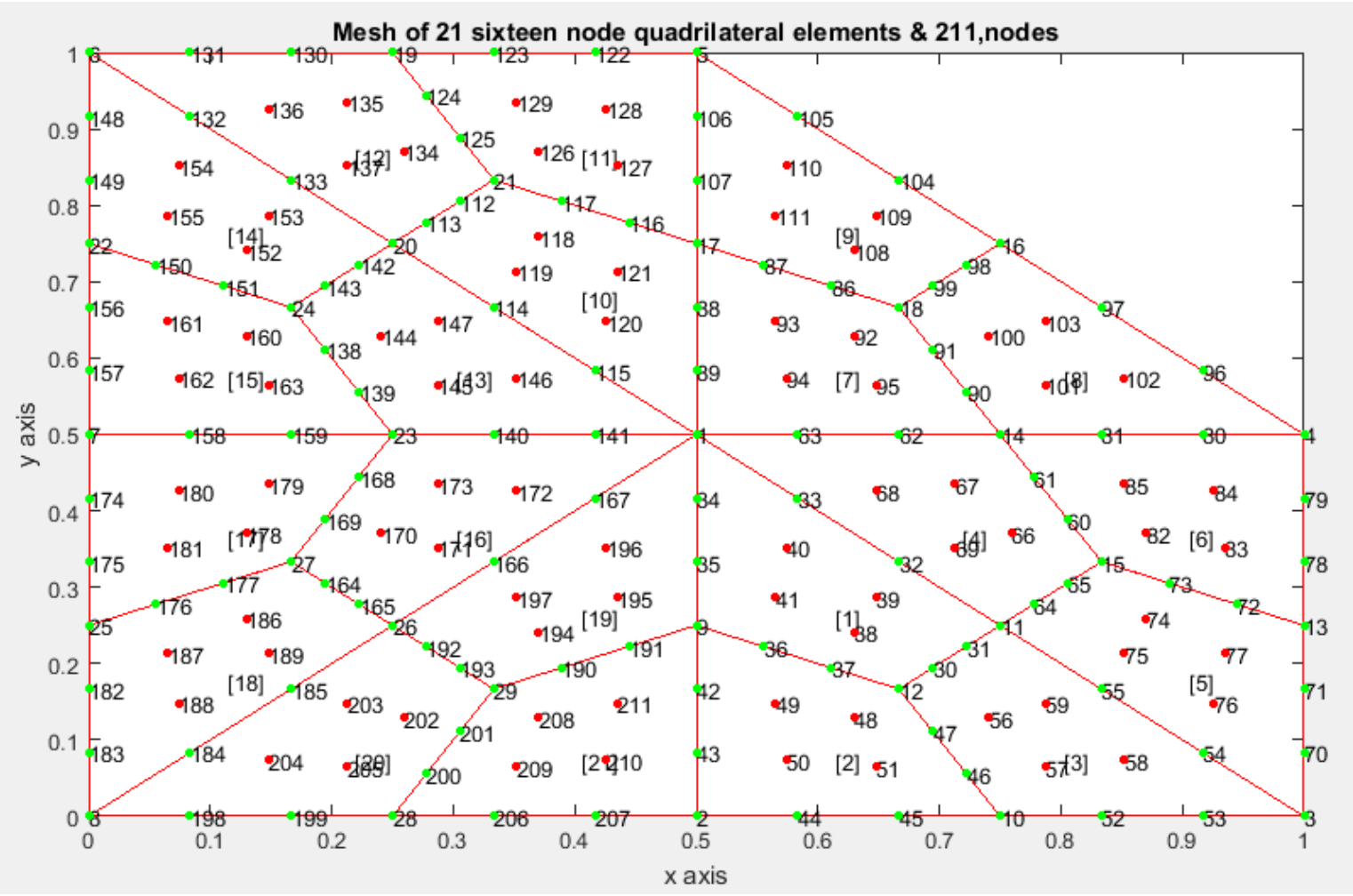


Fig 14b:Initial Mesh for a pentagonal domain seven triangles-16 noded quadrilaterals

Example 5 :a square domain with eight triangles(9-nodes)

$\mathrm{x}=([0 ; 0 ; 1 / 2 ; 1 / 2 ; 1 / 2 ; 0 ;-1 / 2 ;-1 / 2 ;-1 / 2])$

$\mathrm{y}=([0 ;-1 / 2 ;-1 / 2 ; 0 ; 1 / 2 ; 1 / 2 ; 1 / 2 ; 0 ;-1 / 2])$

We use this mesh to solve torsion of a square cross section. We would like to draw countour lines of Prandtl stress function over the entire domain. This is a case of Poisson equation with a constant right hand $\operatorname{side}(=-2)$

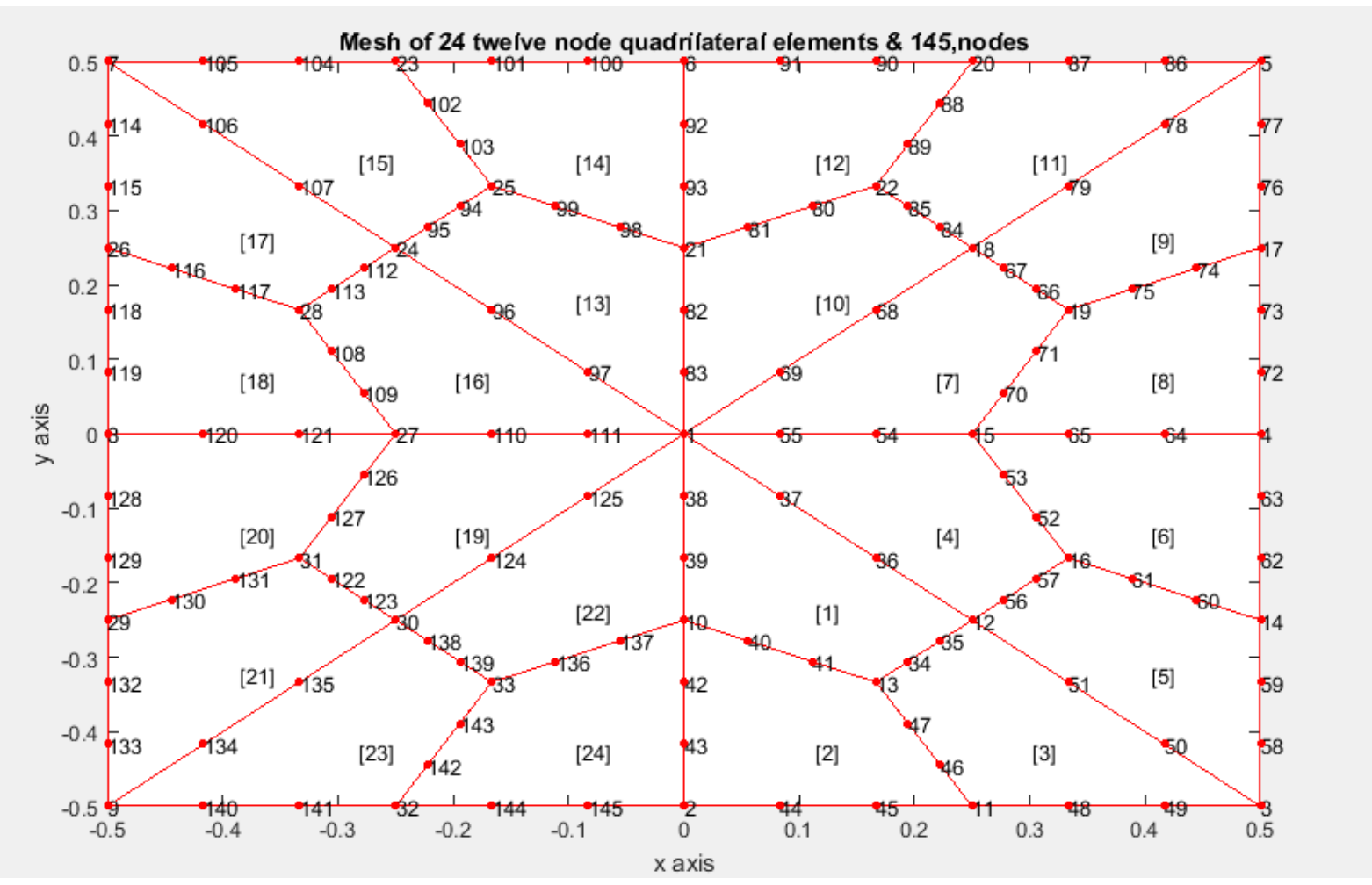

Fig 15a:Initial Mesh for a unit square domain eight triangles-12 noded quadrilaterals

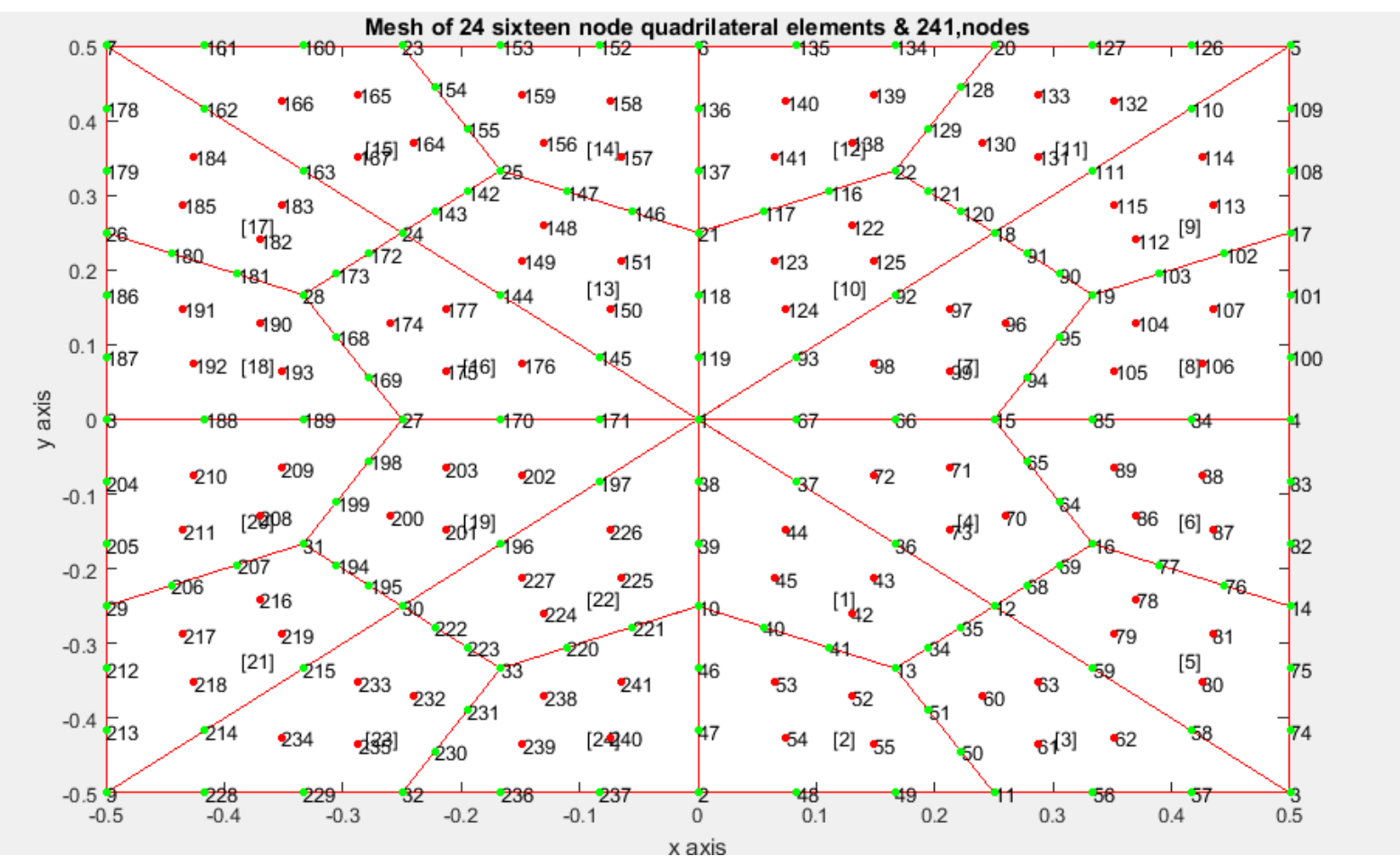

Fig 15b: Initial Mesh for a unit square domain with eight triangles-16 noded quadrilaterals

\subsection{Application Examples}


7.5.1 Mesh Generation Over a Standard Triangle \& a Square cross sections

\section{Examples 1 \&5}

Let us use the explicit integration scheme and the auto mesh generation techniques which are developed in the previous sections to solve the Poisson Equation with Dirichlet boundary value problem:

$-\Delta u=f, x \in \Omega \subset \mathcal{R}^{2}$

$\boldsymbol{u}=\boldsymbol{g}$,

Where $\Omega$ is a triangular or polygonal domain and $\Delta$ is the standard standard Laplace operator

In this section, we examine the application of the proposed explicit integration scheme to the Saint Venant Torsion problem

[24]. Exact solutions of this problem for simple cross sections such as circle, ellipse, equilateral triangle and rectangle have been rigorously derived. These problems are described by the following boundary value problem ;

$\frac{\partial^{2} \phi}{\partial \mathrm{x}^{2}}+\frac{\partial^{2} \phi}{\partial \mathrm{y}^{2}}+2 \mathrm{G} \theta=0 \quad$ in $\mathrm{R}$

$\phi=0$ on $\partial \mathrm{R}$, the boundary of $\mathrm{R}$

where $\phi(x, y)$ is known as Prandtl stress function, $G$ is the shear modulus, $\theta$ is the angle of twist per unit length, $R$ is the cross sectional region and $\partial \mathrm{R}$ is the boundary of $\mathrm{R}$. We choose $\mathrm{G} \theta=1$ for the sake of simplicity. Then the corresponding torisonal constant is given by the equation

$. t_{c}=2 \iint_{R} \phi(x, y) d x d y$

We take $\mathrm{R}$ as the 9-node special quadrilateral meshes described in Examples 1 \&5 in previous section.

In a recent paper[26] a new approach to automatic generation of all quadrilateral mesh for finite analysis is proposed and it was applied to discretise the 1/8-th of the square cross section a triangular region into an all quadrilateral mesh. We have demonstrated the proposed explicit integration scheme to solve the St. Venant Torsion problem for a square cross section. Monotonic convergence from below is observed with known analytical solutions for the Prandtl stress function and the torisonal constant which are expessed in terms of infinite series. This triangular domain is a right isosceles triangle and it was discretised by 8-noded special linear convex quadrilaterals of serendipity family. We have considered this problem again and illustrated the application of 9-node quadrilateral of Lagrange family.

\subsubsection{Mesh Generation Over an Arbitrary Triangular Domain}

Exmple 2

In applications to boundary value problems due to symmetry considerations or otherwise also, we may have to discretize an arbitrary triangle. Our purpose is to have a code which automatically generates convex quadrangulations of the domain by assuming the input as coordinates of the boundary vertices. We use the theory and procedure developed in section 7.2 and section 7.3 for this purpose..

Let us use the explicit integration scheme and the auto mesh generation techniques which are developed in the previous sections to solve the Poisson Equation with Dirichlet boundary value problem:

$\nabla^{2} u=-1, x \epsilon \Omega \subset \mathcal{R}^{2}$

$\boldsymbol{u}=\mathbf{0}$,

............................................................................................(10)

Where $\Omega$ is a regular triangular or polygonal domain and $\nabla^{2}$ is the standard Laplace operator

Example 2

In this example, we would like to consider the linear elastic torsion of an equilateral triangle which is inscribed in a circle of unit radius.

In our recent we considered torsion of an equilateral triangular cross section and it was discretised by 8-noded special linear convex quadrilaterals of serendipity family.Now we would like to illustrate the $t$ St. Venant Torsion problem for an arbitrary triangular cross section by using 9-node special linear convex quadrilaterals of Lagrange family. 


\subsubsection{Mesh Generation over a Convex Polygonal Domain}

In several physical applications in science and engineering, the boundary value problem require meshes generated over convex polygons. Again our aim is to have a code which automatically generates a mesh of 9 noded convex quadrilaterals of Lagrange family for the complex domains such as those in [27-33]. We use the theory and procedure developed in sections 7.2, 7.3 and 7.4 for this purpose..

Example 3(with nonconstant smooth function as right hand side of Poisson equation )

$-\Delta u=2 \pi^{2} \sin (\pi x) \sin (\pi y),(x, y) \epsilon \Omega \subset \mathcal{R}^{2}$

$u(x, 0)=0$, on $y=0,0 \leq x \leq 1$

$u(x, 1)=0$, on $y=1,0 \leq x \leq 1$,

$u(1, y)=0$, on $x=1,0 \leq y \leq 1 / 2$,

$u(x, y)=\sin (\pi x) \sin (\pi y)$, on the line $x=1-0.5 t, y=0.5+0.5 t, 0 \leq t \leq 1$

Where $\Delta$ is a standard Laplace operator and $\Omega$ is a pentagonal domain joining the vertices $\{(\mathbf{0 , 0}),(1,0),(1,0.5),(0.5,1),(0,1)\}$

The exact solution of the above boundary value problem is $u(x, y)=\sin (\pi x) \sin (\pi y)$.

Example 4(with nonconstant smooth function as right hand side of Poisson equation)

$-\Delta u=2 \pi^{2} \sin (\pi x) \sin (\pi y),(x, y) \epsilon \Omega \subset \mathcal{R}^{2}$

$\mathbf{u}=\mathbf{0}$, on the boundary $\partial \Omega$

Where $\Delta$ is a standard Laplace operator and $\Omega$ is a square domain $[0,1]^{2}$.

The following MATLAB codes are written for the purpose of developing automesh generation techniques and explicit integration methods for 12-node Serendipity and 16-node Lagrange family elements.They can be developed by referring our previous works [27-33]

[1]quadrilateralmesh_over_arbitrarytriangle_q12automeshgen.m

[2]quadrilateralmesh_over_arbitrarytriangle_q16automeshgen.m

[3]quadrilateral_mesh4MOINEX_q12.m

[4]quadrilateral_mesh4MOINEX_q16LG.m

[5]D2LaplaceEquationQ12Ex3automeshgenNewContour.m $\sqrt{ }^{*}$

[6]D2LaplaceEquationQ12Ex3automeshgenNewPolygonContour.m $\sqrt{ }{ }^{*}$

[7]D2LaplaceEquationQ16Ex3automeshgenNewContour.m $\sqrt{ }^{*}$

[8]D2LaplaceEquationQ16Ex3automeshgenNewPolygonContour.m $\sqrt{ }$ *

[9]polygonal_domain_coordinates_3rd_orderLG.m

[10]polygonal_domain_coordinates_3rd_order.m

[11]coordinate_special_quadrilaterals_in_stdtriangle_3rd_orderLAGR.m

[12]coordinate_special_quadrilaterals_in_stdtriangle_3rd_order.m

[13]integral_valuesof_localderivative_products.m

[14]nodaladdresses_special_convex_quadrilaterals_trial_3rd_order.m

[15]generate_area_coordinate_over_the_standard_triangle.m

[16]nodaladdresses_special_convex_quadrilaterals_trial_3rd_orderLG.m

[17]D2PoissonEquationQ12MoinEx_MeshgridContourNew.m $\sqrt{ }{ }^{*}$

[18]D2PoissonEquationQ16MoinEx_MeshgridContourNew.m $\sqrt{ }{ }^{*}$

[19]newtonmethod4spquadrileteral.m

[20]parameqnspqd.m

[21]paramdetJspqd.m

[22]paraminvJspqd.m

[23]glsampleptsweights.m

In the above list serial no.s $5,6,7,8,17,18$ are marked as $\sqrt{*}$ and they call the program integral_valuesof_localderivative_products.m at serial no.13 
The programs at serial no.s 19,20,21,22 are called in programs at serial no.s 1718 to solve bilinear equations in two variates and they are required for interpolating physical solutions at mesh grid points to draw the contour lines.

We are also appending these MATLAB programs towards end of this paper.

\subsection{CONCLUSIONS}

This paper presents the explicit integration schemes for a unique(special) linear convex 12- node and 16-node quadrilaterals of Serendipity and Lagrange family elements which can be obtained from an arbitrary linear triangle by joining the centroid to the midpoints of sides of the triangle. The explicit integration scheme proposed for these unique linear convex 9- node quadrilaterals is derived by using the standard transformations in two steps. We first map an arbitrary linear triangle into a standard right isosceles triangle by using the affine linear transformation from global $(\mathrm{x}, \mathrm{y})$ space into a local space $(\mathrm{u}, \mathrm{v})$. We then discritise this standard right isosceles triangle in $(\mathrm{u}, \mathrm{v})$ space into three unique linear convex 9 - node quadrilaterals. We have shown by proving a lemma that any unique linear convex 9-node quadrilateral in (x,y) space can be mapped into one of the unique 9-node quadrilaterals in $(u, v)$ space. We have then mapped these linear convex 9- node quadrilaterals into a 2 -square in the local $(\xi, \eta)$ space by use of the bilinear transformation between $(u, v)$ and $(\xi, \eta)$ space. Using these two mappings, we have established an integral derivative product relation between the linear convex 9-node quadrilaterals in the global (x ,y) space interior to the arbitrary triangle and the linear convex 9- node quadrilaterals in the local $(\mathrm{u}, \mathrm{v})$ space which are interior to the standard right isosceles triangle. We have then shown that the product of global derivative integrals $S^{i, j, e}$ in global (x, y) space can be expressed as a matrix triple product $\mathrm{P} *\left(\mathrm{~K}^{\mathrm{i}, \mathrm{j}, \mathrm{e}}\right) * \mathrm{P}^{\mathrm{T}} *(2 *$ area of the arbitrary triangle in $(\mathrm{x}, \mathrm{y})$ space $)$, in which $\mathrm{P}$ is a geometric properties matrix and $K^{i, j, e}$ is the product of global derivative integrals in $(u, v)$ space, $(i, j=1(1) 12)$. and $(i, j=1(1) 16)$. We have shown that the explicit integration of the global derivative products in $(\mathrm{u}, \mathrm{v})$ space over the unique 12-node and 16-node quadrilaterals is now possible by application of symbolic processing capabilities in MATLAB which are based on MAPLE -V mathematical software package. The proposed explicit integration scheme is a useful technique for boundary value problems governed by either a single or a system of partial differential equations. The physical applications of such problems are numerous in science , engineering, medical, business and social sciences. The well known examples are the Laplace and Poisson equations with suitable boundary conditions and the some examples of system of equations are the plane stress, plane strain and axisymmetric stress analysis, flow through porous media, shallow water circulation, dispersion and viscous incompressible flow etc in the areas of solid and fluid mechanics. We have first demonstrated the proposed explicit integration scheme to solve the St. Venant Torsion problem for an equilateral triangular cross section. Monotonic convergence from below is observed with known analytical solutions for the Prandtl stress function and the torisonal constant. We have demonstrated the proposed explicit integration scheme to solve the Poisson Boundary Value Problem for pentagonal and square domains which are to be considered as simple polygonal domains. Monotonic convergence from below is observed with known analytical solutions for the governing unknown function of Poisson Boundary Value Problem.We have shown the solutions in Tables which list both the FEM and exact solutions.The graphical solutions of nine noded quadrilateral meshes and contour level curves for FEM and exact solutions are also displayed. We conclude that efficient scheme on explicit integration of stiffness matrix and a novel automesh generation technique developed in this paper will be useful for the solution of many physical problems governed by second order partial differential equations.

We hope that the scheme developed in this paper will be useful for the solution of boundary value problems governed by second order partial differential equations with fast convergence and economy for the computational problems.

\section{REFERENCES:}

1. Zienkiewicz O.C, Taylor R.L and Zhu J.Z Finite Element Method, its basis and fundamentals, Elservier, (2005)

2. Bathe K.J Finite Element Procedures, Prentice Hall, Englewood Cliffs, N J (1996)

3. Reddy J.N Finite Element Method, Third Edition, Tata Mc Graw-Hill (2005)

4. Burden R.L and Faires J.D Numerical Analysis, $9^{\text {th }}$ Edition, Brooks/Cole, Cengage Learning (2011)

5. Stroud A.H and Secrest D, Gaussian quadrature formulas, Prentice Hall,Englewood Cliffs, N J, (1966)

6. Stoer J and Bulirsch R, Introduction to Numerical Analysis, Springer-Verlag, New York (1980)

7. Chung T.J Finite Element Analysis in Fluid Dynamics, pp. 191-199, Mc Graw Hill, Scarborough, C A , (1978)

8. Rathod H.T, Some analytical integration formulae for four node isoparametric element, Computer and structures 30(5), pp.1101-1109, (1988)

9. Babu D.K and Pinder G.F, Analytical integration formulae for linear isoparametric finite elements, Int. J. Numer. Methods Eng 20, pp.1153-1166

10. Mizukami A, Some integration formulas for four node isoparametric element Computer Methods in Applied Mechanics and Engineering. 59 pp. 111-21(1986)

11. Okabe M, Analytical integration formulas related to convex quadrilateral finite elements, Computer methods in Applied mechanics and Engineering. 29, pp.201-218 (1981)

12. Griffiths D.V Stiffness matrix of the four node quadrilateral element in closed form, International Journal for Numerical Methods in Engineering. 28, pp.687-703(1996)

13. Rathod H.T and Shafiqul Islam. Md, Integration of rational functions of bivariate polynomial numerators with linear denominators over a $(-1,1)$ square in a local parametric two dimensional space, Computer Methods in Applied Mechanics and Engineering. 161 pp.195-213 (1998)

14. Rathod H.T and Sajedul Karim, Md An explicit integration scheme based on recursion and matrix multiplication for the linear convex quadrilateral elements, International Journal of Computational Engineering Science. 2(1) pp. 95135(2001) 
15. Yagawa G, Ye G.W and Yoshimura S, A numerical integration scheme for finite element method based on symbolic manipulation, International Journal for Numerical Methods in Engineering. 29, pp.1539-1549(1990)

16. Rathod H.T and Shafiqul Islam Md, Some pre-computed numeric arrays for linear convex quadrilateral finite elements, Finite Elements in Analysis and Design 38, pp. 113-136 (2001)

17. Hanselman D and Littlefield B, Mastering MATLAB 7 , Prentice Hall, Happer Saddle River, N J . (2005)

18. Hunt B.H, Lipsman R.L and Rosenberg J.M, A Guide to MATLAB for beginners and experienced users, Cambridge University Press (2005)

19. Char B, Geddes K, Gonnet G, Leong B, Monagan M and Watt S, First Leaves; A tutorial Introduction to Maple V, New York : Springer-Verlag (1992)

20. Eugene D, Mathematica, Schaums Outlines Theory and Problems, Tata Mc Graw Hill (2001)

21. Ruskeepaa H, Mathematica Navigator, Academic Press (2009)

22. Timoshenko S.P and Goodier J.N, Theory of Elasticity, $3^{\text {rd }}$ Edition, Tata Mc graw Hill Edition (2010)

23. Budynas R.G, Applied Strength and Applied Stress Analysis, Second Edition Tata Mc Graw Hill Edition (2011)

24. Roark R.J, Formulas for stress and strain, Mc Graw Hill, New York (1965)

25. Nguyen S.H , An accurate finite element formulation for linear elasticcalculations, Computers and Structures. 42, pp.707-711 (1992)

26. Rathod H.T, Venkatesh.B, Shivaram. K.T,Mamatha.T.M, Numerical Integration over polygonal domains using convex quadrangulation and Gauss Legendre Quadrature Rules, International Journal of Engineering and Computer Science, Vol. 2,issue 8,pp2576-2610(2013)

27. Rathod H.T, Rathod Bharath, Shivaram.K.T,Sugantha Devi.K, A new approach to automatic generation of all quadrilateral mesh for finite analysis, International Journal of Engineering and Computer Science, Vol. 2,issue 12,pp3488-3530(2013)

28. Rathod.H.T, Bharath Rathod, Shivaram K.T , H. Y. Shrivalli , Tara Rathod, K. Sugantha Devi,An explicit finite element integration scheme using automatic mesh generation technique for linear convex quadrilaterals over plane regions , international Journal of Engineering and Computer Science, Vol. 3,issue 4,pp5400-5435 (2014)

29. Rathod.H.T, Bharath Rathod, K.T.Shivaram,Sugantha Devi.K,Tara Rathod, An explicit finite element integration scheme for linear eight node convex quadrilaterals using automatic mesh generation technique over plane regions, international Journal of Engineering and Computer Science, Vol. 3,issue 4,pp5657-5713 (2014)

30. Rathod.H.T, Sugantha Devi.K ,Finite element solution of Poisson equation over polygonal domains using an explicit integration scheme and a novel auto mesh generation technique,,International Journal of Engineering and Computer Science, ISSN:2319-7242,Volume(5),issue 8,August(2016), pp. 17397-17481

31. Rathod.H.T, Sugantha Devi.K,Nagabhushana.C.S , Chudamani.H.M ,Finite element analysis of linear elastic torsion for regular polygons, International Journal of Engineering and Computer Science, ISSN:2319-7242,Volume(5),issue 10,October(2016), pp. 18413-1842

32. Rathod.H.T, Bharath Rathod, Sugantha Devi.K,Hariprasad.A.S, Finite element solution of Poisson Equation over Polygonal Domains using a novel auto mesh generation technique and an explicit integration scheme for eight node linear convex quadrilaterals, International Journal of Engineering and Computer Science, ISSN:2319-7242, Volume (6) Issue 10 October 2017, pp 22632-22787

33. H. T. Rathod,Md.Shafiqul. H. Y. Shrivall , Bharath Ratho K. Sugantha Devi, Finite element solution of Poisson Equation over Polygonal Domains using a novel auto mesh generation technique and an explicit integration scheme for nine node linear convex quadrilateral of Lagrange family, International Journal of Engineering and Computer Science, ISSN:2319-7242, Volume 6 Issue 11 November 2017, Page No. 22869-23058

\section{APPENDIX-1}

We now propose to compute the following integrals(see eqns(52a-b)) and they will be listed in Tables 1a-1i

$K^{i, j, e}=\iint_{\widehat{Q}} G_{u, v}^{i, j, e} \quad d u d v=\left(\begin{array}{cc}\iint_{\widehat{Q}} \frac{\partial N_{i}^{e}}{\partial u} \frac{\partial N_{j}^{e}}{\partial u} d u d v & \iint_{\widehat{Q}} \frac{\partial N_{i}^{e} \frac{\partial N_{j}^{e}}{\partial u}}{\partial v} d u d v \\ \iint_{\widehat{Q}} \frac{\partial N_{i}^{e}}{\partial v} \frac{\partial N_{j}^{e}}{\partial u} d u d v & \iint_{\widehat{Q}} \frac{\partial N_{i}^{e}}{\partial v} \frac{\partial N_{j}^{e}}{\partial v} d u d v\end{array}\right)=\left(\begin{array}{cc}K_{2 i-1,2 j-1}^{e} & K_{2 i-1,2 j}^{e} \\ K_{2 i, 2 j-1}^{e} & K_{2 i, 2 j}^{e}\end{array}\right)(s a y)$

(52a-b)

We map $\widehat{\mathbf{Q}}$ into a 2 -square We map $\widehat{\mathbf{Q}}$ into a 2 - square, $-1 \leq \xi, \eta \leq 1$

in the natural parametric space $(\xi, \eta)$ by using the bilinear transformation from $(u, v)$ to $(\xi, \eta)$

This gives(see eqns(18-20))

$\left(\begin{array}{l}\frac{\partial \mathrm{N}_{\mathrm{i}}^{\mathrm{e}}}{\partial \mathrm{u}} \\ \frac{\partial \mathrm{N}_{\mathrm{i}}^{\mathrm{e}}}{\partial \mathrm{v}}\end{array}\right)=\frac{1}{\mathrm{~J}}\left[\begin{array}{cr}\frac{\partial \mathrm{v}}{\partial \eta} & -\frac{\partial \mathrm{v}}{\partial \xi} \\ -\frac{\partial \mathrm{u}}{\partial \eta} & \frac{\partial \mathrm{u}}{\partial \xi}\end{array}\right]\left[\begin{array}{c}\frac{\partial \mathrm{N}_{\mathrm{i}}^{\mathrm{e}}}{\partial \xi} \\ \frac{\partial \mathrm{N}_{\mathrm{i}}^{\mathrm{e}}}{\partial \eta}\end{array}\right]$

Let us replace the Greek letters $\boldsymbol{\xi}, \boldsymbol{\eta}$ by English letters $\mathbf{r}, \mathbf{s}$ for computing the integrals by using MATLAB programming. With this assumption,we denote the entries of submatrix $\mathbf{K}^{\mathbf{i}, \mathbf{j}, \mathbf{e}}=$ int Jdnidnjuvrs ,(i,j=1(1)9)

and we have from eqn(52a-b):

intJdnidnjuvrs $=\left(\begin{array}{ll}{[\text { int Jdnidnjuvrs }(1,1)} & \text { intJdnidnjuvrs }(1,2)] \\ {[\text { intJdnidnjuvrs }(2,1)} & \text { intJdnidnjuvrs }(2,2)]\end{array}\right)$ 
$\mathrm{K}_{2 \mathrm{i}-1,2 \mathrm{j}-1}^{\mathrm{e}}=\iint_{\widehat{Q}} \frac{\partial N_{\mathrm{i}}^{\mathrm{e}}}{\partial \mathrm{u}} \frac{\partial N_{\mathrm{j}}^{\mathrm{e}}}{\partial \mathrm{u}} \mathrm{dudv}=\operatorname{intJdnidnjuvrs}(1,1), \mathrm{K}_{2 \mathrm{i}-1,2 \mathrm{j}}^{\mathrm{e}}=\iint_{\widehat{Q}} \frac{\partial N_{\mathrm{i}}^{e}}{\partial \mathrm{u}} \frac{\partial N_{\mathrm{i}}^{\mathrm{e}}}{\partial v} \mathrm{dudv}=\operatorname{intJdnidnjuvrs}(1,2)$,

$K_{2 i, 2 j-1}^{e}=\iint_{\widehat{Q}} \frac{\partial N_{i}^{e}}{\partial v} \frac{\partial N_{j}^{e}}{\partial u} d u d v=\operatorname{intJdnidnjuvrs}(2,1), K_{2 i, 2 j}^{e}=\iint_{\widehat{Q}} \frac{\partial N_{i}^{e}}{\partial v} \frac{\partial N_{j}^{e}}{\partial v} d u d v=\operatorname{intJdnidnjuvrs}(2,2)$

Tables of integral values for cubic order Serendipity and Lagrange elements

intJdnidnjuvrs $=\left(\begin{array}{ll}{[\text { intJdnidnjuvrs }(1,1)} & \text { intJdnidnjuvrs }(1,2)] \\ {[\text { intJdnidnjuvrs }(2,1)} & \text { intJdnidnjuvrs }(2,2)]\end{array}\right)$

(I)Cubic order Serendipity Elements

Table-1a

intJdnidnjuvrs, $(\mathbf{i}=\mathbf{1}, \mathbf{j}=\mathbf{1}(\mathbf{1}) \mathbf{1 2})$

intJdn1dn1uvrs $=([$ 1.8785274341641741281222496546, 1.64496677534890315539341209663$]$

[ $1.64496677534890315539341209663,1.8785274341641741281222496546])$

intJdn1dn2uvrs $=([0.2439236805808402079811424029,0.2545641907595989129234164489] ;$

[ $0.1670641907595989129234164489,0.4941299057543551745397431605])$

intJdn1dn3uvrs $=([0.065143728569018129264176158009,-0.08063499940854168581340003167]$

[ - $-0.08063499940854168581340003167,0.065143728569018129264176158009])$

intJdn1dn4uvrs $=([0.4941299057543551745397431605,0.1670641907595989129234164489]$

[ $0.2545641907595989129234164489,0.2439236805808402079811424029])$

intJdn1dn5uvrs $=([-1.91709736541803286033938722609,-0.50073891821327672848240784486]$

[ - $-1.21323891821327672848240784486,-0.489515939250703449364685749])$

intJdn1dn6uvrs $=([0.377654219333168620525401790571,-0.07885728723191145226859121413]$

[ $0.22114271276808854773140878587,0.0471024620459754344696508823])$

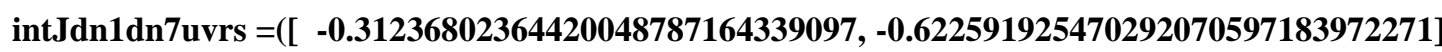

[ - -0.622591925470292070597183972271, $-\mathbf{- 0 . 9 3 2 6 4 1 1 6 7 0 5 1 0 6 7 1 6 8 0 5 0 2 9 5 5 1 2 7 9 ] )}$

intJdn1dn8uvrs $=([\mathbf{0 . 2 2 3 3 4 5 6 8 2 6 0 8 1 1 3 4 2 4 4 5 2 6 7 2 7 5 9 2 9 , 0 . 4 1 5 4 5 8 0 5 2 1 8 5 7 0 0 6 0 3 6 3 4 7 6 0 5 4 9 9 5 7 ]}]$

[ 0.415458052185700603634760549957, 0.3217953823083588462709750703$])$

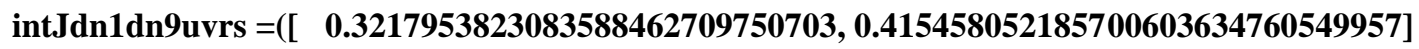

[ 0.415458052185700603634760549957, 0.22334568260811342445267275929])

intJdn1dn10uvrs $=([-\mathbf{0 . 9 3 2 6 4 1 1 6 7 0 5 1 0 6 7 1 6 8 0 5 0 2 9 5 5 1 2 7 9 ,},-\mathbf{0 . 6 2 2 5 9 1 9 2 5 4 7 0 2 9 2 0 7 0 5 9 7 1 8 3 9 7 2 2 7 1 ]}]$

[ - $\mathbf{0 . 6 2 2 5 9 1 9 2 5 4 7 0 2 9 2 0 7 0 5 9 7 1 8 3 9 7 2 2 7 1 , - 0 . 3 1 2 3 6 8 0 2 3 6 4 4 2 0 0 4 8 7 8 7 1 6 4 3 3 9 0 9 7 ] )}$

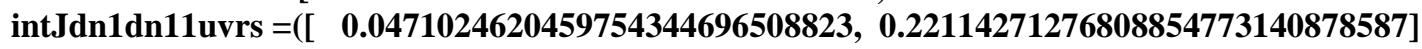

[ - $-\mathbf{0 . 0 7 8 8 5 7 2 8 7 2 3 1 9 1 1 4 5 2 2 6 8 5 9 1 2 1 4 1 3 , 0 . 3 7 7 6 5 4 2 1 9 3 3 3 1 6 8 6 2 0 5 2 5 4 0 1 7 9 0 5 7 1 ] )}$

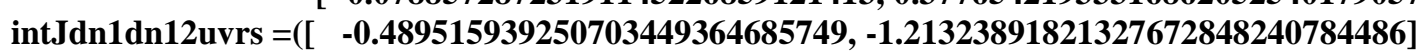

[ - $\mathbf{0 . 5 0 0 7 3 8 9 1 8 2 1 3 2 7 6 7 2 8 4 8 2 4 0 7 8 4 4 8 6 , - 1 . 9 1 7 0 9 7 3 6 5 4 1 8 0 3 2 8 6 0 3 3 9 3 8 7 2 2 6 0 9 ] )}$

Table-1b

intJdnidnjuvrs, $(\mathrm{i}=\mathbf{2}, \mathbf{j}=\mathbf{1}(\mathbf{1}) \mathbf{1 2})$

\begin{abstract}
intJdn2dn1uvrs $=([0.2439236805808402079811424029,0.1670641907595989129234164489]$
[ 0.2545641907595989129234164489, 0.4941299057543551745397431605])

intJdn2dn2uvrs $=([\mathbf{0 . 9 2 7 2 4 7 4 4 1 7 5 2 3 3 4 1 0 5 6 2 2 0 8 0 7 9 5 1 8 9 ,},-\mathbf{0 . 1 5 4 8 1 3 4 1 6 5 3 8 1 6 3 0 2 5 1 6 3 9 3 9 3 5 7 5 9 ]}$

[ -0.15481341653816302516393935759, 0.7947159164781167997293081128$])$

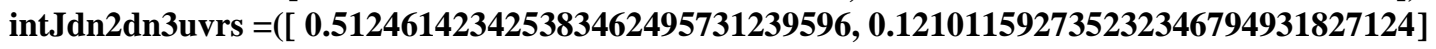

[ $0.033511592735232346794931827124,0.156606852059463586417315376026])$

int Jdn2dn4uvrs $=([\mathbf{0 . 1 6 9 8 5 3 3 9 9 9 2 7 1 2 6 7 7 7 4 3 5 0 4 1 9 5 1 3 6 , 0 . 1 7 9 0 4 5 0 5 5 4 5 0 0 0 2 3 2 6 9 5 7 8 3 1 4 6 2 7 4 4 ]}]$

[ 0.179045055450002326957831462744, 0.16985339992712677743504195136])

intJdn2dn5uvrs $=([0.737450284572716169349341246171,0.36072316479445118067281174443]$

[ $0.06072316479445118067281174443,0.0676185160002392642047802966]$

intJdn2dn6uvrs $=([-\mathbf{- 1 . 8 9 5 0 7 3 4 4 8 1 6 3 2 3 8 9 8 3 1 0 9 8 7 0 6 9 8 5 7 1},-\mathbf{- 0 . 3 2 9 4 7 0 5 9 8 7 6 1 6 1 3 1 6 8 6 8 5 7 5 1 9 4 3 3 3 ]}]$

[ $0.38302940123838683131424805667,-0.0610174037597342509867071996])$

intJdn2dn7uvrs $=([\mathbf{0 . 0 8 9 3 8 1 7 5 9 6 0 7 9 1 3 7 6 1 0 7 2 4 4 1 6 2 2 4 7 1 ,},-\mathbf{0 . 0 3 2 9 4 2 0 1 1 8 9 5 3 6 9 9 8 6 1 7 6 1 7 1 1 7 6 8}]$

[ - -0.7454420118953699861761711768, -1.32051059158026743632811545266])
\end{abstract}

H. T. Rathod, IJECS Volume 7 Issue 1 January 2018 Page No. 23329-23482 
intJdn2dn8uvrs $=([\mathbf{0 . 0 6 6 0 2 8 7 8 5 3 8 5 7 0 3 7 7 3 3 4 7 9 4 2 3 9 0 5 7 9 , 0 . 1 3 2 7 7 3 1 6 5 3 3 1 7 8 0 6 8 4 0 3 8 7 7 2 4 4 1 5 4 1}]$

[ $0.432773165331780684038772441541,0.364521033839059598529804469259])$ intJdn2dn9uvrs $=([-\mathbf{- 0 . 8 2 0 1 3 7 9 7 7 5 7 8 5 0 6 6 3 8 7 0 6 8 1 1 6 9 3 0 8 9}, \mathbf{0 . 1 0 2 2 8 3 0 3 5 3 2 7 1 0 4 6 3 5 4 0 6 1 6 0 2 3 9 3 1 8 6}]$ [ 0.1022830353271046354061602393186, 0.03640416697255654640867267668$])$

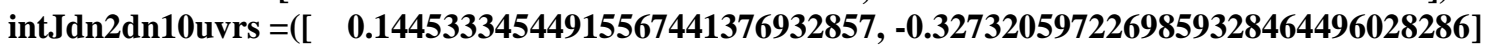
[ - $\mathbf{- 0 . 3 2 7 3 2 0 5 9 7 2 2 6 9 8 5 9 3 2 8 4 6 4 4 9 6 0 2 8 2 8 6 ,},-\mathbf{0 . 1 0 2 7 7 6 0 2 0 5 1 8 1 7 8 5 5 6 4 4 7 2 1 6 8 6 7 0 7 ] )}$

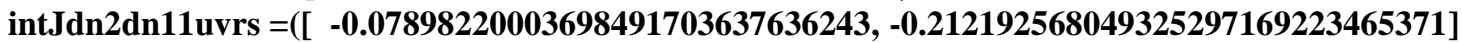
$[\mathbf{- 0 . 2 1 2 1 9 2 5 6 8 0 4 9 3 2 5 2 9 7 1 6 9 2 2 3 4 6 5 3 7 1 ,} \mathbf{0 . 0 6 2 3 6 4 7 5 8 0 4 8 8 0 3 6 2 4 9 7 6 1 2 5 5 2 7 3 7 1 ]})$

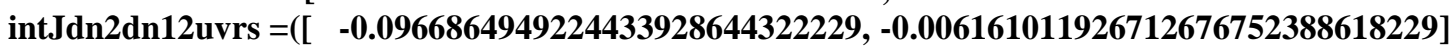
[ -0.006161011926712676752388618229, $-\mathbf{- 0 . 6 6 1 9 1 0 5 3 3 2 2 1 5 4 1 1 2 8 4 7 8 7 5 2 0 5 1 3 7 ] )}$

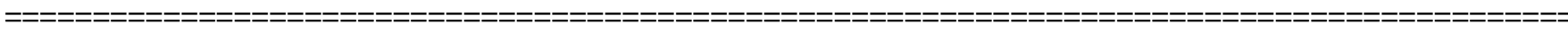

Table-1c

intJdnidnjuvrs, $(\mathrm{i}=\mathbf{3}, \mathrm{j}=\mathbf{1}(\mathbf{1}) \mathbf{1 2})$

intJdn3dn1uvrs $=([\mathbf{0 . 0 6 5 1 4 3 7 2 8 5 6 9 0 1 8 1 2 9 2 6 4 1 7 6 1 5 8 0 0 9 , - 0 . 0 8 0 6 3 4 9 9 9 4 0 8 5 4 1 6 8 5 8 1 3 4 0 0 0 3 1 6 6 9 ]}$

$[\mathbf{- 0 . 0 8 0 6 3 4 9 9 9 4 0 8 5 4 1 6 8 5 8 1 3 4 0 0 0 3 1 6 6 9 ,} \mathbf{0 . 0 6 5 1 4 3 7 2 8 5 6 9 0 1 8 1 2 9 2 6 4 1 7 6 1 5 8 0 0 9}])$

intJdn3dn2uvrs $=([\mathbf{0 . 5 1 2 4 6 1 4 2 3 4 2 5 3 8 3 4 6 2 4 9 5 7 3 1 2 3 9 5 9 6 , 0 . 0 3 3 5 1 1 5 9 2 7 3 5 2 3 2 3 4 6 7 9 4 9 3 1 8 2 7 1 2 4}]$

([ 0.121011592735232346794931827124, 0.156606852059463586417315376026])

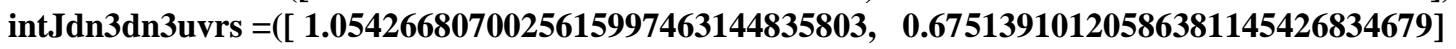

([ $\mathbf{0 . 6 7 5 1 3 9 1 0 1 2 0 5 8 6 3 8 1 1 4 5 4 2 6 8 3 4 6 7 9 , 1 . 0 5 4 2 6 6 8 0 7 0 0 2 5 6 1 5 9 9 7 4 6 3 1 4 4 8 3 5 8 0 3 ]}]$

intJdn3dn4uvrs $=([\mathbf{0 . 1 5 6 6 0 6 8 5 2 0 5 9 4 6 3 5 8 6 4 1 7 3 1 5 3 7 6 0 2 6}, \mathbf{0 . 1 2 1 0 1 1 5 9 2 7 3 5 2 3 2 3 4 6 7 9 4 9 3 1 8 2 7 1 2 4}]$

([ 0.033511592735232346794931827124, 0.512461423425383462495731239596])

intJdn3dn5uvrs $=([\mathbf{0 . 4 2 2 2 0 9 0 7 0 5 4 4 0 7 9 3 5 8 5 0 8 9 6 0 0 0 5 3 2 , 0 . 3 3 8 0 7 9 8 9 0 3 1 7 6 1 9 3 7 8 5 1 7 7 1 5 4 7 2 6 5 7}]$

([ $0.338079890317619378517715472657, \quad 0.1209239805970398938152641344])$

intJdn3dn6uvrs $=([-\mathbf{- 0 . 9 7 8 1 1 3 5 2 1 9 4 1 8 3 9 5 3 0 5 5 7 0 4 6 0 4 9 1 2}, \mathbf{- 0 . 2 7 1 0 7 5 4 5 9 7 0 5 4 7 9 5 2 3 5 0 7 6 4 7 9 0 5 6 2 1}]$

[ - -0.271075459705479523507647905621, $\mathbf{- 0 . 1 6 5 5 9 4 3 6 0 5 1 9 2 3 9 9 7 2 9 2 2 1 6 2 0 7 5 9 ] )}$

intJdn3dn7uvrs $=([\mathbf{- 0 . 0 9 7 4 5 1 5 6 5 5 0 7 8 6 6 3 3 1 9 8 2 0 9 9 3 9 1 9 0 9 ,}, \mathbf{- 0 . 0 0 2 5 3 8 1 0 3 9 5 5 5 8 6 3 8 0 3 3 8 6 9 2 2 4 7 5 3 9}]$

$[\mathbf{- 0 . 3 0 2 5 3 8 1 0 3 9 5 5 5 8 6 3 8 0 3 3 8 6 9 2 2 4 7 5 3 9 ,} \mathbf{0 . 4 6 3 4 4 7 5 5 8 5 3 3 2 1 3 6 1 4 6 9 6 6 8 0 0 8 9 1 6 6 ] )}$

intJdn3dn8uvrs $=([\mathbf{0 . 0 9 9 5 4 3 8 9 7 0 6 0 3 7 3 0 5 3 1 0 7 6 2 4 7 3 8 8 8 4}, \mathbf{- 0 . 6 4 5 2 2 9 9 7 0 2 9 0 4 4 6 8 8 4 2 8 6 7 4 1 3 0 4 2 6 7}]$

[ 0.067270029709553115713258695733, $-\mathbf{- 1 . 6 5 3 4 4 3 8 6 9 8 2 2 1 8 6 8 6 2 5 9 0 7 5 8 7 0 8 1 ] )}$

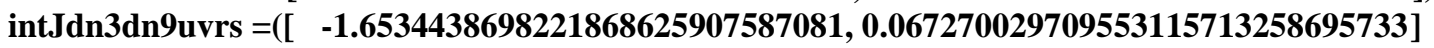

[ -0.645229970290446884286741304267, 0.099543897060373053107624738884])

intJdn3dn10uvrs $=([\mathbf{0 . 4 6 3 4 4 7 5 5 8 5 3 3 2 1 3 6 1 4 6 9 6 6 8 0 0 8 9 1 6 6},-\mathbf{- 0 . 3 0 2 5 3 8 1 0 3 9 5 5 5 8 6 3 8 0 3 3 8 6 9 2 2 4 7 5 3 9}]$

[ - $\mathbf{- 0 . 0 0 2 5 3 8 1 0 3 9 5 5 5 8 6 3 8 0 3 3 8 6 9 2 2 4 7 5 3 9 ,}-\mathbf{- 0 . 0 9 7 4 5 1 5 6 5 5 0 7 8 6 6 3 3 1 9 8 2 0 9 9 3 9 1 9 0 9 ] )}$

intJdn3dn11uvrs $=([-\mathbf{- 0 . 1 6 5 5 9 4 3 6 0 5 1 9 2 3 9 9 7 2 9 2 2 1 6 2 0 7 5 8 9 6},-\mathbf{- 0 . 2 7 1 0 7 5 4 5 9 7 0 5 4 7 9 5 2 3 5 0 7 6 4 7 9 0 5 6 2 1}]$

[ -0.271075459705479523507647905621, $-\mathbf{- 0 . 9 7 8 1 1 3 5 2 1 9 4 1 8 3 9 5 3 0 5 5 7 0 4 6 0 4 9 1 2 ] )}$

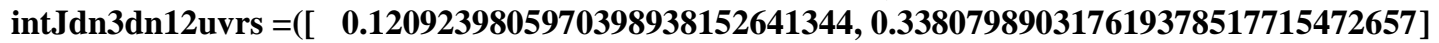

[ 0.338079890317619378517715472657, 0.42220907054407935850896000532])

Table-1d

intJdnidnjuvrs, $(i=4, j=1(1) 12)$

intJdn4dn1uvrs $=([0.4941299057543551745397431605,0.2545641907595989129234164489]$

[ 0.1670641907595989129234164489, 0.2439236805808402079811424029])

intJdn4dn2uvrs $=([\mathbf{0 . 1 6 9 8 5 3 3 9 9 9 2 7 1 2 6 7 7 7 4 3 5 0 4 1 9 5 1 3 6 , 0 . 1 7 9 0 4 5 0 5 5 4 5 0 0 0 2 3 2 6 9 5 7 8 3 1 4 6 2 7 4 4}]$

[ 0.179045055450002326957831462744, 0.16985339992712677743504195136$])$

intJdn4dn3uvrs $=([\mathbf{0 . 1 5 6 6 0 6 8 5 2 0 5 9 4 6 3 5 8 6 4 1 7 3 1 5 3 7 6 0 2 6}, \mathbf{0 . 0 3 3 5 1 1 5 9 2 7 3 5 2 3 2 3 4 6 7 9 4 9 3 1 8 2 7 1 2 4 ]}$

[ 0.121011592735232346794931827124, 0.512461423425383462495731239596])

intJdn4dn4uvrs $=([\mathbf{0 . 7 9 4 7 1 5 9 1 6 4 7 8 1 1 6 7 9 9 7 2 9 3 0 8 1 1 2 8 ,},-\mathbf{0 . 1 5 4 8 1 3 4 1 6 5 3 8 1 6 3 0 2 5 1 6 3 9 3 9 3 5 7 6}]$

[ -0.1548134165381630251639393576, 0.927247441752334105622080795189])

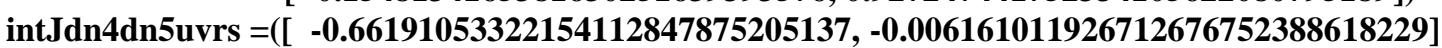

[ -0.006161011926712676752388618229, $\mathbf{- 0 . 0 9 6 6 8 6 4 9 4 9 2 2 4 4 3 3 9 2 8 6 4 4 3 2 2 2 2 9 ] )}$

intJdn4dn6uvrs $=([\mathbf{0 . 0 6 2 3 6 4 7 5 8 0 4 8 8 0 3 6 2 4 9 7 6 1 2 5 5 2 7 3 7 1},-\mathbf{- 0 . 2 1 2 1 9 2 5 6 8 0 4 9 3 2 5 2 9 7 1 6 9 2 2 3 4 6 5 3 7 1}]$

[ -0.212192568049325297169223465371, $-\mathbf{- 0 . 0 7 8 9 8 2 2 0 0 0 3 6 9 8 4 9 1 7 0 3 6 3 7 6 3 6 2 4 3 ] )}$

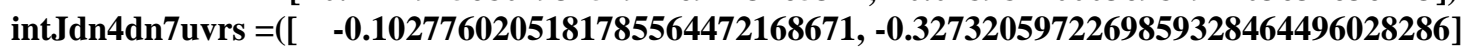

[ -0.3273205972269859328464496028286, 0.14453334544915567441376932857$])$

intJdn4dn8uvrs $=([\mathrm{O} \quad \mathbf{0 3 6 4 0 4 1 6 6 9 7 2 5 5 6 5 4 6 4 0 8 6 7 2 6 7 6 6 8 , 0 . 1 0 2 2 8 3 0 3 5 3 2 7 1 0 4 6 3 5 4 0 6 1 6 0 2 3 9 3 1 8 6}])$

[ 0.1022830353271046354061602393186, - -0.820137977578506638706811693089])

intJdn4dn9uvrs $=([0.364521033839059598529804469259,0.432773165331780684038772441541]$

[ $0.132773165331780684038772441541,0.066028785385703773347942390579])$

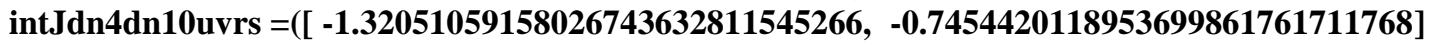

[ -0.0329420118953699861761711768, 0.089381759607913761072441622471])

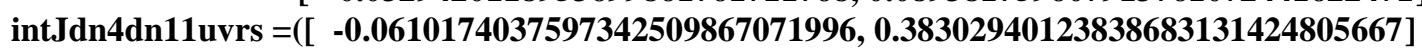

H. T. Rathod, IJECS Volume 7 Issue 1 January 2018 Page No. 23329-23482 
Table-1e

intJdnidnjuvrs, $(i=5, j=1(1) 12)$

intJdn5dn1uvrs $=([-\mathbf{- 1 . 9 1 7 0 9 7 3 6 5 4 1 8 0 3 2 8 6 0 3 3 9 3 8 7 2 2 6 0 9}, \mathbf{- 1 . 2 1 3 2 3 8 9 1 8 2 1 3 2 7 6 7 2 8 4 8 2 4 0 7 8 4 4 8 6}]$

[ -0.50073891821327672848240784486, $-\mathbf{- 0 . 4 8 9 5 1 5 9 3 9 2 5 0 7 0 3 4 4 9 3 6 4 6 8 5 7 4 9 ] )}$

intJdn5dn2uvrs $=([\mathbf{0 . 7 3 7 4 5 0 2 8 4 5 7 2 7 1 6 1 6 9 3 4 9 3 4 1 2 4 6 1 7 1}, \mathbf{0 . 0 6 0 7 2 3 1 6 4 7 9 4 4 5 1 1 8 0 6 7 2 8 1 1 7 4 4 4 3}]$

[ $0.36072316479445118067281174443,0.0676185160002392642047802966])$

intJdn5dn3uvrs $=([\mathbf{0 . 4 2 2 2 0 9 0 7 0 5 4 4 0 7 9 3 5 8 5 0 8 9 6 0 0 0 5 3 2}, \mathbf{0 . 3 3 8 0 7 9 8 9 0 3 1 7 6 1 9 3 7 8 5 1 7 7 1 5 4 7 2 6 5 7}]$

[ 0.338079890317619378517715472657, $\mathbf{0 . 1 2 0 9 2 3 9 8 0 5 9 7 0 3 9 8 9 3 8 1 5 2 6 4 1 3 4 4 ] )}$

intJdn5dn4uvrs $=([-\mathbf{- 0 . 6 6 1 9 1 0 5 3 3 2 2 1 5 4 1 1 2 8 4 7 8 7 5 2 0 5 1 3 7},-\mathbf{- 0 . 0 0 6 1 6 1 0 1 1 9 2 6 7 1 2 6 7 6 7 5 2 3 8 8 6 1 8 2 2 9}]$

[ -0.006161011926712676752388618229, $-\mathbf{- 0 . 0 9 6 6 8 6 4 9 4 9 2 2 4 4 3 3 9 2 8 6 4 4 3 2 2 2 2 9 ]})$

intJdn5dn5uvrs $=([$ 4.20027429051479081211473647017, 1.3438679189477808452044247751$]$

[ $1.3438679189477808452044247751,0.831814504364695263991287102])$

intJdn5dn6uvrs $=([-\mathbf{- 2 . 9 9 7 0 2 4 9 4 4 5 1 0 1 0 2 4 2 3 8 3 2 6 6 7 1 0 9 8 9}, \mathbf{- 0 . 1 3 4 9 8 7 1 9 1 9 1 6 7 0 2 5 5 4 8 7 4 6 1 6 0 2 1}]$

[ -1.147487191916702554874616021, - -0.265306180626066014206434443])

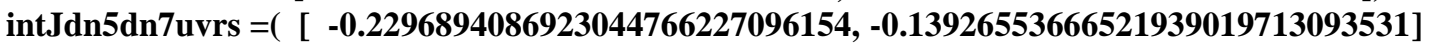

[ -0.13926553666521939019713093531, - -0.03285352909863772097735829779])

intJdn5dn8uvrs $=([-\mathbf{- 0 . 0 3 6 0 8 0 2 5 6 6 5 3 8 6 6 0 3 2 2 6 5 3 9 4 0 8 3 5 1}, \mathbf{- 0 . 1 5 3 9 9 9 6 6 4 4 9 1 3 1 7 5 4 8 3 9 7 9 4 3 0 1 5 2}]$

[ - -0.1539996644913175483979430152, $\mathbf{- 0 . 0 6 8 2 9 7 3 2 0 3 9 0 7 1 6 3 5 8 1 6 8 3 5 1 3 3 0 8 ] )}$

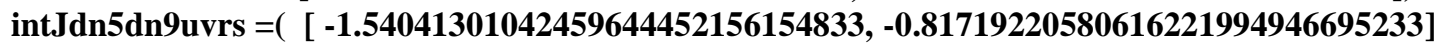

[ -0.81719220580616221994946695233, - $-\mathbf{0 . 2 6 5 8 1 0 8 2 5 9 5 3 6 6 9 1 1 4 7 5 3 5 6 4 2 7 5 3 ] )}$

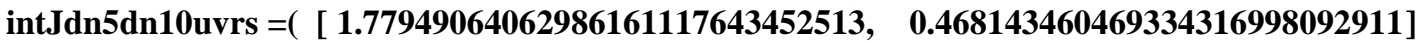

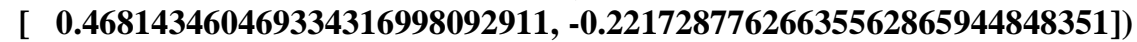

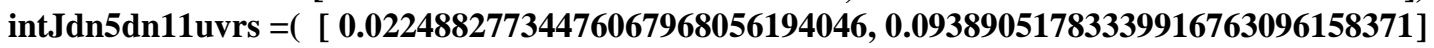

[ 0.09389051783339916763096158371, 0.199539110232382521752505822$])$

intJdn5dn12uvrs $=([\mathbf{0 . 2 2 0 3 0 2 9 5 5 3 1 4 2 3 4 7 3 5 2 3 0 4 3 7 4 4 6 7 , 0 . 1 6 0 1 3 9 5 7 6 6 5 6 8 0 6 2 2 9 6 2 9 9 4 6 8 9 9 1 9}]$

[ 0.16013957665680622962994689919, 0.2203029553142347352304374467])

Table-1f

intJdnidnjuvrs, $i=6, j=1$ (1)12)

intJdn6dn1uvrs $=([\mathbf{0 . 3 7 7 6 5 4 2 1 9 3 3 3 1 6 8 6 2 0 5 2 5 4 0 1 7 9 0 5 7 1}, \mathbf{0 . 2 2 1 1 4 2 7 1 2 7 6 8 0 8 8 5 4 7 7 3 1 4 0 8 7 8 5 8 7}]$ [ - $\mathbf{0 . 0 7 8 8 5 7 2 8 7 2 3 1 9 1 1 4 5 2 2 6 8 5 9 1 2 1 4 1 3 , 0 . 0 4 7 1 0 2 4 6 2 0 4 5 9 7 5 4 3 4 4 6 9 6 5 0 8 8 2 3 ]})$

intJdn6dn2uvrs $=([-\mathbf{~} \mathbf{1 . 8 9 5 0 7 3 4 4 8 1 6 3 2 3 8 9 8 3 1 0 9 8 7 0 6 9 8 5 7 1}, \mathbf{0 . 3 8 3 0 2 9 4 0 1 2 3 8 3 8 6 8 3 1 3 1 4 2 4 8 0 5 6 6 7}]$ [ - -0.32947059876161316868575194333, - 0.0610174037597342509867071996$])$

intJdn6dn3uvrs $=([-\mathbf{- 0 . 9 7 8 1 1 3 5 2 1 9 4 1 8 3 9 5 3 0 5 5 7 0 4 6 0 4 9 1 2 ,}, \mathbf{- 0 . 2 7 1 0 7 5 4 5 9 7 0 5 4 7 9 5 2 3 5 0 7 6 4 7 9 0 5 6 2 1}]$ [ -0.271075459705479523507647905621, $\mathbf{- 0 . 1 6 5 5 9 4 3 6 0 5 1 9 2 3 9 9 7 2 9 2 2 1 6 2 0 7 5 9 ] )}$

intJdn6dn4uvrs $=([\mathbf{0 . 0 6 2 3 6 4 7 5 8 0 4 8 8 0 3 6 2 4 9 7 6 1 2 5 5 2 7 3 7 1},-\mathbf{- 0 . 2 1 2 1 9 2 5 6 8 0 4 9 3 2 5 2 9 7 1 6 9 2 2 3 4 6 5 3 7 1}]$ [ -0.212192568049325297169223465371, - -0.07898220003698491703637636243])

intJdn6dn5uvrs $=([-\mathbf{- 2 . 9 9 7 0 2 4 9 4 4 5 1 0 1 0 2 4 2 3 8 3 2 6 6 7 1 0 9 8 9},-\mathbf{1 . 1 4 7 4 8 7 1 9 1 9 1 6 7 0 2 5 5 4 8 7 4 6 1 6 0 2 1}]$ [ - $\mathbf{- 0 . 1 3 4 9 8 7 1 9 1 9 1 6 7 0 2 5 5 4 8 7 4 6 1 6 0 2 1 , - 0 . 2 6 5 3 0 6 1 8 0 6 2 6 0 6 6 0 1 4 2 0 6 4 3 4 4 4 3 ] )}$

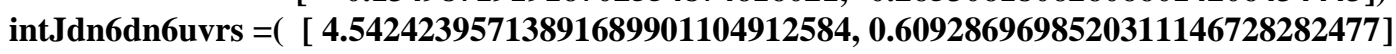
[ $0.6092869698520311146728282477,0.443331612234228602914621619])$

intJdn6dn7uvrs $=([-\mathbf{- 0 . 2 1 8 3 8 7 5 0 8 3 3 0 3 6 3 0 2 3 6 6 6 0 0 3 8 2 1 9 7},-\mathbf{- 0 . 1 0 1 4 6 0 4 7 7 1 6 9 1 0 2 5 7 9 7 9 5 8 4 3 9 3 4 3}]$ [ - -0.1014604771691025797958439343, 0.07828128386135153260806627353])

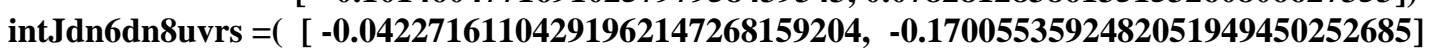
[ - $\mathbf{- 0 . 1 7 0 0 5 5 3 5 9 2 4 8 2 0 5 1 9 4 9 4 5 0 2 5 2 6 8 5 , 0 . 0 6 6 2 9 6 4 3 3 8 0 4 1 0 3 0 6 4 9 4 6 3 7 8 5 1 0 3 4 3 ] )}$ intJdn6dn9uvrs $=([\mathbf{1 . 9 8 5 8 5 5 3 6 5 2 7 4 8 0 8 4 7 3 3 7 2 1 2 6 6 0 5 9 5 7}, \mathbf{0 . 1 7 4 9 5 7 0 8 4 5 6 3 1 3 2 4 4 0 1 3 2 0 3 9 5 1 6 8 3}]$

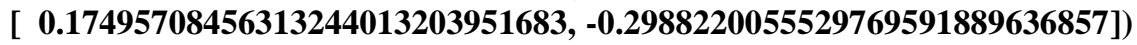
intJdn6dn10uvrs $=([-\mathbf{- 1 . 0 7 1 2 1 7 4 3 3 0 9 5 0 8 0 4 6 6 4 8 9 3 1 2 9 6 3 5}, \mathbf{0 . 2 9 3 7 8 4 0 8 6 6 9 7 6 3 1 7 5 3 0 0 6 2 2 3 3 9 9 9 4}]$ [ 0.29378408669763175300622339994, 0.1779710241491188902309911789]) intJdn6dn11uvrs $=([\mathbf{0 . 0 3 4 2 5 1 0 5 7 0 5 5 4 6 3 9 0 9 4 9 0 3 7 3 3 6 3 4 1 , 0 . 1 2 6 1 8 0 2 8 3 1 3 6 1 4 5 2 9 5 8 0 4 6 4 7 0 0 2 7 4}]$ [ 0.12618028313614529580464700274, 0.03425105705546390949037336341])

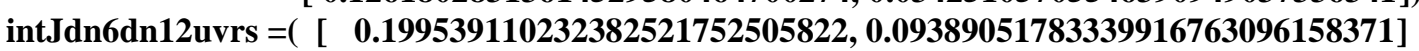
[ 0.09389051783339916763096158371, 0.02248827734476067968056194046]) 


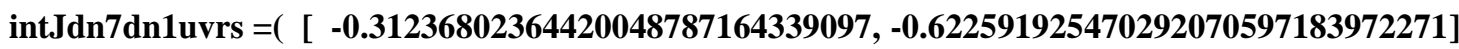
[ - -0.622591925470292070597183972271, $-\mathbf{- 0 . 9 3 2 6 4 1 1 6 7 0 5 1 0 6 7 1 6 8 0 5 0 2 9 5 5 1 2 7 9 ] )}$

intJdn7dn2uvrs $=([0.089381759607913761072441622471,-0.7454420118953699861761711768]$ [ - -0.0329420118953699861761711768, - 1.32051059158026743632811545266$])$ intJdn7dn3uvrs $=([-\mathbf{- 0 . 0 9 7 4 5 1 5 6 5 5 0 7 8 6 6 3 3 1 9 8 2 0 9 9 3 9 1 9 0 9},-\mathbf{- 0 . 3 0 2 5 3 8 1 0 3 9 5 5 5 8 6 3 8 0 3 3 8 6 9 2 2 4 7 5 3 9}]$ [ - $\mathbf{- 0 . 0 0 2 5 3 8 1 0 3 9 5 5 5 8 6 3 8 0 3 3 8 6 9 2 2 4 7 5 3 9 ,} \mathbf{0 . 4 6 3 4 4 7 5 5 8 5 3 3 2 1 3 6 1 4 6 9 6 6 8 0 0 8 9 1 6 6 ] )}$

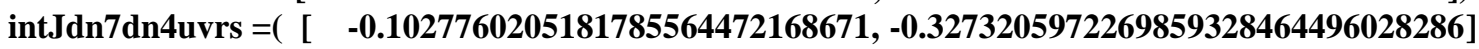
[ - $0.3273205972269859328464496028286, \quad 0.14453334544915567441376932857])$ intJdn7dn5uvrs $=([-\mathbf{- 0 . 2 2 9 6 8 9 4 0 8 6 9 2 3 0 4 4 7 6 6 2 2 7 0 9 6 1 5 4 ,},-\mathbf{0 . 1 3 9 2 6 5 5 3 6 6 6 5 2 1 9 3 9 0 1 9 7 1 3 0 9 3 5 3 1}]$ [ - -0.13926553666521939019713093531, - -0.03285352909863772097735829779]) intJdn7dn6uvrs $=([-\mathbf{- 0 . 2 1 8 3 8 7 5 0 8 3 3 0 3 6 3 0 2 3 6 6 6 0 0 3 8 2 1 9 7}, \mathbf{- 0 . 1 0 1 4 6 0 4 7 7 1 6 9 1 0 2 5 7 9 7 9 5 8 4 3 9 3 4 3}]$ [ - - $0.1014604771691025797958439343,0.07828128386135153260806627353])$

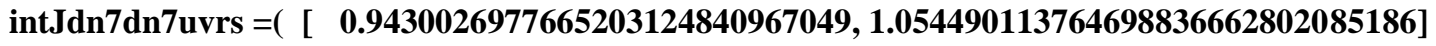
[ 1 .054490113764698836662802085186, 3.17595231695266811253589555038])

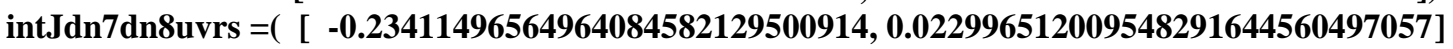
[ - -0.989503487990451708355439502943, - 2.31569161290561082190356514273$])$ intJdn7dn9uvrs $=([0.10963550129394871861715141079,0.19369458821691485109794452082]$ [ 0.19369458821691485109794452082, - -0.06531609748699460034793405982])

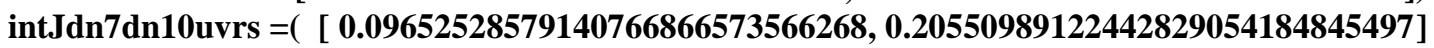
[ $0.20550989122442829054184845497,0.09652528579140766866573566268])$ intJdn7dn11uvrs $=([\mathbf{0 . 1 7 7 9 7 1 0 2 4 1 4 9 1 1 8 8 9 0 2 3 0 9 9 1 1 7 8 9 , 0 . 2 9 3 7 8 4 0 8 6 6 9 7 6 3 1 7 5 3 0 0 6 2 2 3 3 9 9 9 4}]$ [ 0.29378408669763175300622339994, -1.0712174330950804664893129635])

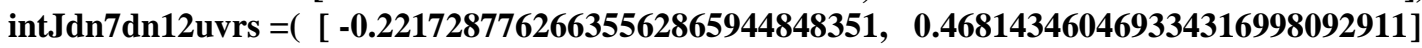
[ $0.468143460469334316998092911,1.77949064062986161117643452513])$

Table-1h

intJdnidnjuvrs, $\mathrm{i}=\mathbf{8}, \mathrm{j}=\mathbf{1}(\mathbf{1}) 12)$

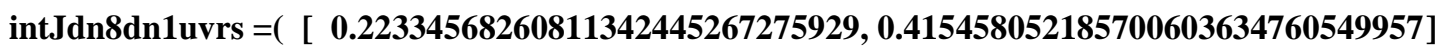
[ $0.415458052185700603634760549957,0.3217953823083588462709750703])$ intJdn8dn2uvrs $=([0.066028785385703773347942390579,0.432773165331780684038772441541]$ [ 0.132773165331780684038772441541, 0.364521033839059598529804469259]) intJdn8dn3uvrs $=([$ 0.099543897060373053107624738884, 0.067270029709553115713258695733$]$ [ - $\mathbf{0 . 6 4 5 2 2 9 9 7 0 2 9 0 4 4 6 8 8 4 2 8 6 7 4 1 3 0 4 2 6 7 , - 1 . 6 5 3 4 4 3 8 6 9 8 2 2 1 8 6 8 6 2 5 9 0 7 5 8 7 0 8 1 ] )}$

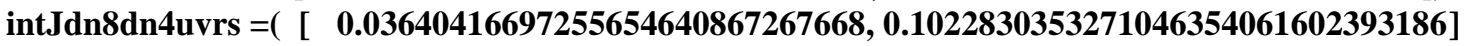
[ 0.1022830353271046354061602393186, -0.820137977578506638706811693089]) intJdn8dn5uvrs $=([-\mathbf{- 0 . 0 3 6 0 8 0 2 5 6 6 5 3 8 6 6 0 3 2 2 6 5 3 9 4 0 8 3 5 1},-\mathbf{0 . 1 5 3 9 9 9 6 6 4 4 9 1 3 1 7 5 4 8 3 9 7 9 4 3 0 1 5 2}]$ [ - -0.1539996644913175483979430152, $-\mathbf{0 . 0 6 8 2 9 7 3 2 0 3 9 0 7 1 6 3 5 8 1 6 8 3 5 1 3 3 0 8 ] )}$ intJdn8dn6uvrs $=([-\mathbf{- 0 . 0 4 2 2 7 1 6 1 1 0 4 2 9 1 9 6 2 1 4 7 2 6 8 1 5 9 2 0 4},-\mathbf{- 0 . 1 7 0 0 5 5 3 5 9 2 4 8 2 0 5 1 9 4 9 4 5 0 2 5 2 6 8 5}]$ [ - $-\mathbf{0 . 1 7 0 0 5 5 3 5 9 2 4 8 2 0 5 1 9 4 9 4 5 0 2 5 2 6 8 5 , 0 . 0 6 6 2 9 6 4 3 3 8 0 4 1 0 3 0 6 4 9 4 6 3 7 8 5 1 0 3 4 3 ] )}$ intJdn8dn7uvrs $=([-\mathbf{0 . 2 3 4 1 1 4 9 6 5 6 4 9 6 4 0 8 4 5 8 2 1 2 9 5 0 0 9 1 4},-\mathbf{- 0 . 9 8 9 5 0 3 4 8 7 9 9 0 4 5 1 7 0 8 3 5 5 4 3 9 5 0 2 9 4 3}]$ [ 0.022996512009548291644560497057, - -2.31569161290561082190356514273]) intJdn8dn8uvrs $=([$ [ $0.57399287653962671437054925866,0.510536435455242956618237924447]$ [ 0.510536435455242956618237924447, 3.606779720827644761992241475244]) intJdn8dn9uvrs $=([-\mathbf{- 0 . 0 5 6 8 9 9 6 4 6 2 2 6 3 0 6 3 3 7 8 3 7 6 2 9 1 1 8 9 , 0 . 2 3 3 7 7 8 3 2 6 7 4 6 7 0 7 3 8 5 0 0 6 7 0 0 8 5 0 1 8}]$ [ 0.23377832674670738500670085018, -0.0568996462263063378376291189]) intJdn8dn10uvrs $=([-\mathbf{- 0 . 0 6 5 3 1 6 0 9 7 4 8 6 9 9 4 6 0 0 3 4 7 9 3 4 0 5 9 8 2}, \mathbf{0 . 1 9 3 6 9 4 5 8 8 2 1 6 9 1 4 8 5 1 0 9 7 9 4 4 5 2 0 8 2}]$ [ 0.19369458821691485109794452082, 0.10963550129394871861715141079]) intJdn8dn11uvrs $=([-\mathbf{- 0 . 2 9 8 8 2 2 0 0 5 5 5 2 9 7 6 9 5 9 1 8 8 9 6 3 6 8 5 7}, \mathbf{0 . 1 7 4 9 5 7 0 8 4 5 6 3 1 3 2 4 4 0 1 3 2 0 3 9 5 1 6 8 3 ]}]$ [ $0.17495708456313244013203951683,1.985855365274808473372126605957])$ intJdn8dn12uvrs $=([-\mathbf{0 . 2 6 5 8 1 0 8 2 5 9 5 3 6 6 9 1 1 4 7 5 3 5 6 4 2 7 5 3 3},-\mathbf{- 0 . 8 1 7 1 9 2 2 0 5 8 0 6 1 6 2 2 1 9 9 4 9 4 6 6 9 5 2 3 3}]$ [ - $\mathbf{0 . 8 1 7 1 9 2 2 0 5 8 0 6 1 6 2 2 1 9 9 4 9 4 6 6 9 5 2 3 3},-\mathbf{1 . 5 4 0 4 1 3 0 1 0 4 2 4 5 9 6 4 4 4 5 2 1 5 6 1 5 4 8 3 3 ] )}$

Table-1i

intJdnidnjuvrs, $\mathrm{i}=\mathbf{9}, \mathrm{j}=\mathbf{1}(\mathbf{1}) 12)$ 
[ $\mathbf{0 . 4 1 5 4 5 8 0 5 2 1 8 5 7 0 0 6 0 3 6 3 4 7 6 0 5 4 9 9 5 7 ,} \mathbf{0 . 2 2 3 3 4 5 6 8 2 6 0 8 1 1 3 4 2 4 4 5 2 6 7 2 7 5 9 2 9 ]})$

intJdn9dn2uvrs $=([-\mathbf{- 0 . 8 2 0 1 3 7 9 7 7 5 7 8 5 0 6 6 3 8 7 0 6 8 1 1 6 9 3 0 8 9}, \mathbf{0 . 1 0 2 2 8 3 0 3 5 3 2 7 1 0 4 6 3 5 4 0 6 1 6 0 2 3 9 3 1 8 6}]$

[ 0.1022830353271046354061602393186, 0.03640416697255654640867267668$])$

intJdn9dn3uvrs $=([-\mathbf{- 1 . 6 5 3 4 4 3 8 6 9 8 2 2 1 8 6 8 6 2 5 9 0 7 5 8 7 0 8 1}, \mathbf{- 0 . 6 4 5 2 2 9 9 7 0 2 9 0 4 4 6 8 8 4 2 8 6 7 4 1 3 0 4 2 6 7}]$

[ 0.067270029709553115713258695733, 0.099543897060373053107624738884])

intJdn9dn4uvrs $=([\mathbf{0 . 3 6 4 5 2 1 0 3 3 8 3 9 0 5 9 5 9 8 5 2 9 8 0 4 4 6 9 2 5 9 , 0 . 1 3 2 7 7 3 1 6 5 3 3 1 7 8 0 6 8 4 0 3 8 7 7 2 4 4 1 5 4 1}]$

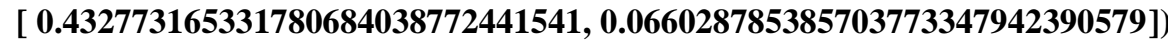

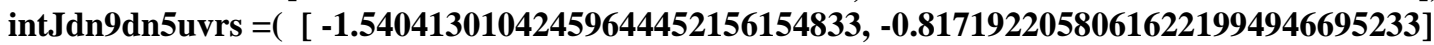

[ -0.81719220580616221994946695233, - $\mathbf{- 0 . 2 6 5 8 1 0 8 2 5 9 5 3 6 6 9 1 1 4 7 5 3 5 6 4 2 7 5 3 ] )}$

intJdn9dn6uvrs $=([\mathbf{1 . 9 8 5 8 5 5 3 6 5 2 7 4 8 0 8 4 7 3 3 7 2 1 2 6 6 0 5 9 5 7}, \mathbf{0 . 1 7 4 9 5 7 0 8 4 5 6 3 1 3 2 4 4 0 1 3 2 0 3 9 5 1 6 8 3}]$

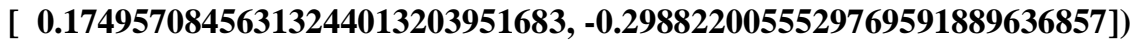

intJdn9dn7uvrs $=([\mathbf{0 . 1 0 9 6 3 5 5 0 1 2 9 3 9 4 8 7 1 8 6 1 7 1 5 1 4 1 0 7 9}, \mathbf{0 . 1 9 3 6 9 4 5 8 8 2 1 6 9 1 4 8 5 1 0 9 7 9 4 4 5 2 0 8 2}]$

[ 0.19369458821691485109794452082, -0.06531609748699460034793405982])

intJdn9dn8uvrs $=([\mathbf{- 0 . 0 5 6 8 9 9 6 4 6 2 2 6 3 0 6 3 3 7 8 3 7 6 2 9 1 1 8 9 , 0 . 2 3 3 7 7 8 3 2 6 7 4 6 7 0 7 3 8 5 0 0 6 7 0 0 8 5 0 1 8}]$

[ 0.23377832674670738500670085018, - $\mathbf{0 . 0 5 6 8 9 9 6 4 6 2 2 6 3 0 6 3 3 7 8 3 7 6 2 9 1 1 8 9 ] )}$

intJdn9dn9uvrs $=([$ 3.606779720827644761992241475244, 0.510536435455242956618237924447]

[ 0.510536435455242956618237924447, 0.57399287653962671437054925866$])$

intJdn9dn10uvrs $=([-\mathbf{- 2 . 3 1 5 6 9 1 6 1 2 9 0 5 6 1 0 8 2 1 9 0 3 5 6 5 1 4 2 7 2 7}, \mathbf{0 . 0 2 2 9 9 6 5 1 2 0 0 9 5 4 8 2 9 1 6 4 4 5 6 0 4 9 7 0 5 7}]$

[ - $\mathbf{- 0 . 9 8 9 5 0 3 4 8 7 9 9 0 4 5 1 7 0 8 3 5 5 4 3 9 5 0 2 9 4 3 , - 0 . 2 3 4 1 1 4 9 6 5 6 4 9 6 4 0 8 4 5 8 2 1 2 9 5 0 0 9 1 4 ] )}$

intJdn9dn11uvrs $=([\mathbf{0 . 0 6 6 2 9 6 4 3 3 8 0 4 1 0 3 0 6 4 9 4 6 3 7 8 5 1 0 3 4 3},-\mathbf{- 0 . 1 7 0 0 5 5 3 5 9 2 4 8 2 0 5 1 9 4 9 4 5 0 2 5 2 6 8 5}]$

[ -0.1700553592482051949450252685, -0.04227161104291962147268159204])

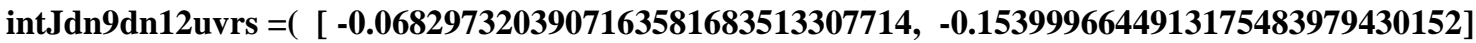

[ -0.1539996644913175483979430152, -0.03608025665386603226539408351])

Table-1j

intJdnidnjuvrs, $\mathrm{i}=\mathbf{1 0}, \mathrm{j}=\mathbf{1}(\mathbf{1}) 12)$

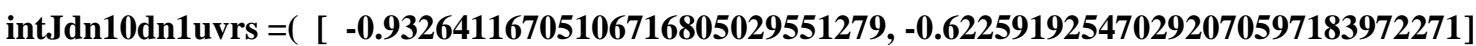

[ -0.622591925470292070597183972271, $-\mathbf{- 0 . 3 1 2 3 6 8 0 2 3 6 4 4 2 0 0 4 8 7 8 7 1 6 4 3 3 9 0 9 7 ] )}$

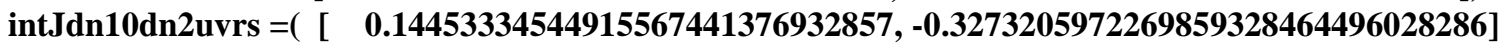
[ -0.3273205972269859328464496028286, $-\mathbf{- 0 . 1 0 2 7 7 6 0 2 0 5 1 8 1 7 8 5 5 6 4 4 7 2 1 6 8 6 7 0 7 ] )}$

intJdn10dn3uvrs $=([\mathrm{0}$.463447558533213614696680089166, $\mathbf{- 0 . 0 0 2 5 3 8 1 0 3 9 5 5 5 8 6 3 8 0 3 3 8 6 9 2 2 4 7 5 3 9}]$ [ - $\mathbf{0 . 3 0 2 5 3 8 1 0 3 9 5 5 5 8 6 3 8 0 3 3 8 6 9 2 2 4 7 5 3 9 ,}-\mathbf{- 0 . 0 9 7 4 5 1 5 6 5 5 0 7 8 6 6 3 3 1 9 8 2 0 9 9 3 9 1 9 0 9 ]})$

intJdn10dn4uvrs $=([-\mathbf{- 1 . 3 2 0 5 1 0 5 9 1 5 8 0 2 6 7 4 3 6 3 2 8 1 1 5 4 5 2 6 6},-\mathbf{- 0 . 0 3 2 9 4 2 0 1 1 8 9 5 3 6 9 9 8 6 1 7 6 1 7 1 1 7 6 8}]$

[ - $\mathbf{0 . 7 4 5 4 4 2 0 1 1 8 9 5 3 6 9 9 8 6 1 7 6 1 7 1 1 7 6 8 , 0 . 0 8 9 3 8 1 7 5 9 6 0 7 9 1 3 7 6 1 0 7 2 4 4 1 6 2 2 4 7 1 ] )}$

intJdn10dn5uvrs $=\left(\left[\begin{array}{l}1.77949064062986161117643452513\end{array}, \quad \mathbf{0 . 4 6 8 1 4 3 4 6 0 4 6 9 3 3 4 3 1 6 9 9 8 0 9 2 9 1 1}\right]\right.$

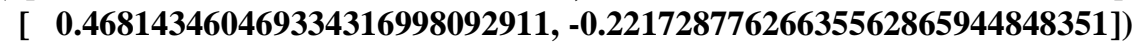

intJdn10dn6uvrs $=([-\mathbf{- 1 . 0 7 1 2 1 7 4 3 3 0 9 5 0 8 0 4 6 6 4 8 9 3 1 2 9 6 3 5}, \mathbf{0 . 2 9 3 7 8 4 0 8 6 6 9 7 6 3 1 7 5 3 0 0 6 2 2 3 3 9 9 9 4}]$

[ 0.29378408669763175300622339994, 0.1779710241491188902309911789])

intJdn10dn7uvrs $=([\mathbf{0 . 0 9 6 5 2 5 2 8 5 7 9 1 4 0 7 6 6 8 6 6 5 7 3 5 6 6 2 6 8}, \mathbf{0 . 2 0 5 5 0 9 8 9 1 2 2 4 4 2 8 2 9 0 5 4 1 8 4 8 4 5 4 9 7}]$

[ 0.20550989122442829054184845497, 0.09652528579140766866573566268])

intJdn10dn8uvrs $=([-\mathbf{- 0 . 0 6 5 3 1 6 0 9 7 4 8 6 9 9 4 6 0 0 3 4 7 9 3 4 0 5 9 8 2 , 0 . 1 9 3 6 9 4 5 8 8 2 1 6 9 1 4 8 5 1 0 9 7 9 4 4 5 2 0 8 2}]$

[ $0.19369458821691485109794452082,0.10963550129394871861715141079])$

intJdn10dn9uvrs $=([-\mathbf{- 2 . 3 1 5 6 9 1 6 1 2 9 0 5 6 1 0 8 2 1 9 0 3 5 6 5 1 4 2 7 2 7},-\mathbf{- 0 . 9 8 9 5 0 3 4 8 7 9 9 0 4 5 1 7 0 8 3 5 5 4 3 9 5 0 2 9 4 3}]$

[ $0.022996512009548291644560497057,-0.23411496564964084582129500914])$

intJdn10dn10uvrs $=([$ 3.17595231695266811253589555038, 1.054490113764698836662802085186$]$ [ 1.054490113764698836662802085186, 0.94300269776652031248409670487$])$

intJdn10dn11uvrs $=([\mathbf{0 . 0 7 8 2 8 1 2 8 3 8 6 1 3 5 1 5 3 2 6 0 8 0 6 6 2 7 3 5 3},-\mathbf{- 0 . 1 0 1 4 6 0 4 7 7 1 6 9 1 0 2 5 7 9 7 9 5 8 4 3 9 3 4 3}]$ [ -0.1014604771691025797958439343, - - $\mathbf{- 2 1 8 3 8 7 5 0 8 3 3 0 3 6 3 0 2 3 6 6 6 0 0 3 8 2 1 9 7 ] )}$

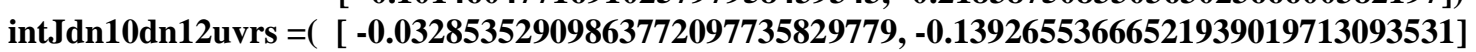

[ -0.13926553666521939019713093531, - - 0.2296894086923044766227096154$])$

Table-1k

intJdnidnjuvrs, $\mathrm{i}=\mathbf{1 1}, \mathrm{j}=\mathbf{1}(\mathbf{1}) 12)$

intJdn11dn1uvrs $=([\mathbf{0 . 0 4 7 1 0 2 4 6 2 0 4 5 9 7 5 4 3 4 4 6 9 6 5 0 8 8 2 3},-\mathbf{- 0 . 0 7 8 8 5 7 2 8 7 2 3 1 9 1 1 4 5 2 2 6 8 5 9 1 2 1 4 1 3}]$

[ 0.22114271276808854773140878587, 0.377654219333168620525401790571])

intJdn11dn2uvrs $=([-\mathbf{- 0 . 0 7 8 9 8 2 2 0 0 0 3 6 9 8 4 9 1 7 0 3 6 3 7 6 3 6 2 4 3 ,}, \mathbf{- 0 . 2 1 2 1 9 2 5 6 8 0 4 9 3 2 5 2 9 7 1 6 9 2 2 3 4 6 5 3 7 1}]$

[ -0.212192568049325297169223465371, 0.062364758048803624976125527371])

intJdn11dn3uvrs $=([\mathbf{- 0 . 1 6 5 5 9 4 3 6 0 5 1 9 2 3 9 9 7 2 9 2 2 1 6 2 0 7 5 8 9 6}, \mathbf{- 0 . 2 7 1 0 7 5 4 5 9 7 0 5 4 7 9 5 2 3 5 0 7 6 4 7 9 0 5 6 2 1}]$

[ -0.271075459705479523507647905621, $-\mathbf{- 0 . 9 7 8 1 1 3 5 2 1 9 4 1 8 3 9 5 3 0 5 5 7 0 4 6 0 4 9 1 2 ] )}$

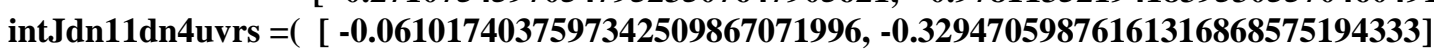

[ 0.38302940123838683131424805667, -1.8950734481632389831098706986])

H. T. Rathod, IJECS Volume 7 Issue 1 January 2018 Page No. 23329-23482 
intJdn11dn5uvrs $=([\mathbf{0 . 0 2 2 4 8 8 2 7 7 3 4 4 7 6 0 6 7 9 6 8 0 5 6 1 9 4 0 4 6 , 0 . 0 9 3 8 9 0 5 1 7 8 3 3 3 9 9 1 6 7 6 3 0 9 6 1 5 8 3 7 1}]$ [ 0.09389051783339916763096158371, 0.199539110232382521752505822$])$

intJdn11dn6uvrs $=([\mathbf{0 . 0 3 4 2 5 1 0 5 7 0 5 5 4 6 3 9 0 9 4 9 0 3 7 3 3 6 3 4 1}, \mathbf{0 . 1 2 6 1 8 0 2 8 3 1 3 6 1 4 5 2 9 5 8 0 4 6 4 7 0 0 2 7 4}]$ [ 0.12618028313614529580464700274, 0.03425105705546390949037336341]) intJdn11dn7uvrs $=\left(\left[\begin{array}{l}0.1779710241491188902309911789,0.29378408669763175300622339994\end{array}\right]\right.$ [ 0.29378408669763175300622339994, - -1.0712174330950804664893129635])

intJdn11dn8uvrs $=([-\mathbf{- 0 . 2 9 8 8 2 2 0 0 5 5 5 2 9 7 6 9 5 9 1 8 8 9 6 3 6 8 5 7}, \mathbf{0 . 1 7 4 9 5 7 0 8 4 5 6 3 1 3 2 4 4 0 1 3 2 0 3 9 5 1 6 8 3}]$ [ $0.17495708456313244013203951683,1.985855365274808473372126605957])$ intJdn11dn9uvrs $=([\mathbf{0 . 0 6 6 2 9 6 4 3 3 8 0 4 1 0 3 0 6 4 9 4 6 3 7 8 5 1 0 3 4 3 ,},-\mathbf{- 0 . 1 7 0 0 5 5 3 5 9 2 4 8 2 0 5 1 9 4 9 4 5 0 2 5 2 6 8 5}]$ [ - -0.1700553592482051949450252685, - $\mathbf{0 . 0 4 2 2 7 1 6 1 1 0 4 2 9 1 9 6 2 1 4 7 2 6 8 1 5 9 2 0 4 ] )}$ intJdn11dn10uvrs $=([\mathbf{0 . 0 7 8 2 8 1 2 8 3 8 6 1 3 5 1 5 3 2 6 0 8 0 6 6 2 7 3 5 3},-\mathbf{- 0 . 1 0 1 4 6 0 4 7 7 1 6 9 1 0 2 5 7 9 7 9 5 8 4 3 9 3 4 3}]$ [ -0.1014604771691025797958439343, - $\mathbf{0 . 2 1 8 3 8 7 5 0 8 3 3 0 3 6 3 0 2 3 6 6 6 0 0 3 8 2 1 9 7 ] )}$ intJdn11dn11uvrs $=([\mathbf{0 . 4 4 3 3 3 1 6 1 2 2 3 4 2 2 8 6 0 2 9 1 4 6 2 1 6 1 9}, \mathbf{0 . 6 0 9 2 8 6 9 6 9 8 5 2 0 3 1 1 1 4 6 7 2 8 2 8 2 4 7 7}]$ [ 0.6092869698520311146728282477, 4.54242395713891689901104912584]) intJdn11dn12uvrs $=\left(\left[\begin{array}{l}-\mathbf{0} .265306180626066014206434443, \\ -\mathbf{- 0 . 1 3 4 9 8 7 1 9 1 9 1 6 7 0 2 5 5 4 8 7 4 6 1 6 0 2 1}]\end{array}\right.\right.$ [ -1.147487191916702554874616021, - -2.99702494451010242383266710989])

Table-11

intJdnidnjuvrs, $\mathrm{i}=\mathbf{1 2}, \mathrm{j}=1$ ( 1)12)

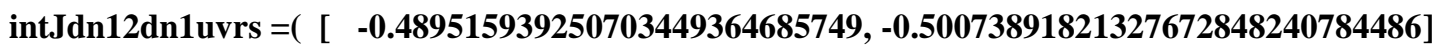

[ -1.21323891821327672848240784486, - -1.91709736541803286033938722609])

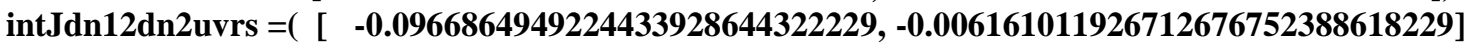
[ - -0.006161011926712676752388618229, - $-\mathbf{0 . 6 6 1 9 1 0 5 3 3 2 2 1 5 4 1 1 2 8 4 7 8 7 5 2 0 5 1 3 7 ] )}$

intJdn12dn3uvrs $=\left(\left[\begin{array}{l}\mathbf{0} .1209239805970398938152641344,0.338079890317619378517715472657\end{array}\right]\right.$ [ $0.338079890317619378517715472657,0.42220907054407935850896000532])$

intJdn12dn4uvrs $=([\mathbf{0 . 0 6 7 6 1 8 5 1 6 0 0 0 2 3 9 2 6 4 2 0 4 7 8 0 2 9 6 6}, \mathbf{0 . 3 6 0 7 2 3 1 6 4 7 9 4 4 5 1 1 8 0 6 7 2 8 1 1 7 4 4 4 3}]$ [ 0.06072316479445118067281174443, 0.737450284572716169349341246171])

intJdn12dn5uvrs $=([\mathbf{0 . 2 2 0 3 0 2 9 5 5 3 1 4 2 3 4 7 3 5 2 3 0 4 3 7 4 4 6 7 , 0 . 1 6 0 1 3 9 5 7 6 6 5 6 8 0 6 2 2 9 6 2 9 9 4 6 8 9 9 2}]$ [ 0.1601395766568062296299468992, 0.2203029553142347352304374467])

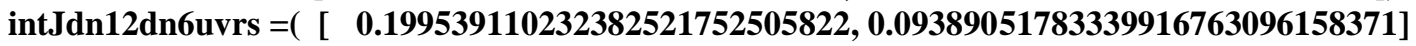
[ 0.09389051783339916763096158371, 0.02248827734476067968056194046])

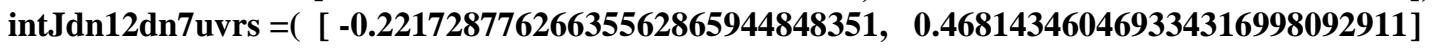
[ $\mathbf{0 . 4 6 8 1 4 3 4 6 0 4 6 9 3 3 4 3 1 6 9 9 8 0 9 2 9 1 1 , 1 . 7 7 9 4 9 0 6 4 0 6 2 9 8 6 1 6 1 1 1 7 6 4 3 4 5 2 5 1 3 ] )}$ intJdn12dn8uvrs $=([\mathbf{- 0 . 2 6 5 8 1 0 8 2 5 9 5 3 6 6 9 1 1 4 7 5 3 5 6 4 2 7 5 3 3},-\mathbf{0 . 8 1 7 1 9 2 2 0 5 8 0 6 1 6 2 2 1 9 9 4 9 4 6 6 9 5 2 3 3}]$ [ - $\mathbf{0 . 8 1 7 1 9 2 2 0 5 8 0 6 1 6 2 2 1 9 9 4 9 4 6 6 9 5 2 3 3 , - 1 . 5 4 0 4 1 3 0 1 0 4 2 4 5 9 6 4 4 4 5 2 1 5 6 1 5 4 8 3 3 ] )}$

intJdn12dn9uvrs $=([-\mathbf{- 0 . 0 6 8 2 9 7 3 2 0 3 9 0 7 1 6 3 5 8 1 6 8 3 5 1 3 3 0 7 7 1 4},-\mathbf{- 0 . 1 5 3 9 9 9 6 6 4 4 9 1 3 1 7 5 4 8 3 9 7 9 4 3 0 1 5 2}]$

[ $\mathbf{- 0 . 1 5 3 9 9 9 6 6 4 4 9 1 3 1 7 5 4 8 3 9 7 9 4 3 0 1 5 2 , - 0 . 0 3 6 0 8 0 2 5 6 6 5 3 8 6 6 0 3 2 2 6 5 3 9 4 0 8 3 5 1 ] )}$

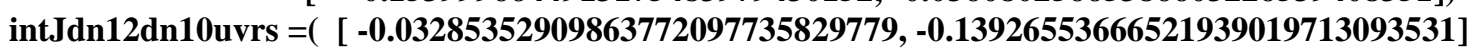
[ -0.13926553666521939019713093531, - -0.2296894086923044766227096154])

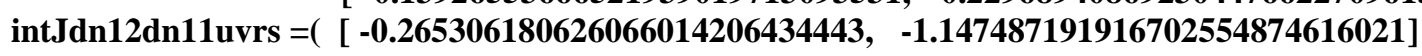
[ -0.134987191916702554874616021, - 2.99702494451010242383266710989$])$ intJdn12dn12uvrs $=([\mathbf{0 . 8 3 1 8 1 4 5 0 4 3 6 4 6 9 5 2 6 3 9 9 1 2 8 7 1 0 2}, \mathbf{1 . 3 4 3 8 6 7 9 1 8 9 4 7 7 8 0 8 4 5 2 0 4 4 2 4 7 7 5 1}]$ [ $1.3438679189477808452044247751,4.20027429051479081211473647017])$

( II)Cubic order Lagrange Elements

Table-2a

intJdnidnjuvrs, $\mathrm{i}=\mathbf{1}, \mathrm{j}=1$ (1)16)

intJdn1dn1uvrs $=([0.701681093145454720870380087,0.675893220631106832241358344]$ [ 0.675893220631106832241358344, 0.701681093145454720870380087])

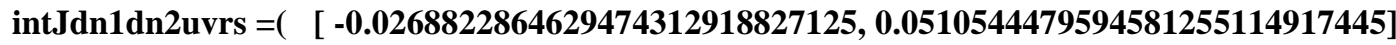
[ - $\mathbf{0 . 0 3 6 4 4 5 5 5 2 0 4 0 5 4 1 8 7 4 4 8 8 5 0 8 2 5 5 5 , 0 . 0 3 2 2 1 2 2 1 1 8 0 4 1 0 7 0 7 5 1 3 3 5 8 3 7 2 7 ]})$

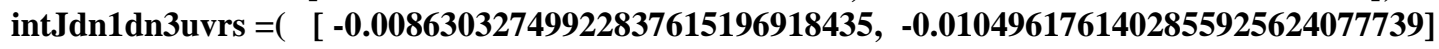
[ -0.0104961761402855925624077739, - $\mathbf{0 . 0 0 8 6 3 0 3 2 7 4 9 9 2 2 8 3 7 6 1 5 1 9 6 9 1 8 4 3 5 ] )}$

intJdn1dn4uvrs $=([\mathbf{0 . 0 3 2 2 1 2 2 1 1 8 0 4 1 0 7 0 7 5 1 3 3 5 8 3 7 2 7}, \mathbf{- 0 . 0 3 6 4 4 5 5 5 2 0 4 0 5 4 1 8 7 4 4 8 8 5 0 8 2 5 5 5}]$ [ 0.051054447959458125511491745, $\mathbf{- 0 . 0 2 6 8 8 2 2 8 6 4 6 2 9 4 7 4 3 1 2 9 1 8 8 2 7 1 2 5 ] )}$

intJdn1dn5uvrs $=([-\mathbf{- 0 . 4 1 2 6 3 3 0 5 4 1 1 7 6 0 0 2 3 0 7 1 6 2 0 6 8 9 8}, \mathbf{0 . 2 6 8 9 4 5 5 2 5 9 8 4 7 7 6 9 8 1 5 4 3 0 2 2 5 3 3}]$ [ - $\mathbf{0 . 4 4 3 5 5 4 4 7 4 0 1 5 2 2 3 0 1 8 4 5 6 9 7 7 4 6 7 , 0 . 1 5 7 8 7 1 6 2 4 5 4 1 3 3 8 7 0 4 7 9 4 5 5 7 8 2 9 ] )}$

intJdn1dn6uvrs $=([\mathbf{0 . 1 1 7 2 5 0 3 9 4 5 3 7 4 0 1 7 4 7 9 6 7 4 6 0 4 9 6 7 , - 0 . 1 4 2 5 9 2 0 7 9 4 7 6 4 9 6 6 7 0 3 8 9 2 5 4 6 8 2}]$ [ 0.157407920523503329610745318, -0.070027089525016846364967113])

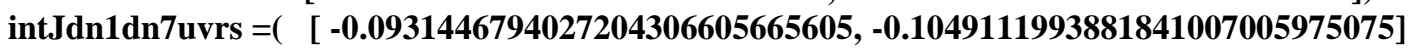


[ -0.1049111993881841007005975075, - $\mathbf{0 . 0 9 2 2 4 5 1 8 8 4 0 8 5 1 9 8 6 2 0 3 0 0 0 4 7 0 4 ] )}$

intJdn1dn8uvrs $=([\mathbf{0 . 0 3 3 5 1 8 2 7 9 7 8 9 7 3 3 4 8 5 4 4 6 8 6 9 5 0 5}, \mathbf{0 . 0 3 9 2 8 1 1 5 3 4 8 6 4 3 1 2 5 5 4 9 6 9 1 0 5 1 6}]$ [ 0.039281153486431255496910516, 0.0318790711453175872507008895])

intJdn1dn9uvrs $=([\mathbf{0 . 0 3 1 8 7 9 0 7 1 1 4 5 3 1 7 5 8 7 2 5 0 7 0 0 8 8 9 5 , 0 . 0 3 9 2 8 1 1 5 3 4 8 6 4 3 1 2 5 5 4 9 6 9 1 0 5 1 5 5}]$

[ $0.0392811534864312554969105155,0.0335182797897334854468695045])$

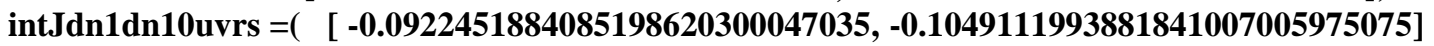

[ -0.1049111993881841007005975075, - 0.0931446794027204306605665605$])$

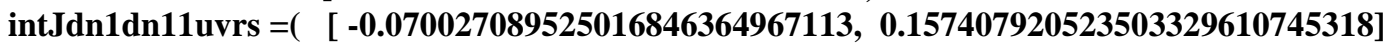

[ - $\mathbf{- 0 . 1 4 2 5 9 2 0 7 9 4 7 6 4 9 6 6 7 0 3 8 9 2 5 4 6 8 2 , 0 . 1 1 7 2 5 0 3 9 4 5 3 7 4 0 1 7 4 7 9 6 7 4 6 0 4 9 6 7 ] )}$

intJdn1dn12uvrs $=([\mathbf{0 . 1 5 7 8 7 1 6 2 4 5 4 1 3 3 8 7 0 4 7 9 4 5 5 7 8 2 9}, \mathbf{- 0 . 4 4 3 5 5 4 4 7 4 0 1 5 2 2 3 0 1 8 4 5 6 9 7 7 4 6 7}]$

[ 0.268945525984776981543022533, - 0.412633054117600230716206898$])$

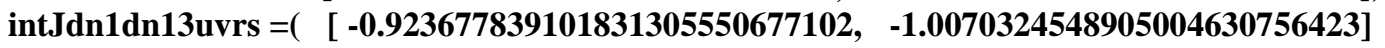

[ -1.0070324548905004630756423, - 0.923677839101831305550677102$])$

intJdn1dn14uvrs $=([\mathbf{0 . 3 3 6 8 5 1 9 9 0 2 2 2 7 2 2 8 6 4 0 9 4 1 7 2 5 1 9 , 0 . 3 8 1 6 7 1 4 0 4 3 5 2 3 9 1 7 1 7 7 1 6 0 5 0 0 0 3}]$

[ 0.381671404352391717716050003, 0.338459011543472358906908599])

intJdn1dn15uvrs $=([\mathbf{- 0 . 1 2 2 4 8 3 2 1 2 2 1 1 6 8 4 0 6 1 6 9 8 3 5 9 3 4 5}, \mathbf{- 0 . 1 4 5 2 6 3 0 9 5 4 3 7 0 7 5 3 9 4 9 5 8 5 5 3 4 9 5}]$

[ - $\mathbf{- 0 . 1 4 5 2 6 3 0 9 5 4 3 7 0 7 5 3 9 4 9 5 8 5 5 3 4 9 5 ,} \mathbf{- 0 . 1 2 2 4 8 3 2 1 2 2 1 1 6 8 4 0 6 1 6 9 8 3 5 9 3 4 5 ]})$

intJdn1dn16uvrs $=([\mathbf{0 . 3 3 8 4 5 9 0 1 1 5 4 3 4 7 2 3 5 8 9 0 6 9 0 8 5 9 9 , 0 . 3 8 1 6 7 1 4 0 4 3 5 2 3 9 1 7 1 7 7 1 6 0 5 0 0 0 3}]$

[ 0.381671404352391717716050003, 0.33685199022272286409417252])

Table-2b

intJdnidnjuvrs, $\mathrm{i}=\mathbf{2}, \mathrm{j}=1$ (1)16)

intJdn2dn1uvrs $=([-\mathbf{- 0 . 0 2 6 8 8 2 2 8 6 4 6 2 9 4 7 4 3 1 2 9 1 8 8 2 7 1 2 5},-\mathbf{- 0 . 0 3 6 4 4 5 5 5 2 0 4 0 5 4 1 8 7 4 4 8 8 5 0 8 2 5 5 5}]$

[ $\mathbf{0 . 0 5 1 0 5 4 4 4 7 9 5 9 4 5 8 1 2 5 5 1 1 4 9 1 7 4 4 5 ,}, \mathbf{0 . 0 3 2 2 1 2 2 1 1 8 0 4 1 0 7 0 7 5 1 3 3 5 8 3 7 2 7 ] )}$

intJdn2dn2uvrs $=\left(\left[\begin{array}{l}\mathbf{0} .211971597991981069039609973,-0.15304657482688411066233853364\end{array}\right]\right.$ [ - $\mathbf{0 . 1 5 3 0 4 6 5 7 4 8 2 6 8 8 4 1 1 0 6 6 2 3 3 8 5 3 3 6 4 ,} \mathbf{0 . 1 9 4 9 3 5 4 6 7 1 1 6 9 3 3 3 8 6 7 6 0 6 9 6 7 7 3 7 8}])$

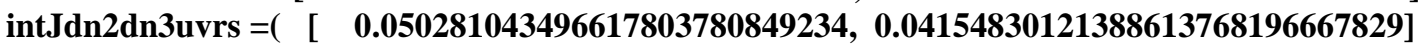
[ -0.04595169878611386231803332171, $\mathbf{- 0 . 0 2 1 0 6 6 6 9 0 8 3 3 0 0 6 0 1 3 5 6 3 8 6 5 6 4 0 8 2 ] )}$

intJdn2dn4uvrs $=([-\mathbf{- 0 . 0 0 0 4 5 9 8 6 7 3 7 1 6 9 6 9 4 2 2 8 8 9 9 2 2 6 7 5}, \mathbf{0 . 0 0 6 9 3 2 7 7 4 6 9 2 8 4 4 5 6 9 7 2 9 5 6 4 6 5 2 2}]$ [ 0.0069327746928445697295646522, - $\mathbf{0 . 0 0 0 4 5 9 8 6 7 3 7 1 6 9 6 9 4 2 2 8 8 9 9 2 2 6 7 5 ] )}$

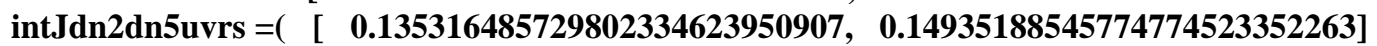

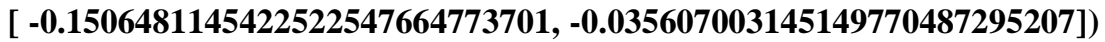

intJdn2dn6uvrs $=([\mathbf{- 0 . 4 8 4 0 6 0 3 3 6 3 5 6 1 4 0 6 4 6 7 6 1 8 0 7 1 1 9}, \mathbf{- 0 . 3 2 2 5 4 0 7 9 1 6 3 2 4 7 7 4 1 0 0 7 9 1 6 7 0 8 5 1}]$ [ 0.3899592083675225899208329149, 0.1378297018364707614848534907])

intJdn2dn7uvrs $=\left(\left[\begin{array}{l}\mathbf{0} .28346871318031592670232799,0.2616521469230527187251402015\end{array}\right]\right.$ [ -0.450847853076947281274859798, $-\mathbf{- 0 . 2 6 4 6 4 7 2 8 9 9 5 6 7 6 4 0 1 9 5 2 3 5 4 8 1 1 ] )}$

intJdn2dn8uvrs $=([\quad-\mathbf{0 . 1 1 4 1 3 7 9 6 2 2 6 6 7 6 3 6 0 6 7 4 7 7 2 0 4 8}, \mathbf{- 0 . 1 2 9 3 5 4 4 8 7 3 7 6 2 8 1 0 5 6 5 1 6 2 7 2 9 3 3}]$ [ 0.17064551262371894348372706704, 0.0803271661318366652125848671])

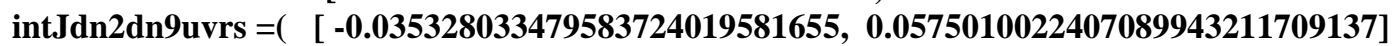
[ 0.0575010022407089943211709137, - -0.0107656396311072669181942109])

intJdn2dn10uvrs $=\left(\left[\begin{array}{l}\mathbf{0} .005100403817923470925719506,-\mathbf{0 . 0 2 4 7 6 1 7 1 7 9 9 9 8 7 7 5 4 3 7 6 9 6 7 1 4 5 6 9}] \\ )\end{array}\right.\right.$ [ - $\mathbf{- 0 . 0 2 4 7 6 1 7 1 7 9 9 9 8 7 7 5 4 3 7 6 9 6 7 1 4 5 6 9 ,} \mathbf{0 . 0 0 0 3 2 7 3 7 0 7 0 6 0 3 6 3 5 9 5 6 3 4 9 3 1 2 9 ] )}$

intJdn2dn11uvrs $=([\quad[\quad-\mathbf{0 . 0 0 8 0 4 9 4 9 8 8 7 2 3 6 9 4 7 9 0 0 4 1 2 3 ,}, \mathbf{- 0 . 0 2 3 9 6 0 9 9 1 4 5 5 1 2 8 5 3 5 8 1 8 6 3 4 8 3 6 1}]$ $[\mathbf{- 0 . 0 2 3 9 6 0 9 9 1 4 5 5 1 2 8 5 3 5 8 1 8 6 3 4 8 3 6 1 ,} \mathbf{0 . 0 0 2 3 5 3 0 4 5 7 9 4 8 5 8 3 6 5 2 6 3 2 3 5 0 8 2 7}])$

intJdn2dn12uvrs $=([\mathbf{0 . 0 2 2 0 3 4 6 6 2 6 2 8 5 9 5 6 9 3 4 0 0 3 0 1 6 9 8 6 , 0 . 0 4 9 5 4 8 7 6 6 3 2 6 6 4 0 7 1 3 4 7 6 1 0 3 3 8 4 2}]$ [ 0.0495487663266407134761033842, $-\mathbf{- 0 . 0 2 0 2 7 0 5 5 0 7 0 5 8 5 7 1 4 6 8 1 3 4 5 2 0 5 7 ] )}$

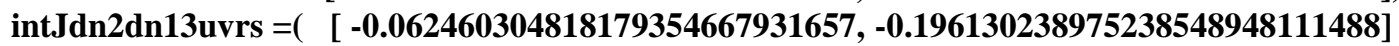
[ -0.196130238975238548948111488, 0.018066447765168896456321824])

intJdn2dn14uvrs $=([-\mathbf{- 0 . 0 0 5 6 5 4 5 4 4 7 1 7 9 1 4 1 4 6 9 9 0 0 7 2 5 9 5}, \mathbf{0 . 4 2 7 6 2 2 3 7 0 3 8 4 5 8 4 4 1 9 7 1 8 4 7 4 4 9 4 1}]$ [ 0.4276223703845844197184744941, - - 0.1539310157132113391331378486$])$

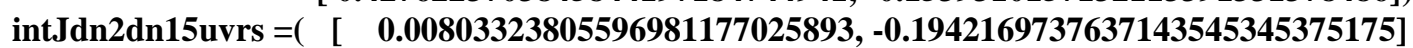
[ - $\mathbf{0 . 1 9 4 2 1 6 9 7 3 7 6 3 7 1 4 3 5 4 5 3 4 5 3 7 5 1 7 5 , 0 . 0 4 1 6 8 1 3 1 4 3 6 7 4 2 5 9 9 8 1 1 1 6 9 6 9 1 1 9 ] )}$

intJdn2dn16uvrs $=([\mathbf{0 . 0 2 0 8 2 6 6 8 9 4 4 4 3 8 9 2 2 1 5 2 9 0 9 3 2 4 6 9}, \quad \mathbf{0 . 0 8 6 3 0 0 0 8 0 8 3 0 6 7 8 1 3 5 9 3 1 2 9 9 1 5 2}]$

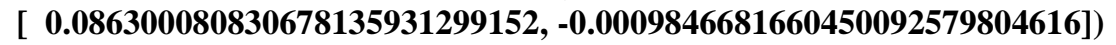

intJdnidnjuvrs, $\mathrm{i}=3, \mathrm{j}=1$ (1)16)

\section{Table-2c}

int

intJdn3dn1uvrs $=([-0.00863032749922837615196918435,-0.0104961761402855925624077739]$

[ - $\mathbf{- 0 . 0 1 0 4 9 6 1 7 6 1 4 0 2 8 5 5 9 2 5 6 2 4 0 7 7 7 3 9 , - 0 . 0 0 8 6 3 0 3 2 7 4 9 9 2 2 8 3 7 6 1 5 1 9 6 9 1 8 4 3 5 ] )}$

intJdn3dn2uvrs $=\left(\left[\begin{array}{l}\mathbf{0 . 0 5 0 2 8 1 0 4 3 4 9 6 6 1 7 8 0 3 7 8 0 8 4 9 2 3 4},-\mathbf{- 0 . 0 4 5 9 5 1 6 9 8 7 8 6 1 1 3 8 6 2 3 1 8 0 3 3 3 2 1 7 1}] \\ {[}\end{array}\right.\right.$ [ 0.04154830121388613768196667829, -0.02106669083300601356386564082])

H. T. Rathod, IJECS Volume 7 Issue 1 January 2018 Page No. 23329-23482 
intJdn3dn3uvrs $=([\mathbf{0 . 2 9 3 4 5 6 4 5 8 2 4 8 5 1 6 1 7 7 2 5 5 5 6 8 1 4 9 1 , 0 . 2 7 2 6 0 5 3 9 5 6 8 2 2 0 4 9 6 9 4 0 4 1 5 2 0 1 6 2 9}]$

[ $0.27260539568220496940415201629,0.2934564582485161772555681491])$ intJdn3dn4uvrs $=([-\mathbf{- 0 . 0 2 1 0 6 6 6 9 0 8 3 3 0 0 6 0 1 3 5 6 3 8 6 5 6 4 0 8 2 , ~ 0 . 0 4 1 5 4 8 3 0 1 2 1 3 8 8 6 1 3 7 6 8 1 9 6 6 6 7 8 2 9}]$ [ -0.04595169878611386231803332171, 0.050281043496617803780849234$])$

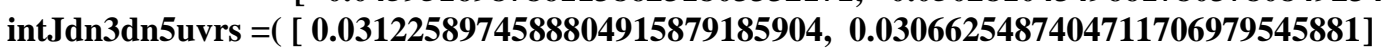
[ $0.0306625487404711706979545881,0.01203062469906479395309804256])$

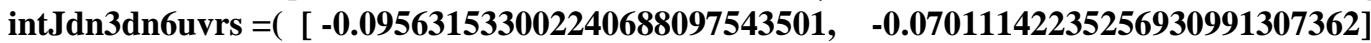
[ - -0.07011142235256930991307362, $\mathbf{- 0 . 0 2 2 9 0 1 9 4 8 5 3 2 9 2 0 1 5 1 0 5 8 7 0 8 3 4 4 7 7 ] )}$

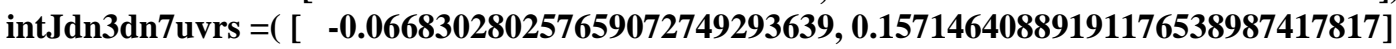
[ - $\mathbf{0 . 1 4 2 8 5 3 5 9 1 1 0 8 0 8 8 2 3 4 6 1 0 1 2 5 8 2 1 8 3 , 0 . 0 9 5 8 3 1 0 7 2 5 9 1 5 1 5 6 5 9 5 9 0 7 3 0 7 0 6 2 ] )}$

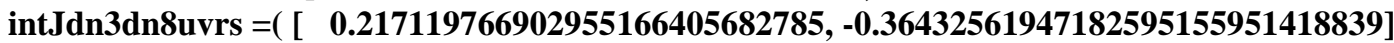

[ $0.34817438052817404844048581161,-\mathbf{0 . 3 5 2 5 4 2 7 2 4 9 1 6 6 8 0 7 3 2 8 3 2 9 8 4 0 2 ]})$ intJdn3dn9uvrs $=\left(\left[\begin{array}{l}{[-0.35254272491668073283298402,0.34817438052817404844048581161}\end{array}\right]\right.$ [ - -0.3643256194718259515595141884, 0.217119766902955166405682785$])$ intJdn3dn10uvrs $=([\mathbf{0 . 0 9 5 8 3 1 0 7 2 5 9 1 5 1 5 6 5 9 5 9 0 7 3 0 7 0 6 2}, \mathbf{- 0 . 1 4 2 8 5 3 5 9 1 1 0 8 0 8 8 2 3 4 6 1 0 1 2 5 8 2 1 8 3}]$ [ 0.15714640889191176538987417817, $-\mathbf{- 0 . 0 6 6 8 3 0 2 8 0 2 5 7 6 5 9 0 7 2 7 4 9 2 9 3 6 3 9 ] )}$

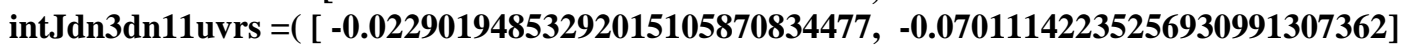

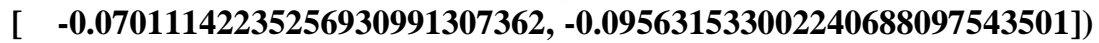
intJdn3dn12uvrs $=([\mathbf{0 . 0 1 2 0 3 0 6 2 4 6 9 9 0 6 4 7 9 3 9 5 3 0 9 8 0 4 2 5 6 , 0 . 0 3 0 6 6 2 5 4 8 7 4 0 4 7 1 1 7 0 6 9 7 9 5 4 5 8 8 1}]$ [ $\mathbf{0 . 0 3 0 6 6 2 5 4 8 7 4 0 4 7 1 1 7 0 6 9 7 9 5 4 5 8 8 1 , 0 . 0 3 1 2 2 5 8 9 7 4 5 8 8 8 0 4 9 1 5 8 7 9 1 8 5 9 0 4 ] )}$

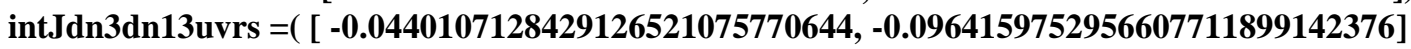
[ -0.0964159752956607711899142376, - -0.0440107128429126521075770644]) intJdn3dn14uvrs $=([$ 0.136999462878579334021389201, 0.2272134136490097198166572636$]$ [ 0.2272134136490097198166572636, 0.0941185558099118441713021744]) intJdn3dn15uvrs $=\left(\left[\begin{array}{l}\mathbf{- 0 . 3 1 9 4 4 8 6 6 4 2 0 1 3 9 3 5 8 4 2 0 4 5 9 7 4 7 9}, \mathbf{- 0 . 5 3 4 9 6 0 5 0 5 5 8 8 0 2 5 6 6 9 8 7 9 5 5 9 8 0 5 1 7}] \\ {[}\end{array}\right.\right.$ [ -0.53496050558802566987955980517, $-\mathbf{- 0 . 3 1 9 4 4 8 6 6 4 2 0 1 3 9 3 5 8 4 2 0 4 5 9 7 4 7 9 ] )}$ intJdn3dn16uvrs $=([\mathbf{0 . 0 9 4 1 1 8 5 5 5 8 0 9 9 1 1 8 4 4 1 7 1 3 0 2 1 7 4 4 , 0 . 2 2 7 2 1 3 4 1 3 6 4 9 0 0 9 7 1 9 8 1 6 6 5 7 2 6 3 6}]$ [ 0.2272134136490097198166572636, 0.136999462878579334021389201])

intJdnidnjuvrs, $\mathrm{i}=4, \mathrm{j}=1$ (1)16)

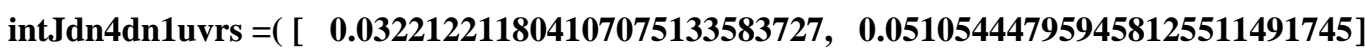
[ -0.0364455520405418744885082555, $\mathbf{- 0 . 0 2 6 8 8 2 2 8 6 4 6 2 9 4 7 4 3 1 2 9 1 8 8 2 7 1 2 5 ] )}$ intJdn4dn2uvrs $=([-\mathbf{- 0 . 0 0 0 4 5 9 8 6 7 3 7 1 6 9 6 9 4 2 2 8 8 9 9 2 2 6 7 5}, \mathbf{0 . 0 0 6 9 3 2 7 7 4 6 9 2 8 4 4 5 6 9 7 2 9 5 6 4 6 5 2 2}]$ [ 0.0069327746928445697295646522, - -0.0004598673716969422889922675]) intJdn4dn3uvrs $=([\mathbf{- 0 . 0 2 1 0 6 6 6 9 0 8 3 3 0 0 6 0 1 3 5 6 3 8 6 5 6 4 0 8 2},-\mathbf{0 . 0 4 5 9 5 1 6 9 8 7 8 6 1 1 3 8 6 2 3 1 8 0 3 3 3 2 1 7 1}]$ [ 0.04154830121388613768196667829, $\mathbf{0 . 0 5 0 2 8 1 0 4 3 4 9 6 6 1 7 8 0 3 7 8 0 8 4 9 2 3 4 ] )}$ intJdn4dn4uvrs $=([\mathbf{0 . 1 9 4 9 3 5 4 6 7 1 1 6 9 3 3 3 8 6 7 6 0 6 9 6 7 7 3 7 8},-\mathbf{- 0 . 1 5 3 0 4 6 5 7 4 8 2 6 8 8 4 1 1 0 6 6 2 3 3 8 5 3 3 6 4}]$ [ - $\mathbf{0 . 1 5 3 0 4 6 5 7 4 8 2 6 8 8 4 1 1 0 6 6 2 3 3 8 5 3 3 6 4 ,} \quad \mathbf{0 . 2 1 1 9 7 1 5 9 7 9 9 1 9 8 1 0 6 9 0 3 9 6 0 9 9 7 3 ] )}$

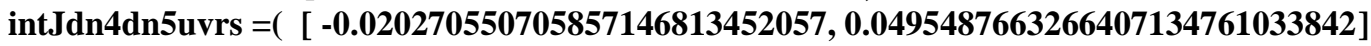
[ 0.0495487663266407134761033842, 0.022034662628595693400301699]) intJdn4dn6uvrs $=([\mathbf{0 . 0 0 2 3 5 3 0 4 5 7 9 4 8 5 8 3 6 5 2 6 3 2 3 5 0 8 3 ,},-\mathbf{0 . 0 2 3 9 6 0 9 9 1 4 5 5 1 2 8 5 3 5 8 1 8 6 3 4 8 3 6}]$ [ -0.023960991455128535818634836, $-\mathbf{- 0 . 0 0 8 0 4 9 4 9 8 8 7 2 3 6 9 4 7 9 0 0 4 1 2 3 0 0 0 2 ]})$ intJdn4dn7uvrs $=([\mathbf{0 . 0 0 0 3 2 7 3 7 0 7 0 6 0 3 6 3 5 9 5 6 3 4 9 3 1 2 8 9 6 1}, \mathbf{- 0 . 0 2 4 7 6 1 7 1 7 9 9 9 8 7 7 5 4 3 7 6 9 6 7 1 4 5 6 9}]$ [ $\mathbf{- 0 . 0 2 4 7 6 1 7 1 7 9 9 9 8 7 7 5 4 3 7 6 9 6 7 1 4 5 6 9 ,} \mathbf{0 . 0 0 5 1 0 0 4 0 3 8 1 7 9 2 3 4 7 0 9 2 5 7 1 9 5 0 6 ] )}$

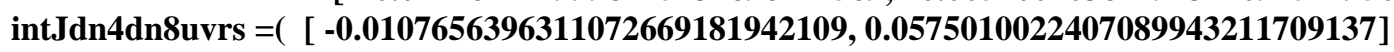
[ 0.0575010022407089943211709137, - $\mathbf{0 . 0 3 5 3 2 8 0 3 3 4 7 9 5 8 3 7 2 4 0 1 9 5 8 1 6 5 5 ] )}$ intJdn4dn9uvrs $=\left(\left[\begin{array}{l}\mathbf{0} .0803271661318366652125848671 \\ \mathbf{0}\end{array}\right.\right.$ [ -0.12935448737628105651627293296, $-\mathbf{- 0 . 1 1 4 1 3 7 9 6 2 2 6 6 7 6 3 6 0 6 7 4 7 7 2 0 4 8}])$

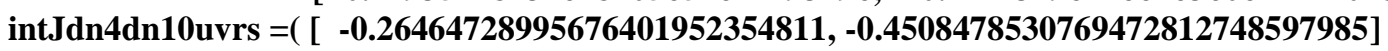
[ 0.2616521469230527187251402015, 0.28346871318031592670232799$])$ intJdn4dn11uvrs $=([\mathbf{0 . 1 3 7 8 2 9 7 0 1 8 3 6 4 7 0 7 6 1 4 8 4 8 5 3 4 9 0 7 , 0 . 3 8 9 9 5 9 2 0 8 3 6 7 5 2 2 5 8 9 9 2 0 8 3 2 9 1 4 9}]$ [ -0.3225407916324774100791670851, -0.484060336356140646761807119])

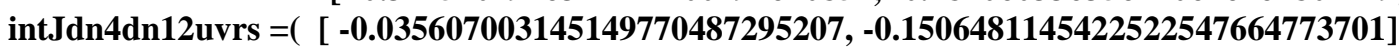
[ 0.1493518854577477452335226299, $\mathbf{0 . 1 3 5 3 1 6 4 8 5 7 2 9 8 0 2 3 3 4 6 2 3 9 5 0 9 0 7 ] )}$ intJdn4dn13uvrs $=([\mathbf{0 . 0 1 8 0 6 6 4 4 7 7 6 5 1 6 8 8 9 6 4 5 6 3 2 1 8 2 4},-\mathbf{- 0 . 1 9 6 1 3 0 2 3 8 9 7 5 2 3 8 5 4 8 9 4 8 1 1 1 4 8 8}]$ [ -0.196130238975238548948111488, $-\mathbf{- 0 . 0 6 2 4 6 0 3 0 4 8 1 8 1 7 9 3 5 4 6 6 7 9 3 1 6 6 ] )}$ intJdn4dn14uvrs $=([-\mathbf{- 0 . 0 0 0 9 8 4 6 6 8 1 6 6 0 4 5 0 0 9 2 5 7 9 8 0 4 6 1 6}, \mathbf{0 . 0 8 6 3 0 0 0 8 0 8 3 0 6 7 8 1 3 5 9 3 1 2 9 9 1 5 2}]$

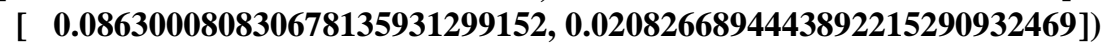
intJdn4dn15uvrs $=([$ o.0416813143674259981116969119, $-\mathbf{- 0 . 1 9 4 2 1 6 9 7 3 7 6 3 7 1 4 3 5 4 5 3 4 5 3 7 5 1 7 5}]$

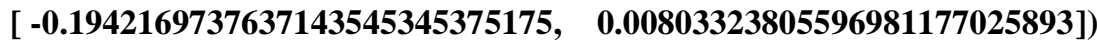

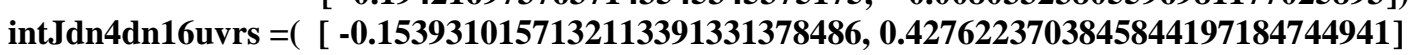

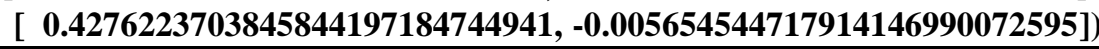


intJdn5dn1uvrs $=([-\mathbf{- 0 . 4 1 2 6 3 3 0 5 4 1 1 7 6 0 0 2 3 0 7 1 6 2 0 6 8 9 8}, \mathbf{- 0 . 4 4 3 5 5 4 4 7 4 0 1 5 2 2 3 0 1 8 4 5 6 9 7 7 4 6 7}]$

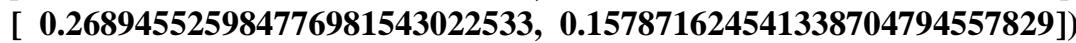
intJdn5dn2uvrs $=([\mathbf{0 . 1 3 5 3 1 6 4 8 5 7 2 9 8 0 2 3 3 4 6 2 3 9 5 0 9 0 7},-\mathbf{- 0 . 1 5 0 6 4 8 1 1 4 5 4 2 2 5 2 2 5 4 7 6 6 4 7 7 3 7 0 1}]$ [ $\mathbf{0 . 1 4 9 3 5 1 8 8 5 4 5 7 7 4 7 7 4 5 2 3 3 5 2 2 6 3 ,} \mathbf{- 0 . 0 3 5 6 0 7 0 0 3 1 4 5 1 4 9 7 7 0 4 8 7 2 9 5 2 0 7 ] )}$ intJdn5dn3uvrs $=([\mathbf{0 . 0 3 1 2 2 5 8 9 7 4 5 8 8 8 0 4 9 1 5 8 7 9 1 8 5 9 0 4}, \mathbf{0 . 0 3 0 6 6 2 5 4 8 7 4 0 4 7 1 1 7 0 6 9 7 9 5 4 5 8 8 1}]$ [ 0.0306625487404711706979545881, 0.01203062469906479395309804256])

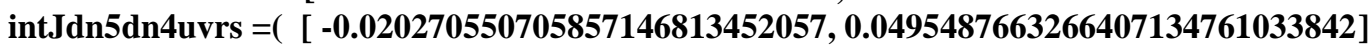
[ 0.0495487663266407134761033842, $\mathbf{0 . 0 2 2 0 3 4 6 6 2 6 2 8 5 9 5 6 9 3 4 0 0 3 0 1 6 9 9 ] )}$

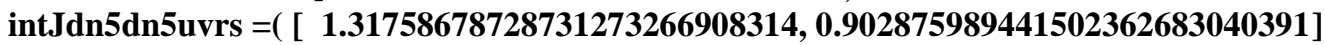
[ 0.902875989441502362683040391, 1.49530824854081722232161367])

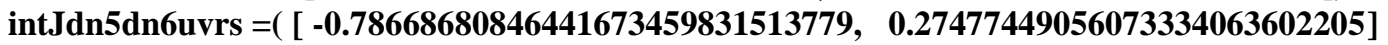
[ - $\mathbf{- 0 . 7 3 7 7 2 5 5 0 9 4 3 9 2 6 6 6 5 9 3 6 3 9 7 7 9 5 4 , - 0 . 2 2 2 0 2 6 4 8 0 8 1 3 6 2 1 3 5 9 9 3 2 4 0 7 8 1 1 ] )}$ intJdn5dn7uvrs $=([0.3065397294444835089245124969,0.272605979766225412235674987]$ [ $\mathbf{0 . 2 7 2 6 0 5 9 7 9 7 6 6 2 2 5 4 1 2 2 3 5 6 7 4 9 8 7 , 0 . 1 1 8 0 1 0 8 0 5 4 6 7 1 7 6 2 1 4 1 4 4 9 1 7 7 3 4 ]})$ intJdn5dn8uvrs $=([-\mathbf{- 0 . 1 1 2 3 6 0 2 7 7 2 5 3 8 5 7 1 7 2 9 6 1 5 2 0 0 0 2},-\mathbf{0 . 1 0 8 5 7 8 3 1 9 4 1 0 5 2 4 8 9 3 0 3 2 4 0 5 2 2 6 4}]$ [ - -0.1085783194105248930324052264, $\mathbf{- 0 . 0 4 2 4 0 3 1 7 3 1 7 2 5 6 1 5 7 2 5 5 4 2 5 4 0 8 4 1 ] )}$

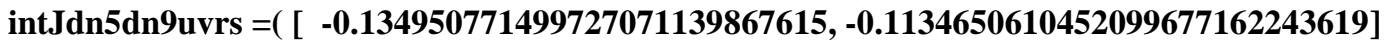
[ -0.1134650610452099677162243619, - - 0.0283674858742885011995552238])

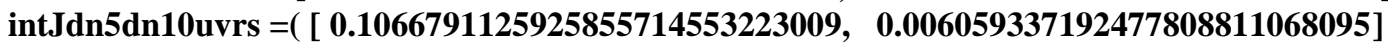

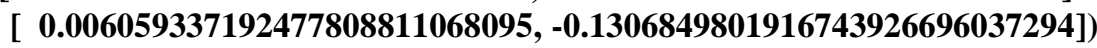

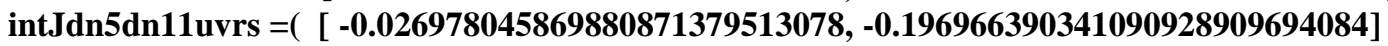
[ - $\mathbf{0 . 1 9 6 9 6 6 3 9 0 3 4 1 0 9 0 9 2 8 9 0 9 6 9 4 0 8 4 , - 0 . 0 7 8 8 9 7 3 5 8 2 4 9 7 5 2 6 0 8 6 6 8 2 7 3 9 8 1 ] )}$ intJdn5dn12uvrs $=([\mathbf{0 . 0 8 9 2 1 1 6 5 4 5 8 1 6 3 8 1 0 6 9 6 8 7 6 6 9 6 , 0 . 4 2 7 1 8 8 8 8 4 7 9 9 0 7 8 0 7 0 1 7 3 9 9 2 3 8 6}]$ [ $\mathbf{0 . 4 2 7 1 8 8 8 8 4 7 9 9 0 7 8 0 7 0 1 7 3 9 9 2 3 8 6 , 0 . 0 8 9 2 1 1 6 5 4 5 8 1 6 3 8 1 0 6 9 6 8 7 6 6 9 6 ] )}$ intJdn5dn13uvrs $=([\mathbf{0 . 4 7 5 5 5 8 9 0 7 8 3 1 9 3 2 0 0 3 1 8 8 7 4 6 4 7 8 , - 0 . 5 3 0 8 4 3 3 5 5 9 8 7 0 5 1 3 2 6 2 0 4 9 8 7 9 2}]$ [ -0.53084335598705132620498792, -1.83117956250893266072281398])

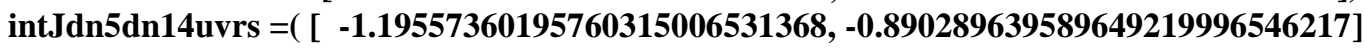
[ - $\mathbf{0 . 8 9 0 2 8 9 6 3 9 5 8 9 6 4 9 2 1 9 9 9 6 5 4 6 2 1 7 , - 0 . 1 4 6 2 3 7 6 7 9 9 0 2 3 8 1 6 6 8 3 4 4 1 4 5 4 8 ] )}$ intJdn5dn15uvrs $=([\mathbf{0 . 4 4 9 8 7 7 6 9 2 1 2 6 6 6 3 6 6 1 6 2 9 5 5 2 3 5 , 0 . 3 7 9 3 6 0 7 4 7 0 7 1 9 4 7 7 4 1 9 6 0 2 3 2 0 4 2}]$ [ 0.379360747071947741960232042, 0.086904547772480103903106692])

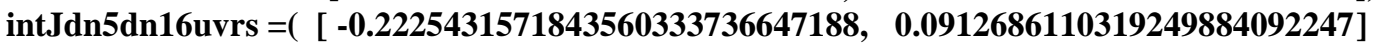
[ $\mathbf{0 . 0 9 1 2 6 8 6 1 1 0 3 1 9 2 4 9 8 8 4 0 9 2 2 4 7 , 0 . 5 3 4 0 3 1 5 5 5 6 2 7 2 5 1 6 9 5 0 9 1 9 8 6 8 8 7 ] )}$

Table-2f

intJdnidnjuvrs, $\mathrm{i}=6, \mathrm{j}=1$ (1)16)

intJdn6dn1uvrs $=([\mathbf{0 . 1 1 7 2 5 0 3 9 4 5 3 7 4 0 1 7 4 7 9 6 7 4 6 0 4 9 6 7 ,} \mathbf{0 . 1 5 7 4 0 7 9 2 0 5 2 3 5 0 3 3 2 9 6 1 0 7 4 5 3 1 8}]$ [ - -0.142592079476496670389254682, $\mathbf{- 0 . 0 7 0 0 2 7 0 8 9 5 2 5 0 1 6 8 4 6 3 6 4 9 6 7 1 1 3 ] )}$

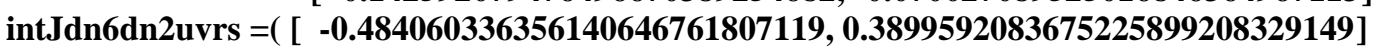
[ - $\mathbf{0 . 3 2 2 5 4 0 7 9 1 6 3 2 4 7 7 4 1 0 0 7 9 1 6 7 0 8 5 1 , 0 . 1 3 7 8 2 9 7 0 1 8 3 6 4 7 0 7 6 1 4 8 4 8 5 3 4 9 0 7 ] )}$

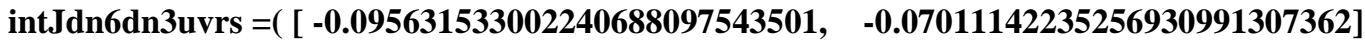
[ - -0.07011142235256930991307362, $\mathbf{- 0 . 0 2 2 9 0 1 9 4 8 5 3 2 9 2 0 1 5 1 0 5 8 7 0 8 3 4 4 7 7 ] )}$

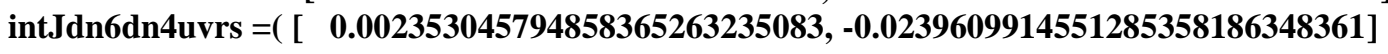

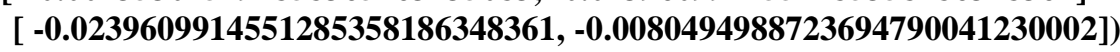
intJdn6dn5uvrs $=([\mathbf{- 0 . 7 8 6 6 8 6 8 0 8 4 6 4 4 1 6 7 3 4 5 9 8 3 1 5 1 3 7 7 9}, \mathbf{- 0 . 7 3 7 7 2 5 5 0 9 4 3 9 2 6 6 6 5 9 3 6 3 9 7 7 9 5 4}]$

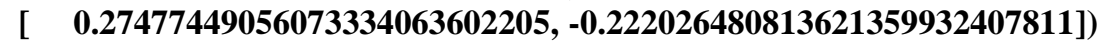
intJdn6dn6uvrs $=([$ 1.332289708203970804992564589, $\mathbf{0 . 6 0 2 0 6 5 3 7 3 5 1 8 4 3 5 1 5 9 5 0 3 1 1 3 5 1 9}]$ [ 0.602065373518435159503113519, 1.141981449369884652860820049])

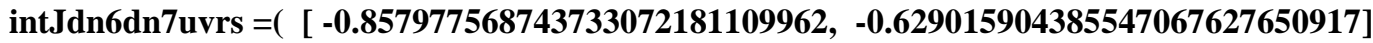
[ -0.629015904385547067627650917, $\mathbf{- 0 . 2 5 4 3 3 7 7 6 0 1 2 1 6 5 3 4 3 9 5 4 4 5 3 5 4 5 3 4 ] )}$ intJdn6dn8uvrs $=([\mathbf{0 . 3 1 5 7 1 1 3 0 2 4 7 3 1 8 7 0 4 1 2 7 7 2 2 0 1 8 9 , 0 . 2 4 8 1 9 9 0 1 8 7 3 6 5 5 9 9 0 8 8 1 3 6 2 3 1 9 7 4}]$ [ 0.2481990187365599088136231974, 0.0837329943403397038665668996])

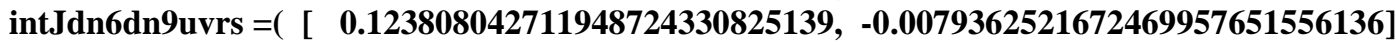
[ -0.0079362521672469957651556136, - -0.11543642609252788831829720852]) intJdn6dn10uvrs $=([-\mathbf{- 0 . 0 4 2 6 1 9 1 8 0 2 3 7 0 6 5 5 2 3 5 3 7 4 3 9 7 2 9 4 , 0 . 0 8 3 2 1 2 1 6 9 6 0 7 4 4 0 5 7 8 2 5 4 9 4 5 8 7 9 1}]$ [ $\mathbf{0 . 0 8 3 2 1 2 1 6 9 6 0 7 4 4 0 5 7 8 2 5 4 9 4 5 8 7 9 1 , 0 . 0 4 9 8 8 2 7 0 0 9 9 0 2 8 4 9 6 7 0 2 3 4 6 4 9 7 8 1 ] )}$ intJdn6dn11uvrs $=([\mathbf{0 . 0 2 7 5 2 7 7 9 1 0 9 3 3 0 7 2 0 8 2 4 8 7 2 5 5 7 5 , 0 . 0 8 9 2 6 8 0 9 8 8 1 6 0 5 2 0 1 6 5 7 8 3 8 7 7 2 6}]$ [ 0.089268098816052016578387726, 0.027527791093307208248725575]) intJdn6dn12uvrs $=([-\mathbf{- 0 . 0 7 8 8 9 7 3 5 8 2 4 9 7 5 2 6 0 8 6 6 8 2 7 3 9 8 1}, \mathbf{- 0 . 1 9 6 9 6 6 3 9 0 3 4 1 0 9 0 9 2 8 9 0 9 6 9 4 0 8 4}]$ [ -0.196966390341090928909694084, $\mathbf{- 0 . 0 2 6 9 7 8 0 4 5 8 6 9 8 8 0 8 7 1 3 7 9 5 1 3 0 7 8 ] )}$ 
intJdn6dn13uvrs $=([\mathbf{0 . 1 0 7 3 6 6 7 0 7 3 6 8 6 7 9 8 5 3 4 1 9 8 8 7 0 3 9}, \mathbf{0 . 8 2 7 4 2 3 7 7 9 1 2 9 9 4 3 6 3 9 1 1 3 0 1 4 0 6 1 ]}$ [ 0.827423779129943639113014061, 0.520202184956697891678232119])

intJdn6dn14uvrs $=([\mathbf{0 . 6 0 1 0 5 8 3 5 7 5 0 6 5 4 3 5 9 4 9 5 6 5 8 2 6 3},-\mathbf{- 0 . 5 1 0 5 9 9 4 6 0 9 5 9 2 0 0 0 5 1 9 7 8 4 5 9 6 5}]$

[ - $\mathbf{- 0 . 5 1 0 5 9 9 4 6 0 9 5 9 2 0 0 0 5 1 9 7 8 4 5 9 6 5 , - 1 . 5 0 1 7 5 2 7 8 7 7 5 6 7 2 0 2 9 1 0 5 1 9 5 5 3 6 ] )}$

intJdn6dn15uvrs $=([-0.26123164244466091271015883,0.106104670667416834133287489]$

[ 0.106104670667416834133287489, 0.449881020809519531356712718])

intJdn6dn16uvrs $=([-\mathbf{- 0 . 0 2 0 2 6 0 9 2 2 1 9 1 8 8 7 1 5 3 9 0 1 8 5 2 4 9},-\mathbf{0 . 3 2 7 3 2 4 3 0 8 2 6 6 8 2 4 5 0 6 5 5 1 3 0 3 4 6}]$

[ -0.32732430826682450655130346, -0.18952780581179438986486843])

Table-2g

intJdnidnjuvrs, $\mathrm{i}=7, \mathrm{j}=1$ (1)16)

intJdn7dn1uvrs $=([-\mathbf{- 0 . 0 9 3 1 4 4 6 7 9 4 0 2 7 2 0 4 3 0 6 6 0 5 6 6 5 6 0 5},-\mathbf{0 . 1 0 4 9 1 1 1 9 9 3 8 8 1 8 4 1 0 0 7 0 0 5 9 7 5 0 7 5}]$

[ - - $\mathbf{0 . 1 0 4 9 1 1 1 9 9 3 8 8 1 8 4 1 0 0 7 0 0 5 9 7 5 0 7 5 ,} \mathbf{- 0 . 0 9 2 2 4 5 1 8 8 4 0 8 5 1 9 8 6 2 0 3 0 0 0 4 7 0 4 ] )}$

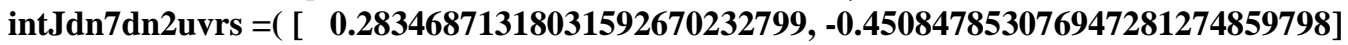

[ 0.2616521469230527187251402015, -0.26464728995676401952354811])

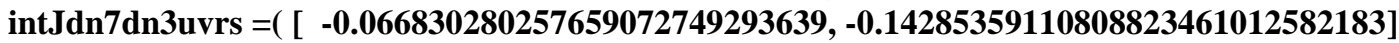

[ $\mathbf{0 . 1 5 7 1 4 6 4 0 8 8 9 1 9 1 1 7 6 5 3 8 9 8 7 4 1 7 8 1 7 ,} \mathbf{0 . 0 9 5 8 3 1 0 7 2 5 9 1 5 1 5 6 5 9 5 9 0 7 3 0 7 0 6 2 ]})$

intJdn7dn4uvrs $=([\mathbf{0 . 0 0 0 3 2 7 3 7 0 7 0 6 0 3 6 3 5 9 5 6 3 4 9 3 1 2 8 9 6 1 ,}, \mathbf{- 0 . 0 2 4 7 6 1 7 1 7 9 9 9 8 7 7 5 4 3 7 6 9 6 7 1 4 5 6 9}]$

[ -0.0247617179998775437696714569, $\mathbf{0 . 0 0 5 1 0 0 4 0 3 8 1 7 9 2 3 4 7 0 9 2 5 7 1 9 5 0 6 ] )}$

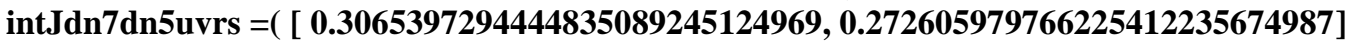

[ 0.272605979766225412235674987, 0.118010805467176214144917734])

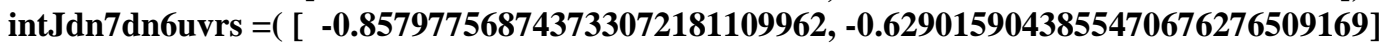

[ -0.6290159043855470676276509169, - $\mathbf{0 . 2 5 4 3 3 7 7 6 0 1 2 1 6 5 3 4 3 9 5 4 4 5 3 5 4 5 3 4 ] )}$

intJdn7dn7uvrs $=\left(\left[\begin{array}{l}\mathbf{2 . 0 0 1 0 8 9 6 5 8 0 5 7 7 2 1 1 0 2 3 9 1 2 6 4 3 2 7 , 0 . 2 7 3 0 9 5 6 7 6 7 7 9 1 6 2 6 5 6 1 0 6 5 4 1 8 6 0 6 8}] \\ {[}\end{array}\right.\right.$

[ 0.27309567677916265610654186068, 0.666504113204159209546195038$])$

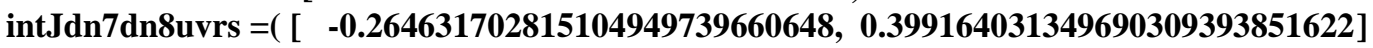

[ - -0.61333596865030969060614837761, - -0.488120340846869169507695406])

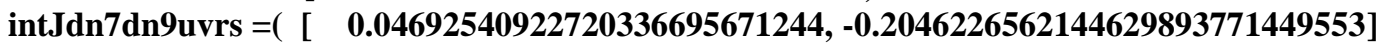

[ -0.2046226562144629893771449553, $\mathbf{0 . 0 5 3 0 2 6 7 3 5 2 0 7 9 8 4 6 2 0 5 3 7 7 5 1 6 6 8 ]})$

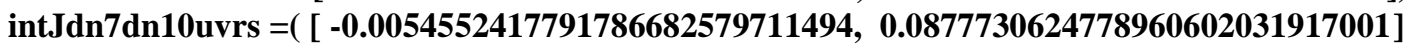

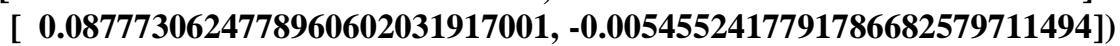

intJdn7dn11uvrs $=([\mathbf{0 . 0 4 9 8 8 2 7 0 0 9 9 0 2 8 4 9 6 7 0 2 3 4 6 4 9 7 8 1}, \mathbf{0 . 0 8 3 2 1 2 1 6 9 6 0 7 4 4 0 5 7 8 2 5 4 9 4 5 8 7 9 1}]$

[ 0.0832121696074405782549458791, - -0.0426191802370655235374397294])

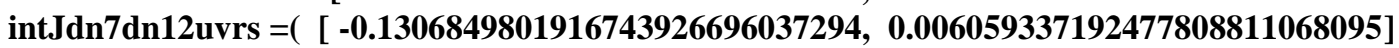

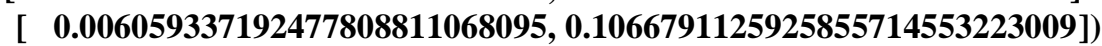

intJdn7dn13uvrs $=\left(\left[\begin{array}{l}\mathbf{0 . 6 2 1 7 9 0 5 4 4 8 2 9 7 7 4 9 0 2 3 9 9 4 6 3 4 8 ,}, \quad \mathbf{0 . 0 3 0 4 4 8 3 2 0 8 4 4 6 3 0 4 5 0 0 4 4 6 6 4 7}] \\ {[}\end{array}\right.\right.$

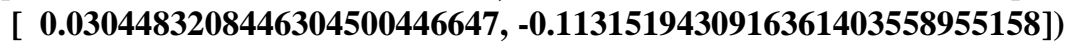

intJdn7dn14uvrs $=([-\mathbf{- 2 . 2 4 5 5 9 1 1 4 4 9 8 2 4 5 3 5 8 7 4 8 5 2 5 6 1 5}, \mathbf{0 . 0 3 2 5 0 2 7 1 2 0 7 9 2 1 7 1 9 3 4 3 3 8 3 6 1 3 7}]$

[ $0.032502712079217193433836137,0.4636424264352335415173399435])$

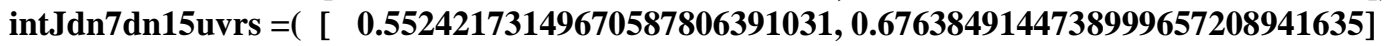

[ 0.6763849144738999657208941635, -0.293283237003299311368713211])

intJdn7dn16uvrs $=([\mathbf{- 0 . 1 9 8 1 3 0 2 5 9 7 6 0 0 0 1 8 3 8 2 8 1 6 8 7 3 4 1 ,}, \mathbf{- 0 . 3 0 4 2 3 3 2 8 2 3 9 7 5 3 3 2 1 6 8 4 4 6 1 8 6 8 6}]$

[ -0.304233282397533216844618686, $\mathbf{0 . 0 4 5 0 6 5 5 1 2 1 2 8 4 0 7 8 4 6 4 0 7 8 2 6 3 8 4 ] )}$

intJdnidnjuvrs, $\mathrm{i}=\mathbf{8}, \mathrm{j}=1$ (1)16)

Table-2h

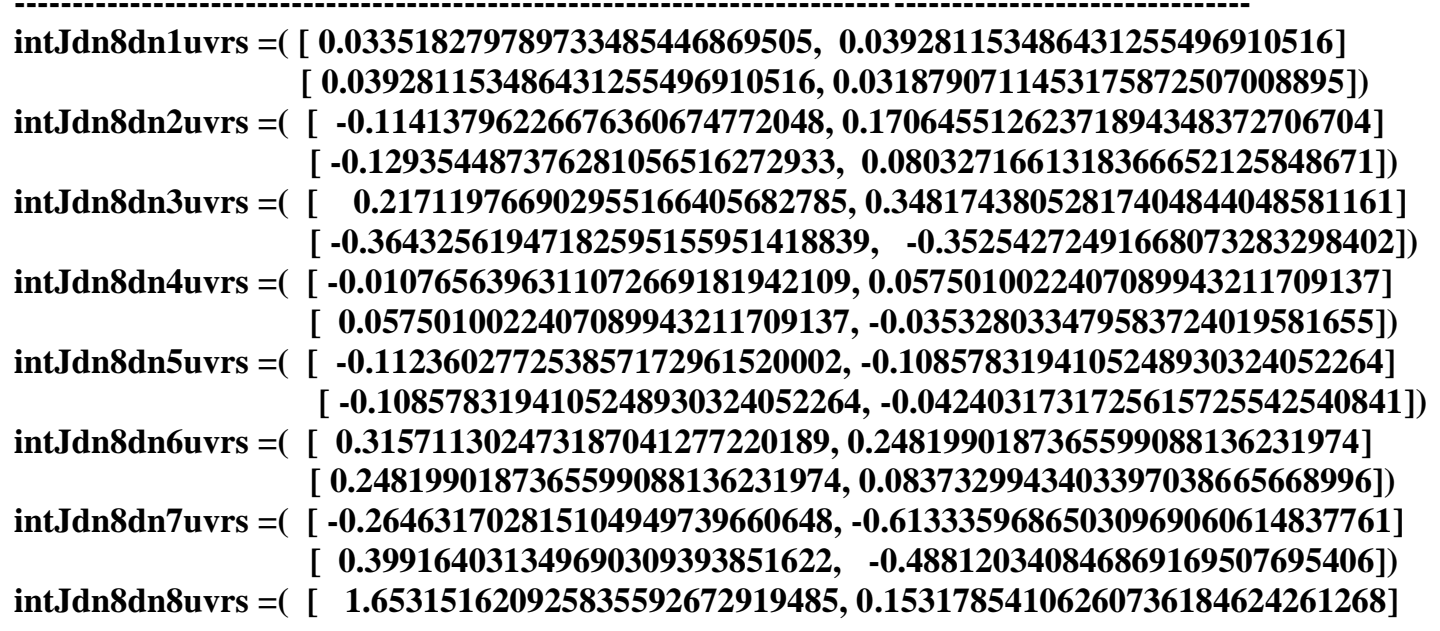

H. T. Rathod, IJECS Volume 7 Issue 1 January 2018 Page No. 23329-23482

Page 23373 
[ $0.15317854106260736184624261268, \quad 0.76711507859866107933357613])$

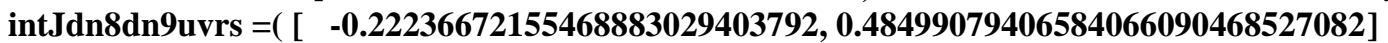

[ 0.48499079406584066090468527082, $-\mathbf{- 0 . 2 2 2 3 6 6 7 2 1 5 5 4 6 8 8 8 3 0 2 9 4 0 3 7 9 2 ] )}$

intJdn8dn10uvrs $=([\mathbf{0 . 0 5 3 0 2 6 7 3 5 2 0 7 9 8 4 6 2 0 5 3 7 7 5 1 6 6 8}, \mathbf{- 0 . 2 0 4 6 2 2 6 5 6 2 1 4 4 6 2 9 8 9 3 7 7 1 4 4 9 5 5 3}]$

$[-\mathbf{- 0 . 2 0 4 6 2 2 6 5 6 2 1 4 4 6 2 9 8 9 3 7 7 1 4 4 9 5 5 3 ,} \mathbf{0 . 0 4 6 9 2 5 4 0 9 2 2 7 2 0 3 3 6 6 9 5 6 7 1 2 4 4 ]})$

intJdn8dn11uvrs $=([-\mathbf{0 . 1 1 5 4 3 6 4 2 6 0 9 2 5 2 7 8 8 8 3 1 8 2 9 7 2 0 8 5 2}, \mathbf{- 0 . 0 0 7 9 3 6 2 5 2 1 6 7 2 4 6 9 9 5 7 6 5 1 5 5 6 1 3 6}]$

[ -0.0079362521672469957651556136, 0.123808042711948724330825139$])$

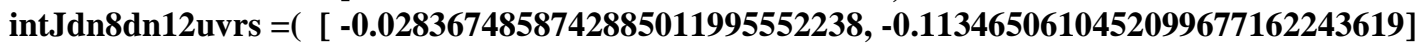

[ -0.1134650610452099677162243619, - $\mathbf{0 . 1 3 4 9 5 0 7 7 1 4 9 9 7 2 7 0 7 1 1 3 9 8 6 7 6 1 5 ] )}$

intJdn8dn13uvrs $=([\mathbf{0 . 0 6 0 3 2 9 0 5 9 1 6 5 4 1 1 5 4 3 3 3 4 6 9 6 0 7 8 , 0 . 3 3 1 2 7 8 7 3 1 1 2 6 1 9 3 7 0 5 0 0 6 3 7 1 9 4 8}]$

[ 0.331278731126193705006371948, 0.178851051525261210302257368])

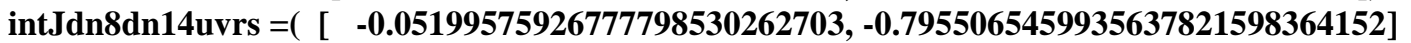

[ - -0.7955065459935637821598364152, $-\mathbf{0 . 4 3 3 2 6 2 2 1 4 1 9 7 9 3 2 0 2 0 3 6 0 2 3 8 3 3 5 ]})$

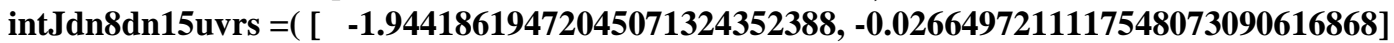

$\left[\begin{array}{rl}-0.0266497211117548073090616868, & \mathbf{0 . 5 5 0 8 4 3 9 7 9 7 6 1 9 9 3 3 4 1 7 6 3 2 7 8 6 7 9}])\end{array}\right.$

intJdn8dn16uvrs $=([\mathbf{0 . 5 3 1 3 9 1 4 0 5 0 1 1 4 5 9 4 6 5 0 4 9 9 9 6 9 3 6}, \mathbf{0 . 0 3 6 8 4 5 3 9 0 7 2 2 8 3 8 2 4 7 6 5 2 7 5 9 2 9 6}]$

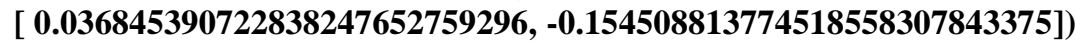

intJdnidnjuvrs, $\mathrm{i}=9, \mathrm{j}=1$ (1)16)

\section{Table-2i}

intJdn9dn1uvrs $=([\mathbf{0 . 0 3 1 8 7 9 0 7 1 1 4 5 3 1 7 5 8 7 2 5 0 7 0 0 8 8 9 5}, \mathbf{0 . 0 3 9 2 8 1 1 5 3 4 8 6 4 3 1 2 5 5 4 9 6 9 1 0 5 1 6}]$ [ 0.039281153486431255496910516, 0.0335182797897334854468695045]) intJdn9dn2uvrs $=([-\mathbf{- 0 . 0 3 5 3 2 8 0 3 3 4 7 9 5 8 3 7 2 4 0 1 9 5 8 1 6 5 5}, \mathbf{0 . 0 5 7 5 0 1 0 0 2 2 4 0 7 0 8 9 9 4 3 2 1 1 7 0 9 1 3 7}]$ [ 0.0575010022407089943211709137, -0.0107656396311072669181942109])

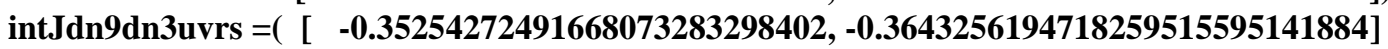
[ $0.34817438052817404844048581161,0.217119766902955166405682785])$ intJdn9dn4uvrs $=\left(\left[\begin{array}{l}\mathbf{0} .0803271661318366652125848671 \\ -\mathbf{- 0 . 1 2 9 3 5 4 4 8 7 3 7 6 2 8 1 0 5 6 5 1 6 2 7 2 9 3 2 9 6}]\end{array}\right.\right.$ [ 0.17064551262371894348372706704, $-\mathbf{- 0 . 1 1 4 1 3 7 9 6 2 2 6 6 7 6 3 6 0 6 7 4 7 7 2 0 4 8 ]})$

intJdn9dn5uvrs $=([-\mathbf{- 0 . 1 3 4 9 5 0 7 7 1 4 9 9 7 2 7 0 7 1 1 3 9 8 6 7 6 1 5},-\mathbf{- 0 . 1 1 3 4 6 5 0 6 1 0 4 5 2 0 9 9 6 7 7 1 6 2 2 4 3 6 2}]$

[ -0.113465061045209967716224362, -0.0283674858742885011995552238])

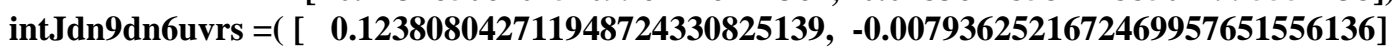
[ -0.0079362521672469957651556136, - $\mathbf{0 . 1 1 5 4 3 6 4 2 6 0 9 2 5 2 7 8 8 8 3 1 8 2 9 7 2 0 8 5 2 ] )}$

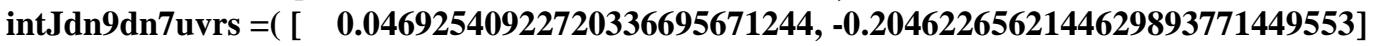

$[-\mathbf{- 0 . 2 0 4 6 2 2 6 5 6 2 1 4 4 6 2 9 8 9 3 7 7 1 4 4 9 5 5 3 ,} \mathbf{0 . 0 5 3 0 2 6 7 3 5 2 0 7 9 8 4 6 2 0 5 3 7 7 5 1 6 6 8 ]})$

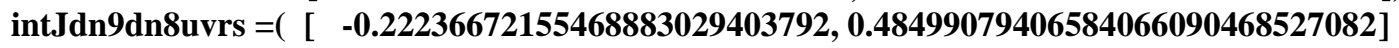

[ 0.48499079406584066090468527082, $-\mathbf{- 0 . 2 2 2 3 6 6 7 2 1 5 5 4 6 8 8 8 3 0 2 9 4 0 3 7 9 2 ] )}$

intJdn9dn9uvrs $=\left(\left[\begin{array}{ll}\mathbf{0} .76711507859866107933357613,0.15317854106260736184624261268\end{array}\right]\right.$

[ $0.15317854106260736184624261268,1.653151620925835592672919485])$

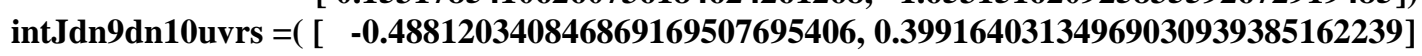

[ -0.61333596865030969060614837761, - -0.264631702815104949739660648])

intJdn9dn11uvrs $=([\mathbf{0 . 0 8 3 7 3 2 9 9 4 3 4 0 3 3 9 7 0 3 8 6 6 5 6 6 8 9 9 6}, \mathbf{0 . 2 4 8 1 9 9 0 1 8 7 3 6 5 5 9 9 0 8 8 1 3 6 2 3 1 9 7 4}]$

[ 0.2481990187365599088136231974, $\mathbf{0 . 3 1 5 7 1 1 3 0 2 4 7 3 1 8 7 0 4 1 2 7 7 2 2 0 1 8 9 ] )}$

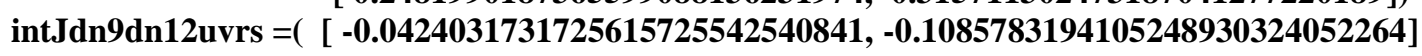

[ -0.1085783194105248930324052264, - $\mathbf{0 . 1 1 2 3 6 0 2 7 7 2 5 3 8 5 7 1 7 2 9 6 1 5 2 0 0 0 2 ] )}$

intJdn9dn13uvrs $=([\mathbf{0 . 1 7 8 8 5 1 0 5 1 5 2 5 2 6 1 2 1 0 3 0 2 2 5 7 3 6 8 , 0 . 3 3 1 2 7 8 7 3 1 1 2 6 1 9 3 7 0 5 0 0 6 3 7 1 9 4 8}]$

[ 0.331278731126193705006371948, 0.060329059165411543334696078])

intJdn9dn14uvrs $=([-\mathbf{- 0 . 1 5 4 5 0 8 8 1 3 7 7 4 5 1 8 5 5 8 3 0 7 8 4 3 3 7 5 , 0 . 0 3 6 8 4 5 3 9 0 7 2 2 8 3 8 2 4 7 6 5 2 7 5 9 2 9 6}]$

[ 0.036845390722838247652759296, 0.531391405011459465049996936])

intJdn9dn15uvrs $=([\mathbf{0 . 5 5 0 8 4 3 9 7 9 7 6 1 9 9 3 3 4 1 7 6 3 2 7 8 6 7 9}, \mathbf{- 0 . 0 2 6 6 4 9 7 2 1 1 1 1 7 5 4 8 0 7 3 0 9 0 6 1 6 8 6 8}]$

[ - $\mathbf{0 . 0 2 6 6 4 9 7 2 1 1 1 1 7 5 4 8 0 7 3 0 9 0 6 1 6 8 6 8 ,}-\mathbf{- 1 . 9 4 4 1 8 6 1 9 4 7 2 0 4 5 0 7 1 3 2 4 3 5 2 3 8 8}])$

intJdn9dn16uvrs $=([-\mathbf{- 0 . 4 3 3 2 6 2 2 1 4 1 9 7 9 3 2 0 2 0 3 6 0 2 3 8 3 3 5},-\mathbf{- 0 . 7 9 5 5 0 6 5 4 5 9 9 3 5 6 3 7 8 2 1 5 9 8 3 6 4 1 5 2}]$

[ - $\mathbf{- 0 . 7 9 5 5 0 6 5 4 5 9 9 3 5 6 3 7 8 2 1 5 9 8 3 6 4 1 5 2 ,}-\mathbf{- 0 . 0 5 1 9 9 5 7 5 9 2 6 7 7 7 7 9 8 5 3 0 2 6 2 7 0 3 ]})$

Table-2j

intJdnidnjuvrs, $\mathrm{i}=10, \mathrm{j}=1$ (1)16)

intJdn10dn1uvrs $=([-\mathbf{- 0 . 0 9 2 2 4 5 1 8 8 4 0 8 5 1 9 8 6 2 0 3 0 0 0 4 7 0 4},-\mathbf{- 0 . 1 0 4 9 1 1 1 9 9 3 8 8 1 8 4 1 0 0 7 0 0 5 9 7 5 0 8}]$

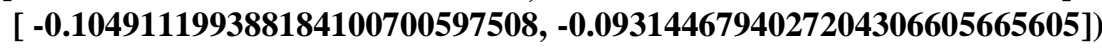

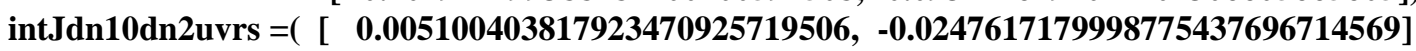
[ -0.0247617179998775437696714569, 0.000327370706036359563493128961])

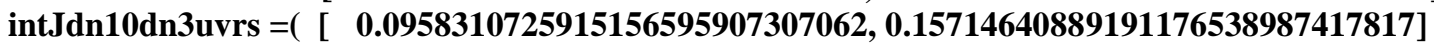

[ -0.14285359110808823461012582183, $-\mathbf{- 0 . 0 6 6 8 3 0 2 8 0 2 5 7 6 5 9 0 7 2 7 4 9 2 9 3 6 3 9 ] )}$

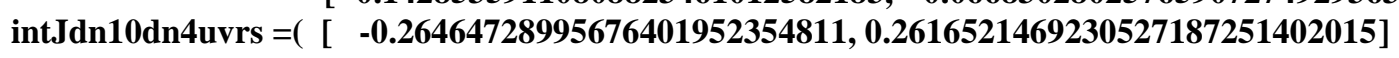

[ - -0.4508478530769472812748597985, 0.28346871318031592670232799$])$

H. T. Rathod, IJECS Volume 7 Issue 1 January 2018 Page No. 23329-23482 
intJdn10dn5uvrs $=([\mathbf{0 . 1 0 6 6 7 9 1 1 2 5 9 2 5 8 5 5 7 1 4 5 5 3 2 2 3 0 1}, \mathbf{0 . 0 0 6 0 5 9 3 3 7 1 9 2 4 7 7 8 0 8 8 1 1 0 6 8 0 9 5}]$ [ $0.006059337192477808811068095,-\mathbf{0 . 1 3 0 6 8 4 9 8 0 1 9 1 6 7 4 3 9 2 6 6 9 6 0 3 7 2 9 4 ]})$

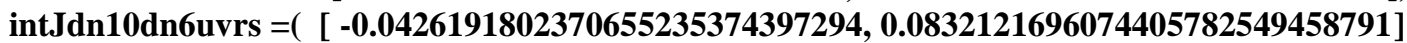
[ $0.0832121696074405782549458791,0.0498827009902849670234649781])$ intJdn10dn7uvrs $=([-\mathbf{- 0 . 0 0 5 4 5 5 2 4 1 7 7 9 1 7 8 6 6 8 2 5 7 9 7 1 1 4 9 4 ,}, \mathbf{0 . 0 8 7 7 7 3 0 6 2 4 7 7 8 9 6 0 6 0 2 0 3 1 9 1 7 0 0 1}]$

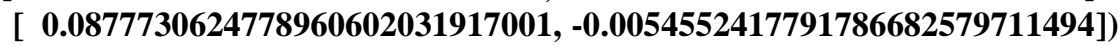

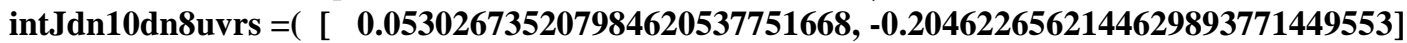

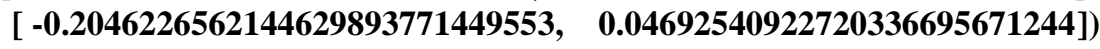
intJdn10dn9uvrs $=([-\mathbf{- 0 . 4 8 8 1 2 0 3 4 0 8 4 6 8 6 9 1 6 9 5 0 7 6 9 5 4 0 6 ,}, \mathbf{- 0 . 6 1 3 3 3 5 9 6 8 6 5 0 3 0 9 6 9 0 6 0 6 1 4 8 3 7 7 6 1}]$ [ 0.39916403134969030939385162239, $-\mathbf{- 0 . 2 6 4 6 3 1 7 0 2 8 1 5 1 0 4 9 4 9 7 3 9 6 6 0 6 4 8 ] )}$ intJdn10dn10uvrs $=([\mathrm{0.6665041132041592095461950376}, \mathbf{0 . 2 7 3 0 9 5 6 7 6 7 7 9 1 6 2 6 5 6 1 0 6 5 4 1 8 6 0 6 8}]$ [ 0.27309567677916265610654186068, 2.001089658057721102391264327]) intJdn10dn11uvrs $=([-\mathbf{- 0 . 2 5 4 3 3 7 7 6 0 1 2 1 6 5 3 4 3 9 5 4 4 5 3 5 4 5 3 4}, \mathbf{- 0 . 6 2 9 0 1 5 9 0 4 3 8 5 5 4 7 0 6 7 6 2 7 6 5 0 9 1 6 9}]$ [ -0.6290159043855470676276509169, $-\mathbf{- 0 . 8 5 7 9 7 7 5 6 8 7 4 3 7 3 3 0 7 2 1 8 1 1 0 9 9 6 2 ] ) ~}$ intJdn10dn12uvrs $=([\mathbf{0 . 1 1 8 0 1 0 8 0 5 4 6 7 1 7 6 2 1 4 1 4 4 9 1 7 7 3 4}, \mathbf{0 . 2 7 2 6 0 5 9 7 9 7 6 6 2 2 5 4 1 2 2 3 5 6 7 4 9 8 7}]$ [ 0.272605979766225412235674987, 0.3065397294444835089245124969])

intJdn10dn13uvrs $=([-\mathbf{- 0 . 1 1 3 1 5 1 9 4 3 0 9 1 6 3 6 1 4 0 3 5 5 8 9 5 5 1 5 8 ,}, \mathbf{0 . 0 3 0 4 4 8 3 2 0 8 4 4 6 3 0 4 5 0 0 4 4 6 6 4 7 ]}$ [ $\mathbf{0 . 0 3 0 4 4 8 3 2 0 8 4 4 6 3 0 4 5 0 0 4 4 6 6 4 7 , 0 . 6 2 1 7 9 0 5 4 4 8 2 9 7 7 4 9 0 2 3 9 9 4 6 3 4 8 ] )}$ intJdn10dn14uvrs $=([\mathbf{0 . 0 4 5 0 6 5 5 1 2 1 2 8 4 0 7 8 4 6 4 0 7 8 2 6 3 8 4},-\mathbf{- 0 . 3 0 4 2 3 3 2 8 2 3 9 7 5 3 3 2 1 6 8 4 4 6 1 8 6 8 6}]$ [ - -0.304233282397533216844618686, $-\mathbf{- 0 . 1 9 8 1 3 0 2 5 9 7 6 0 0 0 1 8 3 8 2 8 1 6 8 7 3 4 1 ]})$

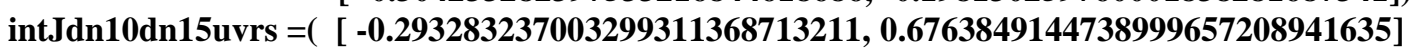
[ 0.6763849144738999657208941635, 0.55242173149670587806391031$])$ intJdn10dn16uvrs $=([\mathbf{0 . 4 6 3 6 4 2 4 2 6 4 3 5 2 3 3 5 4 1 5 1 7 3 3 9 9 4 3 5}, \mathbf{0 . 0 3 2 5 0 2 7 1 2 0 7 9 2 1 7 1 9 3 4 3 3 8 3 6 1 3 7}]$ [ 0.032502712079217193433836137, -2.24559114498245358748525615])

intJdnidnjuvrs, $\mathrm{i}=\mathbf{1 1}, \mathrm{j}=1$ (1)16)

Table-2k

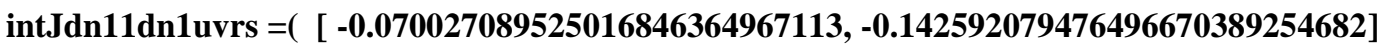
[ $\mathbf{0 . 1 5 7 4 0 7 9 2 0 5 2 3 5 0 3 3 2 9 6 1 0 7 4 5 3 1 8 , 0 . 1 1 7 2 5 0 3 9 4 5 3 7 4 0 1 7 4 7 9 6 7 4 6 0 4 9 6 7 ] )}$ intJdn11dn2uvrs $=([\mathbf{- 0 . 0 0 8 0 4 9 4 9 8 8 7 2 3 6 9 4 7 9 0 0 4 1 2 3 0 0 0 2}, \mathbf{- 0 . 0 2 3 9 6 0 9 9 1 4 5 5 1 2 8 5 3 5 8 1 8 6 3 4 8 3 6 1}]$ $[-\mathbf{0 . 0 2 3 9 6 0 9 9 1 4 5 5 1 2 8 5 3 5 8 1 8 6 3 4 8 3 6 1 , 0 . 0 0 2 3 5 3 0 4 5 7 9 4 8 5 8 3 6 5 2 6 3 2 3 5 0 8 2 7}])$

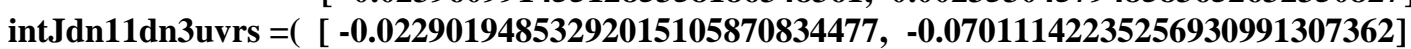
[ - $\mathbf{- 0 . 0 7 0 1 1 1 4 2 2 3 5 2 5 6 9 3 0 9 9 1 3 0 7 3 6 2 ,}, \mathbf{- 0 . 0 9 5 6 3 1 5 3 3 0 0 2 2 4 0 6 8 8 0 9 7 5 4 3 5 0 1 ] )}$ intJdn11dn4uvrs $=([\mathbf{0 . 1 3 7 8 2 9 7 0 1 8 3 6 4 7 0 7 6 1 4 8 4 8 5 3 4 9 0 7}, \mathbf{- 0 . 3 2 2 5 4 0 7 9 1 6 3 2 4 7 7 4 1 0 0 7 9 1 6 7 0 8 5 1}]$ [ 0.3899592083675225899208329149, - $\mathbf{0 . 4 8 4 0 6 0 3 3 6 3 5 6 1 4 0 6 4 6 7 6 1 8 0 7 1 1 9 ] )}$ intJdn11dn5uvrs $=([\mathbf{- 0 . 0 2 6 9 7 8 0 4 5 8 6 9 8 8 0 8 7 1 3 7 9 5 1 3 0 7 8}, \mathbf{- 0 . 1 9 6 9 6 6 3 9 0 3 4 1 0 9 0 9 2 8 9 0 9 6 9 4 0 8 4}]$ [ -0.196966390341090928909694084, - $\mathbf{0 . 0 7 8 8 9 7 3 5 8 2 4 9 7 5 2 6 0 8 6 6 8 2 7 3 9 8 1 ]})$

intJdn11dn6uvrs $=([\mathbf{0 . 0 2 7 5 2 7 7 9 1 0 9 3 3 0 7 2 0 8 2 4 8 7 2 5 5 7 5 , 0 . 0 8 9 2 6 8 0 9 8 8 1 6 0 5 2 0 1 6 5 7 8 3 8 7 7 2 6}]$ [ 0.089268098816052016578387726, 0.027527791093307208248725575]) intJdn11dn7uvrs $=([\mathbf{0 . 0 4 9 8 8 2 7 0 0 9 9 0 2 8 4 9 6 7 0 2 3 4 6 4 9 7 8 1 ,}, \mathbf{0 . 0 8 3 2 1 2 1 6 9 6 0 7 4 4 0 5 7 8 2 5 4 9 4 5 8 7 9 1}]$ [ 0.0832121696074405782549458791, -0.0426191802370655235374397294])

intJdn11dn8uvrs $=([-\mathbf{- 0 . 1 1 5 4 3 6 4 2 6 0 9 2 5 2 7 8 8 8 3 1 8 2 9 7 2 0 8 5 2},-\mathbf{- 0 . 0 0 7 9 3 6 2 5 2 1 6 7 2 4 6 9 9 5 7 6 5 1 5 5 6 1 3 6}]$ [ - $\mathbf{- 0 . 0 0 7 9 3 6 2 5 2 1 6 7 2 4 6 9 9 5 7 6 5 1 5 5 6 1 3 6 ,}, \mathbf{0 . 1 2 3 8 0 8 0 4 2 7 1 1 9 4 8 7 2 4 3 3 0 8 2 5 1 3 9 ] )}$ intJdn11dn9uvrs $=([\mathbf{0 . 0 8 3 7 3 2 9 9 4 3 4 0 3 3 9 7 0 3 8 6 6 5 6 6 8 9 9 6 , 0 . 2 4 8 1 9 9 0 1 8 7 3 6 5 5 9 9 0 8 8 1 3 6 2 3 1 9 7 4}]$ [ 0.2481990187365599088136231974, $\mathbf{0 . 3 1 5 7 1 1 3 0 2 4 7 3 1 8 7 0 4 1 2 7 7 2 2 0 1 8 9 ] )}$ intJdn11dn10uvrs $=([\mathbf{- 0 . 2 5 4 3 3 7 7 6 0 1 2 1 6 5 3 4 3 9 5 4 4 5 3 5 4 5 3 4}, \mathbf{- 0 . 6 2 9 0 1 5 9 0 4 3 8 5 5 4 7 0 6 7 6 2 7 6 5 0 9 1 6 9 ]}$ [ -0.6290159043855470676276509169, $-\mathbf{- 0 . 8 5 7 9 7 7 5 6 8 7 4 3 7 3 3 0 7 2 1 8 1 1 0 9 9 6 2 ] )}$

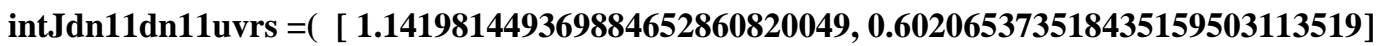
[ 0.602065373518435159503113519, 1.332289708203970804992564589]) intJdn11dn12uvrs $=\left(\left[\begin{array}{ll}-\mathbf{0 . 2 2 2 0 2 6 4 8 0 8 1 3 6 2 1 3 5 9 9 3 2 4 0 7 8 1 1}, & \mathbf{0 . 2 7 4 7 7 4 4 9 0 5 6 0 7 3 3 3 4 0 6 3 6 0 2 2 0 4 6}]\end{array}\right.\right.$ [ - -0.737725509439266659363977954, - 0.78668680846441673459831513779$])$

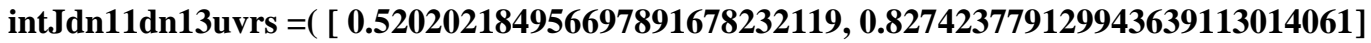
[ 0.827423779129943639113014061, 0.107366707368679853419887039])

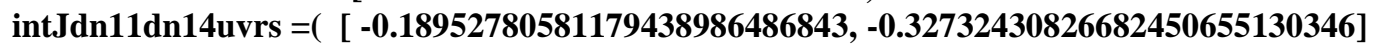

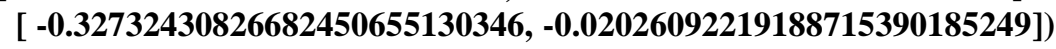
intJdn11dn15uvrs $=([$ [ $0.449881020809519531356712718,0.106104670667416834133287489]$ [ 0.106104670667416834133287489, - -0.26123164244466091271015883]) intJdn11dn16uvrs $=([-\mathbf{- 1 . 5 0 1 7 5 2 7 8 7 7 5 6 7 2 0 2 9 1 0 5 1 9 5 5 3 6},-\mathbf{- 0 . 5 1 0 5 9 9 4 6 0 9 5 9 2 0 0 0 5 1 9 7 8 4 5 9 6 5}]$ [ - $\mathbf{0 . 5 1 0 5 9 9 4 6 0 9 5 9 2 0 0 0 5 1 9 7 8 4 5 9 6 5 ,} \mathbf{0 . 6 0 1 0 5 8 3 5 7 5 0 6 5 4 3 5 9 4 9 5 6 5 8 2 6 3 ]})$ 
intJdn12dn1uvrs $=([\mathbf{0 . 1 5 7 8 7 1 6 2 4 5 4 1 3 3 8 7 0 4 7 9 4 5 5 7 8 2 9}, \mathbf{0 . 2 6 8 9 4 5 5 2 5 9 8 4 7 7 6 9 8 1 5 4 3 0 2 2 5 3 3}]$

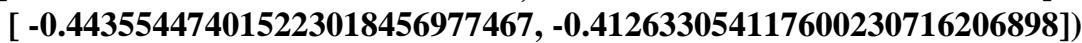

intJdn12dn2uvrs $=([\mathbf{0 . 0 2 2 0 3 4 6 6 2 6 2 8 5 9 5 6 9 3 4 0 0 3 0 1 6 9 8 6 , 0 . 0 4 9 5 4 8 7 6 6 3 2 6 6 4 0 7 1 3 4 7 6 1 0 3 3 8 4 2}]$ [ 0.0495487663266407134761033842, - $\mathbf{0 . 0 2 0 2 7 0 5 5 0 7 0 5 8 5 7 1 4 6 8 1 3 4 5 2 0 5 7 ]})$ intJdn12dn3uvrs $=([\mathbf{0 . 0 1 2 0 3 0 6 2 4 6 9 9 0 6 4 7 9 3 9 5 3 0 9 8 0 4 3 , 0 . 0 3 0 6 6 2 5 4 8 7 4 0 4 7 1 1 7 0 6 9 7 9 5 4 5 8 8 1}]$ [ $\mathbf{0 . 0 3 0 6 6 2 5 4 8 7 4 0 4 7 1 1 7 0 6 9 7 9 5 4 5 8 8 1 , 0 . 0 3 1 2 2 5 8 9 7 4 5 8 8 8 0 4 9 1 5 8 7 9 1 8 5 9 0 4 ] )}$

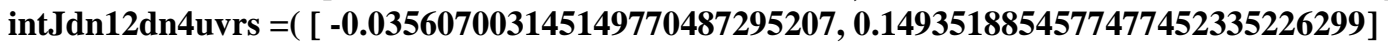
[ - -0.15064811454225225476647737, 0.135316485729802334623950907]) intJdn12dn5uvrs $=([\mathbf{0 . 0 8 9 2 1 1 6 5 4 5 8 1 6 3 8 1 0 6 9 6 8 7 6 6 9 6 , 0 . 4 2 7 1 8 8 8 8 4 7 9 9 0 7 8 0 7 0 1 7 3 9 9 2 3 8 6}]$ [ 0.427188884799078070173992386, $\mathbf{0 . 0 8 9 2 1 1 6 5 4 5 8 1 6 3 8 1 0 6 9 6 8 7 6 6 9 6 ] )}$ intJdn12dn6uvrs $=([-\mathbf{- 0 . 0 7 8 8 9 7 3 5 8 2 4 9 7 5 2 6 0 8 6 6 8 2 7 3 9 8 1}, \mathbf{- 0 . 1 9 6 9 6 6 3 9 0 3 4 1 0 9 0 9 2 8 9 0 9 6 9 4 0 8 4}]$ [ -0.196966390341090928909694084, $\mathbf{- 0 . 0 2 6 9 7 8 0 4 5 8 6 9 8 8 0 8 7 1 3 7 9 5 1 3 0 7 8 ] )}$

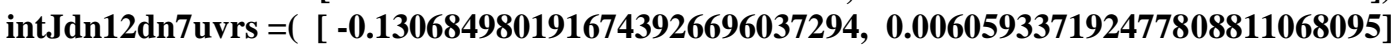
[ $\mathbf{0 . 0 0 6 0 5 9 3 3 7 1 9 2 4 7 7 8 0 8 8 1 1 0 6 8 0 9 5 , 0 . 1 0 6 6 7 9 1 1 2 5 9 2 5 8 5 5 7 1 4 5 5 3 2 2 3 0 0 9 ]})$

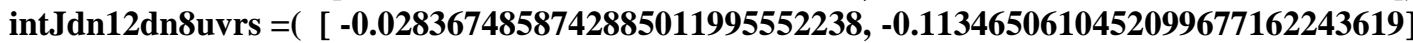
[ -0.1134650610452099677162243619, - $\mathbf{0 . 1 3 4 9 5 0 7 7 1 4 9 9 7 2 7 0 7 1 1 3 9 8 6 7 6 1 5 ] )}$

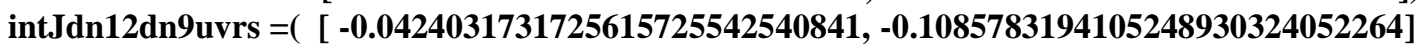
[ - -0.1085783194105248930324052264, $-\mathbf{- 0 . 1 1 2 3 6 0 2 7 7 2 5 3 8 5 7 1 7 2 9 6 1 5 2 0 0 0 2 ] )}$ intJdn12dn10uvrs $=([\mathbf{0 . 1 1 8 0 1 0 8 0 5 4 6 7 1 7 6 2 1 4 1 4 4 9 1 7 7 3 4 ,}, \mathbf{0 . 2 7 2 6 0 5 9 7 9 7 6 6 2 2 5 4 1 2 2 3 5 6 7 4 9 8 7}]$ [ 0.272605979766225412235674987, 0.3065397294444835089245124969])

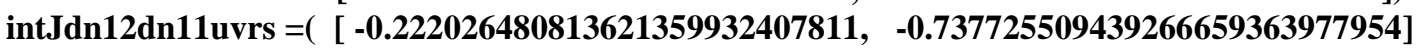
[ $0.274774490560733340636022046,-\mathbf{0 . 7 8 6 6 8 6 8 0 8 4 6 4 4 1 6 7 3 4 5 9 8 3 1 5 1 3 7 7 9 ]})$ intJdn12dn12uvrs $=([$ [ 1.495308248540817222321613675, 0.902875989441502362683040391$]$ [ 0.902875989441502362683040391, 1.317586787287312732669083137]) intJdn12dn13uvrs $=([-\mathbf{- 1 . 8 3 1 1 7 9 5 6 2 5 0 8 9 3 2 6 6 0 7 2 2 8 1 3 9 8 3}, \mathbf{- 0 . 5 3 0 8 4 3 3 5 5 9 8 7 0 5 1 3 2 6 2 0 4 9 8 7 9 1 7}]$ [ - $\mathbf{0 . 5 3 0 8 4 3 3 5 5 9 8 7 0 5 1 3 2 6 2 0 4 9 8 7 9 1 7 ,} \mathbf{0 . 4 7 5 5 5 8 9 0 7 8 3 1 9 3 2 0 0 3 1 8 8 7 4 6 4 7 8 ] )}$

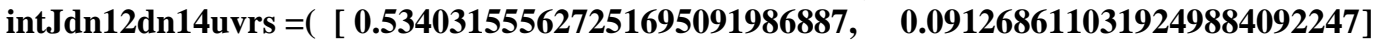

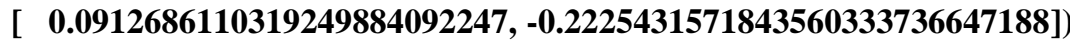
intJdn12dn15uvrs $=\left(\left[\begin{array}{l}\mathbf{0} .086904547772480103903106692,0.379360747071947741960232042\end{array}\right]\right.$ [ 0.379360747071947741960232042, $\mathbf{0 . 4 4 9 8 7 7 6 9 2 1 2 6 6 6 3 6 6 1 6 2 9 5 5 2 3 5 ] )}$

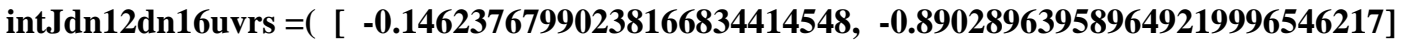
[ -0.890289639589649219996546217, -1.1955736019576031500653136788])

Table-2m

intJdnidnjuvrs, $\mathrm{i}=13, \mathrm{j}=1$ (1)16)

intJdn13dn1uvrs $=([-\mathbf{- 0 . 9 2 3 6 7 7 8 3 9 1 0 1 8 3 1 3 0 5 5 5 0 6 7 7 1}, \mathbf{- 1 . 0 0 7 0 3 2 4 5 4 8 9 0 5 0 0 4 6 3 0 7 5 6 4 2 3}])$ [ -1.0070324548905004630756423, - $\mathbf{0 . 9 2 3 6 7 7 8 3 9 1 0 1 8 3 1 3 0 5 5 5 0 6 7 7 1 ] )}$

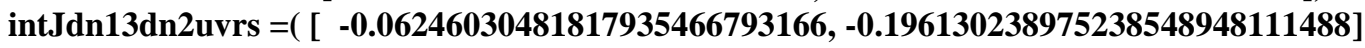
[ - $\mathbf{0 . 1 9 6 1 3 0 2 3 8 9 7 5 2 3 8 5 4 8 9 4 8 1 1 1 4 8 8 ,} \mathbf{0 . 0 1 8 0 6 6 4 4 7 7 6 5 1 6 8 8 9 6 4 5 6 3 2 1 8 2 4 ]})$

intJdn13dn3uvrs $=([-\mathbf{- 0 . 0 4 4 0 1 0 7 1 2 8 4 2 9 1 2 6 5 2 1 0 7 5 7 7 0 6 4 4},-\mathbf{0 . 0 9 6 4 1 5 9 7 5 2 9 5 6 6 0 7 7 1 1 8 9 9 1 4 2 3 7 6}]$ [ -0.0964159752956607711899142376, - $\mathbf{0 . 0 4 4 0 1 0 7 1 2 8 4 2 9 1 2 6 5 2 1 0 7 5 7 7 0 6 4 4 ] )}$

intJdn13dn4uvrs $=([$ o.018066447765168896456321824, $-\mathbf{- 0 . 1 9 6 1 3 0 2 3 8 9 7 5 2 3 8 5 4 8 9 4 8 1 1 1 4 8 8}]$ [ -0.196130238975238548948111488, $\mathbf{- 0 . 0 6 2 4 6 0 3 0 4 8 1 8 1 7 9 3 5 4 6 6 7 9 3 1 6 5 7 ] )}$

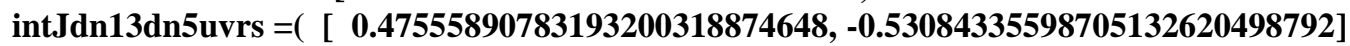
[ - $\mathbf{0 . 5 3 0 8 4 3 3 5 5 9 8 7 0 5 1 3 2 6 2 0 4 9 8 7 9 2 , - 1 . 8 3 1 1 7 9 5 6 2 5 0 8 9 3 2 6 6 0 7 2 2 8 1 3 9 8 ] )}$

intJdn13dn6uvrs $=([\mathbf{0 . 1 0 7 3 6 6 7 0 7 3 6 8 6 7 9 8 5 3 4 1 9 8 8 7 0 3 9}, \mathbf{0 . 8 2 7 4 2 3 7 7 9 1 2 9 9 4 3 6 3 9 1 1 3 0 1 4 0 6 1}]$ [ 0.827423779129943639113014061, 0.520202184956697891678232119]) intJdn13dn7uvrs $=\left(\left[\begin{array}{l}0.62179054482977490239946348, \\ 0.0304483208446304500446647\end{array}\right]\right.$ [ $\mathbf{0 . 0 3 0 4 4 8 3 2 0 8 4 4 6 3 0 4 5 0 0 4 4 6 6 4 7 , - 0 . 1 1 3 1 5 1 9 4 3 0 9 1 6 3 6 1 4 0 3 5 5 8 9 5 5 1 5 8 ] )}$

intJdn13dn8uvrs $=([\mathbf{0 . 0 6 0 3 2 9 0 5 9 1 6 5 4 1 1 5 4 3 3 3 4 6 9 6 0 7 8}, \mathbf{0 . 3 3 1 2 7 8 7 3 1 1 2 6 1 9 3 7 0 5 0 0 6 3 7 1 9 4 8}]$ [ 0.331278731126193705006371948, 0.178851051525261210302257368]) intJdn13dn9uvrs $=\left(\left[\begin{array}{l}0.178851051525261210302257368,0.331278731126193705006371948\end{array}\right]\right.$ [ 0.331278731126193705006371948, 0.060329059165411543334696078])

intJdn13dn10uvrs $=([-\mathbf{- 0 . 1 1 3 1 5 1 9 4 3 0 9 1 6 3 6 1 4 0 3 5 5 8 9 5 5 1 5 8}, \mathbf{0 . 0 3 0 4 4 8 3 2 0 8 4 4 6 3 0 4 5 0 0 4 4 6 6 4 7 ]}$ [ $\mathbf{0 . 0 3 0 4 4 8 3 2 0 8 4 4 6 3 0 4 5 0 0 4 4 6 6 4 7 , 0 . 6 2 1 7 9 0 5 4 4 8 2 9 7 7 4 9 0 2 3 9 9 4 6 3 4 8 ] )}$ intJdn13dn11uvrs $=([\mathbf{0 . 5 2 0 2 0 2 1 8 4 9 5 6 6 9 7 8 9 1 6 7 8 2 3 2 1 1 9}, \mathbf{0 . 8 2 7 4 2 3 7 7 9 1 2 9 9 4 3 6 3 9 1 1 3 0 1 4 0 6 1}]$ [ 0.827423779129943639113014061, 0.107366707368679853419887039]) intJdn13dn12uvrs $=([-\mathbf{- 1 . 8 3 1 1 7 9 5 6 2 5 0 8 9 3 2 6 6 0 7 2 2 8 1 3 9 8 3},-\mathbf{- 0 . 5 3 0 8 4 3 3 5 5 9 8 7 0 5 1 3 2 6 2 0 4 9 8 7 9 1 7}]$ [ - $\mathbf{0 . 5 3 0 8 4 3 3 5 5 9 8 7 0 5 1 3 2 6 2 0 4 9 8 7 9 1 7 ,} \mathbf{0 . 4 7 5 5 5 8 9 0 7 8 3 1 9 3 2 0 0 3 1 8 8 7 4 6 4 7 8 ] )}$ intJdn13dn13uvrs $=([$ 5.220412586998316768421088045, 2.88129572754069028280297951] [ $2.88129572754069028280297951,5.220412586998316768421088045])$

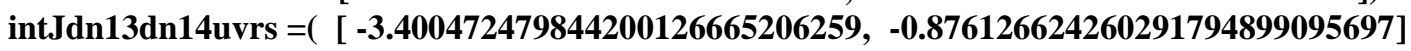

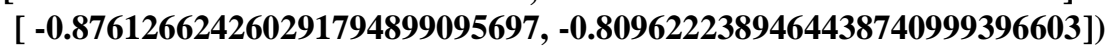

H. T. Rathod, IJECS Volume 7 Issue 1 January 2018 Page No. 23329-23482 
intJdn13dn15uvrs $=([-\mathbf{- 0 . 0 1 8 0 0 2 4 0 9 2 8 7 1 0 6 9 5 5 0 3 0 6 5 1 1 3 8},-\mathbf{- 0 . 9 4 9 9 4 8 5 2 1 1 1 0 9 0 1 2 9 6 7 6 1 1 3 4 2 2 2}]$ [ -0.949948521110901296761134222, - $\mathbf{0 . 0 1 8 0 0 2 4 0 9 2 8 7 1 0 6 9 5 5 0 3 0 6 5 1 1 3 8 ] )}$ intJdn13dn16uvrs $=([-\mathbf{- 0 . 8 0 9 6 2 2 2 3 8 9 4 6 4 4 3 8 7 4 0 9 9 9 3 9 6 6 0 3},-\mathbf{- 0 . 8 7 6 1 2 6 6 2 4 2 6 0 2 9 1 7 9 4 8 9 9 0 9 5 6 9 7}]$ [ - -0.876126624260291794899095697, -3.400472479844200126665206259])

intJdnidnjuvrs, $\mathrm{i}=14, \mathrm{j}=1$ (1)16)

Table-2n

intJdn14dn1uvrs $=([\mathbf{0 . 3 3 6 8 5 1 9 9 0 2 2 2 7 2 2 8 6 4 0 9 4 1 7 2 5 1 9 , 0 . 3 8 1 6 7 1 4 0 4 3 5 2 3 9 1 7 1 7 7 1 6 0 5 0 0 0 3}]$ [ 0.381671404352391717716050003, 0.3384590115434723589069086$])$

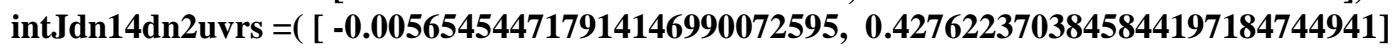
[ 0.4276223703845844197184744941, - $\mathbf{0 . 1 5 3 9 3 1 0 1 5 7 1 3 2 1 1 3 3 9 1 3 3 1 3 7 8 4 8 6 ] )}$

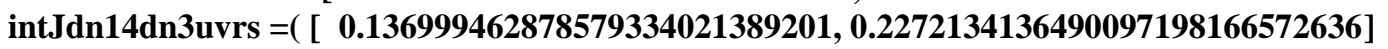
[ 0.2272134136490097198166572636, 0.094118555809911844171302174$])$

intJdn14dn4uvrs $=([-\mathbf{- 0 . 0 0 0 9 8 4 6 6 8 1 6 6 0 4 5 0 0 9 2 5 7 9 8 0 4 6 1 6}, \mathbf{0 . 0 8 6 3 0 0 0 8 0 8 3 0 6 7 8 1 3 5 9 3 1 2 9 9 1 5 1 9}]$ [ $0.0863000808306781359312991519,0.0208266894443892215290932469])$

intJdn14dn5uvrs $=([-\mathbf{- 1 . 1 9 5 5 7 3 6 0 1 9 5 7 6 0 3 1 5 0 0 6 5 3 1 3 6 7 8 8}, \mathbf{- 0 . 8 9 0 2 8 9 6 3 9 5 8 9 6 4 9 2 1 9 9 9 6 5 4 6 2 1 7}]$ [ - -0.890289639589649219996546217, $-\mathbf{- 0 . 1 4 6 2 3 7 6 7 9 9 0 2 3 8 1 6 6 8 3 4 4 1 4 5 4 8 ] )}$

intJdn14dn6uvrs $=([\mathbf{0 . 6 0 1 0 5 8 3 5 7 5 0 6 5 4 3 5 9 4 9 5 6 5 8 2 6 3},-\mathbf{0 . 5 1 0 5 9 9 4 6 0 9 5 9 2 0 0 0 5 1 9 7 8 4 5 9 6 5}]$

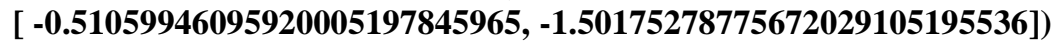

intJdn14dn7uvrs $=([-\mathbf{- 2 . 2 4 5 5 9 1 1 4 4 9 8 2 4 5 3 5 8 7 4 8 5 2 5 6 1 5}, \mathbf{0 . 0 3 2 5 0 2 7 1 2 0 7 9 2 1 7 1 9 3 4 3 3 8 3 6 1 3 7}]$ [ $\mathbf{0 . 0 3 2 5 0 2 7 1 2 0 7 9 2 1 7 1 9 3 4 3 3 8 3 6 1 3 7 , 0 . 4 6 3 6 4 2 4 2 6 4 3 5 2 3 3 5 4 1 5 1 7 3 3 9 9 4 3 5 ] )}$

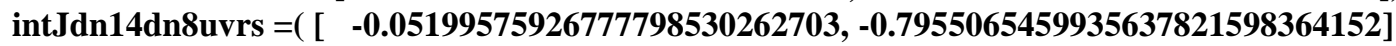
[ - - $0.7955065459935637821598364152,-0.433262214197932020360238335])$

intJdn14dn9uvrs $=([-\mathbf{- 0 . 1 5 4 5 0 8 8 1 3 7 7 4 5 1 8 5 5 8 3 0 7 8 4 3 3 7 5}, \mathbf{0 . 0 3 6 8 4 5 3 9 0 7 2 2 8 3 8 2 4 7 6 5 2 7 5 9 2 9 6}]$ [ $\mathbf{0 . 0 3 6 8 4 5 3 9 0 7 2 2 8 3 8 2 4 7 6 5 2 7 5 9 2 9 6 , 0 . 5 3 1 3 9 1 4 0 5 0 1 1 4 5 9 4 6 5 0 4 9 9 9 6 9 3 6 ] )}$ intJdn14dn10uvrs $=([\mathbf{0 . 0 4 5 0 6 5 5 1 2 1 2 8 4 0 7 8 4 6 4 0 7 8 2 6 3 8 4},-\mathbf{- 0 . 3 0 4 2 3 3 2 8 2 3 9 7 5 3 3 2 1 6 8 4 4 6 1 8 6 8 6}]$ [ -0.304233282397533216844618686, $-\mathbf{- 0 . 1 9 8 1 3 0 2 5 9 7 6 0 0 0 1 8 3 8 2 8 1 6 8 7 3 4 1 ] )}$

intJdn14dn11uvrs $=([-\mathbf{- 0 . 1 8 9 5 2 7 8 0 5 8 1 1 7 9 4 3 8 9 8 6 4 8 6 8 4 3}, \mathbf{- 0 . 3 2 7 3 2 4 3 0 8 2 6 6 8 2 4 5 0 6 5 5 1 3 0 3 4 6}]$

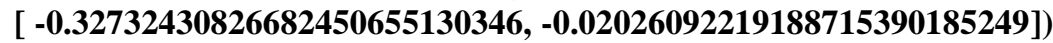

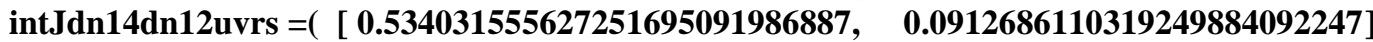

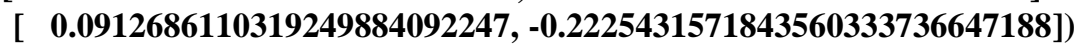

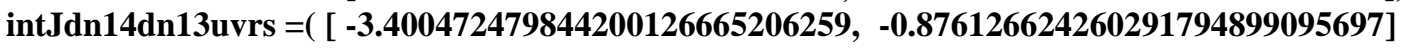
[ -0.876126624260291794899095697, -0.8096222389464438740999396603])

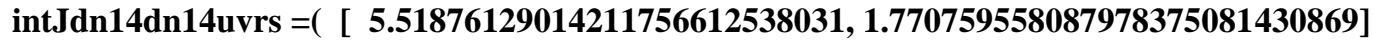
[ $1.770759558087978375081430869,3.929578185962763299703317274])$

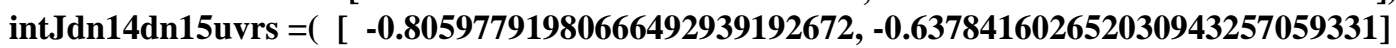
[ -0.637841602652030943257059331, - 2.769794568377644494965093629]) intJdn14dn16uvrs $=([0.877518569823348982633756744,1.287737922980470717927187534]$ [ 1.287737922980470717927187534, 0.877518569823348982633756744])

Table-2o

intJdnidnjuvrs, $\mathrm{i}=15, \mathrm{j}=1$ (1)16)

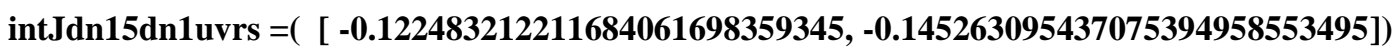
[ -0.145263095437075394958553495, - $\mathbf{0 . 1 2 2 4 8 3 2 1 2 2 1 1 6 8 4 0 6 1 6 9 8 3 5 9 3 4 5 ] )}$ intJdn15dn2uvrs $=\left(\left[\begin{array}{l}\mathbf{0 . 0 0 8 0 3 3 2 3 8 0 5 5 9 6 9 8 1 1 7 7 0 2 5 8 9 3},-\mathbf{0 . 1 9 4 2 1 6 9 7 3 7 6 3 7 1 4 3 5 4 5 3 4 5 3 7 5 1 7 5}] \\ {[}\end{array}\right.\right.$ [ - $\mathbf{0 . 1 9 4 2 1 6 9 7 3 7 6 3 7 1 4 3 5 4 5 3 4 5 3 7 5 1 7 5 ,} \mathbf{0 . 0 4 1 6 8 1 3 1 4 3 6 7 4 2 5 9 9 8 1 1 1 6 9 6 9 1 2 ] )}$

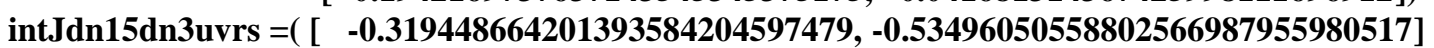
[ - $\mathbf{- 0 . 5 3 4 9 6 0 5 0 5 5 8 8 0 2 5 6 6 9 8 7 9 5 5 9 8 0 5 1 7 ,}-\mathbf{- 0 . 3 1 9 4 4 8 6 6 4 2 0 1 3 9 3 5 8 4 2 0 4 5 9 7 4 7 9 ] )}$

intJdn15dn4uvrs $=([\mathbf{0 . 0 4 1 6 8 1 3 1 4 3 6 7 4 2 5 9 9 8 1 1 1 6 9 6 9 1 1 9},-\mathbf{0 . 1 9 4 2 1 6 9 7 3 7 6 3 7 1 4 3 5 4 5 3 4 5 3 7 5 1 7 5}]$

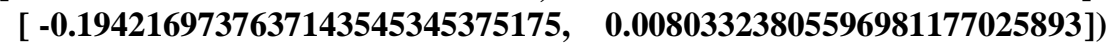

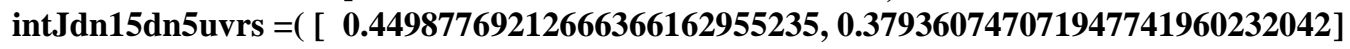
[ 0.379360747071947741960232042, 0.086904547772480103903106692])

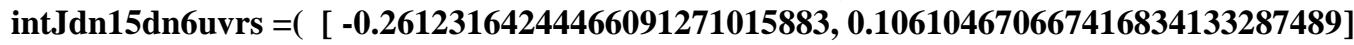
[ 0.106104670667416834133287489, 0.44988102080951953135671272])

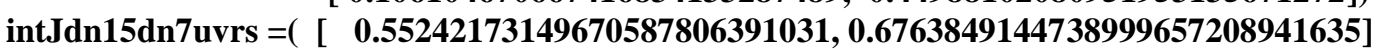
[ 0.6763849144738999657208941635, -0.293283237003299311368713211])

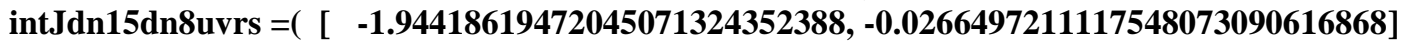
[ -0.0266497211117548073090616868, 0.550843979761993341763278679$])$

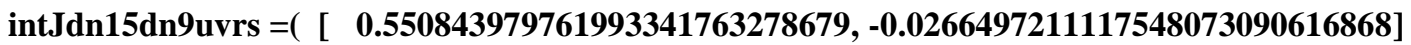
[ -0.0266497211117548073090616868, $-\mathbf{- 1 . 9 4 4 1 8 6 1 9 4 7 2 0 4 5 0 7 1 3 2 4 3 5 2 3 8 8 ] )}$

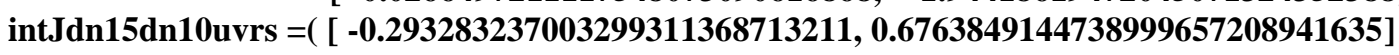
[ 0.6763849144738999657208941635, 0.55242173149670587806391031$])$ 
intJdn15dn11uvrs $=([\mathbf{0 . 4 4 9 8 8 1 0 2 0 8 0 9 5 1 9 5 3 1 3 5 6 7 1 2 7 1 8 , 0 . 1 0 6 1 0 4 6 7 0 6 6 7 4 1 6 8 3 4 1 3 3 2 8 7 4 8 9}]$ [ 0.106104670667416834133287489, -0.26123164244466091271015883]) intJdn15dn12uvrs $=([\mathbf{0 . 0 8 6 9 0 4 5 4 7 7 7 2 4 8 0 1 0 3 9 0 3 1 0 6 6 9 2 , 0 . 3 7 9 3 6 0 7 4 7 0 7 1 9 4 7 7 4 1 9 6 0 2 3 2 0 4 2}]$ [ 0.379360747071947741960232042, 0.44987769212666366162955235$])$ intJdn15dn13uvrs $=([-\mathbf{- 0 . 0 1 8 0 0 2 4 0 9 2 8 7 1 0 6 9 5 5 0 3 0 6 5 1 1 3 8},-\mathbf{- 0 . 9 4 9 9 4 8 5 2 1 1 1 0 9 0 1 2 9 6 7 6 1 1 3 4 2 2 2}]$ [ -0.949948521110901296761134222, - $\mathbf{0 . 0 1 8 0 0 2 4 0 9 2 8 7 1 0 6 9 5 5 0 3 0 6 5 1 1 3 8 ] )}$

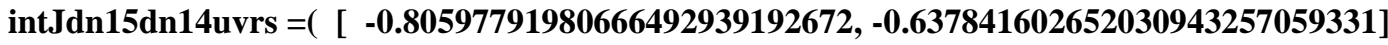
[ -0.637841602652030943257059331, - -2.769794568377644494965093629])

intJdn15dn15uvrs $=([$ 4.39476432366214663601450761, 1.023888052764473488171737195$]$ [ $1.023888052764473488171737195,4.39476432366214663601450761])$ intJdn15dn16uvrs $=([-2.769794568377644494965093629,-\mathbf{0 . 6 3 7 8 4 1 6 0 2 6 5 2 0 3 0 9 4 3 2 5 7 0 5 9 3 3 1}]$ [ -0.637841602652030943257059331, $-\mathbf{- 0 . 8 0 5 9 7 7 9 1 9 8 0 6 6 6 4 9 2 9 3 9 1 9 2 6 7 2 ] ) ~}$

intJdnidnjuvrs, $\mathrm{i}=\mathbf{1 6}, \mathrm{j}=1$ (1)16)

Table-2p

intJdn16dn1uvrs $=\left(\left[\begin{array}{l}\mathbf{0} .3384590115434723589069086 \\ 0.381671404352391717716050003\end{array}\right]\right.$ [ $0.381671404352391717716050003,0.33685199022272286409417252])$

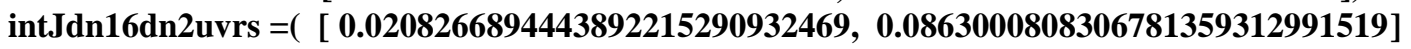
[ 0.0863000808306781359312991519, -0.0009846681660450092579804616])

intJdn16dn3uvrs $=([\mathbf{0 . 0 9 4 1 1 8 5 5 5 8 0 9 9 1 1 8 4 4 1 7 1 3 0 2 1 7 4 4 , 0 . 2 2 7 2 1 3 4 1 3 6 4 9 0 0 9 7 1 9 8 1 6 6 5 7 2 6 3 6}]$ [ 0.2272134136490097198166572636, $\mathbf{0 . 1 3 6 9 9 9 4 6 2 8 7 8 5 7 9 3 3 4 0 2 1 3 8 9 2 0 1 ] )}$

intJdn16dn4uvrs $=([-\mathbf{- 0 . 1 5 3 9 3 1 0 1 5 7 1 3 2 1 1 3 3 9 1 3 3 1 3 7 8 4 8 6}, \mathbf{0 . 4 2 7 6 2 2 3 7 0 3 8 4 5 8 4 4 1 9 7 1 8 4 7 4 4 9 4 1}]$

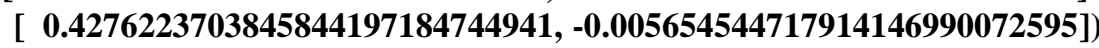

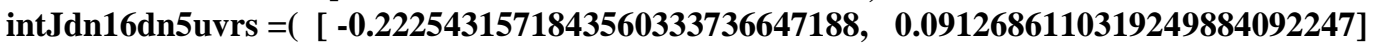
[ $\mathbf{0 . 0 9 1 2 6 8 6 1 1 0 3 1 9 2 4 9 8 8 4 0 9 2 2 4 7 , 0 . 5 3 4 0 3 1 5 5 5 6 2 7 2 5 1 6 9 5 0 9 1 9 8 6 8 8 7 ]})$

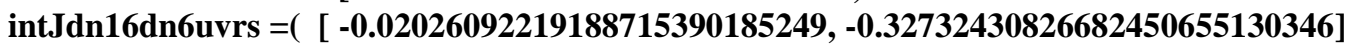
[ -0.32732430826682450655130346, $-\mathbf{- 0 . 1 8 9 5 2 7 8 0 5 8 1 1 7 9 4 3 8 9 8 6 4 8 6 8 4 3 ] )}$ intJdn16dn7uvrs $=([-\mathbf{~} \mathbf{0 . 1 9 8 1 3 0 2 5 9 7 6 0 0 0 1 8 3 8 2 8 1 6 8 7 3 4 1 , - 0 . 3 0 4 2 3 3 2 8 2 3 9 7 5 3 3 2 1 6 8 4 4 6 1 8 6 8 6}]$ $[-\mathbf{0 . 3 0 4 2 3 3 2 8 2 3 9 7 5 3 3 2 1 6 8 4 4 6 1 8 6 8 6 , 0 . 0 4 5 0 6 5 5 1 2 1 2 8 4 0 7 8 4 6 4 0 7 8 2 6 3 8 4 ] )}$

intJdn16dn8uvrs $=([\mathbf{0 . 5 3 1 3 9 1 4 0 5 0 1 1 4 5 9 4 6 5 0 4 9 9 9 6 9 3 6}, \mathbf{0 . 0 3 6 8 4 5 3 9 0 7 2 2 8 3 8 2 4 7 6 5 2 7 5 9 2 9 6}]$ [ 0.036845390722838247652759296, - $\mathbf{0 . 1 5 4 5 0 8 8 1 3 7 7 4 5 1 8 5 5 8 3 0 7 8 4 3 3 7 5 ]})$

intJdn16dn9uvrs $=([-\mathbf{- 0 . 4 3 3 2 6 2 2 1 4 1 9 7 9 3 2 0 2 0 3 6 0 2 3 8 3 3 5}, \mathbf{- 0 . 7 9 5 5 0 6 5 4 5 9 9 3 5 6 3 7 8 2 1 5 9 8 3 6 4 1 5 2}]$

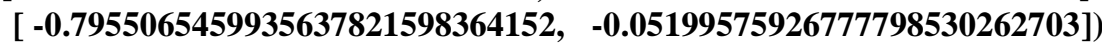

intJdn16dn10uvrs $=([\mathbf{0 . 4 6 3 6 4 2 4 2 6 4 3 5 2 3 3 5 4 1 5 1 7 3 3 9 9 4 3 5}, \mathbf{0 . 0 3 2 5 0 2 7 1 2 0 7 9 2 1 7 1 9 3 4 3 3 8 3 6 1 3 7}]$ [ 0.032502712079217193433836137, -2.24559114498245358748525615])

intJdn16dn11uvrs $=([-\mathbf{~} \mathbf{5 0 1 7 5 2 7 8 7 7 5 6 7 2 0 2 9 1 0 5 1 9 5 5 3 6}, \mathbf{- 0 . 5 1 0 5 9 9 4 6 0 9 5 9 2 0 0 0 5 1 9 7 8 4 5 9 6 5}]$ $[\mathbf{- 0 . 5 1 0 5 9 9 4 6 0 9 5 9 2 0 0 0 5 1 9 7 8 4 5 9 6 5 ,} \mathbf{0 . 6 0 1 0 5 8 3 5 7 5 0 6 5 4 3 5 9 4 9 5 6 5 8 2 6 3 ]})$

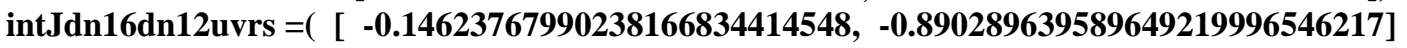
[ -0.890289639589649219996546217, - 1.1955736019576031500653136788$])$

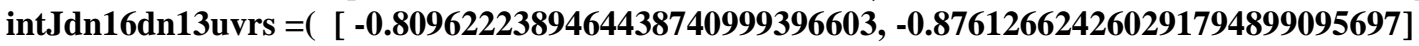
[ - -0.876126624260291794899095697, -3.400472479844200126665206259]) intJdn16dn14uvrs $=([\mathbf{0 . 8 7 7 5 1 8 5 6 9 8 2 3 3 4 8 9 8 2 6 3 3 7 5 6 7 4 4}, 1.287737922980470717927187534]$ [ 1.287737922980470717927187534, 0.877518569823348982633756744]) intJdn16dn15uvrs $=([-\mathbf{- 2 . 7 6 9 7 9 4 5 6 8 3 7 7 6 4 4 4 9 4 9 6 5 0 9 3 6 2 9}, \mathbf{- 0 . 6 3 7 8 4 1 6 0 2 6 5 2 0 3 0 9 4 3 2 5 7 0 5 9 3 3 1}]$ [ -0.637841602652030943257059331, - $\mathbf{0 . 8 0 5 9 7 7 9 1 9 8 0 6 6 6 4 9 2 9 3 9 1 9 2 6 7 2 ] )}$ intJdn16dn16uvrs $=([$ 3.929578185962763299703317274, 1.770759558087978375081430869$]$ [ 1.770759558087978375081430869, 5.51876129014211756612538031]) 


\section{SOME SAMPLE RESULTS TABLES\& FIGURES)}

Table $2 \mathrm{a}$

PRESENT PAPER: FEM SOLUTION FOR 12 node and 16 node cubic elements)

PREVIOUS PAPER: FEM SOLUTION FOR 9 node quadratic Lagrange element)

Torsion of a square cross section modeled as a right isoscles triangle: $\mathrm{R}$

, where $\left.\left.\mathrm{R}=\{\mathrm{x}, \mathrm{y}) \mid 0 \leq x, y \leq \frac{1}{2}, 0 \leq x-y\right) \leq 1 / 2\right\}$

nnode $=9$, Nine node -linear convex quadrilaterals of Lagrange family elememts

nnode $=12$, Twelve node -linear convex quadrilaterals of Serendipity family elememts

nnode $=16$, Sixteen node -linear convex quadrilaterals of Lagrange family elememts

exact solution of torisonal constant $=0.140577014955156$ )

nnode $=$ number of nodes in the triangular region $\mathrm{R}$

nel=number of elements in the region $\mathrm{R}$

FEM solution for Prandtl Stress function

Mesh No nnode nel nnel Torisonal constant maximum absolute error
1) $\begin{array}{llll}19 & 3 & 9 & 0.140226269123952\end{array}$
$\begin{array}{lllll}37 & 3 & 16 & 0.140564616238274 & 0.000361119966844\end{array}$

$\begin{array}{cccccc}2) & 61 & 12 & 9 & 0.140549995991093 & 0.000283838209277359 \\ 79 & 12 & 12 & 0.140527987183054 & 0.000386966456726 \\ 127 & 12 & 16 & 0.140576254134519 & 0.000089194283500\end{array}$
3) $127 \quad 27 \quad 9 \quad 0.140571151900467$
$\begin{array}{llll}163 & 27 & 12 & 0.140563898437538\end{array}$
0.000125431921097136
$\begin{array}{llll}271 & 27 & 16 & 0.140576864992942\end{array}$
0.000188851358012
0.000040770762286

4) $\begin{array}{ccccc}217 & 48 & 9 & 0.140575044191592 & 7.22896125021954 \mathrm{e}-005 \\ 277 & 48 & 12 & 0.140571630576105 & 0.000115489036847 \\ 469 & 48 & 16 & 0.140576967538218 & 0.000020792323046\end{array}$

\begin{tabular}{|c|c|c|c|}
\hline 5) 331 & 75 & 90.140576171269977 & $4.498537060151 \mathrm{e}-005$ \\
\hline 421 & 75 & 120.140574220427089 & 0.000080483984142 \\
\hline 721 & 75 & 160.140576995539002 & 0.000014277421181 \\
\hline
\end{tabular}

\begin{tabular}{|c|c|c|c|}
\hline 6) 469 & 108 & 90.140576593774739 & $2.98546615531262 \mathrm{e}-005$ \\
\hline 595 & 108 & 120.140575338318066 & 0.000058263173270 \\
\hline 1027 & 108 & 160.140577005593141 & 0.000011806028713 \\
\hline
\end{tabular}

\begin{tabular}{|c|c|c|c|c|}
\hline 7) 631 & 147 & 9 & 0.140576781096277 & $2.23280855678916 \mathrm{e}-005$ \\
\hline 799 & 147 & 12 & 0.140575906857497 & 0.000045873905107 \\
\hline 1387 & 147 & 16 & 0.140577009902267 & 0.000009955551927 \\
\hline
\end{tabular}

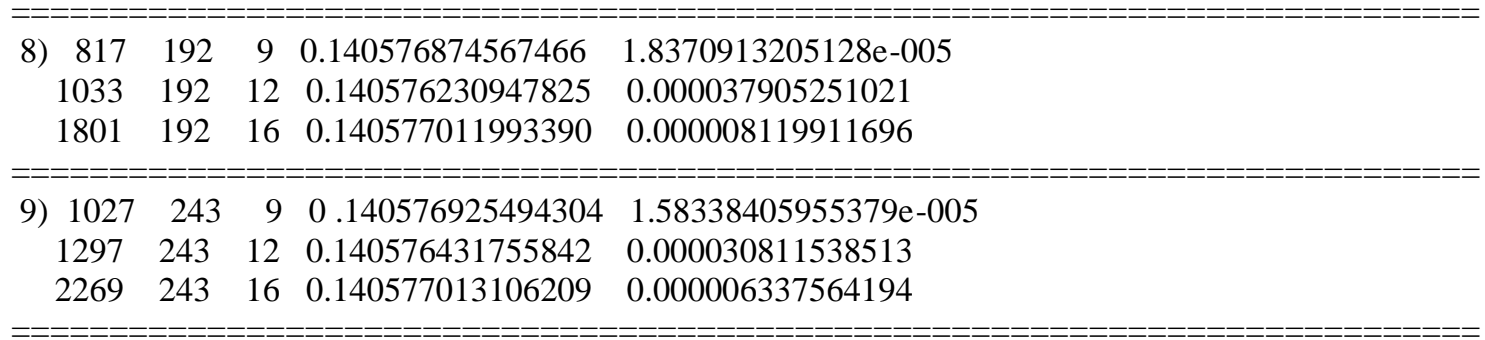

$\begin{array}{cccccc}\text { 10) } 1261 & 300 & 9 & 0.140576955193951 & 1.38273942157716 \mathrm{e}-005 \\ 1591 & 300 & 12 & 0.140576564296181 & 0.000024665504597 \\ 2791 & 300 & 16 & 0.140577013742108 & 0.000004703818292\end{array}$



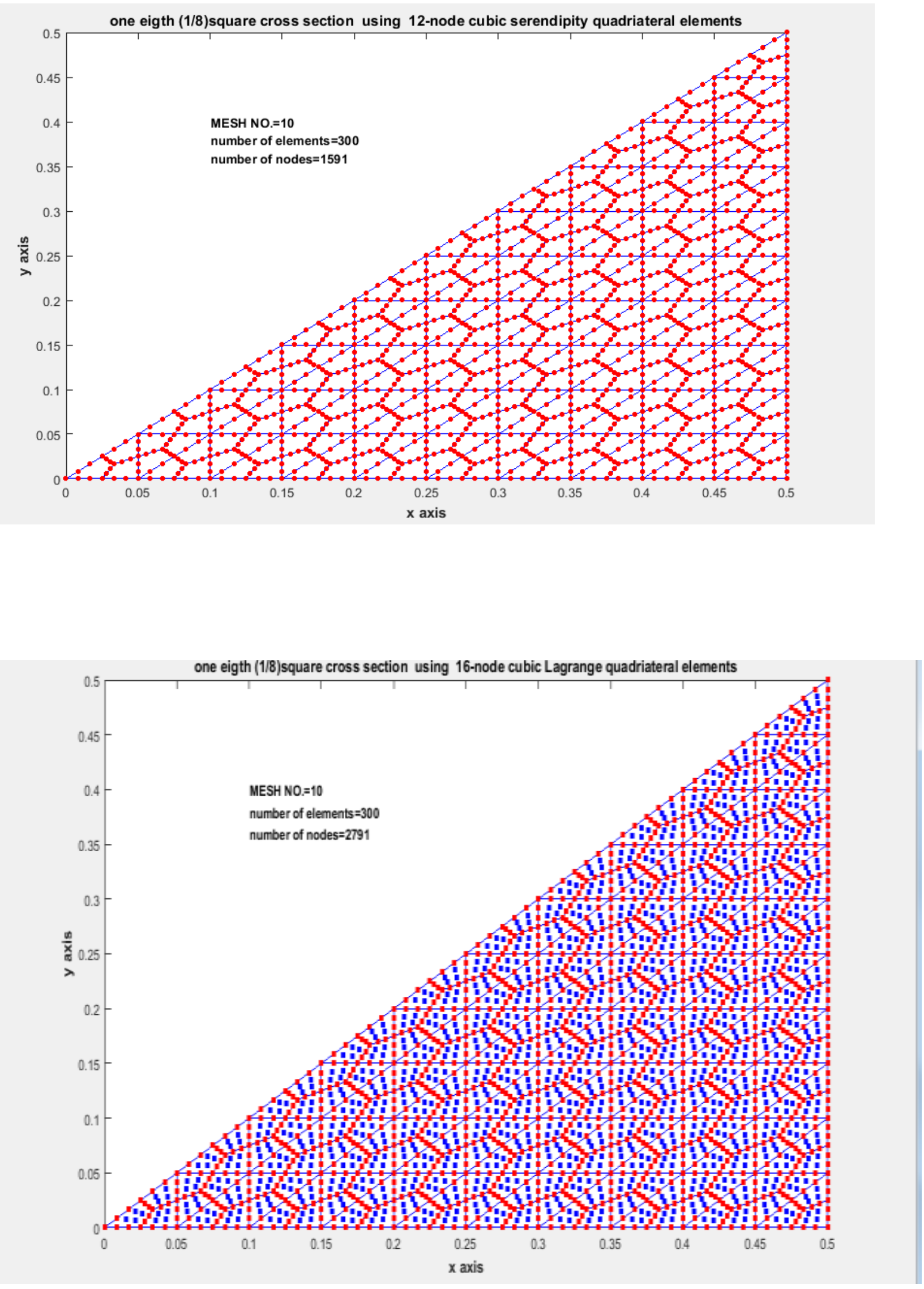

Table-3a 


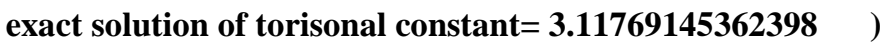

PRESENT PAPER: FEM SOLUTION FOR 12 node and 16 node cubic elements)

PREVIOUS PAPER: FEM SOLUTION FOR 9 node quadratic Lagrange element)

Linear elastic torsion of an equilateral triangle $T$ which is inscribed in a circle of unit radius.

Equilateral triangle $\mathbf{T}$ has coordinates for vertices as

$\mathrm{x}=[-\sqrt{3} ; \sqrt{3} ; 0])$

$\mathrm{y}=[-1 ;-1 ; 2])$

nnode $=$ number of nodes in the triangular region $T$

nel=number of elements in the region $T$

FEM solution for of Prandtl Stress function

Mesh No nnode nel nnel Torisonal constant maximum absolute error

1

$\begin{array}{cccccc} & 19 & 3 & 9 & \mathbf{3 . 1 0 7 3 9 4 0 7 5 7 8 9 2 4} \\ 25 & 3 & 12 & \mathbf{3 . 0 3 2 1 1 0 0 9 1 1 8 9 7 5 6} & \mathbf{0 . 0 2 9 1 4 3 2 3 9 8 1 4 2 6} \\ 37 & 3 & 16 & \mathbf{3 . 1 1 7 6 9 1 4 5 3 6 2 3 9 8 0} & \mathbf{0 . 0 0 0 0 0 0 0 0 0 0 0 0 0 0 0}\end{array}$

0.00317246382279579

$\begin{array}{lllll}37 & 3 & 16 & 3.117691453623980 & 0.000000000000000\end{array}$

$\begin{array}{llllllll}2 & 61 & & 12 & & 9 & 3.11704786750931 & 0.000396557977849543 \\ & & & 79 & 12 & 12 & 3.110644194853498 & 0.006021532419355\end{array}$

$\begin{array}{llllll}3 & 127 & 27 & 9 & 3.11756432550257 & 0.000117498660104104\end{array}$

$\begin{array}{lllll}163 & 27 & 12 & 3.115753983225545 & \mathbf{0 . 0 0 2 0 2 1 1 5 4 3 2 3 7 1 9}\end{array}$

\begin{tabular}{|c|c|c|c|c|c|}
\hline 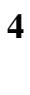 & $\begin{array}{c}217 \\
277\end{array}$ & $\begin{array}{c}48 \\
48\end{array}$ & $\begin{array}{c}9 \\
12\end{array}$ & $\begin{array}{l}\text { 3.11765122949181 } \\
\mathbf{3 . 1 1 6 8 3 9 7 5 7 4 3 0 2 9 4}\end{array}$ & $\begin{array}{l}\text { 4.95697472316092e-005 } \\
\mathbf{0 . 0 0 1 3 0 6 2 1 4 8 9 3 3 0 4}\end{array}$ \\
\hline & $\begin{array}{l}331 \\
421\end{array}$ & $\begin{array}{l}75 \\
75\end{array}$ & $\begin{array}{c}9 \\
12\end{array}$ & $\begin{array}{l}\text { 3.11767497781946 } \\
\text { 3.117217781295634 }\end{array}$ & $\begin{array}{l}2.53797105838616 \mathrm{e}-005 \\
0.000901007607025\end{array}$ \\
\hline
\end{tabular}

Example:TORSION OF AN EQUILATERAL TRIANGULAR CROSS SECTION 
Contour level curves for FEM solution of Sixteen Noded Special Quadrilateral Elements
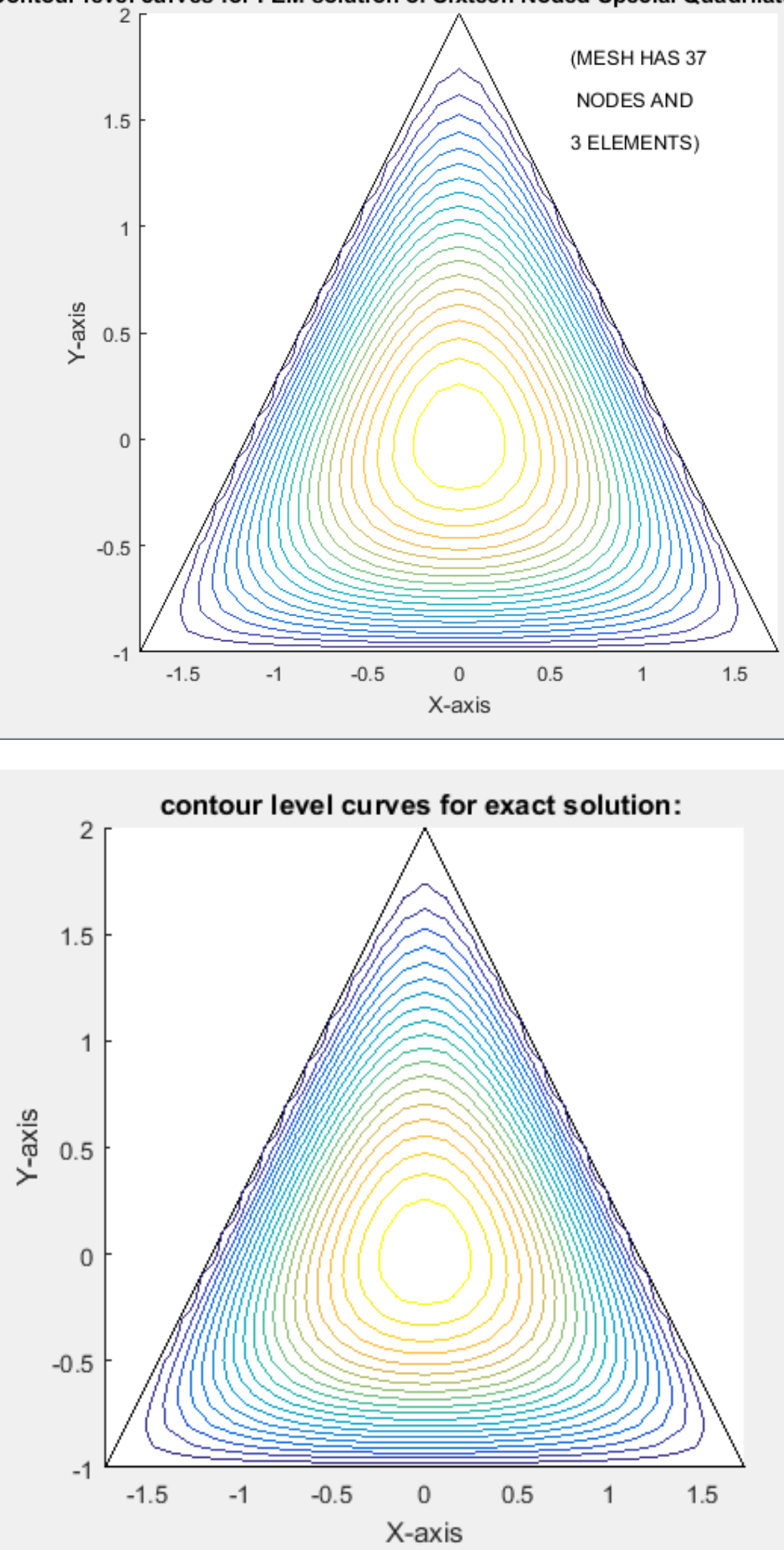
Contour level curves for FEM solution of Sixteen Noded Special Quadrilateral Elements

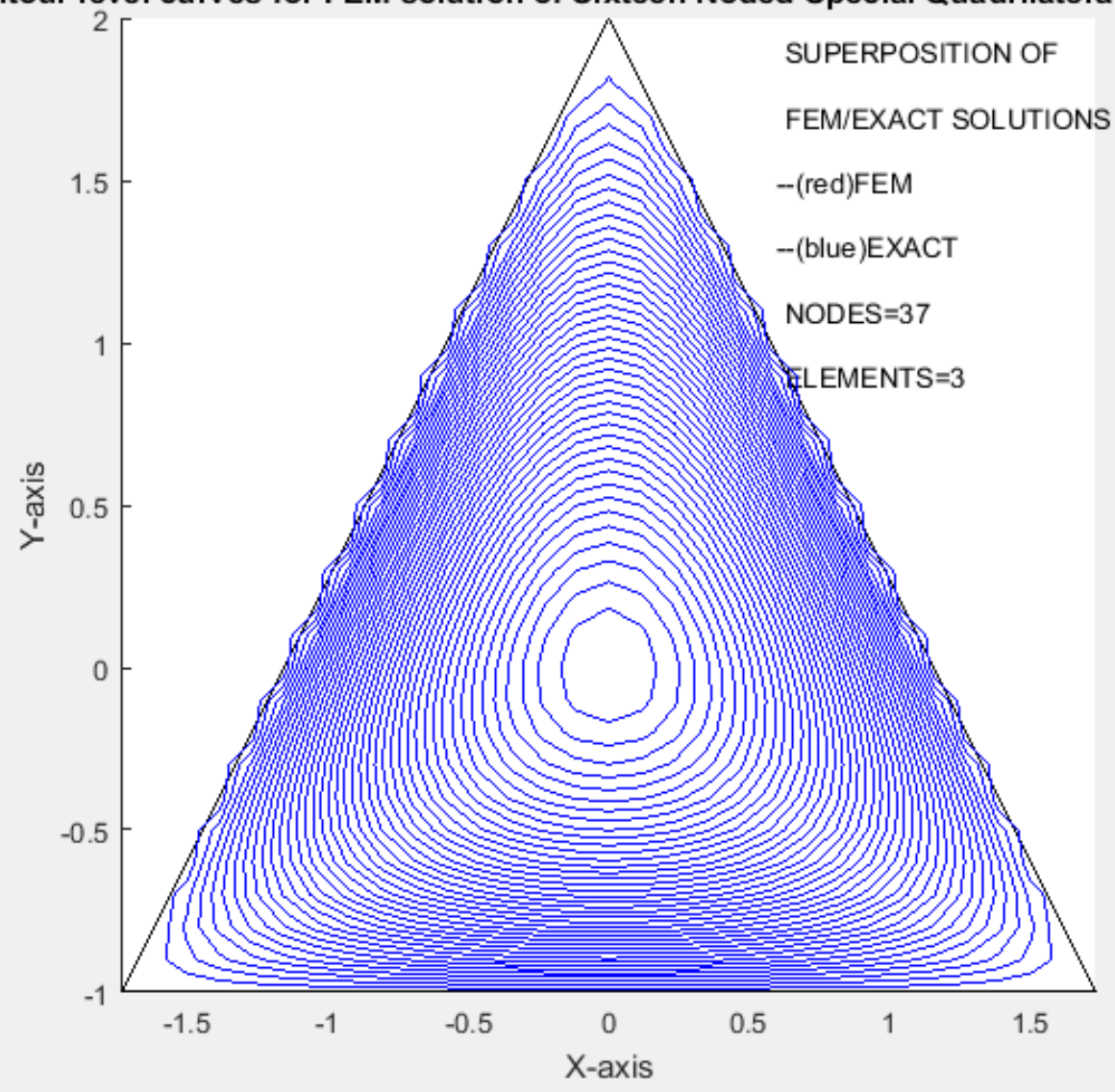

equilateral triangular cross section using 12-node cubic serendipity quadriateral elements

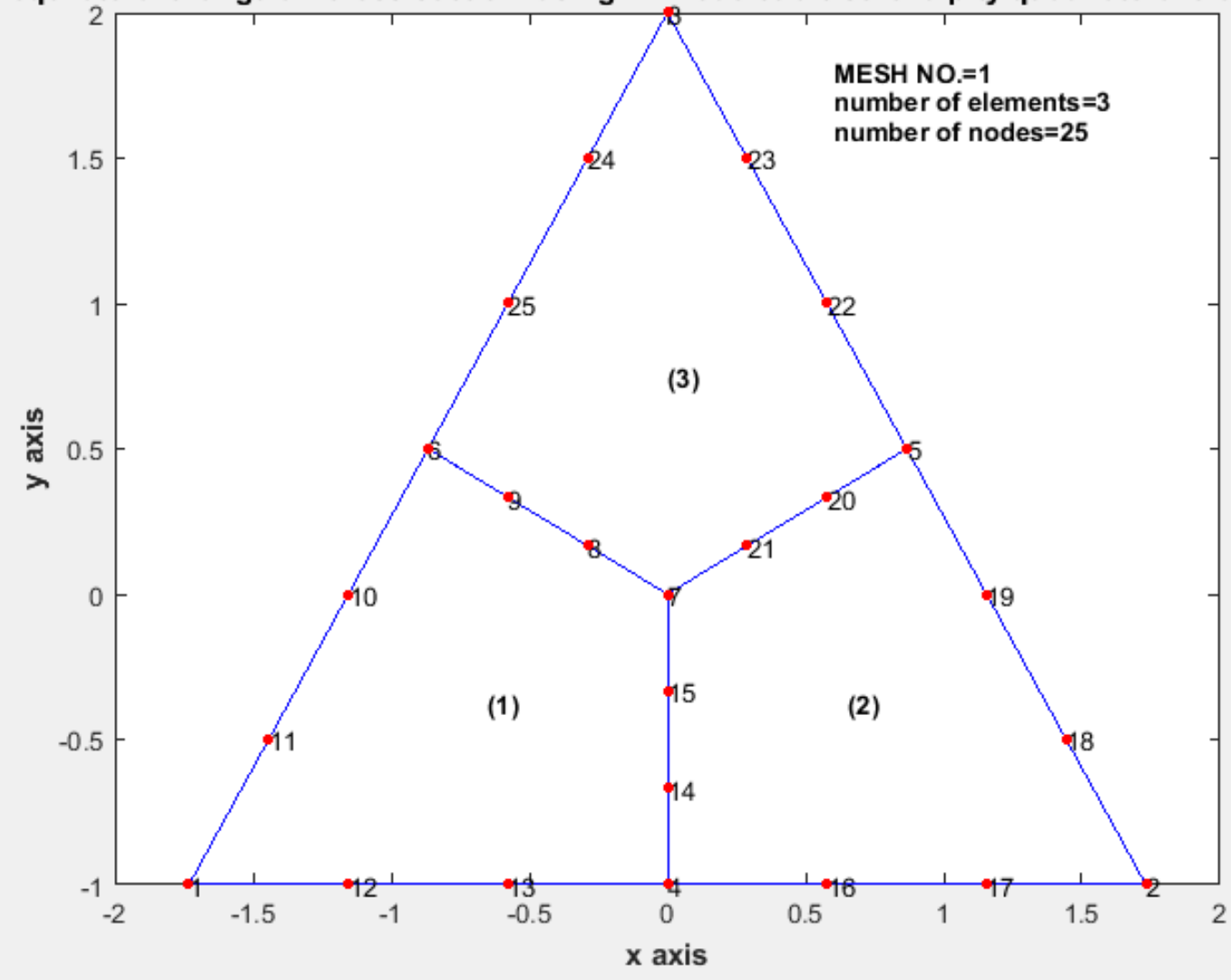



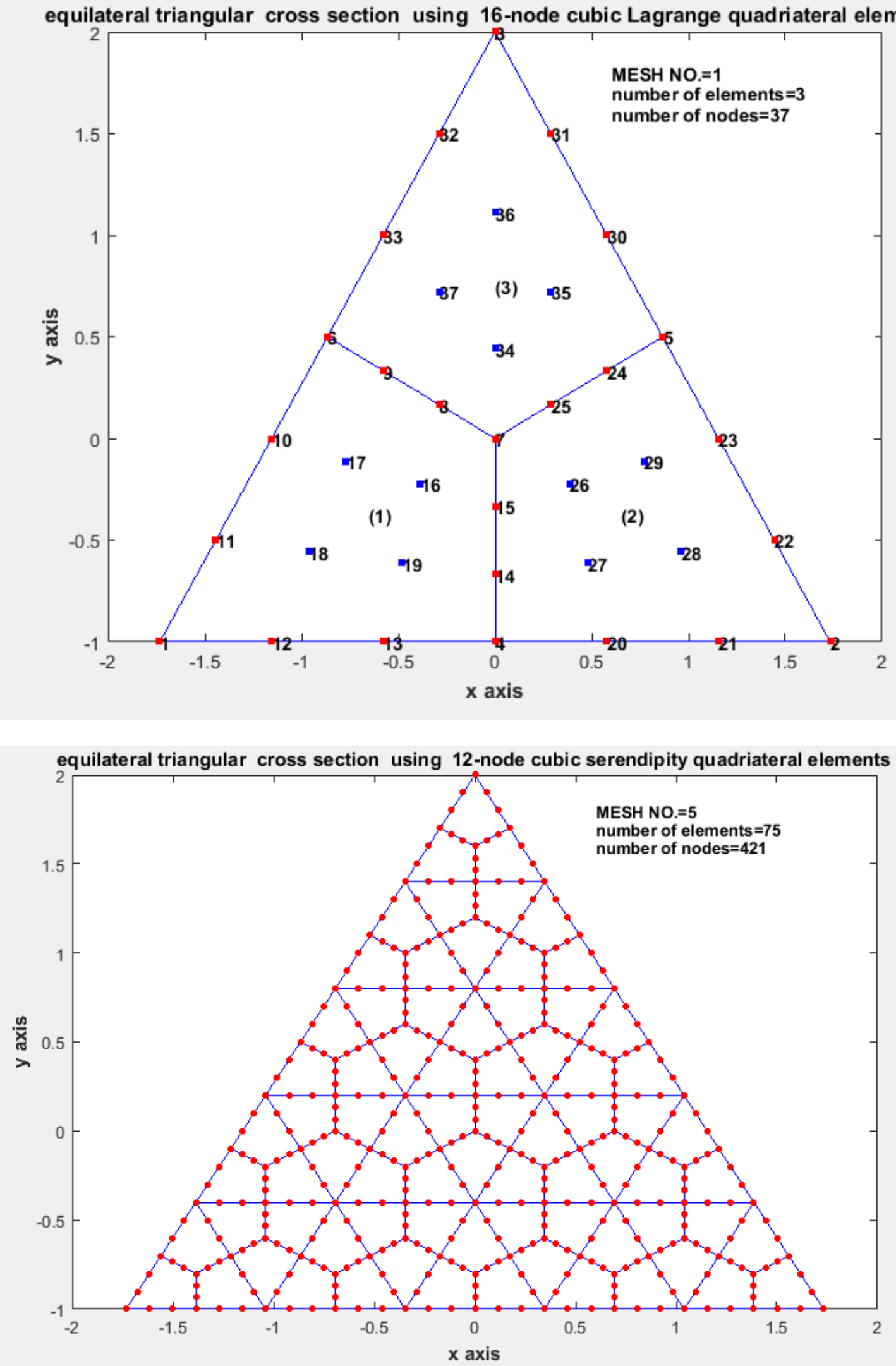


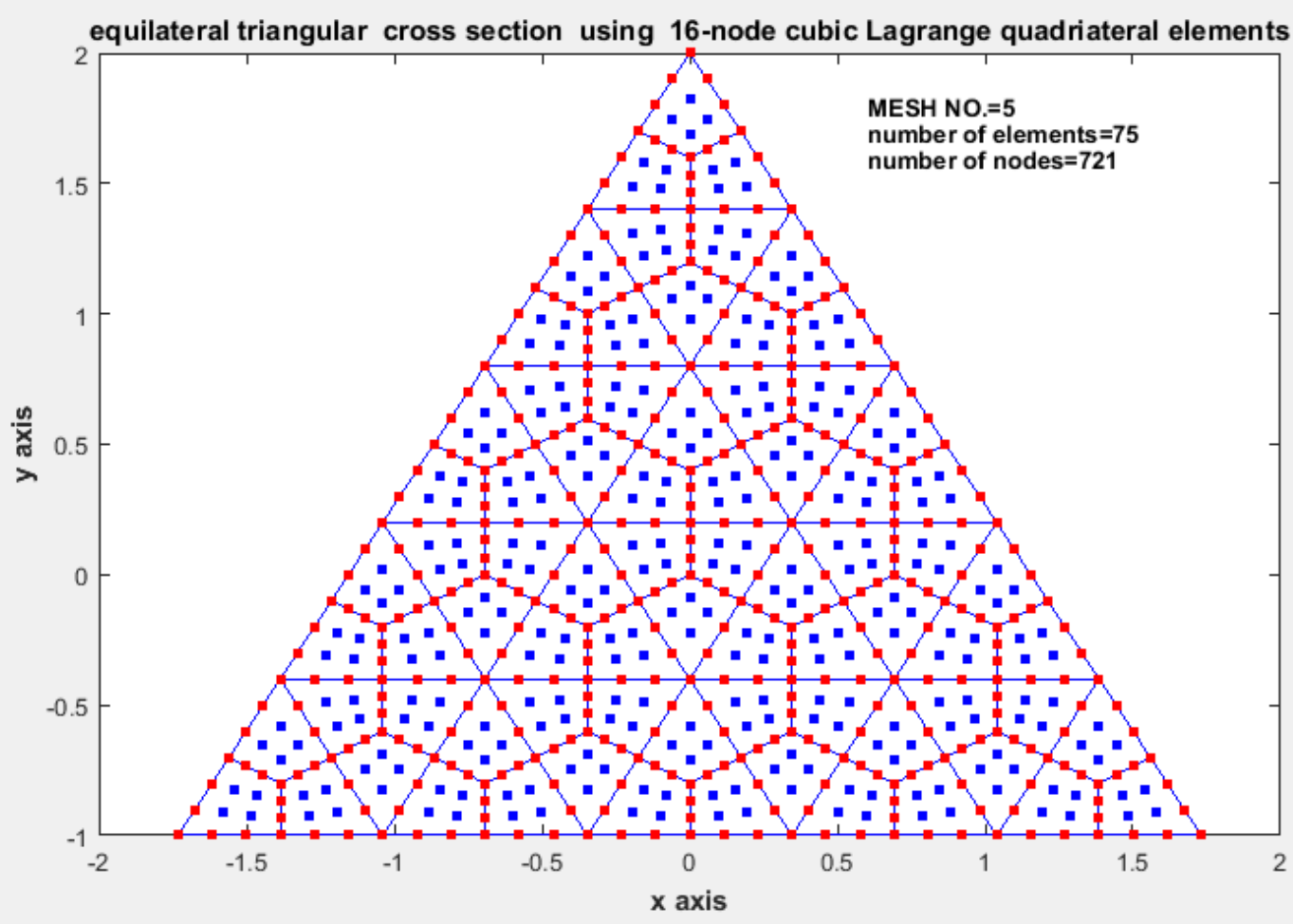

Example5:TORSION OF A SQUARE CROSS SECTION

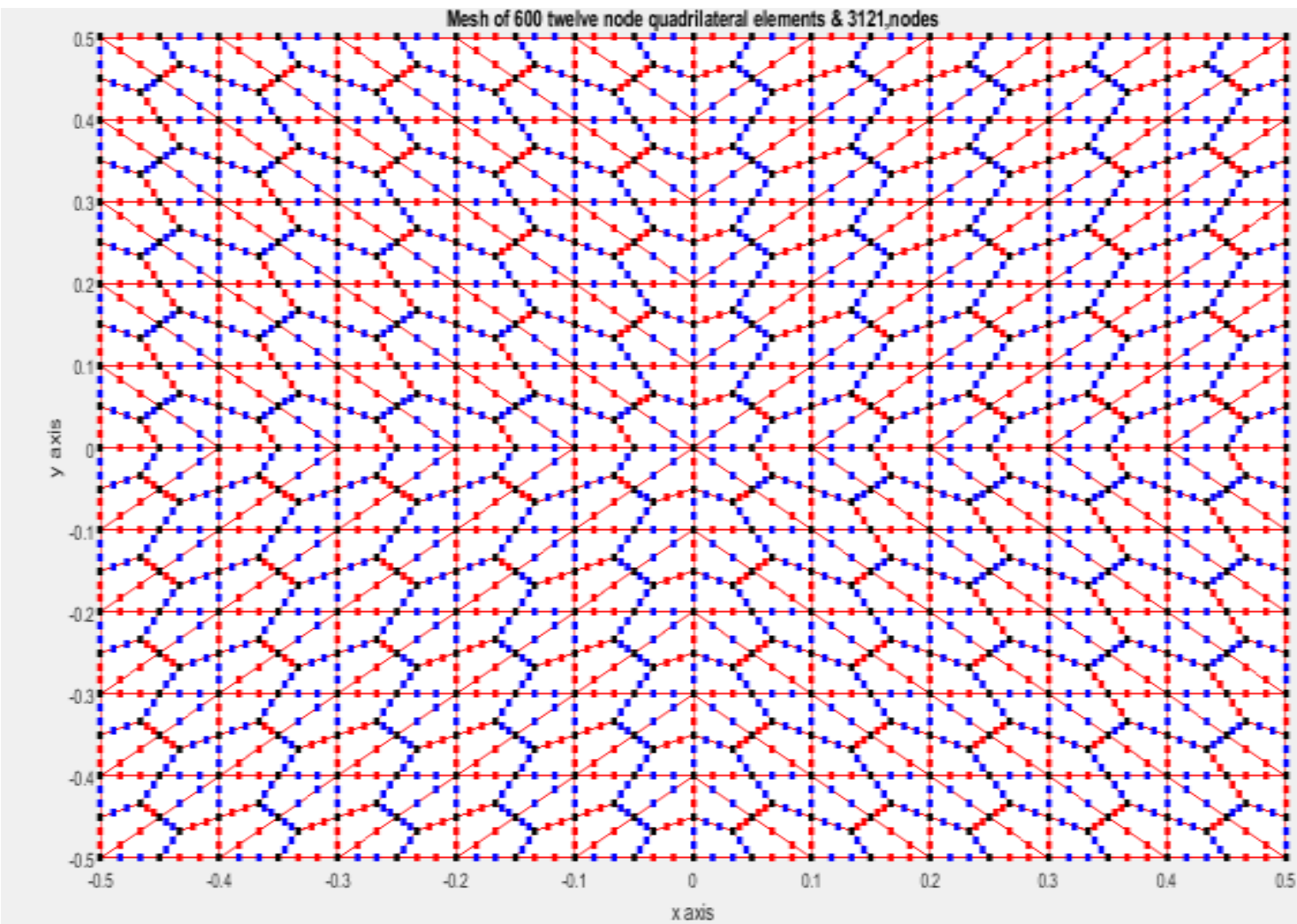


Contour level curves for FEM solution of Twelve Noded Special Quadrilateral Elements

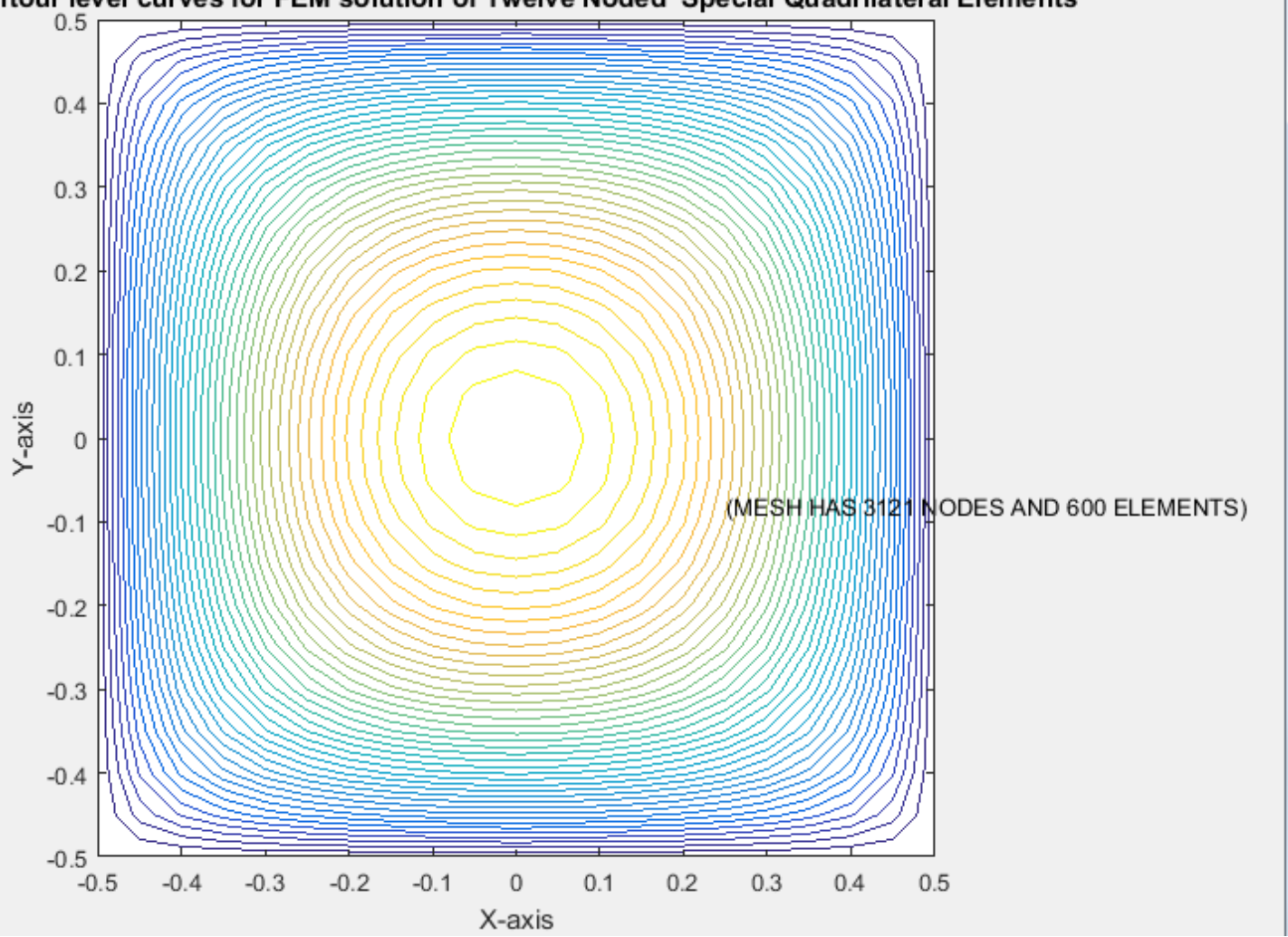




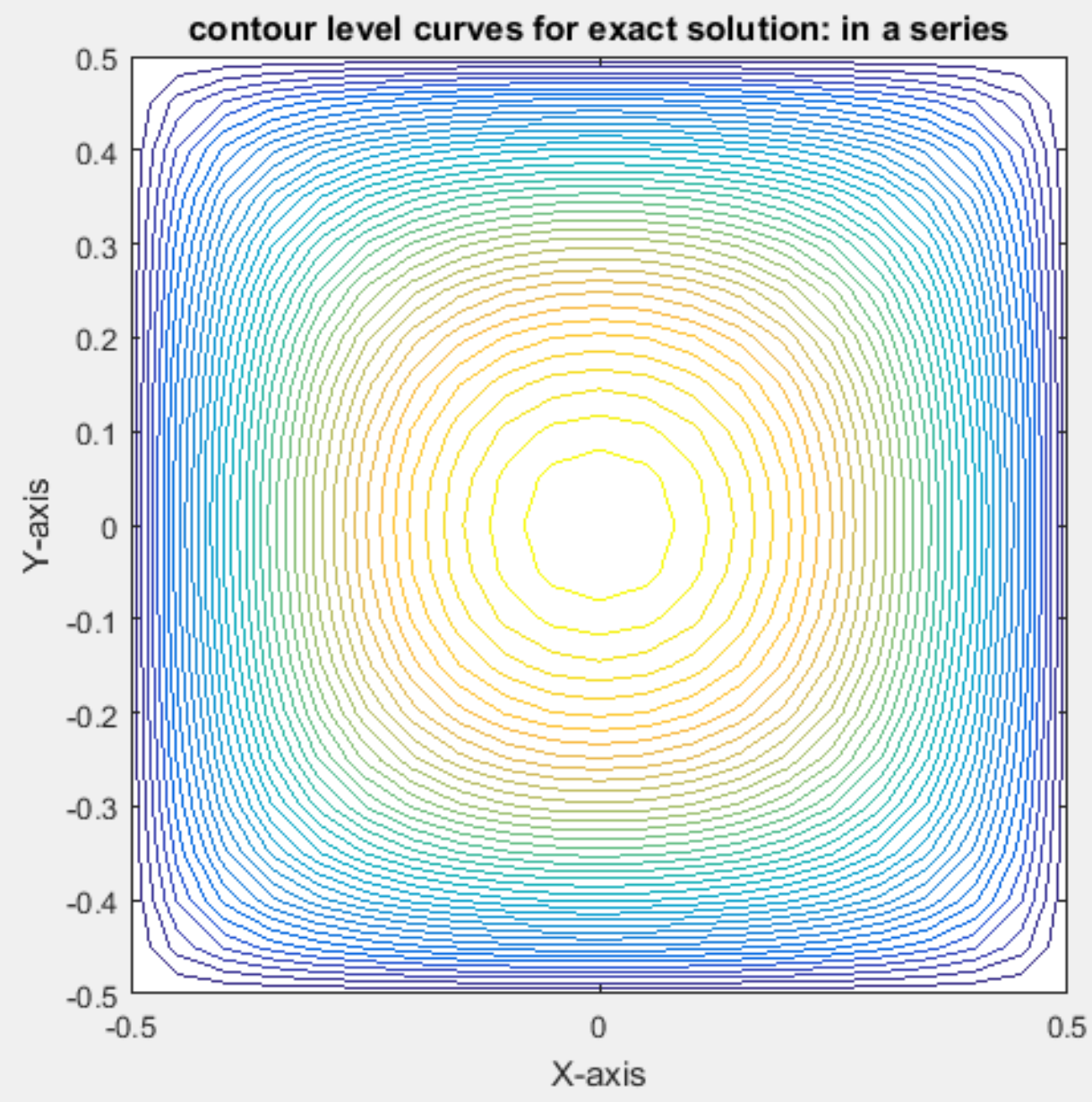

Contour level curves for FEM(red)\&exact(green) Twelve Noded Special Quadrilateral Elements

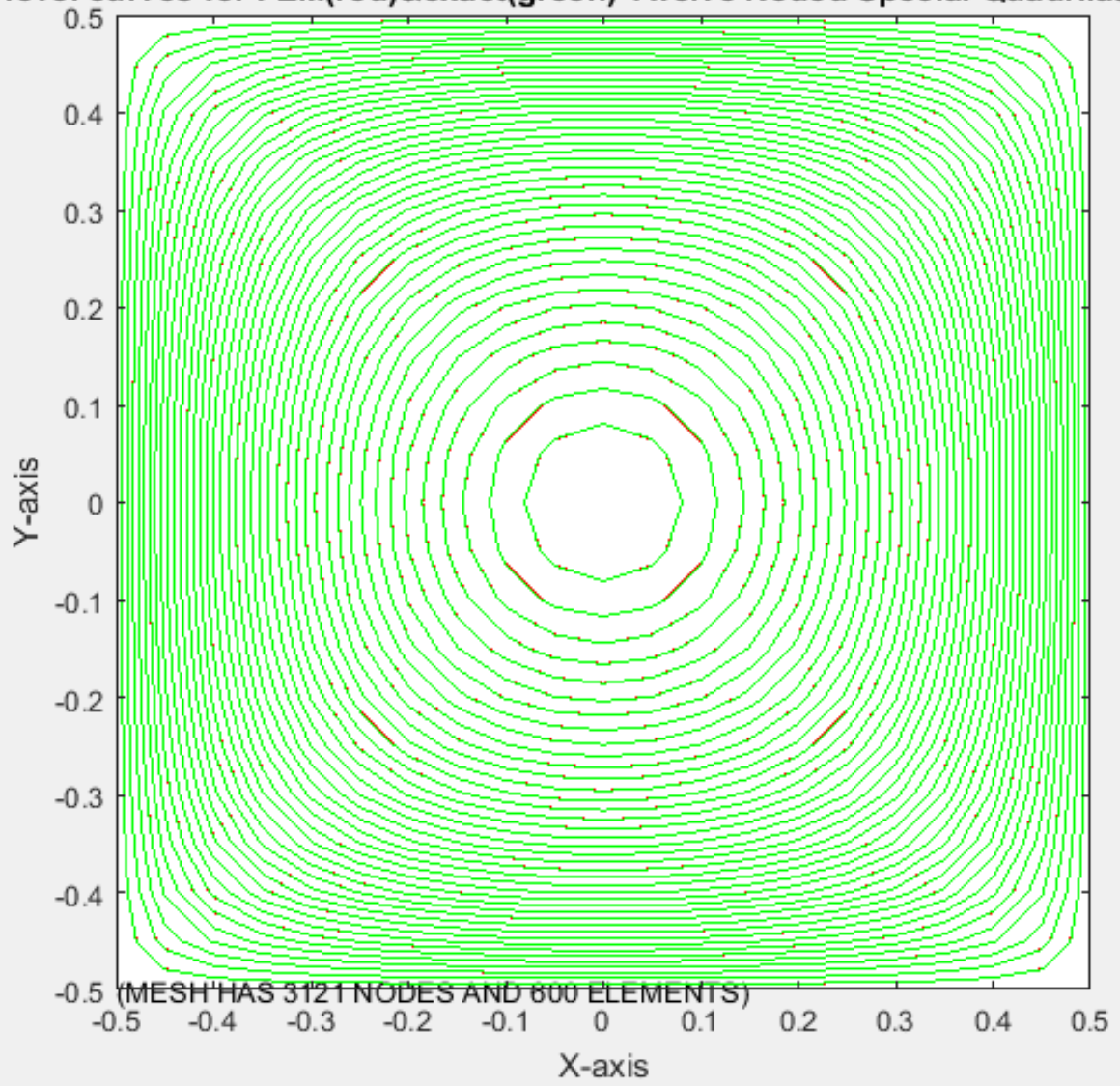



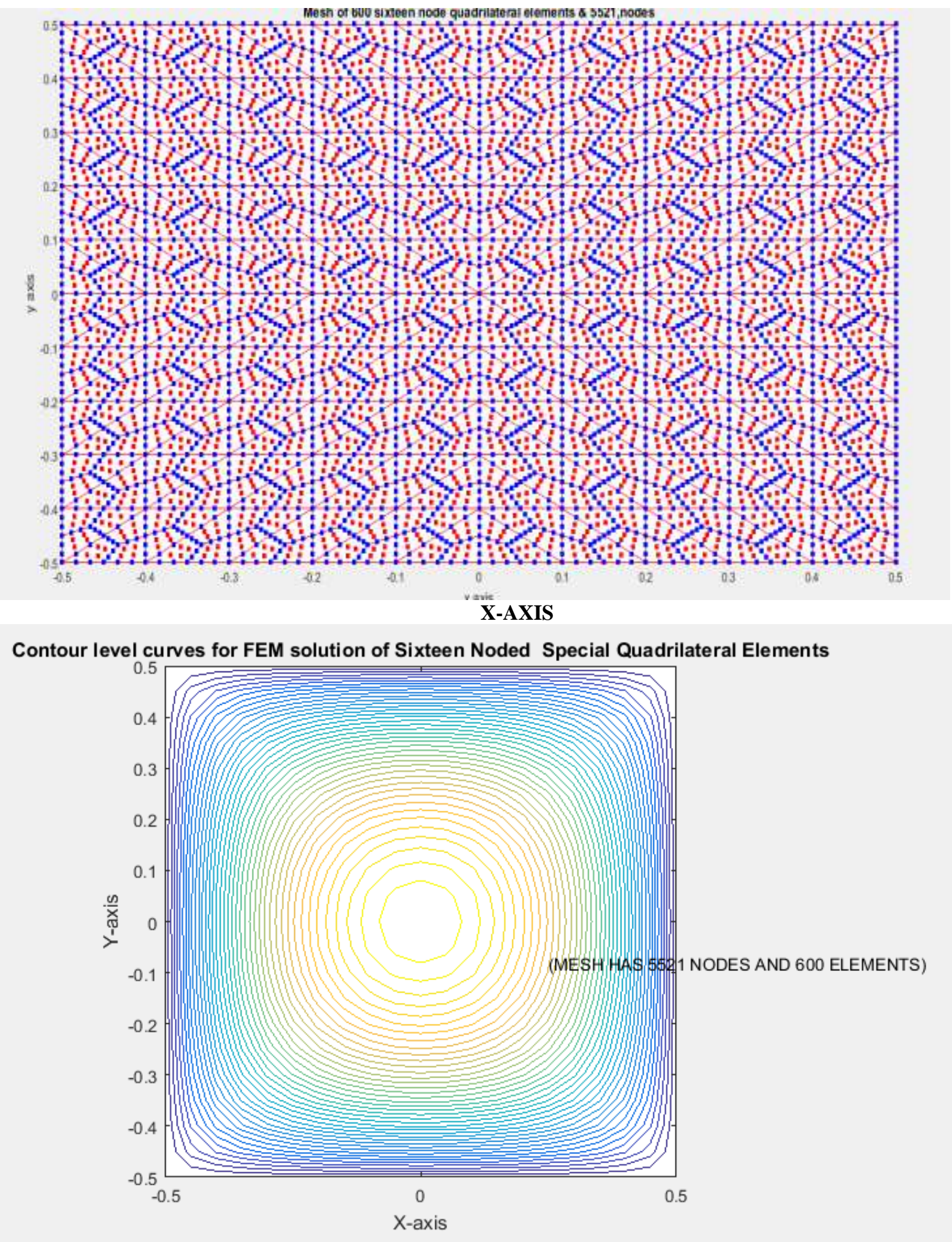


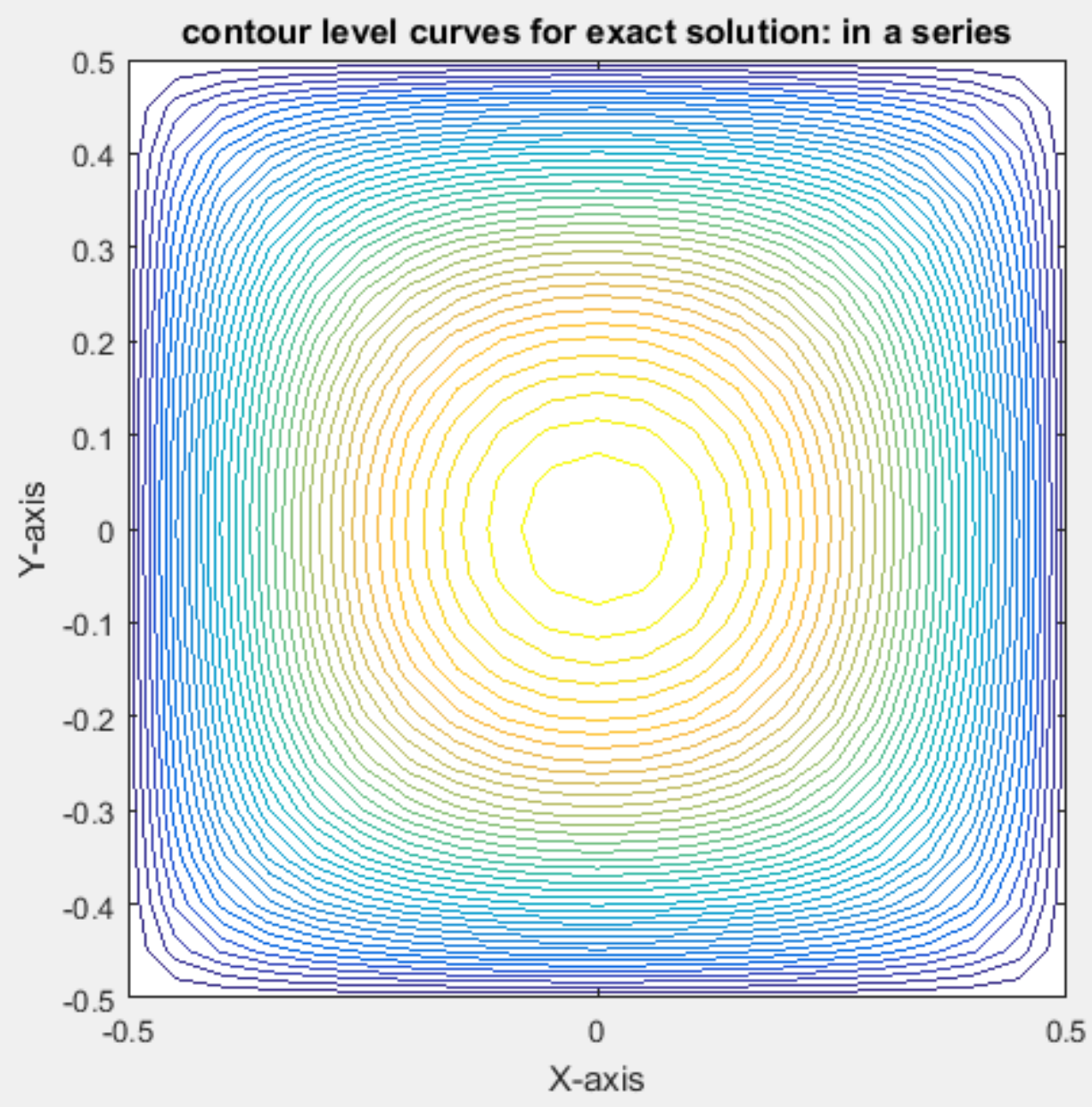

Contour level curves for FEM(red)\&exact(green) Sixteen Noded Special Quadrilateral Elements

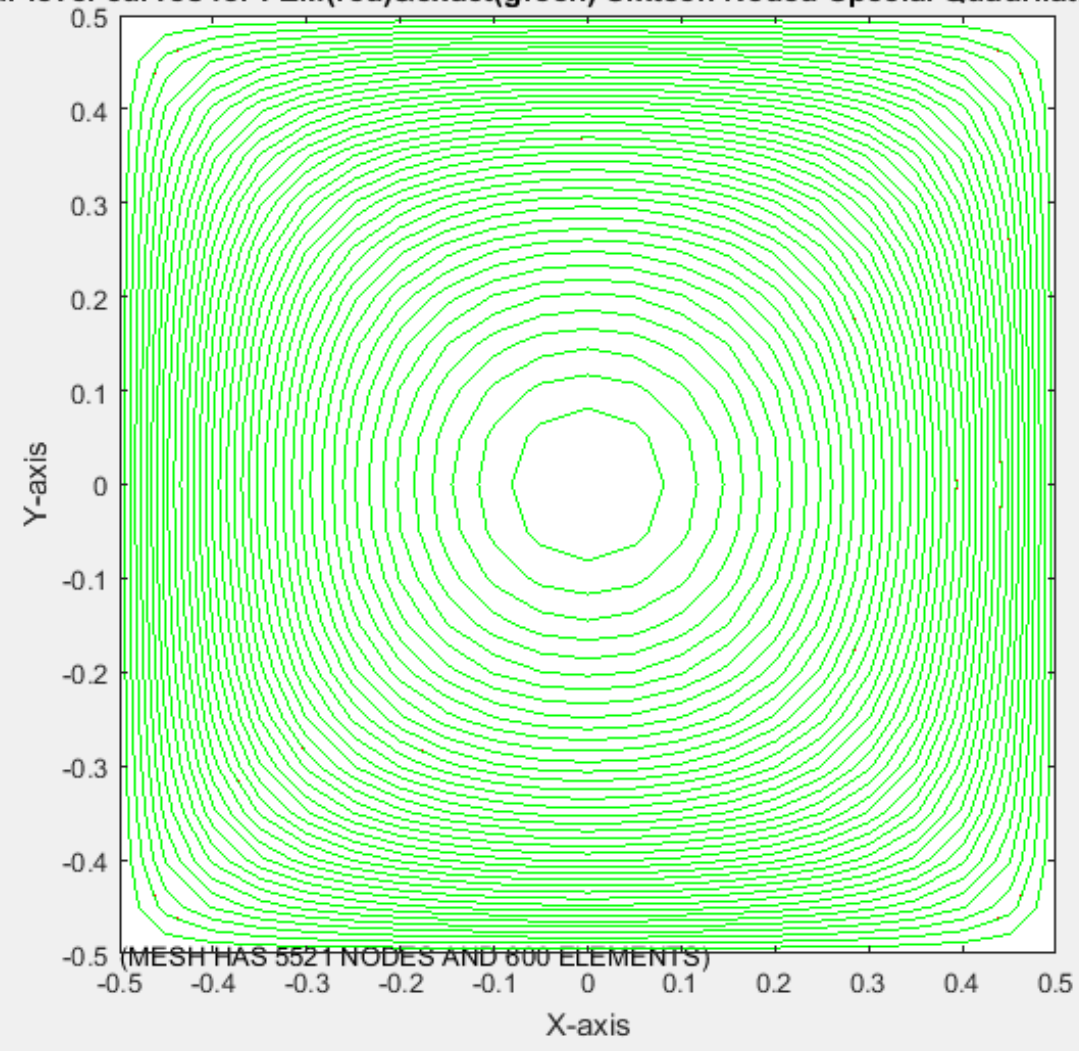

Solution of Poisson Boundary Value Problems Over Polygonal Domains

MESHES AND CONTOUR LEVEL CURVES FOR A PENTAGONAL DOMAIN WITH 12-NOODED QUADRILATERAL ELEMENTS

Mesh with 525 twelve noded quadrilateral elements \& no. of nodes $=2731$

H. T. Rathod, IJECS Volume 7 Issue 1 January 2018 Page No. 23329-23482 

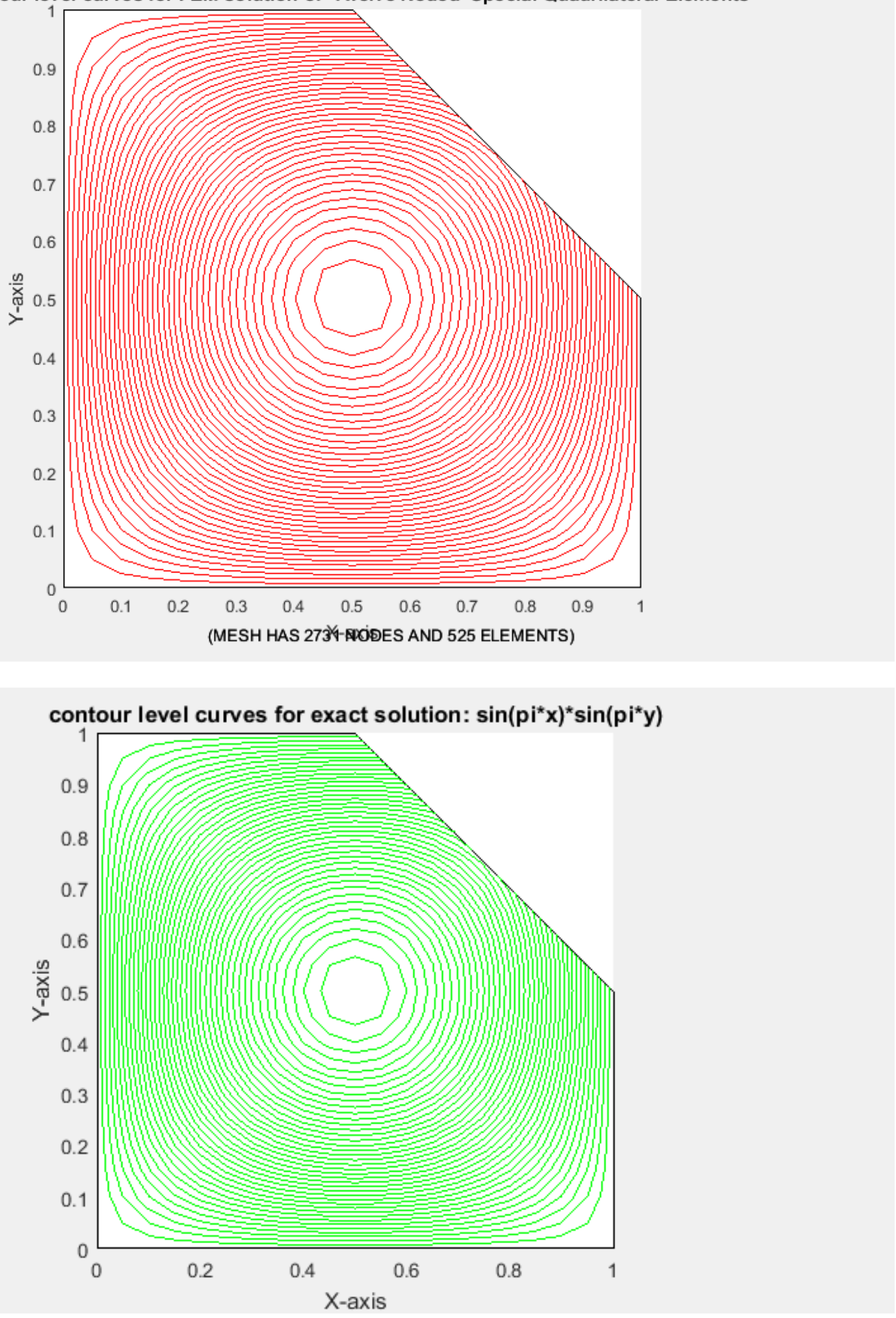


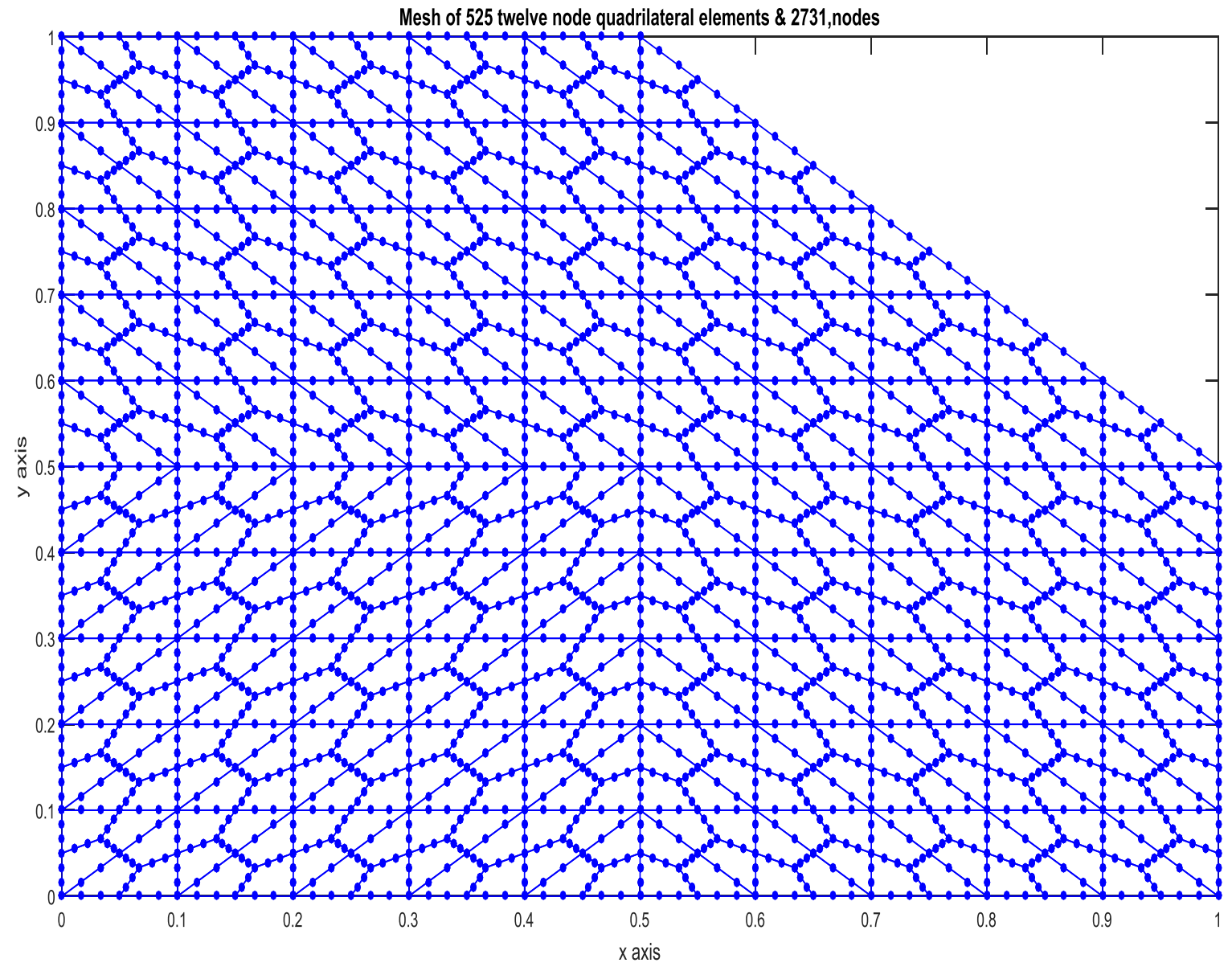

\section{MESHES AND CONTOUR LEVEL CURVES FOR A SQUARE DOMAIN WITH TWELVE NOODED} QUADRILATERAL ELEMENTS

Mesh with 600 twelve noded quadrilateral elements \& no. of nodes $=3121$

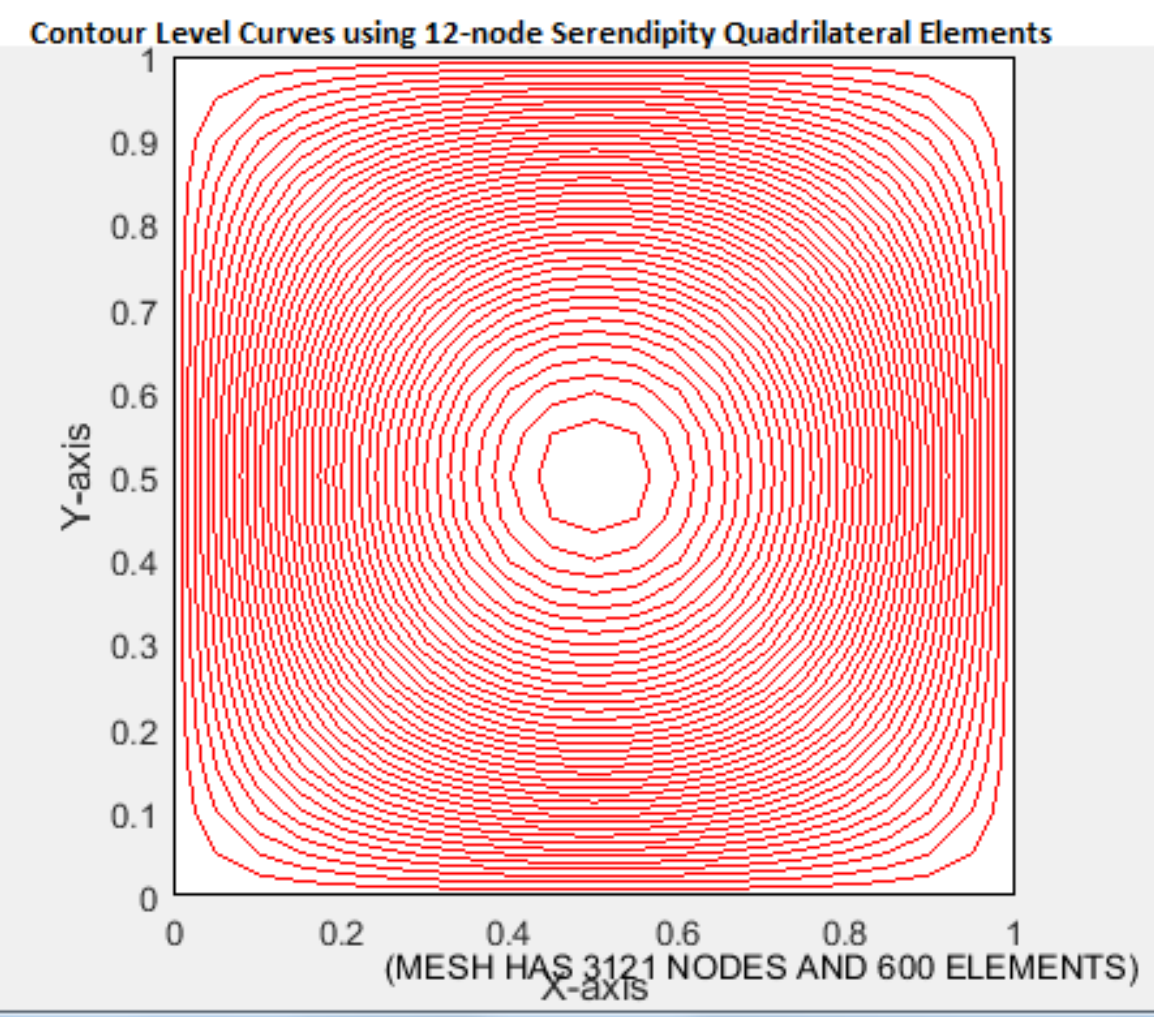



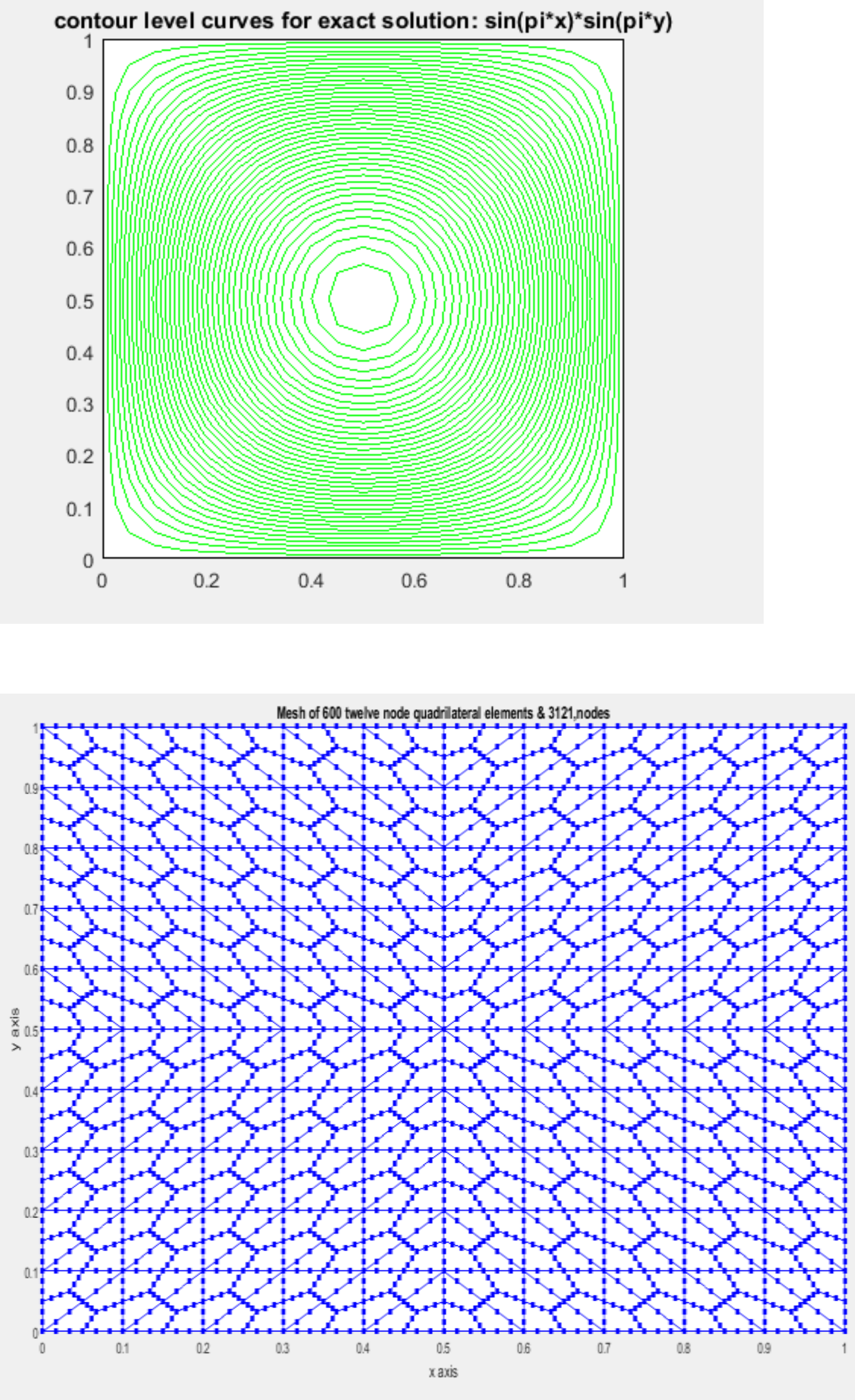

MESHES AND CONTOUR LEVEL CURVES FOR A PENTAGONAL DOMAIN WITH 16-NOODED QUADRILATERAL ELEMENTS 

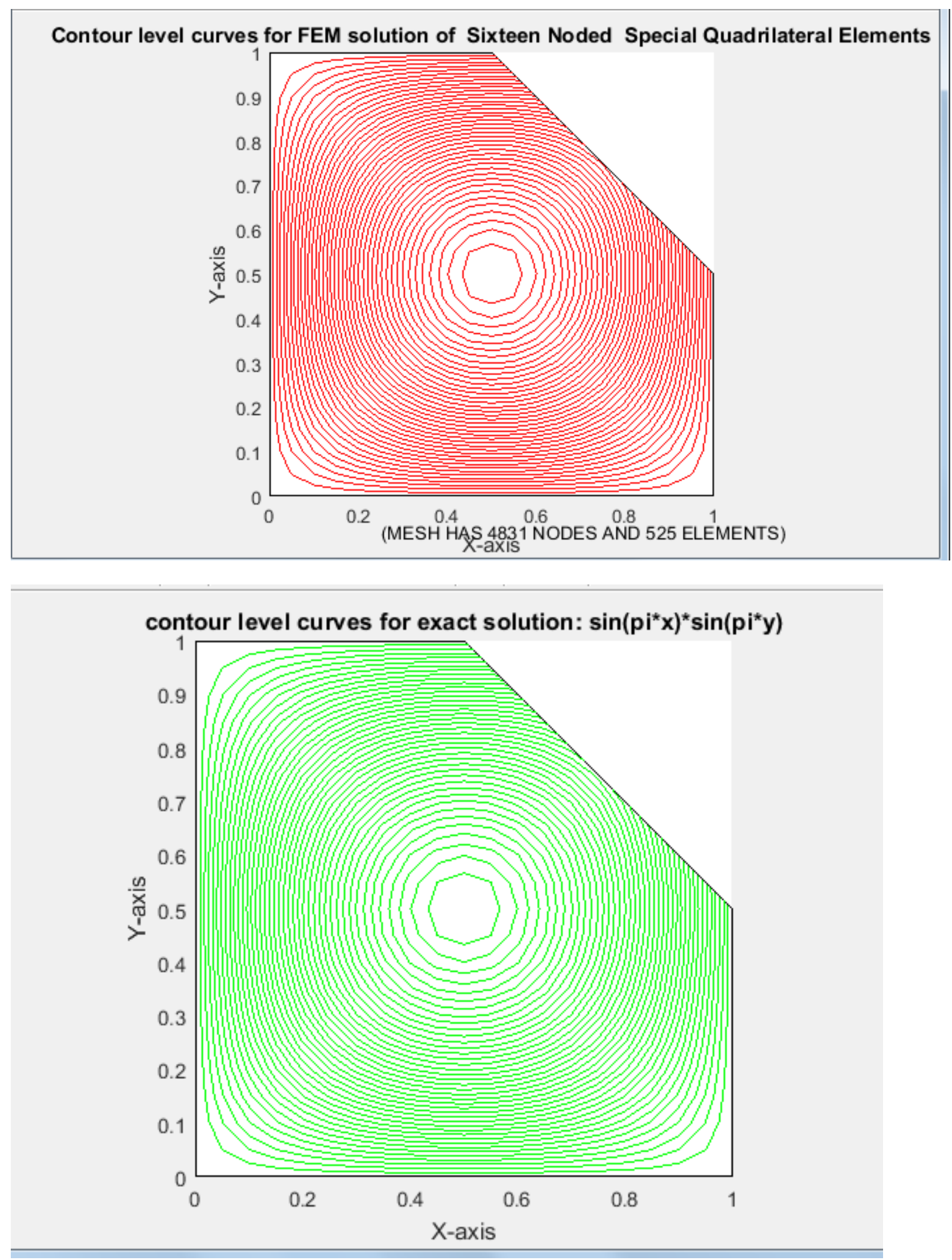


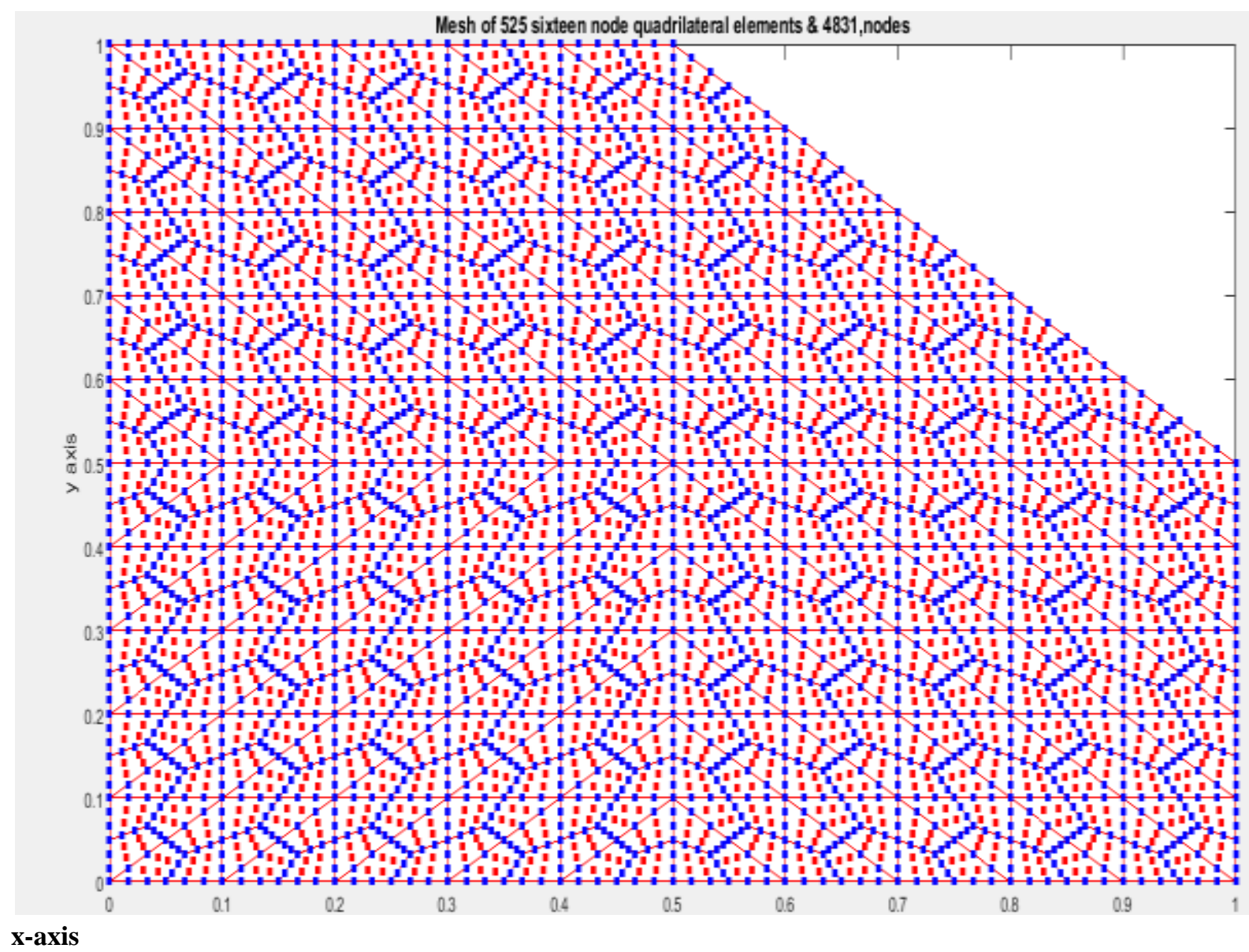

MESHES AND CONTOUR LEVEL CURVES FOR A SQUARE DOMAIN WITH SIXTEEN NOODED QUADRILATERAL ELEMENTS

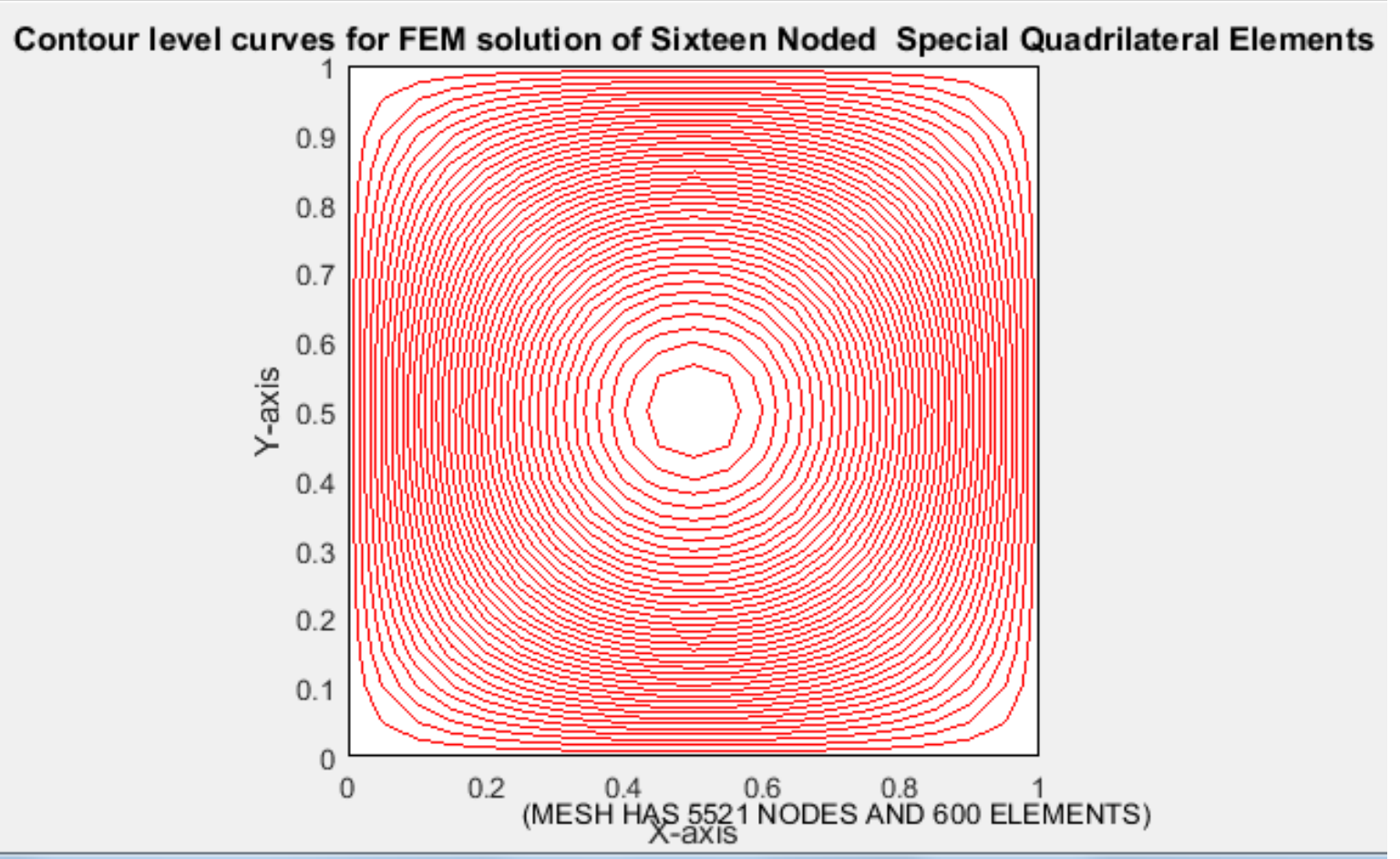



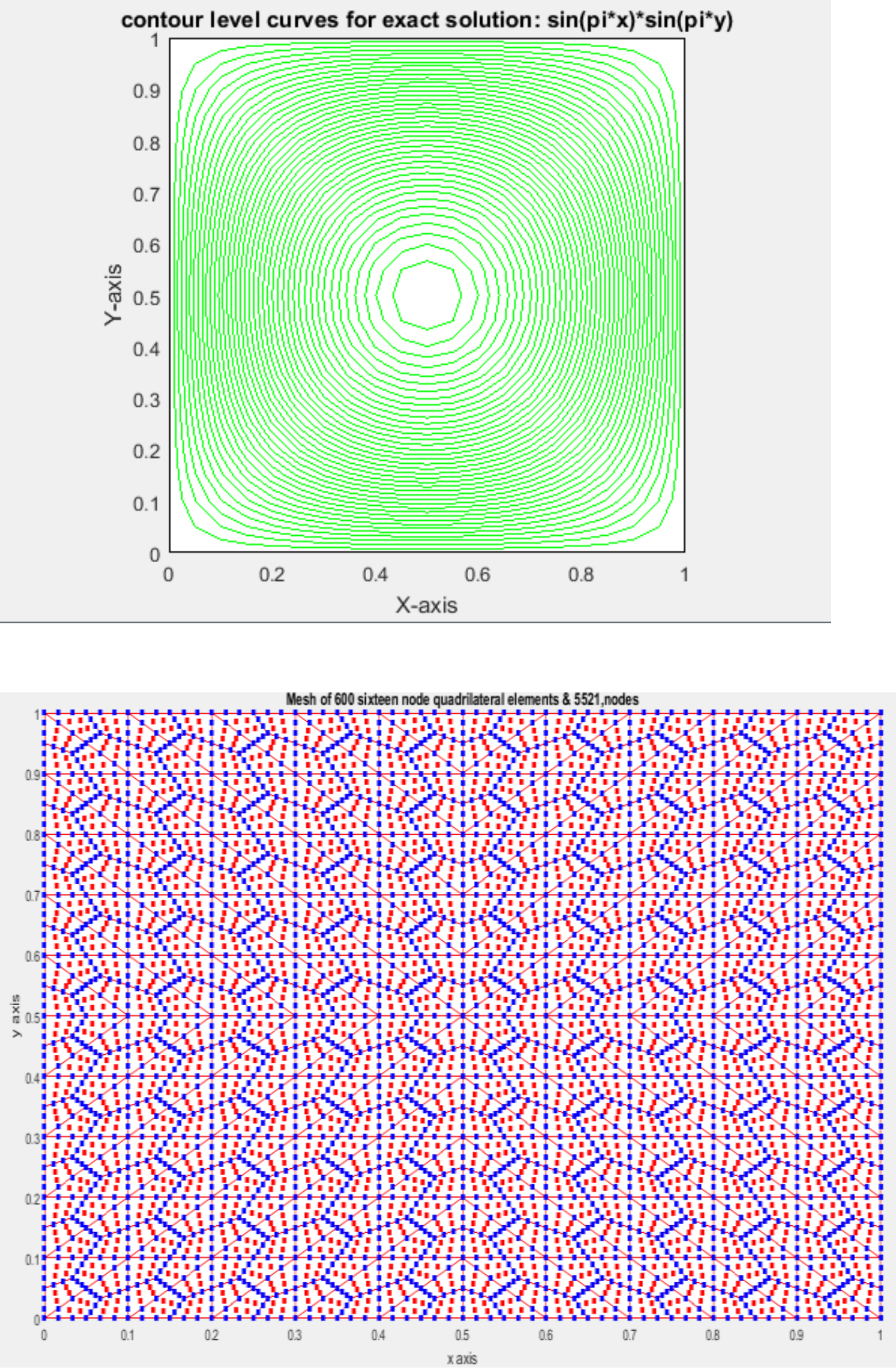

MATLAB PROGRAMS FOR 12-NODE SERENDIPITY AND 16-NODE LAGRANGE ELEMENTS LIST OF PROGRAMS

[1]quadrilateralmesh_over_arbitrarytriangle_q12automeshgen.m

[2]quadrilateralmesh_over_arbitrarytriangle_q16automeshgen.m 
[3]quadrilateral_mesh4MOINEX_q12.m

[4]quadrilateral_mesh4MOINEX_q16LG.m

[5]D2LaplaceEquationQ12Ex3automeshgenNewContour.m $\sqrt{ }{ }^{*}$

[6]D2LaplaceEquationQ12Ex3automeshgenNewPolygonContour.m $\sqrt{ }{ }^{*}$

[7]D2LaplaceEquationQ16Ex3automeshgenNewContour.m $\sqrt{ }^{*}$

[8]D2LaplaceEquationQ16Ex3automeshgenNewPolygonContour.m $\sqrt{ }$ *

[9]polygonal_domain_coordinates_3rd_orderLG.m

[10]polygonal_domain_coordinates_3rd_order.m

[11]coordinate_special_quadrilaterals_in_stdtriangle_3rd_orderLAGR.m

[12]coordinate_special_quadrilaterals_in_stdtriangle_3rd_order.m

[13]integral_valuesof_localderivative_products.m

[14]nodaladdresses_special_convex_quadrilaterals_trial_3rd_order.m

[15]generate_area_coordinate_over_the_standard_triangle.m

[16]nodaladdresses_special_convex_quadrilaterals_trial_3rd_orderLG.m

[17]D2PoissonEquationQ12MoinEx_MeshgridContourNew.m $\sqrt{ }^{*}$

[18]D2PoissonEquationQ16MoinEx_MeshgridContourNew.m $\sqrt{ }$ *

[19]newtonmethod4spquadrileteral.m

[20]parameqnspqd.m

[21]paramdetJspqd.m

[22]paraminvJspqd.m

[23] glsampleptsweights.m

MATLAB CODES

[1]quadrilateralmesh_over_arbitrarytriangle_q12automeshgen.m

function[]=quadrilateralmesh_over_arbitrarytriangle_q12automeshgen(mmesh,nmesh,tri)

$\%$ quadrilateralmesh_over_arbitrarytriangle_q9automeshgen $(1,1,4)$

\%quadrilateralmesh_over_arbitrarytriangle_q12automeshgen $(1,1,4)$

clf

switch tri

case $1 \%$ standard triangle

$\mathbf{x x}=\operatorname{sym}([0 ; 1 ; 0])$

$\operatorname{yy}=\operatorname{sym}([0 ; 0 ; 1])$

case 2

$\mathrm{xx}=\operatorname{sym}([0 ; 1 / 2 ; 1 / 2])$

$\operatorname{yy}=\operatorname{sym}([0 ; 0 ; 1 / 2])$

case $3 \%$ equilateral triangle

$\mathbf{x x}=\operatorname{sym}([0 ; 1 ; 1 / 2])$

yy=sym([0;0;sqrt(3)/2] $)$

case $4 \%$ equilateral triangle

$\mathbf{x x}=\operatorname{sym}([-\operatorname{sqrt}(3) ; \operatorname{sqrt}(3) ; 0])$

$\operatorname{yy}=\operatorname{sym}([-1 ;-1 ; 2])$

end

for mesh=mmesh:nmesh

figure(mesh)

ndiv $=2 *$ mesh;

$\%[$ eln,nodetel,nodes,nnode]=nodaladdresses4Lagrangespecial_convex_quadrilaterals_3rd_order(ndiv)

[eln,nodetel,nodes,nnode]=nodaladdresses_special_convex_quadrilaterals_3rd_order(ndiv);

$\%[$ coord,gcoord] $=$ coordinate_rtisoscelestriangle00_h0_hh_2ndorder(ndiv);

$\%[$ coord,gcoord $]=$ coordinate_arbitrarytriangle_3rdorderLAGR(xx,yy,ndiv)

[coord,gcoord]=coordinate_arbitrarytriangle_3rdorder(xx,yy,ndiv)

$[$ nel,nnel $]=\operatorname{size}($ nodes $)$

for $i=1$ :nel

NN(i,1)=i;

end

table1 $=[\mathrm{NN}$ nodes $]$

[nnode, dimension] $=$ size $($ gcoord $)$

$\%$ plot the mesh for the generated data

$\% \mathrm{x}$ and $\mathrm{y}$ coordinates 
$\operatorname{xcoord}(1:$ nnode, 1$)=\operatorname{gcoord}(1:$ nnode, 1$)$; $\operatorname{ycoord}(1:$ nnode, 1$)=\operatorname{gcoord}(1:$ nnode, 2$)$;

\% extract coordinates for each element

for $i=1$ :nel

for $\mathbf{j}=\mathbf{1}$ :nnel

$x(\mathbf{1}, \mathbf{j})=x \operatorname{coord}(\operatorname{nodes}(\mathbf{i}, \mathbf{j}), \mathbf{1})$;

$\mathbf{y}(\mathbf{1}, \mathbf{j})=\mathbf{y c o o r d}(\operatorname{nodes}(\mathbf{i}, \mathbf{j}), \mathbf{1})$;

end; $\% \mathrm{j}$ loop

$\operatorname{xvec}(1,1: 5)=[x(1,1), x(1,2), x(1,3), x(1,4), x(1,1)]$;

$\operatorname{yvec}(1,1: 5)=[\mathbf{y}(1,1), y(1,2), y(1,3), y(1,4), y(1,1)]$;

$\%$ axis equal

switch tri

case 1

axis tight

$\mathbf{x m i n}=0 ; x \max =1 ; y \min =0 ; y \max =1$;

$\operatorname{axis}([\mathbf{x m i n}, \mathbf{x m a x}, \mathbf{y m i n}, \mathbf{y m a x}])$;

case 2

axis tight

$\mathrm{xmin}=0 ; \mathrm{xmax}=1 / 2 ; \mathrm{ymin}=0 ; \mathrm{ymax}=1 / 2$;

$\operatorname{axis}([\mathbf{x m i n}, \mathbf{x m a x}, y \min , y \max ])$;

case 3

axis tight

$\mathrm{xmin}=0 ; \mathrm{xmax}=1 ; \mathrm{ymin}=0 ; \mathrm{ymax}=1$;

$\operatorname{axis}([x \min , \mathbf{x m a x}, \mathbf{y m i n}, y \max ])$;

case 4

axis tight

$\mathrm{xmin}=-2 ; \mathrm{xmax}=2 ; y \min =-1 ; y \max =2$;

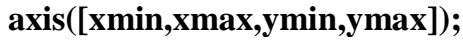

end

figure(ndiv/2)

plot(xvec,yvec,'r-'); \% plot element

$\%$ plot(xvec,yvec); \% plot element

hold on;

$\%$ place element number

$\operatorname{mid} x=\operatorname{mean}(\operatorname{xvec}(1,1: 4))$;

$\operatorname{midy}=\operatorname{mean}(\operatorname{yvec}(1,1: 4))$;

if $\operatorname{mesh}<=2$

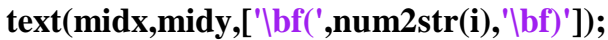

end

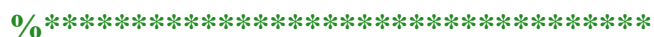

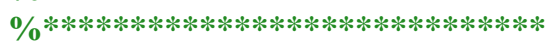

end; \% i loop

switch tri

case 1

xlabel('Ibfx axis')

ylabel(''bfy axis')

st1=' 'bfone eigth (1/8)square cross section ';

st2=' using ';

st3='12-node cubic serendipity ';

st4='quadriateral';

st5=' elements'

title([st1,st2,st3,st4,st5])

case 2

xlabel(''bfx axis')

ylabel('ไbfy axis')

st1='\bfone eigth (1/8)square cross section ';

st2=' using ';

st3='12-node cubic serendipity ';

st4='quadriateral';

st5 $\mathbf{=}^{\prime}$ elements'

title([st1,st2,st3,st4,st5]) 
case 3

xlabel('Ibfx axis')

ylabel('Ibfy axis')

st1=' \bfequilateral triangular cross section ';

st2=' using ';

st3='12-node cubic serendipity ';

st4='quadriateral';

st5=' elements'

title([st1,st2,st3,st4,st5])

case 4

xlabel('Ibfx axis')

ylabel(')'bfy axis')

st1=' \bfequilateral triangular cross section ';

st2=' using ';

st3='12-node cubic serendipity ';

st4='quadriateral';

st5 $=$ ' elements'

title([st1,st2,st3,st4,st5])

end

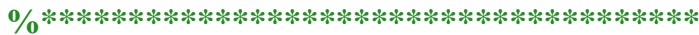

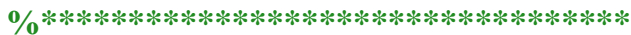

switch tri

case 1

$\operatorname{text}(\mathbf{0 . 6 , 0 . 8},[$ ' $\backslash$ bfMESH NO.=',num2str(mesh)]

$\operatorname{text}(0.6,0.75,[' \backslash b f$ number of elements=',num2str(nel)])

$\operatorname{text}(\mathbf{0 . 6 , 0 . 7 0},[' \backslash b$ fnumber of nodes=',num2str(nnode)])

case 2

$\operatorname{text}(0.1,0.4,[' \backslash b f M E S H$ NO.=',num2str(mesh)])

$\operatorname{text}(0.1,0.38,[' \backslash b f n u m b e r$ of elements=',num2str(nel)])

$\operatorname{text}(0.1,0.36$, ['Vbfnumber of nodes=',num2str(nnode)])

case 3

$\operatorname{text}(0.65,0.8,[' \backslash b f M E S H ~ N O .=', n u m 2 s t r(m e s h)])$

$\operatorname{text}(\mathbf{0 . 6 5 , 0 . 7 5}$, ['Vbfnumber of elements=',num2str(nel)])

$\operatorname{text}(\mathbf{0 . 6 5 , 0 . 7 0},[' \backslash b$ fnumber of nodes=',num2str(nnode)])

case 4

$\operatorname{text}(0.6,1.8,[' \backslash b f M E S H ~ N O .=', n u m 2 s t r(m e s h)])$

$\operatorname{text}(0.6,1.7,[' \backslash$ bf number of elements=',num2str(nel)])

$\operatorname{text}(\mathbf{0 . 6 , 1 . 6},[$ ' $\backslash$ bfnumber of nodes=',num2str(nnode)])

end

$\%$ put node numbers

for $\mathbf{j} \mathbf{j}=\mathbf{1}$ :nnode

if $\operatorname{mesh}<=2$

\%text(gcoord(jj,1),gcoord(jj,2),['ไbf.',num2str(jj)]);

$\operatorname{text}(\operatorname{gcoord}(\mathbf{j j}, \mathbf{1}), \operatorname{gcoord}(\mathbf{j j}, 2),[$ num2str(jj)]);

else

\%text(gcoord(jj,1),gcoord(jj,2),['ไbf.']);

$\operatorname{text}\left(\operatorname{gcoord}(\mathbf{j j}, 1), \operatorname{gcoord}(\mathbf{j j}, 2),\left[{ }^{\prime} \backslash b f f^{\prime}\right]\right)$;

end

end

hold on

for $i=1$ :nel

for $\mathrm{j}=1$ :nnel

$x(\mathbf{1}, \mathbf{j})=x \operatorname{coord}(\operatorname{nodes}(\mathbf{i}, \mathbf{j}), \mathbf{1})$;

$\mathbf{y}(\mathbf{1}, \mathbf{j})=\operatorname{ycoord}(\operatorname{nodes}(\mathbf{i}, \mathbf{j}), \mathbf{1})$;

end; $\%$ j loop

figure(mesh),scatter(x(1,1:nnel),y(1,1:nnel),20, 'filled','b')

\%figure(mesh),scatter(x(1,1:nnel),y(1,1:nnel), 'MarkerFaceColor','g')

\% figure(mesh),scatter(x(1,nnel-3:nnel),y(1,nnel-3:nnel),'MarkerFaceColor','r')

end \% i loop

\% figure(mesh),scatter(gcoord(:,1),gcoord(:,2),'MarkerFaceColor','g')

$\%$ axis off

$\% * * * * * * * * * * * * * * * * * * * * * * * * * * * * * * * * * * * * * * * *$ 
end \% for nmesh-the number of meshes

[2]quadrilateralmesh_over_arbitrarytriangle_q16automeshgen.m

function[]=quadrilateralmesh_over_arbitrarytriangle_q16automeshgen(mmesh,nmesh,tri)

\%quadrilateralmesh_over_arbitrarytriangle_q9automeshgen $(1,1,4)$

\%quadrilateralmesh_over_arbitrarytriangle_q16automeshgen $(1,1,4)$

clf

switch tri

case $1 \%$ standard triangle

$\mathrm{xx}=\operatorname{sym}([0 ; 1 ; 0])$

yy=sym $([0 ; 0 ; 1])$

case 2

$\mathrm{xx}=\operatorname{sym}([0 ; 1 / 2 ; 1 / 2])$

$\mathrm{yy}=\operatorname{sym}([0 ; 0 ; 1 / 2])$

case $3 \%$ equilateral triangle

$\mathrm{xx}=\operatorname{sym}([0 ; 1 ; 1 / 2])$

$\mathrm{yy}=\operatorname{sym}([0 ; 0 ; \operatorname{sqrt}(3) / 2])$

case $4 \%$ equilateral triangle

$\mathrm{xx}=\operatorname{sym}([-\operatorname{sqrt}(3) ; \operatorname{sqrt}(3) ; 0])$

$\operatorname{yy}=\operatorname{sym}([-1 ;-1 ; 2])$

end

for mesh=mmesh:nmesh

figure(mesh)

ndiv $=2 *$ mesh;

[eln,nodetel,nodes,nnode]=nodaladdresses4Lagrangespecial_convex_quadrilaterals_3rd_order(ndiv)

$\%$ [coord,gcoord] =coordinate_rtisoscelestriangle00_h0_hh_2ndorder(ndiv);

[coord,gcoord]=coordinate_arbitrarytriangle_3rdorderLAGR(xx,yy,ndiv)

$[$ nel,nnel] $=$ size $($ nodes $)$

for $i=1:$ nel

$\mathrm{NN}(\mathrm{i}, 1)=\mathrm{i}$;

end

table $1=[\mathrm{NN}$ nodes $]$

[nnode, dimension] $=$ size $($ gcoord $)$

$\%$ plot the mesh for the generated data

$\% \mathrm{x}$ and $\mathrm{y}$ coordinates

$\mathrm{xcoord}(1:$ nnode, 1$)=\operatorname{gcoord}(1:$ nnode, 1$)$;

$\operatorname{ycoord}(1:$ nnode, 1$)=\operatorname{gcoord}(1:$ nnode, 2$)$;

$\%$ extract coordinates for each element

for $\mathrm{i}=1:$ nel

for $\mathrm{j}=1$ :nnel

$x(1, j)=x \operatorname{coord}(\operatorname{nodes}(i, j), 1)$;

$y(1, j)=y \operatorname{coord}(\operatorname{nodes}(i, j), 1)$;

end; $\%$ j loop

$\mathrm{xvec}(1,1: 5)=[\mathrm{x}(1,1), \mathrm{x}(1,2), \mathrm{x}(1,3), \mathrm{x}(1,4), \mathrm{x}(1,1)]$;

$\operatorname{yvec}(1,1: 5)=[\mathrm{y}(1,1), \mathrm{y}(1,2), \mathrm{y}(1,3), \mathrm{y}(1,4), \mathrm{y}(1,1)]$;

$\%$ axis equal

switch tri

case 1

axis tight

$\mathrm{xmin}=0 ; \mathrm{xmax}=1 ; \mathrm{ymin}=0 ; \mathrm{ymax}=1$;

$\operatorname{axis}([\mathrm{xmin}, \mathrm{xmax}, \mathrm{ymin}, \mathrm{ymax}])$;

case 2

axis tight 
$\mathrm{x} \min =0 ; \mathrm{xmax}=1 / 2 ; \mathrm{ymin}=0 ; \mathrm{ymax}=1 / 2$;

axis([xmin,xmax,ymin,ymax]);

case 3

axis tight

$\mathrm{x} \min =0 ; \mathrm{xmax}=1 ; \mathrm{ymin}=0 ; \mathrm{ymax}=1$;

axis([xmin, xmax,ymin,ymax]);

case 4

axis tight

$\mathrm{x} \min =-2 ; \mathrm{xmax}=2 ; \mathrm{ymin}=-1 ; \mathrm{ymax}=2$;

axis([xmin, xmax,ymin,ymax]);

end

plot(xvec,yvec,'b-');\%plot element

hold on;

$\%$ place element number

$\operatorname{mid} x=\operatorname{mean}(\mathrm{xvec}(1,1: 4))$;

$\operatorname{midy}=\operatorname{mean}(\operatorname{yvec}(1,1: 4))$;

if $\mathrm{mesh}<=2$

text(midx,midy,['lbf(',num2str(i),'lbf)']);

end

end;\% loop

switch tri

case 1

xlabel('Ibfx axis')

ylabel('Ibfy axis')

st1='\bfone eigth (1/8)square cross section ';

st2=' using ';

st3='16-node cubic Lagrange ';

st4='quadriateral';

st5=' elements'

title([st1,st2,st3,st4,st5])

case 2

xlabel('Ibfx axis')

ylabel('Ibfy axis')

st $1=$ '\bfone eigth (1/8)square cross section ';

st2=' using ';

st3='16-node cubic Lagrange ';

st4='quadriateral';

st5=' elements'

title([st1,st2,st3,st4,st5])

case 3

xlabel('Ibfx axis')

ylabel('Ibfy axis')

st1='\bfequilateral triangular cross section ';

st2=' using ';

st3='16-node cubic Lagrange ';

st4='quadriateral';

st5=' elements'

title([st1,st2,st3,st4,st5])

case 4

xlabel('Ibfx axis')

ylabel('Ibfy axis')

st1='\bfequilateral triangular cross section ';

st2=' using ';

st3='16-node cubic Lagrange ';

st4='quadriateral';

st5=' elements'

title([st1,st2,st3,st4,st5])

end

switch tri

case 1

$\operatorname{text}(0.6,0.8,[$ '\bfMESH NO.=',num2str(mesh)]

text $(0.6,0.75$, ['lbfnumber of elements=',num2str(nel)]) 
case 2

$\operatorname{text}(0.1,0.4,[$ '\bfMESH NO.=',num2str(mesh)])

$\operatorname{text}(0.1,0.38,[$ ' $\mathrm{b}$ fnumber of elements=',num2str(nel)])

text $(0.1,0.36$, [''bfnumber of nodes=',num 2 str(nnode)])

case 3

text $(0.65,0.8,[$ '।bfMESH NO.=',num2str(mesh)])

$\operatorname{text}(0.65,0.75$, ['lbfnumber of elements=',num2str(nel)])

text $(0.65,0.70,[$ ' $\backslash$ bfnumber of nodes=',num2str(nnode) $]$ )

case 4

text $(0.6,1.8,[$ '।bfMESH NO.=',num2str(mesh)])

$\operatorname{text}(0.6,1.7,[$ '।bfnumber of elements=',num2str(nel)])

text $(0.6,1.6,[$ '।bfnumber of nodes=',num2str(nnode)])

end

\%put node numbers

for $\mathrm{jj}=1$ :nnode

if $\operatorname{mesh}<=2$

text $\left(\operatorname{gcoord}(\mathrm{jj}, 1), \operatorname{gcoord}(\mathrm{jj}, 2),\left[{ }^{\prime} \mid b f^{\prime}\right.\right.$, num2str(jj)]);

else

$\operatorname{text}\left(\operatorname{gcoord}(\mathrm{jj}, 1), \operatorname{gcoord}(\mathrm{jj}, 2),\left[{ }^{\prime} \mid \mathrm{bf} '\right]\right)$;

end

end

hold on

for $i=1:$ nel

for $\mathrm{j}=1$ :nnel

$x(1, j)=x \operatorname{coord}(\operatorname{nodes}(i, j), 1)$;

$y(1, j)=y \operatorname{coord}(\operatorname{nodes}(i, j), 1)$;

end;\%j loop

figure(mesh),scatter(x(1,1:nnel-4),y(1,1:nnel-4),20,'filled','r')

figure(mesh),scatter(x(1,nnel-3:nnel),y(1,nnel-3:nnel),20,'filled','b')

end \% i loop

\%figure(mesh),scatter(gcoord(:,1),gcoord(:,2),'MarkerFaceColor','g')

$\%$ axis off

end \% for nmesh-the number of meshes

\section{[3]quadrilateral_mesh4MOINEX_q12.m}

function[]=quadrilateral_mesh4MOINEX_q12(n1,n2,n3,nmax,numtri,ndiv,mesh,xlength,ylength)

$\%$ clc

$\%$ clf

$\%(1)=$ generate 2 -D quadrilateral mesh

$\%$ for a rectangular shape of domain

\%quadrilateral_mesh_q4(xlength,ylength)

$\%$ xnode=number of nodes along $\mathrm{x}$-axis

$\%$ ynode=number of nodes along $\mathrm{y}$-axis

$\%$ xzero $=\mathrm{x}$-coord of bottom left corner

$\%$ yzero=y-coord of bottom left corner

\%xlength=size of domain alog $\mathrm{x}$-axis

\%ylength=size of domain alog $\mathrm{y}$-axis

\%quadrilateral_mesh4MOINEX_q16LG([1;1;1;1;1;1;1],[2;3;4;5;6;7;8],[3;4;5;6;7;8;2],8,1,2,1,1,1)

\%quadrilateral_mesh4MOINEX_q12([1;1;1;1;1;1;1],[2;3;4;5;6;7;8],[3;4;5;6;7;8;2],8,1,2,1,1,1)

\%quadrilateral_mesh4MOINEX_q12([1;1;1;1;1;1;1;1],[2;3;4;5;6;7;8;9],[3;4;5;6;7;8;9;2],9,1,2,4,1,1)

[coord,gcoord,nodes,nodetel,nnode,nel]=polygonal_domain_coordinates_3rd_order(n1,n2,n3,nmax,numtri,ndiv,mesh)

[nel,nnel]=size(nodes);

$\operatorname{disp}([x l e n g t h, y l e n g t h, n n o d e, n e l, n n e l])$

$\% \operatorname{gcoord}(i, j)$, where i->node no. and $\mathrm{j}->\mathrm{x}$ or $\mathrm{y}$

$\%$

$\%$ plot the mesh for the generated data

$\% \mathrm{x}$ and y coordinates

$\operatorname{xcoord}(:, 1)=\operatorname{gcoord}(:, 1)$;

$\operatorname{ycoord}(:, 1)=\operatorname{gcoord}(:, 2)$;

$\%$ extract coordinates for each element 
clf

for $i=1:$ nel

for $\mathrm{j}=1$ :nnel

$x(1, j)=x \operatorname{coord}(\operatorname{nodes}(i, j), 1)$;

$y(1, j)=y \operatorname{coord}(\operatorname{nodes}(i, j), 1)$;

end;\%j loop

$\mathrm{xvec}(1,1: 5)=[\mathrm{x}(1,1), \mathrm{x}(1,2), \mathrm{x}(1,3), \mathrm{x}(1,4), \mathrm{x}(1,1)]$;

$\operatorname{yvec}(1,1: 5)=[\mathrm{y}(1,1), \mathrm{y}(1,2), \mathrm{y}(1,3), \mathrm{y}(1,4), \mathrm{y}(1,1)]$;

axis tight

switch mesh

case 1

axis([0 xlength 0 ylength $])$

case 2

axis([0 xlength 0 ylength])

case 3

axis([0 xlength 0 ylength $])$

case 4

axis([-xlength/2 xlength/2 -ylength/2 ylength/2])

end

figure(ndiv/2)

plot(xvec,yvec,'r-');\%plot element

hold on;

$\%$ place element number

$\operatorname{mid} x=\operatorname{mean}(\operatorname{xvec}(1,1: 4))$

$\operatorname{midy}=\operatorname{mean}(\mathrm{yvec}(1,1: 4))$

if $\operatorname{ndiv}<=2$

text(midx+.01, midy-.03, ['[',num2str(i),']']);

end

end;\%i loop

xlabel('x axis')

ylabel('y axis')

st1='Mesh of ';

st2=num2str(nel);

st3=' twelve node ';

st4='quadrilateral';

st5=' elements \& ';

st6=num 2 str(nnode);

st7=',nodes'

title([st1,st2,st3,st4,st5,st6,st7])

$\%$ put node numbers

disp(nnode)

if $\operatorname{ndiv}<=2$

for $\mathrm{jj}=1$ :nnode

text(gcoord(jj,1),gcoord(jj,2),[num2str(jj)]);

end

hold on

\%figure(1),scatter(gcoord(:,1),gcoord(:,2),15,'filled','g')

hold on

for $\mathrm{i}=1$ :nel

for $\mathrm{j}=1$ :nnel

$x(1, j)=x \operatorname{coord}(\operatorname{nodes}(i, j), 1)$;

$y(1, j)=y \operatorname{coord}(\operatorname{nodes}(i, j), 1)$

end;\%j loop

figure(1),scatter(x(1,1:nnel),y(1,1:nnel),20,'filled','r')

\%figure(1),scatter(x(1,1:nnel-4),y(1,1:nnel-4),15,'filled','g')

\%figure(1),scatter(x(1,nnel-3:nnel),y(1,nnel-3:nnel),15,'filled','r')

end \% loop

end

$\%$ axis off

$\%$ if ndiv $>=4$

$\%$ for $\mathrm{jj}=1$ :nnode

$\%$ text(gcoord(jj,1),gcoord(ji,2),['o']); 
hold on

\%figure(ndiv/2), scatter(gcoord(:,1),gcoord(:,2),'MarkerFaceColor','g')

$\%$ figure(ndiv/2), scatter(gcoord(:,1), gcoord(:,2), 15,'g')

if ndiv $>=4$

\%figure(ndiv/2), scatter(gcoord(:,1),gcoord(:,2), 15,'filled','g')

hold on

for $i=1$ :nel

for $\mathrm{j}=1$ :nnel

$x(1, j)=x \operatorname{coord}(\operatorname{nodes}(i, j), 1)$;

$y(1, j)=y \operatorname{coord}(\operatorname{nodes}(i, j), 1)$;

end; \% j loop

figure(ndiv/2),scatter(x(1,1:4),y(1,1:4),20,'filled','k')

hold on

figure(ndiv/2),scatter(x(1,5:6),y(1,5:6),20,'filled','b')

hold on

figure(ndiv/2),scatter(x(1,7:8),y(1,7:8),20,'filled','r')

hold on

figure(ndiv/2), scatter(x(1,9:10),y(1,9:10),20,'filled','b')

hold on

figure(ndiv/2),scatter(x(1,11:12),y(1,11:12),20,'filled','r')

hold on

\%figure(ndiv/2), scatter(x(1,1:nnel-4),y(1,1:nnel-4),15,'filled','g')

\%figure(ndiv/2), scatter(x(1,nnel-3:nnel),y(1,nnel-3:nnel),15,'filled','r')

end \% i loop

end

[4]quadrilateral_mesh4MOINEX_q16LG.m

function[]=quadrilateral_mesh4MOINEX_q16LG(n1,n2,n3,nmax,numtri,ndiv,mesh,xlength,ylength)

$\%$ clc

$\%$ clf

$\%(1)=$ generate 2 -D quadrilateral mesh

$\%$ for a rectangular shape of domain

\%quadrilateral_mesh_q4(xlength,ylength)

$\%$ xnode=number of nodes along $\mathrm{x}$-axis

$\%$ ynode=number of nodes along $\mathrm{y}$-axis

$\% x z e r o=x$-coord of bottom left corner

$\%$ yzero=y-coord of bottom left corner

$\% x$ length=size of domain alog $\mathrm{x}$-axis

\%ylength=size of domain alog $y$-axis

\%quadrilateral_mesh4MOINEX_q16LG([1;1;1;1;1;1;1;1],[2;3;4;5;6;7;8;9],[3;4;5;6;7;8;9;2],9,1,2,4,1,1)

\%quadrilateral_mesh4MOINEX_q16LG([1;1;1;1;1;1;1],[2;3;4;5;6;7;8],[3;4;5;6;7;8;2],8,1,2,1,1,1)

\%quadrilateral_mesh4MOINEX_q16LG([1;1;1;1;1;1;1;1],[2;3;4;5;6;7;8;9],[3;4;5;6;7;8;9;2],9,1,2,2,1,1)

\%quadrilateral_mesh4MOINEX_q16LG( $([1 ; 1 ; 1 ; 1 ; 1 ; 1 ; 1 ; 1],[2 ; 3 ; 4 ; 5 ; 6 ; 7 ; 8 ; 9],[3 ; 4 ; 5 ; 6 ; 7 ; 8 ; 9 ; 2], 9,25,10,2,1,1)$

[coord,gcoord,nodes,nodetel,nnode,nel]=polygonal_domain_coordinates_3rd_orderLG(n1,n2,n3,nmax,numtri,ndiv,mesh)

[nel,nnel]=size(nodes);

$\operatorname{disp}([x l e n g t h, y l e n g t h$, nnode,nel,nnel])

$\%$ plot the mesh for the generated data

$\% \mathrm{x}$ and $\mathrm{y}$ coordinates

$\operatorname{xcoord}(:, 1)=\operatorname{gcoord}(:, 1)$;

$\operatorname{ycoord}(:, 1)=\operatorname{gcoord}(:, 2)$;

\%extract coordinates for each element

clf

for $\mathrm{i}=1$ :nel

for $\mathrm{j}=1$ :nnel

$x(1, j)=x \operatorname{coord}(\operatorname{nodes}(i, j), 1)$;

$y(1, j)=\operatorname{ycoord}(\operatorname{nodes}(i, j), 1)$;

end; \% j loop

$\mathrm{xvec}(1,1: 5)=[\mathrm{x}(1,1), \mathrm{x}(1,2), \mathrm{x}(1,3), \mathrm{x}(1,4), \mathrm{x}(1,1)]$;

$\operatorname{yvec}(1,1: 5)=[\mathrm{y}(1,1), \mathrm{y}(1,2), \mathrm{y}(1,3), \mathrm{y}(1,4), \mathrm{y}(1,1)]$;

axis tight

switch mesh

case 1

axis([0 xlength 0 ylength $])$

case 2 
axis([0 xlength 0 ylength $])$

case 3

axis([0 xlength 0 ylength])

case 4

axis([-xlength/2 xlength/2 -ylength/2 ylength/2])

end

figure(ndiv/2)

plot(xvec,yvec, 'r');\%plot element

hold on;

$\%$ place element number

$\operatorname{midx}=\operatorname{mean}(\operatorname{xvec}(1,1: 4))$

midy $=$ mean $(\operatorname{yvec}(1,1: 4))$

if ndiv $<=2$

text(midx +.01 , midy-.03,['[',num2str(i),']']);

end

end; \% i loop

xlabel('x axis')

ylabel('y axis')

st1='Mesh of ';

st2=num2str(nel);

st3=' sixteen node ';

st4='quadrilateral';

st5=' elements \& ';

st6=num $2 \operatorname{str}($ nnode);

st7=',nodes'

title([st1,st2,st3,st4,st5,st6,st7])

$\%$ put node numbers

disp(nnode)

if $\operatorname{ndiv}<=2$

for $\mathrm{jj}=1$ :nnode

text $(\operatorname{gcoord}(\mathrm{jj}, 1), \operatorname{gcoord}(\mathrm{jj}, 2),[\operatorname{num} 2 \mathrm{str}(\mathrm{jj})])$;

end

hold on

\%figure(1),scatter(gcoord(:,1),gcoord(:,2),15,'filled','g')

hold on

for $i=1:$ nel

for $\mathrm{j}=1$ :nnel

$x(1, j)=x \operatorname{coord}(\operatorname{nodes}(i, j), 1)$;

$y(1, j)=y \operatorname{coord}(\operatorname{nodes}(i, j), 1)$;

end; \% j loop

figure(1),scatter(x(1,1:nnel-4),y(1,1:nnel-4),20,'filled','b')

figure(1),scatter(x(1,nnel-3:nnel),y(1,nnel-3:nnel),20,'filled','r')

end $\%$ i loop

end

\%axis off

$\%$ if ndiv $>=4$

$\%$ for $\mathrm{jj}=1$ :nnode

\%text(gcoord(jj,1), gcoord(jj,2),['o']);

$\%$ end

\%end

hold on

\%figure(ndiv/2), scatter(gcoord(:,1),gcoord(:,2),'MarkerFaceColor','g')

\%figure(ndiv/2), scatter(gcoord(:,1),gcoord(:,2),15,'g')

if ndiv $>=4$

\%figure(ndiv/2),scatter(gcoord(:,1),gcoord(:,2),15,'filled','g')

hold on

for $\mathrm{i}=1$ :nel

for $\mathrm{j}=1$ :nnel

$x(1, j)=x \operatorname{coord}(\operatorname{nodes}(i, j), 1)$;

$y(1, j)=y \operatorname{coord}(\operatorname{nodes}(i, j), 1)$;

end;\%j loop

figure(ndiv/2),scatter(x(1,1:nnel-4),y(1,1:nnel-4),20,'filled','b') 
figure(ndiv/2),scatter(x(1,nnel-3:nnel),y(1,nnel-3:nnel),20,'filled','r')

end $\%$ i loop

end

[5]D2LaplaceEquationQ12Ex3automeshgenNewContour.m

function[]=D2LaplaceEquationQ12Ex3automeshgenNewContour(n1,n2,n3,numtri,ndiv,mesh)

\%function[]=improvedLaplaceEquationQuad8twodimensionEx3_explicitvfnmesh(nel,nnode,nnel,ndof,quadtype,mesh)

\%note that input vlues of $\mathrm{X}$ and $\mathrm{Y}$ must be symbolic constants

$\%$ for the example triangle input for $\mathrm{X}$ is sym([-1/2 1/2 0$])$

$\%$ for the example triangle input for $\mathrm{Y}$ is $\operatorname{sym}([00 \mathrm{sqrt}(3 / 4)])$

$\%$ LaplaceEquationQ4twoD(3,sym([-1/2 1/2 0]),sym([0 $0 \operatorname{sqrt}(3 / 4)]))$

\%syms ff ss f sk N NN table1 table2

\%D2LaplaceEquationQ12Ex3automeshgenNewContour(1,2,3,1,2,1)

$\%$ D2LaplaceEquationQ12Ex3automeshgenNewContour(1,2,3,1,2,2)

$\%$

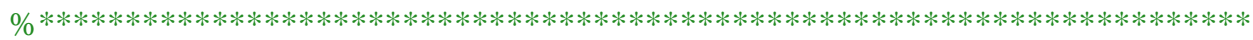

syms coord

syms x y

ndof $=1$;

switch mesh

case 1

$\mathrm{x}=\operatorname{sym}([0 ; 1 / 2 ; 1 / 2])$

$\mathrm{y}=\operatorname{sym}([0 ; 0 ; 1 / 2])$

case $2 \%$ isoscles triangle(torsion of an equilateral triangle,each side $=2 * \operatorname{sqrt}(3)$ )

$\mathrm{x}=\operatorname{sym}([-\operatorname{sqrt}(3) ; \operatorname{sqrt}(3) ; 0])$

$\mathrm{y}=\operatorname{sym}([-1 ;-1 ; 2])$

end

syms ui vi wi xi yi

[ui,vi,wi]=coordinate_special_quadrilaterals_in_stdtriangle_3rd_order(ndiv)

$\% \operatorname{disp}([u$ ui vi wi])

$\mathrm{N}=$ length(ui);

$\mathrm{NN}=(1: \mathrm{N})^{\prime}$;

$\mathrm{X}$

$\mathrm{y}$

$\mathrm{x} 1=\mathrm{x}(\mathrm{n} 1,1) ; \mathrm{x} 2=\mathrm{x}(\mathrm{n} 2,1) ; \mathrm{x} 3=\mathrm{x}(\mathrm{n} 3,1) ; \mathrm{y} 1=\mathrm{y}(\mathrm{n} 1,1) ; \mathrm{y} 2=\mathrm{y}(\mathrm{n} 2,1) ; \mathrm{y} 3=\mathrm{y}(\mathrm{n} 3,1) ;$

for $\mathrm{i}=1: \mathrm{N}$

xxi $(\mathrm{i}, 1)=x 1+(\mathrm{x} 2-\mathrm{x} 1) * \mathrm{ui}(\mathrm{i}, 1)+(\mathrm{x} 3-\mathrm{x} 1) * \mathrm{vi}(\mathrm{i}, 1)$;

yyi $(\mathrm{i}, 1)=y 1+(y 2-y 1) * u i(i, 1)+(y 3-y 1) * v i(i, 1)$;

end

$\% \operatorname{disp}('$

$\% \operatorname{disp}\left(' \mathrm{NN}\right.$ xi yi'$\left.^{\prime}\right)$

$\% \operatorname{disp}([\mathrm{NN}$ xi yi])

$\%$ disp ('

$\% \operatorname{coord}(:, 1)=(\operatorname{xxi}(:, 1))$;

$\% \operatorname{coord}(:, 2)=($ yyi $(:, 1))$;

$\operatorname{gcoord}(:, 1)=\operatorname{double}(\operatorname{xxi}(:, 1))$;

$\operatorname{gcoord}(:, 2)=\operatorname{double}($ yyi $(:, 1))$

[eln,nodetel,nodes,nnode]=nodaladdresses_special_convex_quadrilaterals_3rd_order(ndiv);

[nel,nnel]=size(nodes);

$\%$ disp([nel nnode nnel ndof])

format long $g$

for $\mathrm{i}=1$ :nel

$\mathrm{N}(\mathrm{i}, 1)=\mathrm{i}$;

end

for $\mathrm{i}=1$ :nel

$\mathrm{NN}(\mathrm{i}, 1)=\mathrm{i}$;

end

sdof=nnode $*$ ndof; 
$\mathrm{ff}=($ zeros $($ sdof, 1$)) ; \mathrm{ss}=($ zeros $($ sdof,sdof $))$;

format long $g$

for $\mathrm{i}=1$ :nel

$\mathrm{N}(\mathrm{i}, 1)=\mathrm{i}$;

end

$\%$ radius of the hole $=1.25 \mathrm{~cm}$

\%input data for nodal coordinate values

$\% \operatorname{gcoord}(i, j)$, where i->node no. and j->x or y

$\%$ table $1=[\mathrm{N}$ nodes $]$

$\%[$ nel,nnel $]=\operatorname{size}($ nodes $)$;

\% ****************************************************************8

$\%$ \%********************************************

switch mesh

case 1

$\mathrm{nnn}=0$;

for $n=1$ :nnode

if $\operatorname{gcoord}(\mathrm{nn}, 1)==(1 / 2)$

$\mathrm{nnn}=\mathrm{nnn}+1$;

bcdof $(\mathrm{nnn}, 1)=\mathrm{nn}$;

end

end

format long $\mathrm{g}$

$\mathrm{k} 1$ =double $(0.14057701495515551037840396020329)$;

$\mathrm{xi}=($ zeros $($ nnode, 1$))$;

$\mathrm{a} 0=8 / \mathrm{pi}^{\wedge} 3$;

for $\mathrm{m}=1$ :nnode

$\mathrm{gx}=(\mathrm{gcoord}(\mathrm{m}, 1)) ; \mathrm{gy}=(\mathrm{gcoord}(\mathrm{m}, 2)) ; \mathrm{rr}=(0) ;$

for $\mathrm{n}=1: 2: 99$

$\mathrm{rr}=\mathrm{rr}+(-1)^{\wedge}((\mathrm{n}-1) / 2)^{*}(1-(\cosh (\mathrm{n} * \mathrm{pi} * \mathrm{gy}) / \cosh (\mathrm{n} * \mathrm{pi} / 2))) * \cos (\mathrm{n} * \mathrm{pi} * \mathrm{gx}) / \mathrm{n}^{\wedge} 3$;

end

$\operatorname{xi}(\mathrm{m}, 1)=(\mathrm{a} 0 * \mathrm{rr})$

end

mm=length(bcdof);

case $2 \%$ torsion of an equilateral triangle

$\mathrm{nnn}=0$;

\%boundary conditions on side 1

for $n n=1$ :nnode

$\mathrm{xnn}=\operatorname{gcoord}(\mathrm{nn}, 1) ; \mathrm{ynn}=\mathrm{g} \operatorname{coord}(\mathrm{nn}, 2)$;

if $((\mathrm{ynn}+1)<1 . e-5)$

$\mathrm{nnn}=\mathrm{nnn}+1$;

$\operatorname{bcdof}(\mathrm{nnn}, 1)=\mathrm{nn}$;

$\operatorname{bcval}(\mathrm{nnn}, 1)=0$;

end

end

\%boundary conditions on side 2

for $n n=1$ :nnode

$\mathrm{xnn}=\operatorname{gcoord}(\mathrm{nn}, 1) ; \mathrm{ynn}=\operatorname{gcoord}(\mathrm{nn}, 2)$;

if $((-(\operatorname{sqrt}(3)) * x n n-y n n+2)<1 . e-5)$

$\mathrm{nnn}=\mathrm{nnn}+1$;

$\operatorname{bcdof}(\mathrm{nnn}, 1)=\mathrm{nn}$;

$\operatorname{bcval}(\mathrm{nnn}, 1)=0$;

end

end

\%boundary conditions on side 3

for $\mathrm{nn}=1$ :nnode

$\mathrm{xnn}=\operatorname{gcoord}(\mathrm{nn}, 1) ; \mathrm{ynn}=\operatorname{gcoord}(\mathrm{nn}, 2)$;

if $(((\operatorname{sqrt}(3)) * x n n-y n n+2)<1 . e-5)$

$\mathrm{nnn}=\mathrm{nnn}+1$

$\operatorname{bcdof}(\mathrm{nnn}, 1)=\mathrm{nn}$;

$\operatorname{bcval}(\mathrm{nnn}, 1)=0$;

end 


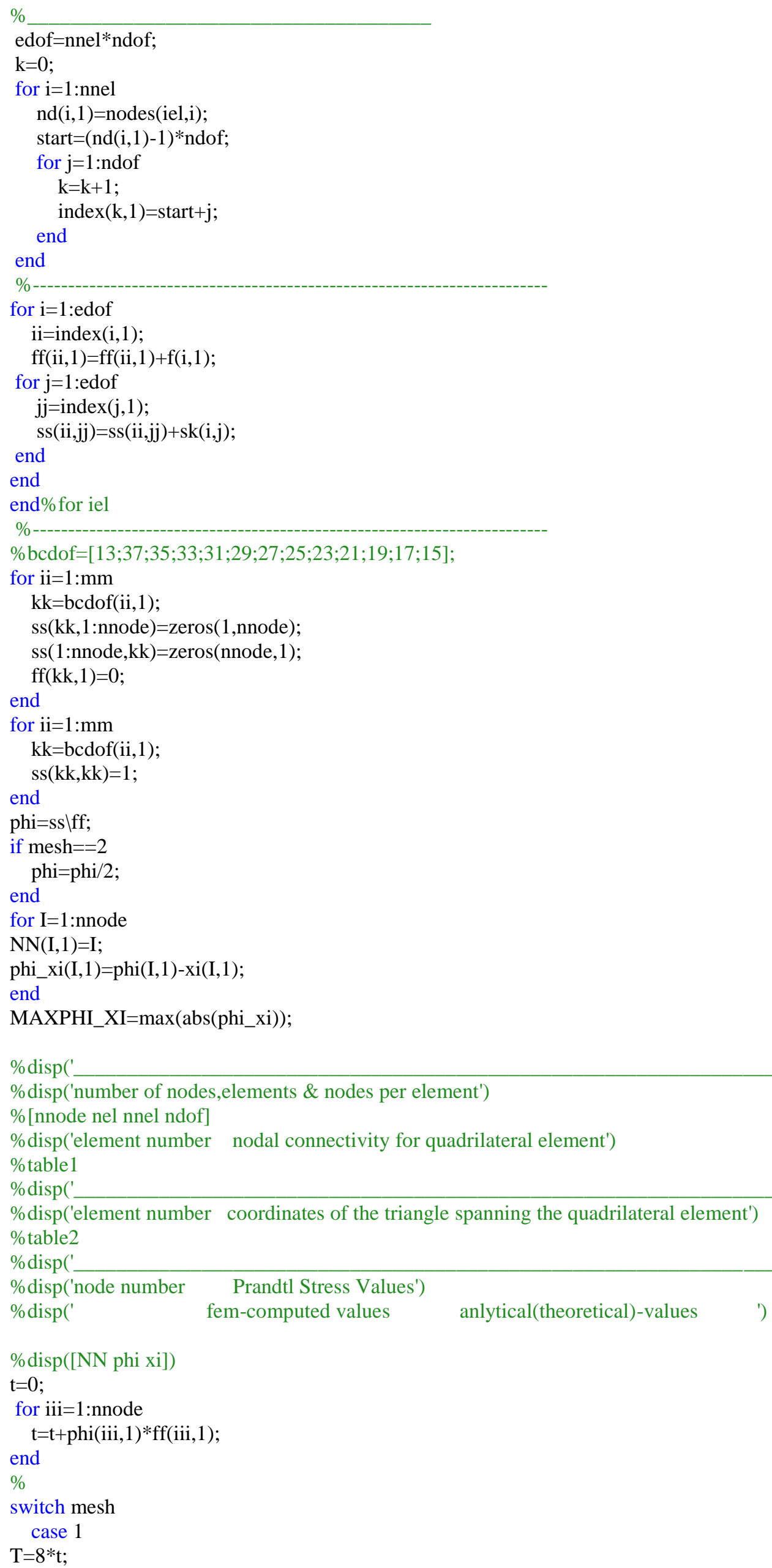


case 2

end

$\mathrm{T}=2 * \mathrm{t}$

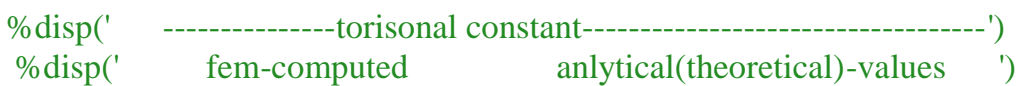

$\operatorname{disp}('$

$\operatorname{disp}([$ nnode nel nnel ] $)$

$\operatorname{disp}([$ T k1 MAXPHI_XI ])

$\operatorname{disp}(-$

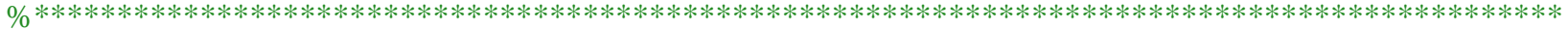

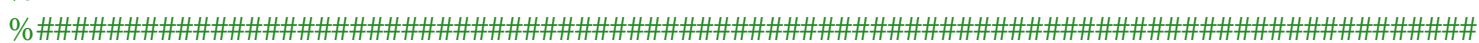

if $(\mathrm{mesh}==2)$

$[\mathrm{x}, \mathrm{y}]=\operatorname{meshgrid}(-\operatorname{sqrt}(3):(1 / 15) * \operatorname{sqrt}(3): \operatorname{sqrt}(3),-1:(0.1): 2)$;

$\mathrm{z}=(\mathrm{zeros}(31,31))$

for $i=1: 31$

for $\mathrm{j}=1: 31$

for iel=1:nel

$\mathrm{XX}=\mathrm{Xx}(\mathrm{iel}, 1: 3)$

YY=yy(iel,1:3);

$\% \operatorname{disp}([\mathrm{X} Y])$

$\mathrm{xa}=\mathrm{XX}(1,1)$;

$\mathrm{xb}=\mathrm{XX}(1,2)$;

$\mathrm{Xc}=\mathrm{XX}(1,3)$;

ya $=Y Y(1,1)$;

$\mathrm{yb}=\mathrm{YY}(1,2)$;

$\mathrm{yc}=\mathrm{YY}(1,3)$;

$\mathrm{aLPa}=\mathrm{xb} * \mathrm{yc}-\mathrm{xc} * \mathrm{yb}$

$\mathrm{aLPb}=\mathrm{xc} * \mathrm{ya}-\mathrm{xa} * \mathrm{yc}$

bta=yb-yc;btb=yc-ya;

$\mathrm{gma}=\mathrm{xc}-\mathrm{xb} ; \mathrm{gmb}=\mathrm{xa}-\mathrm{xc}$;

delabc $=$ gmb $*$ bta-gma*btb;

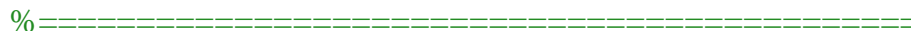

$\%$ node numbers of quadrilateral

nd1=nodes(iel,1);nd2=nodes(iel,2);nd3=nodes(iel,3);nd4=nodes(iel,4);

nd5=nodes(iel,5);nd6=nodes(iel,6);nd7=nodes(iel,7);nd8=nodes(iel,8);

nd9=nodes(iel,9); nd10=nodes(iel,10);nd11=nodes(iel,11);nd12=nodes(iel,12);

\%nd13=nodes(iel,13);nd14=nodes(iel,14);nd15=nodes(iel,15);nd16=nodes(iel,16);

$\%$ coordinates of quadrilateral $(\mathrm{u}, \mathrm{v})$

$\mathrm{u}(1,1)=\operatorname{gcoord}(\mathrm{nd} 1,1) ; \mathrm{u}(2,1)=\operatorname{gcoord}(\mathrm{nd} 2,1) ; \mathrm{u}(3,1)=\operatorname{gcoord}(\mathrm{nd} 3,1) ; \mathrm{u}(4,1)=\operatorname{gcoord}(\mathrm{nd} 4,1)$;

$\mathrm{v}(1,1)=\operatorname{gcoord}(\mathrm{nd} 1,2) ; \mathrm{v}(2,1)=\operatorname{gcoord}(\mathrm{nd} 2,2) ; \mathrm{v}(3,1)=\operatorname{gcoord}(\mathrm{nd} 3,2) ; \mathrm{v}(4,1)=\operatorname{gcoord}(\mathrm{nd} 4,2)$;

$\%$ coordinates of the $\operatorname{grid}(\mathrm{x}, \mathrm{y})$

in=inpolygon $(x(i, j), y(i, j), u, v)$;

if (in==1)

$\mathrm{X}=\mathrm{x}(\mathrm{i}, \mathrm{j}) ; \mathrm{Y}=\mathrm{y}(\mathrm{i}, \mathrm{j})$;

$\mathrm{p}=(\mathrm{aLPa}+\mathrm{bta} * \mathrm{X}+\mathrm{gma} * \mathrm{Y}) /$ delabc;

$\mathrm{q}=(\mathrm{aLPb}+\mathrm{btb} * \mathrm{X}+\mathrm{gmb} * \mathrm{Y}) /$ delabc;

$\mathrm{t} 0=[0 ; 0]$;

$\%[\mathrm{t}]=$ convexquadrilateral_coordinates $(\mathrm{u}, \mathrm{v}, \mathrm{X}, \mathrm{Y})$;

$\%[\mathrm{t}]=$ solveconvexquadrilateral_coordinates $(\mathrm{u}, \mathrm{v}, \mathrm{X}, \mathrm{Y})$;

$\%[\mathrm{t}]=$ convexquadrilateral_coordinatesnew $(\mathrm{u}, \mathrm{v}, \mathrm{X}, \mathrm{Y})$;

$\%[\mathrm{t}]=$ convexquadrilateral_naturalcoordinates $([1 / 3 ; 0 ; 0 ; 1 / 2],[1 / 3 ; 1 / 2 ; 0 ; 0], \mathrm{U}, \mathrm{V})$

[t,iter] = newtonmethod4spquadrileteral(t0,p,q,'parameqnspqd','paramdetJspqd','paraminvJspqd');

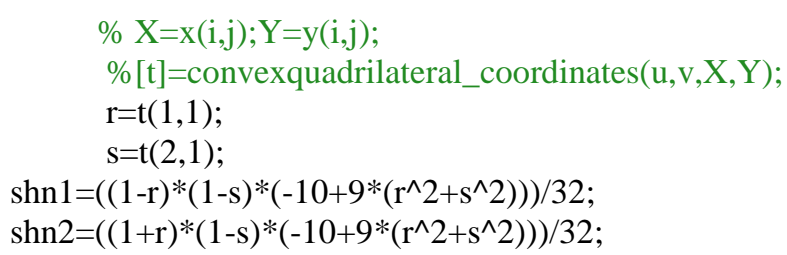


$\operatorname{shn} 3=\left((1+r) *(1+s) *\left(-10+9 *\left(r^{\wedge} 2+s^{\wedge} 2\right)\right)\right) / 32 ;$

$\operatorname{shn} 4=\left((1-\mathrm{r}) *(1+\mathrm{s}) *\left(-10+9 *\left(\mathrm{r}^{\wedge} 2+\mathrm{s}^{\wedge} 2\right)\right)\right) / 32$;

$\operatorname{shn} 5=(9 / 32) *\left((1-s) *\left(1-r^{\wedge} 2\right) *(1-3 * r)\right)$;

$\operatorname{shn} 6=(9 / 32) *\left((1-s) *\left(1-r^{\wedge} 2\right) *(1+3 * r)\right)$;

$\operatorname{shn} 7=(9 / 32) *\left((1+\mathrm{r}) *\left(1-\mathrm{s}^{\wedge} 2\right) *(1-3 * \mathrm{~s})\right)$;

$\operatorname{shn} 8=(9 / 32) *\left((1+\mathrm{r}) *\left(1-\mathrm{s}^{\wedge} 2\right) *(1+3 * \mathrm{~s})\right)$;

$\operatorname{shn} 9=(9 / 32) *\left((1+\mathrm{s}) *\left(1-\mathrm{r}^{\wedge} 2\right) *(1+3 * \mathrm{r})\right)$;

$\operatorname{shn} 10=(9 / 32) *\left((1+\mathrm{s}) *\left(1-\mathrm{r}^{\wedge} 2\right) *(1-3 * \mathrm{r})\right)$;

$\operatorname{shn} 11=(9 / 32) *\left((1-r) *\left(1-s^{\wedge} 2\right) *(1+3 * s)\right)$;

$\operatorname{shn} 12=(9 / 32) *\left((1-r) *\left(1-s^{\wedge} 2\right) *(1-3 * s)\right)$;

PHI(i,j)=shn $1 *$ phi(nd1,1)+shn2*phi(nd2,1)+shn3*phi(nd3,1)+shn4*phi(nd4,1)+shn5*phi(nd5,1)+shn6*phi(nd6,1)+shn7*phi(nd7,

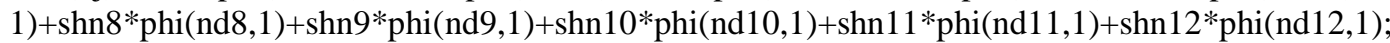
$\mathrm{z}(\mathrm{i}, \mathrm{j})=((\mathrm{Y}+1) *((\operatorname{sqrt}(3)) * \mathrm{X}-\mathrm{Y}+2) *(-(\operatorname{sqrt}(3)) * \mathrm{X}-\mathrm{Y}+2)) / 12 ;$

break end $\%$ if (in==1)

end $\%$ for iel

\% THE PROGRAM EXECUTION JUMPS TO HERE if (in==1) end $\%$ for $\mathrm{j}$

end $\%$ for $\mathrm{i}$

$\% \mathrm{z}=\sin (\mathrm{pi} * \mathrm{x}) \cdot * \sin \left(\mathrm{pi}^{*} \mathrm{y}\right)$

$\% \mathrm{z}=(\operatorname{zeros}(31,31))$;

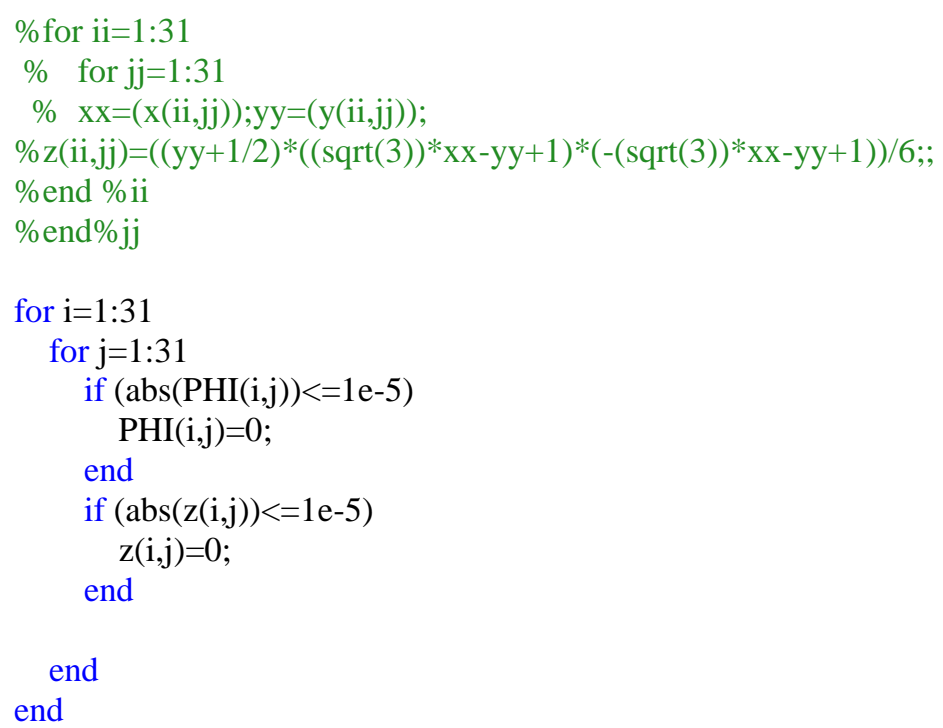

end $\%(\operatorname{mesh}==2)$

switch mesh

case 2

clf,figure(1)

clf,figure(2)

clf,figure(3)

figure(1)

$\mathrm{x}=[-\operatorname{sqrt}(3) ; \operatorname{sqrt}(3) ; 0]$;

$\mathrm{y}=[-1 ;-1 ; 2]$;

$\operatorname{patch}\left(\mathrm{x}, \mathrm{y},{ }^{\prime} \mathrm{w}\right)$

hold on

$\%[\mathrm{x}, \mathrm{y}]=$ meshgrid $(0: 1: 1,0: 0.1: 1)$

$[\mathrm{x}, \mathrm{y}]=$ meshgrid( $\left(-\operatorname{sqrt}(3):(1 / 15)^{*} \operatorname{sqrt}(3): \operatorname{sqrt}(3),-1:(0.1): 2\right)$;

$\% \mathrm{y}((\mathrm{y}>1 / 2) \&(\mathrm{y}<=1) \&(\mathrm{x}>1 / 2) \&(\mathrm{x}<=1) \&(\mathrm{x}+\mathrm{y}>3 / 2))=\mathrm{NaN}$;

$\% \% \mathrm{y}((\mathrm{y}>-1 / 2) \&(\mathrm{y}<=1) \&(\mathrm{x}>0) \&(\mathrm{x}<=(\operatorname{sqrt}(3) / 2)) \&((-\operatorname{sqrt}(3) * \mathrm{x}-\mathrm{y}+1)<0))=\mathrm{NaN}$;

$\% \% \mathrm{y}((\mathrm{y}>-1 / 2) \&(\mathrm{y}<=1) \&(\mathrm{x}>(-\operatorname{sqrt}(3) / 2)) \&(\mathrm{x}<=0) \&((\operatorname{sqrt}(3) * \mathrm{x}-\mathrm{y}+1)<0))=\mathrm{NaN}$;

$\%[\mathrm{c}, \mathrm{h}]=\operatorname{contour}(\mathrm{x}, \mathrm{y}, \mathrm{PHI})$

contour(x,y,PHI,20)

xlabel('X-axis');

ylabel('Y-axis');

\%clabel(c,h); 
axis square

st1='Contour level curves for ';

st2='FEM solution of ';

st3='twelve Noded ';

st4='Special Quadrilateral';

st5=' Elements'

title([st1,st2,st3,st4,st5])

sst $1=$ '(MESH HAS '

sst $2=$ num 2 str(nnode)

sst3 $=$ ' NODES'

sst4=' AND

sst5=num2str(nel)

sst6=' ELEMENTS)'

$\operatorname{text}(0.6,1.8,[\mathrm{sst} 1 \mathrm{sst} 2])$

$\operatorname{text}(0.6,1.6,[\mathrm{sst} 3 \mathrm{sst} 4])$

$\operatorname{text}(0.6,1.4,[\mathrm{sst} 5 \mathrm{sst} 6])$

$\% \operatorname{text}(0.25,-.08,[\mathrm{sst} 1 \mathrm{sst} 2 \mathrm{sst} 3 \mathrm{sst} 4 \mathrm{sst} 5 \mathrm{sst} 6])$

$\%$

figure(2)

$\% \mathrm{x}=\left[\begin{array}{llllll}0.0 & 1.0 & 1.0 & 0.5 & 0.0\end{array}\right]$;

$\% y=\left[\begin{array}{lllll}0.0 & 0.0 & 0.5 & 1.0 & 1.0\end{array}\right]$

$\mathrm{x}=[-\operatorname{sqrt}(3) ; \operatorname{sqrt}(3) ; 0]$;

$\mathrm{y}=[-1 ;-1 ; 2]$;

$\operatorname{patch}\left(\mathrm{x}, \mathrm{y}, \mathrm{w}^{\prime}\right)$

hold on

$\%[\mathrm{x}, \mathrm{y}]=\operatorname{meshgrid}(0: 1: 1,0: 0.1: 1)$

$\% y((y>1 / 2) \&(y<=1) \&(x>1 / 2) \&(x<=1) \&(x+y>3 / 2))=N a N$;

$\%[\mathrm{c}, \mathrm{h}]=$ contour $(\mathrm{x}, \mathrm{y}, \mathrm{z})$

$[x, y]=$ meshgrid( $(-\operatorname{sqrt}(3):(1 / 15) * \operatorname{sqrt}(3): \operatorname{sqrt}(3),-1:(0.1): 2)$;

contour(x,y,z,20)

xlabel('X-axis');

ylabel('Y-axis');

\%clabel(c,h);

axis square

title('contour level curves for exact solution: ')

hold off

figure(3)

$\% \mathrm{x}=\left[\begin{array}{llllll}0.0 & 1.0 & 1.0 & 0.5 & 0.0\end{array}\right]$;

$\% \mathrm{y}=\left[\begin{array}{lllll}0.0 & 0.0 & 0.5 & 1.0 & 1.0\end{array}\right]$;

$\mathrm{x}=[-\operatorname{sqrt}(3) ; \operatorname{sqrt}(3) ; 0]$;

$\mathrm{y}=[-1 ;-1 ; 2]$;

$\operatorname{patch}\left(\mathrm{x}, \mathrm{y}, \mathrm{w}^{\prime}\right)$

hold on

$[\mathrm{x}, \mathrm{y}]=$ meshgrid( $(-\operatorname{sqrt}(3):(1 / 15) * \operatorname{sqrt}(3): \operatorname{sqrt}(3),-1:(0.1): 2)$;

$\%[\mathrm{x}, \mathrm{y}]=$ meshgrid $(0: 1: 1,0: 0.1: 1)$

$\% \mathrm{y}((\mathrm{y}>1 / 2) \&(\mathrm{y}<=1) \&(\mathrm{x}>1 / 2) \&(\mathrm{x}<=1) \&(\mathrm{x}+\mathrm{y}>3 / 2))=\mathrm{NaN}$;

$\% \% \mathrm{y}((\mathrm{y}>-1 / 2) \&(\mathrm{y}<=1) \&(\mathrm{x}>0) \&(\mathrm{x}<=(\operatorname{sqrt}(3) / 2)) \&((-\operatorname{sqrt}(3) * \mathrm{x}-\mathrm{y}+1)<0))=\mathrm{NaN}$;

$\% \% \mathrm{y}((\mathrm{y}>-1 / 2) \&(\mathrm{y}<=1) \&(\mathrm{x}>(-\operatorname{sqrt}(3) / 2)) \&(\mathrm{x}<=0) \&((\operatorname{sqrt}(3) * \mathrm{x}-\mathrm{y}+1)<0))=\mathrm{NaN}$;

contour(x,y,PHI,'r-')

xlabel('X-axis');

ylabel('Y-axis');

$\% \operatorname{clabel}(\mathrm{c}, \mathrm{h})$;

axis square

st1='Contour level curves for ';

st2='FEM solution of ';

st3='Nine Noded ';

st4='Special Quadrilateral';

st5=' Elements'

title([st1,st2,st3,st4,st5])

sst $1=$ ' NODES $=$ '

sst $2=$ num $2 \operatorname{str}($ nnode $)$

sst3=' ELEMENTS=' 
sst4=num2str(nel)

$\operatorname{text}(0.6,1.1,[\mathrm{sst} 1 \mathrm{sst} 2])$

$\operatorname{text}(0.6, .9,[\mathrm{sst} 3 \mathrm{sst} 4])$

hold on

$\%[\mathrm{x}, \mathrm{y}]=\operatorname{meshgrid}(0: .1: 1,0: 0.1: 1)$

$\%[\mathrm{c}, \mathrm{h}]=$ contour $\left(\mathrm{x}, \mathrm{y}, \mathrm{z}, \mathrm{g}^{-}{ }^{\prime}\right)$

contour(x,y,z,'b-')

\%xlabel('X-axis');

\%ylabel('Y-axis');

$\%$ clabel(c,h);

axis square

text $\left(0.6,1.9,{ }^{\prime}\{\right.$ SUPERPOSITION OF $\left.\} '\right)$

text $(0.6,1.7, '\{$ FEM/EXACT SOLUTIONS $\}$ ')

$\operatorname{text}\left(0.6,1.5\right.$, ,--(red)FEM ') $^{-}$

text(0.6,1.3,'--(blue)EXACT')

$\mathrm{mm}=0$;

for $\mathrm{i}=1: 31$

for $j=1: 31$

$\mathrm{mm}=\mathrm{mm}+1$;

femsoln $(\mathrm{mm}, 1)=\mathrm{PHI}(\mathrm{i}, \mathrm{j})$;

$\operatorname{exactsoln}(\mathrm{mm}, 1)=\mathrm{z}(\mathrm{i}, \mathrm{j})$;

end

end

end

$\%$ [femsoln exactsoln]

format long

$\operatorname{disp}('-$

disp('number of nodes,elements \& nodes per element')

$\operatorname{disp}([$ nnode nel nnel ])

disp('torisonal constants(fem=phi\&exact=xi) error(max(abs(phi_xi))')

$\% \operatorname{disp}('-$

$\%$ disp([nnode nel nnel ])

disp([T k1 MAXPHI_XI ])

$\operatorname{disp}('-$

[ 6]D2LaplaceEquationQ12Ex3automeshgenNewPolygonContour.m

function[]=D2LaplaceEquationQ12Ex3automeshgenNewPolygonContour(n1,n2,n3,nmax,numtri,ndiv,mesh)

$\%$ ndiv $=2,4,6,8, \ldots \ldots$.

\%polygonal_domain_coordinates([1;1;1;1;1;1;1],[2;3;4;5;6;7;8],[3;4;5;6;7;8;2],8,1,2)

\%polygonal_domain_coordinates([1;1;1;1;1;1;1],[2;3;4;5;6;7;8],[3;4;5;6;7;8;2],8,4,4)

\%D2LaplaceEquationQ4MoinExautomeshgen(n1,n2,n3,nmax,numtri,ndiv)

\%D2LaplaceEquationQ4MoinExautomeshgen([1;1;1;1;1;1;1],[2;3;4;5;6;7;8],[3;4;5;6;7;8;2],8,1,2,1)

\%D2LaplaceEquationQ4MoinExautomeshgen([1;1;1;1;1;1;1],[2;3;4;5;6;7;8],[3;4;5;6;7;8;2],8,4,4,1)

\%D2LaplaceEquationQ4MoinExautomeshgen $([1 ; 1 ; 1 ; 1 ; 1 ; 1 ; 1 ; 1],[2 ; 3 ; 4 ; 5 ; 6 ; 7 ; 8 ; 9],[3 ; 4 ; 5 ; 6 ; 7 ; 8 ; 9 ; 2], 9,1,2,2)$

\%D2LaplaceEquationQ4MoinExautomeshgen([1;1;1;1;1;1;1;1],[2;3;4;5;6;7;8;9],[3;4;5;6;7;8;9;2],9,4,4,2)

\%quadrilateral_mesh4MOINEX_q4(n1,n2,n3,nmax,numtri,ndiv,mesh,xlength,ylength) $([1 ; 1 ; 1 ; 1 ; 1 ; 1 ; 1 ; 1],[2 ; 3 ; 4 ; 5 ; 6 ; 7 ; 8 ; 9],[3 ; 4 ; 5 ; 6$ $; 7 ; 8 ; 9 ; 2], 9,1,2,2,1,1)$

\%D2POISSONEQUATION_NODALINTERPOLATION_VALUES(n1,n2,n3,nmax,numtri,ndiv,mesh)([1;1;1;1;1;1;1;1],[2;3;4;5 ;6;7;8;9],[3;4;5;6;7;8;9;2],9,1,2,2)

\%D2LaplaceEquationQ4MoinExautomeshgen([1;1;1;1;1;1;1;1],[2;3;4;5;6;7;8;9],[3;4;5;6;7;8;9;2],9,100,20,2)

\%D2PoissonEquationQ4MoinEx_MeshgridContour([1;1;1;1;1;1;1;1],[2;3;4;5;6;7;8;9],[3;4;5;6;7;8;9;2],9,1,2,2)

$\%$ D2PoissonEquationQ4MoinEx_MeshgridContour([1;1;1;1;1;1;1],[2;3;4;5;6;7;8],[3;4;5;6;7;8;2],8,1,2,1)

\%D2PoissonEquationQ4MoinEx_MeshgridContour([1;1;1;1;1;1;1],[2;3;4;5;6;7;8],[3;4;5;6;7;8;2],8,4,4,1)

\%D2PoissonEquationQ4MoinEx_MeshgridContour([1;1;1;1;1;1;1],[2;3;4;5;6;7;8],[3;4;5;6;7;8;2],8,9,6,1)

\%D2PoissonEquationQ4MoinEx_MeshgridContour([1;1;1;1;1;1;1],[2;3;4;5;6;7;8],[3;4;5;6;7;8;2],8,16,8,1)

\%D2PoissonEquationQ4MoinEx_MeshgridContour([1;1;1;1;1;1;1],[2;3;4;5;6;7;8],[3;4;5;6;7;8;2],8,25,10,1)

\%D2PoissonEquationQ8MoinEx_MeshgridContour([1;1;1;1;1;1;1;1],[2;3;4;5;6;7;8;9],[3;4;5;6;7;8;9;2],9,1,2,2)

\%D2PoissonEquationQ8MoinEx_MeshgridContour([1;1;1;1;1;1;1],[2;3;4;5;6;7;8],[3;4;5;6;7;8;2],8,1,2,1)

\%D2PoissonEquationQ9MoinEx_MeshgridContour([1;1;1;1;1;1;1],[2;3;4;5;6;7;8],[3;4;5;6;7;8;2],8,1,2,1)

\%D2PoissonEquationQ9MoinEx_MeshgridContour([1;1;1;1;1;1;1;1],[2;3;4;5;6;7;8;9],[3;4;5;6;7;8;9;2],9,1,2,2)

\%D2PoissonEquationQ9MoinEx_MeshgridContour([1;1;1;1;1;1;1],[2;3;4;5;6;7;8],[3;4;5;6;7;8;2],8,1,2,1)

\%\%D2PoissonEquationQ9MoinEx_MeshgridContour([1;1;1;1;1;1;1],[2;3;4;5;6;7;8],[3;4;5;6;7;8;2],8,1,2,1,1,10)

\%D2PoissonEquationQ9MoinEx_MeshgridContour([1;1;1;1;1;1;1;1],[2;3;4;5;6;7;8;9],[3;4;5;6;7;8;9;2],9,1,2,2,1,10)

\%D2LaplaceEquationQ9Ex3automeshgenNewPolygon( $([1 ; 1 ; 1 ; 1 ; 1 ; 1 ; 1 ; 1],[2 ; 3 ; 4 ; 5 ; 6 ; 7 ; 8 ; 9],[3 ; 4 ; 5 ; 6 ; 7 ; 8 ; 9 ; 2], 9,1,2,4)$ 
$\%$ [coord,gcoord,nodes,nodetel,nnode,nel]=polygonal_domain_coordinates(n1,n2,n3,nmax,numtri,ndiv,mesh)

$\%$ nnel=4;

[coord,gcoord,nodes,nodetel,nnode,nel]=polygonal_domain_coordinates_3rd_order(n1,n2,n3,nmax,numtri,ndiv,mesh)

nnel $=12$;

ndof $=1$;

$\%$ nc $=(\text { ndiv } / 2)^{\wedge} 2$;

$\%$ nnode $=($ ndiv +1$) *(\operatorname{ndiv}+2) / 2+n c$;

$\%$ nel= $3 *$ nc;

sdof $=$ nnode $*$ ndof;

$\mathrm{ff}=($ zeros $($ sdof, 1$)) ; \mathrm{ss}=($ zeros $($ sdof,sdof $))$;

$\%$ nnode $=17$, nel $=12$,nnel $=4$, ndof $=1$

$\%>>$ LaplaceEquationQuad4twodimension $(12,17,4,1)$

$\%$

$\%$ Ex1:nnode $=41$,nel=36, nnel=4,nodf $=1$

$\%>>$ LaplaceEquationQuad4twodimensionEx 1 $(36,41,4,1)$

$\%>>$ improvedLaplaceEquationQuad4twodimensionEx1_explicit(36,41,4,1)

$\%$ Ex2: nnode $=83$, nel $=69$, ,nnel $=4$, nodf $=1$

$\%$ >>improvedLaplace EquationQuad4twodimensionEx2_explicit(69,83,4,1)\#

$\%$ >>improvedLaplaceEquationQuad4twodimensionEx2_explicitfnmesh(69,83,4,1)\#

\%improvedLaplaceEquationQuad4twodimensionEx2_explicitvfnmesh $(72,87,4,1)$ \#new

\%improvedLaplaceEquationQuad4twodimensionEx3_explicitmesh(nel=3,nnode=7,nnel=4,ndof=1)

\%improvedLaplaceEquationQuad4twodimensionEx3_explicitmesh(nel,nnode,nnel=4,ndof=1,quadtype=0/3,mesh=1,2,3...)

\%improvedLaplaceEquationQuad4twodimensionEx3_explicitmesh(nel=12,nnode=19,nnel=4,ndof=1, quadtype=0/3, mesh=3)

\%improvedLaplaceEquationQuad4twodimensionEx3_explicitmesh(nel=27,nnode $=37$, nnel $=4$, ndof $=1$, quadtype $=0 / 3$, mesh $=4$ )

\%improvedLaplaceEquationQuad4twodimensionEx3_explicitmesh(nel=48,nnode=61,nnel=4,ndof=1,quadtype=0/3, mesh=5)

\%improvedLaplaceEquationQuad4twodimensionEx3_explicitmesh(nel=75,nnode=91,nnel=4,ndof=1,quadtype=0/3,mesh=6)

\%improvedLaplaceEquationQuad4twodimensionEx3_explicitmesh $(108,127,4,1,3,7)$

\%improvedLaplaceEquationQuad4twodimensionEx3_explicitmesh $(147,169,4,1,3,8)$

\%improvedLaplaceEquationQuad4twodimensionEx3_explicitmesh(192,217,4,1,3,9)

\%improvedLaplaceEquationQuad4twodimensionEx3_explicitmesh $(243,271,4,1,3,10)$

$\operatorname{disp}([$ nel nnode nnel ndof])

format long $g$

for $i=1:$ nel

$\mathrm{N}(\mathrm{i}, 1)=\mathrm{i}$;

end

for $i=1:$ nel

$\mathrm{NN}(\mathrm{i}, 1)=\mathrm{i}$;

end

$\%[$ coord,gcoord] $=$ coordinate_rtisoscelestriangle00_h0_hh(ndiv);

$\%[$ nodetel,nodes]=nodaladdresses4special_convex_quadrilaterals(ndiv)

$\%$

$\%$ bcdof $=[2 ; 5 ; 3]$

\%boundary conditions-1

switch mesh

case 4

\%boundary conditions-2

nnn $=0$;

for $n n=1$ :nnode

$\mathrm{xnn}=\operatorname{coord}(\mathrm{nn}, 1) ; \mathrm{ynn}=\operatorname{gcoord}(\mathrm{nn}, 2)$;

if $(\mathrm{xnn}==-1 / 2) \&((\mathrm{ynn}>=-1 / 2) \&(\mathrm{ynn}<=1 / 2))$

$\mathrm{nnn}=\mathrm{nnn}+1$;

$\operatorname{bcdof}(\mathrm{nnn}, 1)=\mathrm{nn}$;

$\operatorname{bcval}(\mathrm{nnn}, 1)=0$;

end

end

\%boundary conditions-2

for $\mathrm{nn}=1$ :nnode

$\mathrm{xnn}=\operatorname{gcoord}(\mathrm{nn}, 1) ; \mathrm{ynn}=\operatorname{coord}(\mathrm{nn}, 2)$; 


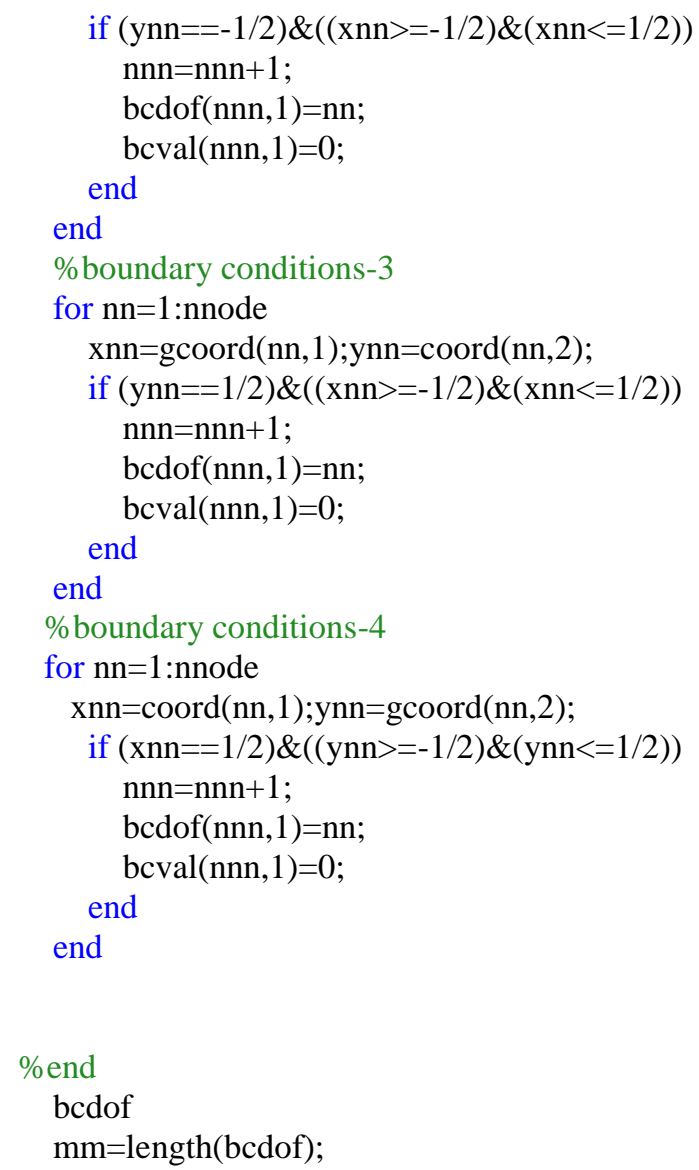


$\mathrm{X}=\mathrm{xx}(\mathrm{iel}, 1: 3)$;

$\mathrm{Y}=\mathrm{yy}(\mathrm{iel}, 1: 3)$;

$\% \operatorname{disp}([\mathrm{X} \mathrm{Y}])$

$\mathrm{xa}=\mathrm{X}(1,1)$;

$\mathrm{xb}=\mathrm{X}(1,2)$

$\mathrm{xc}=\mathrm{X}(1,3)$;

ya $=Y(1,1)$

$\mathrm{yb}=\mathrm{Y}(1,2)$

$\mathrm{yc}=\mathrm{Y}(1,3)$;

bta $=y b-y c ; b t b=y c-y a ;$

gma $=x c-x b ; g m b=x a-x c$;

delabc $=$ gmb $*$ bta-gma*btb;

$\mathrm{G}=$ [bta btb;gma gmb]/delabc;

$\mathrm{GT}=[$ bta gma;btb gmb]/delabc;

$\mathrm{Q}=\mathrm{GT} * \mathrm{G}$;

$\operatorname{sk}(1: 12,1: 12)=(\operatorname{zeros}(12,12))$;

for $\mathrm{i}=1: 12$

for $\mathrm{j}=\mathrm{i}: 12$

$\operatorname{sk}(\mathrm{i}, \mathrm{j})=(\operatorname{delabc} * \operatorname{sum}(\operatorname{sum}(\mathrm{Q} . *(\operatorname{int} J \operatorname{dndn}(2 * \mathrm{i}-1: 2 * \mathrm{i}, 2 * \mathrm{j}-1: 2 * \mathrm{j})))))$;

$\operatorname{sk}(\mathrm{j}, \mathrm{i})=\operatorname{sk}(\mathrm{i}, \mathrm{j})$;

end

end

$\% \mathrm{f}=[5 / 144 ; 1 / 24 ; 7 / 144 ; 1 / 24] *(2 *$ delabc $)$;

$\% \mathrm{f}=[-7 / 432 ;-1 / 72 ;-5 / 432 ;-1 / 72 ; 11 / 216 ; 13 / 216 ; 13 / 216 ; 11 / 216] *(2 *$ delabc $)$

$\mathrm{f}=[-7 / 360 ;-1 / 48 ;-1 / 45 ;-1 / 48 ; 23 / 960 ; 1 / 30 ; 7 / 240 ; 37 / 960 ; 37 / 960 ; 7 / 240 ; 1 / 30 ; 23 / 960] *(2 *$ delabc $)$

$\% \mathrm{f}=[19 / 11520 ; 1 / 384 ; 41 / 11520 ; 1 / 384 ; 1 / 192 ; 29 / 3840 ; 31 / 3840 ; 1 / 96 ; 1 / 96 ; 31 / 3840 ; 29 / 3840 ; 1 / 192 ; 21 / 1280 ; 3 / 128 ; 39 / 1280 ; 3 / 128] *($

$2 *$ delabc);

$\%$

edof=nnel*ndof;

$\mathrm{k}=0$;

for $\mathrm{i}=1$ :nnel

$\operatorname{nd}(\mathrm{i}, 1)=\operatorname{nodes}(\mathrm{iel}, \mathrm{i})$;

start $=($ nd $(\mathrm{i}, 1)-1) *$ ndof;

for $\mathrm{j}=1$ :ndof

$\mathrm{k}=\mathrm{k}+1$;

index $(\mathrm{k}, 1)=\mathrm{start}+\mathrm{j}$;

end

end

for $i=1$ :edof

$\mathrm{ii}=\operatorname{index}(\mathrm{i}, 1)$;

$\mathrm{ff}(\mathrm{ii}, 1)=\mathrm{ff}(\mathrm{ii}, 1)+\mathrm{f}(\mathrm{i}, 1)$;

for $\mathrm{j}=1$ :edof

$\mathrm{jj}=\mathrm{index}(\mathrm{j}, 1)$;

$s s(i i, j j)=s s(i i, j j)+s k(i, j)$;

end

end

end \% for iel

\%bcdof $=[13 ; 37 ; 35 ; 33 ; 31 ; 29 ; 27 ; 25 ; 23 ; 21 ; 19 ; 17 ; 15]$;

for $\mathrm{ii}=1: \mathrm{mm}$

$\mathrm{kk}=\mathrm{bcdof}(\mathrm{ii}, 1)$;

ss $(\mathrm{kk}, 1:$ nnode $)=$ zeros $(1$, nnode $)$;

ss $(1:$ nnode, $\mathrm{kk})=$ zeros $($ nnode, 1$)$;

$\mathrm{ff}(\mathrm{kk}, 1)=0$;

end

for $\mathrm{ii}=1: \mathrm{mm}$

kk=bcdof(ii,1);

$\mathrm{ss}(\mathrm{kk}, \mathrm{kk})=1$;

end

phi=ss $\backslash f f ;$ 
phi=double(phi);

[phi xi]

for $\mathrm{I}=1$ :nnode

$\mathrm{NN}(\mathrm{I}, 1)=\mathrm{I}$;

phi_xi(I,1)=phi(I,1)-xi(I,1);

end

MAXPHI_XI=max(abs(phi_xi));

$\operatorname{disp}('$

disp('number of nodes,elements \& nodes per element')

[nnode nel nnel ndof]

$\operatorname{disp}('$

$\operatorname{disp}('$

fem-computed values

anlytical(theoretical)-values

$\operatorname{disp}([\mathrm{NN}$ phi xi] $)$

$\operatorname{disp}\left(^{\prime}\right.$

disp('number of nodes, elements \& nodes per element')

[nnode nel nnel ndof]

nodes

gcoord

\% ***********************************************************

$\operatorname{disp}([\mathrm{NN}$ phi xi] $)$

$\mathrm{t}=0$;

for iii=1:nnode

$\mathrm{t}=\mathrm{t}+$ phi(iii, 1$) * \mathrm{ff}(\mathrm{iii}, 1)$;

end

$\mathrm{T}=\mathrm{t}$;

$\operatorname{disp}('-$

disp('number of nodes,elements \& nodes per element')

disp([nnode nel nnel ])

disp('torisonal constants $\left(\right.$ fem=phi\&exact=xi) error $\left(\max (\operatorname{abs}(\text { phi_xi }))^{\prime}\right)$

$\%$ disp (

$\%$ disp([nnode nel nnel ])

disp([T k1 MAXPHI_XI ])

$\operatorname{disp}('-$

$\%$ end

$\operatorname{disp}('$

disp('number of nodes,elements \& nodes per element')

[nnode nel nnel ndof]

\% $* * * * * * * * * * * * * * * * * * * * * * * * * * * * * * * * * * * * * * * * * * * *$

$[\mathrm{x}, \mathrm{y}]=$ meshgrid( $(-1 / 2: 0.05: 1 / 2,-1 / 2: 0.05: 1 / 2)$;

$\mathrm{a} 0=8 / \mathrm{pi}^{\wedge} 3$;

for $\mathrm{i}=1: 21$

for $\mathrm{j}=1: 21$

for iel=1:nel

$\mathrm{XX}=\mathrm{xx}($ iel, $1: 3)$

YY=yy(iel,1:3);

$\% \operatorname{disp}([\mathrm{X} Y])$

$\mathrm{xa}=\mathrm{XX}(1,1)$;

$\mathrm{xb}=\mathrm{XX}(1,2)$;

$\mathrm{xc}=\mathrm{XX}(1,3)$;

ya $=Y Y(1,1)$;

$\mathrm{yb}=\mathrm{YY}(1,2)$;

$\mathrm{yc}=\mathrm{YY}(1,3)$;

$\mathrm{aLPa}=x b^{*} \mathrm{yc}-\mathrm{xc} * \mathrm{yb}$;

$\mathrm{aLPb}=\mathrm{xc} * \mathrm{ya}-\mathrm{xa}^{*} \mathrm{yc}$;

bta=yb-yc;btb=yc-ya;

gma $=x c-x b ; g m b=x a-x c$; 


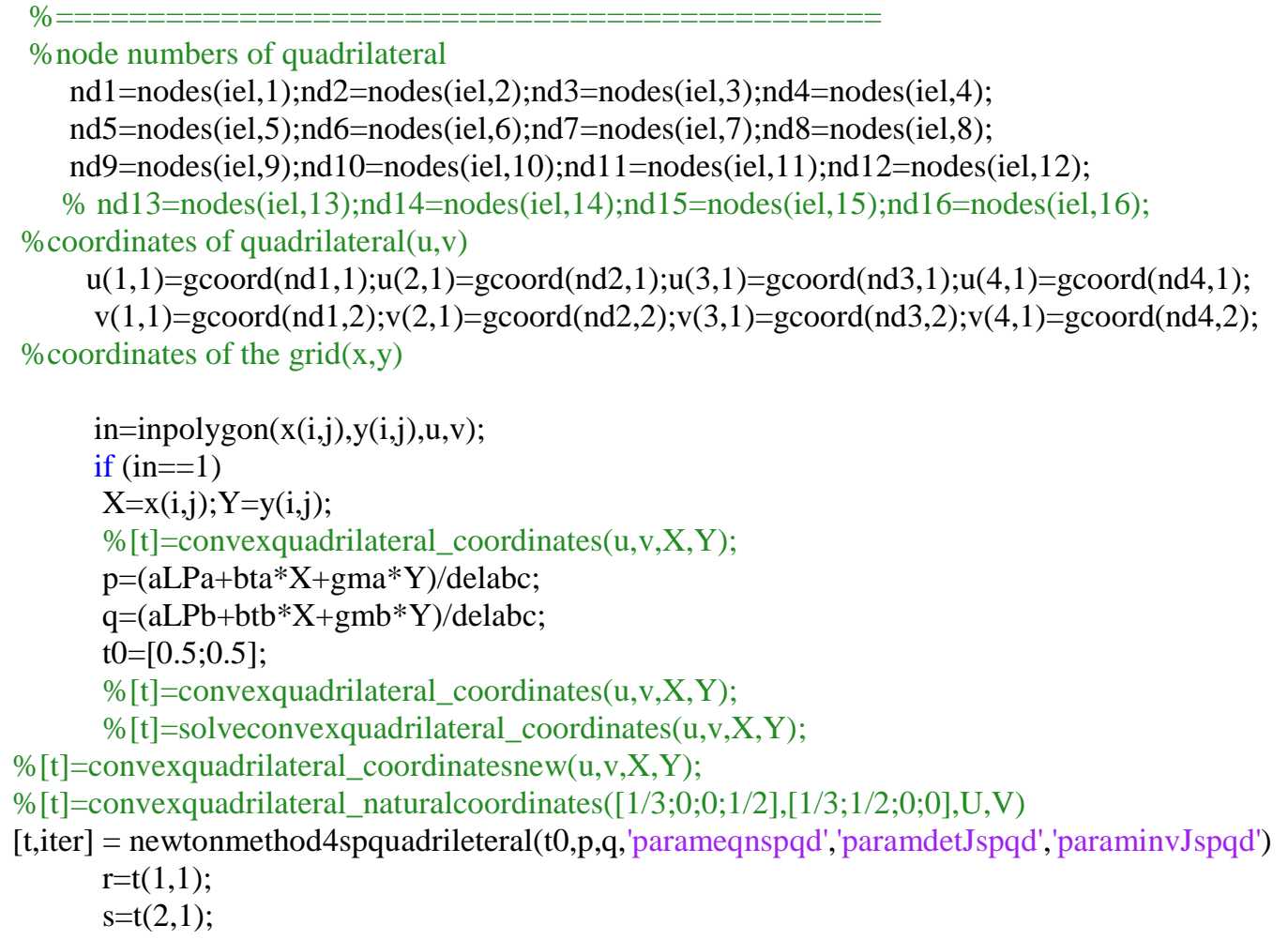

PHI $(\mathrm{i}, \mathrm{j})=\operatorname{shn} 1 * \operatorname{phi}(\mathrm{nd} 1,1)+\operatorname{shn} 2 * \operatorname{phi}(\mathrm{nd} 2,1)+\operatorname{shn} 3 * \operatorname{phi}(\mathrm{nd} 3,1)+\operatorname{shn} 4 * \operatorname{phi}(\mathrm{nd} 4,1)+\operatorname{shn} 5 *$ phi(nd5,1)+shn6*phi(nd6,1)+shn7*phi(nd7, 1)+shn $8 * \operatorname{phi}($ nd 8,1$)+\operatorname{shn} 9 * \operatorname{phi}($ nd 9,1$)+\operatorname{shn} 10 * \operatorname{phi}($ nd10,1)+shn $11 * \operatorname{phi}($ nd11,1) $+\operatorname{shn} 12 * \operatorname{phi}(\operatorname{nd} 12,1)$;

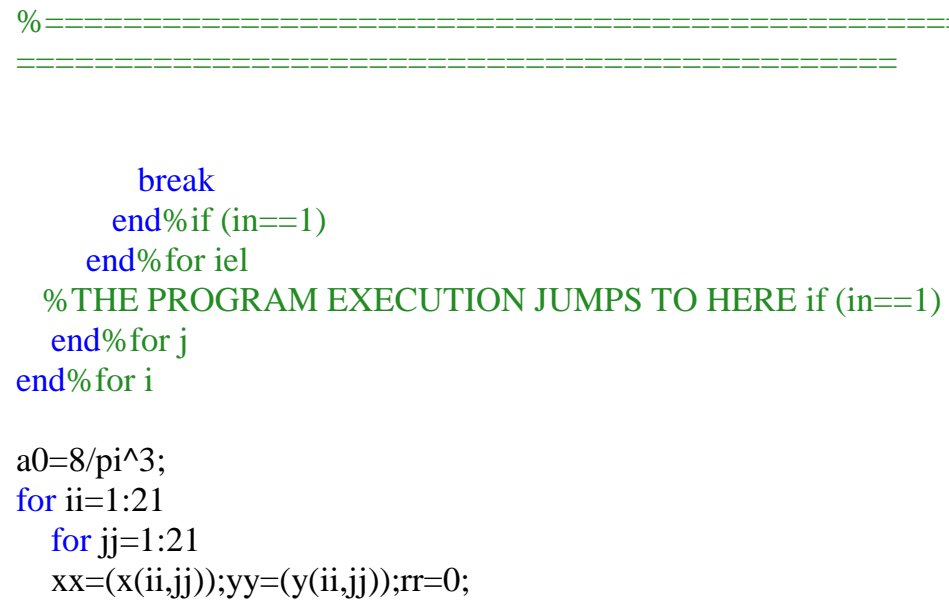




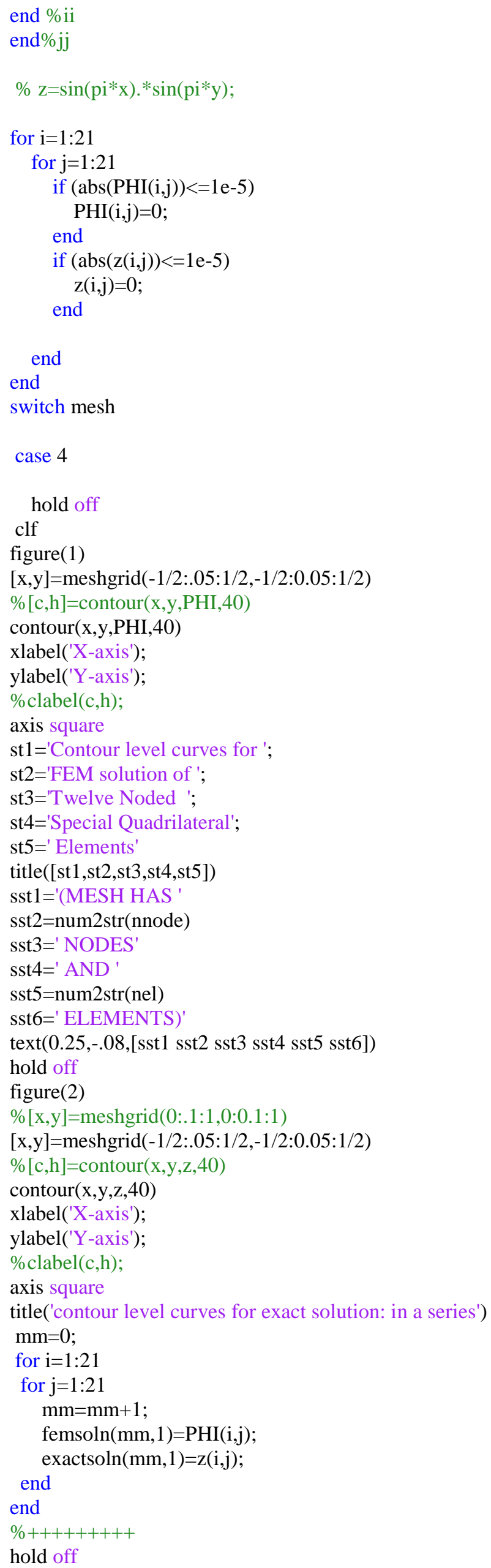


figure(3)

$[\mathrm{x}, \mathrm{y}]=$ meshgrid( $(-1 / 2: .05: 1 / 2,-1 / 2: 0.05: 1 / 2)$

$\%[\mathrm{x}, \mathrm{y}]=$ meshgrid(-sqrt(2)/2:(0.1)*sqrt(2)/2: $\operatorname{sqrt}(2) / 2,-\operatorname{sqrt}(2) / 2:(0.1) * \operatorname{sqrt}(2) / 2: \operatorname{sqrt}(2) / 2)$;

$\%[\mathrm{c}, \mathrm{h}]=$ contour $\left(\mathrm{x}, \mathrm{y}, \mathrm{PHI}, \mathrm{r}^{-}\right)$

contour(x,y,PHI,40,'r-')

xlabel('X-axis');

ylabel('Y-axis');

\%clabel(c,h);

axis square

st1='Contour level curves for ';

st2='FEM(red)\&exact(green) ';

st3='Twelve Noded ';

st4='Special Quadrilateral';

st5=' Elements'

title([st1,st2,st3,st4,st5])

sst $1=$ '(MESH HAS '

sst2 $=$ num $2 \operatorname{str}($ nnode $)$

sst3 =' NODES'

sst4=' AND

sst5=num2str(nel)

sst6=' ELEMENTS)'

text(-1/2,-1/2,[sst1 sst2 sst3 sst4 sst5 sst6])

hold on

$\%[\mathrm{x}, \mathrm{y}]=\operatorname{meshgrid}(0: 1: 1,0: 0.1: 1)$

$\%[\mathrm{c}, \mathrm{h}]=$ contour $\left(\mathrm{x}, \mathrm{y}, \mathrm{z}, \mathrm{g}_{-}{ }^{\prime}\right)$

contour(x,y,z,40,'g-')

\%xlabel('X-axis');

\%ylabel('Y-axis');

$\%$ clabel(c,h);

axis square

end \%switch mesh

$\%$ [femsoln exactsoln]

disp('number of nodes,elements \& nodes per element')

[nnode nel nnel ndof]

$[1 \operatorname{phi}(1,1) \times \mathrm{xi}(1,1)]$

$\operatorname{disp}('$

disp('number of nodes,elements \& nodes per element')

[nnode nel nnel ndof]

$\operatorname{disp}('$

$\operatorname{disp}('$

fem-computed values

anlytical(theoretical)-values

')

$\operatorname{disp}([\mathrm{NN}$ phi xi] $)$

$\operatorname{disp}$ (' $^{-}$

disp('number of nodes,elements \& nodes per element')

[nnode nel nnel ndof]

if $\operatorname{mesh}==4$

$\%$ [phi xi]

for $\mathrm{I}=1$ :nnode

$\mathrm{NN}(\mathrm{I}, 1)=\mathrm{I}$;

phi_xi(I,1)=phi(I,1)-xi(I,1);

end

MAXPHI_XI=max $(\operatorname{abs}($ double $($ phi_xi $)))$;

format long e

$\mathrm{t}=0$;

for $\mathrm{iii}=1$ :nnode

$\mathrm{t}=\mathrm{t}+$ phi( $(\mathrm{iii}, 1) * \mathrm{ff}(\mathrm{iii}, 1)$;

end

$\mathrm{T}=\mathrm{t}$;

$\operatorname{disp}('-$

disp('number of nodes,elements \& nodes per element') 
$\operatorname{disp}([$ nnode nel nnel ] $)$

disp('torisonal constants $\left(\right.$ fem=phi\&exact=xi) error $\left(\max (\operatorname{abs}(\text { phi_xi }))^{\prime}\right)$

$\%$ disp('--

$\%$ disp([nnode nel nnel ])

$\operatorname{disp}([$ T k1 MAXPHI_XI ] $)$

$\operatorname{disp}($

end

$\operatorname{disp}('$

disp('number of nodes, elements \& nodes per element')

[nnode nel nnel ndof]

for $\mathrm{ncpt}=1$ :nel

c1=nodes(ncpt,1);c2=nodes(ncpt,2);c3=nodes(ncpt,3);c4=nodes(ncpt,4);

elcentr $(\mathrm{ncpt}, 1)=\mathrm{ncpt}$;

$\operatorname{phicpt}(\mathrm{ncpt}, 1)=(\operatorname{phi}(\mathrm{c} 1,1)+\operatorname{phi}(\mathrm{c} 2,1)+\operatorname{phi}(\mathrm{c} 3,1)+\operatorname{phi}(\mathrm{c} 4,1)) / 4$;

$\operatorname{xicpt}(\mathrm{ncpt}, 1)=(\mathrm{xi}(\mathrm{c} 1,1)+\mathrm{xi}(\mathrm{c} 2,1)+\mathrm{xi}(\mathrm{c} 3,1)+\mathrm{xi}(\mathrm{c} 4,1)) / 4$;

end

for $\mathrm{I}=1$ :nel

elnumm $(\mathrm{I}, 1)=\mathrm{I}$;

end

$\operatorname{disp}('$

disp(' serial no center point

fem-computed values

anlytical(theoretical)-values

')

disp([elnumm elcentr phicpt xicpt])

$\operatorname{disp}('$

format compact

$\%====$

$\operatorname{disp}('$ ')

disp('number of nodes,elements \& nodes per element')

[nnode nel nnel ndof]

$\operatorname{disp}('$

disp(' ELM FEM SOLUTION EXACT SOLUTION ELM FEM SOLUTION EXACT SOLUTION ELCENTNODE FEM SOLUTION EXACT SOLUTION ')

$\operatorname{disp}('-$

for $\mathrm{I}=1: 3$ :nel

$\% \mathrm{~A}=[\operatorname{elcentr}(\mathrm{I}) \operatorname{phicpt}(\mathrm{I}) \operatorname{xicpt}(\mathrm{I})] ; \mathrm{B}=[\operatorname{elcentr}(\mathrm{I}+1) \operatorname{phicpt}(\mathrm{I}+1) \operatorname{xicpt}(\mathrm{I}+1)] ; \mathrm{C}=[\operatorname{elcentr}(\mathrm{I}+2) \operatorname{phicpt}(\mathrm{I}+2) \operatorname{xicpt}(\mathrm{I}+2)]$;

$\% \operatorname{disp}([\operatorname{elcentr}(\mathrm{I}) \operatorname{phicpt}(\mathrm{I}) \operatorname{xicpt}(\mathrm{I}) \operatorname{elcentr}(\mathrm{I}+1) \operatorname{phicpt}(\mathrm{I}+1) \operatorname{xicpt}(\mathrm{I}+1)])$

$\% \operatorname{disp}([$ elcentr($(\mathrm{I}+2) \operatorname{phicpt}(\mathrm{I}+2) \operatorname{xicpt}(\mathrm{I}+2)])$

$\% \operatorname{disp}([\mathrm{A} \mathrm{B} \mathrm{C}])$

fprintf('In\%5d \%18.14f \%18.14f \%5d \%18.14f \%18.14f \%5d \%18.14f \% 18.14f',elcentr(I), phicpt(I), xicpt(I), elcentr(I+1), phicpt(I+1), xicpt(I+1), elcentr(I+2), phicpt(I+2), xicpt(I+2));

end

fprintf('ไn')

$\operatorname{disp}(-$

[7]D2LaplaceEquationQ16Ex3automeshgenNewContour.m

function[]=D2LaplaceEquationQ16Ex3automeshgenNewContour(n1,n2,n3,numtri,ndiv,mesh)

\%function[]=improvedLaplaceEquationQuad8twodimensionEx3_explicitvfnmesh(nel,nnode,nnel,ndof,quadtype,mesh)

$\%$ note that input vlues of $\mathrm{X}$ and $\mathrm{Y}$ must be symbolic constants

$\%$ for the example triangle input for $\mathrm{X}$ is $\operatorname{sym}([-1 / 21 / 20])$

$\%$ for the example triangle input for $\mathrm{Y}$ is $\operatorname{sym}([00 \mathrm{sqrt}(3 / 4)])$

\%LaplaceEquationQ4twoD(3,sym([-1/2 1/2 0 $]), \operatorname{sym}\left(\left[\begin{array}{lll}0 & 0 & \operatorname{sqrt}(3 / 4)]))\end{array}\right.\right.$

\%syms ff ss f sk N NN table1 table2

\%D2LaplaceEquationQ16Ex3automeshgenNew $(1,2,3,1,2,1)$

$\%$ D2LaplaceEquationQ16Ex3automeshgenNew $(1,2,3,1,2,2)$

$\%$ D2LaplaceEquationQ16Ex3automeshgenNewContour $(1,2,3,1,2,1)$

$\%$ D2LaplaceEquationQ16Ex3automeshgenNewContour(1,2,3,1,2,2)

syms coord

ndof $=1$;

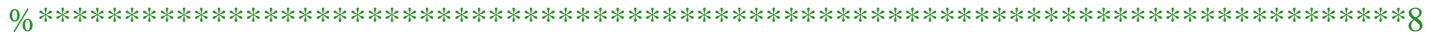

\% $* * * * * * * * * * * * * * * * * * * * * * * * * * * * * * * * * * * * * * * * * * * * * * * * * * * * * * * * * * * * * * * * * * * * * *$ 
syms coord

syms x y

ndof $=1$;

switch mesh

case 1

$\mathrm{x}=\operatorname{sym}([0 ; 1 / 2 ; 1 / 2])$

$\mathrm{y}=\operatorname{sym}([0 ; 0 ; 1 / 2])$

case $2 \%$ an equilateral triangle,each side $=2 * \operatorname{sqrt}(3)$

$\mathrm{x}=\operatorname{sym}([-\operatorname{sqrt}(3) ; \operatorname{sqrt}(3) ; 0])$

$\mathrm{y}=\operatorname{sym}([-1 ;-1 ; 2])$

end

syms ui vi wi xi yi

$\%[u i, v i, w i]=$ coordinate_special_quadrilaterals_in_stdtriangle_2nd_order(ndiv);

$\%[$ ui,vi,wi]=coordinate_special_quadrilaterals_in_stdtriangle_2nd_orderLAGR(ndiv)

[ui,vi,wi]=coordinate_special_quadrilaterals_in_stdtriangle_3rd_orderLAGR(ndiv)

$\% \operatorname{disp}([u$ ui vi wi])

$\mathrm{N}=$ length(ui);

$\mathrm{NN}=(1: \mathrm{N})^{\prime}$;

$\mathrm{x}$

$\mathrm{y}$

$\mathrm{x} 1=\mathrm{x}(\mathrm{n} 1,1) ; \mathrm{x} 2=\mathrm{x}(\mathrm{n} 2,1) ; \mathrm{x} 3=\mathrm{x}(\mathrm{n} 3,1) ; \mathrm{y} 1=\mathrm{y}(\mathrm{n} 1,1) ; \mathrm{y} 2=\mathrm{y}(\mathrm{n} 2,1) ; \mathrm{y} 3=\mathrm{y}(\mathrm{n} 3,1) ;$

for $\mathrm{i}=1: \mathrm{N}$

$x x i(i, 1)=x 1+(x 2-x 1) * u i(i, 1)+(x 3-x 1) * v i(i, 1)$;

yyi $(\mathrm{i}, 1)=y 1+(y 2-y 1) * u i(i, 1)+(y 3-y 1) * v i(i, 1)$;

end

$\% \operatorname{disp}('$

$\% \operatorname{disp}(' \mathrm{NN} \quad$ xi yi')

$\% \operatorname{disp}([\mathrm{NN}$ xi yi])

$\% \operatorname{disp}('$

$\% \operatorname{coord}(:, 1)=(\operatorname{xxi}(:, 1))$;

$\% \operatorname{coord}(:, 2)=($ yyi $(:, 1))$;

$\operatorname{gcoord}(:, 1)=\operatorname{double}(\mathrm{xxi}(:, 1))$;

$\operatorname{gcoord}(:, 2)=\operatorname{double}(\operatorname{yyi}(:, 1))$;

$\%$ disp(gcoord);

$\%[$ eln,nodetel,nodes,nnode]=nodaladdresses_special_convex_quadrilaterals_2nd_order(ndiv);

$\%[$ eln,nodetel,nodes,nnode]=nodaladdresses4Lagrangespecial_convex_quadrilaterals_2nd_order(ndiv);

[eln,nodetel,nodes,nnode]=nodaladdresses4Lagrangespecial_convex_quadrilaterals_3rd_order(ndiv);

$\%[$ ui,vi,wi]=coordinate_special_quadrilaterals_in_stdtriangle_3rd_orderLAGR(n)

$\%[$ eln,nodetel,nodes,nnode]=nodaladdresses4Lagrangespecial_convex_quadrilaterals_3rd_order(n)

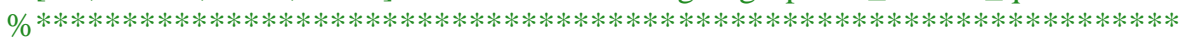

$\%$ syms coord

$\%$ ndof $=1$;

$\%[$ eln,nodetel,nodes,nnode]=nodaladdresses4Lagrangespecial_convex_quadrilaterals_2nd_order(ndiv);

$\%$ [coord,gcoord]=coordinate_rtisoscelestriangle00_h0_hh_2ndorderLAGR(ndiv);

$\%[$ coord,gcoord] $=$ coordinate_rtisoscelestriangle00_h0_hh(ndiv);

$\%[$ nodetel,nodes]=nodaladdresses4special_convex_quadrilaterals(ndiv)

[nel,nnel]=size(nodes);

$\%$ disp([nel nnode nnel ndof])

format long $g$

for $i=1:$ nel

$\mathrm{N}(\mathrm{i}, 1)=\mathrm{i}$;

end

for $i=1$ :nel

$\mathrm{NN}(\mathrm{i}, 1)=\mathrm{i}$;

end 
sdof=nnode $*$ ndof;

$\mathrm{ff}=($ zeros $($ sdof, 1$)) ; \mathrm{ss}=($ zeros $($ sdof,sdof $))$;

format long $g$

for $i=1$ :nel

$\mathrm{N}(\mathrm{i}, 1)=\mathrm{i}$;

end

\%radius of the hole $=1.25 \mathrm{~cm}$

\%input data for nodal coordinate values

$\% \operatorname{gcoord}(\mathrm{i}, \mathrm{j})$, where $\mathrm{i}->$ node no. and $\mathrm{j}->\mathrm{x}$ or $\mathrm{y}$

$\%$

table $1=[\mathrm{N}$ nodes $]$

[nel,nnel]=size(nodes);

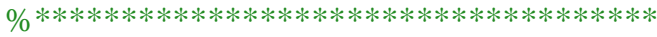

switch mesh

case 1

$\mathrm{nnn}=0$;

for $n n=1$ :nnode

if $\operatorname{gcoord}(\mathrm{nn}, 1)==(1 / 2)$

$\mathrm{nnn}=\mathrm{nnn}+1$;

$\operatorname{bcdof}(\mathrm{nnn}, 1)=\mathrm{nn}$;

end

end

format long $\mathrm{g}$

$\mathrm{k} 1$ =double $(0.14057701495515551037840396020329)$;

$\mathrm{xi}=($ zeros $($ nnode, 1$))$;

$\mathrm{a} 0=8 / \mathrm{pi}^{\wedge} 3$;

for $\mathrm{m}=1$ :nnode

$\mathrm{gx}=(\operatorname{gcoord}(\mathrm{m}, 1)) ; \mathrm{gy}=(\operatorname{gcoord}(\mathrm{m}, 2)) ; \mathrm{rr}=(0) ;$

for $\mathrm{n}=1: 2: 99$

$\mathrm{rr}=\mathrm{rr}+(-1)^{\wedge}((\mathrm{n}-1) / 2)^{*}(1-(\cosh (\mathrm{n} * \mathrm{pi} * \mathrm{gy}) / \cosh (\mathrm{n} * \mathrm{pi} / 2))) * \cos (\mathrm{n} * \mathrm{pi} * \mathrm{gx}) / \mathrm{n}^{\wedge} 3$;

end

$\operatorname{xi}(\mathrm{m}, 1)=(\mathrm{a} 0 * \mathrm{rr})$;

end

mm=length(bcdof);

case $2 \%$ torsion of an equilateral triangle

$\mathrm{nnn}=0$;

\%boundary conditions on side 1

for $n n=1$ :nnode

$\mathrm{xnn}=\operatorname{gcoord}(\mathrm{nn}, 1) ; \mathrm{ynn}=\operatorname{gcoord}(\mathrm{nn}, 2)$;

if $((y n n+1)<1 . e-5)$

$\mathrm{nnn}=\mathrm{nnn}+1$;

$\operatorname{bcdof}(\mathrm{nnn}, 1)=\mathrm{nn}$;

$\operatorname{bcval}(\mathrm{nnn}, 1)=0$;

end

end

\%boundary conditions on side 2

for $n n=1$ :nnode

$\mathrm{xnn}=\mathrm{g} \operatorname{coord}(\mathrm{nn}, 1) ; \mathrm{ynn}=\mathrm{g} \operatorname{coord}(\mathrm{nn}, 2)$;

if $((-(\operatorname{sqrt}(3)) * x n n-y n n+2)<1 . e-5)$

nnn=nnn+1;

$\operatorname{bcdof}(\mathrm{nnn}, 1)=\mathrm{nn}$;

$\operatorname{bcval}(\mathrm{nnn}, 1)=0$;

end

end

\%boundary conditions on side 3

for $\mathrm{nn}=1$ :nnode

$\mathrm{xnn}=\operatorname{gcoord}(\mathrm{nn}, 1) ; \mathrm{ynn}=\operatorname{gcoord}(\mathrm{nn}, 2)$;

if $(((\operatorname{sqrt}(3)) * x n n-y n n+2)<1 . e-5)$

$\mathrm{nnn}=\mathrm{nnn}+1$

$\operatorname{bcdof}(\mathrm{nnn}, 1)=\mathrm{nn}$;

$\operatorname{bcval}(\mathrm{nnn}, 1)=0$; 


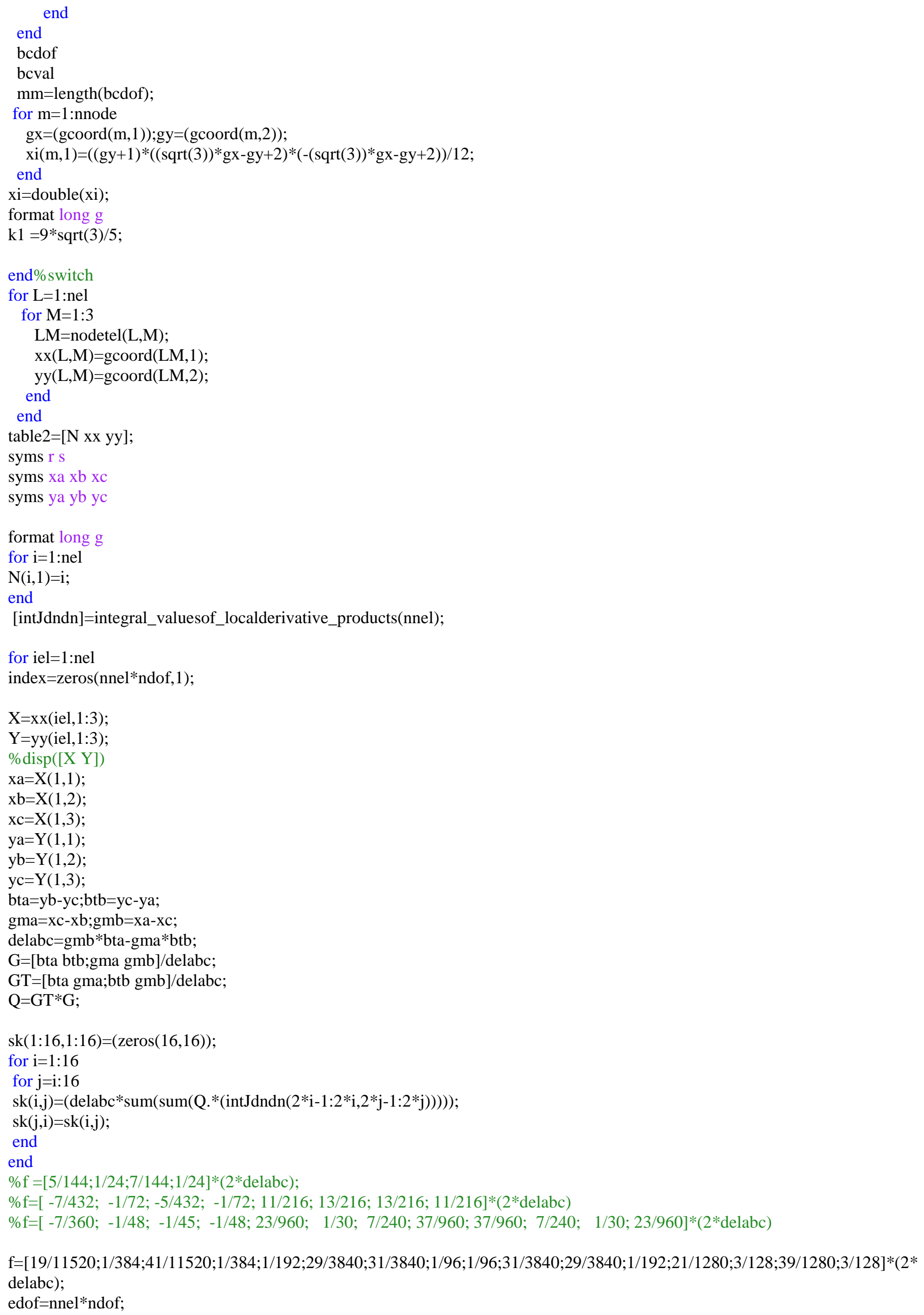




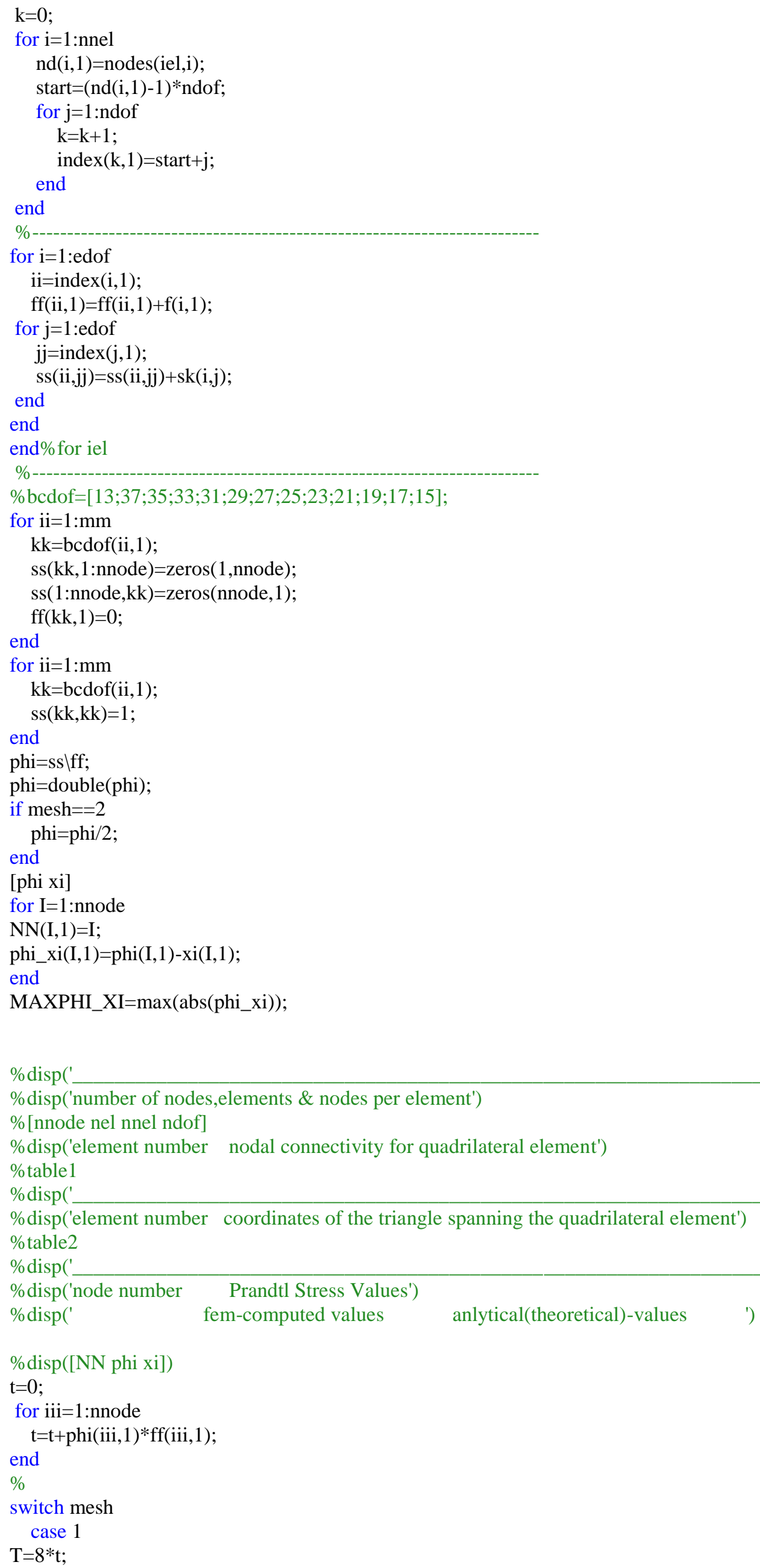


case 2

end

$\% \operatorname{disp}('$

$\% \operatorname{disp}('$

fem-computed

torisonal constant

$\operatorname{disp}('-$

disp([nnode nel nnel ])

$\operatorname{disp}([\mathrm{T}$ k1 MAXPHI_XI ])

$\operatorname{disp}(-$ anlytical(theoretical)-values ')

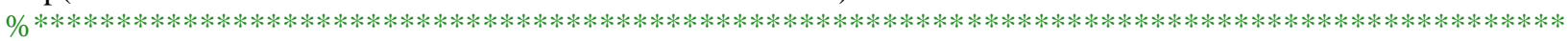

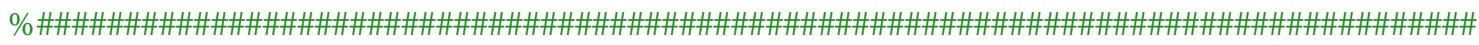

if $(\mathrm{mesh}==2)$

$[\mathrm{x}, \mathrm{y}]=\operatorname{meshgrid}\left(-\operatorname{sqrt}(3):(1 / 15)^{*} \operatorname{sqrt}(3): \operatorname{sqrt}(3),-1:(0.1): 2\right)$;

$\mathrm{z}=(\mathrm{zeros}(31,31))$;

for $i=1: 31$

for $\mathrm{j}=1: 31$

for iel $=1:$ nel

$\%========$
$X X=\operatorname{xx}($ iel, $1: 3)$

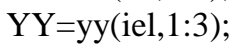

$\% \operatorname{disp}([\mathrm{X} Y])$

$\mathrm{xa}=\mathrm{XX}(1,1)$;

$\mathrm{xb}=\mathrm{XX}(1,2)$;

$\mathrm{xc}=\mathrm{XX}(1,3)$;

ya $=Y Y(1,1)$;

$\mathrm{yb}=\mathrm{YY}(1,2)$;

$\mathrm{yc}=\mathrm{YY}(1,3)$;

$\mathrm{aLPa}=\mathrm{xb} * \mathrm{yc}-\mathrm{xc} * \mathrm{yb}$;

$\mathrm{aLPb}=\mathrm{xc} * \mathrm{ya}-\mathrm{xa} * \mathrm{yc}$;

bta $=y b-y c ; b t b=y c-y a ;$

gma $=x c-x b ; g m b=x a-x c ;$

delabc $=$ gmb $*$ bta-gma*btb;

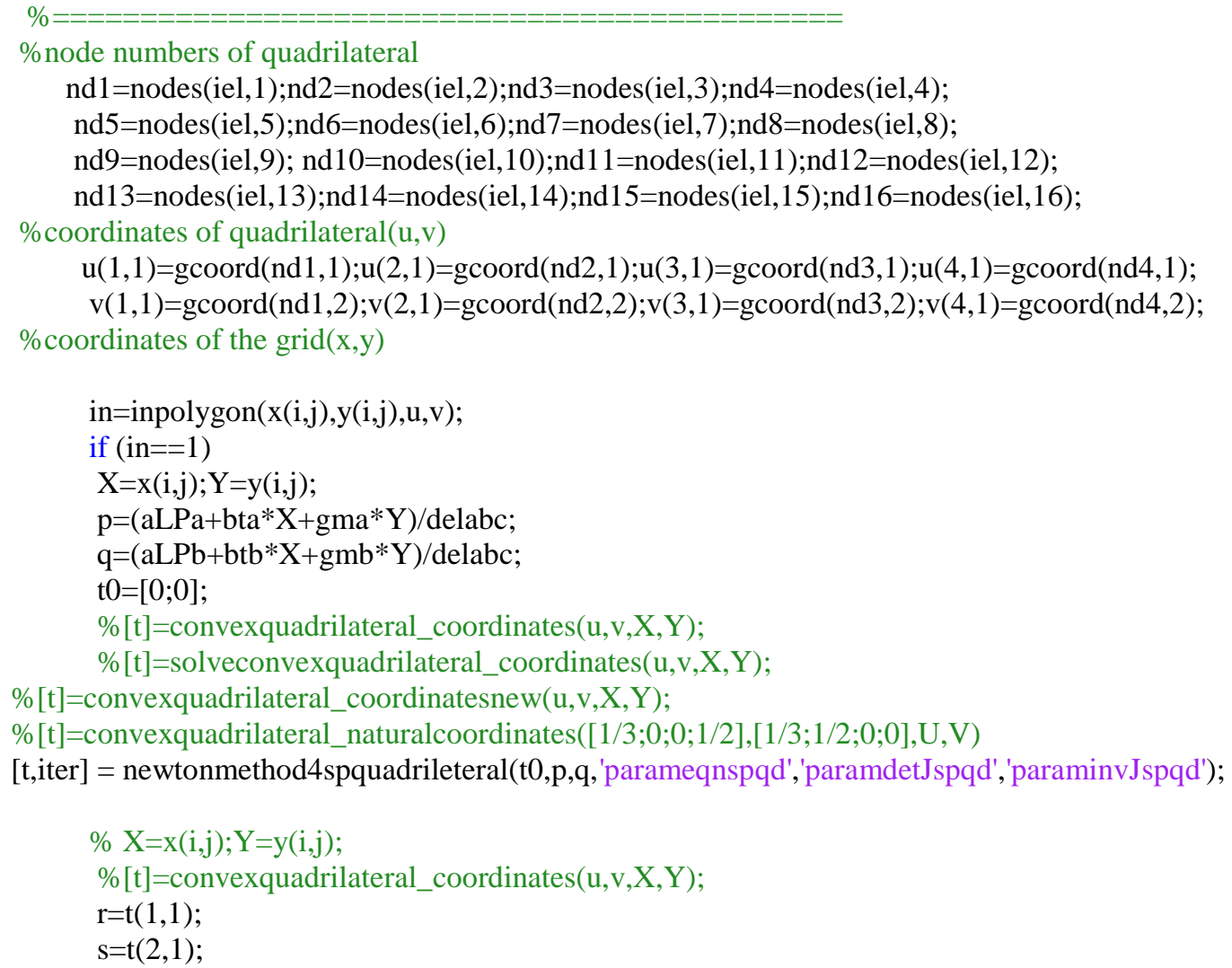

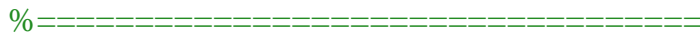

$\mathrm{h} 1 \mathrm{r}=-9 *(\mathrm{r}+1 / 3) *(\mathrm{r}-1 / 3) *(\mathrm{r}-1) / 16$

$\mathrm{h} 2 \mathrm{r}=27 *(\mathrm{r}+1) *(\mathrm{r}-1 / 3) *(\mathrm{r}-1) / 16$ 
$\mathrm{h} 3 \mathrm{r}=-27 *(\mathrm{r}+1) *(\mathrm{r}+1 / 3) *(\mathrm{r}-1) / 16$

$\mathrm{h} 4 \mathrm{r}=9 *(\mathrm{r}+1) *(\mathrm{r}+1 / 3) *(\mathrm{r}-1 / 3) / 16$

$\mathrm{h} 1 \mathrm{~s}=-9 *(\mathrm{~s}+1 / 3) *(\mathrm{~s}-1 / 3) *(\mathrm{~s}-1) / 16$

$\mathrm{h} 2 \mathrm{~s}=27 *(\mathrm{~s}+1) *(\mathrm{~s}-1 / 3) *(\mathrm{~s}-1) / 16$

$\mathrm{h} 3 \mathrm{~s}=-27 *(\mathrm{~s}+1) *(\mathrm{~s}+1 / 3) *(\mathrm{~s}-1) / 16$

$\mathrm{h} 4 \mathrm{~s}=9 *(\mathrm{~s}+1) *(\mathrm{~s}+1 / 3) *(\mathrm{~s}-1 / 3) / 16$

$\%$

$\operatorname{shn} 1=h 1 \mathrm{r} * \mathrm{~h} 1 \mathrm{~s} ; \operatorname{shn} 5=\mathrm{h} 2 \mathrm{r} * \mathrm{~h} 1 \mathrm{~s} ; \mathrm{shn} 6=\mathrm{h} 3 \mathrm{r} * \mathrm{~h} 1 \mathrm{~s} ; \mathrm{shn} 2=\mathrm{h} 4 \mathrm{r} * \mathrm{~h} 1 \mathrm{~s}$;

shn12=h1r*h2s;shn13=h2r*h2s;shn14=h3r*h2s;shn7=h4r*h2s;

shn11=h1r*h3s;shn16=h2r*h3s;shn15=h3r*h3s;shn8=h4r*h3s;

shn4=h1r*h4s;shn10=h2r*h4s;shn9=h3r*h4s;shn3=h4r*h4s;

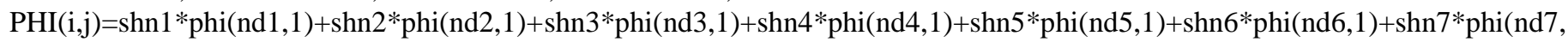

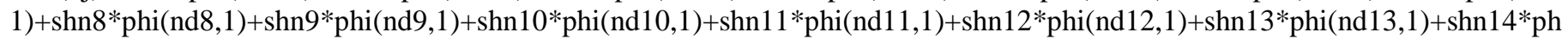
i(nd14,1)+shn15*phi(nd15,1)+shn16*phi(nd16,1);

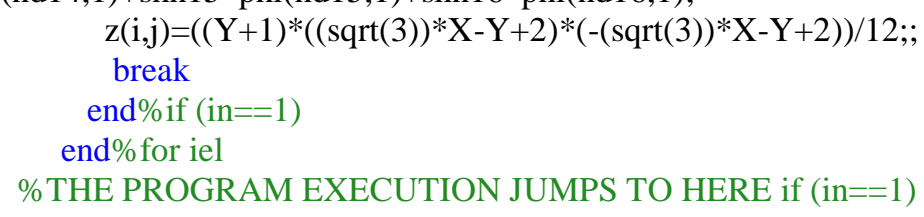


axis square

st1='Contour level curves for ';

st2='FEM solution of ';

st3='Sixteen Noded ';

st4='Special Quadrilateral';

st5=' Elements'

title([st1,st2,st3,st4,st5])

sst $1=$ '(MESH HAS '

sst $2=$ num 2 str(nnode)

sst3=' NODES'

sst4=' AND

sst5=num2str(nel)

sst6=' ELEMENTS)'

$\operatorname{text}(0.6,1.8,[\mathrm{sst} 1 \mathrm{sst} 2])$

$\operatorname{text}(0.6,1.6,[\mathrm{sst} 3 \mathrm{sst} 4])$

$\operatorname{text}(0.6,1.4,[\mathrm{sst} 5 \mathrm{sst} 6])$

$\%$ text $(0.25,-.08,[$ sst1 sst2 sst3 sst4 sst5 sst6])

$\%$

figure(2)

$\% \mathrm{x}=\left[\begin{array}{llllll}0.0 & 1.0 & 1.0 & 0.5 & 0.0\end{array}\right]$;

$\% y=\left[\begin{array}{lllll}0.0 & 0.0 & 0.5 & 1.0 & 1.0\end{array}\right]$;

$\mathrm{x}=[-\operatorname{sqrt}(3) ; \operatorname{sqrt}(3) ; 0]$;

$\mathrm{y}=[-1 ;-1 ; 2]$;

$\operatorname{patch}\left(\mathrm{x}, \mathrm{y}, \mathrm{w}^{\prime}\right)$

hold on

$\%[\mathrm{x}, \mathrm{y}]=\operatorname{meshgrid}(0: 1: 1,0: 0.1: 1)$

$\% y((y>1 / 2) \&(y<=1) \&(x>1 / 2) \&(x<=1) \&(x+y>3 / 2))=N a N$;

$\%[\mathrm{c}, \mathrm{h}]=$ contour $(\mathrm{x}, \mathrm{y}, \mathrm{z})$

$[x, y]=$ meshgrid( $(-\operatorname{sqrt}(3):(1 / 15) * \operatorname{sqrt}(3): \operatorname{sqrt}(3),-1:(0.1): 2)$;

contour(x,y,z,20)

xlabel('X-axis');

ylabel('Y-axis');

\%clabel(c,h);

axis square

title('contour level curves for exact solution: ')

hold off

figure(3)

$\% \mathrm{x}=\left[\begin{array}{lllll}0.0 & 1.0 & 1.0 & 0.5 & 0.0\end{array}\right]$;

$\% \mathrm{y}=\left[\begin{array}{lllll}0.0 & 0.0 & 0.5 & 1.0 & 1.0\end{array}\right]$;

$\mathrm{x}=[-\operatorname{sqrt}(3) ; \operatorname{sqrt}(3) ; 0]$;

$\mathrm{y}=[-1 ;-1 ; 2]$;

$\operatorname{patch}\left(\mathrm{x}, \mathrm{y},{ }^{\prime} \mathrm{w}\right)$

hold on

$[\mathrm{x}, \mathrm{y}]=$ meshgrid( $(-\operatorname{sqrt}(3):(1 / 15) * \operatorname{sqrt}(3): \operatorname{sqrt}(3),-1:(0.1): 2)$;

$\%[\mathrm{x}, \mathrm{y}]=$ meshgrid $(0: 1: 1,0: 0.1: 1)$

$\% \mathrm{y}((\mathrm{y}>1 / 2) \&(\mathrm{y}<=1) \&(\mathrm{x}>1 / 2) \&(\mathrm{x}<=1) \&(\mathrm{x}+\mathrm{y}>3 / 2))=\mathrm{NaN}$

$\% \% \mathrm{y}((\mathrm{y}>-1 / 2) \&(\mathrm{y}<=1) \&(\mathrm{x}>0) \&(\mathrm{x}<=(\operatorname{sqrt}(3) / 2)) \&((-\operatorname{sqrt}(3) * \mathrm{x}-\mathrm{y}+1)<0))=\mathrm{NaN}$;

$\% \% \mathrm{y}((\mathrm{y}>-1 / 2) \&(\mathrm{y}<=1) \&(\mathrm{x}>(-\operatorname{sqrt}(3) / 2)) \&(\mathrm{x}<=0) \&((\operatorname{sqrt}(3) * \mathrm{x}-\mathrm{y}+1)<0))=\mathrm{NaN}$;

contour(x,y,PHI,40,'r-')

xlabel('X-axis');

ylabel('Y-axis');

$\% \operatorname{clabel}(\mathrm{c}, \mathrm{h})$;

axis square

st1='Contour level curves for ';

st2='FEM solution of ';

st3='Sixteen Noded ';

st4='Special Quadrilateral';

st5=' Elements'

title([st1,st2,st3,st4,st5])

sst $1=$ ' NODES $=$ '

sst $2=$ num 2 str(nnode)

sst3=' ELEMENTS=' 
sst4=num $2 \operatorname{str}($ nel $)$

text $(0.6,1.1$, [sst1 sst2] $)$

$\operatorname{text}(0.6, .9,[\mathrm{sst} 3 \mathrm{sst} 4])$

hold on

$\%[\mathrm{x}, \mathrm{y}]=\operatorname{meshgrid}(0: 1: 1,0: 0.1: 1)$

$\%[\mathrm{c}, \mathrm{h}]=$ contour $\left(\mathrm{x}, \mathrm{y}, \mathrm{z}, \mathrm{g}_{-}{ }^{\prime}\right)$

contour(x,y,z,40,'b-')

\%xlabel('X-axis');

\%ylabel('Y-axis');

$\%$ clabel(c,h);

axis square

text $(0.6,1.9, '\{$ SUPERPOSITION OF $\}$ ')

text $(0.6,1.7, '\{$ FEM/EXACT SOLUTIONS $\} ')$

$\operatorname{text}\left(0.6,1.5,{ }^{\prime}--(\mathrm{red}) \mathrm{FEM}\right.$ ')

text(0.6,1.3,'--(blue)EXACT')

$\mathrm{mm}=0$;

for $\mathrm{i}=1: 31$

for $j=1: 31$

$\mathrm{mm}=\mathrm{mm}+1$

femsoln $(m m, 1)=P H I(i, j)$;

$\operatorname{exactsoln}(\mathrm{mm}, 1)=\mathrm{z}(\mathrm{i}, \mathrm{j})$;

end

end

end

$\%$ [femsoln exactsoln]

format long

$\operatorname{disp}('-$

disp('number of nodes,elements \& nodes per element')

disp([nnode nel nnel ])

disp('torisonal constants(fem=phi\&exact=xi) error(max (abs(phi_xi) $)$ ')

$\% \operatorname{disp}('-$

$\%$ disp([nnode nel nnel ])

disp([T k1 MAXPHI_XI ])

$\operatorname{disp}('-$

[8]D2LaplaceEquationQ16Ex3automeshgenNewPolygonContour.m

function[]=D2LaplaceEquationQ16Ex3automeshgenNewPolygonContour(n1,n2,n3,nmax,numtri,ndiv,mesh)

$\%$ ndiv $=2,4,6,8, \ldots$

\%D2LaplaceEquationQ16Ex3automeshgenNewPolygonContour([1;1;1;1;1;1;1;1],[2;3;4;5;6;7;8;9],[3;4;5;6;7;8;9;2],9,1,2,4)

$\%$ D2LaplaceEquationQ16Ex3automeshgenNewPolygonContour $([1 ; 1 ; 1 ; 1 ; 1 ; 1 ; 1 ; 1],[2 ; 3 ; 4 ; 5 ; 6 ; 7 ; 8 ; 9],[3 ; 4 ; 5 ; 6 ; 7 ; 8 ; 9 ; 2], 9,25,10,4)$

syms coord

[coord,gcoord,nodes,nodetel,nnode,nel]=polygonal_domain_coordinates_3rd_orderLG(n1,n2,n3,nmax,numtri,ndiv,mesh) nnel=16;

ndof $=1$;

sdof=nnode*ndof;

$\mathrm{ff}=($ zeros $($ sdof, 1$)) ; \mathrm{ss}=($ zeros $($ sdof,sdof $))$;

$\operatorname{disp}([$ nel nnode nnel ndof] $)$

format long $g$

for $\mathrm{i}=1$ :nel

$\mathrm{N}(\mathrm{i}, 1)=\mathrm{i}$;

end

for $\mathrm{i}=1$ :nel

$\mathrm{NN}(\mathrm{i}, 1)=\mathrm{i}$;

end

$\%[$ coord,gcoord] $=$ coordinate_rtisoscelestriangle00_h0_hh(ndiv);

$\%[$ nodetel,nodes]=nodaladdresses4special_convex_quadrilaterals(ndiv)

$\%$

$\%$ bcdof $=[2 ; 5 ; 3]$

\%boundary conditions-1

switch mesh 
case 4

\%boundary conditions- 2

$\mathrm{nnn}=0$;

for $\mathrm{nn}=1$ :nnode

$\mathrm{xnn}=\operatorname{coord}(\mathrm{nn}, 1) ; \mathrm{ynn}=\operatorname{gcoord}(\mathrm{nn}, 2)$;

if $(\mathrm{xnn}==-1 / 2) \&((\mathrm{ynn}>=-1 / 2) \&(\mathrm{ynn}<=1 / 2))$

$\mathrm{nnn}=\mathrm{nnn}+1$;

bcdof $(\mathrm{nnn}, 1)=\mathrm{nn}$;

$\operatorname{bcval}(n n n, 1)=0$;

end

end

\%boundary conditions-2

for $\mathrm{nn}=1$ :nnode

$\mathrm{xnn}=\operatorname{gcoord}(\mathrm{nn}, 1) ; \mathrm{ynn}=\operatorname{coord}(\mathrm{nn}, 2)$;

if $(\mathrm{ynn}==-1 / 2) \&((\mathrm{xnn}>=-1 / 2) \&(\mathrm{xnn}<=1 / 2))$

$\mathrm{nnn}=\mathrm{nnn}+1$;

$\operatorname{bcdof}(\mathrm{nnn}, 1)=\mathrm{nn}$;

$\operatorname{bcval}(n n n, 1)=0$;

end

end

\%boundary conditions-3

for $n n=1$ :nnode

$\mathrm{xnn}=\operatorname{gcoord}(\mathrm{nn}, 1) ; \mathrm{ynn}=\operatorname{coord}(\mathrm{nn}, 2)$;

if $(\mathrm{ynn}==1 / 2) \&((\mathrm{xnn}>=-1 / 2) \&(\mathrm{xnn}<=1 / 2))$

$\mathrm{nnn}=\mathrm{nnn}+1$;

$\operatorname{bcdof}(\mathrm{nnn}, 1)=\mathrm{nn}$;

$\operatorname{bcval}(\mathrm{nnn}, 1)=0$;

end

end

\%boundary conditions-4

for $\mathrm{nn}=1$ :nnode

$\mathrm{xnn}=\operatorname{coord}(\mathrm{nn}, 1) ; \mathrm{ynn}=\operatorname{gcoord}(\mathrm{nn}, 2)$;

if $(\mathrm{xnn}==1 / 2) \&((\mathrm{ynn}>=-1 / 2) \&(\mathrm{ynn}<=1 / 2))$

$\mathrm{nnn}=\mathrm{nnn}+1$;

$\operatorname{bcdof}(\mathrm{nnn}, 1)=\mathrm{nn}$;

$\operatorname{bcval}(\mathrm{nnn}, 1)=0$;

end

end

$\%$ end

bcdof

$\mathrm{mm}=$ length(bcdof);

format long $\mathrm{g}$

$\mathrm{k} 1$ =double $(0.14057701495515551037840396020329)$;

$\mathrm{xi}=($ zeros $($ nnode, 1$))$;

$\mathrm{a} 0=8 / \mathrm{pi}^{\wedge} 3$;

for $\mathrm{m}=1$ :nnode

$\mathrm{gx}=(\mathrm{gcoord}(\mathrm{m}, 1)) ; \mathrm{gy}=(\mathrm{gcoord}(\mathrm{m}, 2)) ; \mathrm{rr}=(0) ;$

for $\mathrm{n}=1: 2: 99$

$\mathrm{rr}=\mathrm{rr}+(-1)^{\wedge}((\mathrm{n}-1) / 2)^{*}(1-(\cosh (\mathrm{n} * \mathrm{pi} * \mathrm{gy}) / \cosh (\mathrm{n} * \mathrm{pi} / 2))) * \cos \left(\mathrm{n}^{*} \mathrm{pi}^{*} \mathrm{gx}\right) / \mathrm{n}^{\wedge} 3$;

end

$\operatorname{xi}(\mathrm{m}, 1)=(\mathrm{a} 0 * \mathrm{rr})$;

end

end \%switch

for $\mathrm{L}=1$ :nel

for $M=1: 3$

$\mathrm{LM}=\operatorname{nodetel}(\mathrm{L}, \mathrm{M})$;

$\mathrm{xx}(\mathrm{L}, \mathrm{M})=\operatorname{gcoord}(\mathrm{LM}, 1)$;

$\mathrm{yy}(\mathrm{L}, \mathrm{M})=\operatorname{gcoord}(\mathrm{LM}, 2)$;

end

end 
$\%$

$\%$ ng=10

table $2=[\mathrm{N}$ xx yy $]$;

$\% \operatorname{disp}([\mathrm{xx} y \mathrm{y}])$

$\%$

[intJdndn]=integral_valuesof_localderivative_products(nnel);

for iel=1:nel

index $=$ zeros(nnel*ndof,1);

$\mathrm{X}=\mathrm{xx}(\mathrm{iel}, 1: 3)$;

$\mathrm{Y}=\mathrm{yy}(\mathrm{iel}, 1: 3)$;

$\% \operatorname{disp}([\mathrm{X} Y])$

$\mathrm{xa}=\mathrm{X}(1,1)$;

$\mathrm{xb}=\mathrm{X}(1,2)$;

$\mathrm{xc}=\mathrm{X}(1,3)$;

ya $=Y(1,1)$

$\mathrm{yb}=\mathrm{Y}(1,2)$;

$\mathrm{yc}=\mathrm{Y}(1,3)$;

bta $=y b-y c ; b t b=y c-y a ;$

gma $=x c-x b ; g m b=x a-x c$;

delabc $=$ gmb*bta-gma*btb;

$\mathrm{G}=[\mathrm{bta}$ btb;gma gmb]/delabc;

$\mathrm{GT}=[\mathrm{bta}$ gma;btb gmb]/delabc;

$\mathrm{Q}=\mathrm{GT} * \mathrm{G}$;

$\operatorname{sk}(1: 16,1: 16)=(\operatorname{zeros}(16,16))$;

for $i=1: 16$

for $\mathrm{j}=\mathrm{i}: 16$

$\operatorname{sk}(\mathrm{i}, \mathrm{j})=(\operatorname{delabc} * \operatorname{sum}(\operatorname{sum}(\mathrm{Q} . *(\operatorname{int} \operatorname{Jdndn}(2 * \mathrm{i}-1: 2 * \mathrm{i}, 2 * \mathrm{j}-1: 2 * \mathrm{j})))))$;

$\operatorname{sk}(\mathrm{j}, \mathrm{i})=\mathrm{sk}(\mathrm{i}, \mathrm{j})$;

end

end

$\% \mathrm{f}=[5 / 144 ; 1 / 24 ; 7 / 144 ; 1 / 24] *(2 *$ delabc $)$;

$\% \mathrm{f}=[-7 / 432 ;-1 / 72 ;-5 / 432 ;-1 / 72 ; 11 / 216 ; 13 / 216 ; 13 / 216 ; 11 / 216] *(2 *$ delabc $)$

$\% \mathrm{f}=[-7 / 360 ;-1 / 48 ;-1 / 45 ;-1 / 48 ; 23 / 960 ; 1 / 30 ; 7 / 240 ; 37 / 960 ; 37 / 960 ; 7 / 240 ; 1 / 30 ; 23 / 960] *(2 *$ delabc $)$

$\mathrm{f}=[19 / 11520 ; 1 / 384 ; 41 / 11520 ; 1 / 384 ; 1 / 192 ; 29 / 3840 ; 31 / 3840 ; 1 / 96 ; 1 / 96 ; 31 / 3840 ; 29 / 3840 ; 1 / 192 ; 21 / 1280 ; 3 / 128 ; 39 / 1280 ; 3 / 128] *(2 *$ delabc);

$\%$

edof=nnel*ndof;

$\mathrm{k}=0$;

for $\mathrm{i}=1:$ nnel

$\operatorname{nd}(\mathrm{i}, 1)=\operatorname{nodes}(\mathrm{iel}, \mathrm{i})$;

start $=($ nd $(\mathrm{i}, 1)-1) *$ ndof;

for $\mathrm{j}=1$ :ndof

$\mathrm{k}=\mathrm{k}+1$;

index $(\mathrm{k}, 1)=\operatorname{start}+\mathrm{j}$

end

end

for $i=1$ :edof

ii=index $(\mathrm{i}, 1)$;

ff(ii, 1$)=f f(i i, 1)+f(i, 1)$;

for $\mathrm{j}=1$ :edof

$\mathrm{jj}=\operatorname{index}(\mathrm{j}, 1)$;

$s s(i i, j j)=s s(i i, j j)+s k(i, j)$;

end

end

end $\%$ for iel 
$\%$

\%bcdof $=[13 ; 37 ; 35 ; 33 ; 31 ; 29 ; 27 ; 25 ; 23 ; 21 ; 19 ; 17 ; 15]$;

for $\mathrm{ii}=1: \mathrm{mm}$

$\mathrm{kk}=\mathrm{bcdof}(\mathrm{ii}, 1)$;

$\mathrm{ss}(\mathrm{kk}, 1:$ nnode $)=$ zeros $(1$, nnode $)$;

ss $(1:$ nnode, $\mathrm{kk})=$ zeros $($ nnode, 1$)$;

$\mathrm{ff}(\mathrm{kk}, 1)=0$;

end

for $\mathrm{ii}=1: \mathrm{mm}$

$\mathrm{kk}=\mathrm{bcdof}(\mathrm{ii}, 1)$;

$\mathrm{ss}(\mathrm{kk}, \mathrm{kk})=1$;

end

phi=ss $\backslash f f ;$

phi=double(phi);

[phi xi]

for $\mathrm{I}=1$ :nnode

$\mathrm{NN}(\mathrm{I}, 1)=\mathrm{I}$

phi_xi(I,1)=phi(I,1)-xi(I,1);

end

MAXPHI_XI=max(abs(phi_xi));

$\operatorname{disp}('$

disp('number of nodes, elements \& nodes per element')

[nnode nel nnel ndof]

$\operatorname{disp}('$

fem-computed values

anlytical(theoretical)-values

$\operatorname{disp}('$

$\operatorname{disp}([\mathrm{NN}$ phi xi] $)$

$\operatorname{disp}('$

disp('number of nodes, elements \& nodes per element')

[nnode nel nnel ndof]

nodes

gcoord

$\operatorname{disp}([\mathrm{NN}$ phi xi] $)$

$\mathrm{t}=0$;

for iii=1:nnode

$\mathrm{t}=\mathrm{t}+$ phi(iii, 1$) * \mathrm{ff}(\mathrm{iii}, 1)$;

end

$\mathrm{T}=\mathrm{t}$;

$\operatorname{disp}('$

disp('number of nodes,elements \& nodes per element')

$\operatorname{disp}([$ nnode nel nnel ])

disp('torisonal constants(fem=phi\&exact=xi) error $\left(\max \left(\operatorname{abs}\left(p h i \_x i\right)\right)\right.$ ')

$\% \operatorname{disp}('-$

$\%$ disp([nnode nel nnel ])

$\operatorname{disp}([\mathrm{T}$ k1 MAXPHI_XI ])

$\operatorname{disp}(-$

$\%$ end

$\operatorname{disp}('$

disp('number of nodes, elements \& nodes per element')

[nnode nel nnel ndof]

$\% * * * * * * * * * * * * * * * * * * * * * * * * * * * * * * * * * * * * * * *$

$[\mathrm{x}, \mathrm{y}]=$ meshgrid(-1/2:0.05:1/2,-1/2:0.05:1/2);

$\mathrm{a} 0=8 / \mathrm{pi}^{\wedge} 3$;

for $i=1: 21$

for $j=1: 21$

for iel=1:nel

$\mathrm{XX}=\mathrm{xx}(\mathrm{iel}, 1: 3)$; 


$$
\mathrm{xx}=(\mathrm{x}(\mathrm{ii}, \mathrm{jj})) ; \mathrm{yy}=(\mathrm{y}(\mathrm{ii}, \mathrm{jj})) ; \mathrm{rr}=(0)
$$

for $\mathrm{n}=1: 2: 99$

$\mathrm{rr}=\mathrm{rr}+(-1)^{\wedge}((\mathrm{n}-1) / 2)^{*}(1-(\cosh (\mathrm{n} * \mathrm{pi} * \mathrm{yy}) / \cosh (\mathrm{n} * \mathrm{pi} / 2))) * \cos (\mathrm{n} * \mathrm{pi} * \mathrm{xx}) / \mathrm{n}^{\wedge} 3$;

end

$\mathrm{z}(\mathrm{ii}, \mathrm{jj})=(\mathrm{a} 0 * \mathrm{rr})$;

end \% ii

end $\% \mathrm{jj}$

$\% \mathrm{z}=\sin \left(\mathrm{pi}^{*} \mathrm{x}\right) . * \sin \left(\mathrm{pi}^{*} \mathrm{y}\right)$

for $i=1: 21$

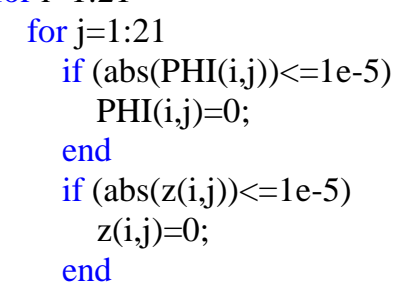

end

end

switch mesh

case 4

hold off

clf

figure(1)

$[\mathrm{x}, \mathrm{y}]=$ meshgrid( $(-1 / 2: .05: 1 / 2,-1 / 2: 0.05: 1 / 2)$

$\%[\mathrm{c}, \mathrm{h}]=$ contour $(\mathrm{x}, \mathrm{y}, \mathrm{PHI})$

contour(x,y,PHI,40)

xlabel('X-axis');

ylabel('Y-axis');

$\%$ clabel $(\mathrm{c}, \mathrm{h})$;

axis square

st1='Contour level curves for ';

st2='FEM solution of ';

st3='Sixteen Noded ';

st4='Special Quadrilateral';

st5=' Elements'

title([st1,st2,st3,st4,st5])

sst1='(MESH HAS '

sst2=num $2 \operatorname{str}($ nnode $)$

sst3=' NODES'

sst4=' AND

sst5=num2str(nel)

sst6=' ELEMENTS)'

text(0.25,-.08,[sst1 sst2 sst3 sst4 sst5 sst6])

hold on

figure(2)

$\%[\mathrm{x}, \mathrm{y}]=$ meshgrid $(0: .1: 1,0: 0.1: 1)$

$[\mathrm{x}, \mathrm{y}]=$ meshgrid $(-1 / 2: .05: 1 / 2,-1 / 2: 0.05: 1 / 2)$

$\%[\mathrm{c}, \mathrm{h}]=\operatorname{contour}(\mathrm{x}, \mathrm{y}, \mathrm{z})$

contour $(\mathrm{x}, \mathrm{y}, \mathrm{z}, 40)$

xlabel('X-axis');

ylabel('Y-axis');

$\%$ clabel $(\mathrm{c}, \mathrm{h})$;

axis square

title('contour level curves for exact solution: in a series')

$\mathrm{mm}=0$;

for $i=1: 21$

for $\mathrm{j}=1: 21$

$\mathrm{mm}=\mathrm{mm}+1$;

femsoln $(\mathrm{mm}, 1)=\mathrm{PHI}(\mathrm{i}, \mathrm{j})$; 


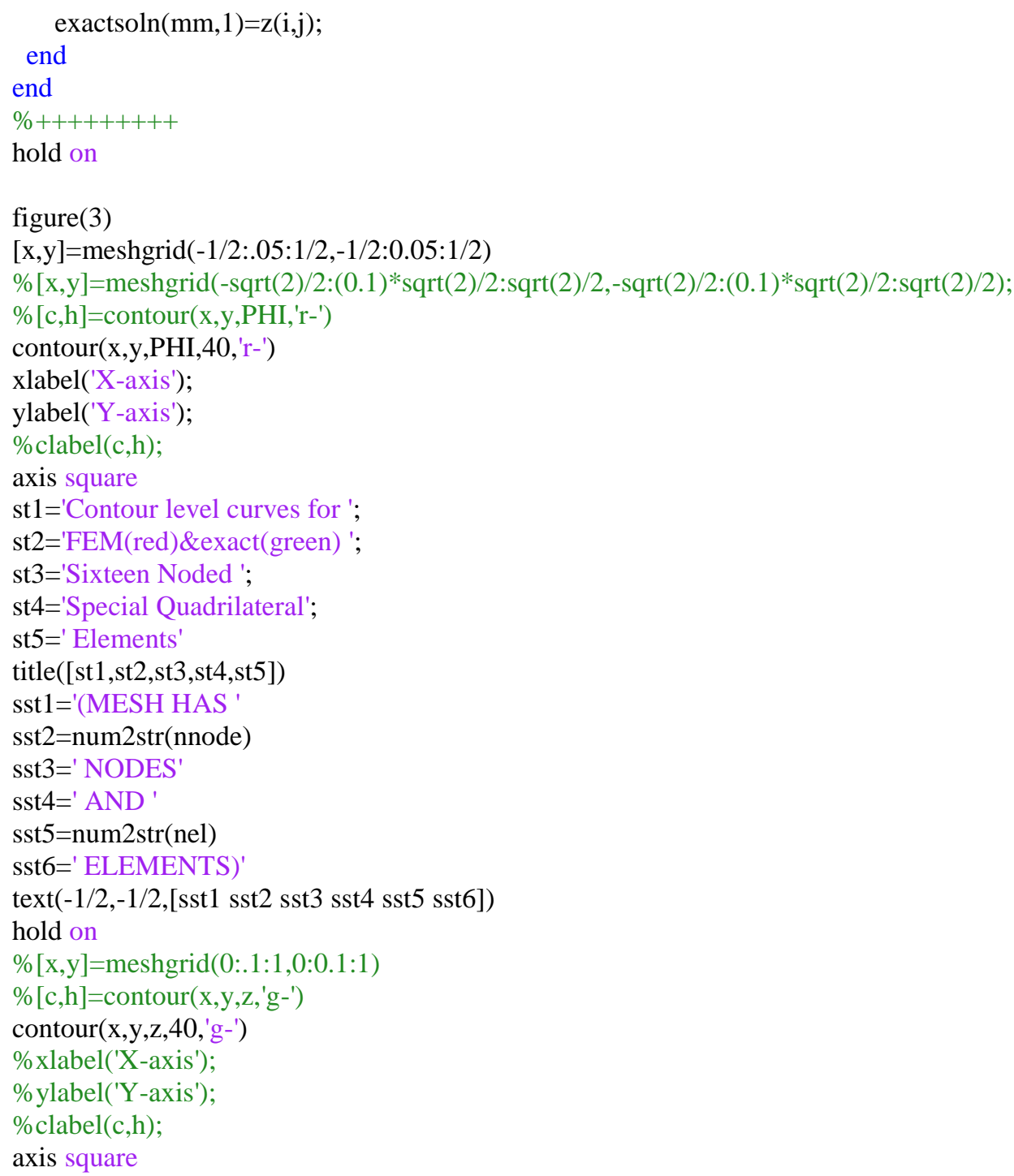

disp('number of nodes, elements \& nodes per element')

[nnode nel nnel ndof]

if mesh $==4$

$\%$ [phi xi]

for $\mathrm{I}=1$ :nnode

$\mathrm{NN}(\mathrm{I}, 1)=\mathrm{I}$;

phi_xi(I,1)=phi(I,1)-xi(I,1);

end

MAXPHI_XI=max $(\operatorname{abs}($ double(phi_xi $)))$;

$\mathrm{t}=0$; 
for iii=1:nnode

$\mathrm{t}=\mathrm{t}+$ phi(iii, 1$) * \mathrm{ff}(\mathrm{iii}, 1)$;

end

$\mathrm{T}=\mathrm{t}$;

$\operatorname{disp}('$

disp('number of nodes,elements \& nodes per element')

disp([nnode nel nnel ])

disp('torisonal constants(fem=phi\&exact=xi) error $\left(\max \left(\operatorname{abs}\left(p h i \_x i\right)\right)\right.$ ')

$\%$ disp('-

$\%$ disp([nnode nel nnel ])

$\operatorname{disp}([$ T k1 MAXPHI_XI ] $)$

$\operatorname{disp}($

end

$\operatorname{disp}('$

disp('number of nodes,elements \& nodes per element')

[nnode nel nnel ndof]

for $\mathrm{ncpt}=1$ :nel

$\mathrm{c} 1=\operatorname{nodes}(\mathrm{ncpt}, 13) ; \mathrm{c} 2=\operatorname{nodes}(\mathrm{ncpt}, 14) ; \mathrm{c} 3=\operatorname{nodes}(\mathrm{ncpt}, 15) ; \mathrm{c} 4=\operatorname{nodes}(\mathrm{ncpt}, 16)$;

elcentr $(\mathrm{ncpt}, 1)=\mathrm{ncpt}$;

$\operatorname{phicpt}(\mathrm{ncpt}, 1)=(\operatorname{phi}(\mathrm{c} 1,1)+\operatorname{phi}(\mathrm{c} 2,1)+\operatorname{phi}(\mathrm{c} 3,1)+\operatorname{phi}(\mathrm{c} 4,1)) / 4$;

$\operatorname{xicpt}(\mathrm{ncpt}, 1)=(\mathrm{xi}(\mathrm{c} 1,1)+\mathrm{xi}(\mathrm{c} 2,1)+\mathrm{xi}(\mathrm{c} 3,1)+\mathrm{xi}(\mathrm{c} 4,1)) / 4$;

end

for $\mathrm{I}=1$ :nel

elnumm $(\mathrm{I}, 1)=\mathrm{I}$;

end

$\operatorname{disp}('$

$\operatorname{disp}($ ' serial no center point

fem-computed values

anlytical(theoretical)-values

') ')

$\operatorname{disp}([$ elnumm elcentr phicpt xicpt $])$

$\operatorname{disp}('$

format compact

$\%====$
=====

$\operatorname{disp}\left(^{\prime}\right.$ ')

disp('number of nodes,elements \& nodes per element')

[nnode nel nnel ndof]

$\operatorname{disp}('$

')

\section{disp(' NODE FEM SOLUTION EXACT SOLUTION NODE FEM SOLUTION EXACT SOLUTION NODE FEM} SOLUTION EXACT SOLUTION ')

$\operatorname{disp}('-$ -)

for $\mathrm{I}=1: 3$ :nel

$\% \mathrm{~A}=[\operatorname{elcentr}(\mathrm{I}) \operatorname{phicpt}(\mathrm{I}) \operatorname{xicpt}(\mathrm{I})] ; \mathrm{B}=[\operatorname{elcentr}(\mathrm{I}+1) \operatorname{phicpt}(\mathrm{I}+1) \operatorname{xicpt}(\mathrm{I}+1)] ; \mathrm{C}=[\operatorname{elcentr}(\mathrm{I}+2) \operatorname{phicpt}(\mathrm{I}+2) \operatorname{xicpt}(\mathrm{I}+2)]$;

$\% \operatorname{disp}([\operatorname{elcentr}(\mathrm{I}) \operatorname{phicpt}(\mathrm{I}) \operatorname{xicpt}(\mathrm{I}) \operatorname{elcentr}(\mathrm{I}+1) \operatorname{phicpt}(\mathrm{I}+1) \operatorname{xicpt}(\mathrm{I}+1)])$

$\% \operatorname{disp}([\operatorname{elcentr}(\mathrm{I}+2) \operatorname{phicpt}(\mathrm{I}+2) \operatorname{xicpt}(\mathrm{I}+2)])$

$\% \operatorname{disp}([\mathrm{A} \mathrm{B} \mathrm{C}])$

fprintf('In\%5d \%18.14f \%18.14f \%5d \%18.14f \%18.14f \%5d \%18.14f \%18.14f',elcentr(I), phicpt(I), xicpt(I), elcentr(I+1), phicpt(I+1), xicpt(I+1), elcentr(I+2), phicpt(I+2), xicpt(I+2));

end

fprintf('ไn')

$\operatorname{disp}(-$

\section{[9]polygonal_domain_coordinates_3rd_orderLG.m}

function[coord,gcoord,nodes,nodetel,nnode,nel]=polygonal_domain_coordinates_3rd_orderLG(n1,n2,n3,nmax,numtri,n,mesh)

$\%$ n1=node number at $(0,0)$ for a choosen triangle

$\%$ n2=node number at $(1,0)$ for a choosen triangle

$\%$ n3=node number at $(0,1)$ for a choosen triangle

$\%$ eln=6-node triangles with centroid

$\%$ spqd=4-node special convex quadrilateral

$\%$ n must be even,i.e.n $=2,4,6, \ldots \ldots .$. i.e number of divisions

$\%$ nmax $=$ one plus the number of segments of the polygon

$\%$ nmax $=$ the number of segments of the polygon plus a node interior to the polygon 
$\%$ numtri=number of T6 triangles in each segment i.e a triangle formed by

$\%$ joining the end poits of the segment to the interior point(e.g:the centroid) of the polygon

$\%[$ eln,spqd] $=$ nodaladdresses_special_convex_quadrilaterals_trial $(\mathrm{n} 1=1, \mathrm{n} 2=2, \mathrm{n} 3=3, \mathrm{nmax}=3, \mathrm{n}=2,4,6, \ldots)$

$\%[$ eln,spqd] $=$ nodaladdresses_special_convex_quadrilaterals_trial $([1 ; 1 ; 1 ; 1],[2 ; 3 ; 4 ; 5],[3 ; 4 ; 5 ; 2], 5,1,2)$

$\%[$ eln,spqd] $=$ nodaladdresses_special_convex_quadrilaterals_trial $([1 ; 1 ; 1 ; 1],[2 ; 3 ; 4 ; 5],[3 ; 4 ; 5 ; 2], 5,4,4)$

$\%[$ eln,spqd] $=$ nodaladdresses_special_convex_quadrilaterals_trial $([1 ; 1 ; 1 ; 1],[2 ; 3 ; 4 ; 5],[3 ; 4 ; 5 ; 2], 5,9,6)$

$\%[$ eln,spqd] $=$ nodaladdresses_special_convex_quadrilaterals_trial $([1 ; 1 ; 1 ; 1],[2 ; 3 ; 4 ; 5],[3 ; 4 ; 5 ; 2], 5,16,8)$

$\%$ [coord,gcoord,nodes,nodetel,nnode,nel]=polygonal_domain_coordinates_2nd_order $([1 ; 1 ; 1 ; 1],[2 ; 3 ; 4 ; 5],[3 ; 4 ; 5 ; 2], 5,1,2,3)$

$\%[$ coord,gcoord,nodes,nodetel,nnode,nel] $=$ polygonal_domain_coordinates_2nd_order $([1 ; 1 ; 1 ; 1 ; 1 ; 1 ; 1 ; 1],[2 ; 3 ; 4 ; 5 ; 6 ; 7 ; 8 ; 9],[3 ; 4 ; 5 ; 6 ;$

$7 ; 8 ; 9 ; 2], 9,1,2,2)$

$\%$ PARVIZ MOIN EXAMPLE

$\%$ [coord,gcoord,nodes,nodetel,nnode,nel]=polygonal_domain_coordinates_2nd_order $([1 ; 1 ; 1 ; 1 ; 1 ; 1 ; 1],[2 ; 3 ; 4 ; 5 ; 6 ; 7 ; 8],[3 ; 4 ; 5 ; 6 ; 7 ; 8 ;$

2], $8,1,2,1)$

$\%[$ coord,gcoord,nodes,nodetel,nnode,nel]=polygonal_domain_coordinates_2nd_order([1;1;1;1;1;1;1],[2;3;4;5;6;7;8],[3;4;5;6;7;8;

2], $8,4,4,1)$

$\%[$ coord,gcoord,nodes,nodetel,nnode,nel]=polygonal_domain_coordinates_3rd_order([1;1; $1 ; 1],[2 ; 3 ; 4 ; 5],[3 ; 4 ; 5 ; 2], 5,1,2,3)$

syms U V W xi yi

syms x y

switch mesh

case $1 \%$ domain with seven triangles( 8 -nodes)

$\mathrm{x}=\operatorname{sym}([1 / 2 ; 1 / 2 ; 1 ; 1 ; 1 / 2 ; 0 ; 0 ; 0]) \%$ for MOIN EXAMPLE

$\mathrm{y}=\operatorname{sym}([1 / 2 ; 0 ; 0 ; 1 / 2 ; 1 ; 1 ; 1 / 2 ; 0]) \%$ for MOIN EXAMPLE

case $2 \%$ square domain with eight triangles(9-nodes)

$\mathrm{x}=\operatorname{sym}([1 / 2 ; 1 / 2 ; 1 ; 1 ; 1 ; 1 / 2 ; 0 ; 0 ; 0]) \% \mathrm{FOR}$ UNIT SQUARE

$\mathrm{y}=\operatorname{sym}([1 / 2 ; 0 ; 0 ; 1 / 2 ; 1 ; 1 ; 1 ; 1 / 2 ; 0]) \%$ FOR UNIT SQUARE

case $3 \%$ square domain with four triangles(5-nodes)

$\mathrm{x}=\operatorname{sym}([1 / 2 ; 0 ; 1 ; 1 ; 0])$

$\mathrm{y}=\operatorname{sym}([1 / 2 ; 0 ; 0 ; 1 ; 1])$

case $4 \%$ square domain with eight triangles(9-nodes)

$\%$

$\mathrm{x}=\operatorname{sym}([0 ; 0 ; 1 / 2 ; 1 / 2 ; 1 / 2 ; 0 ;-1 / 2 ;-1 / 2 ;-1 / 2]) \% \mathrm{FOR}$ UNIT SQUARE

$\mathrm{y}=\operatorname{sym}([0 ;-1 / 2 ;-1 / 2 ; 0 ; 1 / 2 ; 1 / 2 ; 1 / 2 ; 0 ;-1 / 2]) \%$ FOR UNIT SQUARE

end

[eln,spqd,rrr,nodes,nodetel]=nodaladdresses_special_convex_quadrilaterals_trial_3rd_orderLG(n1,n2,n3,nmax,numtri,n); $[\mathrm{U}, \mathrm{V}, \mathrm{W}]=$ generate_area_coordinate_over_the_standard_triangle(n);

ss1='number of 6-node triangles with centroid=';

$[\mathrm{p} 1, \mathrm{q} 1]=\operatorname{size}(\mathrm{eln})$;

$\operatorname{disp}([\mathrm{ss} 1$ num2str(p1)])

$\%$

eln

$\%$

ss2='number of special convex quadrilaterals elements\&nodes per element =';

[nel,nnel]=size(spqd);

$\operatorname{disp}([\mathrm{ss} 2$ num2str(nel) ',' num2str(nnel)])

$\%$

spqd

$\%$

nnode $=\max (\max (\operatorname{spqd}))$;

ss3='number of nodes of the triangular domain\& number of special quadrilaterals=';

$\operatorname{disp}([\mathrm{ss} 3$ num2str(nnode) ',' num2str(nel)])

xi(1:nnode, 1$)=z e r o s($ nnode, 1$) ; y i(1:$ nnode, 1$)=$ zeros $($ nnode, 1$)$;

nitri=nmax-1;

for itri=1:nitri

disp('vertex nodes of the itri triangle')

[n1(itri,1) n2(itri,1) n3(itri,1)]

$\mathrm{x} 1=\mathrm{x}(\mathrm{n} 1(\mathrm{itri}, 1), 1)$

$\mathrm{x} 2=\mathrm{x}(\mathrm{n} 2(\mathrm{itri}, 1), 1)$

$\mathrm{x} 3=\mathrm{x}(\mathrm{n} 3(\mathrm{itri}, 1), 1)$

$\%$ 
$\mathrm{y} 1=\mathrm{y}(\mathrm{n} 1(\mathrm{itri}, 1), 1)$

$\mathrm{y} 2=\mathrm{y}(\mathrm{n} 2(\mathrm{itri}, 1), 1)$

y3=y(n3(itri, 1), 1)

$\operatorname{rrr}(:,:$,itri $)$

$\mathrm{U}^{\prime}$

$\mathrm{V}^{\prime}$

$\mathrm{W}^{\prime}$

$\mathrm{kk}=0$;

for $\mathrm{ii}=1: n+1$

for $\mathrm{jj}=1:(n+1)-(\mathrm{ii}-1)$

$\mathrm{kk}=\mathrm{kk}+1$;

$\mathrm{mm}=\mathrm{rrr}(\mathrm{ii}, \mathrm{jj}$,itri);

$\mathrm{uu}=\mathrm{U}(\mathrm{kk}, 1) ; \mathrm{vv}=\mathrm{V}(\mathrm{kk}, 1) ; \mathrm{ww}=\mathrm{W}(\mathrm{kk}, 1)$;

$\mathrm{xi}(\mathrm{mm}, 1)=\mathrm{x} 1 * \mathrm{ww}+\mathrm{x} 2 * \mathrm{uu}+\mathrm{x} 3 *^{*} \mathrm{vv}$;

$\mathrm{yi}(\mathrm{mm}, 1)=\mathrm{y} 1 * \mathrm{ww}+\mathrm{y} 2 * \mathrm{uu}+\mathrm{y} 3 * \mathrm{vv}$;

end $\%$ for jj

end $\%$ for ii

[xi yi]

$\%$ add coordinates of centroid

ne $=(\mathrm{n} / 2)^{\wedge} 2$;

$\%$ stdnode $=\mathrm{kk}$;

for $\mathrm{iii}=1+(\mathrm{itri}-1) *$ ne:ne*itri

$\% \mathrm{kk}=\mathrm{kk}+1$;

node1 $=\operatorname{eln}($ iii, 1$)$

node2 $=\operatorname{eln}($ iii, 2$)$

node $3=\operatorname{eln}($ iii, 3$)$

$\mathrm{mm}=\mathrm{eln}(\mathrm{iii}, 7)$

$\mathrm{xi}(\mathrm{mm}, 1)=(\mathrm{xi}($ node 1,1$)+\mathrm{xi}($ node 2,1$)+x i($ node 3,1$)) / 3$;

$\mathrm{yi}(\mathrm{mm}, 1)=(\mathrm{yi}($ node 1,1$)+\mathrm{yi}(\operatorname{node} 2,1)+\mathrm{yi}(\operatorname{node} 3,1)) / 3$;

end \%for iii

[xi yi]

end $\%$ for itri=1:nitri

for $\mathrm{mmm}=1$ :nel

$\mathrm{mmm} 1=\operatorname{nodes}(\mathrm{mmm}, 1)$

$\mathrm{mmm} 2=\operatorname{nodes}(\mathrm{mmm}, 2)$

$\mathrm{mmm} 3=\operatorname{nodes}(\mathrm{mmm}, 3)$

$\mathrm{mmm} 4=\operatorname{nodes}(\mathrm{mmm}, 4)$

$\mathrm{mmm} 5=\operatorname{nodes}(\mathrm{mmm}, 5)$

mmm6=nodes $(\mathrm{mmm}, 6)$

$\mathrm{mmm} 7=\operatorname{nodes}(\mathrm{mmm}, 7)$

$\mathrm{mmm} 8=\operatorname{nodes}(\mathrm{mmm}, 8)$

$\mathrm{mmm} 9=\operatorname{nodes}(\mathrm{mmm}, 9)$

$\mathrm{mmm10}=\operatorname{nodes}(\mathrm{mmm}, 10)$

$\operatorname{mmm} 11=\operatorname{nodes}(\mathrm{mmm}, 11)$

mmm12=nodes $(\mathrm{mmm}, 12)$

$\%$

mmm13=nodes $(\mathrm{mmm}, 13)$

mmm14=nodes $(\mathrm{mmm}, 14)$

$\operatorname{mmm} 15=\operatorname{nodes}(\mathrm{mmm}, 15)$

mmm16=nodes $(\mathrm{mmm}, 16)$

$\%$

$\mathrm{xi1}=\mathrm{xi}(\mathrm{mmm} 1,1)$

$\mathrm{xi} 2=x i(\mathrm{mmm} 2,1)$

$\mathrm{xi} 3=\mathrm{xi}(\mathrm{mmm} 3,1)$

$\mathrm{xi} 4=\mathrm{xi}(\mathrm{mmm} 4,1)$

$\%$

yi1=yi $(\mathrm{mmm} 1,1)$

yi2=yi $(\mathrm{mmm} 2,1)$

yi3=yi $(\mathrm{mmm} 3,1)$

yi4=yi $(\mathrm{mmm} 4,1)$

$\%$

$\mathrm{xi}(\mathrm{mmm} 5,1)=(2 * \mathrm{xi} 1+\mathrm{xi} 2) / 3 ; \mathrm{xi}(\mathrm{mmm} 6,1)=(\mathrm{xi} 1+2 * \mathrm{xi} 2) / 3$;

$\mathrm{xi}(\mathrm{mmm} 7,1)=(2 * x i 2+\mathrm{xi} 3) / 3 ; \mathrm{xi}(\mathrm{mmm} 8,1)=(\mathrm{xi} 2+2 * \mathrm{xi} 3) / 3$; 
$\mathrm{xi}(\mathrm{mmm} 9,1)=(2 * x i 3+x i 4) / 3 ; x i(\mathrm{mmm} 10,1)=(x i 3+2 * x i 4) / 3$;

$\mathrm{xi}(\mathrm{mmm} 11,1)=(2 * \mathrm{xi} 4+\mathrm{xi} 1) / 3 ; \mathrm{xi}(\mathrm{mmm} 12,1)=(\mathrm{xi} 4+2 * \mathrm{xi} 1) / 3$;

$\mathrm{xi}(\mathrm{mmm} 13,1)=(4 * \mathrm{xi} 1+2 * x i 2+\mathrm{xi} 3+2 * x i 4) / 9$;

$\mathrm{xi}(\mathrm{mmm} 14,1)=(2 * \mathrm{xi} 1+4 * \mathrm{xi} 2+2 * \mathrm{xi} 3+\mathrm{xi} 4) / 9$

$\mathrm{xi}(\mathrm{mmm} 15,1)=(\mathrm{xi} 1+2 * x i 2+4 * x i 3+2 * x i 4) / 9$;

$\mathrm{xi}(\mathrm{mmm} 16,1)=(2 * \mathrm{xi} 1+\mathrm{xi} 2+2 * \mathrm{xi} 3+4 * \mathrm{xi} 4) / 9$;

$\mathrm{yi}(\mathrm{mmm} 5,1)=(2 * \mathrm{yi} 1+\mathrm{yi} 2) / 3 ; \mathrm{yi}(\mathrm{mmm} 6,1)=(\mathrm{yi} 1+2 * \mathrm{yi} 2) / 3$;

$\mathrm{yi}(\mathrm{mmm} 7,1)=(2 * \mathrm{yi} 2+\mathrm{yi} 3) / 3 ; \mathrm{yi}(\mathrm{mmm} 8,1)=(\mathrm{yi} 2+2 * \mathrm{yi} 3) / 3$;

$\mathrm{yi}(\mathrm{mmm} 9,1)=(2 * \mathrm{yi} 3+\mathrm{yi} 4) / 3 ; \mathrm{yi}(\mathrm{mmm} 10,1)=(\mathrm{yi} 3+2 * \mathrm{yi} 4) / 3$;

$\mathrm{yi}(\mathrm{mmm} 11,1)=(2 * \mathrm{yi} 4+\mathrm{yi} 1) / 3 ; \mathrm{yi}(\mathrm{mmm} 12,1)=(\mathrm{yi} 4+2 * \mathrm{yi} 1) / 3$;

yi $(\mathrm{mmm} 13,1)=(4 * \mathrm{yi} 1+2 * \mathrm{yi} 2+\mathrm{yi} 3+2 * \mathrm{yi} 4) / 9$;

yi $(\mathrm{mmm} 14,1)=(2 * \mathrm{yi} 1+4 * \mathrm{yi} 2+2 * \mathrm{yi} 3+\mathrm{yi} 4) / 9$;

$\mathrm{yi}(\mathrm{mmm} 15,1)=(\mathrm{yi} 1+2 * \mathrm{yi} 2+4 * \mathrm{yi} 3+2 * \mathrm{yi} 4) / 9$;

yi $(\mathrm{mmm} 16,1)=(2 * \mathrm{yi} 1+\mathrm{yi} 2+2 * \mathrm{yi} 3+4 * \mathrm{yi} 4) / 9$;

end \% for nel

$\%[x i(18,1)$ yi $(18,1)]$

$\mathrm{N}=(1: \text { nnode })^{\prime}$

[N xi yi]

$\%$

$\operatorname{coord}(:, 1)=(x i(:, 1))$;

$\operatorname{coord}(:, 2)=(\mathrm{yi}(:, 1))$;

$\operatorname{gcoord}(:, 1)=\operatorname{double}(x i(:, 1))$;

$\operatorname{gcoord}(:, 2)=\operatorname{double}(\mathrm{yi}(:, 1))$;

$\% \operatorname{disp}($ gcoord $)$

\section{[10]polygonal_domain_coordinates_3rd_order.m}

function[coord,gcoord,nodes,nodetel,nnode,nel]=polygonal_domain_coordinates_3rd_order(n1,n2,n3,nmax,numtri,n,mesh)

$\%$ n1=node number at $(0,0)$ for a choosen triangle

$\%$ n2=node number at $(1,0)$ for a choosen triangle

$\%$ n3=node number at $(0,1)$ for a choosen triangle

$\%$ eln=6-node triangles with centroid

$\%$ spqd=4-node special convex quadrilateral

$\%$ m must be even,i.e.n $=2,4,6, \ldots \ldots .$. i.e number of divisions

$\%$ nmax $=$ one plus the number of segments of the polygon

$\%$ nmax =the number of segments of the polygon plus a node interior to the polygon

$\%$ numtri=number of $\mathrm{T} 6$ triangles in each segment i.e a triangle formed by

$\%$ joining the end poits of the segment to the interior point(e.g:the centroid) of the polygon

\%PARVIZ MOIN EXAMPLE

syms U V W xi yi

syms x y

switch mesh

case $1 \%$ domain with seven triangles( 8 -nodes)

$\mathrm{x}=\operatorname{sym}([1 / 2 ; 1 / 2 ; 1 ; 1 ; 1 / 2 ; 0 ; 0 ; 0]) \%$ for MOIN EXAMPLE

$\mathrm{y}=\operatorname{sym}([1 / 2 ; 0 ; 0 ; 1 / 2 ; 1 ; 1 ; 1 / 2 ; 0]) \%$ for MOIN EXAMPLE

case $2 \%$ square domain with eight triangles(9-nodes)

$\mathrm{x}=\operatorname{sym}([1 / 2 ; 1 / 2 ; 1 ; 1 ; 1 ; 1 / 2 ; 0 ; 0 ; 0]) \% \mathrm{FOR}$ UNIT SQUARE

$\mathrm{y}=\operatorname{sym}([1 / 2 ; 0 ; 0 ; 1 / 2 ; 1 ; 1 ; 1 ; 1 / 2 ; 0]) \%$ FOR UNIT SQUARE

case 3\%square domain with four triangles(5-nodes)

$\mathrm{x}=\operatorname{sym}([1 / 2 ; 0 ; 1 ; 1 ; 0])$

$\mathrm{y}=\operatorname{sym}([1 / 2 ; 0 ; 0 ; 1 ; 1])$

case $4 \%$ square domain with eight triangles(9-nodes)

$\begin{array}{llllllllll}\% & 1 & 2 & 3 & 4 & 5 & 6 & 7 & 8 & 9\end{array}$

$\mathrm{x}=\operatorname{sym}([0 ; 0 ; 1 / 2 ; 1 / 2 ; 1 / 2 ; 0 ;-1 / 2 ;-1 / 2 ;-1 / 2]) \% \mathrm{FOR}$ UNIT SQUARE

$\mathrm{y}=\operatorname{sym}([0 ;-1 / 2 ;-1 / 2 ; 0 ; 1 / 2 ; 1 / 2 ; 1 / 2 ; 0 ;-1 / 2]) \%$ FOR UNIT SQUARE

end

[eln,spqd,rrr,nodes,nodetel]=nodaladdresses_special_convex_quadrilaterals_trial_3rd_order(n1,n2,n3,nmax,numtri,n);

$[\mathrm{U}, \mathrm{V}, \mathrm{W}]=$ generate_area_coordinate_over_the_standard_triangle $(\mathrm{n})$;

ss1='number of 6-node triangles with centroid=';

$[\mathrm{p} 1, \mathrm{q} 1]=\operatorname{size}(\mathrm{eln})$;

$\operatorname{disp}([\mathrm{ss} 1$ num2str(p1)])

$\%$ 
eln

$\%$

ss2='number of special convex quadrilaterals elements\&nodes per element =';

[nel,nnel] $=$ size $($ spqd $)$;

$\operatorname{disp}([\mathrm{ss} 2$ num2str(nel) ',' num2str(nnel)])

$\%$

spqd

$\%$

nnode $=\max (\max (\operatorname{spqd}))$;

ss $3=$ 'number of nodes of the triangular domain \& number of special quadrilaterals=';

disp([ss3 num2str(nnode) ',' num2str(nel)])

xi $(1:$ nnode, 1$)=$ zeros $($ nnode, 1$) ; y i(1:$ nnode, 1$)=$ zeros $($ nnode, 1$)$;

nitri=nmax-1;

for itri=1:nitri

disp('vertex nodes of the itri triangle')

[n1(itri,1) n2(itri,1) n3(itri,1)]

$\mathrm{x} 1=\mathrm{x}(\mathrm{n} 1(\mathrm{itri}, 1), 1)$

$\mathrm{x} 2=\mathrm{x}(\mathrm{n} 2(\mathrm{itri}, 1), 1)$

$\mathrm{x} 3=\mathrm{x}(\mathrm{n} 3(\mathrm{itri}, 1), 1)$

$\%$

$\mathrm{y} 1=\mathrm{y}(\mathrm{n} 1(\mathrm{itri}, 1), 1)$

$\mathrm{y} 2=\mathrm{y}(\mathrm{n} 2(\mathrm{itri}, 1), 1)$

$\mathrm{y} 3=\mathrm{y}(\mathrm{n} 3(\mathrm{itri}, 1), 1)$

$\operatorname{rrr}(:,:$,itri $)$

$\mathrm{U}^{\prime}$

$\mathrm{V}^{\prime}$

$\mathrm{W}^{\prime}$

$\mathrm{kk}=0$;

for $\mathrm{ii}=1: \mathrm{n}+1$

for $\mathrm{jj}=1:(\mathrm{n}+1)-(\mathrm{ii}-1)$

$\mathrm{kk}=\mathrm{kk}+1$

$\mathrm{mm}=\mathrm{rrr}(\mathrm{ii}, \mathrm{jj}$,itri);

$\mathrm{uu}=\mathrm{U}(\mathrm{kk}, 1) ; \mathrm{vv}=\mathrm{V}(\mathrm{kk}, 1) ; \mathrm{ww}=\mathrm{W}(\mathrm{kk}, 1) ;$

$\mathrm{xi}(\mathrm{mm}, 1)=\mathrm{x} 1 * \mathrm{ww}+\mathrm{x} 2 * \mathrm{uu}+\mathrm{x} 3 * \mathrm{vv}$;

$\mathrm{yi}(\mathrm{mm}, 1)=\mathrm{y} 1 * \mathrm{ww}+\mathrm{y} 2 * \mathrm{uu}+\mathrm{y} 3 * \mathrm{vv}$

end $\%$ for $\mathrm{jj}$

end $\%$ for ii

[xi yi]

$\%$ add coordinates of centroid

ne $=(\mathrm{n} / 2)^{\wedge} 2$

$\%$ stdnode $=\mathrm{kk}$;

for iii=1+(itri-1)*ne:ne*itri

$\% \mathrm{kk}=\mathrm{kk}+1$;

node1 $=\operatorname{eln}($ iii, 1$)$

node $2=\operatorname{eln}($ iii, 2$)$

node3=eln(iii,3)

$\mathrm{mm}=\mathrm{eln}(\mathrm{iii}, 7)$

$\mathrm{xi}(\mathrm{mm}, 1)=(\mathrm{xi}($ node 1,1$)+x \mathrm{i}($ node 2,1$)+x i($ node 3,1$)) / 3$;

$\mathrm{yi}(\mathrm{mm}, 1)=(\mathrm{yi}(\operatorname{node} 1,1)+\mathrm{yi}(\operatorname{node} 2,1)+\mathrm{yi}(\operatorname{node} 3,1)) / 3$;

end \%for iii

[xi yi]

end $\%$ for itri=1:nitri

for $\mathrm{mmm}=1:$ nel

$\mathrm{mmm} 1=\operatorname{nodes}(\mathrm{mmm}, 1)$

$\mathrm{mmm} 2=\operatorname{nodes}(\mathrm{mmm}, 2)$

$\mathrm{mmm} 3=\operatorname{nodes}(\mathrm{mmm}, 3)$

$\mathrm{mmm} 4=\operatorname{nodes}(\mathrm{mmm}, 4)$

$\mathrm{mmm} 5=\operatorname{nodes}(\mathrm{mmm}, 5)$

$\mathrm{mmm} 6=\operatorname{nodes}(\mathrm{mmm}, 6)$

$\mathrm{mmm} 7=\operatorname{nodes}(\mathrm{mmm}, 7)$

$\mathrm{mmm} 8=\operatorname{nodes}(\mathrm{mmm}, 8)$ 
$\mathrm{mmm} 9=\operatorname{nodes}(\mathrm{mmm}, 9)$

$\operatorname{mmm} 10=\operatorname{nodes}(\mathrm{mmm}, 10)$

$\operatorname{mmm} 11=\operatorname{nodes}(\mathrm{mmm}, 11)$

$\operatorname{mmm} 12=\operatorname{nodes}(\mathrm{mmm}, 12)$

$\mathrm{xi} 1=\mathrm{xi}(\mathrm{mmm} 1,1)$

$\mathrm{xi} 2=\mathrm{xi}(\mathrm{mmm} 2,1)$

$\mathrm{xi} 3=\mathrm{xi}(\mathrm{mmm} 3,1)$

$\mathrm{xi} 4=\mathrm{xi}(\mathrm{mmm} 4,1)$

$\%$

yi1=yi $(\mathrm{mmm} 1,1)$

yi2 $=$ yi $(\mathrm{mmm} 2,1)$

yi3 $=y i(m m m 3,1)$

yi4=yi $(\mathrm{mmm} 4,1)$

$\%$

$\mathrm{xi}(\mathrm{mmm} 5,1)=(2 * \mathrm{xi} 1+\mathrm{xi} 2) / 3 ; \mathrm{xi}(\mathrm{mmm} 6,1)=(\mathrm{xi} 1+2 * \mathrm{xi} 2) / 3$;

$\mathrm{xi}(\mathrm{mmm} 7,1)=(2 * \mathrm{xi} 2+\mathrm{xi} 3) / 3 ; \mathrm{xi}(\mathrm{mmm} 8,1)=(\mathrm{xi} 2+2 * \mathrm{xi} 3) / 3$;

$\mathrm{xi}(\mathrm{mmm} 9,1)=(2 * \mathrm{xi} 3+\mathrm{xi} 4) / 3 ; \mathrm{xi}(\mathrm{mmm} 10,1)=(\mathrm{xi} 3+2 * \mathrm{xi} 4) / 3$;

$\mathrm{xi}(\mathrm{mmm} 11,1)=(2 * x i 4+x i 1) / 3 ; x i(\mathrm{mmm} 12,1)=(x i 4+2 * x i 1) / 3$;

$\mathrm{yi}(\mathrm{mmm} 5,1)=(2 * \mathrm{yi} 1+\mathrm{yi} 2) / 3 ; \mathrm{yi}(\mathrm{mmm} 6,1)=(\mathrm{yi} 1+2 * \mathrm{yi} 2) / 3$;

$\mathrm{yi}(\mathrm{mmm} 7,1)=(2 * \mathrm{yi} 2+\mathrm{yi} 3) / 3 ; \mathrm{yi}(\mathrm{mmm} 8,1)=(\mathrm{yi} 2+2 * \mathrm{yi} 3) / 3$;

$\mathrm{yi}(\mathrm{mmm} 9,1)=(2 * \mathrm{yi} 3+\mathrm{yi} 4) / 3 ; \mathrm{yi}(\mathrm{mmm} 10,1)=(\mathrm{yi} 3+2 * \mathrm{yi} 4) / 3$;

$\mathrm{yi}(\mathrm{mmm} 11,1)=(2 * \mathrm{yi} 4+\mathrm{yi} 1) / 3 ; \mathrm{yi}(\mathrm{mmm} 12,1)=(\mathrm{yi} 4+2 * \mathrm{yi} 1) / 3$;

end $\%$ for nel

$\%[x i(18,1)$ yi $(18,1)]$

$\mathrm{N}=(1: \text { nnode })^{\prime}$

[N xi yi]

$\%$

$\operatorname{coord}(:, 1)=(x i(:, 1))$;

$\operatorname{coord}(:, 2)=(\mathrm{yi}(:, 1))$;

$\operatorname{gcoord}(:, 1)=\operatorname{double}(x i(:, 1))$;

$\operatorname{gcoord}(:, 2)=\operatorname{double}(\mathrm{yi}(:, 1))$;

$\% \operatorname{disp}($ gcoord $)$

[11]coordinate_special_quadrilaterals_in_stdtriangle_3rd_orderLAGR.m

function[ui,vi,wi]=coordinate_special_quadrilaterals_in_stdtriangle_3rd_orderLAGR(n)

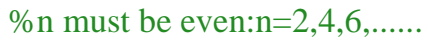

syms ui vi wi

ui $(1: 3,1)=[0 ; 1 ; 0]$;

$\operatorname{vi}(1: 3,1)=[0 ; 0 ; 1]$

$\operatorname{wi}(1: 3,1)=[1 ; 0 ; 0]$;

if $(\mathrm{n}-1)>0$

$\mathrm{kk}=3$;

for $\mathrm{i}=1: \mathrm{n}-1$

$\mathrm{kk}=\mathrm{kk}+1$;

ui $(\mathrm{kk}, 1)=\operatorname{sym}(\mathrm{i} / \mathrm{n})$;

$\operatorname{vi}(\mathrm{kk}, 1)=\operatorname{sym}(0)$;

$\operatorname{wi}(k k, 1)=\operatorname{sym}(1-u i(k k, 1)-v i(k k, 1))$;

end

$\mathrm{kkk}=\mathrm{kk}$;

for $\mathrm{ii}=1: \mathrm{n}-1$

$\mathrm{kkk}=\mathrm{kkk}+1$;

ui $(\mathrm{kkk}, 1)=\operatorname{sym}((\mathrm{n}-\mathrm{ii}) / \mathrm{n})$;

$\operatorname{vi}(\mathrm{kkk}, 1)=\operatorname{sym}(1-(\mathrm{n}-\mathrm{ii}) / \mathrm{n})$;

wi $(\mathrm{kkk}, 1)=0$;

end;

$\mathrm{kkkk}=\mathrm{kkk}$;

for iii=1:n-1

$\mathrm{kkkk}=\mathrm{kkkk+1}$;

ui $(\mathrm{kkkk}, 1)=0$;

$\operatorname{vi}(k k k k, 1)=\operatorname{sym}(1-\mathrm{iii} / \mathrm{n})$;

wi $(k k k k, 1)=\operatorname{sym}($ iii $/ \mathrm{n})$;

end

end $\%$ if $(n-1)>0$

if $(n-2)>0$ 


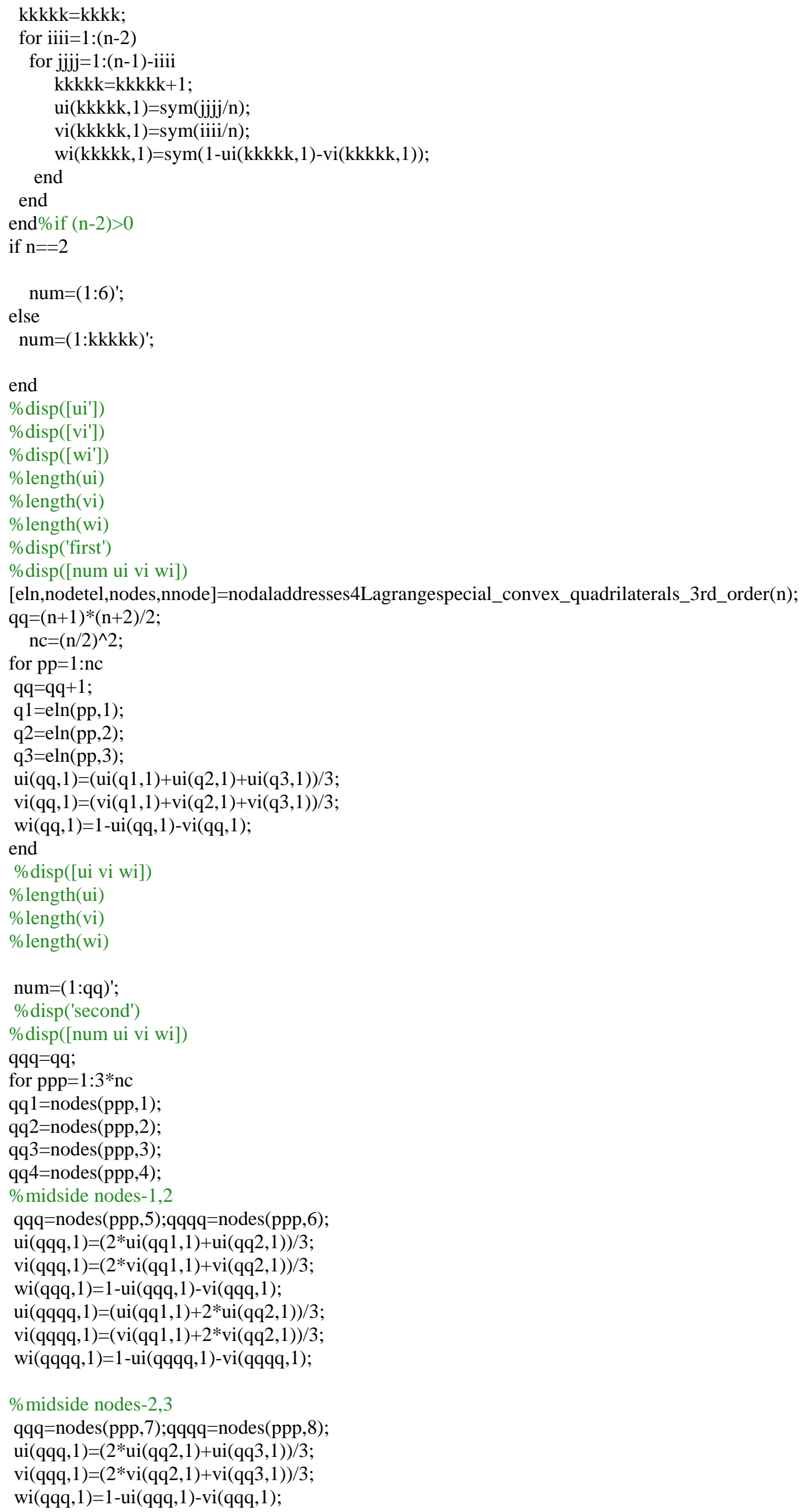


ui $(q q q q, 1)=(\operatorname{ui}(q q 2,1)+2 * u i(q q 3,1)) / 3$;

$\operatorname{vi}(q q q q, 1)=(\operatorname{vi}(q q 2,1)+2 * \operatorname{vi}(q q 3,1)) / 3$;

wi(qqqq, 1)=1-ui(qqqq,1)-vi(qqqq,1);

$\%$ midside nodes- 3,4

qqq=nodes(ppp,9);qqqq=nodes(ppp,10);

ui $(q q q, 1)=(2 * u i(q q 3,1)+u i(q q 4,1)) / 3$;

$\operatorname{vi}(q q q, 1)=(2 * v i(q q 3,1)+v i(q q 4,1)) / 3$;

wi(qqq,1)=1-ui(qqq,1)-vi(qqq,1);

ui $(q q q q, 1)=($ ui $(q q 3,1)+2 * u i(q q 4,1)) / 3$;

$\operatorname{vi}(q q q q, 1)=(\operatorname{vi}(q q 3,1)+2 * \operatorname{vi}(q q 4,1)) / 3$;

wi(qqqq,1)=1-ui(qqqq,1)-vi(qqqq,1);

$\%$ midside nodes- 4,1

$\mathrm{qqq}=\operatorname{nodes}(\mathrm{ppp}, 11) ; \mathrm{qqqq}=\operatorname{nodes}(\mathrm{ppp}, 12)$;

ui $(q q q, 1)=(2 * u i(q q 4,1)+u i(q q 1,1)) / 3$;

$\operatorname{vi}(q q q, 1)=(2 * v i(q q 4,1)+v i(q q 1,1)) / 3$;

wi(qqq, 1)=1-ui(qqq,1)-vi(qqq,1);

ui $(q q q q, 1)=($ ui $(q q 4,1)+2 * u i(q q 1,1)) / 3$;

$\operatorname{vi}(q q q q, 1)=(\operatorname{vi}(q q 4,1)+2 * \operatorname{vi}(q q 1,1)) / 3$;

wi(qqqq,1)=1-ui(qqqq,1)-vi(qqqq,1);

\%interior nodes

$\mathrm{q} 1=\operatorname{nodes}(\mathrm{ppp}, 13)$

$\mathrm{q} 2=\operatorname{nodes}(\mathrm{ppp}, 14)$;

$\mathrm{q} 3=\operatorname{nodes}(\mathrm{ppp}, 15)$;

$\mathrm{q} 4=\operatorname{nodes}(\mathrm{ppp}, 16)$

$\%$

ui (q1,1) $=(4 * u i(q q 1,1)+2 * u i(q q 2,1)+u i(q q 3,1)+2 * u i(q q 4,1)) / 9$;

$\operatorname{vi}(\mathrm{q} 1,1)=(4 * \mathrm{vi}(\mathrm{qq} 1,1)+2 * \mathrm{vi}(\mathrm{qq} 2,1)+\mathrm{vi}(\mathrm{qq} 3,1)+2 * \mathrm{vi}(\mathrm{qq} 4,1)) / 9$;

wi(q1,1)=1-ui(q1,1)-vi(q1,1);

$\%$

ui (q2,1) $=(2 * u i(q q 1,1)+4 * u i(q q 2,1)+2 * u i(q q 3,1)+u i(q q 4,1)) / 9$; $\operatorname{vi}(\mathrm{q} 2,1)=(2 * \mathrm{vi}(\mathrm{qq} 1,1)+4 * \mathrm{vi}(\mathrm{qq} 2,1)+2 * \operatorname{vi}(\mathrm{qq} 3,1)+\mathrm{vi}(\mathrm{qq} 4,1)) / 9$; wi(q2,1)=1-ui(q2,1)-vi(q2,1);

$\%$

ui $(q 3,1)=($ ui $(q q 1,1)+2 * u i(q q 2,1)+4 * u i(q q 3,1)+2 * u i(q q 4,1)) / 9$; $\operatorname{vi}(\mathrm{q} 3,1)=(\operatorname{vi}(\mathrm{qq} 1,1)+2 * \mathrm{vi}(\mathrm{qq} 2,1)+4 * \mathrm{vi}(\mathrm{qq} 3,1)+2 * \mathrm{vi}(\mathrm{qq} 4,1)) / 9$; wi(q3,1)=1-ui(q3,1)-vi(q3,1);

$\%$

ui $(\mathrm{q} 4,1)=(2 *$ ui $(\mathrm{qq} 1,1)+$ ui $(\mathrm{qq} 2,1)+2 *$ ui (qq3,1)+4*ui(qq4,1))/9; $\operatorname{vi}(\mathrm{q} 4,1)=(2 * \mathrm{vi}(\mathrm{qq} 1,1)+\mathrm{vi}(\mathrm{qq} 2,1)+2 * \mathrm{vi}(\mathrm{qq} 3,1)+4 * \mathrm{vi}(\mathrm{qq} 4,1)) / 9$; wi $(q 4,1)=1$-ui $(q 4,1)$-vi(q4,1);

end

$\operatorname{maxnode}=\max (\max (\operatorname{nodes}(1: 3 * \mathrm{nc}, 1: 16)))$;

num $=(1: \text { maxnode })^{\prime}$;

$\%$ disp(['maximum value of node number=',num2str(maxnode)]

\%disp('node ui vi wi')

$\%$ disp([num ui vi wi])

[12]coordinate_special_quadrilaterals_in_stdtriangle_3rd_order.m

function[ui,vi,wi]=coordinate_special_quadrilaterals_in_stdtriangle_3rd_order(n)

$\%$ must be even: $n=2,4,6, \ldots \ldots$

syms ui vi wi

ui $(1: 3,1)=[0 ; 1 ; 0]$;

$\operatorname{vi}(1: 3,1)=[0 ; 0 ; 1] ;$

$\operatorname{wi}(1: 3,1)=[1 ; 0 ; 0]$;

if $(\mathrm{n}-1)>0$

$\mathrm{kk}=3$;

for $\mathrm{i}=1: \mathrm{n}-1$

$\mathrm{kk}=\mathrm{kk}+1$;

ui $(\mathrm{kk}, 1)=\operatorname{sym}(\mathrm{i} / \mathrm{n})$;

$\operatorname{vi}(\mathrm{kk}, 1)=\operatorname{sym}(0)$;

wi(kk,1)=sym(1-ui(kk,1)-vi(kk,1));

end

$\mathrm{kkk}=\mathrm{kk}$;

for ii $=1: \mathrm{n}-1$

$\mathrm{kkk}=\mathrm{kkk}+1$; 


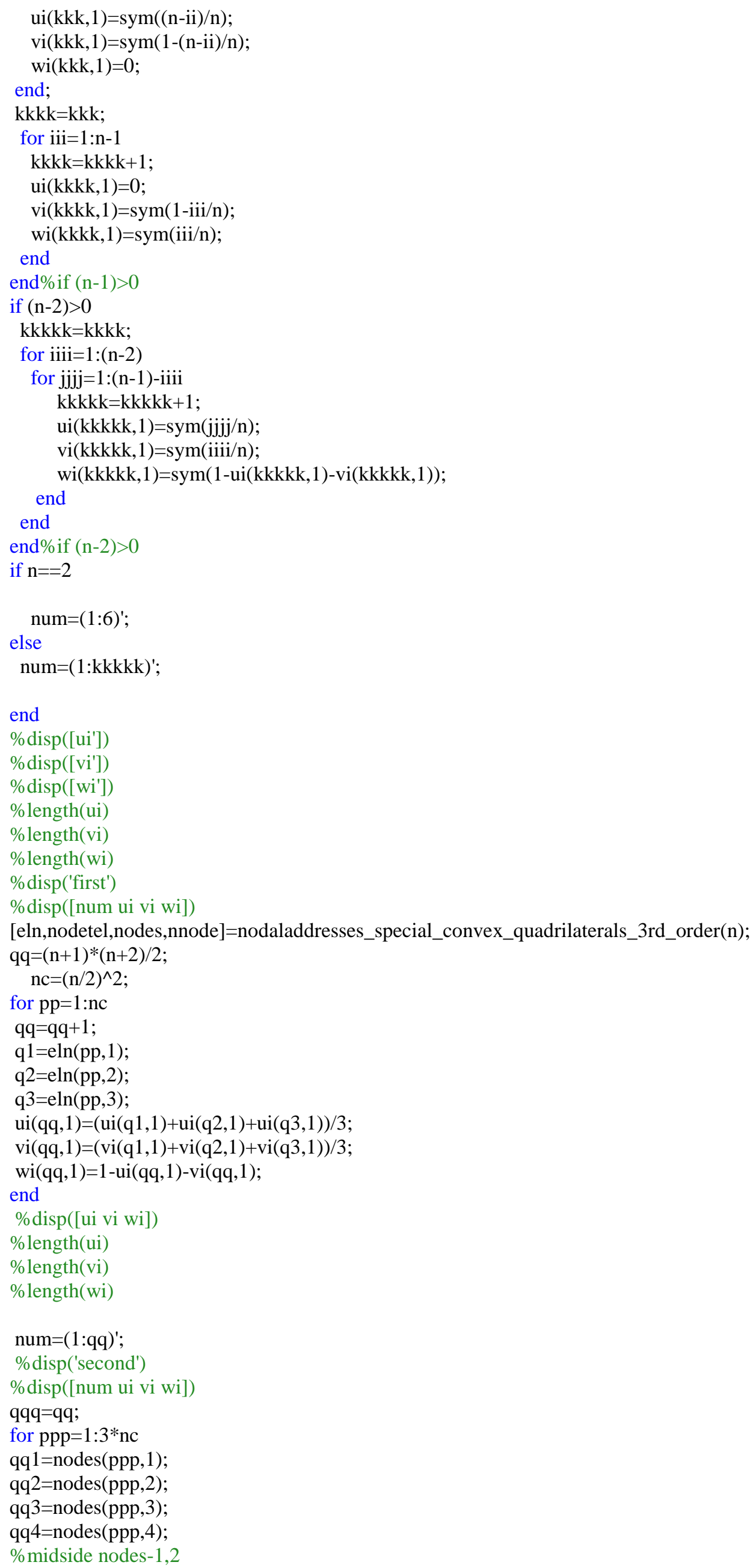


qqq=nodes(ppp,5);qqqq=nodes(ppp,6); ui $(q q q, 1)=(2 * u i(q q 1,1)+u i(q q 2,1)) / 3$; $\operatorname{vi}(q q q, 1)=(2 * \operatorname{vi}(q q 1,1)+\operatorname{vi}(q q 2,1)) / 3$; wi(qqq, 1)=1-ui(qqq,1)-vi(qqq, 1); ui $(q q q q, 1)=($ ui $(q q 1,1)+2 * u i(q q 2,1)) / 3$; $\operatorname{vi}(q q q q, 1)=(\operatorname{vi}(q q 1,1)+2 * \operatorname{vi}(q q 2,1)) / 3$; wi(qqqq,1)=1-ui(qqqq,1)-vi(qqqq,1);

$\%$ midside nodes-2,3

qqq=nodes(ppp,7);qqqq=nodes(ppp,8); ui $(q q q, 1)=(2 * u i(q q 2,1)+u i(q q 3,1)) / 3$; $\operatorname{vi}(q q q, 1)=(2 * \operatorname{vi}(q q 2,1)+\operatorname{vi}(q q 3,1)) / 3$; wi(qqq, 1)=1-ui(qqq, 1)-vi(qqq, 1); ui $(q q q q, 1)=($ ui $(q q 2,1)+2 * u i(q q 3,1)) / 3$; $\operatorname{vi}(q q q q, 1)=(\operatorname{vi}(q q 2,1)+2 * \operatorname{vi}(q q 3,1)) / 3$; wi(qqqq,1)=1-ui(qqqq,1)-vi(qqqq,1); $\%$ midside nodes-3,4 qqq=nodes $(p p p, 9) ; q q q q=\operatorname{nodes}(p p p, 10)$; ui $(q q q, 1)=(2 * u i(q q 3,1)+u i(q q 4,1)) / 3$; vi(qqq, 1$)=(2 * \operatorname{vi}(q q 3,1)+v i(q q 4,1)) / 3$; wi $(q q q, 1)=1$-ui(qqq,1)-vi(qqq, 1); ui $(q q q q, 1)=($ ui $(q q 3,1)+2 * u i(q q 4,1)) / 3$; $\operatorname{vi}(q q q q, 1)=(\operatorname{vi}(q q 3,1)+2 * \operatorname{vi}(q q 4,1)) / 3$; wi(qqqq, 1)=1-ui(qqqq,1)-vi(qqqq,1); $\%$ midside nodes-4, 1

$\mathrm{qqq}=\operatorname{nodes}(\mathrm{ppp}, 11) ; \mathrm{qqqq}=\operatorname{nodes}(\mathrm{ppp}, 12)$; ui $(q q q, 1)=(2 * u i(q q 4,1)+u i(q q 1,1)) / 3$; $\operatorname{vi}(q q q, 1)=(2 * \operatorname{vi}(q q 4,1)+\operatorname{vi}(q q 1,1)) / 3$; wi(qqq, 1)=1-ui(qqq,1)-vi(qqq,1); ui $(q q q q, 1)=($ ui $(q q 4,1)+2 * u i(q q 1,1)) / 3$; $\operatorname{vi}(q q q q, 1)=(\operatorname{vi}(q q 4,1)+2 * \operatorname{vi}(q q 1,1)) / 3$; wi(qqqq,1)=1-ui(qqqq,1)-vi(qqqq,1); end

$\operatorname{maxnode}=\max (\max (\operatorname{nodes}(1: 3 * \mathrm{nc}, 1: 12)))$; num $=(1: \text { maxnode })^{\prime}$;

$\%$ disp(['maximum value of node number=',num2str(maxnode)])

$\% \operatorname{disp}('$ node ui vi wi')

$\% \operatorname{disp}([$ num ui vi wi])

\section{[13]integral_valuesof_localderivative_products.m}

function[intJdndn]=integral_valuesof_localderivative_products(nnel)

switch nnel

case 12

\%integral values of local derivative products

intJdn1dn1uvrs $=\left[\operatorname{vpa}(\operatorname{sym}(' \quad 1.8785274341641741281222496546 ')) \operatorname{vpa}\left(\operatorname{sym}\left({ }^{\prime} 1.64496677534890315539341209663 '\right)\right) ; \ldots\right.$ vpa(sym(' 1.64496677534890315539341209663')) vpa(sym(' 1.8785274341641741281222496546'))];

intJdn1dn2uvrs $=\left[\operatorname{vpa}\left(\operatorname{sym}\left({ }^{\prime} .24392368058084020798114240290 '\right)\right) \operatorname{vpa}\left(\operatorname{sym}\left({ }^{\prime} .25456419075959891292341644890 '\right)\right) ; \ldots\right.$ vpa(sym(' .16706419075959891292341644890')) vpa(sym(' .4941299057543551745397431605'))];

intJdn1dn3uvrs =[vpa(sym(' .65143728569018129264176158009e-1')) vpa(sym(' -.8063499940854168581340003167e-1'));... vpa(sym(' -.8063499940854168581340003167e-1')) vpa(sym(' .65143728569018129264176158009e-1'))];

intJdn1dn4uvrs $=\left[\operatorname{vpa}\left(\operatorname{sym}\left({ }^{\prime} .4941299057543551745397431605 '\right)\right) \operatorname{vpa}\left(\operatorname{sym}\left({ }^{\prime} .16706419075959891292341644890 '\right)\right) ; \ldots\right.$ $\left.\operatorname{vpa}\left(\operatorname{sym}\left({ }^{\prime} .25456419075959891292341644890 '\right)\right) \operatorname{vpa}\left(\operatorname{sym}\left({ }^{\prime} .24392368058084020798114240290 '\right)\right)\right]$;

intJdn1dn5uvrs $=\left[\operatorname{vpa}\left(\operatorname{sym}\left({ }^{\prime}-1.91709736541803286033938722609 '\right)\right) \mathrm{vpa}\left(\operatorname{sym}\left({ }^{\prime}-.50073891821327672848240784486 '\right)\right) ; \ldots\right.$ $\operatorname{vpa}(\operatorname{sym}('-1.21323891821327672848240784486 '))$ vpa(sym(' $\quad-.489515939250703449364685749 '))]$;

intJdn1dn6uvrs $=\left[\operatorname{vpa}\left(\operatorname{sym}\left({ }^{\prime} \quad .377654219333168620525401790571^{\prime}\right)\right) \operatorname{vpa}\left(\operatorname{sym}\left({ }^{\prime}-.7885728723191145226859121413 \mathrm{e}-1^{\prime}\right)\right) ; \ldots\right.$ vpa(sym(' .22114271276808854773140878587')) vpa(sym(' .471024620459754344696508823e-1'))];

intJdn1dn7uvrs $=\left[\operatorname{vpa}\left(\operatorname{sym}\left({ }^{\prime}-.31236802364420048787164339097 '\right)\right) \operatorname{vpa}\left(\operatorname{sym}\left({ }^{\prime}-.622591925470292070597183972271 '\right)\right) ; \ldots\right.$ vpa(sym(' -.622591925470292070597183972271')) vpa(sym(' -.93264116705106716805029551279'))];

intJdn1dn8uvrs $=\left[\operatorname{vpa}\left(\operatorname{sym}\left({ }^{\prime} .22334568260811342445267275929 '\right)\right) \operatorname{vpa}\left(\operatorname{sym}\left({ }^{\prime} .415458052185700603634760549957 '\right)\right) ; \ldots\right.$ $\left.\operatorname{vpa}\left(\operatorname{sym}\left({ }^{\prime} .415458052185700603634760549957 '\right)\right) \operatorname{vpa}\left(\operatorname{sym}\left({ }^{\prime} .321795382308358846270975070300 '\right)\right)\right] ;$

intJdn1dn9uvrs $=\left[\operatorname{vpa}\left(\operatorname{sym}\left({ }^{\prime} .321795382308358846270975070300 '\right)\right) \operatorname{vpa}\left(\operatorname{sym}\left({ }^{\prime} .415458052185700603634760549957 '\right)\right) ; \ldots\right.$ vpa(sym(' .415458052185700603634760549957')) vpa(sym(' .22334568260811342445267275929'))];

intJdn1dn10uvrs $=\left[\operatorname{vpa}\left(\operatorname{sym}\left({ }^{\prime}-.93264116705106716805029551279 '\right)\right) \operatorname{vpa}(\operatorname{sym}('-.622591925470292070597183972271 ')) ; \ldots\right.$ vpa(sym(' -.622591925470292070597183972271')) vpa(sym(' -.31236802364420048787164339097'))];

intJdn1dn11uvrs =[vpa(sym(' $\left.\left.\quad .471024620459754344696508823 \mathrm{e}-1^{\prime}\right)\right)$ vpa(sym(' $\left.\left..22114271276808854773140878587 '\right)\right) ; \ldots$ 
$\left.\operatorname{vpa}\left(\operatorname{sym}\left({ }^{\prime}-.7885728723191145226859121413 e-1^{\prime}\right)\right) \operatorname{vpa}\left(\operatorname{sym}\left({ }^{\prime} \quad .377654219333168620525401790571 '\right)\right)\right]$;

intJdn1dn12uvrs $=\left[\operatorname{vpa}\left(\operatorname{sym}\left({ }^{\prime} \quad-.489515939250703449364685749 '\right)\right) \operatorname{vpa}\left(\operatorname{sym}\left({ }^{\prime}-1.21323891821327672848240784486 '\right)\right) ; \ldots\right.$ $\operatorname{vpa}\left(\operatorname{sym}\left({ }^{\prime}-.50073891821327672848240784486 '\right)\right)$ vpa(sym(' -1.91709736541803286033938722609'))];

intJdn2dn1uvrs $=\left[\operatorname{vpa}\left(\operatorname{sym}\left({ }^{\prime} .24392368058084020798114240290 '\right)\right) \operatorname{vpa}\left(\operatorname{sym}\left({ }^{\prime} .16706419075959891292341644890 '\right)\right) ; \ldots\right.$ $\operatorname{vpa}(\operatorname{sym}(' .25456419075959891292341644890 ')) \operatorname{vpa}(\operatorname{sym}(' .4941299057543551745397431605 '))] ;$

intJdn2dn2uvrs $=\left[\operatorname{vpa}\left(\operatorname{sym}\left({ }^{\prime} .927247441752334105622080795189 '\right)\right) \operatorname{vpa}\left(\operatorname{sym}\left({ }^{\prime}-.15481341653816302516393935759 '\right)\right) ; \ldots\right.$ $\left.\operatorname{vpa}\left(\operatorname{sym}\left({ }^{\prime}-.15481341653816302516393935759 '\right)\right) \operatorname{vpa}\left(\operatorname{sym}\left({ }^{\prime} \quad .7947159164781167997293081128^{\prime}\right)\right)\right]$;

intJdn2dn3uvrs =[vpa(sym(' $\quad .512461423425383462495731239596 ')) \operatorname{vpa}\left(\operatorname{sym}\left({ }^{\prime} \quad .121011592735232346794931827124 '\right)\right) ; \ldots$ $\left.\operatorname{vpa}\left(\operatorname{sym}\left({ }^{\prime} .33511592735232346794931827124 \mathrm{e}-1^{\prime}\right)\right) \operatorname{vpa}(\operatorname{sym}(' \quad .156606852059463586417315376026 '))\right]$;

intJdn2dn4uvrs $=\left[\operatorname{vpa}\left(\operatorname{sym}\left({ }^{\prime} .16985339992712677743504195136 '\right)\right) \operatorname{vpa}\left(\operatorname{sym}\left({ }^{\prime} .179045055450002326957831462744 '\right)\right) ; \ldots\right.$. $\operatorname{vpa}(\operatorname{sym}(' .179045055450002326957831462744 '))$ vpa(sym(' .16985339992712677743504195136'))];

intJdn2dn5uvrs $=\left[\operatorname{vpa}\left(\operatorname{sym}\left({ }^{\prime} .737450284572716169349341246171 '\right)\right) \operatorname{vpa}\left(\operatorname{sym}\left({ }^{\prime} \quad .36072316479445118067281174443 '\right)\right) ; \ldots\right.$ vpa(sym(' .6072316479445118067281174443e-1')) vpa(sym(' .676185160002392642047802966e-1'))];

intJdn2dn6uvrs $=\left[\operatorname{vpa}\left(\operatorname{sym}\left({ }^{\prime}-1.895073448163238983109870698571^{\prime}\right)\right) \mathrm{vpa}\left(\operatorname{sym}\left({ }^{\prime} \quad-.32947059876161316868575194333 '\right)\right) ; \ldots\right.$ vpa(sym(' .38302940123838683131424805667')) vpa(sym(' -.610174037597342509867071996e-1'))];

intJdn2dn7uvrs =[vpa(sym(' .89381759607913761072441622471e-1')) vpa(sym(' -.3294201189536998617617117680e-1'));... $\operatorname{vpa}(\operatorname{sym}(' \quad-.74544201189536998617617117680$ ')) vpa(sym(' $-1.32051059158026743632811545266 '))]$;

intJdn2dn8uvrs $=\left[\operatorname{vpa}\left(\operatorname{sym}\left({ }^{\prime} .66028785385703773347942390579 \mathrm{e}-1^{\prime}\right)\right) \operatorname{vpa}\left(\operatorname{sym}\left({ }^{\prime} \quad .132773165331780684038772441541 '\right)\right) ; \ldots\right.$ $\operatorname{vpa(sym('~.432773165331780684038772441541'))\operatorname {vpa}(\operatorname {sym}('\quad .364521033839059598529804469259'))];}$

intJdn2dn9uvrs =[vpa(sym(' -.820137977578506638706811693089')) vpa(sym(' .1022830353271046354061602393186')); ... $\operatorname{vpa}(\operatorname{sym}(' .1022830353271046354061602393186 '))$ vpa(sym(' .3640416697255654640867267668e-1'))];

intJdn2dn10uvrs $=\left[\operatorname{vpa}\left(\operatorname{sym}\left(' \quad .14453334544915567441376932857^{\prime}\right)\right) \operatorname{vpa}\left(\operatorname{sym}\left({ }^{\prime}-.3273205972269859328464496028286 '\right)\right) ; \ldots\right.$. $\left.\operatorname{vpa}\left(\operatorname{sym}\left({ }^{\prime}-.3273205972269859328464496028286 '\right)\right) \operatorname{vpa}\left(\operatorname{sym}\left({ }^{\prime} \quad-.10277602051817855644721686707 '\right)\right)\right]$;

intJdn2dn11uvrs $=\left[\operatorname{vpa}\left(\operatorname{sym}\left({ }^{\prime}-.7898220003698491703637636243 e-1^{\prime}\right)\right) \operatorname{vpa}\left(\operatorname{sym}\left({ }^{\prime}-.212192568049325297169223465371 '\right)\right) ; \ldots\right.$ vpa(sym(' -.212192568049325297169223465371')) vpa(sym(' .62364758048803624976125527371e-1'))];

intJdn2dn12uvrs $=\left[\operatorname{vpa}\left(\operatorname{sym}\left({ }^{\prime} \quad-.966864949224433928644322229 \mathrm{e}-1^{\prime}\right)\right) \operatorname{vpa}\left(\operatorname{sym}\left({ }^{\prime}-.6161011926712676752388618229 \mathrm{e}-2\right.\right.\right.$ ') $) ; \ldots$ vpa(sym(' -.6161011926712676752388618229e-2')) vpa(sym(' $\quad-.66191053322154112847875205137$ '))];

intJdn3dn1uvrs $=\left[\operatorname{vpa}\left(\operatorname{sym}\left(' \quad .65143728569018129264176158009 \mathrm{e}-1^{\prime}\right)\right) \mathrm{vpa}\left(\operatorname{sym}\left({ }^{\prime}-.80634999408541685813400031669 \mathrm{e}-1^{\prime}\right)\right) ; \ldots\right.$ $\left.\operatorname{vpa}\left(\operatorname{sym}\left({ }^{\prime}-.80634999408541685813400031669 \mathrm{e}-1^{\prime}\right)\right) \operatorname{vpa}\left(\operatorname{sym}\left({ }^{\prime} .65143728569018129264176158009 \mathrm{e}-1^{\prime}\right)\right)\right]$;

intJdn3dn2uvrs $=\left[\operatorname{vpa}\left(\operatorname{sym}\left({ }^{\prime} \quad .512461423425383462495731239596 '\right)\right) \operatorname{vpa}\left(\operatorname{sym}\left({ }^{\prime} .33511592735232346794931827124 \mathrm{e}-1^{\prime}\right)\right) ; \ldots\right.$ vpa(sym(' .121011592735232346794931827124')) vpa(sym(' .156606852059463586417315376026'))];

intJdn3dn3uvrs $=\left[\operatorname{vpa}(\operatorname{sym}(' 1.0542668070025615997463144835803 ')) \operatorname{vpa}\left(\operatorname{sym}\left({ }^{\prime} \quad .675139101205863811454268346790 '\right)\right) ; \ldots\right.$ vpa(sym(' .675139101205863811454268346790')) vpa(sym(' 1.0542668070025615997463144835803'))];

intJdn3dn4uvrs $=\left[\operatorname{vpa}\left(\operatorname{sym}\left({ }^{\prime} \quad .156606852059463586417315376026 '\right)\right) \operatorname{vpa}\left(\operatorname{sym}\left({ }^{\prime} \quad .121011592735232346794931827124 '\right)\right) ; \ldots\right.$ $\left.\operatorname{vpa}\left(\operatorname{sym}\left({ }^{\prime} .33511592735232346794931827124 \mathrm{e}-1^{\prime}\right)\right) \operatorname{vpa}(\operatorname{sym}(' \quad .512461423425383462495731239596 '))\right]$;

intJdn3dn5uvrs $=\left[\operatorname{vpa}\left(\operatorname{sym}\left({ }^{\prime} .42220907054407935850896000532 '\right)\right) \operatorname{vpa}\left(\operatorname{sym}\left({ }^{\prime} .338079890317619378517715472657 '\right)\right) ; \ldots\right.$ $\left.\operatorname{vpa}\left(\operatorname{sym}\left({ }^{\prime} .338079890317619378517715472657 '\right)\right) \operatorname{vpa}\left(\operatorname{sym}\left({ }^{\prime} .12092398059703989381526413440 '\right)\right)\right] ;$

intJdn3dn6uvrs $=\left[\operatorname{vpa}\left(\operatorname{sym}\left({ }^{\prime}-.97811352194183953055704604912^{\prime}\right)\right) \operatorname{vpa}\left(\operatorname{sym}\left({ }^{\prime}-.271075459705479523507647905621 '\right)\right) ; \ldots\right.$ $\operatorname{vpa}(\operatorname{sym}('-.271075459705479523507647905621$ ')) vpa(sym(' -.16559436051923997292216207590'))];

intJdn3dn7uvrs $=\left[\operatorname{vpa}\left(\operatorname{sym}\left({ }^{\prime}-.97451565507866331982099391909 \mathrm{e}-1^{\prime}\right)\right) \mathrm{vpa}\left(\operatorname{sym}\left({ }^{\prime}-.2538103955586380338692247539 \mathrm{e}-2^{\prime}\right)\right) ; \ldots\right.$ $\operatorname{vpa}(\operatorname{sym}(' \quad-.302538103955586380338692247539 '))$ vpa(sym(' $.463447558533213614696680089166 '))]$;

intJdn3dn8uvrs =[vpa(sym(' .99543897060373053107624738884e-1')) vpa(sym(' -.645229970290446884286741304267'));... $\left.\operatorname{vpa}\left(\operatorname{sym}\left(' .67270029709553115713258695733 \mathrm{e}-1^{\prime}\right)\right) \operatorname{vpa}\left(\operatorname{sym}\left({ }^{\prime}-1.653443869822186862590758708100 '\right)\right)\right]$;

intJdn3dn9uvrs $=\left[\operatorname{vpa}\left(\operatorname{sym}\left({ }^{\prime}-1.653443869822186862590758708100 '\right)\right) \operatorname{vpa}\left(\operatorname{sym}\left({ }^{\prime} .67270029709553115713258695733 \mathrm{e}-1^{\prime}\right)\right) ; \ldots\right.$ $\operatorname{vpa}(\operatorname{sym}('-.645229970290446884286741304267$ ')) vpa(sym(' .99543897060373053107624738884e-1'))];

intJdn3dn10uvrs $=\left[\operatorname{vpa}\left(\operatorname{sym}\left({ }^{\prime} \quad .463447558533213614696680089166 '\right)\right) \operatorname{vpa}\left(\operatorname{sym}\left({ }^{\prime} \quad-.302538103955586380338692247539 '\right)\right) ; \ldots\right.$ $\left.\operatorname{vpa}\left(\operatorname{sym}\left({ }^{\prime}-.2538103955586380338692247539 \mathrm{e}-2^{\prime}\right)\right) \operatorname{vpa}\left(\operatorname{sym}\left({ }^{\prime}-.97451565507866331982099391909 \mathrm{e}-1^{\prime}\right)\right)\right]$;

intJdn3dn11uvrs $=\left[\operatorname{vpa}\left(\operatorname{sym}\left({ }^{\prime}-.165594360519239972922162075896 '\right)\right) \operatorname{vpa}\left(\operatorname{sym}\left({ }^{\prime}-.271075459705479523507647905621 '\right)\right) ; \ldots\right.$ $\left.\operatorname{vpa}\left(\operatorname{sym}\left({ }^{\prime}-.271075459705479523507647905621 '\right)\right) \operatorname{vpa}\left(\operatorname{sym}\left({ }^{\prime}-.97811352194183953055704604912 '\right)\right)\right]$;

intJdn3dn12uvrs $=\left[\operatorname{vpa}\left(\operatorname{sym}\left({ }^{\prime} .12092398059703989381526413440 '\right)\right) \operatorname{vpa}\left(\operatorname{sym}\left({ }^{\prime} .338079890317619378517715472657 '\right)\right) ; \ldots\right.$. $\left.\operatorname{vpa}\left(\operatorname{sym}\left({ }^{\prime} .338079890317619378517715472657 '\right)\right) \operatorname{vpa}\left(\operatorname{sym}\left({ }^{\prime} .42220907054407935850896000532 '\right)\right)\right] ;$

intJdn4dn1uvrs $=\left[\operatorname{vpa}\left(\operatorname{sym}\left({ }^{\prime} .4941299057543551745397431605 '\right)\right) \mathrm{vpa}\left(\operatorname{sym}\left({ }^{\prime} .25456419075959891292341644890^{\prime}\right)\right) ; \ldots\right.$ vpa(sym(' .16706419075959891292341644890')) vpa(sym(' .24392368058084020798114240290'))];

intJdn4dn2uvrs $=\left[\operatorname{vpa}\left(\operatorname{sym}\left({ }^{\prime} .16985339992712677743504195136 '\right)\right) \operatorname{vpa}\left(\operatorname{sym}\left({ }^{\prime} .179045055450002326957831462744 '\right)\right) ; \ldots\right.$. $\operatorname{vpa}\left(\operatorname{sym}\left({ }^{\prime} .179045055450002326957831462744 '\right)\right)$ vpa(sym(' .16985339992712677743504195136'))];

intJdn4dn3uvrs =[vpa(sym(' $.156606852059463586417315376026 '))$ vpa(sym(' .33511592735232346794931827124e-1'));... vpa(sym(' .121011592735232346794931827124')) vpa(sym(' .512461423425383462495731239596'))];

intJdn4dn4uvrs $=\left[\operatorname{vpa}(\operatorname{sym}(' \quad .7947159164781167997293081128 ')) \operatorname{vpa}\left(\operatorname{sym}\left({ }^{\prime} \quad-.1548134165381630251639393576 '\right)\right) ; \ldots\right.$ vpa(sym(' -.1548134165381630251639393576')) vpa(sym(' .927247441752334105622080795189'))];

intJdn4dn5uvrs =[vpa(sym(' -.66191053322154112847875205137')) vpa(sym(' -.6161011926712676752388618229e-2'));... vpa(sym(' -.6161011926712676752388618229e-2')) vpa(sym(' -.966864949224433928644322229e-1'))];

intJdn4dn6uvrs $=\left[\operatorname{vpa}\left(\operatorname{sym}\left({ }^{\prime} .62364758048803624976125527371 \mathrm{e}-1^{\prime}\right)\right) \mathrm{vpa}\left(\operatorname{sym}\left({ }^{\prime}-.212192568049325297169223465371 '\right)\right) ; \ldots\right.$ vpa(sym(' -.212192568049325297169223465371')) vpa(sym(' -.7898220003698491703637636243e-1'))];

intJdn4dn7uvrs =[vpa(sym(' $\left.\left.\quad-.1027760205181785564472168671^{\prime}\right)\right) \operatorname{vpa}\left(\operatorname{sym}\left({ }^{\prime}-.3273205972269859328464496028286 '\right)\right) ; \ldots$ $\left.\operatorname{vpa}\left(\operatorname{sym}\left({ }^{\prime}-.3273205972269859328464496028286 '\right)\right) \operatorname{vpa}\left(\operatorname{sym}\left({ }^{\prime} \quad .14453334544915567441376932857 '\right)\right)\right]$; 
intJdn4dn8uvrs $=\left[\operatorname{vpa}\left(\operatorname{sym}\left({ }^{\prime} .3640416697255654640867267668 \mathrm{e}-1^{\prime}\right)\right) \operatorname{vpa}\left(\operatorname{sym}\left({ }^{\prime} .1022830353271046354061602393186 '\right)\right) ; \ldots\right.$ vpa(sym(' .1022830353271046354061602393186')) vpa(sym(' -.820137977578506638706811693089'))];

intJdn4dn9uvrs $=\left[\operatorname{vpa}\left(\operatorname{sym}\left({ }^{\prime} \quad .364521033839059598529804469259 '\right)\right) \mathrm{vpa}\left(\operatorname{sym}\left({ }^{\prime} \quad .432773165331780684038772441541^{\prime}\right)\right) ; \ldots\right.$ vpa(sym(' .132773165331780684038772441541')) vpa(sym('.66028785385703773347942390579e-1'))];

intJdn4dn10uvrs $=\left[\operatorname{vpa}\left(\operatorname{sym}\left({ }^{\prime} \quad-1.32051059158026743632811545266 '\right)\right)\right.$ vpa(sym(' $\left.\left.\quad-.74544201189536998617617117680 '\right)\right) ; \ldots$. vpa(sym(' -.3294201189536998617617117680e-1')) vpa(sym(' .89381759607913761072441622471e-1'))];

intJdn4dn11uvrs $=\left[\operatorname{vpa}\left(\operatorname{sym}\left({ }^{\prime}-.610174037597342509867071996 \mathrm{e}-1^{\prime}\right)\right) \operatorname{vpa}\left(\operatorname{sym}\left({ }^{\prime} \quad .38302940123838683131424805667 '\right)\right) ; \ldots\right.$ $\left.\operatorname{vpa}(\operatorname{sym}('-.32947059876161316868575194333 ')) \operatorname{vpa}\left(\operatorname{sym}\left({ }^{\prime}-1.8950734481632389831098706986 '\right)\right)\right]$;

intJdn4dn12uvrs =[vpa(sym(' .676185160002392642047802966e-1')) vpa(sym(' .6072316479445118067281174443e-1')); ... vpa(sym(' .36072316479445118067281174443')) vpa(sym(' .737450284572716169349341246171'))];

intJdn5dn1uvrs $=\left[\operatorname{vpa}\left(\operatorname{sym}\left({ }^{\prime}-1.91709736541803286033938722609 '\right)\right) \mathrm{vpa}\left(\operatorname{sym}\left({ }^{\prime}-1.21323891821327672848240784486 '\right)\right) ; . .\right.$. vpa(sym(' -.50073891821327672848240784486')) vpa(sym('.$- .489515939250703449364685749 '))]$;

intJdn5dn2uvrs =[vpa(sym(' .737450284572716169349341246171')) vpa(sym(' .6072316479445118067281174443e-1'));... vpa(sym(' .36072316479445118067281174443')) vpa(sym(' .676185160002392642047802966e-1'))];

intJdn5dn3uvrs $=\left[\operatorname{vpa}(\operatorname{sym}(' \quad .42220907054407935850896000532 ')) \mathrm{vpa}\left(\operatorname{sym}\left({ }^{\prime} .338079890317619378517715472657 '\right)\right) ; \ldots\right.$ vpa(sym(' .338079890317619378517715472657')) vpa(sym(' .12092398059703989381526413440'))];

intJdn5dn4uvrs =[vpa(sym(' -.66191053322154112847875205137')) vpa(sym(' -.6161011926712676752388618229e-2'));... vpa(sym(' -.6161011926712676752388618229e-2')) vpa(sym(' -.966864949224433928644322229e-1'))];

intJdn5dn5uvrs $=\left[\operatorname{vpa}\left(\operatorname{sym}\left({ }^{\prime} 4.20027429051479081211473647017 '\right)\right) \operatorname{vpa}\left(\operatorname{sym}\left({ }^{\prime} 1.3438679189477808452044247751^{\prime}\right)\right) ; \ldots\right.$ vpa(sym(' 1.3438679189477808452044247751')) vpa(sym(' .831814504364695263991287102'))];

intJdn5dn6uvrs $=\left[\operatorname{vpa}\left(\operatorname{sym}\left({ }^{\prime}-2.99702494451010242383266710989 '\right)\right) \operatorname{vpa}\left(\operatorname{sym}\left({ }^{\prime} \quad-.1349871919167025548746160210 '\right)\right) ; \ldots\right.$. $\left.\operatorname{vpa}\left(\operatorname{sym}\left('-1.1474871919167025548746160210^{\prime}\right)\right) \operatorname{vpa}\left(\operatorname{sym}\left({ }^{\prime} \quad-.265306180626066014206434443 '\right)\right)\right]$;

intJdn5dn7uvrs $=\left[\operatorname{vpa}\left(\operatorname{sym}\left({ }^{\prime} \quad-.22968940869230447662270961540 '\right)\right) \operatorname{vpa}\left(\operatorname{sym}\left({ }^{\prime} \quad-.13926553666521939019713093531 '\right)\right) ; \ldots\right.$ vpa(sym(' -.13926553666521939019713093531')) vpa(sym(' -.3285352909863772097735829779e-1'))];

intJdn5dn8uvrs =[vpa(sym(' -.3608025665386603226539408351e-1')) vpa(sym(' $\quad-.15399966449131754839794301520 ')) ; \ldots$ vpa(sym(' -.15399966449131754839794301520')) vpa(sym(' -.682973203907163581683513308e-1'))];

intJdn5dn9uvrs $=\left[\operatorname{vpa}\left(\operatorname{sym}\left({ }^{\prime}-1.54041301042459644452156154833 '\right)\right) \operatorname{vpa}\left(\operatorname{sym}\left({ }^{\prime}-.81719220580616221994946695233 '\right)\right) ; \ldots\right.$ $\left.\operatorname{vpa}(\operatorname{sym}('-.81719220580616221994946695233 ')) \mathrm{vpa}\left(\operatorname{sym}\left({ }^{\prime} \quad-.2658108259536691147535642753 '\right)\right)\right]$;

intJdn5dn10uvrs $=\left[\operatorname{vpa}\left(\operatorname{sym}\left({ }^{\prime} 1.77949064062986161117643452513 '\right)\right) \operatorname{vpa}\left(\operatorname{sym}\left({ }^{\prime} \quad .46814346046933431699809291100 '\right)\right) ; \ldots\right.$ vpa(sym(' .46814346046933431699809291100')) vpa(sym(' -.22172877626635562865944848351'))];

intJdn5dn11uvrs $=\left[\operatorname{vpa}\left(\operatorname{sym}\left({ }^{\prime} .2248827734476067968056194046 \mathrm{e}-1^{\prime}\right)\right) \operatorname{vpa}\left(\operatorname{sym}\left({ }^{\prime} .9389051783339916763096158371 \mathrm{e}-1^{\prime}\right)\right) ; \ldots\right.$ vpa(sym(' .9389051783339916763096158371e-1')) vpa(sym(' .19953911023238252175250582200'))];

intJdn5dn12uvrs $=\left[\operatorname{vpa}\left(\operatorname{sym}\left({ }^{\prime} \quad .2203029553142347352304374467^{\prime}\right)\right) \operatorname{vpa}\left(\operatorname{sym}\left({ }^{\prime} .16013957665680622962994689919^{\prime}\right)\right) ; \ldots\right.$ $\operatorname{vpa}(\operatorname{sym}(' .16013957665680622962994689919 '))$ vpa(sym(' .2203029553142347352304374467'))];

intJdn6dn1uvrs $=\left[\operatorname{vpa}\left(\operatorname{sym}\left({ }^{\prime} \quad .377654219333168620525401790571 '\right)\right) \operatorname{vpa}\left(\operatorname{sym}\left({ }^{\prime} \quad .22114271276808854773140878587 '\right)\right) ; \ldots\right.$ vpa(sym(' -.7885728723191145226859121413e-1')) vpa(sym(' .471024620459754344696508823e-1'))];

intJdn6dn2uvrs =[vpa(sym(' -1.895073448163238983109870698571')) vpa(sym(' .38302940123838683131424805667'));... vpa(sym(' -.32947059876161316868575194333')) vpa(sym(' -.610174037597342509867071996e-1'))];

intJdn6dn3uvrs $=\left[\operatorname{vpa}\left(\operatorname{sym}\left({ }^{\prime}-.97811352194183953055704604912^{\prime}\right)\right) \operatorname{vpa}\left(\operatorname{sym}\left({ }^{\prime}-.271075459705479523507647905621 '\right)\right) ; \ldots\right.$ vpa(sym(' -.271075459705479523507647905621')) vpa(sym(' -.16559436051923997292216207590'))];

intJdn6dn4uvrs =[vpa(sym(' .62364758048803624976125527371e-1')) vpa(sym(' -.212192568049325297169223465371'));... $\left.\operatorname{vpa}(\operatorname{sym}('-.212192568049325297169223465371 ')) \operatorname{vpa}\left(\operatorname{sym}\left({ }^{\prime}-.7898220003698491703637636243 \mathrm{e}-1^{\prime}\right)\right)\right]$;

intJdn6dn5uvrs $=\left[\operatorname{vpa}\left(\operatorname{sym}\left({ }^{\prime}-2.99702494451010242383266710989 '\right)\right) \mathrm{vpa}\left(\operatorname{sym}\left({ }^{\prime}-1.1474871919167025548746160210 '\right)\right) ; \ldots\right.$ $\operatorname{vpa}(\operatorname{sym}(' \quad-.1349871919167025548746160210 '))$ vpa(sym(' $\quad-.265306180626066014206434443 '))]$;

intJdn6dn6uvrs $=[\operatorname{vpa}(\operatorname{sym}(' 4.54242395713891689901104912584 ')) \operatorname{vpa}(\operatorname{sym}(' \quad .6092869698520311146728282477 ')) ; \ldots$ vpa(sym(' .6092869698520311146728282477')) vpa(sym(' .443331612234228602914621619'))];

intJdn6dn7uvrs $=\left[\operatorname{vpa}\left(\operatorname{sym}\left({ }^{\prime}-.21838750833036302366600382197 '\right)\right) \operatorname{vpa}\left(\operatorname{sym}\left({ }^{\prime} \quad-.10146047716910257979584393430 '\right)\right) ; \ldots\right.$. vpa(sym(' -.10146047716910257979584393430')) vpa(sym(' .7828128386135153260806627353e-1'))];

intJdn6dn8uvrs =[vpa(sym(' -.4227161104291962147268159204e-1')) vpa(sym(' $\quad-.17005535924820519494502526850 ')) ; \ldots$ $\left.\operatorname{vpa}\left(\operatorname{sym}\left({ }^{\prime} \quad-.17005535924820519494502526850 '\right)\right) \mathrm{vpa}\left(\operatorname{sym}\left({ }^{\prime} .66296433804103064946378510343 \mathrm{e}-1^{\prime}\right)\right)\right]$;

intJdn6dn9uvrs $=\left[\operatorname{vpa}\left(\operatorname{sym}\left({ }^{\prime} 1.985855365274808473372126605957 '\right)\right) \mathrm{vpa}\left(\operatorname{sym}\left({ }^{\prime} \quad .17495708456313244013203951683 '\right)\right) ; \ldots\right.$ $\left.\operatorname{vpa}(\operatorname{sym}(' \quad .17495708456313244013203951683 ')) \operatorname{vpa}\left(\operatorname{sym}\left({ }^{\prime}-.29882200555297695918896368570 '\right)\right)\right]$;

intJdn6dn10uvrs $=\left[\operatorname{vpa}\left(\operatorname{sym}\left({ }^{\prime}-1.071217433095080466489312963500 '\right)\right) \operatorname{vpa}(\operatorname{sym}(' \quad .29378408669763175300622339994 ')) ; \ldots\right.$ vpa(sym(' .29378408669763175300622339994')) vpa(sym(' .1779710241491188902309911789'))];

intJdn6dn11uvrs $=\left[\operatorname{vpa}\left(\operatorname{sym}\left({ }^{\prime} .3425105705546390949037336341 \mathrm{e}-1^{\prime}\right)\right) \operatorname{vpa}\left(\operatorname{sym}\left({ }^{\prime} \quad .12618028313614529580464700274 '\right)\right) ; \ldots\right.$. vpa(sym(' .12618028313614529580464700274')) vpa(sym(' .3425105705546390949037336341e-1'))];

intJdn6dn12uvrs $=\left[\operatorname{vpa}\left(\operatorname{sym}\left({ }^{\prime} \quad .19953911023238252175250582200 '\right)\right) \operatorname{vpa}\left(\operatorname{sym}\left({ }^{\prime} .9389051783339916763096158371 \mathrm{e}-1^{\prime}\right)\right) ; \ldots\right.$ vpa(sym(' .9389051783339916763096158371e-1')) vpa(sym(' .2248827734476067968056194046e-1'))];

intJdn7dn1uvrs $=\left[\operatorname{vpa}\left(\operatorname{sym}\left({ }^{\prime}-.31236802364420048787164339097 '\right)\right) \operatorname{vpa}\left(\operatorname{sym}\left({ }^{\prime}-.622591925470292070597183972271 '\right)\right) ; \ldots\right.$ vpa(sym(' -.622591925470292070597183972271')) vpa(sym(' -.93264116705106716805029551279'))];

intJdn7dn2uvrs $=\left[\operatorname{vpa}\left(\operatorname{sym}\left({ }^{\prime} .89381759607913761072441622471 \mathrm{e}-1^{\prime}\right)\right) \operatorname{vpa}\left(\operatorname{sym}\left({ }^{\prime} \quad-.74544201189536998617617117680 '\right)\right) ; \ldots\right.$ $\left.\operatorname{vpa}\left(\operatorname{sym}\left('-.3294201189536998617617117680 \mathrm{e}-1^{\prime}\right)\right) \operatorname{vpa}(\operatorname{sym}('-1.32051059158026743632811545266 '))\right]$;

intJdn7dn3uvrs $=\left[\operatorname{vpa}\left(\operatorname{sym}\left({ }^{\prime}-.97451565507866331982099391909 \mathrm{e}-1^{\prime}\right)\right) \operatorname{vpa}\left(\operatorname{sym}\left({ }^{\prime} \quad-.302538103955586380338692247539 '\right)\right) ; \ldots\right.$ vpa(sym(' -.2538103955586380338692247539e-2')) vpa(sym(' .463447558533213614696680089166'))];

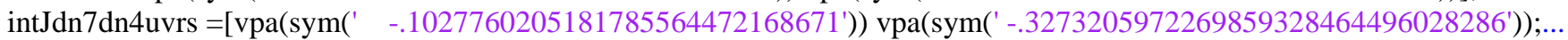


vpa(sym(' -.3273205972269859328464496028286')) vpa(sym(' .14453334544915567441376932857'))];

intJdn7dn5uvrs $=\left[\operatorname{vpa}\left(\operatorname{sym}\left({ }^{\prime} \quad-.22968940869230447662270961540 '\right)\right) \operatorname{vpa}\left(\operatorname{sym}\left({ }^{\prime} \quad-.13926553666521939019713093531 '\right)\right) ; \ldots\right.$ $\operatorname{vpa}\left(\operatorname{sym}\left({ }^{\prime}-.13926553666521939019713093531\right.\right.$ ')) vpa(sym(' -.3285352909863772097735829779e-1'))];

intJdn7dn6uvrs $=\left[\operatorname{vpa}\left(\operatorname{sym}\left({ }^{\prime}-.21838750833036302366600382197 '\right)\right) \operatorname{vpa}\left(\operatorname{sym}\left({ }^{\prime} \quad-.10146047716910257979584393430 '\right)\right) ; \ldots\right.$ vpa(sym(' -.10146047716910257979584393430')) vpa(sym(' .7828128386135153260806627353e-1'))];

intJdn7dn7uvrs $=\left[\operatorname{vpa}(\operatorname{sym}(' \quad .9430026977665203124840967049 ')) \operatorname{vpa}\left(\operatorname{sym}\left({ }^{\prime} 1.054490113764698836662802085186 '\right)\right) ; \ldots\right.$ $\operatorname{vpa}(\operatorname{sym}(' 1.054490113764698836662802085186 '))$ vpa(sym(' $\left.\left.\left.3.17595231695266811253589555038^{\prime}\right)\right)\right]$;

intJdn7dn8uvrs =[vpa(sym(' -.23411496564964084582129500914')) vpa(sym(' .22996512009548291644560497057e-1'));... $\left.\operatorname{vpa}\left(\operatorname{sym}\left({ }^{\prime}-.989503487990451708355439502943 '\right)\right) \operatorname{vpa}\left(\operatorname{sym}\left({ }^{\prime}-2.31569161290561082190356514273 '\right)\right)\right]$;

intJdn7dn9uvrs =[vpa(sym(' $.10963550129394871861715141079 ')) \mathrm{vpa}\left(\operatorname{sym}\left({ }^{\prime} \quad .19369458821691485109794452082 '\right)\right) ; \ldots$ vpa(sym(' .19369458821691485109794452082')) vpa(sym(' -.6531609748699460034793405982e-1'))];

intJdn7dn10uvrs $=\left[\operatorname{vpa}\left(\operatorname{sym}\left({ }^{\prime} .9652528579140766866573566268 \mathrm{e}-1^{\prime}\right)\right) \operatorname{vpa}\left(\operatorname{sym}\left({ }^{\prime} \quad .20550989122442829054184845497 '\right)\right) ; \ldots\right.$ vpa(sym(' .20550989122442829054184845497')) vpa(sym(' .9652528579140766866573566268e-1'))];

intJdn7dn11uvrs $=\left[\operatorname{vpa}(\operatorname{sym}(' \quad .17797102414911889023099117890 ')) \operatorname{vpa}\left(\operatorname{sym}\left({ }^{\prime} .29378408669763175300622339994 '\right)\right) ; \ldots\right.$ vpa(sym(' .29378408669763175300622339994')) vpa(sym(' -1.0712174330950804664893129635'))];

intJdn7dn12uvrs $=\left[\operatorname{vpa}\left(\operatorname{sym}\left({ }^{\prime}-.22172877626635562865944848351 '\right)\right) \operatorname{vpa}\left(\operatorname{sym}\left({ }^{\prime} .46814346046933431699809291100 '\right)\right) ; \ldots\right.$ vpa(sym(' .46814346046933431699809291100')) vpa(sym(' 1.77949064062986161117643452513'))];

intJdn8dn1uvrs $=\left[\operatorname{vpa}\left(\operatorname{sym}\left({ }^{\prime} \quad .22334568260811342445267275929 '\right)\right) \operatorname{vpa}\left(\operatorname{sym}\left({ }^{\prime} .415458052185700603634760549957 '\right)\right) ; \ldots\right.$ $\left.\operatorname{vpa}\left(\operatorname{sym}\left({ }^{\prime} .415458052185700603634760549957 '\right)\right) \operatorname{vpa}\left(\operatorname{sym}\left({ }^{\prime} .321795382308358846270975070300 '\right)\right)\right]$;

intJdn8dn2uvrs =[vpa(sym(' .66028785385703773347942390579e-1')) vpa(sym(' .432773165331780684038772441541'));...

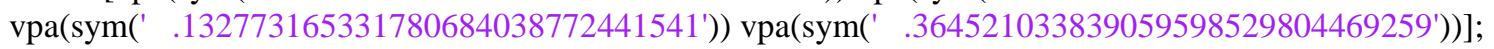

intJdn8dn3uvrs $=\left[\operatorname{vpa}\left(\operatorname{sym}\left({ }^{\prime} .99543897060373053107624738884 \mathrm{e}-1^{\prime}\right)\right)\right.$ vpa( $\left.\operatorname{sym}\left({ }^{\prime} .67270029709553115713258695733 \mathrm{e}-1^{\prime}\right)\right) ; \ldots$ $\operatorname{vpa}\left(\operatorname{sym}\left({ }^{\prime}-.645229970290446884286741304267 '\right)\right)$ vpa(sym(' $\left.\left.\left.-1.653443869822186862590758708100 '\right)\right)\right]$;

intJdn8dn4uvrs =[vpa(sym(' .3640416697255654640867267668e-1')) vpa(sym(' .1022830353271046354061602393186'));... $\left.\operatorname{vpa}\left(\operatorname{sym}\left({ }^{\prime} .1022830353271046354061602393186 '\right)\right) \operatorname{vpa}\left(\operatorname{sym}\left({ }^{\prime}-.820137977578506638706811693089 '\right)\right)\right]$;

intJdn8dn5uvrs $=\left[\operatorname{vpa}\left(\operatorname{sym}\left({ }^{\prime}-.3608025665386603226539408351 \mathrm{e}-1^{\prime}\right)\right) \operatorname{vpa}\left(\operatorname{sym}\left({ }^{\prime} \quad-.15399966449131754839794301520 '\right)\right) ; \ldots\right.$ vpa(sym(' -.15399966449131754839794301520')) vpa(sym(' -.682973203907163581683513308e-1'))];

intJdn8dn6uvrs =[vpa(sym(' -.4227161104291962147268159204e-1')) vpa(sym(' $\quad-.17005535924820519494502526850 ')) ; \ldots$ $\left.\operatorname{vpa}\left(\operatorname{sym}\left({ }^{\prime}-.17005535924820519494502526850 '\right)\right) \mathrm{vpa}\left(\operatorname{sym}\left({ }^{\prime} .66296433804103064946378510343 \mathrm{e}-1^{\prime}\right)\right)\right]$;

intJdn8dn7uvrs $=\left[\operatorname{vpa}\left(\operatorname{sym}\left({ }^{\prime} \quad-.23411496564964084582129500914 '\right)\right) \operatorname{vpa}\left(\operatorname{sym}\left({ }^{\prime} \quad-.989503487990451708355439502943 '\right)\right) ; \ldots\right.$ $\left.\operatorname{vpa}\left(\operatorname{sym}\left({ }^{\prime} .22996512009548291644560497057 \mathrm{e}-1^{\prime}\right)\right) \operatorname{vpa}\left(\operatorname{sym}\left({ }^{\prime}-2.31569161290561082190356514273 '\right)\right)\right]$;

intJdn8dn8uvrs $=\left[\operatorname{vpa}\left(\operatorname{sym}\left({ }^{\prime} \quad .57399287653962671437054925866 '\right)\right) \operatorname{vpa}\left(\operatorname{sym}\left({ }^{\prime} \quad .510536435455242956618237924447 '\right)\right) ; \ldots\right.$ $\operatorname{vpa}(\operatorname{sym}(' .510536435455242956618237924447 '))$ vpa(sym(' $3.606779720827644761992241475244 '))]$;

intJdn8dn9uvrs $=\left[\operatorname{vpa}\left(\operatorname{sym}\left({ }^{\prime}-.5689964622630633783762911890 \mathrm{e}-1^{\prime}\right)\right) \mathrm{vpa}\left(\operatorname{sym}\left({ }^{\prime} \quad .23377832674670738500670085018 '\right)\right) ; \ldots\right.$ vpa(sym(' .23377832674670738500670085018')) vpa(sym(' -.5689964622630633783762911890e-1'))];

intJdn8dn10uvrs $=\left[\operatorname{vpa}\left(\operatorname{sym}\left('-.6531609748699460034793405982 \mathrm{e}-1^{\prime}\right)\right) \operatorname{vpa}\left(\operatorname{sym}\left(' \quad .19369458821691485109794452082^{\prime}\right)\right) ; \ldots\right.$ vpa(sym(' .19369458821691485109794452082')) vpa(sym(' .10963550129394871861715141079'))];

intJdn8dn11uvrs $=\left[\operatorname{vpa}\left(\operatorname{sym}\left({ }^{\prime}-.29882200555297695918896368570 '\right)\right) \operatorname{vpa}\left(\operatorname{sym}\left({ }^{\prime} \quad .17495708456313244013203951683 '\right)\right) ; \ldots\right.$ vpa(sym(' .17495708456313244013203951683')) vpa(sym(' 1.985855365274808473372126605957'))];

intJdn8dn12uvrs $=\left[\operatorname{vpa}\left(\operatorname{sym}\left({ }^{\prime}-.26581082595366911475356427533^{\prime}\right)\right) \operatorname{vpa}\left(\operatorname{sym}\left({ }^{\prime} \quad-.81719220580616221994946695233 '\right)\right) ; \ldots\right.$ vpa(sym(' -.81719220580616221994946695233')) vpa(sym(' -1.54041301042459644452156154833'))];

intJdn9dn1uvrs $=\left[\operatorname{vpa}\left(\operatorname{sym}\left({ }^{\prime} .321795382308358846270975070300 '\right)\right) \operatorname{vpa}\left(\operatorname{sym}\left({ }^{\prime} .415458052185700603634760549957 '\right)\right) ; \ldots\right.$ $\left.\operatorname{vpa}\left(\operatorname{sym}\left({ }^{\prime} .415458052185700603634760549957 '\right)\right) \operatorname{vpa}\left(\operatorname{sym}\left({ }^{\prime} .22334568260811342445267275929 '\right)\right)\right] ;$

intJdn9dn2uvrs =[vpa(sym(' -.820137977578506638706811693089')) vpa(sym(' .1022830353271046354061602393186'));... vpa(sym(' .1022830353271046354061602393186')) vpa(sym(' .3640416697255654640867267668e-1'))];

intJdn9dn3uvrs =[vpa(sym(' -1.653443869822186862590758708100')) vpa(sym(' -.645229970290446884286741304267'));... $\left.\operatorname{vpa}\left(\operatorname{sym}\left({ }^{\prime} .67270029709553115713258695733 \mathrm{e}-1^{\prime}\right)\right) \operatorname{vpa}\left(\operatorname{sym}\left({ }^{\prime} .99543897060373053107624738884 \mathrm{e}-1^{\prime}\right)\right)\right]$;

intJdn9dn4uvrs $=\left[\operatorname{vpa}\left(\operatorname{sym}\left({ }^{\prime} \quad .364521033839059598529804469259 '\right)\right)\right.$ vpa(sym(' $\left.\left..132773165331780684038772441541 '\right)\right) ; \ldots$ vpa(sym(' .432773165331780684038772441541')) vpa(sym(' .66028785385703773347942390579e-1'))];

intJdn9dn5uvrs $=\left[\operatorname{vpa}\left(\operatorname{sym}\left({ }^{\prime}-1.54041301042459644452156154833 '\right)\right) \operatorname{vpa}\left(\operatorname{sym}\left({ }^{\prime}-.81719220580616221994946695233 '\right)\right) ; \ldots\right.$ vpa(sym(' -.81719220580616221994946695233')) vpa(sym(' -.2658108259536691147535642753'))];

intJdn9dn6uvrs $=\left[\operatorname{vpa}\left(\operatorname{sym}\left({ }^{\prime} 1.985855365274808473372126605957 '\right)\right) \operatorname{vpa}(\operatorname{sym}(' \quad .17495708456313244013203951683 ')) ; \ldots\right.$ vpa(sym(' .17495708456313244013203951683')) vpa(sym(' -.29882200555297695918896368570'))];

intJdn9dn7uvrs $=\left[\operatorname{vpa}\left(\operatorname{sym}\left({ }^{\prime} \quad .10963550129394871861715141079 '\right)\right) \operatorname{vpa}(\operatorname{sym}(' \quad .19369458821691485109794452082 ')) ; \ldots\right.$ vpa(sym(' .19369458821691485109794452082')) vpa(sym(' -.6531609748699460034793405982e-1'))];

intJdn9dn8uvrs $=\left[\operatorname{vpa}\left(\operatorname{sym}\left({ }^{\prime}-.5689964622630633783762911890 \mathrm{e}-1^{\prime}\right)\right) \mathrm{vpa}\left(\operatorname{sym}\left({ }^{\prime} \quad .23377832674670738500670085018 '\right)\right) ; \ldots\right.$ vpa(sym(' .23377832674670738500670085018')) vpa(sym(' -.5689964622630633783762911890e-1'))];

intJdn9dn9uvrs =[vpa(sym(' 3.606779720827644761992241475244')) vpa(sym(' .510536435455242956618237924447'));... $\left.\operatorname{vpa}(\operatorname{sym}(' .510536435455242956618237924447 ')) \mathrm{vpa}\left(\operatorname{sym}\left({ }^{\prime} \quad .57399287653962671437054925866 '\right)\right)\right]$;

intJdn9dn10uvrs $=\left[\operatorname{vpa}\left(\operatorname{sym}\left({ }^{\prime}-2.315691612905610821903565142727 '\right)\right) \mathrm{vpa}\left(\operatorname{sym}\left({ }^{\prime} .22996512009548291644560497057 \mathrm{e}-1^{\prime}\right)\right) ; \ldots\right.$ $\left.\operatorname{vpa}\left(\operatorname{sym}\left({ }^{\prime}-.989503487990451708355439502943 '\right)\right) \operatorname{vpa}(\operatorname{sym}(' \quad-.23411496564964084582129500914 '))\right]$;

intJdn9dn11uvrs =[vpa(sym(' .66296433804103064946378510343e-1')) vpa(sym(' $\quad-.17005535924820519494502526850 ')) ; \ldots$ $\left.\operatorname{vpa}\left(\operatorname{sym}\left({ }^{\prime} \quad-.17005535924820519494502526850 '\right)\right) \operatorname{vpa}\left(\operatorname{sym}\left('-.4227161104291962147268159204 \mathrm{e}-1^{\prime}\right)\right)\right]$;

intJdn9dn12uvrs =[vpa(sym(' -.682973203907163581683513307714e-1')) vpa(sym(' $\quad-.15399966449131754839794301520 ')) ; \ldots$ $\operatorname{vpa}\left(\operatorname{sym}\left({ }^{\prime} \quad-.15399966449131754839794301520\right.\right.$ ')) vpa(sym(' $\left.\left.\left.\quad-.3608025665386603226539408351 \mathrm{e}-1^{\prime}\right)\right)\right]$; 
intJdn10dn1uvrs $=\left[\operatorname{vpa}\left(\operatorname{sym}\left({ }^{\prime}-.93264116705106716805029551279 '\right)\right) \operatorname{vpa}\left(\operatorname{sym}\left({ }^{\prime}-.622591925470292070597183972271 '\right)\right) ; \ldots\right.$ $\operatorname{vpa}(\operatorname{sym}('-.622591925470292070597183972271 ')) \operatorname{vpa}(\operatorname{sym}('-.31236802364420048787164339097 '))]$;

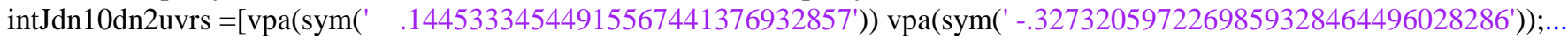
$\left.\operatorname{vpa}\left(\operatorname{sym}\left({ }^{\prime}-.3273205972269859328464496028286 '\right)\right) \operatorname{vpa}(\operatorname{sym}(' \quad-.10277602051817855644721686707 '))\right]$;

intJdn10dn3uvrs $=\left[\operatorname{vpa}(\operatorname{sym}(' \quad .463447558533213614696680089166 ')) \operatorname{vpa}\left(\operatorname{sym}\left({ }^{\prime} \quad-.2538103955586380338692247539 \mathrm{e}-22^{\prime}\right)\right) ; \ldots\right.$ $\left.\operatorname{vpa}\left(\operatorname{sym}\left({ }^{\prime} \quad-.302538103955586380338692247539 '\right)\right) \operatorname{vpa}\left(\operatorname{sym}\left({ }^{\prime}-.97451565507866331982099391909 \mathrm{e}-1^{\prime}\right)\right)\right]$;

intJdn10dn4uvrs $=\left[\operatorname{vpa}\left(\operatorname{sym}\left({ }^{\prime}-1.32051059158026743632811545266 '\right)\right) \operatorname{vpa}\left(\operatorname{sym}\left({ }^{\prime}-.3294201189536998617617117680 e-1 '\right)\right) ; \ldots\right.$ $\left.\operatorname{vpa}\left(\operatorname{sym}\left({ }^{\prime} \quad-.74544201189536998617617117680 '\right)\right) \operatorname{vpa}\left(\operatorname{sym}\left(' .89381759607913761072441622471 \mathrm{e}-1^{\prime}\right)\right)\right]$;

intJdn10dn5uvrs $=\left[\operatorname{vpa}\left(\operatorname{sym}\left({ }^{\prime} 1.77949064062986161117643452513 '\right)\right) \operatorname{vpa}\left(\operatorname{sym}\left({ }^{\prime} .46814346046933431699809291100 '\right)\right) ; \ldots\right.$. vpa(sym(' .46814346046933431699809291100')) vpa(sym(' -.22172877626635562865944848351'))];

intJdn10dn6uvrs $=\left[\operatorname{vpa}\left(\operatorname{sym}\left({ }^{\prime}-1.071217433095080466489312963500 '\right)\right) \operatorname{vpa}\left(\operatorname{sym}\left({ }^{\prime} \quad .29378408669763175300622339994 '\right)\right) ; \ldots\right.$ vpa(sym(' .29378408669763175300622339994')) vpa(sym(' .1779710241491188902309911789'))];

intJdn10dn7uvrs =[vpa(sym(' .9652528579140766866573566268e-1')) vpa(sym(' .20550989122442829054184845497')); ...

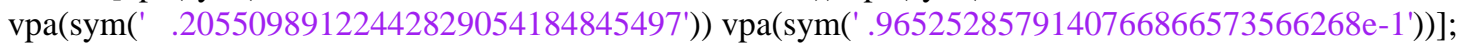

intJdn10dn8uvrs =[vpa(sym(' -.6531609748699460034793405982e-1')) vpa(sym(' .19369458821691485109794452082'));... vpa(sym(' .19369458821691485109794452082')) vpa(sym(' .10963550129394871861715141079'))];

intJdn10dn9uvrs $=\left[\operatorname{vpa}\left(\operatorname{sym}\left({ }^{\prime}-2.315691612905610821903565142727 '\right)\right) \operatorname{vpa}\left(\operatorname{sym}\left({ }^{\prime} \quad-.989503487990451708355439502943 '\right)\right) ; \ldots\right.$ $\left.\operatorname{vpa}\left(\operatorname{sym}\left({ }^{\prime} .22996512009548291644560497057 \mathrm{e}-1^{\prime}\right)\right) \operatorname{vpa}\left(\operatorname{sym}\left({ }^{\prime} \quad-.23411496564964084582129500914 '\right)\right)\right]$;

intJdn10dn10uvrs $=\left[\operatorname{vpa}\left(\operatorname{sym}\left({ }^{\prime} 3.17595231695266811253589555038 '\right)\right) \mathrm{vpa}\left(\operatorname{sym}\left({ }^{\prime} 1.054490113764698836662802085186 '\right)\right) ; \ldots\right.$ vpa(sym(' 1.054490113764698836662802085186')) vpa(sym(' .94300269776652031248409670487'))];

intJdn10dn11uvrs =[vpa(sym(' .7828128386135153260806627353e-1')) vpa(sym(' -.10146047716910257979584393430'));... $\left.\operatorname{vpa}\left(\operatorname{sym}\left({ }^{\prime}-.10146047716910257979584393430 '\right)\right) \operatorname{vpa}\left(\operatorname{sym}\left({ }^{\prime}-.21838750833036302366600382197 '\right)\right)\right]$;

intJdn10dn12uvrs $=\left[\operatorname{vpa}\left(\operatorname{sym}\left({ }^{\prime}-.3285352909863772097735829779 \mathrm{e}-1^{\prime}\right)\right) \operatorname{vpa}\left(\operatorname{sym}\left({ }^{\prime} \quad-.13926553666521939019713093531 '\right)\right) ; \ldots\right.$ $\left.\operatorname{vpa}(\operatorname{sym}(' \quad-.13926553666521939019713093531 ')) \mathrm{vpa}\left(\operatorname{sym}\left({ }^{\prime} \quad-.22968940869230447662270961540 '\right)\right)\right]$;

intJdn11dn1uvrs =[vpa(sym(' .471024620459754344696508823e-1')) vpa(sym(' -.7885728723191145226859121413e-1'));...

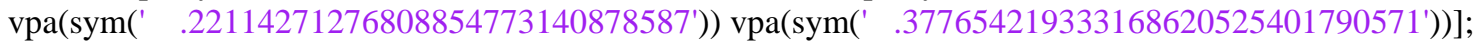

intJdn11dn2uvrs $=\left[\operatorname{vpa}\left(\operatorname{sym}\left({ }^{\prime}-.7898220003698491703637636243 e-1^{\prime}\right)\right) \operatorname{vpa}\left(\operatorname{sym}\left({ }^{\prime}-.212192568049325297169223465371 '\right)\right) ; \ldots\right.$ vpa(sym(' -.212192568049325297169223465371')) vpa(sym(' .62364758048803624976125527371e-1'))];

intJdn11dn3uvrs $=\left[\operatorname{vpa}\left(\operatorname{sym}\left({ }^{\prime}-.165594360519239972922162075896 '\right)\right) \mathrm{vpa}\left(\operatorname{sym}\left({ }^{\prime}-.2710754597054795235076479056211^{\prime}\right)\right) ; \ldots\right.$ $\left.\operatorname{vpa}(\operatorname{sym}('-.271075459705479523507647905621 ')) \operatorname{vpa}\left(\operatorname{sym}\left({ }^{\prime}-.97811352194183953055704604912 '\right)\right)\right]$;

intJdn11dn4uvrs $=\left[\operatorname{vpa}\left(\operatorname{sym}\left('-.610174037597342509867071996 \mathrm{e}-1^{\prime}\right)\right) \operatorname{vpa}\left(\operatorname{sym}\left({ }^{\prime}-.32947059876161316868575194333 '\right)\right) ; \ldots\right.$ vpa(sym(' .38302940123838683131424805667')) vpa(sym(' -1.8950734481632389831098706986'))];

intJdn11dn5uvrs $=\left[\operatorname{vpa}\left(\operatorname{sym}\left({ }^{\prime} .2248827734476067968056194046 \mathrm{e}-1^{\prime}\right)\right) \operatorname{vpa}\left(\operatorname{sym}\left({ }^{\prime} .9389051783339916763096158371 \mathrm{e}-1^{\prime}\right)\right) ; \ldots\right.$ $\left.\operatorname{vpa}(\operatorname{sym}(' .9389051783339916763096158371 \mathrm{e}-1 ')) \operatorname{vpa}\left(\operatorname{sym}\left({ }^{\prime} \quad .19953911023238252175250582200 '\right)\right)\right]$;

intJdn11dn6uvrs $=\left[\operatorname{vpa}\left(\operatorname{sym}\left({ }^{\prime} .3425105705546390949037336341 \mathrm{e}-1^{\prime}\right)\right) \operatorname{vpa}\left(\operatorname{sym}\left({ }^{\prime} \quad .12618028313614529580464700274 '\right)\right) ; \ldots\right.$ vpa(sym(' .12618028313614529580464700274')) vpa(sym(' .3425105705546390949037336341e-1'))];

intJdn11dn7uvrs $=\left[\operatorname{vpa}\left(\operatorname{sym}\left({ }^{\prime} .17797102414911889023099117890 '\right)\right) \operatorname{vpa}(\operatorname{sym}(' \quad .29378408669763175300622339994 ')) ; \ldots\right.$. $\operatorname{vpa}(\operatorname{sym}('$.29378408669763175300622339994')) vpa(sym(' -1.0712174330950804664893129635'))];

intJdn11dn8uvrs $=\left[\operatorname{vpa}\left(\operatorname{sym}\left({ }^{\prime} \quad-.29882200555297695918896368570 '\right)\right) \operatorname{vpa}\left(\operatorname{sym}\left({ }^{\prime} \quad .17495708456313244013203951683 '\right)\right) ; \ldots\right.$ vpa(sym(' .17495708456313244013203951683')) vpa(sym(' 1.985855365274808473372126605957'))];

intJdn11dn9uvrs $=\left[\operatorname{vpa}\left(\operatorname{sym}(' .66296433804103064946378510343 \mathrm{e}-1\right.\right.$ ') $) \mathrm{vpa}\left(\operatorname{sym}\left({ }^{\prime} \quad-.17005535924820519494502526850 '\right)\right) ; \ldots$ $\operatorname{vpa}(\operatorname{sym}('-.17005535924820519494502526850$ ')) vpa(sym(' -.4227161104291962147268159204e-1'))];

intJdn11dn10uvrs =[vpa(sym(' .7828128386135153260806627353e-1')) vpa(sym(' -.10146047716910257979584393430'));... vpa(sym(' -.10146047716910257979584393430')) vpa(sym(' -.21838750833036302366600382197'))];

intJdn11dn11uvrs $=\left[\operatorname{vpa}\left(\operatorname{sym}\left({ }^{\prime} \quad .443331612234228602914621619^{\prime}\right)\right) \mathrm{vpa}\left(\operatorname{sym}\left({ }^{\prime} \quad .6092869698520311146728282477 '\right)\right) ; \ldots\right.$ vpa(sym(' .6092869698520311146728282477')) vpa(sym(' 4.54242395713891689901104912584'))];

intJdn11dn12uvrs $=\left[\operatorname{vpa}\left(\operatorname{sym}\left({ }^{\prime} \quad-.265306180626066014206434443 '\right)\right) \operatorname{vpa}\left(\operatorname{sym}\left({ }^{\prime} \quad-.1349871919167025548746160210 '\right)\right) ; \ldots\right.$ vpa(sym(' -1.1474871919167025548746160210')) vpa(sym(' -2.99702494451010242383266710989'))];

intJdn12dn1uvrs $=\left[\operatorname{vpa}\left(\operatorname{sym}\left({ }^{\prime} \quad-.489515939250703449364685749 '\right)\right) \operatorname{vpa}\left(\operatorname{sym}\left({ }^{\prime} \quad-.50073891821327672848240784486 '\right)\right) ; \ldots\right.$ $\operatorname{vpa}(\operatorname{sym}('-1.21323891821327672848240784486 '))$ vpa(sym(' -1.91709736541803286033938722609'))];

intJdn12dn2uvrs $=\left[\operatorname{vpa}\left(\operatorname{sym}\left({ }^{\prime}-.966864949224433928644322229 \mathrm{e}-1^{\prime}\right)\right) \mathrm{vpa}\left(\operatorname{sym}\left({ }^{\prime}-.6161011926712676752388618229 \mathrm{e}-22^{\prime}\right)\right) ; \ldots\right.$ vpa(sym(' -.6161011926712676752388618229e-2')) vpa(sym(' -.66191053322154112847875205137'))];

intJdn12dn3uvrs $=\left[\operatorname{vpa}\left(\operatorname{sym}\left({ }^{\prime} .12092398059703989381526413440 '\right)\right) \operatorname{vpa}\left(\operatorname{sym}\left({ }^{\prime} .338079890317619378517715472657 '\right)\right) ; \ldots\right.$. $\operatorname{vpa}(\operatorname{sym}(' .338079890317619378517715472657 ')) \operatorname{vpa}(\operatorname{sym}(' .42220907054407935850896000532 '))]$;

intJdn12dn4uvrs $=\left[\operatorname{vpa}\left(\operatorname{sym}\left({ }^{\prime} \quad .676185160002392642047802966 \mathrm{e}-1^{\prime}\right)\right) \operatorname{vpa}\left(\operatorname{sym}\left({ }^{\prime} \quad .36072316479445118067281174443{ }^{\prime}\right)\right) ; \ldots\right.$ vpa(sym(' .6072316479445118067281174443e-1')) vpa(sym(' .737450284572716169349341246171'))];

intJdn12dn5uvrs $=\left[\operatorname{vpa}\left(\operatorname{sym}\left({ }^{\prime} .2203029553142347352304374467^{\prime}\right)\right) \operatorname{vpa}\left(\operatorname{sym}\left({ }^{\prime} .1601395766568062296299468992 '\right)\right) ; \ldots\right.$. $\operatorname{vpa}(\operatorname{sym}(' .1601395766568062296299468992 ')) \mathrm{vpa}(\operatorname{sym}('$ '.2203029553142347352304374467'))];

intJdn12dn6uvrs $=\left[\operatorname{vpa}(\operatorname{sym}(' \quad .19953911023238252175250582200 ')) \operatorname{vpa}\left(\operatorname{sym}\left({ }^{\prime} .9389051783339916763096158371 \mathrm{e}-1 '\right)\right) ; \ldots\right.$ vpa(sym(' .9389051783339916763096158371e-1')) vpa(sym(' .2248827734476067968056194046e-1'))];

intJdn12dn7uvrs $=\left[\operatorname{vpa}\left(\operatorname{sym}\left({ }^{\prime}-.22172877626635562865944848351^{\prime}\right)\right) \operatorname{vpa}\left(\operatorname{sym}\left({ }^{\prime} .46814346046933431699809291100 '\right)\right) ; \ldots\right.$ vpa(sym(' .46814346046933431699809291100')) vpa(sym(' 1.77949064062986161117643452513'))];

intJdn12dn8uvrs $=\left[\operatorname{vpa}\left(\operatorname{sym}\left({ }^{\prime}-.26581082595366911475356427533^{\prime}\right)\right) \operatorname{vpa}\left(\operatorname{sym}\left({ }^{\prime}-.81719220580616221994946695233 '\right)\right) ; \ldots\right.$ vpa(sym(' -.81719220580616221994946695233')) vpa(sym(' -1.54041301042459644452156154833'))];

intJdn12dn9uvrs =[vpa(sym(' -.682973203907163581683513307714e-1')) vpa(sym(' $\quad-.15399966449131754839794301520 ')) ; \ldots$ 
$\left.\operatorname{vpa}\left(\operatorname{sym}\left({ }^{\prime} \quad-.15399966449131754839794301520 '\right)\right) \operatorname{vpa}\left(\operatorname{sym}\left(' \quad-.3608025665386603226539408351 \mathrm{e}-1^{\prime}\right)\right)\right]$; intJdn12dn10uvrs $=\left[\operatorname{vpa}\left(\operatorname{sym}\left('-.3285352909863772097735829779 \mathrm{e}-1^{\prime}\right)\right) \operatorname{vpa}\left(\operatorname{sym}\left({ }^{\prime} \quad-.13926553666521939019713093531 '\right)\right) ; . .\right.$. vpa(sym(' -.13926553666521939019713093531')) vpa(sym(' $-.22968940869230447662270961540 '))]$;

intJdn12dn11uvrs $=\left[\operatorname{vpa}\left(\operatorname{sym}\left({ }^{\prime} \quad-.265306180626066014206434443 '\right)\right) \operatorname{vpa}\left(\operatorname{sym}\left({ }^{\prime}-1.1474871919167025548746160210 '\right)\right) ; \ldots\right.$ vpa(sym(' -.1349871919167025548746160210')) vpa(sym(' -2.99702494451010242383266710989'))]; intJdn12dn12uvrs $=\left[\operatorname{vpa}\left(\operatorname{sym}\left({ }^{\prime} \quad .831814504364695263991287102^{\prime}\right)\right) \operatorname{vpa}\left(\operatorname{sym}\left({ }^{\prime} 1.3438679189477808452044247751^{\prime}\right)\right) ; \ldots\right.$ vpa(sym(' 1.3438679189477808452044247751')) vpa(sym(' 4.20027429051479081211473647017'))];

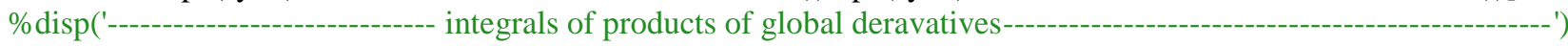

intJdndn=[intJdn1dn1uvrs intJdn1dn2uvrs intJdn1dn3uvrs intJdn1dn4uvrs intJdn1dn5uvrs intJdn1dn6uvrs intJdn1dn7uvrs intJdn1dn8uvrs intJdn1dn9uvrs intJdn1dn10uvrs intJdn1dn11uvrs intJdn1dn12uvrs; ... intJdn2dn1uvrs intJdn2dn2uvrs intJdn2dn3uvrs intJdn2dn4uvrs intJdn2dn5uvrs intJdn2dn6uvrs intJdn2dn7uvrs intJdn2dn8uvrs intJdn2dn9uvrs intJdn2dn10uvrs intJdn2dn11uvrs intJdn2dn12uvrs;... intJdn3dn1uvrs intJdn3dn2uvrs intJdn3dn3uvrs intJdn3dn4uvrs intJdn3dn5uvrs intJdn3dn6uvrs intJdn3dn7uvrs intJdn3dn8uvrs intJdn3dn9uvrs intJdn3dn10uvrs intJdn3dn11uvrs intJdn3dn12uvrs;... intJdn4dn1uvrs intJdn4dn2uvrs intJdn4dn3uvrs intJdn4dn4uvrs intJdn4dn5uvrs intJdn4dn6uvrs intJdn4dn7uvrs intJdn4dn8uvrs intJdn4dn9uvrs intJdn4dn10uvrs intJdn4dn11uvrs intJdn4dn12uvrs;... intJdn5dn1uvrs intJdn5dn2uvrs intJdn5dn3uvrs intJdn5dn4uvrs intJdn5dn5uvrs intJdn5dn6uvrs intJdn5dn7uvrs intJdn5dn8uvrs intJdn5dn9uvrs intJdn5dn10uvrs intJdn5dn11uvrs intJdn5dn12uvrs;... intJdn6dn1uvrs intJdn6dn2uvrs intJdn6dn3uvrs intJdn6dn4uvrs intJdn6dn5uvrs intJdn6dn6uvrs intJdn6dn7uvrs intJdn6dn8uvrs intJdn6dn9uvrs intJdn6dn10uvrs intJdn6dn11uvrs intJdn6dn12uvrs;... intJdn7dn1uvrs intJdn7dn2uvrs intJdn7dn3uvrs intJdn7dn4uvrs intJdn7dn5uvrs intJdn7dn6uvrs intJdn7dn7uvrs intJdn7dn8uvrs intJdn7dn9uvrs intJdn7dn10uvrs intJdn7dn11uvrs intJdn7dn12uvrs; ... intJdn8dn1uvrs intJdn8dn2uvrs intJdn8dn3uvrs intJdn8dn4uvrs intJdn8dn5uvrs intJdn8dn6uvrs intJdn8dn7uvrs intJdn8dn8uvrs intJdn8dn9uvrs intJdn8dn10uvrs intJdn8dn11uvrs intJdn8dn12uvrs;... intJdn9dn1uvrs intJdn9dn2uvrs intJdn9dn3uvrs intJdn9dn4uvrs intJdn9dn5uvrs intJdn9dn6uvrs intJdn9dn7uvrs intJdn9dn8uvrs intJdn9dn9uvrs intJdn9dn10uvrs intJdn9dn11uvrs intJdn9dn12uvrs; ... intJdn10dn1uvrs intJdn10dn2uvrs intJdn10dn3uvrs intJdn10dn4uvrs intJdn10dn5uvrs intJdn10dn6uvrs intJdn10dn7uvrs intJdn10dn8uvrs intJdn10dn9uvrs intJdn10dn10uvrs intJdn10dn11uvrs intJdn10dn12uvrs; ... intJdn11dn1uvrs intJdn11dn2uvrs intJdn11dn3uvrs intJdn11dn4uvrs intJdn11dn5uvrs intJdn11dn6uvrs intJdn11dn7uvrs intJdn11dn8uvrs intJdn11dn9uvrs intJdn11dn10uvrs intJdn11dn11uvrs intJdn11dn12uvrs;... intJdn12dn1uvrs intJdn12dn2uvrs intJdn12dn3uvrs intJdn12dn4uvrs intJdn12dn5uvrs intJdn12dn6uvrs intJdn12dn7uvrs intJdn12dn8uvrs intJdn12dn9uvrs intJdn12dn10uvrs intJdn12dn11uvrs intJdn12dn12uvrs];

intJdndn=double(intJdndn);

case 16

intJdn1dn1uvrs $=\left[\operatorname{vpa}\left(\operatorname{sym}\left({ }^{\prime} .701681093145454720870380087^{\prime}\right)\right) \quad \operatorname{vpa}\left(\operatorname{sym}\left({ }^{\prime} .675893220631106832241358344 '\right)\right) ; \ldots\right.$ $\operatorname{vpa}(\operatorname{sym}(' .675893220631106832241358344 ')) \quad v p a(\operatorname{sym}(' .701681093145454720870380087 '))]$; intJdn1dn2uvrs $=\left[\operatorname{vpa}\left(\operatorname{sym}\left({ }^{\prime}-.268822864629474312918827125 \mathrm{e}-1^{\prime}\right)\right) \quad \operatorname{vpa}\left(\operatorname{sym}\left({ }^{\prime} .510544479594581255114917445 \mathrm{e}-1{ }^{\prime}\right)\right) ; \ldots\right.$ vpa(sym('-.364455520405418744885082555e-1')) vpa(sym(' .32212211804107075133583727e-1'))]; intJdn1dn3uvrs $=\left[\operatorname{vpa}\left(\operatorname{sym}\left({ }^{\prime}-.863032749922837615196918435 \mathrm{e}-2^{\prime}\right)\right) \quad \operatorname{vpa}\left(\operatorname{sym}\left({ }^{\prime}-.104961761402855925624077739 \mathrm{e}-1^{\prime}\right)\right) ; \ldots\right.$ $\left.\operatorname{vpa}\left(\operatorname{sym}\left('-.104961761402855925624077739 \mathrm{e}-11^{\prime}\right)\right) \quad \operatorname{vpa}\left(\operatorname{sym}\left('-.863032749922837615196918435 \mathrm{e}-2{ }^{\prime}\right)\right)\right]$; intJdn1dn4uvrs $=\left[\operatorname{vpa}\left(\operatorname{sym}\left({ }^{\prime} \quad .32212211804107075133583727 \mathrm{e}-1^{\prime}\right)\right) \quad \operatorname{vpa}\left(\operatorname{sym}\left({ }^{\prime}-.364455520405418744885082555 e-1^{\prime}\right)\right) ; \ldots\right.$ $\left.\operatorname{vpa}\left(\operatorname{sym}\left(' \quad .51054447959458125511491745 \mathrm{e}-1^{\prime}\right)\right) \quad \operatorname{vpa}\left(\operatorname{sym}\left('-.268822864629474312918827125 \mathrm{e}-1^{\prime}\right)\right)\right]$; intJdn1dn5uvrs $=\left[\operatorname{vpa}\left(\operatorname{sym}\left({ }^{\prime}-.412633054117600230716206898^{\prime}\right)\right) \quad \operatorname{vpa}\left(\operatorname{sym}\left({ }^{\prime} .268945525984776981543022533 '\right)\right) ; \ldots\right.$ $\operatorname{vpa}(\operatorname{sym}('-.443554474015223018456977467 ')) \quad \operatorname{vpa}(\operatorname{sym}(' .157871624541338704794557829 '))]$; intJdn1dn6uvrs $=\left[\operatorname{vpa}\left(\operatorname{sym}\left({ }^{\prime} \quad .1172503945374017479674604967 '\right)\right) \quad \operatorname{vpa}\left(\operatorname{sym}\left({ }^{\prime} \quad-.142592079476496670389254682 '\right)\right) ; \ldots\right.$ vpa(sym(' .157407920523503329610745318')) vpa(sym('-.70027089525016846364967113e-1'))]; intJdn1dn7uvrs $=\left[\operatorname{vpa}\left(\operatorname{sym}\left({ }^{\prime}-.931446794027204306605665605 \mathrm{e}-1^{\prime}\right)\right) \quad \operatorname{vpa}\left(\operatorname{sym}\left({ }^{\prime} \quad-.1049111993881841007005975075 '\right)\right) ; \ldots\right.$ $\left.\operatorname{vpa}(\operatorname{sym}('-.1049111993881841007005975075 ')) \quad \operatorname{vpa}\left(\operatorname{sym}\left({ }^{\prime}-.92245188408519862030004704 \mathrm{e}-1^{\prime}\right)\right)\right]$; intJdn1dn8uvrs =[vpa(sym(' .33518279789733485446869505e-1')) vpa(sym(' .39281153486431255496910516e-1'));... vpa(sym(' .39281153486431255496910516e-1')) vpa(sym(' .318790711453175872507008895e-1'))]; intJdn1dn9uvrs $=\left[\operatorname{vpa}\left(\operatorname{sym}\left({ }^{\prime} .318790711453175872507008895 \mathrm{e}-1^{\prime}\right)\right) \quad \operatorname{vpa}\left(\operatorname{sym}\left({ }^{\prime} .392811534864312554969105155 \mathrm{e}-1^{\prime}\right)\right) ; \ldots\right.$ $\left.\operatorname{vpa}\left(\operatorname{sym}\left({ }^{\prime} .392811534864312554969105155 \mathrm{e}-1^{\prime}\right)\right) \quad \operatorname{vpa}\left(\operatorname{sym}\left(' .335182797897334854468695045 \mathrm{e}-1^{\prime}\right)\right)\right]$; intJdn1dn10uvrs $=\left[\operatorname{vpa}\left(\operatorname{sym}\left({ }^{\prime}-.922451884085198620300047035 \mathrm{e}-1^{\prime}\right)\right) \quad v p a\left(\operatorname{sym}\left({ }^{\prime} \quad-.1049111993881841007005975075 '\right)\right) ; \ldots\right.$ vpa(sym(' -.1049111993881841007005975075')) vpa(sym(' -.931446794027204306605665605e-1'))]; intJdn1dn11uvrs $=\left[\operatorname{vpa}\left(\operatorname{sym}\left({ }^{\prime}-.70027089525016846364967113 \mathrm{e}-1^{\prime}\right)\right) \quad \operatorname{vpa}\left(\operatorname{sym}\left({ }^{\prime} \quad .157407920523503329610745318^{\prime}\right)\right) ; \ldots\right.$ $\left.\operatorname{vpa}\left(\operatorname{sym}\left({ }^{\prime} \quad-.142592079476496670389254682 '\right)\right) \quad \operatorname{vpa}(\operatorname{sym}(' \quad .1172503945374017479674604967 '))\right]$; intJdn1dn12uvrs $=\left[\operatorname{vpa}\left(\operatorname{sym}\left({ }^{\prime} \quad .157871624541338704794557829 '\right)\right) \quad \operatorname{vpa}\left(\operatorname{sym}\left({ }^{\prime}-.443554474015223018456977467 '\right)\right) ; \ldots\right.$ vpa(sym(' .268945525984776981543022533')) vpa(sym(' -.412633054117600230716206898'))]; intJdn1dn13uvrs $=\left[\operatorname{vpa}\left(\operatorname{sym}\left({ }^{\prime}-.923677839101831305550677102^{\prime}\right)\right) \quad \operatorname{vpa}\left(\operatorname{sym}\left({ }^{\prime}-1.00703245489050046307564230 '\right)\right) ; \ldots\right.$ $\left.\operatorname{vpa}(\operatorname{sym}('-1.00703245489050046307564230 ')) \quad \operatorname{vpa}\left(\operatorname{sym}\left('-.923677839101831305550677102^{\prime}\right)\right)\right]$; intJdn1dn14uvrs $=\left[\operatorname{vpa}\left(\operatorname{sym}\left({ }^{\prime} .336851990222722864094172519^{\prime}\right)\right) \quad \operatorname{vpa}\left(\operatorname{sym}\left({ }^{\prime} .381671404352391717716050003 '\right)\right) ; \ldots\right.$ $\left.\operatorname{vpa}\left(\operatorname{sym}\left({ }^{\prime} .381671404352391717716050003 '\right)\right) \quad \operatorname{vpa}\left(\operatorname{sym}\left({ }^{\prime} .338459011543472358906908599 '\right)\right)\right]$; intJdn1dn15uvrs $=\left[\operatorname{vpa}\left(\operatorname{sym}\left({ }^{\prime}-.122483212211684061698359345^{\prime}\right)\right) \quad \operatorname{vpa}\left(\operatorname{sym}\left({ }^{\prime}-.145263095437075394958553495 '\right)\right) ; \ldots\right.$ $\left.\operatorname{vpa}(\operatorname{sym}('-.145263095437075394958553495 ')) \quad \operatorname{vpa}\left(\operatorname{sym}\left({ }^{\prime}-.122483212211684061698359345^{\prime}\right)\right)\right]$; 
intJdn1dn16uvrs $=\left[\operatorname{vpa}\left(\operatorname{sym}\left({ }^{\prime} .338459011543472358906908599 '\right)\right) \quad \operatorname{vpa}\left(\operatorname{sym}\left({ }^{\prime} .381671404352391717716050003 '\right)\right) ; \ldots\right.$

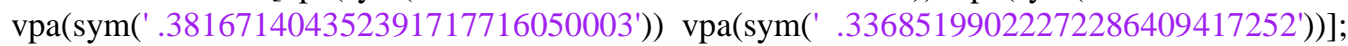

intJdn2dn1uvrs $=\left[\operatorname{vpa}\left(\operatorname{sym}\left({ }^{\prime}-.268822864629474312918827125 \mathrm{e}-1^{\prime}\right)\right) \quad \operatorname{vpa}\left(\operatorname{sym}\left({ }^{\prime}-.364455520405418744885082555 \mathrm{e}-1^{\prime}\right)\right) ; \ldots\right.$ $\left.\operatorname{vpa}\left(\operatorname{sym}\left(' \quad .510544479594581255114917445 \mathrm{e}-1^{\prime}\right)\right) \quad \operatorname{vpa}\left(\operatorname{sym}\left(' \quad .32212211804107075133583727 \mathrm{e}-1^{\prime}\right)\right)\right]$;

intJdn2dn2uvrs $=\left[\operatorname{vpa}(\operatorname{sym}(' \quad .211971597991981069039609973 ')) \quad \operatorname{vpa}\left(\operatorname{sym}\left({ }^{\prime}-.15304657482688411066233853364 '\right)\right) ; \ldots\right.$ vpa(sym(' -.15304657482688411066233853364')) vpa(sym(' .19493546711693338676069677378'))]; intJdn2dn3uvrs $=\left[\operatorname{vpa}\left(\operatorname{sym}\left(' \quad .502810434966178037808492340 \mathrm{e}-1^{\prime}\right)\right) \quad \operatorname{vpa}\left(\operatorname{sym}\left({ }^{\prime} \quad .4154830121388613768196667829 \mathrm{e}-1^{\prime}\right)\right) ; \ldots\right.$ $\operatorname{vpa}\left(\operatorname{sym}\left('-.4595169878611386231803332171 \mathrm{e}-1^{\prime}\right)\right) \quad \operatorname{vpa}\left(\operatorname{sym}\left({ }^{\prime}-.2106669083300601356386564082 \mathrm{e}-1\right.\right.$ '))]; intJdn2dn4uvrs $=\left[\operatorname{vpa}\left(\operatorname{sym}\left({ }^{\prime}-.4598673716969422889922675 \mathrm{e}-3^{\prime}\right)\right) \quad v p a\left(\operatorname{sym}\left({ }^{\prime} .69327746928445697295646522 \mathrm{e}-2^{\prime}\right)\right) ; . .\right.$. vpa(sym(' .69327746928445697295646522e-2')) vpa(sym(' -.4598673716969422889922675e-3'))]; intJdn2dn5uvrs $=\left[\operatorname{vpa}\left(\operatorname{sym}\left({ }^{\prime} \quad .135316485729802334623950907 '\right)\right) \quad v p a\left(\operatorname{sym}\left({ }^{\prime} \quad .149351885457747745233522630 '\right)\right) ; \ldots\right.$ $\left.\operatorname{vpa}(\operatorname{sym}('-.1506481145422522547664773701 ')) \quad \operatorname{vpa}\left(\operatorname{sym}\left('-.356070031451497704872952070 \mathrm{e}-1^{\prime}\right)\right)\right]$; intJdn2dn6uvrs $=\left[\operatorname{vpa}\left(\operatorname{sym}\left({ }^{\prime}-.484060336356140646761807119^{\prime}\right)\right) \quad \operatorname{vpa}\left(\operatorname{sym}\left({ }^{\prime}-.3225407916324774100791670851 '\right)\right) ; .\right.$. vpa(sym(' .3899592083675225899208329149')) vpa(sym(' .1378297018364707614848534907'))]; intJdn2dn7uvrs $=\left[\operatorname{vpa}\left(\operatorname{sym}\left({ }^{\prime} \quad .283468713180315926702327990 '\right)\right) \quad \operatorname{vpa}\left(\operatorname{sym}\left({ }^{\prime} .2616521469230527187251402015 '\right)\right) ; \ldots\right.$ $\left.\operatorname{vpa}\left(\operatorname{sym}\left({ }^{\prime}-.450847853076947281274859798 '\right)\right) \quad v p a(\operatorname{sym}('-.2646472899567640195235481100 '))\right]$; intJdn2dn8uvrs $=\left[\operatorname{vpa}\left(\operatorname{sym}\left({ }^{\prime} \quad-.11413796226676360674772048^{\prime}\right)\right) \quad \operatorname{vpa}\left(\operatorname{sym}\left({ }^{\prime} \quad-.129354487376281056516272933 '\right)\right) ; \ldots\right.$ vpa(sym(' .17064551262371894348372706704')) vpa(sym(' .8032716613183666521258486710e-1'))]; intJdn2dn9uvrs $=\left[\operatorname{vpa}\left(\operatorname{sym}\left({ }^{\prime} \quad-.35328033479583724019581655 \mathrm{e}-1^{\prime}\right)\right) \quad \operatorname{vpa}\left(\operatorname{sym}\left({ }^{\prime} \quad .575010022407089943211709137 \mathrm{e}-1^{\prime}\right)\right) ; \ldots\right.$ $\left.\operatorname{vpa}\left(\operatorname{sym}\left(' \quad .575010022407089943211709137 \mathrm{e}-1^{\prime}\right)\right) \quad \operatorname{vpa}\left(\operatorname{sym}\left({ }^{\prime}-.1076563963110726691819421090 \mathrm{e}-1^{\prime}\right)\right)\right]$; intJdn2dn10uvrs $=\left[\operatorname{vpa}\left(\operatorname{sym}\left(' \quad .51004038179234709257195060 \mathrm{e}-2^{\prime}\right)\right) \quad \operatorname{vpa}\left(\operatorname{sym}\left({ }^{\prime}-.247617179998775437696714569 \mathrm{e}-1^{\prime}\right)\right) ; \ldots\right.$ $\left.\operatorname{vpa}\left(\operatorname{sym}\left('-.247617179998775437696714569 \mathrm{e}-1^{\prime}\right)\right) \quad \operatorname{vpa}\left(\operatorname{sym}\left(' \quad .327370706036359563493129 \mathrm{e}-3{ }^{\prime}\right)\right)\right]$; intJdn2dn11uvrs $=\left[\operatorname{vpa}\left(\operatorname{sym}\left({ }^{\prime} \quad-.8049498872369479004123000 \mathrm{e}-2^{\prime}\right)\right) \quad v p a\left(\operatorname{sym}\left({ }^{\prime}-.239609914551285358186348361 \mathrm{e}-1^{\prime}\right)\right) ; \ldots\right.$ $\operatorname{vpa}(\operatorname{sym}('-.239609914551285358186348361 \mathrm{e}-1 ')) \quad v p a(\operatorname{sym}(' \quad .23530457948583652632350827 \mathrm{e}-2$ '))]; intJdn2dn12uvrs $=\left[\operatorname{vpa}\left(\operatorname{sym}\left({ }^{\prime} .220346626285956934003016986 \mathrm{e}-1^{\prime}\right)\right) \quad \operatorname{vpa}\left(\operatorname{sym}\left({ }^{\prime} .495487663266407134761033842 \mathrm{e}-11^{\prime}\right)\right) ; \ldots\right.$ $\left.\operatorname{vpa}\left(\operatorname{sym}\left(' .495487663266407134761033842 \mathrm{e}-1^{\prime}\right)\right) \quad \operatorname{vpa}\left(\operatorname{sym}\left('-.202705507058571468134520570 \mathrm{e}-1^{\prime}\right)\right)\right]$; intJdn2dn13uvrs $=\left[\operatorname{vpa}\left(\operatorname{sym}\left({ }^{\prime}-.624603048181793546679316570 \mathrm{e}-1^{\prime}\right)\right) \quad \operatorname{vpa}\left(\operatorname{sym}\left({ }^{\prime} \quad-.196130238975238548948111488 '\right)\right) ; \ldots\right.$ vpa(sym(' -.196130238975238548948111488')) vpa(sym(' .18066447765168896456321824e-1'))]; intJdn2dn14uvrs $=\left[\operatorname{vpa}\left(\operatorname{sym}\left({ }^{\prime}-.5654544717914146990072595 \mathrm{e}-2^{\prime}\right)\right) \quad \operatorname{vpa}\left(\operatorname{sym}\left({ }^{\prime} .42762237038458441971847449411^{\prime}\right)\right) ; \ldots\right.$ vpa(sym(' .4276223703845844197184744941')) vpa(sym(' -.1539310157132113391331378486'))]; intJdn2dn15uvrs $=\left[\operatorname{vpa}\left(\operatorname{sym}\left({ }^{\prime} \quad .8033238055969811770258930 \mathrm{e}-2^{\prime}\right)\right) \quad \operatorname{vpa}\left(\operatorname{sym}\left({ }^{\prime} \quad-.1942169737637143545345375175 '\right)\right) ; \ldots\right.$ vpa(sym(' -.1942169737637143545345375175')) vpa(sym('.416813143674259981116969119e-1'))]; intJdn2dn16uvrs $=\left[\operatorname{vpa}\left(\operatorname{sym}\left({ }^{\prime} .208266894443892215290932469 \mathrm{e}-1^{\prime}\right)\right) \quad \operatorname{vpa}\left(\operatorname{sym}\left({ }^{\prime} .86300080830678135931299152 \mathrm{e}-1^{\prime}\right)\right) ; .\right.$. vpa(sym(' .86300080830678135931299152e-1')) vpa(sym(' -.9846681660450092579804616e-3'))]; intJdn3dn1uvrs $=\left[\operatorname{vpa}\left(\operatorname{sym}\left({ }^{\prime}-.863032749922837615196918435 \mathrm{e}-2^{\prime}\right)\right) \quad v p a\left(\operatorname{sym}\left({ }^{\prime}-.104961761402855925624077739 \mathrm{e}-1^{\prime}\right)\right) ; \ldots\right.$ $\left.\operatorname{vpa}\left(\operatorname{sym}\left('-.104961761402855925624077739 \mathrm{e}-1^{\prime}\right)\right) \quad \operatorname{vpa}\left(\operatorname{sym}\left('-.863032749922837615196918435 \mathrm{e}-2^{\prime}\right)\right)\right]$; intJdn3dn2uvrs $=\left[\operatorname{vpa}\left(\operatorname{sym}\left({ }^{\prime} \quad .502810434966178037808492340 \mathrm{e}-1^{\prime}\right)\right) \quad \operatorname{vpa}\left(\operatorname{sym}\left({ }^{\prime}-.4595169878611386231803332171 \mathrm{e}-1^{\prime}\right)\right) ; \ldots\right.$ $\operatorname{vpa}\left(\operatorname{sym}\left(' .4154830121388613768196667829 \mathrm{e}-1\right.\right.$ ')) $\left.\operatorname{vpa}\left(\operatorname{sym}\left({ }^{\prime}-.2106669083300601356386564082 \mathrm{e}-1^{\prime}\right)\right)\right]$; intJdn3dn3uvrs $=\left[\operatorname{vpa}\left(\operatorname{sym}\left({ }^{\prime} .2934564582485161772555681491 '\right)\right) \quad \operatorname{vpa}\left(\operatorname{sym}\left({ }^{\prime} .27260539568220496940415201629 '\right)\right) ; \ldots\right.$ $\operatorname{vpa}(\operatorname{sym}(' .27260539568220496940415201629 ')) \quad v p a(\operatorname{sym}(' .2934564582485161772555681491 '))]$; intJdn3dn4uvrs =[vpa(sym(' -.2106669083300601356386564082e-1')) vpa(sym(' .4154830121388613768196667829e-1'));... $\left.\operatorname{vpa}(\operatorname{sym}('-.4595169878611386231803332171 \mathrm{e}-1 ')) \quad v p a\left(\operatorname{sym}\left(' \quad .502810434966178037808492340 \mathrm{e}-1{ }^{\prime}\right)\right)\right]$; intJdn3dn5uvrs $=\left[\operatorname{vpa}\left(\operatorname{sym}\left({ }^{\prime} .312258974588804915879185904 \mathrm{e}-1^{\prime}\right)\right) \quad \operatorname{vpa}\left(\operatorname{sym}\left({ }^{\prime} \quad .306625487404711706979545881 \mathrm{e}-1^{\prime}\right)\right) ; \ldots\right.$ $\left.\operatorname{vpa}\left(\operatorname{sym}\left(' .306625487404711706979545881 \mathrm{e}-1^{\prime}\right)\right) \operatorname{vpa}\left(\operatorname{sym}\left({ }^{\prime} .1203062469906479395309804256 \mathrm{e}-1^{\prime}\right)\right)\right]$;

intJdn3dn6uvrs $=\left[\operatorname{vpa}\left(\operatorname{sym}\left({ }^{\prime} \quad-.95631533002240688097543501 \mathrm{e}-1^{\prime}\right)\right) \quad v p a\left(\operatorname{sym}\left({ }^{\prime}-.701114223525693099130736200 \mathrm{e}-1^{\prime}\right)\right) ; .\right.$. vpa(sym(' -.701114223525693099130736200e-1')) vpa(sym(' -.2290194853292015105870834477e-1'))]; intJdn3dn7uvrs $=\left[\operatorname{vpa}\left(\operatorname{sym}\left({ }^{\prime}-.66830280257659072749293639 \mathrm{e}-1^{\prime}\right)\right) \quad \operatorname{vpa}\left(\operatorname{sym}\left({ }^{\prime} .15714640889191176538987417817^{\prime}\right)\right) ; \ldots\right.$ $\left.\operatorname{vpa}(\operatorname{sym}('-.14285359110808823461012582183 ')) \quad \operatorname{vpa}\left(\operatorname{sym}\left(' .958310725915156595907307062 \mathrm{e}-1^{\prime}\right)\right)\right]$; intJdn3dn8uvrs $=\left[\operatorname{vpa}\left(\operatorname{sym}\left(' \quad .217119766902955166405682785^{\prime}\right)\right) \quad \operatorname{vpa}\left(\operatorname{sym}\left({ }^{\prime}-.36432561947182595155951418839 '\right)\right) ; .\right.$. $\operatorname{vpa}(\operatorname{sym}(' .34817438052817404844048581161 ')) \quad v p a(\operatorname{sym}(' \quad-.352542724916680732832984020 '))]$; intJdn3dn9uvrs $=\left[\operatorname{vpa}\left(\operatorname{sym}\left({ }^{\prime}-.352542724916680732832984020 '\right)\right) \quad \operatorname{vpa}\left(\operatorname{sym}\left({ }^{\prime} .34817438052817404844048581161 '\right)\right) ; \ldots\right.$ $\left.\operatorname{vpa}\left(\operatorname{sym}\left({ }^{\prime}-.3643256194718259515595141884 '\right)\right) \quad v p a(\operatorname{sym}(' \quad .217119766902955166405682785 '))\right]$; intJdn3dn10uvrs $=\left[\operatorname{vpa}\left(\operatorname{sym}\left(' .958310725915156595907307062 \mathrm{e}-1^{\prime}\right)\right) \quad \operatorname{vpa}\left(\operatorname{sym}\left({ }^{\prime}-.14285359110808823461012582183 '\right)\right) ; \ldots\right.$ vpa(sym(' .15714640889191176538987417817')) vpa(sym(' -.66830280257659072749293639e-1'))]; intJdn3dn11uvrs $=\left[\operatorname{vpa}\left(\operatorname{sym}\left({ }^{\prime}-.2290194853292015105870834477 \mathrm{e}-1^{\prime}\right)\right) \quad \operatorname{vpa}\left(\operatorname{sym}\left({ }^{\prime}-.701114223525693099130736200 \mathrm{e}-1^{\prime}\right)\right) ; \ldots\right.$ $\left.\operatorname{vpa}(\operatorname{sym}('-.701114223525693099130736200 \mathrm{e}-1 ')) \quad \operatorname{vpa}\left(\operatorname{sym}\left({ }^{\prime} \quad-.95631533002240688097543501 \mathrm{e}-1^{\prime}\right)\right)\right]$; intJdn3dn12uvrs $=\left[\operatorname{vpa}\left(\operatorname{sym}\left({ }^{\prime} .1203062469906479395309804256 \mathrm{e}-1^{\prime}\right)\right) \quad \operatorname{vpa}\left(\operatorname{sym}\left({ }^{\prime} .306625487404711706979545881 \mathrm{e}-1^{\prime}\right)\right) ; \ldots\right.$ vpa(sym(' .306625487404711706979545881e-1')) vpa(sym(' .312258974588804915879185904e-1'))]; intJdn3dn13uvrs $=\left[\operatorname{vpa}\left(\operatorname{sym}\left({ }^{\prime}-.440107128429126521075770644 \mathrm{e}-1^{\prime}\right)\right) \quad \operatorname{vpa}\left(\operatorname{sym}\left({ }^{\prime}-.964159752956607711899142376 \mathrm{e}-1^{\prime}\right)\right) ; \ldots\right.$ $\left.\operatorname{vpa}\left(\operatorname{sym}\left('-.964159752956607711899142376 \mathrm{e}-1^{\prime}\right)\right) \quad \operatorname{vpa}\left(\operatorname{sym}\left({ }^{\prime}-.440107128429126521075770644 \mathrm{e}-1^{\prime}\right)\right)\right]$; intJdn3dn14uvrs $=\left[\operatorname{vpa}\left(\operatorname{sym}\left({ }^{\prime} \quad .136999462878579334021389201^{\prime}\right)\right) \quad \operatorname{vpa}\left(\operatorname{sym}\left({ }^{\prime} \quad .2272134136490097198166572636 '\right)\right) ; \ldots\right.$ vpa(sym(' .2272134136490097198166572636')) vpa(sym(' .941185558099118441713021744e-1'))]; intJdn3dn15uvrs $=\left[\operatorname{vpa}\left(\operatorname{sym}\left({ }^{\prime} \quad-.319448664201393584204597479 '\right)\right) \quad \operatorname{vpa}\left(\operatorname{sym}\left({ }^{\prime}-.53496050558802566987955980517 '\right)\right) ; \ldots\right.$ $\operatorname{vpa}(\operatorname{sym}('-.53496050558802566987955980517 ')) \quad \operatorname{vpa}\left(\operatorname{sym}\left(\left(^{\prime} \quad-.319448664201393584204597479 '\right)\right)\right]$; 
intJdn3dn16uvrs $=\left[\operatorname{vpa}\left(\operatorname{sym}\left({ }^{\prime} .941185558099118441713021744 \mathrm{e}-1^{\prime}\right)\right) \quad \operatorname{vpa}\left(\operatorname{sym}\left({ }^{\prime} \quad .2272134136490097198166572636 '\right)\right) ; \ldots\right.$ vpa(sym(' .2272134136490097198166572636')) vpa(sym(' .136999462878579334021389201'))]; intJdn4dn1uvrs $=\left[\operatorname{vpa}\left(\operatorname{sym}\left({ }^{\prime} \quad .32212211804107075133583727 \mathrm{e}-1^{\prime}\right)\right) \quad \operatorname{vpa}\left(\operatorname{sym}\left({ }^{\prime} \quad .51054447959458125511491745 \mathrm{e}-11^{\prime}\right)\right) ; \ldots\right.$ $\operatorname{vpa}(\operatorname{sym}('-.364455520405418744885082555 e-1 ')) \quad \operatorname{vpa}(\operatorname{sym}('-.268822864629474312918827125 e-1 '))]$; intJdn4dn2uvrs $=\left[\operatorname{vpa}\left(\operatorname{sym}\left({ }^{\prime}-.4598673716969422889922675 \mathrm{e}-3^{\prime}\right)\right) \quad v p a\left(\operatorname{sym}\left({ }^{\prime} .69327746928445697295646522 \mathrm{e}-2^{\prime}\right)\right) ; . .\right.$. $\left.\operatorname{vpa}\left(\operatorname{sym}\left(' .69327746928445697295646522 \mathrm{e}-2^{\prime}\right)\right) \operatorname{vpa}\left(\operatorname{sym}\left({ }^{\prime}-.4598673716969422889922675 \mathrm{e}-3 '\right)\right)\right]$; intJdn4dn3uvrs $=\left[\operatorname{vpa}\left(\operatorname{sym}\left({ }^{\prime}-.2106669083300601356386564082 \mathrm{e}-1^{\prime}\right)\right) \quad v p a\left(\operatorname{sym}\left({ }^{\prime}-.4595169878611386231803332171 \mathrm{e}-1^{\prime}\right)\right) ; \ldots\right.$ vpa(sym(' .4154830121388613768196667829e-1')) vpa(sym(' .502810434966178037808492340e-1'))]; intJdn4dn4uvrs $=\left[\operatorname{vpa}\left(\operatorname{sym}\left({ }^{\prime} .19493546711693338676069677378 '\right)\right) \quad v p a\left(\operatorname{sym}\left({ }^{\prime}-.15304657482688411066233853364 '\right)\right) ; \ldots\right.$. $\operatorname{vpa}(\operatorname{sym}('-.15304657482688411066233853364 ')) \quad v p a(\operatorname{sym}(' \quad .211971597991981069039609973 '))]$; intJdn4dn5uvrs $=\left[\operatorname{vpa}\left(\operatorname{sym}\left({ }^{\prime}-.202705507058571468134520570 \mathrm{e}-1^{\prime}\right)\right) \quad \operatorname{vpa}\left(\operatorname{sym}\left({ }^{\prime} .495487663266407134761033842 \mathrm{e}-1^{\prime}\right)\right) ; \ldots\right.$ $\left.\operatorname{vpa}\left(\operatorname{sym}\left(' .495487663266407134761033842 \mathrm{e}-1^{\prime}\right)\right) \quad \operatorname{vpa}\left(\operatorname{sym}\left({ }^{\prime} \quad .22034662628595693400301699 \mathrm{e}-1^{\prime}\right)\right)\right]$; intJdn4dn6uvrs $=\left[\operatorname{vpa}\left(\operatorname{sym}\left(' \quad .2353045794858365263235083 e-2^{\prime}\right)\right) \quad \operatorname{vpa}\left(\operatorname{sym}\left({ }^{\prime}-.23960991455128535818634836 e-1 '\right)\right) ; \ldots\right.$ $\operatorname{vpa}\left(\operatorname{sym}\left('-.23960991455128535818634836 \mathrm{e}-1^{\prime}\right)\right) \quad \operatorname{vpa}(\operatorname{sym}('-.80494988723694790041230002 \mathrm{e}-2$ '))]; intJdn4dn7uvrs $=\left[\operatorname{vpa}\left(\operatorname{sym}\left({ }^{\prime} .327370706036359563493128961 \mathrm{e}-3^{\prime}\right)\right) \quad \operatorname{vpa}\left(\operatorname{sym}\left({ }^{\prime}-.247617179998775437696714569 \mathrm{e}-1^{\prime}\right)\right) ; \ldots\right.$ $\left.\operatorname{vpa}\left(\operatorname{sym}\left('-.247617179998775437696714569 \mathrm{e}-1^{\prime}\right)\right) \quad v p a\left(\operatorname{sym}\left(' \quad .51004038179234709257195060 \mathrm{e}-2^{\prime}\right)\right)\right]$; intJdn4dn8uvrs $=\left[\operatorname{vpa}\left(\operatorname{sym}\left({ }^{\prime}-.1076563963110726691819421090 \mathrm{e}-1^{\prime}\right)\right) \quad \operatorname{vpa}\left(\operatorname{sym}\left({ }^{\prime} \quad .575010022407089943211709137 \mathrm{e}-1^{\prime}\right)\right) ; \ldots\right.$ $\operatorname{vpa}\left(\operatorname{sym}\left(' \quad .575010022407089943211709137 \mathrm{e}-1\right.\right.$ ')) $\operatorname{vpa}\left(\operatorname{sym}\left({ }^{\prime} \quad-.35328033479583724019581655 \mathrm{e}-1\right.\right.$ '))]; intJdn4dn9uvrs $=\left[\operatorname{vpa}\left(\operatorname{sym}\left({ }^{\prime} .8032716613183666521258486710 \mathrm{e}-1^{\prime}\right)\right) \quad \operatorname{vpa}\left(\operatorname{sym}\left({ }^{\prime} \quad .17064551262371894348372706704 '\right)\right) ; \ldots\right.$ $\left.\operatorname{vpa}\left(\operatorname{sym}\left({ }^{\prime}-.12935448737628105651627293296 '\right)\right) \operatorname{vpa}\left(\operatorname{sym}\left({ }^{\prime} \quad-.114137962266763606747720480 '\right)\right)\right]$; intJdn4dn10uvrs $=\left[\operatorname{vpa}\left(\operatorname{sym}\left({ }^{\prime}-.2646472899567640195235481100^{\prime}\right)\right) \quad \operatorname{vpa}\left(\operatorname{sym}\left({ }^{\prime}-.4508478530769472812748597985^{\prime}\right)\right) ; \ldots\right.$ $\left.\operatorname{vpa}\left(\operatorname{sym}\left({ }^{\prime} .2616521469230527187251402015 '\right)\right) \quad v p a(\operatorname{sym}(' \quad .283468713180315926702327990 '))\right]$; intJdn4dn11uvrs $=\left[\operatorname{vpa}\left(\operatorname{sym}\left({ }^{\prime} .1378297018364707614848534907 '\right)\right) \quad \operatorname{vpa}(\operatorname{sym}(' \quad .3899592083675225899208329149 ')) ; \ldots\right.$ $\operatorname{vpa}(\operatorname{sym}('-.3225407916324774100791670851 ')) \quad \operatorname{vpa}(\operatorname{sym}('-.484060336356140646761807119 '))]$; intJdn4dn12uvrs $=\left[\operatorname{vpa}\left(\operatorname{sym}\left({ }^{\prime}-.356070031451497704872952070 \mathrm{e}-1^{\prime}\right)\right) \quad v p a\left(\operatorname{sym}\left({ }^{\prime} \quad-.1506481145422522547664773701 '\right)\right) ; \ldots\right.$ vpa(sym(' .1493518854577477452335226299')) vpa(sym(' .1353164857298023346239509070'))]; intJdn4dn13uvrs $=\left[\operatorname{vpa}\left(\operatorname{sym}\left({ }^{\prime} .18066447765168896456321824 \mathrm{e}-1^{\prime}\right)\right) \quad \operatorname{vpa}\left(\operatorname{sym}\left({ }^{\prime}-.196130238975238548948111488 '\right)\right) ; \ldots\right.$ $\left.\operatorname{vpa}(\operatorname{sym}('-.196130238975238548948111488 ')) \quad \operatorname{vpa}\left(\operatorname{sym}\left('-.6246030481817935466793166 \mathrm{e}-1^{\prime}\right)\right)\right]$; intJdn4dn14uvrs $=\left[\operatorname{vpa}\left(\operatorname{sym}\left({ }^{\prime}-.9846681660450092579804616 \mathrm{e}-3^{\prime}\right)\right) \quad \operatorname{vpa}\left(\operatorname{sym}\left({ }^{\prime} .86300080830678135931299152 \mathrm{e}-1^{\prime}\right)\right) ; \ldots\right.$ $\operatorname{vpa}\left(\operatorname{sym}\left(' .86300080830678135931299152 \mathrm{e}-1\right.\right.$ ')) $\left.\operatorname{vpa}\left(\operatorname{sym}\left({ }^{\prime} .208266894443892215290932469 \mathrm{e}-11^{\prime}\right)\right)\right]$; intJdn4dn15uvrs $=\left[\operatorname{vpa}\left(\operatorname{sym}\left(' .416813143674259981116969119 \mathrm{e}-1^{\prime}\right)\right) \quad \operatorname{vpa}\left(\operatorname{sym}\left({ }^{\prime}-.1942169737637143545345375175 '\right)\right) ; \ldots\right.$. $\operatorname{vpa}(\operatorname{sym}('-.1942169737637143545345375175 ')) \quad \operatorname{vpa}(\operatorname{sym}(' \quad .8033238055969811770258930 \mathrm{e}-2 '))]$; intJdn4dn16uvrs $=\left[\operatorname{vpa}\left(\operatorname{sym}\left({ }^{\prime}-.1539310157132113391331378486 '\right)\right) \quad \operatorname{vpa}\left(\operatorname{sym}\left({ }^{\prime} .4276223703845844197184744941 '\right)\right) ; . .\right.$. $\left.\operatorname{vpa}(\operatorname{sym}(' .4276223703845844197184744941 ')) \quad \operatorname{vpa}\left(\operatorname{sym}\left({ }^{\prime}-.5654544717914146990072595 e-2 '\right)\right)\right]$; intJdn5dn1uvrs $=\left[\operatorname{vpa}\left(\operatorname{sym}\left({ }^{\prime}-.412633054117600230716206898^{\prime}\right)\right) \quad \operatorname{vpa}\left(\operatorname{sym}\left({ }^{\prime}-.443554474015223018456977467 '\right)\right) ; \ldots\right.$ $\operatorname{vpa}(\operatorname{sym}(' .268945525984776981543022533 '))$ vpa(sym(' .157871624541338704794557829'))]; intJdn5dn2uvrs $=\left[\operatorname{vpa}\left(\operatorname{sym}\left({ }^{\prime} \quad .135316485729802334623950907 '\right)\right) \quad \operatorname{vpa}\left(\operatorname{sym}\left({ }^{\prime} \quad-.1506481145422522547664773701 '\right)\right) ; \ldots\right.$ $\left.\operatorname{vpa}(\operatorname{sym}(' \quad .149351885457747745233522630 ')) \quad \operatorname{vpa}\left(\operatorname{sym}\left('-.356070031451497704872952070 \mathrm{e}-1^{\prime}\right)\right)\right]$; intJdn5dn3uvrs $=\left[\operatorname{vpa}\left(\operatorname{sym}\left({ }^{\prime} .312258974588804915879185904 \mathrm{e}-1^{\prime}\right)\right) \quad v p a\left(\operatorname{sym}\left({ }^{\prime} .306625487404711706979545881 \mathrm{e}-11^{\prime}\right)\right) ; \ldots\right.$ $\left.\operatorname{vpa}\left(\operatorname{sym}\left(' .306625487404711706979545881 \mathrm{e}-1^{\prime}\right)\right) \quad \operatorname{vpa}\left(\operatorname{sym}\left(' .1203062469906479395309804256 \mathrm{e}-1^{\prime}\right)\right)\right]$; intJdn5dn4uvrs $=\left[\operatorname{vpa}\left(\operatorname{sym}\left({ }^{\prime}-.202705507058571468134520570 \mathrm{e}-1^{\prime}\right)\right) \quad \operatorname{vpa}\left(\operatorname{sym}\left({ }^{\prime} .495487663266407134761033842 \mathrm{e}-1{ }^{\prime}\right)\right) ; \ldots\right.$ $\left.\operatorname{vpa}\left(\operatorname{sym}\left(' .495487663266407134761033842 \mathrm{e}-1^{\prime}\right)\right) \quad \operatorname{vpa}\left(\operatorname{sym}\left(' \quad .22034662628595693400301699 \mathrm{e}-1^{\prime}\right)\right)\right]$; intJdn5dn5uvrs $=\left[\operatorname{vpa}\left(\operatorname{sym}\left({ }^{\prime} 1.31758678728731273266908314^{\prime}\right)\right) \quad \operatorname{vpa}\left(\operatorname{sym}\left({ }^{\prime} .902875989441502362683040391 '\right)\right) ; \ldots\right.$ $\operatorname{vpa}(\operatorname{sym}(' .902875989441502362683040391 ')) \operatorname{vpa}(\operatorname{sym}(' 1.49530824854081722232161367 '))]$; intJdn5dn6uvrs $=\left[\operatorname{vpa}\left(\operatorname{sym}\left({ }^{\prime}-.78668680846441673459831513779 '\right)\right) \quad \operatorname{vpa}\left(\operatorname{sym}\left({ }^{\prime} \quad .27477449056073334063602205 '\right)\right) ; \ldots\right.$ $\left.\operatorname{vpa}(\operatorname{sym}(' \quad-.737725509439266659363977954 ')) \quad \operatorname{vpa}\left(\operatorname{sym}\left(' \quad-.222026480813621359932407811^{\prime}\right)\right)\right]$; intJdn5dn7uvrs $=\left[\operatorname{vpa}\left(\operatorname{sym}\left({ }^{\prime} .3065397294444835089245124969 '\right)\right) \quad \operatorname{vpa}\left(\operatorname{sym}\left({ }^{\prime} .272605979766225412235674987 '\right)\right) ; \ldots\right.$ $\operatorname{vpa}(\operatorname{sym}(' .272605979766225412235674987 '))$ vpa(sym(' .118010805467176214144917734'))]; intJdn5dn8uvrs $=\left[\operatorname{vpa}\left(\operatorname{sym}\left({ }^{\prime} \quad-.1123602772538571729615200020 '\right)\right) \quad \operatorname{vpa}\left(\operatorname{sym}\left({ }^{\prime} \quad-.1085783194105248930324052264 '\right)\right) ; .\right.$. $\left.\operatorname{vpa}(\operatorname{sym}('-.1085783194105248930324052264 ')) \quad \operatorname{vpa}\left(\operatorname{sym}\left({ }^{\prime}-.424031731725615725542540841 \mathrm{e}-1^{\prime}\right)\right)\right]$; intJdn5dn9uvrs $=\left[\operatorname{vpa}\left(\operatorname{sym}\left({ }^{\prime} \quad-.1349507714997270711398676150 '\right)\right) \quad \operatorname{vpa}\left(\operatorname{sym}\left({ }^{\prime} \quad-.1134650610452099677162243619 '\right)\right) ; \ldots\right.$ $\left.\operatorname{vpa}(\operatorname{sym}('-.1134650610452099677162243619 ')) \quad \operatorname{vpa}\left(\operatorname{sym}\left({ }^{\prime}-.283674858742885011995552238 \mathrm{e}-1^{\prime}\right)\right)\right]$; intJdn5dn10uvrs $=\left[\operatorname{vpa}\left(\operatorname{sym}\left({ }^{\prime} .1066791125925855714553223009 '\right)\right) \quad \operatorname{vpa}(\operatorname{sym}(' \quad .6059337192477808811068095 e-2 ')) ; \ldots\right.$ $\operatorname{vpa}(\operatorname{sym}('$.6059337192477808811068095e-2')) vpa(sym(' -.1306849801916743926696037294'))]; intJdn5dn11uvrs $=\left[\operatorname{vpa}\left(\operatorname{sym}\left({ }^{\prime}-.26978045869880871379513078 \mathrm{e}-1^{\prime}\right)\right) \quad \operatorname{vpa}\left(\operatorname{sym}\left({ }^{\prime} \quad-.196966390341090928909694084 '\right)\right) ; \ldots\right.$ vpa(sym(' -.196966390341090928909694084')) vpa(sym(' -.78897358249752608668273981e-1'))]; intJdn5dn12uvrs $=\left[\operatorname{vpa}\left(\operatorname{sym}\left({ }^{\prime} .8921165458163810696876696 \mathrm{e}-1^{\prime}\right)\right) \quad \operatorname{vpa}\left(\operatorname{sym}\left({ }^{\prime} .427188884799078070173992386 '\right)\right) ; \ldots\right.$ vpa(sym(' .427188884799078070173992386')) vpa(sym(' .8921165458163810696876696e-1'))]; intJdn5dn13uvrs $=\left[\operatorname{vpa}\left(\operatorname{sym}\left({ }^{\prime} \quad .475558907831932003188746478^{\prime}\right)\right) \quad \operatorname{vpa}\left(\operatorname{sym}\left({ }^{\prime} \quad-.53084335598705132620498792 '\right)\right) ; \ldots\right.$ $\left.\operatorname{vpa}\left(\operatorname{sym}\left({ }^{\prime}-.53084335598705132620498792 '\right)\right) \operatorname{vpa}(\operatorname{sym}('-1.83117956250893266072281398 '))\right]$;

intJdn5dn14uvrs $=\left[\operatorname{vpa}\left(\operatorname{sym}\left({ }^{\prime}-1.19557360195760315006531368^{\prime}\right)\right) \quad \operatorname{vpa}\left(\operatorname{sym}\left({ }^{\prime}-.890289639589649219996546217 '\right)\right) ; \ldots\right.$ vpa(sym(' -.890289639589649219996546217')) vpa(sym(' -.146237679902381668344145480'))]; intJdn5dn15uvrs $=\left[\operatorname{vpa}\left(\operatorname{sym}\left({ }^{\prime} \quad .449877692126663661629552350 '\right)\right) \quad \operatorname{vpa}\left(\operatorname{sym}\left({ }^{\prime} \quad .379360747071947741960232042 '\right)\right) ; \ldots\right.$ $\left.\operatorname{vpa}(\operatorname{sym}(' \quad .379360747071947741960232042 ')) \quad \operatorname{vpa}\left(\operatorname{sym}\left({ }^{\prime} .86904547772480103903106692 \mathrm{e}-1^{\prime}\right)\right)\right]$; 
intJdn5dn16uvrs $=\left[\operatorname{vpa}\left(\operatorname{sym}\left({ }^{\prime}-.2225431571843560333736647188^{\prime}\right)\right) \quad \operatorname{vpa}\left(\operatorname{sym}\left(' .91268611031924988409224700 \mathrm{e}-1^{\prime}\right)\right) ; \ldots\right.$ vpa(sym('.91268611031924988409224700e-1')) vpa(sym(' .534031555627251695091986887'))];

intJdn6dn1uvrs $=\left[\operatorname{vpa}\left(\operatorname{sym}\left({ }^{\prime} \quad .1172503945374017479674604967 '\right)\right) \quad \operatorname{vpa}\left(\operatorname{sym}\left({ }^{\prime} \quad .157407920523503329610745318^{\prime}\right)\right) ; \ldots\right.$ vpa(sym(' -.142592079476496670389254682')) vpa(sym(' -.70027089525016846364967113e-1'))];

intJdn6dn2uvrs $=\left[\operatorname{vpa}\left(\operatorname{sym}\left({ }^{\prime}-.484060336356140646761807119^{\prime}\right)\right) \quad \operatorname{vpa}\left(\operatorname{sym}\left({ }^{\prime} .3899592083675225899208329149 '\right)\right) ; .\right.$. $\operatorname{vpa}(\operatorname{sym}('-.3225407916324774100791670851 ')) \quad \operatorname{vpa}(\operatorname{sym}(' .1378297018364707614848534907 '))]$;

intJdn6dn3uvrs $=\left[\operatorname{vpa}\left(\operatorname{sym}\left({ }^{\prime} \quad-.95631533002240688097543501 \mathrm{e}-1^{\prime}\right)\right) \quad \operatorname{vpa}\left(\operatorname{sym}\left({ }^{\prime} \quad-.701114223525693099130736200 \mathrm{e}-1^{\prime}\right)\right) ; \ldots\right.$ $\left.\operatorname{vpa}(\operatorname{sym}('-.701114223525693099130736200 \mathrm{e}-1 ')) \quad \operatorname{vpa}\left(\operatorname{sym}\left({ }^{\prime}-.2290194853292015105870834477 \mathrm{e}-11^{\prime}\right)\right)\right]$;

intJdn6dn4uvrs $=\left[\operatorname{vpa}\left(\operatorname{sym}\left({ }^{\prime} \quad .2353045794858365263235083 \mathrm{e}-2^{\prime}\right)\right) \quad \operatorname{vpa}\left(\operatorname{sym}\left({ }^{\prime}-.239609914551285358186348361 \mathrm{e}-1^{\prime}\right)\right) ; .\right.$. $\operatorname{vpa}(\operatorname{sym}('-.239609914551285358186348361 \mathrm{e}-1 ')) \quad v p a(\operatorname{sym}('-.80494988723694790041230002 \mathrm{e}-2$ '))]; intJdn6dn5uvrs $=\left[\operatorname{vpa}\left(\operatorname{sym}\left({ }^{\prime}-.78668680846441673459831513779 '\right)\right) \quad \operatorname{vpa}\left(\operatorname{sym}\left({ }^{\prime} \quad-.737725509439266659363977954 '\right)\right) ; .\right.$. $\left.\operatorname{vpa}(\operatorname{sym}(' \quad .27477449056073334063602205 ')) \quad v p a\left(\operatorname{sym}\left(' \quad-.222026480813621359932407811^{\prime}\right)\right)\right]$; intJdn6dn6uvrs $=\left[\operatorname{vpa}\left(\operatorname{sym}\left({ }^{\prime} 1.332289708203970804992564589 '\right)\right) \quad \operatorname{vpa}\left(\operatorname{sym}\left({ }^{\prime} \quad .602065373518435159503113519 '\right)\right) ; \ldots\right.$ $\operatorname{vpa}(\operatorname{sym}(' .602065373518435159503113519 '))$ vpa(sym(' 1.141981449369884652860820049'))]; intJdn6dn7uvrs $=\left[\operatorname{vpa}\left(\operatorname{sym}\left({ }^{\prime}-.857977568743733072181109962 '\right)\right) \quad v p a\left(\operatorname{sym}\left({ }^{\prime}-.629015904385547067627650917 '\right)\right) ; \ldots\right.$ $\operatorname{vpa}(\operatorname{sym}('-.629015904385547067627650917 ')) \operatorname{vpa}(\operatorname{sym}('-.2543377601216534395445354534 '))]$; intJdn6dn8uvrs $=\left[\operatorname{vpa}\left(\operatorname{sym}\left({ }^{\prime} \quad .315711302473187041277220189 '\right)\right) \quad \operatorname{vpa}\left(\operatorname{sym}\left({ }^{\prime} \quad .2481990187365599088136231974 '\right)\right) ; \ldots\right.$ vpa(sym(' .2481990187365599088136231974')) vpa(sym(' .837329943403397038665668996e-1'))]; intJdn6dn9uvrs $=\left[\operatorname{vpa}(\operatorname{sym}(' \quad .123808042711948724330825139 ')) \quad \operatorname{vpa}\left(\operatorname{sym}\left(\left(^{\prime}-.79362521672469957651556136 \mathrm{e}-2^{\prime}\right)\right) ; \ldots\right.\right.$ $\operatorname{vpa}\left(\operatorname{sym}('-.79362521672469957651556136 \mathrm{e}-2\right.$ ') $\left.) \quad \operatorname{vpa}\left(\operatorname{sym}\left({ }^{\prime}-.11543642609252788831829720852 '\right)\right)\right]$;

intJdn6dn10uvrs $=\left[\operatorname{vpa}\left(\operatorname{sym}\left({ }^{\prime}-.426191802370655235374397294 \mathrm{e}-1^{\prime}\right)\right) \quad \operatorname{vpa}\left(\operatorname{sym}\left({ }^{\prime} .832121696074405782549458791 \mathrm{e}-1^{\prime}\right)\right) ; \ldots\right.$ $\left.\operatorname{vpa}\left(\operatorname{sym}\left(' .832121696074405782549458791 \mathrm{e}-11^{\prime}\right)\right) \operatorname{vpa}\left(\operatorname{sym}\left(' .498827009902849670234649781 \mathrm{e}-11^{\prime}\right)\right)\right]$;

intJdn6dn11uvrs =[vpa(sym(' .27527791093307208248725575e-1')) vpa(sym(' .89268098816052016578387726e-1'));... $\left.\operatorname{vpa}\left(\operatorname{sym}\left(' .89268098816052016578387726 \mathrm{e}-1^{\prime}\right)\right) \operatorname{vpa}\left(\operatorname{sym}\left({ }^{\prime} .27527791093307208248725575 \mathrm{e}-1^{\prime}\right)\right)\right]$; intJdn6dn12uvrs $=\left[\operatorname{vpa}\left(\operatorname{sym}\left({ }^{\prime}-.78897358249752608668273981 \mathrm{e}-1^{\prime}\right)\right) \quad \operatorname{vpa}\left(\operatorname{sym}\left({ }^{\prime} \quad-.196966390341090928909694084 '\right)\right) ; \ldots\right.$ vpa(sym(' -.196966390341090928909694084')) vpa(sym(' -.26978045869880871379513078e-1'))]; intJdn6dn13uvrs $=\left[\operatorname{vpa}\left(\operatorname{sym}\left({ }^{\prime} .107366707368679853419887039 '\right)\right) \quad \operatorname{vpa}\left(\operatorname{sym}\left({ }^{\prime} .827423779129943639113014061^{\prime}\right)\right) ; \ldots\right.$ $\left.\operatorname{vpa}\left(\operatorname{sym}\left(' .827423779129943639113014061^{\prime}\right)\right) \quad \operatorname{vpa}\left(\operatorname{sym}\left(' .520202184956697891678232119^{\prime}\right)\right)\right]$; intJdn6dn14uvrs $=\left[\operatorname{vpa}\left(\operatorname{sym}\left({ }^{\prime} \quad .60105835750654359495658263 '\right)\right) \quad \operatorname{vpa}\left(\operatorname{sym}\left({ }^{\prime} \quad-.510599460959200051978459650 '\right)\right) ; \ldots\right.$ $\operatorname{vpa}(\operatorname{sym}('-.510599460959200051978459650 ')) \quad v p a(\operatorname{sym}('-1.501752787756720291051955360 '))]$; intJdn6dn15uvrs $=\left[\operatorname{vpa}\left(\operatorname{sym}\left({ }^{\prime}-.26123164244466091271015883^{\prime}\right)\right) \quad \operatorname{vpa}\left(\operatorname{sym}\left({ }^{\prime} .106104670667416834133287489 '\right)\right) ; \ldots\right.$ $\left.\operatorname{vpa}(\operatorname{sym}(' .106104670667416834133287489 ')) \quad \operatorname{vpa}\left(\operatorname{sym}\left(' .449881020809519531356712718^{\prime}\right)\right)\right]$; intJdn6dn16uvrs $=\left[\operatorname{vpa}\left(\operatorname{sym}\left({ }^{\prime}-.20260922191887153901852490 \mathrm{e}-1^{\prime}\right)\right) \quad \operatorname{vpa}\left(\operatorname{sym}\left({ }^{\prime} \quad-.327324308266824506551303460 '\right)\right) ; \ldots\right.$ $\operatorname{vpa}(\operatorname{sym}(' \quad-.327324308266824506551303460 ')) \quad \operatorname{vpa}(\operatorname{sym}(' \quad-.189527805811794389864868430 '))]$; intJdn7dn1uvrs $=\left[\operatorname{vpa}\left(\operatorname{sym}\left({ }^{\prime}-.931446794027204306605665605 e-1^{\prime}\right)\right) \quad \operatorname{vpa}\left(\operatorname{sym}\left({ }^{\prime} \quad-.1049111993881841007005975075 '\right)\right) ; \ldots\right.$ $\left.\operatorname{vpa}(\operatorname{sym}(' \quad-.1049111993881841007005975075 ')) \quad \operatorname{vpa}\left(\operatorname{sym}\left({ }^{\prime}-.92245188408519862030004704 \mathrm{e}-1^{\prime}\right)\right)\right]$; intJdn7dn2uvrs $=\left[\operatorname{vpa}\left(\operatorname{sym}\left({ }^{\prime} \quad .283468713180315926702327990 '\right)\right) \quad \operatorname{vpa}\left(\operatorname{sym}\left({ }^{\prime}-.450847853076947281274859798 '\right)\right) ; \ldots\right.$ $\operatorname{vpa}\left(\operatorname{sym}\left(' .2616521469230527187251402015^{\prime}\right)\right)$ vpa(sym(' -.2646472899567640195235481100'))]; intJdn7dn3uvrs $=\left[\operatorname{vpa}\left(\operatorname{sym}\left({ }^{\prime}-.66830280257659072749293639 e-1^{\prime}\right)\right) \quad \operatorname{vpa}\left(\operatorname{sym}\left({ }^{\prime}-.14285359110808823461012582183 '\right)\right) ; \ldots\right.$ vpa(sym(' .15714640889191176538987417817')) vpa(sym(' .958310725915156595907307062e-1'))]; intJdn7dn4uvrs $=\left[\operatorname{vpa}\left(\operatorname{sym}\left({ }^{\prime} .327370706036359563493128961 \mathrm{e}-3^{\prime}\right)\right) \quad \operatorname{vpa}\left(\operatorname{sym}\left({ }^{\prime}-.247617179998775437696714569 \mathrm{e}-1^{\prime}\right)\right) ; \ldots\right.$ vpa(sym(' -.247617179998775437696714569e-1')) vpa(sym(' .51004038179234709257195060e-2'))]; intJdn7dn5uvrs $=\left[\operatorname{vpa}\left(\operatorname{sym}\left({ }^{\prime} .3065397294444835089245124969 '\right)\right) \quad \operatorname{vpa}\left(\operatorname{sym}\left({ }^{\prime} .272605979766225412235674987 '\right)\right) ; \ldots\right.$ $\operatorname{vpa}(\operatorname{sym}(' .272605979766225412235674987 ')) \operatorname{vpa}(\operatorname{sym}(' .118010805467176214144917734 '))]$; intJdn7dn6uvrs $=\left[\operatorname{vpa}\left(\operatorname{sym}\left({ }^{\prime} \quad-.857977568743733072181109962 '\right)\right) \quad v p a\left(\operatorname{sym}\left({ }^{\prime}-.6290159043855470676276509169 '\right)\right) ; . .\right.$. $\left.\operatorname{vpa}\left(\operatorname{sym}\left('-.6290159043855470676276509169^{\prime}\right)\right) \quad \operatorname{vpa}(\operatorname{sym}('-.2543377601216534395445354534 '))\right]$; intJdn7dn7uvrs $=\left[\operatorname{vpa}\left(\operatorname{sym}\left({ }^{\prime} 2.001089658057721102391264327 '\right)\right) \quad \operatorname{vpa}\left(\operatorname{sym}\left({ }^{\prime} .27309567677916265610654186068 '\right)\right) ; \ldots\right.$ $\operatorname{vpa}(\operatorname{sym}(' .27309567677916265610654186068 '))$ vpa(sym(' .666504113204159209546195038'))]; intJdn7dn8uvrs $=\left[\operatorname{vpa}\left(\operatorname{sym}\left({ }^{\prime} \quad-.264631702815104949739660648 '\right)\right) \quad \operatorname{vpa}\left(\operatorname{sym}\left({ }^{\prime} \quad .399164031349690309393851622 '\right)\right) ; \ldots\right.$ vpa(sym(' -.61333596865030969060614837761')) vpa(sym(' -.4881203408468691695076954060'))]; intJdn7dn9uvrs $=\left[\operatorname{vpa}\left(\operatorname{sym}\left({ }^{\prime} .46925409227203366956712440 \mathrm{e}-1^{\prime}\right)\right) \quad \operatorname{vpa}\left(\operatorname{sym}\left({ }^{\prime}-.2046226562144629893771449553 '\right)\right) ; \ldots\right.$ vpa(sym(' -.2046226562144629893771449553')) vpa(sym(' .530267352079846205377516680e-1'))]; intJdn7dn10uvrs $=\left[\operatorname{vpa}\left(\operatorname{sym}\left({ }^{\prime}-.54552417791786682579711494 \mathrm{e}-2^{\prime}\right)\right) \quad v p a\left(\operatorname{sym}\left({ }^{\prime} .877730624778960602031917001 \mathrm{e}-1^{\prime}\right)\right) ; \ldots\right.$ $\left.\operatorname{vpa}\left(\operatorname{sym}\left(' .877730624778960602031917001 \mathrm{e}-1^{\prime}\right)\right) \quad \operatorname{vpa}\left(\operatorname{sym}\left('-.54552417791786682579711494 \mathrm{e}-2^{\prime}\right)\right)\right]$; intJdn7dn11uvrs =[vpa(sym(' .498827009902849670234649781e-1')) vpa(sym(' .832121696074405782549458791e-1'));... $\left.\operatorname{vpa}\left(\operatorname{sym}\left(' .832121696074405782549458791 \mathrm{e}-1^{\prime}\right)\right) \quad \operatorname{vpa}\left(\operatorname{sym}\left('-.426191802370655235374397294 \mathrm{e}-1^{\prime}\right)\right)\right]$; intJdn7dn12uvrs $=\left[\operatorname{vpa}\left(\operatorname{sym}\left({ }^{\prime}-.1306849801916743926696037294 '\right)\right) \quad \operatorname{vpa}\left(\operatorname{sym}\left({ }^{\prime} \quad .6059337192477808811068095 \mathrm{e}-2^{\prime}\right)\right) ; \ldots\right.$ $\operatorname{vpa}(\operatorname{sym}('$.6059337192477808811068095e-2')) vpa(sym(' .1066791125925855714553223009'))]; intJdn7dn13uvrs $=\left[\operatorname{vpa}\left(\operatorname{sym}\left({ }^{\prime} \quad .621790544829774902399463480 '\right)\right) \quad \operatorname{vpa}\left(\operatorname{sym}\left({ }^{\prime} .30448320844630450044664700 \mathrm{e}-1^{\prime}\right)\right) ; \ldots\right.$ $\operatorname{vpa}\left(\operatorname{sym}\left(' .30448320844630450044664700 \mathrm{e}-1^{\prime}\right)\right)$ vpa(sym(' -.1131519430916361403558955158'))]; intJdn7dn14uvrs $=\left[\operatorname{vpa}\left(\operatorname{sym}\left({ }^{\prime}-2.24559114498245358748525615^{\prime}\right)\right) \quad \operatorname{vpa}\left(\operatorname{sym}\left({ }^{\prime} .32502712079217193433836137 \mathrm{e}-1^{\prime}\right)\right) ; \ldots\right.$ $\operatorname{vpa}\left(\operatorname{sym}\left(' .32502712079217193433836137 \mathrm{e}-1^{\prime}\right)\right)$ vpa(sym(' .4636424264352335415173399435'))]; intJdn7dn15uvrs $=\left[\operatorname{vpa}\left(\operatorname{sym}\left({ }^{\prime} \quad .55242173149670587806391031^{\prime}\right)\right) \quad \operatorname{vpa}\left(\operatorname{sym}\left({ }^{\prime} .6763849144738999657208941635 '\right)\right) ; \ldots\right.$ $\left.\operatorname{vpa}(\operatorname{sym}(' .6763849144738999657208941635 ')) \quad \operatorname{vpa}\left(\operatorname{sym}\left('-.293283237003299311368713211^{\prime}\right)\right)\right]$; intJdn7dn16uvrs $=\left[\operatorname{vpa}\left(\operatorname{sym}\left({ }^{\prime}-.198130259760001838281687341 '\right)\right) \quad \operatorname{vpa}\left(\operatorname{sym}\left({ }^{\prime}-.304233282397533216844618686 '\right)\right) ; \ldots\right.$ 
$\left.\operatorname{vpa}\left(\operatorname{sym}\left({ }^{\prime}-.304233282397533216844618686 '\right)\right) \quad \operatorname{vpa}\left(\operatorname{sym}\left({ }^{\prime} .45065512128407846407826384 \mathrm{e}-1^{\prime}\right)\right)\right]$;

intJdn8dn1uvrs $=\left[\operatorname{vpa}\left(\operatorname{sym}\left({ }^{\prime} .33518279789733485446869505 e-1 '\right)\right) \quad \operatorname{vpa}\left(\operatorname{sym}\left({ }^{\prime} .39281153486431255496910516 \mathrm{e}-1^{\prime}\right)\right) ; \ldots\right.$ vpa(sym(' .39281153486431255496910516e-1')) vpa(sym(' .318790711453175872507008895e-1'))];

intJdn8dn2uvrs $=\left[\operatorname{vpa}\left(\operatorname{sym}\left({ }^{\prime} \quad-.11413796226676360674772048 '\right)\right) \quad \operatorname{vpa}\left(\operatorname{sym}\left({ }^{\prime} \quad .17064551262371894348372706704 '\right)\right) ; \ldots\right.$ $\left.\operatorname{vpa}(\operatorname{sym}(' \quad-.129354487376281056516272933 ')) \quad v p a\left(\operatorname{sym}\left(' .8032716613183666521258486710 \mathrm{e}-1^{\prime}\right)\right)\right]$;

intJdn8dn3uvrs $=\left[\operatorname{vpa}\left(\operatorname{sym}\left(' \quad .217119766902955166405682785^{\prime}\right)\right) \quad \operatorname{vpa}\left(\operatorname{sym}\left({ }^{\prime} \quad .34817438052817404844048581161 '\right)\right) ; \ldots\right.$ $\left.\operatorname{vpa}\left(\operatorname{sym}\left({ }^{\prime}-.36432561947182595155951418839 '\right)\right) \quad \operatorname{vpa}\left(\operatorname{sym}\left({ }^{\prime} \quad-.352542724916680732832984020 '\right)\right)\right]$;

intJdn8dn4uvrs $=\left[\operatorname{vpa}\left(\operatorname{sym}\left({ }^{\prime}-.1076563963110726691819421090 \mathrm{e}-1^{\prime}\right)\right) \quad \operatorname{vpa}\left(\operatorname{sym}\left({ }^{\prime} \quad .575010022407089943211709137 \mathrm{e}-11^{\prime}\right)\right) ; \ldots\right.$ vpa(sym(' .575010022407089943211709137e-1')) vpa(sym(' $\left.\left.\left.\quad-.35328033479583724019581655 \mathrm{e}-1^{\prime}\right)\right)\right]$; intJdn8dn5uvrs $=\left[\operatorname{vpa}(\operatorname{sym}(' \quad-.1123602772538571729615200020 ')) \quad \operatorname{vpa}\left(\operatorname{sym}\left({ }^{\prime} \quad-.1085783194105248930324052264 '\right)\right) ; \ldots\right.$ $\operatorname{vpa}(\operatorname{sym}(' \quad-.1085783194105248930324052264 ')) \operatorname{vpa}(\operatorname{sym}('-.424031731725615725542540841 \mathrm{e}-1$ '))];

intJdn8dn6uvrs $=\left[\operatorname{vpa}\left(\operatorname{sym}\left({ }^{\prime} \quad .315711302473187041277220189 '\right)\right) \quad \operatorname{vpa}\left(\operatorname{sym}\left({ }^{\prime} \quad .2481990187365599088136231974 '\right)\right) ; \ldots\right.$ vpa(sym(' .2481990187365599088136231974')) vpa(sym(' .837329943403397038665668996e-1'))]; intJdn8dn7uvrs $=\left[\operatorname{vpa}\left(\operatorname{sym}\left({ }^{\prime} \quad-.264631702815104949739660648^{\prime}\right)\right) \quad \operatorname{vpa}\left(\operatorname{sym}\left({ }^{\prime}-.61333596865030969060614837761 '\right)\right) ; \ldots\right.$ $\left.\operatorname{vpa}(\operatorname{sym}(' \quad .399164031349690309393851622 ')) \quad \operatorname{vpa}\left(\operatorname{sym}\left({ }^{\prime}-.4881203408468691695076954060 '\right)\right)\right]$; intJdn8dn8uvrs $=\left[\operatorname{vpa}\left(\operatorname{sym}\left(' 1.653151620925835592672919485^{\prime}\right)\right) \quad \operatorname{vpa}\left(\operatorname{sym}\left({ }^{\prime} .15317854106260736184624261268 '\right)\right) ; \ldots\right.$ $\operatorname{vpa}(\operatorname{sym}(' .15317854106260736184624261268 ')) \quad v p a(\operatorname{sym}(' \quad .767115078598661079333576130 '))]$; intJdn8dn9uvrs $=\left[\operatorname{vpa}\left(\operatorname{sym}\left({ }^{\prime}-.222366721554688830294037920 '\right)\right) \quad \operatorname{vpa}\left(\operatorname{sym}\left({ }^{\prime} .48499079406584066090468527082 '\right)\right) ; \ldots\right.$ $\operatorname{vpa}(\operatorname{sym}(' .48499079406584066090468527082 '))$ vpa(sym(' -.222366721554688830294037920'))]; intJdn8dn10uvrs $=\left[\operatorname{vpa}\left(\operatorname{sym}\left(' .530267352079846205377516680 \mathrm{e}-1^{\prime}\right)\right) \quad \operatorname{vpa}\left(\operatorname{sym}\left({ }^{\prime}-.2046226562144629893771449553 '\right)\right) ; \ldots\right.$ $\operatorname{vpa}(\operatorname{sym}('-.2046226562144629893771449553 ')) \operatorname{vpa}(\operatorname{sym}(' .46925409227203366956712440 \mathrm{e}-1$ '))]; intJdn8dn11uvrs $=\left[\operatorname{vpa}\left(\operatorname{sym}\left({ }^{\prime}-.11543642609252788831829720852 '\right)\right) \quad \operatorname{vpa}\left(\operatorname{sym}\left({ }^{\prime}-.79362521672469957651556136 \mathrm{e}-2 '\right)\right) ; \ldots\right.$ $\left.\operatorname{vpa}\left(\operatorname{sym}\left('-.79362521672469957651556136 \mathrm{e}-2^{\prime}\right)\right) \quad \operatorname{vpa}\left(\operatorname{sym}\left({ }^{\prime} \quad .123808042711948724330825139 '\right)\right)\right]$; intJdn8dn12uvrs $=\left[\operatorname{vpa}\left(\operatorname{sym}\left({ }^{\prime}-.283674858742885011995552238 \mathrm{e}-1^{\prime}\right)\right) \quad \operatorname{vpa}\left(\operatorname{sym}\left({ }^{\prime} \quad-.1134650610452099677162243619 '\right)\right) ; \ldots\right.$. $\left.\operatorname{vpa}(\operatorname{sym}(' \quad-.1134650610452099677162243619 ')) \quad \operatorname{vpa}\left(\operatorname{sym}\left({ }^{\prime} \quad-.1349507714997270711398676150 '\right)\right)\right]$; intJdn8dn13uvrs $=\left[\operatorname{vpa}\left(\operatorname{sym}\left({ }^{\prime} .60329059165411543334696078 \mathrm{e}-1^{\prime}\right)\right) \quad \operatorname{vpa}\left(\operatorname{sym}\left({ }^{\prime} \quad .331278731126193705006371948 '\right)\right) ; \ldots\right.$ $\operatorname{vpa}(\operatorname{sym}(' \quad .331278731126193705006371948 ')) \operatorname{vpa}(\operatorname{sym}(' \quad .178851051525261210302257368 '))]$; intJdn8dn14uvrs $=\left[\operatorname{vpa}\left(\operatorname{sym}\left({ }^{\prime}-.5199575926777798530262703 \mathrm{e}-1^{\prime}\right)\right) \quad \operatorname{vpa}\left(\operatorname{sym}\left({ }^{\prime}-.7955065459935637821598364152 '\right)\right) ; \ldots\right.$ vpa(sym(' -.7955065459935637821598364152')) vpa(sym(' -.433262214197932020360238335'))]; intJdn8dn15uvrs $=\left[\operatorname{vpa}\left(\operatorname{sym}\left({ }^{\prime} \quad-1.94418619472045071324352388 '\right)\right) \quad \operatorname{vpa}\left(\operatorname{sym}\left({ }^{\prime}-.266497211117548073090616868 \mathrm{e}-1^{\prime}\right)\right) ; \ldots\right.$ $\left.\operatorname{vpa}\left(\operatorname{sym}\left('-.266497211117548073090616868 \mathrm{e}-1^{\prime}\right)\right) \quad v p a(\operatorname{sym}(' \quad .550843979761993341763278679 '))\right]$; intJdn8dn16uvrs $=\left[\operatorname{vpa}\left(\operatorname{sym}\left({ }^{\prime} \quad .531391405011459465049996936 '\right)\right) \quad v p a\left(\operatorname{sym}\left({ }^{\prime} .36845390722838247652759296 e-1 '\right)\right) ; \ldots\right.$ vpa(sym(' .36845390722838247652759296e-1')) vpa(sym(' -.154508813774518558307843375'))]; intJdn9dn1uvrs =[vpa(sym(' .318790711453175872507008895e-1')) vpa(sym(' .39281153486431255496910516e-1'));... $\left.\operatorname{vpa}\left(\operatorname{sym}\left(' .39281153486431255496910516 \mathrm{e}-1^{\prime}\right)\right) \operatorname{vpa}\left(\operatorname{sym}\left({ }^{\prime} .335182797897334854468695045 \mathrm{e}-1^{\prime}\right)\right)\right]$; intJdn9dn2uvrs $=\left[\operatorname{vpa}\left(\operatorname{sym}\left({ }^{\prime} \quad-.35328033479583724019581655 \mathrm{e}-1^{\prime}\right)\right) \quad \operatorname{vpa}\left(\operatorname{sym}\left({ }^{\prime} \quad .575010022407089943211709137 \mathrm{e}-1^{\prime}\right)\right) ; \ldots\right.$ $\left.\operatorname{vpa}\left(\operatorname{sym}\left(' \quad .575010022407089943211709137 \mathrm{e}-1^{\prime}\right)\right) \quad \operatorname{vpa}\left(\operatorname{sym}\left({ }^{\prime}-.1076563963110726691819421090 \mathrm{e}-1^{\prime}\right)\right)\right]$; intJdn9dn3uvrs $=\left[\operatorname{vpa}\left(\operatorname{sym}\left({ }^{\prime}-.352542724916680732832984020^{\prime}\right)\right) \quad \operatorname{vpa}\left(\operatorname{sym}\left({ }^{\prime}-.3643256194718259515595141884 '\right)\right) ; \ldots\right.$ $\left.\operatorname{vpa}\left(\operatorname{sym}\left(' .34817438052817404844048581161^{\prime}\right)\right) \quad v p a\left(\operatorname{sym}\left(' \quad .217119766902955166405682785^{\prime}\right)\right)\right]$; intJdn9dn4uvrs $=\left[\operatorname{vpa}\left(\operatorname{sym}\left({ }^{\prime} .8032716613183666521258486710 \mathrm{e}-1^{\prime}\right)\right) \quad \operatorname{vpa}\left(\operatorname{sym}\left({ }^{\prime}-.12935448737628105651627293296 '\right)\right) ; . .\right.$. vpa(sym(' .17064551262371894348372706704')) vpa(sym(' $\quad-.114137962266763606747720480 '))]$; intJdn9dn5uvrs $=\left[\operatorname{vpa}\left(\operatorname{sym}\left({ }^{\prime} \quad-.1349507714997270711398676150 '\right)\right) \quad \operatorname{vpa}\left(\operatorname{sym}\left({ }^{\prime} \quad-.113465061045209967716224362 '\right)\right) ; \ldots\right.$ $\left.\operatorname{vpa}(\operatorname{sym}(' \quad-.113465061045209967716224362 ')) \quad \operatorname{vpa}\left(\operatorname{sym}\left('-.283674858742885011995552238 \mathrm{e}-1^{\prime}\right)\right)\right]$; intJdn9dn6uvrs $=\left[\operatorname{vpa}(\operatorname{sym}(' \quad .123808042711948724330825139 ')) \quad \operatorname{vpa}\left(\operatorname{sym}\left({ }^{\prime}-.79362521672469957651556136 \mathrm{e}-2^{\prime}\right)\right) ; \ldots\right.$ $\left.\operatorname{vpa}(\operatorname{sym}('-.79362521672469957651556136 \mathrm{e}-2 ')) \quad \operatorname{vpa}\left(\operatorname{sym}\left({ }^{\prime}-.11543642609252788831829720852 '\right)\right)\right]$; intJdn9dn7uvrs $=\left[\operatorname{vpa}\left(\operatorname{sym}\left({ }^{\prime} \quad .46925409227203366956712440 \mathrm{e}-1^{\prime}\right)\right) \quad \operatorname{vpa}\left(\operatorname{sym}\left({ }^{\prime}-.2046226562144629893771449553 '\right)\right) ; \ldots\right.$ $\left.\operatorname{vpa}\left(\operatorname{sym}\left({ }^{\prime}-.2046226562144629893771449553 '\right)\right) \quad \operatorname{vpa}\left(\operatorname{sym}\left({ }^{\prime} .530267352079846205377516680 \mathrm{e}-1^{\prime}\right)\right)\right]$; intJdn9dn8uvrs $=\left[\operatorname{vpa}\left(\operatorname{sym}\left({ }^{\prime}-.222366721554688830294037920^{\prime}\right)\right) \quad \operatorname{vpa}\left(\operatorname{sym}\left({ }^{\prime} .48499079406584066090468527082^{\prime}\right)\right) ; \ldots\right.$ $\left.\operatorname{vpa}\left(\operatorname{sym}\left({ }^{\prime} .48499079406584066090468527082 '\right)\right) \operatorname{vpa}\left(\operatorname{sym}\left({ }^{\prime}-.222366721554688830294037920 '\right)\right)\right]$; intJdn9dn9uvrs $=\left[\operatorname{vpa}\left(\operatorname{sym}\left({ }^{\prime} \quad .767115078598661079333576130 '\right)\right) \quad \operatorname{vpa}\left(\operatorname{sym}\left({ }^{\prime} .15317854106260736184624261268 '\right)\right) ; \ldots\right.$ $\operatorname{vpa}\left(\operatorname{sym}\left({ }^{\prime} .15317854106260736184624261268 '\right)\right)$ vpa(sym(' 1.653151620925835592672919485'))]; intJdn9dn10uvrs $=\left[\operatorname{vpa}\left(\operatorname{sym}\left({ }^{\prime}-.4881203408468691695076954060^{\prime}\right)\right) \quad \operatorname{vpa}\left(\operatorname{sym}\left({ }^{\prime} .39916403134969030939385162239 '\right)\right) ; \ldots\right.$ $\left.\operatorname{vpa}(\operatorname{sym}('-.61333596865030969060614837761 ')) \quad v p a\left(\operatorname{sym}\left({ }^{\prime} \quad-.264631702815104949739660648 '\right)\right)\right]$; intJdn9dn11uvrs $=\left[\operatorname{vpa}\left(\operatorname{sym}\left({ }^{\prime} .837329943403397038665668996 \mathrm{e}-1^{\prime}\right)\right) \quad \operatorname{vpa}\left(\operatorname{sym}\left({ }^{\prime} \quad .2481990187365599088136231974 '\right)\right) ; \ldots\right.$ $\operatorname{vpa}\left(\operatorname{sym}\left({ }^{\prime} .2481990187365599088136231974 '\right)\right)$ vpa(sym(' .315711302473187041277220189'))]; intJdn9dn12uvrs $=\left[\operatorname{vpa}\left(\operatorname{sym}\left({ }^{\prime}-.424031731725615725542540841 \mathrm{e}-1\right.\right.\right.$ ') $) \quad \operatorname{vpa}\left(\operatorname{sym}\left({ }^{\prime} \quad-.1085783194105248930324052264 '\right)\right) ; \ldots$ $\left.\operatorname{vpa}(\operatorname{sym}(' \quad-.1085783194105248930324052264 ')) \quad \operatorname{vpa}\left(\operatorname{sym}\left({ }^{\prime} \quad-.1123602772538571729615200020 '\right)\right)\right]$; intJdn9dn13uvrs $=\left[\operatorname{vpa}\left(\operatorname{sym}\left({ }^{\prime} \quad .178851051525261210302257368^{\prime}\right)\right) \quad \operatorname{vpa}\left(\operatorname{sym}\left({ }^{\prime} \quad .331278731126193705006371948 '\right)\right) ; \ldots\right.$ $\left.\operatorname{vpa}(\operatorname{sym}(' \quad .331278731126193705006371948 ')) \quad v p a\left(\operatorname{sym}\left(' .60329059165411543334696078 \mathrm{e}-1^{\prime}\right)\right)\right]$; intJdn9dn14uvrs $=\left[\operatorname{vpa}\left(\operatorname{sym}\left({ }^{\prime}-.154508813774518558307843375^{\prime}\right)\right) \quad \operatorname{vpa}\left(\operatorname{sym}\left({ }^{\prime} .36845390722838247652759296 \mathrm{e}-1^{\prime}\right)\right) ; \ldots\right.$ $\operatorname{vpa}\left(\operatorname{sym}\left(' .36845390722838247652759296 \mathrm{e}-1^{\prime}\right)\right)$ vpa(sym(' .531391405011459465049996936'))]; intJdn9dn15uvrs $=\left[\operatorname{vpa}\left(\operatorname{sym}\left({ }^{\prime} \quad .550843979761993341763278679 '\right)\right) \quad \operatorname{vpa}\left(\operatorname{sym}\left({ }^{\prime}-.266497211117548073090616868 \mathrm{e}-1^{\prime}\right)\right) ; \ldots\right.$ $\left.\operatorname{vpa}\left(\operatorname{sym}\left('-.266497211117548073090616868 \mathrm{e}-1^{\prime}\right)\right) \quad \operatorname{vpa}\left(\operatorname{sym}\left({ }^{\prime} \quad-1.94418619472045071324352388 '\right)\right)\right]$; intJdn9dn16uvrs $=\left[\operatorname{vpa}\left(\operatorname{sym}\left({ }^{\prime}-.433262214197932020360238335^{\prime}\right)\right) \quad \operatorname{vpa}\left(\operatorname{sym}\left({ }^{\prime}-.7955065459935637821598364152 '\right)\right) ; \ldots\right.$ 
$\left.\operatorname{vpa}(\operatorname{sym}('-.7955065459935637821598364152 ')) \quad \operatorname{vpa}\left(\operatorname{sym}\left('-.5199575926777798530262703 \mathrm{e}-1^{\prime}\right)\right)\right]$; intJdn10dn1uvrs $=\left[\operatorname{vpa}\left(\operatorname{sym}\left({ }^{\prime}-.92245188408519862030004704 \mathrm{e}-11^{\prime}\right)\right) \quad \operatorname{vpa}\left(\operatorname{sym}\left({ }^{\prime} \quad-.104911199388184100700597508 '\right)\right) ; \ldots\right.$ vpa(sym(' -.104911199388184100700597508')) vpa(sym(' -.931446794027204306605665605e-1'))]; intJdn10dn2uvrs $=\left[\operatorname{vpa}\left(\operatorname{sym}\left({ }^{\prime} \quad .51004038179234709257195060 \mathrm{e}-2^{\prime}\right)\right) \quad \operatorname{vpa}\left(\operatorname{sym}\left({ }^{\prime}-.247617179998775437696714569 \mathrm{e}-1^{\prime}\right)\right) ; . .\right.$. vpa(sym(' -.247617179998775437696714569e-1')) vpa(sym(' .327370706036359563493128961e-3'))]; intJdn10dn3uvrs $=\left[\operatorname{vpa}\left(\operatorname{sym}\left({ }^{\prime} .958310725915156595907307062 \mathrm{e}-1^{\prime}\right)\right) \quad \operatorname{vpa}\left(\operatorname{sym}\left({ }^{\prime} .15714640889191176538987417817 '\right)\right) ; \ldots\right.$ $\left.\operatorname{vpa}(\operatorname{sym}('-.14285359110808823461012582183 ')) \operatorname{vpa}\left(\operatorname{sym}\left('-.66830280257659072749293639 \mathrm{e}-1^{\prime}\right)\right)\right]$; intJdn10dn4uvrs $=\left[\operatorname{vpa}\left(\operatorname{sym}\left({ }^{\prime}-.2646472899567640195235481100^{\prime}\right)\right) \quad \operatorname{vpa}\left(\operatorname{sym}\left({ }^{\prime} .2616521469230527187251402015^{\prime}\right)\right) ; \ldots\right.$ $\left.\operatorname{vpa}\left(\operatorname{sym}\left({ }^{\prime}-.4508478530769472812748597985^{\prime}\right)\right) \quad \operatorname{vpa}\left(\operatorname{sym}\left({ }^{\prime} \quad .283468713180315926702327990 '\right)\right)\right]$; intJdn10dn5uvrs $=\left[\operatorname{vpa}\left(\operatorname{sym}\left({ }^{\prime} \quad .106679112592585571455322301 '\right)\right) \quad \operatorname{vpa}\left(\operatorname{sym}\left({ }^{\prime} .6059337192477808811068095 \mathrm{e}-2 '\right)\right) ; \ldots\right.$ vpa(sym(' .6059337192477808811068095e-2')) vpa(sym(' -.1306849801916743926696037294'))]; intJdn10dn6uvrs $=\left[\operatorname{vpa}\left(\operatorname{sym}\left({ }^{\prime}-.426191802370655235374397294 \mathrm{e}-1^{\prime}\right)\right) \quad \operatorname{vpa}\left(\operatorname{sym}\left({ }^{\prime} .832121696074405782549458791 \mathrm{e}-1^{\prime}\right)\right) ; \ldots\right.$ $\left.\operatorname{vpa}\left(\operatorname{sym}\left(' .832121696074405782549458791 \mathrm{e}-1^{\prime}\right)\right) \quad \operatorname{vpa}\left(\operatorname{sym}\left({ }^{\prime} .498827009902849670234649781 \mathrm{e}-1^{\prime}\right)\right)\right]$; intJdn10dn7uvrs $=\left[\operatorname{vpa}\left(\operatorname{sym}\left({ }^{\prime}-.54552417791786682579711494 \mathrm{e}-2^{\prime}\right)\right) \quad \operatorname{vpa}\left(\operatorname{sym}\left({ }^{\prime} .877730624778960602031917001 \mathrm{e}-1^{\prime}\right)\right) ; \ldots\right.$ $\operatorname{vpa}\left(\operatorname{sym}\left(' .877730624778960602031917001 \mathrm{e}-1^{\prime}\right)\right) \quad \operatorname{vpa}(\operatorname{sym}('-.54552417791786682579711494 \mathrm{e}-2$ '))]; intJdn10dn8uvrs $=\left[\operatorname{vpa}\left(\operatorname{sym}\left(' .530267352079846205377516680 \mathrm{e}-1^{\prime}\right)\right) \quad \operatorname{vpa}\left(\operatorname{sym}\left({ }^{\prime}-.2046226562144629893771449553 '\right)\right) ; \ldots\right.$ $\operatorname{vpa}(\operatorname{sym}('-.2046226562144629893771449553 '))$ vpa(sym(' .46925409227203366956712440e-1'))]; intJdn10dn9uvrs $=\left[\operatorname{vpa}\left(\operatorname{sym}\left({ }^{\prime}-.4881203408468691695076954060 '\right)\right) \quad \operatorname{vpa}\left(\operatorname{sym}\left(\left(^{\prime}-.61333596865030969060614837761 '\right)\right) ; \ldots\right.\right.$ vpa(sym(' .39916403134969030939385162239')) vpa(sym(' -.264631702815104949739660648'))]; intJdn10dn10uvrs $=\left[\operatorname{vpa}\left(\operatorname{sym}\left({ }^{\prime} .6665041132041592095461950376 '\right)\right) \quad \operatorname{vpa}\left(\operatorname{sym}\left({ }^{\prime} .27309567677916265610654186068 '\right)\right) ; . .\right.$. vpa(sym(' .27309567677916265610654186068')) vpa(sym(' 2.001089658057721102391264327'))]; intJdn10dn11uvrs $=\left[\operatorname{vpa}\left(\operatorname{sym}\left({ }^{\prime}-.2543377601216534395445354534 '\right)\right) \quad \operatorname{vpa}\left(\operatorname{sym}\left({ }^{\prime}-.6290159043855470676276509169 '\right)\right) ; \ldots\right.$ vpa(sym(' -.6290159043855470676276509169')) vpa(sym(' -.857977568743733072181109962'))]; intJdn10dn12uvrs $=\left[\operatorname{vpa}\left(\operatorname{sym}\left({ }^{\prime} .118010805467176214144917734 '\right)\right) \quad \operatorname{vpa}\left(\operatorname{sym}\left({ }^{\prime} .272605979766225412235674987 '\right)\right) ; \ldots\right.$ $\operatorname{vpa}(\operatorname{sym}(' .272605979766225412235674987 ')) \operatorname{vpa}(\operatorname{sym}(' .3065397294444835089245124969 '))]$; intJdn10dn13uvrs $=\left[\operatorname{vpa}\left(\operatorname{sym}\left({ }^{\prime}-.1131519430916361403558955158 '\right)\right) \quad \operatorname{vpa}\left(\operatorname{sym}\left({ }^{\prime} .30448320844630450044664700 \mathrm{e}-1^{\prime}\right)\right) ; \ldots\right.$ $\left.\operatorname{vpa}\left(\operatorname{sym}\left({ }^{\prime} .30448320844630450044664700 \mathrm{e}-1 '\right)\right) \operatorname{vpa}(\operatorname{sym}(' \quad .621790544829774902399463480 '))\right]$;

intJdn10dn14uvrs $=\left[\operatorname{vpa}\left(\operatorname{sym}\left({ }^{\prime} .45065512128407846407826384 \mathrm{e}-1^{\prime}\right)\right) \quad \operatorname{vpa}\left(\operatorname{sym}\left({ }^{\prime} \quad-.304233282397533216844618686 '\right)\right) ; \ldots\right.$ $\operatorname{vpa}(\operatorname{sym}('-.304233282397533216844618686 ')) \quad \operatorname{vpa}(\operatorname{sym}('-.198130259760001838281687341 '))]$; intJdn10dn15uvrs $=\left[\operatorname{vpa}\left(\operatorname{sym}\left({ }^{\prime}-.293283237003299311368713211^{\prime}\right)\right) \quad \operatorname{vpa}\left(\operatorname{sym}\left({ }^{\prime} .6763849144738999657208941635 '\right)\right) ; \ldots\right.$ vpa(sym(' .6763849144738999657208941635')) vpa(sym(' .55242173149670587806391031'))]; intJdn10dn16uvrs $=\left[\operatorname{vpa}\left(\operatorname{sym}\left({ }^{\prime} .4636424264352335415173399435^{\prime}\right)\right) \quad \operatorname{vpa}\left(\operatorname{sym}\left({ }^{\prime} .32502712079217193433836137 \mathrm{e}-1^{\prime}\right)\right) ; \ldots\right.$ vpa(sym('.32502712079217193433836137e-1')) vpa(sym(' -2.24559114498245358748525615'))]; intJdn11dn1uvrs $=\left[\operatorname{vpa}\left(\operatorname{sym}\left({ }^{\prime}-.70027089525016846364967113 \mathrm{e}-1^{\prime}\right)\right) \quad \operatorname{vpa}\left(\operatorname{sym}\left({ }^{\prime} \quad-.142592079476496670389254682 '\right)\right) ; \ldots\right.$ vpa(sym(' .157407920523503329610745318')) vpa(sym(' .1172503945374017479674604967'))]; intJdn11dn2uvrs =[vpa(sym(' - $\left.\left..80494988723694790041230002 \mathrm{e}-2^{\prime}\right)\right) \quad \operatorname{vpa}\left(\operatorname{sym}\left({ }^{\prime}-.239609914551285358186348361 \mathrm{e}-1^{\prime}\right)\right) ; \ldots$ vpa(sym(' -.239609914551285358186348361e-1')) vpa(sym(' .23530457948583652632350827e-2'))]; intJdn11dn3uvrs $=\left[\operatorname{vpa}\left(\operatorname{sym}\left({ }^{\prime}-.2290194853292015105870834477 \mathrm{e}-1^{\prime}\right)\right) \quad \operatorname{vpa}\left(\operatorname{sym}\left({ }^{\prime}-.701114223525693099130736200 \mathrm{e}-1^{\prime}\right)\right) ; \ldots\right.$ $\left.\operatorname{vpa}\left(\operatorname{sym}\left({ }^{\prime}-.701114223525693099130736200 \mathrm{e}-1^{\prime}\right)\right) \quad \operatorname{vpa}\left(\operatorname{sym}\left({ }^{\prime} \quad-.95631533002240688097543501 \mathrm{e}-1^{\prime}\right)\right)\right]$; intJdn11dn4uvrs $=\left[\operatorname{vpa}\left(\operatorname{sym}\left({ }^{\prime} .1378297018364707614848534907 '\right)\right) \quad \operatorname{vpa}\left(\operatorname{sym}\left({ }^{\prime}-.3225407916324774100791670851^{\prime}\right)\right) ; \ldots\right.$ $\operatorname{vpa}(\operatorname{sym}(' .3899592083675225899208329149 ')) \quad \operatorname{vpa}(\operatorname{sym}('-.484060336356140646761807119 '))]$; intJdn11dn5uvrs $=\left[\operatorname{vpa}\left(\operatorname{sym}\left({ }^{\prime}-.26978045869880871379513078 \mathrm{e}-1^{\prime}\right)\right) \quad \operatorname{vpa}\left(\operatorname{sym}\left({ }^{\prime} \quad-.196966390341090928909694084 '\right)\right) ; \ldots\right.$ $\left.\operatorname{vpa}(\operatorname{sym}('-.196966390341090928909694084 ')) \operatorname{vpa}\left(\operatorname{sym}\left('-.78897358249752608668273981 \mathrm{e}-1^{\prime}\right)\right)\right]$; intJdn11dn6uvrs $=\left[\operatorname{vpa}\left(\operatorname{sym}\left({ }^{\prime} .27527791093307208248725575 \mathrm{e}-1^{\prime}\right)\right) \quad \operatorname{vpa}\left(\operatorname{sym}\left({ }^{\prime} .89268098816052016578387726 \mathrm{e}-1^{\prime}\right)\right) ; . .\right.$. $\left.\operatorname{vpa}\left(\operatorname{sym}\left(' .89268098816052016578387726 \mathrm{e}-1^{\prime}\right)\right) \quad \operatorname{vpa}\left(\operatorname{sym}\left({ }^{\prime} .27527791093307208248725575 \mathrm{e}-1^{\prime}\right)\right)\right]$; intJdn11dn7uvrs $=\left[\operatorname{vpa}\left(\operatorname{sym}\left({ }^{\prime} .498827009902849670234649781 \mathrm{e}-1^{\prime}\right)\right) \quad \operatorname{vpa}\left(\operatorname{sym}\left({ }^{\prime} .832121696074405782549458791 \mathrm{e}-11^{\prime}\right)\right) ; \ldots\right.$ $\left.\operatorname{vpa}\left(\operatorname{sym}\left(' .832121696074405782549458791 \mathrm{e}-1^{\prime}\right)\right) \quad \operatorname{vpa}\left(\operatorname{sym}\left({ }^{\prime}-.426191802370655235374397294 \mathrm{e}-1^{\prime}\right)\right)\right]$; intJdn11dn8uvrs $=\left[\operatorname{vpa}\left(\operatorname{sym}\left({ }^{\prime}-.11543642609252788831829720852 '\right)\right) \quad \operatorname{vpa}\left(\operatorname{sym}\left({ }^{\prime}-.79362521672469957651556136 \mathrm{e}-2^{\prime}\right)\right) ; \ldots\right.$ $\left.\operatorname{vpa}\left(\operatorname{sym}\left({ }^{\prime}-.79362521672469957651556136 \mathrm{e}-2^{\prime}\right)\right) \operatorname{vpa}\left(\operatorname{sym}\left({ }^{\prime} \quad .123808042711948724330825139 '\right)\right)\right]$; intJdn11dn9uvrs =[vpa(sym(' .837329943403397038665668996e-1')) vpa(sym(' .2481990187365599088136231974'));... $\operatorname{vpa}(\operatorname{sym}(' \quad .2481990187365599088136231974 '))$ vpa(sym(' .315711302473187041277220189'))]; intJdn11dn10uvrs $=\left[\operatorname{vpa}\left(\operatorname{sym}\left({ }^{\prime}-.2543377601216534395445354534 '\right)\right) \quad \operatorname{vpa}\left(\operatorname{sym}\left({ }^{\prime}-.6290159043855470676276509169 '\right)\right) ; \ldots\right.$ $\operatorname{vpa}(\operatorname{sym}('-.6290159043855470676276509169 ')) \operatorname{vpa}(\operatorname{sym}('-.857977568743733072181109962 '))]$; intJdn11dn11uvrs $=\left[\operatorname{vpa}\left(\operatorname{sym}\left({ }^{\prime} 1.141981449369884652860820049 '\right)\right) \quad \operatorname{vpa}\left(\operatorname{sym}\left({ }^{\prime} .602065373518435159503113519 '\right)\right) ; \ldots\right.$ $\operatorname{vpa}(\operatorname{sym}(' .602065373518435159503113519 '))$ vpa(sym(' 1.332289708203970804992564589'))]; intJdn11dn12uvrs $=\left[\operatorname{vpa}\left(\operatorname{sym}\left({ }^{\prime} \quad-.222026480813621359932407811^{\prime}\right)\right) \quad \operatorname{vpa}(\operatorname{sym}(' \quad .274774490560733340636022046 ')) ; \ldots\right.$ $\operatorname{vpa}(\operatorname{sym}('-.737725509439266659363977954 ')) \quad v p a(\operatorname{sym}('-.78668680846441673459831513779 '))]$; intJdn11dn13uvrs $=\left[\operatorname{vpa}\left(\operatorname{sym}\left({ }^{\prime} .520202184956697891678232119^{\prime}\right)\right) \quad \operatorname{vpa}\left(\operatorname{sym}\left({ }^{\prime} .827423779129943639113014061^{\prime}\right)\right) ; \ldots\right.$ $\left.\operatorname{vpa}\left(\operatorname{sym}\left({ }^{\prime} .827423779129943639113014061^{\prime}\right)\right) \quad \operatorname{vpa}\left(\operatorname{sym}\left({ }^{\prime} .107366707368679853419887039 '\right)\right)\right]$; intJdn11dn14uvrs $=\left[\operatorname{vpa}\left(\operatorname{sym}\left({ }^{\prime} \quad-.189527805811794389864868430 '\right)\right) \quad v p a\left(\operatorname{sym}\left({ }^{\prime} \quad-.327324308266824506551303460 '\right)\right) ; \ldots\right.$ $\left.\operatorname{vpa}(\operatorname{sym}(' \quad-.327324308266824506551303460 ')) \quad v p a\left(\operatorname{sym}\left('-.20260922191887153901852490 \mathrm{e}-1^{\prime}\right)\right)\right]$; intJdn11dn15uvrs $=\left[\operatorname{vpa}\left(\operatorname{sym}\left({ }^{\prime} .449881020809519531356712718 '\right)\right) \quad \operatorname{vpa}\left(\operatorname{sym}\left({ }^{\prime} .106104670667416834133287489 '\right)\right) ; \ldots\right.$ $\operatorname{vpa}\left(\operatorname{sym}\left({ }^{\prime} .106104670667416834133287489 '\right)\right)$ vpa(sym(' -.26123164244466091271015883'))]; intJdn11dn16uvrs $=\left[\operatorname{vpa}\left(\operatorname{sym}\left({ }^{\prime}-1.501752787756720291051955360 '\right)\right) \quad \operatorname{vpa}\left(\operatorname{sym}\left({ }^{\prime}-.510599460959200051978459650 '\right)\right) ; \ldots\right.$ 
$\left.\operatorname{vpa}\left(\operatorname{sym}\left({ }^{\prime}-.510599460959200051978459650 '\right)\right) \quad \operatorname{vpa}\left(\operatorname{sym}\left({ }^{\prime} \quad .60105835750654359495658263 '\right)\right)\right]$; intJdn12dn1uvrs $=\left[\operatorname{vpa}\left(\operatorname{sym}\left({ }^{\prime} \quad .157871624541338704794557829 '\right)\right) \quad \operatorname{vpa}\left(\operatorname{sym}\left({ }^{\prime} .268945525984776981543022533 '\right)\right) ; \ldots\right.$ $\operatorname{vpa}(\operatorname{sym}('-.443554474015223018456977467 ')) \quad \operatorname{vpa}(\operatorname{sym}('-.412633054117600230716206898 '))]$; intJdn12dn2uvrs $=\left[\operatorname{vpa}\left(\operatorname{sym}\left({ }^{\prime} .220346626285956934003016986 \mathrm{e}-1^{\prime}\right)\right) \quad \operatorname{vpa}\left(\operatorname{sym}\left({ }^{\prime} .495487663266407134761033842 \mathrm{e}-1^{\prime}\right)\right) ; \ldots\right.$ $\operatorname{vpa}\left(\operatorname{sym}\left(' .495487663266407134761033842 \mathrm{e}-1^{\prime}\right)\right) \quad \operatorname{vpa}(\operatorname{sym}('-.20270550705857146813452057 \mathrm{e}-1$ '))]; intJdn12dn3uvrs $=\left[\operatorname{vpa}\left(\operatorname{sym}\left({ }^{\prime} .12030624699064793953098043 \mathrm{e}-1^{\prime}\right)\right) \quad \operatorname{vpa}\left(\operatorname{sym}\left({ }^{\prime} .306625487404711706979545881 \mathrm{e}-1^{\prime}\right)\right) ; \ldots\right.$ $\operatorname{vpa}(\operatorname{sym}(' .306625487404711706979545881 \mathrm{e}-1$ ')) $\operatorname{vpa}(\operatorname{sym}(' .312258974588804915879185904 \mathrm{e}-1$ '))]; intJdn12dn4uvrs $=\left[\operatorname{vpa}\left(\operatorname{sym}\left({ }^{\prime}-.356070031451497704872952070 \mathrm{e}-1^{\prime}\right)\right) \quad \operatorname{vpa}\left(\operatorname{sym}\left({ }^{\prime} \quad .1493518854577477452335226299 '\right)\right) ; \ldots\right.$ $\operatorname{vpa}(\operatorname{sym}(' \quad-.150648114542252254766477370 ')) \quad \operatorname{vpa}(\operatorname{sym}(' \quad .1353164857298023346239509070 '))]$; intJdn12dn5uvrs $=\left[\operatorname{vpa}(\operatorname{sym}(' .8921165458163810696876696 \mathrm{e}-1 ')) \quad \operatorname{vpa}\left(\operatorname{sym}\left({ }^{\prime} .427188884799078070173992386 '\right)\right) ; \ldots\right.$ vpa(sym(' .427188884799078070173992386')) vpa(sym('.8921165458163810696876696e-1'))]; intJdn12dn6uvrs $=\left[\operatorname{vpa}\left(\operatorname{sym}\left({ }^{\prime}-.78897358249752608668273981 \mathrm{e}-1^{\prime}\right)\right) \quad \operatorname{vpa}\left(\operatorname{sym}\left({ }^{\prime} \quad-.196966390341090928909694084 '\right)\right) ; \ldots\right.$ vpa(sym(' -.196966390341090928909694084')) vpa(sym(' -.26978045869880871379513078e-1'))]; intJdn12dn7uvrs $=[\operatorname{vpa}(\operatorname{sym}('-.1306849801916743926696037294 ')) \quad \operatorname{vpa}(\operatorname{sym}(' \quad .6059337192477808811068095 \mathrm{e}-2$ ') $) ; \ldots$ $\operatorname{vpa}(\operatorname{sym}(' .6059337192477808811068095 \mathrm{e}-2 ')) \quad \operatorname{vpa}(\operatorname{sym}(' .1066791125925855714553223009 '))]$; intJdn12dn8uvrs $=\left[\operatorname{vpa}\left(\operatorname{sym}\left({ }^{\prime}-.283674858742885011995552238 \mathrm{e}-1^{\prime}\right)\right) \quad \operatorname{vpa}\left(\operatorname{sym}\left({ }^{\prime} \quad-.1134650610452099677162243619 '\right)\right) ; .\right.$. $\left.\operatorname{vpa}(\operatorname{sym}(' \quad-.1134650610452099677162243619 ')) \quad \operatorname{vpa}\left(\operatorname{sym}\left({ }^{\prime} \quad-.1349507714997270711398676150 '\right)\right)\right]$; intJdn12dn9uvrs $=\left[\operatorname{vpa}\left(\operatorname{sym}\left({ }^{\prime}-.424031731725615725542540841 \mathrm{e}-1 '\right)\right) \quad v p a\left(\operatorname{sym}\left({ }^{\prime} \quad-.1085783194105248930324052264 '\right)\right) ; \ldots\right.$ $\left.\operatorname{vpa}(\operatorname{sym}(' \quad-.1085783194105248930324052264 ')) \quad \operatorname{vpa}\left(\operatorname{sym}\left({ }^{\prime} \quad-.1123602772538571729615200020 '\right)\right)\right]$; intJdn12dn10uvrs $=\left[\operatorname{vpa}\left(\operatorname{sym}\left({ }^{\prime} .118010805467176214144917734 '\right)\right) \quad \operatorname{vpa}\left(\operatorname{sym}\left({ }^{\prime} .272605979766225412235674987 '\right)\right) ; \ldots\right.$ $\operatorname{vpa}(\operatorname{sym}(' .272605979766225412235674987 ')) \quad v p a(\operatorname{sym}(' .3065397294444835089245124969 '))]$; intJdn12dn11uvrs $=\left[\operatorname{vpa}\left(\operatorname{sym}\left({ }^{\prime} \quad-.222026480813621359932407811^{\prime}\right)\right) \quad \operatorname{vpa}\left(\operatorname{sym}\left({ }^{\prime} \quad-.737725509439266659363977954 '\right)\right) ; \ldots\right.$ vpa(sym(' .274774490560733340636022046')) vpa(sym('-.78668680846441673459831513779'))]; intJdn12dn12uvrs $=\left[\operatorname{vpa}\left(\operatorname{sym}\left({ }^{\prime} 1.495308248540817222321613675^{\prime}\right)\right) \quad \operatorname{vpa}\left(\operatorname{sym}\left({ }^{\prime} .902875989441502362683040391 '\right)\right) ; \ldots\right.$ $\operatorname{vpa}(\operatorname{sym}(' .902875989441502362683040391 ')) \quad v p a(\operatorname{sym}(' 1.317586787287312732669083137 '))]$; intJdn12dn13uvrs $=\left[\operatorname{vpa}\left(\operatorname{sym}\left({ }^{\prime}-1.831179562508932660722813983 '\right)\right) \quad \operatorname{vpa}\left(\operatorname{sym}\left({ }^{\prime}-.530843355987051326204987917 '\right)\right) ; \ldots\right.$ vpa(sym(' -.530843355987051326204987917')) vpa(sym(' .475558907831932003188746478'))]; intJdn12dn14uvrs $=\left[\operatorname{vpa}\left(\operatorname{sym}\left({ }^{\prime} \quad .534031555627251695091986887 '\right)\right) \quad \operatorname{vpa}\left(\operatorname{sym}\left(\left(^{\prime} .91268611031924988409224700 \mathrm{e}-1^{\prime}\right)\right) ; \ldots\right.\right.$ vpa(sym(' .91268611031924988409224700e-1')) vpa(sym(' -.2225431571843560333736647188'))]; intJdn12dn15uvrs $=\left[\operatorname{vpa}\left(\operatorname{sym}\left({ }^{\prime} .86904547772480103903106692 \mathrm{e}-1^{\prime}\right)\right) \quad \operatorname{vpa}\left(\operatorname{sym}\left({ }^{\prime} \quad .379360747071947741960232042^{\prime}\right)\right) ; \ldots\right.$ vpa(sym(' .379360747071947741960232042')) vpa(sym(' .449877692126663661629552350'))]; intJdn12dn16uvrs $=\left[\operatorname{vpa}\left(\operatorname{sym}\left({ }^{\prime} \quad-.146237679902381668344145480 '\right)\right) \quad \operatorname{vpa}\left(\operatorname{sym}\left({ }^{\prime} \quad-.890289639589649219996546217 '\right)\right) ; \ldots\right.$ vpa(sym(' -.890289639589649219996546217')) vpa(sym(' -1.1955736019576031500653136788'))]; intJdn13dn1uvrs $=\left[\operatorname{vpa}\left(\operatorname{sym}\left({ }^{\prime}-.92367783910183130555067710^{\prime}\right)\right) \quad \operatorname{vpa}\left(\operatorname{sym}\left({ }^{\prime}-1.00703245489050046307564230 '\right)\right) ; \ldots\right.$ $\left.\operatorname{vpa}(\operatorname{sym}('-1.00703245489050046307564230 ')) \quad v p a\left(\operatorname{sym}\left({ }^{\prime}-.92367783910183130555067710 '\right)\right)\right]$; intJdn13dn2uvrs $=\left[\operatorname{vpa}\left(\operatorname{sym}\left({ }^{\prime}-.6246030481817935466793166 \mathrm{e}-1^{\prime}\right)\right) \quad \operatorname{vpa}\left(\operatorname{sym}\left({ }^{\prime} \quad-.196130238975238548948111488 '\right)\right) ; \ldots\right.$ $\left.\operatorname{vpa}(\operatorname{sym}('-.196130238975238548948111488 ')) \quad \operatorname{vpa}\left(\operatorname{sym}\left(' .18066447765168896456321824 \mathrm{e}-1^{\prime}\right)\right)\right]$; intJdn13dn3uvrs $=\left[\operatorname{vpa}\left(\operatorname{sym}\left({ }^{\prime}-.440107128429126521075770644 \mathrm{e}-1^{\prime}\right)\right) \quad \operatorname{vpa}\left(\operatorname{sym}\left({ }^{\prime}-.964159752956607711899142376 \mathrm{e}-1^{\prime}\right)\right) ; \ldots\right.$ $\left.\operatorname{vpa}\left(\operatorname{sym}\left('-.964159752956607711899142376 \mathrm{e}-1^{\prime}\right)\right) \quad \operatorname{vpa}\left(\operatorname{sym}\left({ }^{\prime}-.440107128429126521075770644 \mathrm{e}-1^{\prime}\right)\right)\right]$; intJdn13dn4uvrs $=\left[\operatorname{vpa}\left(\operatorname{sym}\left({ }^{\prime} \quad .18066447765168896456321824 \mathrm{e}-1^{\prime}\right)\right) \quad \operatorname{vpa}\left(\operatorname{sym}\left({ }^{\prime} \quad-.196130238975238548948111488 '\right)\right) ; \ldots\right.$ vpa(sym(' -.196130238975238548948111488')) vpa(sym(' -.624603048181793546679316570e-1'))]; intJdn13dn5uvrs $=\left[\operatorname{vpa}(\operatorname{sym}(' \quad .47555890783193200318874648 ')) \quad \operatorname{vpa}\left(\operatorname{sym}\left({ }^{\prime} \quad-.53084335598705132620498792 '\right)\right) ; \ldots\right.$ $\operatorname{vpa}(\operatorname{sym}('-.53084335598705132620498792 ')) \operatorname{vpa}(\operatorname{sym}('-1.83117956250893266072281398 '))]$;

intJdn13dn6uvrs $=\left[\operatorname{vpa}\left(\operatorname{sym}\left({ }^{\prime} .107366707368679853419887039 '\right)\right) \quad \operatorname{vpa}\left(\operatorname{sym}\left(\left(^{\prime} .827423779129943639113014061^{\prime}\right)\right) ; \ldots\right.\right.$ $\left.\operatorname{vpa}\left(\operatorname{sym}\left(' .827423779129943639113014061^{\prime}\right)\right) \quad \operatorname{vpa}\left(\operatorname{sym}\left({ }^{\prime} .520202184956697891678232119^{\prime}\right)\right)\right]$; intJdn13dn7uvrs $=\left[\operatorname{vpa}\left(\operatorname{sym}\left({ }^{\prime} \quad .62179054482977490239946348 '\right)\right) \quad \operatorname{vpa}\left(\operatorname{sym}\left({ }^{\prime} .30448320844630450044664700 \mathrm{e}-1^{\prime}\right)\right) ; \ldots\right.$ $\operatorname{vpa}(\operatorname{sym}(' .30448320844630450044664700 \mathrm{e}-1 ')) \operatorname{vpa}(\operatorname{sym}('-.1131519430916361403558955158 '))]$; intJdn13dn8uvrs $=\left[\operatorname{vpa}\left(\operatorname{sym}\left({ }^{\prime} .60329059165411543334696078 \mathrm{e}-1^{\prime}\right)\right) \quad \operatorname{vpa}\left(\operatorname{sym}\left({ }^{\prime} \quad .331278731126193705006371948 '\right)\right) ; \ldots\right.$ $\left.\operatorname{vpa}\left(\operatorname{sym}\left(' \quad .331278731126193705006371948^{\prime}\right)\right) \operatorname{vpa}\left(\operatorname{sym}\left({ }^{\prime} \quad .178851051525261210302257368 '\right)\right)\right]$; intJdn13dn9uvrs $=\left[\operatorname{vpa}\left(\operatorname{sym}\left({ }^{\prime} \quad .178851051525261210302257368^{\prime}\right)\right) \quad \operatorname{vpa}\left(\operatorname{sym}\left({ }^{\prime} \quad .331278731126193705006371948 '\right)\right) ; \ldots\right.$ vpa(sym(' .331278731126193705006371948')) vpa(sym(' .60329059165411543334696078e-1'))]; intJdn13dn10uvrs $=\left[\operatorname{vpa}\left(\operatorname{sym}\left({ }^{\prime}-.1131519430916361403558955158^{\prime}\right)\right) \quad \operatorname{vpa}\left(\operatorname{sym}\left({ }^{\prime} .30448320844630450044664700 \mathrm{e}-1^{\prime}\right)\right) ; \ldots\right.$ vpa(sym('.30448320844630450044664700e-1')) vpa(sym(' .621790544829774902399463480'))]; intJdn13dn11uvrs $=\left[\operatorname{vpa}\left(\operatorname{sym}\left({ }^{\prime} .520202184956697891678232119^{\prime}\right)\right) \quad \operatorname{vpa}\left(\operatorname{sym}\left({ }^{\prime} .827423779129943639113014061^{\prime}\right)\right) ; \ldots\right.$ $\left.\operatorname{vpa}\left(\operatorname{sym}\left({ }^{\prime} .827423779129943639113014061^{\prime}\right)\right) \quad \operatorname{vpa}\left(\operatorname{sym}\left({ }^{\prime} .107366707368679853419887039 '\right)\right)\right]$; intJdn13dn12uvrs =[vpa(sym(' -1.831179562508932660722813983')) vpa(sym(' -.530843355987051326204987917'));... vpa(sym(' -.530843355987051326204987917')) vpa(sym(' .475558907831932003188746478'))]; intJdn13dn13uvrs $=\left[\operatorname{vpa}\left(\operatorname{sym}\left({ }^{\prime} 5.220412586998316768421088045^{\prime}\right)\right) \quad \operatorname{vpa}\left(\operatorname{sym}\left({ }^{\prime} 2.88129572754069028280297951^{\prime}\right)\right) ; \ldots\right.$ $\left.\operatorname{vpa}\left(\operatorname{sym}\left(' 2.88129572754069028280297951^{\prime}\right)\right) \operatorname{vpa}\left(\operatorname{sym}\left(' 5.220412586998316768421088045^{\prime}\right)\right)\right]$; intJdn13dn14uvrs =[vpa(sym(' -3.400472479844200126665206259')) vpa(sym(' -.876126624260291794899095697'));... $\operatorname{vpa}(\operatorname{sym}('-.876126624260291794899095697 '))$ vpa(sym(' -.8096222389464438740999396603'))]; intJdn13dn15uvrs $=\left[\operatorname{vpa}\left(\operatorname{sym}\left({ }^{\prime}-.18002409287106955030651138 \mathrm{e}-1^{\prime}\right)\right) \quad \operatorname{vpa}\left(\operatorname{sym}\left({ }^{\prime} \quad-.949948521110901296761134222^{\prime}\right)\right) ; \ldots\right.$ vpa(sym(' -.949948521110901296761134222')) vpa(sym('-.18002409287106955030651138e-1'))]; intJdn13dn16uvrs =[vpa(sym(' -.8096222389464438740999396603')) vpa(sym(' - $876126624260291794899095697 ')) ; \ldots$ 
$\left.\operatorname{vpa}\left(\operatorname{sym}\left({ }^{\prime}-.876126624260291794899095697 '\right)\right) \quad v p a(\operatorname{sym}('-3.400472479844200126665206259 '))\right]$; intJdn14dn1uvrs $=\left[\operatorname{vpa}\left(\operatorname{sym}\left({ }^{\prime} .336851990222722864094172519 '\right)\right) \quad \operatorname{vpa}\left(\operatorname{sym}\left({ }^{\prime} .381671404352391717716050003 '\right)\right) ; \ldots\right.$ $\operatorname{vpa}(\operatorname{sym}(' .381671404352391717716050003 '))$ vpa(sym(' .33845901154347235890690860'))]; intJdn14dn2uvrs $=\left[\operatorname{vpa}\left(\operatorname{sym}\left({ }^{\prime}-.5654544717914146990072595 \mathrm{e}-2^{\prime}\right)\right) \quad \operatorname{vpa}\left(\operatorname{sym}\left({ }^{\prime} .4276223703845844197184744941 '\right)\right) ; \ldots\right.$ $\operatorname{vpa}(\operatorname{sym}(' .4276223703845844197184744941 ')) \quad \operatorname{vpa}(\operatorname{sym}('-.1539310157132113391331378486 '))]$; intJdn14dn3uvrs $=\left[\operatorname{vpa}\left(\operatorname{sym}\left({ }^{\prime} \quad .136999462878579334021389201 '\right)\right) \quad \operatorname{vpa}\left(\operatorname{sym}\left({ }^{\prime} .2272134136490097198166572636 '\right)\right) ; \ldots\right.$

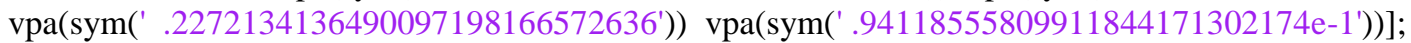
intJdn14dn4uvrs =[vpa(sym(' -.9846681660450092579804616e-3')) vpa(sym(' .863000808306781359312991519e-1'));... $\left.\operatorname{vpa}\left(\operatorname{sym}\left({ }^{\prime} .863000808306781359312991519 \mathrm{e}-1^{\prime}\right)\right) \quad \operatorname{vpa}\left(\operatorname{sym}\left(' .208266894443892215290932469 \mathrm{e}-1^{\prime}\right)\right)\right]$; intJdn14dn5uvrs $=\left[\operatorname{vpa}(\operatorname{sym}('-1.1955736019576031500653136788 ')) \quad \operatorname{vpa}\left(\operatorname{sym}\left({ }^{\prime} \quad-.890289639589649219996546217 '\right)\right) ; \ldots\right.$ vpa(sym(' -.890289639589649219996546217')) vpa(sym('.$- .14623767990238166834414548 '))]$; intJdn14dn6uvrs $=\left[\operatorname{vpa}\left(\operatorname{sym}\left({ }^{\prime} \quad .60105835750654359495658263 '\right)\right) \quad \operatorname{vpa}\left(\operatorname{sym}\left({ }^{\prime} \quad-.510599460959200051978459650 '\right)\right) ; \ldots\right.$ $\left.\operatorname{vpa}(\operatorname{sym}('-.510599460959200051978459650 ')) \quad v p a\left(\operatorname{sym}\left({ }^{\prime}-1.501752787756720291051955360 '\right)\right)\right]$; intJdn14dn7uvrs $=\left[\operatorname{vpa}\left(\operatorname{sym}\left({ }^{\prime}-2.24559114498245358748525615^{\prime}\right)\right) \quad \operatorname{vpa}\left(\operatorname{sym}\left({ }^{\prime} .32502712079217193433836137 \mathrm{e}-1^{\prime}\right)\right) ; \ldots\right.$ $\left.\operatorname{vpa}\left(\operatorname{sym}\left({ }^{\prime} .32502712079217193433836137 \mathrm{e}-1^{\prime}\right)\right) \quad \operatorname{vpa}(\operatorname{sym}(' .4636424264352335415173399435 '))\right]$; intJdn14dn8uvrs $=\left[\operatorname{vpa}\left(\operatorname{sym}\left({ }^{\prime}-.5199575926777798530262703 e-1^{\prime}\right)\right) \quad \operatorname{vpa}\left(\operatorname{sym}\left(\left(^{\prime}-.7955065459935637821598364152 '\right)\right) ; \ldots\right.\right.$ $\left.\operatorname{vpa}(\operatorname{sym}('-.7955065459935637821598364152 ')) \quad \operatorname{vpa}\left(\operatorname{sym}\left('-.433262214197932020360238335^{\prime}\right)\right)\right]$; intJdn14dn9uvrs $=\left[\operatorname{vpa}\left(\operatorname{sym}\left({ }^{\prime}-.154508813774518558307843375^{\prime}\right)\right) \quad \operatorname{vpa}\left(\operatorname{sym}\left({ }^{\prime} .36845390722838247652759296 e-1 '\right)\right) ; \ldots\right.$ $\left.\operatorname{vpa}\left(\operatorname{sym}\left(' .36845390722838247652759296 \mathrm{e}-1^{\prime}\right)\right) \quad \operatorname{vpa}(\operatorname{sym}(' \quad .531391405011459465049996936 '))\right]$; intJdn14dn10uvrs $=\left[\operatorname{vpa}\left(\operatorname{sym}\left({ }^{\prime} .45065512128407846407826384 \mathrm{e}-1^{\prime}\right)\right) \quad \operatorname{vpa}\left(\operatorname{sym}\left({ }^{\prime} \quad-.304233282397533216844618686 '\right)\right) ; \ldots\right.$ $\operatorname{vpa}(\operatorname{sym}('-.304233282397533216844618686 ')) \quad \operatorname{vpa}(\operatorname{sym}('-.198130259760001838281687341 '))]$; intJdn14dn11uvrs $=\left[\operatorname{vpa}\left(\operatorname{sym}\left({ }^{\prime} \quad-.189527805811794389864868430 '\right)\right) \quad v p a\left(\operatorname{sym}\left({ }^{\prime} \quad-.327324308266824506551303460 '\right)\right) ; . .\right.$. vpa(sym(' -.327324308266824506551303460')) vpa(sym(' -.20260922191887153901852490e-1'))]; intJdn14dn12uvrs $=\left[\operatorname{vpa}\left(\operatorname{sym}\left({ }^{\prime} \quad .534031555627251695091986887 '\right)\right) \quad \operatorname{vpa}\left(\operatorname{sym}\left({ }^{\prime} .91268611031924988409224700 \mathrm{e}-1^{\prime}\right)\right) ; \ldots\right.$ vpa(sym(' .91268611031924988409224700e-1')) vpa(sym(' -.2225431571843560333736647188'))]; intJdn14dn13uvrs $=\left[\operatorname{vpa}\left(\operatorname{sym}\left({ }^{\prime}-3.400472479844200126665206259^{\prime}\right)\right) \quad \operatorname{vpa}\left(\operatorname{sym}\left({ }^{\prime}-.876126624260291794899095697 '\right)\right) ; \ldots\right.$ vpa(sym(' -.876126624260291794899095697')) vpa(sym(' -.8096222389464438740999396603'))]; intJdn14dn14uvrs $=\left[\operatorname{vpa}\left(\operatorname{sym}\left({ }^{\prime} 5.51876129014211756612538031^{\prime}\right)\right) \quad \operatorname{vpa}\left(\operatorname{sym}\left({ }^{\prime} 1.770759558087978375081430869 '\right)\right) ; \ldots\right.$. vpa(sym(' 1.770759558087978375081430869')) vpa(sym(' 3.929578185962763299703317274'))]; intJdn14dn15uvrs $=\left[\operatorname{vpa}\left(\operatorname{sym}\left({ }^{\prime} \quad-.80597791980666492939192672 '\right)\right) \quad \operatorname{vpa}\left(\operatorname{sym}\left({ }^{\prime} \quad-.637841602652030943257059331 '\right)\right) ; \ldots\right.$ $\operatorname{vpa}(\operatorname{sym}('-.637841602652030943257059331 ')) \quad v p a(\operatorname{sym}('-2.769794568377644494965093629 '))]$; intJdn14dn16uvrs $=\left[\operatorname{vpa}\left(\operatorname{sym}\left({ }^{\prime} .877518569823348982633756744 '\right)\right) \quad \operatorname{vpa}\left(\operatorname{sym}\left({ }^{\prime} 1.287737922980470717927187534 '\right)\right) ; \ldots\right.$ $\operatorname{vpa}(\operatorname{sym}(' 1.287737922980470717927187534 ')) \quad \operatorname{vpa}(\operatorname{sym}(' \quad .877518569823348982633756744 '))]$; intJdn15dn1uvrs $=\left[\operatorname{vpa}\left(\operatorname{sym}\left({ }^{\prime}-.122483212211684061698359345^{\prime}\right)\right) \quad \operatorname{vpa}\left(\operatorname{sym}\left({ }^{\prime}-.145263095437075394958553495 '\right)\right) ; \ldots\right.$ $\left.\operatorname{vpa}\left(\operatorname{sym}\left({ }^{\prime}-.145263095437075394958553495^{\prime}\right)\right) \quad \operatorname{vpa}\left(\operatorname{sym}\left({ }^{\prime}-.122483212211684061698359345^{\prime}\right)\right)\right]$; intJdn15dn2uvrs $=\left[\operatorname{vpa}\left(\operatorname{sym}\left({ }^{\prime} \quad .8033238055969811770258930 \mathrm{e}-2^{\prime}\right)\right) \quad \operatorname{vpa}\left(\operatorname{sym}\left({ }^{\prime}-.1942169737637143545345375175 '\right)\right) ; \ldots\right.$ $\left.\operatorname{vpa}\left(\operatorname{sym}\left({ }^{\prime}-.1942169737637143545345375175 '\right)\right) \quad \operatorname{vpa}\left(\operatorname{sym}\left(' .41681314367425998111696912 \mathrm{e}-1^{\prime}\right)\right)\right]$; intJdn15dn3uvrs $=\left[\operatorname{vpa}\left(\operatorname{sym}\left({ }^{\prime} \quad-.319448664201393584204597479 '\right)\right) \quad v p a\left(\operatorname{sym}\left({ }^{\prime}-.53496050558802566987955980517 '\right)\right) ; \ldots\right.$ $\left.\operatorname{vpa}(\operatorname{sym}('-.53496050558802566987955980517 ')) \operatorname{vpa}\left(\operatorname{sym}\left({ }^{\prime} \quad-.319448664201393584204597479 '\right)\right)\right]$; intJdn15dn4uvrs $=\left[\operatorname{vpa}\left(\operatorname{sym}\left(' .416813143674259981116969119 \mathrm{e}-1^{\prime}\right)\right) \quad \operatorname{vpa}\left(\operatorname{sym}\left({ }^{\prime}-.1942169737637143545345375175 '\right)\right) ; \ldots\right.$ $\left.\operatorname{vpa}\left(\operatorname{sym}\left({ }^{\prime}-.1942169737637143545345375175 '\right)\right) \quad \operatorname{vpa}\left(\operatorname{sym}\left({ }^{\prime} \quad .8033238055969811770258930 \mathrm{e}-2^{\prime}\right)\right)\right]$; intJdn15dn5uvrs $=\left[\operatorname{vpa}\left(\operatorname{sym}\left({ }^{\prime} \quad .449877692126663661629552350 '\right)\right) \quad \operatorname{vpa}\left(\operatorname{sym}\left({ }^{\prime} \quad .379360747071947741960232042^{\prime}\right)\right) ; \ldots\right.$ $\left.\operatorname{vpa}(\operatorname{sym}(' \quad .379360747071947741960232042 ')) \quad \operatorname{vpa}\left(\operatorname{sym}\left(' .86904547772480103903106692 \mathrm{e}-1^{\prime}\right)\right)\right]$; intJdn15dn6uvrs $=\left[\operatorname{vpa}\left(\operatorname{sym}\left({ }^{\prime}-.26123164244466091271015883 '\right)\right) \quad \operatorname{vpa}\left(\operatorname{sym}\left({ }^{\prime} .106104670667416834133287489 '\right)\right) ; \ldots\right.$ $\operatorname{vpa}\left(\operatorname{sym}\left({ }^{\prime} .106104670667416834133287489 '\right)\right)$ vpa(sym(' .44988102080951953135671272'))]; intJdn15dn7uvrs $=\left[\operatorname{vpa}\left(\operatorname{sym}\left({ }^{\prime} \quad .55242173149670587806391031^{\prime}\right)\right) \quad \operatorname{vpa}\left(\operatorname{sym}\left({ }^{\prime} .6763849144738999657208941635 '\right)\right) ; \ldots\right.$ $\left.\operatorname{vpa}(\operatorname{sym}(' .6763849144738999657208941635 ')) \quad \operatorname{vpa}\left(\operatorname{sym}\left('-.293283237003299311368713211^{\prime}\right)\right)\right]$; intJdn15dn8uvrs $=\left[\operatorname{vpa}\left(\operatorname{sym}\left({ }^{\prime} \quad-1.94418619472045071324352388 '\right)\right) \quad \operatorname{vpa}\left(\operatorname{sym}\left({ }^{\prime}-.266497211117548073090616868 \mathrm{e}-1^{\prime}\right)\right) ; \ldots\right.$ $\left.\operatorname{vpa}\left(\operatorname{sym}\left('-.266497211117548073090616868 \mathrm{e}-1^{\prime}\right)\right) \quad \operatorname{vpa}(\operatorname{sym}(' \quad .550843979761993341763278679 '))\right]$; intJdn15dn9uvrs $=\left[\operatorname{vpa}\left(\operatorname{sym}\left({ }^{\prime} \quad .550843979761993341763278679 '\right)\right) \quad \operatorname{vpa}\left(\operatorname{sym}\left({ }^{\prime}-.266497211117548073090616868 \mathrm{e}-1^{\prime}\right)\right) ; \ldots\right.$ $\left.\operatorname{vpa}\left(\operatorname{sym}\left({ }^{\prime}-.266497211117548073090616868 \mathrm{e}-1^{\prime}\right)\right) \quad \operatorname{vpa}\left(\operatorname{sym}\left({ }^{\prime} \quad-1.94418619472045071324352388 '\right)\right)\right]$; intJdn15dn10uvrs $=\left[\operatorname{vpa}\left(\operatorname{sym}\left({ }^{\prime}-.293283237003299311368713211^{\prime}\right)\right) \quad \operatorname{vpa}\left(\operatorname{sym}\left({ }^{\prime} .6763849144738999657208941635 '\right)\right) ; \ldots\right.$ $\left.\operatorname{vpa}\left(\operatorname{sym}\left({ }^{\prime} .6763849144738999657208941635^{\prime}\right)\right) \quad \operatorname{vpa}\left(\operatorname{sym}\left({ }^{\prime} \quad .55242173149670587806391031 '\right)\right)\right]$; intJdn15dn11uvrs $=\left[\operatorname{vpa}\left(\operatorname{sym}\left({ }^{\prime} .449881020809519531356712718^{\prime}\right)\right) \quad \operatorname{vpa}\left(\operatorname{sym}\left({ }^{\prime} .106104670667416834133287489 '\right)\right) ; \ldots\right.$ $\left.\operatorname{vpa}(\operatorname{sym}(' .106104670667416834133287489 ')) \quad \operatorname{vpa}\left(\operatorname{sym}\left('-.26123164244466091271015883^{\prime}\right)\right)\right]$; intJdn15dn12uvrs $=\left[\operatorname{vpa}\left(\operatorname{sym}\left({ }^{\prime} .86904547772480103903106692 \mathrm{e}-1^{\prime}\right)\right) \quad \operatorname{vpa}\left(\operatorname{sym}\left({ }^{\prime} \quad .379360747071947741960232042 '\right)\right) ; \ldots\right.$ $\operatorname{vpa}(\operatorname{sym}(' \quad .379360747071947741960232042 ')) \quad \operatorname{vpa}(\operatorname{sym}(' \quad .449877692126663661629552350 '))]$; intJdn15dn13uvrs $=\left[\operatorname{vpa}\left(\operatorname{sym}\left({ }^{\prime}-.18002409287106955030651138 \mathrm{e}-1^{\prime}\right)\right) \quad \operatorname{vpa}\left(\operatorname{sym}\left({ }^{\prime} \quad-.949948521110901296761134222 '\right)\right) ; \ldots\right.$ $\left.\operatorname{vpa}(\operatorname{sym}(' \quad-.949948521110901296761134222 ')) \operatorname{vpa}\left(\operatorname{sym}\left({ }^{\prime}-.18002409287106955030651138 \mathrm{e}-1^{\prime}\right)\right)\right]$;

intJdn15dn14uvrs $=\left[\operatorname{vpa}\left(\operatorname{sym}\left({ }^{\prime} \quad-.80597791980666492939192672 '\right)\right) \quad \operatorname{vpa}\left(\operatorname{sym}\left({ }^{\prime} \quad-.637841602652030943257059331 '\right)\right) ; \ldots\right.$ $\operatorname{vpa}(\operatorname{sym}('-.637841602652030943257059331 ')) \quad v p a(\operatorname{sym}('-2.769794568377644494965093629 '))]$; intJdn15dn15uvrs $=\left[\operatorname{vpa}\left(\operatorname{sym}\left({ }^{\prime} 4.39476432366214663601450761^{\prime}\right)\right) \quad \operatorname{vpa}\left(\operatorname{sym}\left({ }^{\prime} 1.023888052764473488171737195 '\right)\right) ; \ldots\right.$ $\operatorname{vpa}(\operatorname{sym}(' 1.023888052764473488171737195 ')) \operatorname{vpa}(\operatorname{sym}(' 4.39476432366214663601450761 '))]$; intJdn15dn16uvrs $=\left[\operatorname{vpa}(\operatorname{sym}('-2.769794568377644494965093629 ')) \quad \operatorname{vpa}\left(\operatorname{sym}\left({ }^{\prime}-.637841602652030943257059331 '\right)\right) ; . .\right.$. 
$\left.\operatorname{vpa}\left(\operatorname{sym}\left({ }^{\prime}-.637841602652030943257059331 '\right)\right) \quad \operatorname{vpa}\left(\operatorname{sym}\left({ }^{\prime} \quad-.80597791980666492939192672 '\right)\right)\right]$; intJdn16dn1uvrs $=\left[\operatorname{vpa}\left(\operatorname{sym}\left({ }^{\prime} .33845901154347235890690860^{\prime}\right)\right) \quad v p a\left(\operatorname{sym}\left({ }^{\prime} .381671404352391717716050003 '\right)\right) ; \ldots\right.$ $\operatorname{vpa}(\operatorname{sym}(' .381671404352391717716050003 '))$ vpa(sym(' .33685199022272286409417252'))]; intJdn16dn2uvrs $=\left[\operatorname{vpa}\left(\operatorname{sym}\left({ }^{\prime} .208266894443892215290932469 \mathrm{e}-1^{\prime}\right)\right) \quad \operatorname{vpa}\left(\operatorname{sym}\left({ }^{\prime} .863000808306781359312991519 \mathrm{e}-1^{\prime}\right)\right) ; \ldots\right.$ $\left.\operatorname{vpa}\left(\operatorname{sym}\left({ }^{\prime} .863000808306781359312991519 \mathrm{e}-1^{\prime}\right)\right) \quad \operatorname{vpa}\left(\operatorname{sym}\left({ }^{\prime}-.9846681660450092579804616 \mathrm{e}-3 '\right)\right)\right]$; intJdn16dn3uvrs $=\left[\operatorname{vpa}\left(\operatorname{sym}\left({ }^{\prime} .941185558099118441713021744 \mathrm{e}-1^{\prime}\right)\right) \quad \operatorname{vpa}\left(\operatorname{sym}\left({ }^{\prime} \quad .2272134136490097198166572636 '\right)\right) ; .\right.$. vpa(sym(' .2272134136490097198166572636')) vpa(sym(' .136999462878579334021389201'))]; intJdn16dn4uvrs $=\left[\operatorname{vpa}\left(\operatorname{sym}\left({ }^{\prime}-.1539310157132113391331378486 '\right)\right) \quad \operatorname{vpa}\left(\operatorname{sym}\left({ }^{\prime} \quad .4276223703845844197184744941 '\right)\right) ; \ldots\right.$ $\left.\operatorname{vpa}(\operatorname{sym}(' .4276223703845844197184744941 ')) \operatorname{vpa}\left(\operatorname{sym}\left({ }^{\prime}-.5654544717914146990072595 e-2 '\right)\right)\right]$; intJdn16dn5uvrs $=\left[\operatorname{vpa}\left(\operatorname{sym}\left({ }^{\prime}-.2225431571843560333736647188 '\right)\right) \quad \operatorname{vpa}\left(\operatorname{sym}\left({ }^{\prime} .91268611031924988409224700 \mathrm{e}-1^{\prime}\right)\right) ; \ldots\right.$ $\left.\operatorname{vpa}\left(\operatorname{sym}\left(' .91268611031924988409224700 \mathrm{e}-1^{\prime}\right)\right) \quad \operatorname{vpa}(\operatorname{sym}(' \quad .534031555627251695091986887 '))\right]$; intJdn16dn6uvrs $=\left[\operatorname{vpa}\left(\operatorname{sym}\left({ }^{\prime}-.20260922191887153901852490 \mathrm{e}-1^{\prime}\right)\right) \quad \operatorname{vpa}\left(\operatorname{sym}\left({ }^{\prime} \quad-.32732430826682450655130346 '\right)\right) ; .\right.$. $\operatorname{vpa}(\operatorname{sym}(' \quad-.32732430826682450655130346 '))$ vpa(sym(' $-.189527805811794389864868430 '))]$; intJdn16dn7uvrs $=\left[\operatorname{vpa}\left(\operatorname{sym}\left({ }^{\prime}-.198130259760001838281687341 '\right)\right) \quad \operatorname{vpa}\left(\operatorname{sym}\left({ }^{\prime}-.304233282397533216844618686 '\right)\right) ; \ldots\right.$ $\operatorname{vpa}(\operatorname{sym}('-.304233282397533216844618686 '))$ vpa(sym(' .45065512128407846407826384e-1'))]; intJdn16dn8uvrs $=\left[\operatorname{vpa}\left(\operatorname{sym}\left({ }^{\prime} \quad .531391405011459465049996936 '\right)\right) \quad v p a\left(\operatorname{sym}\left({ }^{\prime} .36845390722838247652759296 e-1 '\right)\right)\right.$; $\left.\operatorname{vpa}\left(\operatorname{sym}\left(' .36845390722838247652759296 \mathrm{e}-1^{\prime}\right)\right) \operatorname{vpa}\left(\operatorname{sym}\left({ }^{\prime}-.154508813774518558307843375^{\prime}\right)\right)\right]$; intJdn16dn9uvrs $=\left[\operatorname{vpa}\left(\operatorname{sym}\left({ }^{\prime}-.433262214197932020360238335^{\prime}\right)\right) \quad \operatorname{vpa}\left(\operatorname{sym}\left(\left(^{\prime}-.7955065459935637821598364152 '\right)\right) ; \ldots\right.\right.$ $\left.\operatorname{vpa}(\operatorname{sym}('-.7955065459935637821598364152 ')) \quad \operatorname{vpa}\left(\operatorname{sym}\left('-.5199575926777798530262703 \mathrm{e}-1^{\prime}\right)\right)\right]$; intJdn16dn10uvrs $=\left[\operatorname{vpa}\left(\operatorname{sym}\left({ }^{\prime} .4636424264352335415173399435^{\prime}\right)\right) \quad \operatorname{vpa}\left(\operatorname{sym}\left({ }^{\prime} .32502712079217193433836137 \mathrm{e}-1^{\prime}\right)\right) ; \ldots\right.$ $\left.\operatorname{vpa}\left(\operatorname{sym}\left(' .32502712079217193433836137 \mathrm{e}-1^{\prime}\right)\right) \operatorname{vpa}\left(\operatorname{sym}\left({ }^{\prime}-2.24559114498245358748525615^{\prime}\right)\right)\right]$; intJdn16dn11uvrs $=\left[\operatorname{vpa}\left(\operatorname{sym}\left({ }^{\prime}-1.501752787756720291051955360 '\right)\right) \quad v p a\left(\operatorname{sym}\left({ }^{\prime}-.510599460959200051978459650 '\right)\right) ; \ldots\right.$ $\left.\operatorname{vpa}\left(\operatorname{sym}\left({ }^{\prime}-.510599460959200051978459650 '\right)\right) \quad \operatorname{vpa}(\operatorname{sym}(' \quad .60105835750654359495658263 '))\right]$; intJdn16dn12uvrs $=\left[\operatorname{vpa}\left(\operatorname{sym}\left({ }^{\prime} \quad-.146237679902381668344145480 '\right)\right) \quad v p a\left(\operatorname{sym}\left({ }^{\prime} \quad-.890289639589649219996546217 '\right)\right) ; \ldots\right.$ vpa(sym(' -.890289639589649219996546217')) vpa(sym(' -1.1955736019576031500653136788'))]; intJdn16dn13uvrs $=\left[\operatorname{vpa}\left(\operatorname{sym}\left({ }^{\prime}-.8096222389464438740999396603 '\right)\right) \quad \operatorname{vpa}\left(\operatorname{sym}\left({ }^{\prime} \quad-.876126624260291794899095697^{\prime}\right)\right) ; \ldots\right.$ vpa(sym(' -.876126624260291794899095697')) vpa(sym(' -3.400472479844200126665206259'))]; intJdn16dn14uvrs $=\left[\operatorname{vpa}\left(\operatorname{sym}\left({ }^{\prime} .877518569823348982633756744 '\right)\right) \quad \operatorname{vpa}\left(\operatorname{sym}\left({ }^{\prime} 1.287737922980470717927187534 '\right)\right) ; \ldots\right.$ vpa(sym(' 1.287737922980470717927187534')) vpa(sym(' .877518569823348982633756744'))]; intJdn16dn15uvrs $=\left[\operatorname{vpa}\left(\operatorname{sym}\left({ }^{\prime}-2.769794568377644494965093629 '\right)\right) \quad \operatorname{vpa}\left(\operatorname{sym}\left({ }^{\prime}-.637841602652030943257059331 '\right)\right) ; \ldots\right.$ vpa(sym(' -.637841602652030943257059331')) vpa(sym(' -.80597791980666492939192672'))]; intJdn16dn16uvrs $=\left[\operatorname{vpa}\left(\operatorname{sym}\left({ }^{\prime} 3.929578185962763299703317274 '\right)\right) \quad \operatorname{vpa}\left(\operatorname{sym}\left({ }^{\prime} 1.770759558087978375081430869 '\right)\right) ; \ldots\right.$ vpa(sym(' 1.770759558087978375081430869')) vpa(sym(' 5.51876129014211756612538031'))]; \%disp('------------------------ integrals of products of global deravatives

intJdndn $(1: 2,1: 32)=[$ intJdn1dn1uvrs intJdn1dn2uvrs intJdn1dn3uvrs intJdn1dn4uvrs intJdn1dn5uvrs intJdn1dn6uvrs intJdn1dn7uvrs intJdn1dn8uvrs intJdn1dn9uvrs intJdn1dn10uvrs intJdn1dn11uvrs intJdn1dn12uvrs ... intJdn1dn13uvrs intJdn1dn14uvrs intJdn1dn15uvrs intJdn1dn16uvrs];

intJdndn $(3: 4,1: 32)=[$ intJdn2dn1 uvrs intJdn2dn2uvrs intJdn2dn3uvrs intJdn2dn4uvrs intJdn2dn5uvrs intJdn2dn6uvrs intJdn2dn7uvrs intJdn2dn8uvrs intJdn2dn9uvrs intJdn2dn10uvrs intJdn2dn11uvrs intJdn2dn12uvrs ... intJdn2dn13uvrs intJdn2dn14uvrs intJdn2dn15uvrs intJdn2dn16uvrs];

intJdndn $(5: 6,1: 32)=[$ intJdn3dn1uvrs int Jdn3dn2uvrs intJdn3dn3uvrs intJdn3dn4uvrs intJdn3dn5uvrs intJdn3dn6uvrs intJdn3dn7uvrs intJdn3dn8uvrs intJdn3dn9uvrs intJdn3dn10uvrs intJdn3dn11uvrs intJdn3dn12uvrs ... intJdn3dn13uvrs intJdn3dn14uvrs intJdn3dn15uvrs intJdn3dn16uvrs];

intJdndn $(7: 8,1: 32)=[$ intJdn4dn1 uvrs intJdn4dn2uvrs intJdn4dn3uvrs intJdn4dn4uvrs intJdn4dn5uvrs intJdn4dn6uvrs intJdn4dn7uvrs intJdn4dn8uvrs intJdn4dn9uvrs intJdn4dn10uvrs intJdn4dn11uvrs intJdn4dn12uvrs ... intJdn4dn13uvrs intJdn4dn14uvrs intJdn4dn15uvrs intJdn4dn16uvrs];

intJdndn $(9: 10,1: 32)=[$ intJdn $5 \mathrm{dn} 1$ uvrs intJdn5dn2uvrs intJdn5dn3uvrs intJdn5dn4uvrs int Jdn5dn5uvrs intJdn5dn6uvrs intJdn5dn7uvrs intJdn5dn8uvrs intJdn5dn9uvrs intJdn5dn10uvrs intJdn5dn11uvrs intJdn5dn12uvrs ... intJdn5dn13uvrs intJdn5dn14uvrs intJdn5dn15uvrs intJdn5dn16uvrs];

intJdndn(11:12,1:32)=[intJdn6dn1uvrs intJdn6dn2uvrs intJdn6dn3uvrs intJdn6dn4uvrs intJdn6dn5uvrs intJdn6dn6uvrs intJdn6dn7uvrs intJdn6dn8uvrs intJdn6dn9uvrs intJdn6dn10uvrs intJdn6dn11uvrs intJdn6dn12uvrs ... intJdn6dn13uvrs intJdn6dn14uvrs intJdn6dn15uvrs intJdn6dn16uvrs];

intJdndn(13:14,1:32)=[intJdn7dn1uvrs intJdn7dn2uvrs intJdn7dn3uvrs intJdn7dn4uvrs intJdn7dn5uvrs intJdn7dn6uvrs intJdn7dn7uvrs intJdn7dn8uvrs intJdn7dn9uvrs intJdn7dn10uvrs intJdn7dn11uvrs intJdn7dn12uvrs ... intJdn7dn13uvrs intJdn7dn14uvrs intJdn7dn15uvrs intJdn7dn16uvrs];

intJdndn(15:16,1:32)=[intJdn8dn1uvrs intJdn8dn2uvrs intJdn8dn3uvrs intJdn8dn4uvrs intJdn8dn5uvrs intJdn8dn6uvrs intJdn8dn7uvrs intJdn8dn8uvrs intJdn8dn9uvrs intJdn8dn10uvrs intJdn8dn11uvrs intJdn8dn12uvrs ... intJdn8dn13uvrs intJdn8dn14uvrs intJdn8dn15uvrs intJdn8dn16uvrs];

intJdndn(17:18,1:32)=[intJdn9dn1uvrs intJdn9dn2uvrs intJdn9dn3uvrs intJdn9dn4uvrs intJdn9dn5uvrs intJdn9dn6uvrs intJdn9dn7uvrs intJdn9dn8uvrs intJdn9dn9uvrs intJdn9dn10uvrs intJdn9dn11uvrs intJdn9dn12uvrs ... intJdn9dn13uvrs intJdn9dn14uvrs intJdn9dn15uvrs intJdn9dn16uvrs];

intJdndn(19:20,1:32)=[intJdn10dn1uvrs intJdn10dn2uvrs intJdn10dn3uvrs intJdn10dn4uvrs intJdn10dn5uvrs intJdn10dn6uvrs intJdn10dn7uvrs intJdn10dn8uvrs intJdn10dn9uvrs intJdn10dn10uvrs intJdn10dn11uvrs intJdn10dn12uvrs ... intJdn10dn13uvrs intJdn10dn14uvrs intJdn10dn15uvrs intJdn10dn16uvrs]; 
intJdndn(21:22,1:32)=[intJdn11dn1uvrs intJdn11dn2uvrs intJdn11dn3uvrs intJdn11dn4uvrs intJdn11dn5uvrs intJdn11dn6uvrs intJdn11dn7uvrs intJdn11dn8uvrs intJdn11dn9uvrs intJdn11dn10uvrs intJdn11dn11uvrs intJdn11dn12uvrs ... intJdn11dn13uvrs intJdn11dn14uvrs intJdn11dn15uvrs intJdn11dn16uvrs];

intJdndn(23:24,1:32)=[intJdn12dn1uvrs intJdn12dn2uvrs intJdn12dn3uvrs intJdn12dn4uvrs intJdn12dn5uvrs intJdn12dn6uvrs intJdn12dn7uvrs intJdn12dn8uvrs intJdn12dn9uvrs intJdn12dn10uvrs intJdn12dn11uvrs intJdn12dn12uvrs ... intJdn12dn13uvrs intJdn12dn14uvrs intJdn12dn15uvrs intJdn12dn16uvrs];

intJdndn(25:26,1:32)=[intJdn13dn1uvrs intJdn13dn2uvrs intJdn13dn3uvrs intJdn13dn4uvrs intJdn13dn5uvrs intJdn13dn6uvrs intJdn13dn7uvrs intJdn13dn8uvrs intJdn13dn9uvrs intJdn13dn10uvrs intJdn13dn11uvrs intJdn13dn12uvrs ... intJdn13dn13uvrs intJdn13dn14uvrs intJdn13dn15uvrs intJdn13dn16uvrs];

intJdndn(27:28,1:32)=[intJdn14dn1uvrs intJdn14dn2uvrs intJdn14dn3uvrs intJdn14dn4uvrs intJdn14dn5uvrs intJdn14dn6uvrs intJdn14dn7uvrs intJdn14dn8uvrs intJdn14dn9uvrs intJdn14dn10uvrs intJdn14dn11uvrs intJdn14dn12uvrs ... intJdn14dn13uvrs intJdn14dn14uvrs intJdn14dn15uvrs intJdn14dn16uvrs];

intJdndn(29:30,1:32)=[intJdn15dn1uvrs intJdn15dn2uvrs intJdn15dn3uvrs intJdn15dn4uvrs intJdn15dn5uvrs intJdn15dn6uvrs intJdn15dn7uvrs intJdn15dn8uvrs intJdn15dn9uvrs intJdn15dn10uvrs intJdn15dn11uvrs intJdn15dn12uvrs ... intJdn15dn13uvrs intJdn15dn14uvrs intJdn15dn15uvrs intJdn15dn16uvrs];

intJdndn(31:32,1:32)=[intJdn16dn1uvrs intJdn16dn2uvrs intJdn16dn3uvrs intJdn16dn4uvrs intJdn16dn5uvrs intJdn16dn6uvrs intJdn16dn7uvrs intJdn16dn8uvrs intJdn16dn9uvrs intJdn16dn10uvrs intJdn16dn11uvrs intJdn16dn12uvrs ... intJdn16dn13uvrs intJdn16dn14uvrs intJdn16dn15uvrs intJdn16dn16uvrs];

intJdndn=double(intJdndn);

end

\section{[14]nodaladdresses_special_convex_quadrilaterals_trial_3rd_order.m}

function[eln,spqd,rrr,nodes,nodetel]=nodaladdresses_special_convex_quadrilaterals_trial_3rd_order(n1,n2,n3,nmax,numtri,n)

$\%$ n1=node number at $(0,0)$ for a choosen triangle

$\%$ n2=node number at $(1,0)$ for a choosen triangle

$\%$ n3=node number at $(0,1)$ for a choosen triangle

$\%$ eln=6-node triangles with centroid

$\%$ spqd=4-node special convex quadrilateral

$\%$ m must be even,i.e.n=2,4,6, ,......i.e number of divisions

$\%$ nmax $=$ one plus the number of segments of the polygon

$\%$ nmax $=$ the number of segments of the polygon plus a node interior to the polygon

$\%$ numtri=number of T6 triangles in each segment i.e a triangle formed by

$\%$ joining the end poits of the segment to the interior point(e.g:the centroid) of the polygon

$\%$ PARVIZ MOIN EXAMPLE

$\%[$ eln,spqd,rrr,nodes,nodetel]=nodaladdresses_special_convex_quadrilaterals_trial_3rd_order([1;1; 1;1],[2;3;4;5],[3;4;5;2],5,1,2)

$\%$ syms mst_trix

ne $=0$;

nitri=nmax-1;

for itri=1:nitri

$\operatorname{elm}(1:(n+1) *(n+2) / 2,1)=\operatorname{zeros}((n+1) *(n+2) / 2,1)$

$\operatorname{elm}(1,1)=n 1($ itri, 1$)$

$\operatorname{elm}(\mathrm{n}+1,1)=\mathrm{n} 2(\mathrm{itri}, 1)$

$\operatorname{elm}((n+1) *(n+2) / 2,1)=n 3($ itri, 1$)$

disp('vertex nodes of the itri triangle')

[n1(itri,1) n2(itri,1) n3(itri,1)]

if itri==1

$\mathrm{kk}=\mathrm{nmax}$;

for $\mathrm{k}=2: \mathrm{n}$

$\mathrm{kk}=\mathrm{kk}+1$

$\operatorname{elm}(\mathrm{k}, 1)=\mathrm{kk}$

end

disp('base nodes=')

$\% \operatorname{elm}(2: \mathrm{n})$

edgen $1 \mathrm{n} 2(1: \mathrm{n}+1, \mathrm{itri})=\operatorname{elm}(1: \mathrm{n}+1,1)$

end $\%$ itri $==1$

if itri> 1

$\operatorname{elm}(1: n+1,1)=$ edgen $1 n 3(1: n+1$, itri-1);

end $\%$ if itri $>1$

if itri $==1$

$\operatorname{lmax}=\mathrm{nmax}+3 *(\mathrm{n}-1)$;

end $\%$ if itri $==1$

if (itri $>1) \&($ itri< $<$ nitri $)$

$\operatorname{lmax}=\mathrm{nmax}+2 *(\mathrm{n}-1)$;

end $\%$ if (itri $>1) \&($ itri $<$ nitri $)$

$\operatorname{mmax}=\mathrm{nmax}$;

if itri $==1$ 
$\operatorname{mmax}=\max (\max (\operatorname{edgen} 1 \mathrm{n} 2(1: \mathrm{n}+1,1)))$

end $\%$ itri $==1$

disp('right edge nodes')

$\mathrm{nni}=\mathrm{n}+1 ; \mathrm{hh}=1 ; \mathrm{qq}(1,1)=\mathrm{n} 2(\mathrm{itri}, 1)$;

for $i=0:(n-2)$

$\mathrm{hh}=\mathrm{hh}+1$;

nni=nni+(n-i);

$\operatorname{elm}(n n i, 1)=(\operatorname{mmax}+1)+\mathrm{i}$;

$\mathrm{qq}(\mathrm{hh}, 1)=(\operatorname{mmax}+1)+\mathrm{i}$;

end

$\mathrm{qq}(\mathrm{n}+1,1)=\mathrm{n} 3($ itri, 1$)$;

edgen $2 n 3(1: n+1$, itri $)=q q$;

if itri<nitri

disp('left edge nodes')

nni=1;gg=1;pp $(1,1)=\mathrm{n} 1($ itri, 1$)$;

for $\mathrm{i}=0:(\mathrm{n}-2)$

$\mathrm{gg}=\mathrm{gg}+1$;

nni $=$ nni $+(n-i)+1$;

$\operatorname{elm}($ nni, 1$)=\operatorname{lmax}-\mathrm{i}$;

$\mathrm{pp}(\mathrm{gg}, 1)=\operatorname{lmax}-\mathrm{i}$;

end

$\mathrm{pp}(\mathrm{n}+1,1)=\mathrm{n} 3($ itri, 1$)$;

edgen $1 \mathrm{n} 3(1: \mathrm{n}+1, \mathrm{itri})=\mathrm{pp}$

end $\%$ if itri<nitri

if itri==nitri

disp('left edge nodes')

nni=1;gg=1;

for $\mathrm{i}=0:(\mathrm{n}-2)$

$\mathrm{gg}=\mathrm{gg}+1$;

nni=nni $+(n-i)+1$;

$\operatorname{elm}(\mathrm{nni}, 1)=\operatorname{edgen} 1 \mathrm{n} 2(\mathrm{gg}, 1)$;

end

$\% \mathrm{pp}(\mathrm{n}+1,1)=\mathrm{n} 3(\mathrm{itri}, 1)$;

\%edgen $1 \mathrm{n} 3(1: \mathrm{n}+1$, itri $)=\mathrm{pp}$

end $\%$ if itri==nitri

if itri==nitri

$\operatorname{lmax}=\max (\max ($ edgen $2 \mathrm{n} 3(1: \mathrm{n}+1, \mathrm{itri})))$;

end $\%$ if itri==nitri

disp('interior nodes')

$\mathrm{nni}=1 ; \mathrm{jj}=0$;

for $\mathrm{i}=0:(\mathrm{n}-3)$

nni=nni+(n-i)+1;

for $j=1:(n-2-i)$

$\mathrm{jj}=\mathrm{jj}+1$;

nnj=nni+j;

$\operatorname{elm}(n n j, 1)=\operatorname{lmax}+\mathrm{jj}$;

[nnj lmax+jj];

$$
\text { end }
$$

end

$\% \operatorname{disp}(\mathrm{elm})$

$\%$ disp(length(elm));

$\mathrm{jj}=0 ; \mathrm{kk}=0$;

for $\mathrm{j}=0: n-1$

$\mathrm{jj}=\mathrm{j}+1$;

for $\mathrm{k}=1:(\mathrm{n}+1)-\mathrm{j}$

$\mathrm{kk}=\mathrm{kk}+1$;

row_nodes $(\mathrm{jj}, \mathrm{k})=\mathrm{elm}(\mathrm{kk}, 1)$;

end

end

row_nodes $(\mathrm{n}+1,1)=\mathrm{n} 3$ (itri,1);

\% for $\mathrm{jj}=(\mathrm{n}+1):-1: 1$

$\% \quad($ row_nodes $(\mathrm{jj},:))$; 
$\%$ [row_nodes]

rr=row_nodes;

rr

$\operatorname{rrr}(:, ;$, itri $)=r r$;

disp('element computations')

if $\operatorname{rem}(\mathrm{n}, 2)==0$

$\mathrm{N}=\mathrm{n}+1$;

for $\mathrm{k}=1: 2: \mathrm{n}-1$

$\mathrm{N}=\mathrm{N}-2$;

$\mathrm{i}=\mathrm{k}$;

for $\mathrm{j}=1: 2: \mathrm{N}$

ne $=$ ne +1

$\operatorname{eln}(\mathrm{ne}, 1)=\operatorname{rr}(\mathrm{i}, \mathrm{j})$;

$\operatorname{eln}(n e, 2)=\operatorname{rr}(i, j+2)$;

$\operatorname{eln}(n e, 3)=\operatorname{rr}(i+2, j)$;

$\operatorname{eln}(n e, 4)=\operatorname{rr}(i, j+1)$;

$\operatorname{eln}(n e, 5)=\operatorname{rr}(i+1, j+1)$;

$\operatorname{eln}(n e, 6)=\operatorname{rr}(i+1, j)$;

end $\% \mathrm{j}$

$\%$ me $=$ ne

$\% \mathrm{~N}-2$

if $(\mathrm{N}-2)>0$

for $\mathrm{jj}=1: 2: \mathrm{N}-2$

ne $=$ ne +1

$\operatorname{eln}(n e, 1)=\operatorname{rr}(i+2, j j+2)$;

$\operatorname{eln}(n e, 2)=\operatorname{rr}(i+2, j j)$;

$\operatorname{eln}(n e, 3)=\operatorname{rr}(i, j j+2)$;

$\operatorname{eln}(n e, 4)=\operatorname{rr}(i+2, j j+1) ;$

eln $(n e, 5)=\operatorname{rr}(i+1, j j+1)$;

$\operatorname{eln}(n e, 6)=\operatorname{rr}(\mathrm{i}+1, \mathrm{jj}+2)$;

end $\% \mathrm{jj}$

end $\%$ if $(\mathrm{N}-2)>0$

end $\% \mathrm{k}$

end $\%$ if $\operatorname{rem}(n, 2)==0$

ne

\%for $\mathrm{kk}=1: \mathrm{ne}$

$\%[\mathrm{eln}(\mathrm{kk}, 1: 6)]$

$\%$ end

\%add node numbers for element centroids

nnd $=\max (\max (\mathrm{eln}))$

if $(n>3)$

for $\mathrm{kkk}=1+($ itri-1)*numtri:ne

nnd $=$ nnd +1 ;

$\operatorname{eln}(\mathrm{kkk}, 7)=\mathrm{nnd}$;

end

end

if $n==2$

for $\mathrm{kkk}=\mathrm{itri}$ :ne

nnd $=$ nnd +1 ;

$\operatorname{eln}(\mathrm{kkk}, 7)=\mathrm{nnd}$;

end

end

$\operatorname{nmax}=\max (\max (\operatorname{eln}))$;

$\%$ nel=mm;

$\%$

$\%$ ne

$\%$ spqd

end \%itri 
$\%$ to generate special quadrilaterals

$\mathrm{mm}=0$;

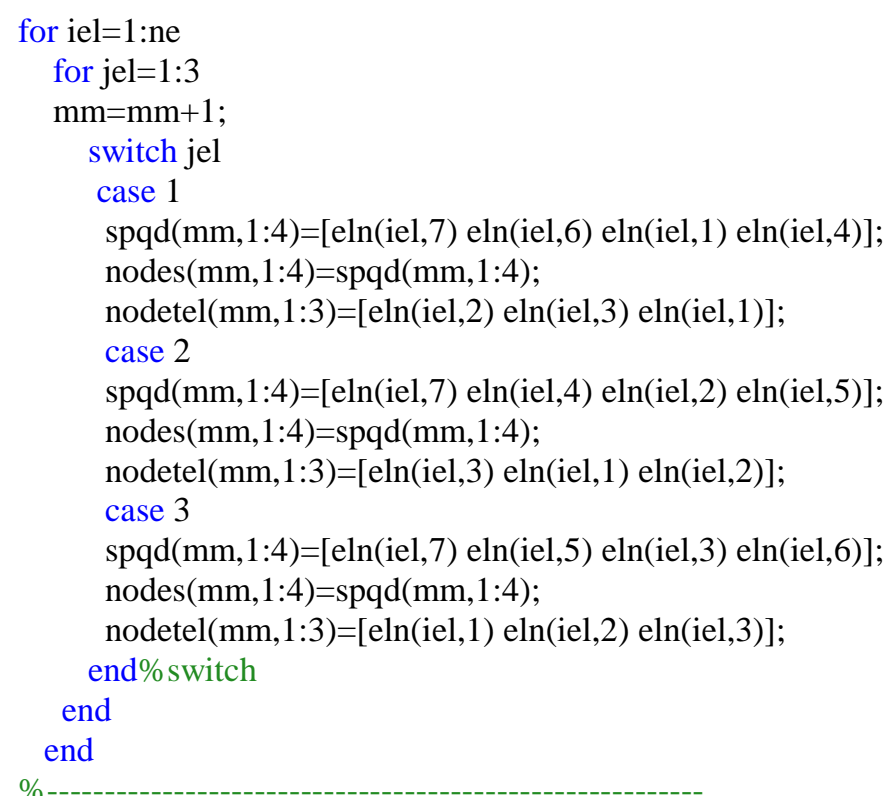

for inum $=1$ :nnd

for jnum=1:nnd

trisect(inum,jnum) $=0$; end

end

nd=nnd;

for $\mathrm{mmm}=1: \mathrm{mm}$

$\mathrm{mmm} 1=\operatorname{nodes}(\mathrm{mmm}, 1)$;

$\mathrm{mmm} 2=\operatorname{nodes}(\mathrm{mmm}, 2)$;

$\operatorname{mmm} 3=\operatorname{nodes}(\mathrm{mmm}, 3)$;

mmm4 $=\operatorname{nodes}(\mathrm{mmm}, 4)$;

$\%$ midpoint side-1 of 4-node special quadrilateral

if $((\operatorname{trisect}(\mathrm{mmm} 1, \mathrm{mmm} 2)==0) \&(\operatorname{trisect}(\mathrm{mmm} 2, \mathrm{mmm} 1)==0))$

$\mathrm{nd}=\mathrm{nd}+1$;

$\operatorname{trisect}(\mathrm{mmm} 1, \mathrm{mmm} 2)=\mathrm{nd}$;

nd $=$ nd +1 ;

$\operatorname{trisect}(\mathrm{mmm} 2, \mathrm{mmm} 1)=\mathrm{nd}$;

end

$\%$ midpoint side-2 of 4-node special quadrilateral

if $((\operatorname{trisect}(\mathrm{mmm} 2, \mathrm{mmm} 3)==0) \&(\operatorname{trisect}(\mathrm{mmm} 3, \mathrm{mmm} 2)==0))$ $\mathrm{nd}=\mathrm{nd}+1$;

$\operatorname{trisect}(\mathrm{mmm} 2, \mathrm{mmm} 3)=\mathrm{nd}$;

$\mathrm{nd}=\mathrm{nd}+1$;

$\operatorname{trisect}(\mathrm{mmm} 3, \mathrm{mmm} 2)=\mathrm{nd}$;

end

$\%$ midpoint side-3 of 4-node special quadrilateral

if $((\operatorname{trisect}(\mathrm{mmm} 3, \mathrm{mmm} 4)==0) \&(\operatorname{trisect}(\mathrm{mmm} 4, \mathrm{mmm} 3)==0))$

nd $=\mathrm{nd}+1$;

$\operatorname{trisect}(\mathrm{mmm} 3, \mathrm{mmm} 4)=\mathrm{nd}$;

nd $=$ nd +1 ;

$\operatorname{trisect}(\mathrm{mmm} 4, \mathrm{mmm} 3)=\mathrm{nd}$;

end

$\%$ midpoint side-4 of 4-node special quadrilateral

if $((\operatorname{trisect}(\mathrm{mmm} 4, \mathrm{mmm} 1)==0) \&(\operatorname{trisect}(\mathrm{mmm} 1, \mathrm{mmm} 4)==0))$

nd $=$ nd +1 ;

$\operatorname{trisect}(\mathrm{mmm} 4, \mathrm{mmm} 1)=\mathrm{nd}$;

nd $=$ nd +1 ;

$\operatorname{trisect}(\mathrm{mmm} 1, \mathrm{mmm} 4)=\mathrm{nd}$;

end

$\operatorname{nodes}(\mathrm{mmm}, 5)=\operatorname{trisect}(\mathrm{mmm} 1, \mathrm{mmm} 2)$;

$\operatorname{nodes}(\mathrm{mmm}, 6)=\operatorname{trisect}(\mathrm{mmm} 2, \mathrm{mmm} 1)$; 
$\%$

$\operatorname{nodes}(\mathrm{mmm}, 7)=\operatorname{trisect}(\mathrm{mmm} 2, \mathrm{mmm} 3)$;

$\operatorname{nodes}(\mathrm{mmm}, 8)=\operatorname{trisect}(\mathrm{mmm} 3, \mathrm{mmm} 2)$;

$\%$

$\operatorname{nodes}(\mathrm{mmm}, 9)=\operatorname{trisect}(\mathrm{mmm} 3, \mathrm{mmm} 4)$;

$\operatorname{nodes}(\mathrm{mmm}, 10)=\operatorname{trisect}(\mathrm{mmm} 4, \mathrm{mmm} 3)$;

$\%$

$\operatorname{nodes}(\mathrm{mmm}, 11)=\operatorname{trisect}(\mathrm{mmm} 4, \mathrm{mmm} 1)$;

$\operatorname{nodes}(\mathrm{mmm}, 12)=\operatorname{trisect}(\mathrm{mmm} 1, \mathrm{mmm} 4)$;

end $\%$ for

nnode=nd;

nel=mm;

spqd=nodes;

ss1='number of 6-node triangles with centroid=';

$[\mathrm{p} 1, \mathrm{q} 1]=\operatorname{size}(\mathrm{eln})$;

$\operatorname{disp}([\mathrm{ss} 1$ num2str(p1)])

$\%$

eln

$\%$

ss2='number of special convex quadrilaterals elements\&nodes per element =';

[nel,nnel]=size(spqd);

$\operatorname{disp}([\mathrm{ss} 2$ num2str(nel) ',' num2str(nnel)])

$\%$

nnode $=\max (\max (\operatorname{spqd}))$;

ss3='number of nodes of the triangular domain\& number of special quadrilaterals=';

disp([ss3 num2str(nnode) ',' num2str(nel)])

[15]generate_area_coordinate_over_the_standard_triangle.m

function$[\mathrm{U}, \mathrm{V}, \mathrm{W}]=$ generate_area_coordinate_over_the_standard_triangle(n)

syms ui vi wi $\mathrm{U} \mathrm{V}$ W

$\mathrm{kk}=0$;

for $\mathrm{j}=1: \mathrm{n}+1$

for $\mathrm{i}=1:(\mathrm{n}+1)-(\mathrm{j}-1)$

$\mathrm{kk}=\mathrm{kk}+1$

ui $(\mathrm{i}, \mathrm{j})=(\mathrm{i}-1) / \mathrm{n}$;

$\operatorname{vi}(\mathrm{i}, \mathrm{j})=(\mathrm{j}-1) / \mathrm{n}$;

wi(i,j)=1-ui(i,j)-vi(i,j);

$\mathrm{U}(\mathrm{kk}, 1)=\mathrm{ui}(\mathrm{i}, \mathrm{j})$;

$\mathrm{V}(\mathrm{kk}, 1)=\mathrm{vi}(\mathrm{i}, \mathrm{j})$;

$\mathrm{W}(\mathrm{kk}, 1)=\mathrm{wi}(\mathrm{i}, \mathrm{j})$;

end

end

[16]nodaladdresses_special_convex_quadrilaterals_trial_3rd_orderLG.m

function[eln,spqd,rrr,nodes,nodetel]=nodaladdresses_special_convex_quadrilaterals_trial_3rd_orderLG(n1,n2,n3,nmax,numtri,n)

$\%$ n1=node number at $(0,0)$ for a choosen triangle

$\%$ n2=node number at $(1,0)$ for a choosen triangle

$\%$ n3=node number at $(0,1)$ for a choosen triangle

$\%$ eln=6-node triangles with centroid

$\%$ spqd=4-node special convex quadrilateral

$\%$ n must be even,i.e.n=2,4,6,.......i.e number of divisions

$\%$ nmax $=$ one plus the number of segments of the polygon

$\%$ nmax =the number of segments of the polygon plus a node interior to the polygon

$\%$ numtri=number of $\mathrm{T} 6$ triangles in each segment i.e a triangle formed by

$\%$ joining the end poits of the segment to the interior point(e.g:the centroid) of the polygon

\%PARVIZ MOIN EXAMPLE

$\%[$ eln,spqd,rrr,nodes,nodetel]=nodaladdresses_special_convex_quadrilaterals_trial_3rd_orderLG([1; $1 ; 1 ; 1],[2 ; 3 ; 4 ; 5],[3 ; 4 ; 5 ; 2], 5,1$, 2)

\%syms mst_tri x

ne=0;

nitri=nmax-1;

for itri=1:nitri

$\operatorname{elm}(1:(n+1) *(n+2) / 2,1)=\operatorname{zeros}((n+1) *(n+2) / 2,1)$ 


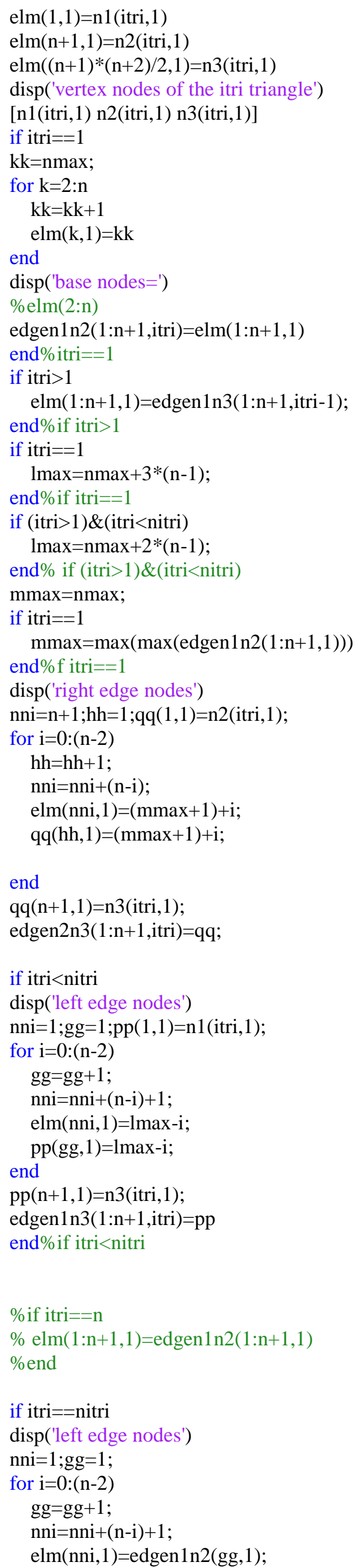


end

$\% p p(n+1,1)=n 3($ itri, 1$)$;

\%edgen $1 \mathrm{n} 3(1: \mathrm{n}+1, \mathrm{itri})=\mathrm{pp}$

end $\%$ if itri==nitri

if itri==nitri

$\operatorname{lmax}=\max (\max ($ edgen2n3(1:n+1,itri $)))$;

end $\%$ if itri==nitri

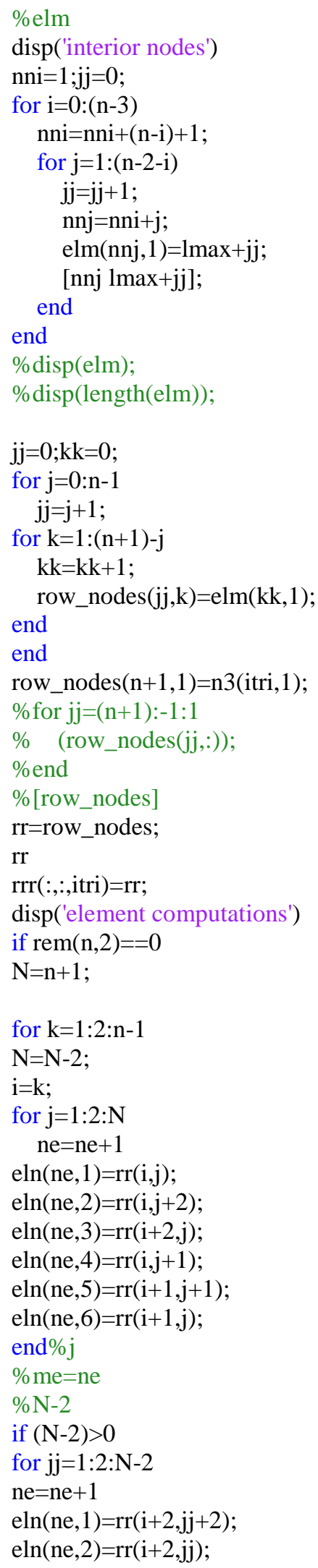



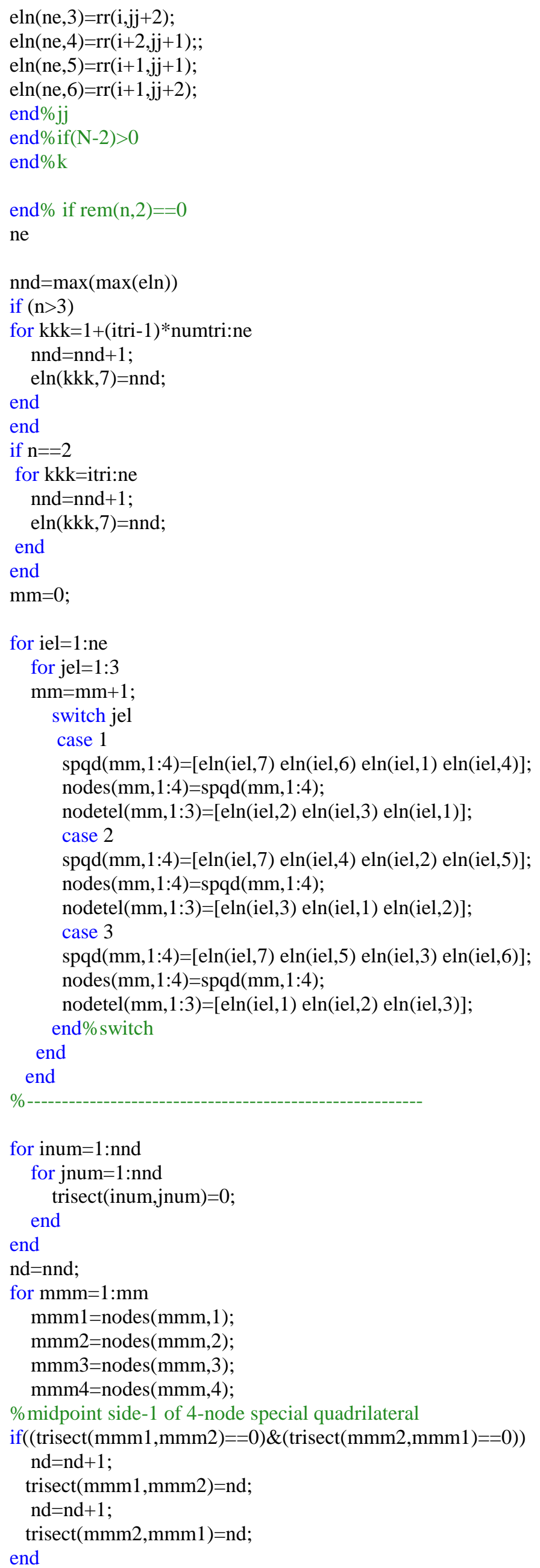
\% midpoint side-2 of 4-node special quadrilateral

$\operatorname{if}((\operatorname{trisect}(\mathrm{mmm} 2, \mathrm{mmm} 3)==0) \&(\operatorname{trisect}(\mathrm{mmm} 3, \mathrm{mmm} 2)==0))$

nd $=$ nd +1 ;

$\operatorname{trisect}(\mathrm{mmm} 2, \mathrm{mmm} 3)=\mathrm{nd}$;

nd $=$ nd +1 ;

$\operatorname{trisect}(\mathrm{mmm} 3, \mathrm{mmm} 2)=\mathrm{nd}$;

end

$\%$ midpoint side-3 of 4-node special quadrilateral

if $((\operatorname{trisect}(\mathrm{mmm} 3, \mathrm{mmm} 4)==0) \&(\operatorname{trisect}(\mathrm{mmm} 4, \mathrm{mmm} 3)==0))$

$\mathrm{nd}=\mathrm{nd}+1$;

$\operatorname{trisect}(\mathrm{mmm} 3, \mathrm{mmm} 4)=\mathrm{nd}$;

$\mathrm{nd}=\mathrm{nd}+1$;

$\operatorname{trisect}(\mathrm{mmm} 4, \mathrm{mmm} 3)=\mathrm{nd}$;

end

$\%$ midpoint side-4 of 4-node special quadrilateral

if $((\operatorname{trisect}(\mathrm{mmm} 4, \mathrm{mmm} 1)==0) \&(\operatorname{trisect}(\mathrm{mmm} 1, \mathrm{mmm} 4)==0))$

nd $=$ nd +1 ;

$\operatorname{trisect}(\mathrm{mmm} 4, \mathrm{mmm} 1)=\mathrm{nd}$;

$\mathrm{nd}=\mathrm{nd}+1$;

$\operatorname{trisect}(\mathrm{mmm} 1, \mathrm{mmm} 4)=\mathrm{nd}$;

end

$\operatorname{nodes}(\mathrm{mmm}, 5)=\operatorname{trisect}(\mathrm{mmm} 1, \mathrm{mmm} 2)$;

$\operatorname{nodes}(\mathrm{mmm}, 6)=\operatorname{trisect}(\mathrm{mmm} 2, \mathrm{mmm} 1)$;

$\%$

$\operatorname{nodes}(\mathrm{mmm}, 7)=\operatorname{trisect}(\mathrm{mmm} 2, \mathrm{mmm} 3)$;

$\operatorname{nodes}(\mathrm{mmm}, 8)=\operatorname{trisect}(\mathrm{mmm} 3, \mathrm{mmm} 2)$;

$\%$

$\operatorname{nodes}(\mathrm{mmm}, 9)=\operatorname{trisect}(\mathrm{mmm} 3, \mathrm{mmm} 4)$;

$\operatorname{nodes}(\mathrm{mmm}, 10)=\operatorname{trisect}(\mathrm{mmm} 4, \mathrm{mmm} 3)$;

$\%$

$\operatorname{nodes}(\mathrm{mmm}, 11)=\operatorname{trisect}(\mathrm{mmm} 4, \mathrm{mmm} 1)$;

$\operatorname{nodes}(\mathrm{mmm}, 12)=\operatorname{trisect}(\mathrm{mmm} 1, \mathrm{mmm} 4)$;

$\%$

nd $=$ nd $+1 ; \operatorname{nodes}(\operatorname{mmm}, 13)=$ nd;

$\mathrm{nd}=\mathrm{nd}+1 ; \operatorname{nodes}(\mathrm{mmm}, 14)=\mathrm{nd}$;

$\mathrm{nd}=\mathrm{nd}+1 ; \operatorname{nodes}(\mathrm{mmm}, 15)=\mathrm{nd}$

$\mathrm{nd}=\mathrm{nd}+1 ; \operatorname{nodes}(\mathrm{mmm}, 16)=\mathrm{nd}$;

end $\%$ for

nnode=nd;

nel=mm;

spqd=nodes;

ss1='number of 6-node triangles with centroid=';

[p1,q1]=size(eln);

$\operatorname{disp}([\mathrm{ss} 1$ num2str(p1)])

$\%$

eln

$\%$

ss2='number of special convex quadrilaterals elements\&nodes per element =';

$[$ nel,nnel $]=\operatorname{size}($ spqd $)$;

$\operatorname{disp}([\mathrm{ss} 2$ num2str(nel) ',' num2str(nnel)])

$\%$

nnode $=\max (\max (\operatorname{spqd}))$;

ss3='number of nodes of the triangular domain\& number of special quadrilaterals=';

disp([ss3 num2str(nnode) ',' num2str(nel)])

[17]D2PoissonEquationQ12MoinEx_MeshgridContourNew.m

function[]=D2PoissonEquationQ12MoinEx_MeshgridContourNew(n1,n2,n3,nmax,numtri,ndiv,mesh)

$\%$ ndiv $=2,4,6,8$,

\%D2PoissonEquationQ12MoinEx_MeshgridContourNew([1;1;1;1;1;1;1],[2;3;4;5;6;7;8],[3;4;5;6;7;8;2],8,1,2,1)

\%D2PoissonEquationQ12MoinEx_MeshgridContourNew([1;1;1;1;1;1;1;1],[2;3;4;5;6;7;8;9],[3;4;5;6;7;8;9;2],9,1,2,2)

clc

syms coord

$\%[$ coord,gcoord,nodes,nodetel,nnode,nel]=polygonal_domain_coordinates(n1,n2,n3,nmax,numtri,ndiv,mesh) 
\%nnel=4;

[coord,gcoord,nodes,nodetel,nnode,nel]=polygonal_domain_coordinates_3rd_order(n1,n2,n3,nmax,numtri,ndiv,mesh) nnel=12;

ndof $=1$;

$\%$ nc $=(\text { ndiv/2 })^{\wedge} 2$

$\%$ nnode $=($ ndiv +1$) *($ ndiv +2$) / 2+n c ;$

$\%$ nel=3*nc;

sdof=nnode $*$ ndof;

$\mathrm{ff}=($ zeros $($ sdof, 1$)) ; \mathrm{ss}=($ zeros $($ sdof,sdof $))$;

$\operatorname{disp}([$ nel nnode nnel ndof])

format long $g$

for $\mathrm{i}=1$ :nel

$\mathrm{N}(\mathrm{i}, 1)=\mathrm{i}$;

end

for $i=1$ :nel

$\mathrm{NN}(\mathrm{i}, 1)=\mathrm{i}$;

end

switch mesh

case 1

\%boundary conditions- 1

$\mathrm{nnn}=0$;

for $\mathrm{nn}=1$ :nnode

$\mathrm{xnn}=\operatorname{gcoord}(\mathrm{nn}, 1) ; \mathrm{ynn}=\operatorname{gcoord}(\mathrm{nn}, 2)$;

if $(\mathrm{xnn}==0) \&((\mathrm{ynn}>=0) \&(\mathrm{ynn}<=1))$

$\mathrm{nnn}=\mathrm{nnn}+1$;

$\operatorname{bcdof}(\mathrm{nnn}, 1)=\mathrm{nn}$;

$\operatorname{bcval}(\mathrm{nnn}, 1)=0$;

end

end

\%boundary conditions-2

for $\mathrm{nn}=1$ :nnode

$\mathrm{xnn}=\mathrm{g} \operatorname{coord}(\mathrm{nn}, 1) ; \mathrm{ynn}=\mathrm{g} \operatorname{coord}(\mathrm{nn}, 2) ;$

if $(\mathrm{ynn}==0) \&((\mathrm{xnn}>=0) \&(\mathrm{xnn}<=1))$

$\mathrm{nnn}=\mathrm{nnn}+1$;

bcdof $(\mathrm{nnn}, 1)=\mathrm{nn}$;

$\operatorname{bcval}(\mathrm{nnn}, 1)=0$;

end

end

\%boundary conditions-3

for $\mathrm{nn}=1$ :nnode

$\mathrm{xnn}=\operatorname{gcoord}(\mathrm{nn}, 1) ; \mathrm{ynn}=\operatorname{gcoord}(\mathrm{nn}, 2)$;

if $(\mathrm{ynn}==1) \&((\mathrm{xnn}>=0) \&(\mathrm{xnn}<=1 / 2))$

$\mathrm{nnn}=\mathrm{nnn}+1$;

$\operatorname{bcdof}(\mathrm{nnn}, 1)=\mathrm{nn}$;

$\operatorname{bcval}(\mathrm{nnn}, 1)=0$;

end

end

\%boundary conditions-4

for $\mathrm{nn}=1$ :nnode

$\mathrm{xnn}=\operatorname{gcoord}(\mathrm{nn}, 1) ; \mathrm{ynn}=\operatorname{gcoord}(\mathrm{nn}, 2)$;

if $(\mathrm{xnn}==1) \&((\mathrm{ynn}>=0) \&(\mathrm{ynn}<=1 / 2))$

$\mathrm{nnn}=\mathrm{nnn}+1$;

$\operatorname{bcdof}(\mathrm{nnn}, 1)=\mathrm{nn}$;

$\operatorname{bcval}(\mathrm{nnn}, 1)=0$;

end

end

\%boundary conditions-5

for $\mathrm{nn}=1$ :nnode

$\mathrm{xnn}=\operatorname{coord}(\mathrm{nn}, 1) ; \mathrm{ynn}=\operatorname{coord}(\mathrm{nn}, 2) ;$

if $((x n n+y n n)==3 / 2)$

$\mathrm{nnn}=\mathrm{nnn}+1$;

$\operatorname{bcdof}(\mathrm{nnn}, 1)=\mathrm{nn}$;

$\operatorname{bcval}(\mathrm{nnn}, 1)=\operatorname{double}\left(\left(\sin \left(\mathrm{pi}^{*} \mathrm{xnn}\right)\right) *(\sin (\mathrm{pi} * \mathrm{ynn}))\right)$

end

end 
case 2

$\mathrm{nnn}=0$;

for $\mathrm{nn}=1$ :nnode

$\mathrm{xnn}=\operatorname{coord}(\mathrm{nn}, 1) ; \mathrm{ynn}=\operatorname{gcoord}(\mathrm{nn}, 2)$;

if $(\mathrm{xnn}==0) \&((\mathrm{ynn}>=0) \&(\mathrm{ynn}<=1))$

$\mathrm{nnn}=\mathrm{nnn}+1$;

$\operatorname{bcdof}(\mathrm{nnn}, 1)=\mathrm{nn}$;

$\operatorname{bcval}(\mathrm{nnn}, 1)=0$;

end

end

\%boundary conditions-2

for $n n=1$ :nnode

$\mathrm{xnn}=\operatorname{gcoord}(\mathrm{nn}, 1) ; \mathrm{ynn}=\operatorname{coord}(\mathrm{nn}, 2)$;

if $(\mathrm{ynn}==0) \&((\mathrm{xnn}>=0) \&(\mathrm{xnn}<=1))$

$\mathrm{nnn}=\mathrm{nnn}+1$;

bcdof $(\mathrm{nnn}, 1)=\mathrm{nn}$;

$\operatorname{bcval}(n n n, 1)=0$;

end

end

\%boundary conditions-3

for $\mathrm{nn}=1$ :nnode

$\mathrm{xnn}=\operatorname{gcoord}(\mathrm{nn}, 1) ; \mathrm{ynn}=\operatorname{coord}(\mathrm{nn}, 2)$;

if $(\mathrm{ynn}==1) \&((\mathrm{xnn}>=0) \&(\mathrm{xnn}<=1))$

$\mathrm{nnn}=\mathrm{nnn}+1$;

bcdof $(\mathrm{nnn}, 1)=\mathrm{nn}$;

$\operatorname{bcval}(\mathrm{nnn}, 1)=0$;

end

end

\%boundary conditions-4

for $\mathrm{nn}=1$ :nnode

$\mathrm{xnn}=\operatorname{coord}(\mathrm{nn}, 1) ; \mathrm{ynn}=\operatorname{gcoord}(\mathrm{nn}, 2)$;

if $(\mathrm{xnn}==1) \&((\mathrm{ynn}>=0) \&(\mathrm{ynn}<=1))$

$\mathrm{nnn}=\mathrm{nnn}+1$;

bcdof $(\mathrm{nnn}, 1)=\mathrm{nn}$;

$\operatorname{bcval}(\mathrm{nnn}, 1)=0$;

end

end

end

bcdof

mm=length(bcdof);

format long $g$

\%analytical solution

$\mathrm{xi}=($ zeros $($ nnode, 1$))$;

for $m=1$ :nnode

$\mathrm{xm}=(\operatorname{gcoord}(\mathrm{m}, 1)) ; \mathrm{ym}=(\operatorname{gcoord}(\mathrm{m}, 2))$;

$\mathrm{xi}(\mathrm{m}, 1)=\sin (\mathrm{pi} * \mathrm{xm}) * \sin \left(\mathrm{pi}^{*} \mathrm{ym}\right)$;

end

for $\mathrm{L}=1:$ nel

for $M=1: 3$

$\mathrm{LM}=\operatorname{nodetel}(\mathrm{L}, \mathrm{M})$;

$\mathrm{xx}(\mathrm{L}, \mathrm{M})=\operatorname{gcoord}(\mathrm{LM}, 1)$;

$\mathrm{yy}(\mathrm{L}, \mathrm{M})=\operatorname{gcoord}(\mathrm{LM}, 2)$;

end

end

$\%$

ng $=10$

$[\mathrm{sp}, \mathrm{wt}]=\mathrm{glsampleptsweights}(\mathrm{ng})$

table2 $=[\mathrm{N}$ xx yy $]$;

$\% \operatorname{disp}([\mathrm{xx} y \mathrm{y}])$

\%integral values of local derivative products 
[intJdndn]=integral_valuesof_localderivative_products(nnel);

for iel=1:nel

index=zeros(nnel*ndof,1);

$\mathrm{X}=\mathrm{xx}(\mathrm{iel}, 1: 3)$;

$\mathrm{Y}=\mathrm{yy}(\mathrm{iel}, 1: 3)$;

$\% \operatorname{disp}([\mathrm{X} \mathrm{Y}])$

$\mathrm{xa}=\mathrm{X}(1,1)$;

$\mathrm{xb}=\mathrm{X}(1,2)$

$\mathrm{xc}=\mathrm{X}(1,3)$;

ya $=Y(1,1)$

$\mathrm{yb}=\mathrm{Y}(1,2)$

$\mathrm{yc}=\mathrm{Y}(1,3)$;

bta $=y b-y c ; b t b=y c-y a ;$

gma $=x c-x b ; g m b=x a-x c$;

delabc $=$ gmb $*$ bta-gma*btb;

$\mathrm{G}=[\mathrm{bta}$ btb;gma gmb]/delabc;

$\mathrm{GT}=[\mathrm{bta}$ gma;btb gmb]/delabc;

$\mathrm{Q}=\mathrm{GT} * \mathrm{G}$;

$\operatorname{sk}(1: 12,1: 12)=($ zeros $(12,12))$;

for $i=1: 12$

for $\mathrm{j}=\mathrm{i}: 12$

$\operatorname{sk}(\mathrm{i}, \mathrm{j})=(\operatorname{delabc} * \operatorname{sum}(\operatorname{sum}(\mathrm{Q} . *(\operatorname{intJdndn}(2 * \mathrm{i}-1: 2 * \mathrm{i}, 2 * \mathrm{j}-1: 2 * \mathrm{j})))))$;

$\operatorname{sk}(\mathrm{j}, \mathrm{i})=\operatorname{sk}(\mathrm{i}, \mathrm{j})$;

end

end

$\% \mathrm{f}=[5 / 144 ; 1 / 24 ; 7 / 144 ; 1 / 24] *(2 *$ delabc $)$;

$\mathrm{xe}(1,1)=(\mathrm{xa}+\mathrm{xb}+\mathrm{xc}) / 3$

$\mathrm{xe}(2,1)=(\mathrm{xa}+\mathrm{xc}) / 2$;

$\mathrm{xe}(3,1)=\mathrm{xa}$;

$\mathrm{xe}(4,1)=(\mathrm{xa}+\mathrm{xb}) / 2$;

$\%$

$\mathrm{ye}(1,1)=(\mathrm{ya}+\mathrm{yb}+\mathrm{yc}) / 3$;

$\mathrm{ye}(2,1)=(\mathrm{ya}+\mathrm{yc}) / 2$;

ye $(3,1)=y a$;

$\mathrm{ye}(4,1)=(\mathrm{ya}+\mathrm{yb}) / 2$;

$\%$

[sp,wt $]=$ glsampleptsweights $(\mathrm{ng})$;

xe1=xe $(1,1) ; \mathrm{xe} 2=\mathrm{xe}(2,1) ; \mathrm{xe} 3=\mathrm{xe}(3,1) ; \mathrm{xe} 4=\mathrm{xe}(4,1)$;

ye $1=y e(1,1) ; y e 2=y e(2,1) ; y e 3=y e(3,1) ; y e 4=y e(4,1)$;

$\mathrm{f}(1: 12,1)=$ zeros $(12,1)$

for $\mathrm{i}=1: \mathrm{ng}$

$s i=s p(i, 1) ; w i=w t(i, 1)$;

for $\mathrm{j}=1$ :ng $s j=s p(j, 1) ; w j=w t(j, 1) ;$

$\mathrm{n} 1 \mathrm{ij}=\left((1-\mathrm{si}) *(1-\mathrm{sj}) *\left(-10+9 *\left(\mathrm{si}^{\wedge} 2+\mathrm{sj}^{\wedge} 2\right)\right)\right) / 32$

$\mathrm{n} 2 \mathrm{ij}=\left((1+\mathrm{si}) *(1-\mathrm{sj}) *\left(-10+9 *\left(\mathrm{si}^{\wedge} 2+\mathrm{sj}^{\wedge} 2\right)\right)\right) / 32$;

$\mathrm{n} 3 \mathrm{ij}=\left((1+\mathrm{si}) *(1+\mathrm{sj}) *\left(-10+9 *\left(\mathrm{si}^{\wedge} 2+\mathrm{sj}^{\wedge} 2\right)\right)\right) / 32$

$\mathrm{n} 4 \mathrm{ij}=\left((1-\mathrm{si}) *(1+\mathrm{sj}) *\left(-10+9 *\left(\mathrm{si}^{\wedge} 2+\mathrm{sj} \mathrm{j}^{\wedge} 2\right)\right)\right) / 32$;

$\mathrm{n} 5 \mathrm{ij}=(9 / 32) *\left((1-\mathrm{sj}) *\left(1-\mathrm{si}^{\wedge} 2\right) *(1-3 * \mathrm{si})\right)$;

$\mathrm{n} 6 \mathrm{ij}=(9 / 32) *\left((1-\mathrm{sj}) *\left(1-\mathrm{si}^{\wedge} 2\right) *(1+3 * \mathrm{si})\right)$

$\mathrm{n} 7 \mathrm{ij}=(9 / 32) *\left((1+\mathrm{si}) *\left(1-\mathrm{sj}^{\wedge} 2\right) *(1-3 * \mathrm{sj})\right)$;

$\mathrm{n} 8 \mathrm{ij}=(9 / 32) *\left((1+\mathrm{si}) *\left(1-\mathrm{sj}^{\wedge} 2\right) *(1+3 * \mathrm{sj})\right)$;

$\mathrm{n} 9 \mathrm{ij}=(9 / 32) *\left((1+\mathrm{sj}) *\left(1-\mathrm{si}^{\wedge} 2\right) *(1+3 * \mathrm{si})\right)$;

$\mathrm{n} 10 \mathrm{ij}=(9 / 32) *\left((1+\mathrm{sj}) *\left(1-\mathrm{si}^{\wedge} 2\right)^{*}(1-3 * \mathrm{si})\right)$;

$\mathrm{n} 11 \mathrm{ij}=(9 / 32)^{*}\left((1-\mathrm{si}) *\left(1-\mathrm{sj}^{\wedge} 2\right)^{*}\left(1+3^{*} \mathrm{sj}\right)\right)$;

$\mathrm{n} 12 \mathrm{ij}=(9 / 32) *\left((1-\mathrm{si}) *\left(1-\mathrm{sj}^{\wedge} 2\right) *(1-3 * \mathrm{sj})\right)$;

$\%$

$\mathrm{N} 1 \mathrm{ij}=(((1-\mathrm{si}) *(1-\mathrm{sj}))) / 4$;

$\mathrm{N} 2 \mathrm{ij}=(((1+\mathrm{si}) *(1-\mathrm{sj}))) / 4$;

$\mathrm{N} 3 \mathrm{ij}=(((1+\mathrm{si}) *(1+\mathrm{sj}))) / 4$;

$\mathrm{N} 4 \mathrm{ij}=(((1-\mathrm{si}) *(1+\mathrm{sj}))) / 4$;

$x e i j=x e 1 * N 1 i j+x e 2 * N 2 i j+x e 3 * N 3 i j+x e 4 * N 4 i j$; 
yeij=ye $1 * N 1 i j+y e 2 * N 2 i j+y e 3 * N 3 i j+y e 4 * N 4 i j$

f1i=n1ij*(2*pi^2)*sin(pi*xeij)*sin(pi*yeij)*(4+si+sj)/96;

$\mathrm{f} 2 \mathrm{i}=\mathrm{n} 2 \mathrm{ij} *\left(2 * \mathrm{pi}^{\wedge} 2\right) * \sin \left(\mathrm{pi}^{*} \mathrm{xeij}\right) * \sin \left(\mathrm{pi}^{*}\right.$ yeij)*(4+si+sj)/96;

$\mathrm{f} 3 \mathrm{i}=\mathrm{n} 3 \mathrm{ij} *\left(2 * \mathrm{pi}^{\wedge} 2\right) * \sin (\mathrm{pi} * \mathrm{xe} \mathrm{j}) * \sin (\mathrm{pi}$ (yeij)*(4+si+sj)/96;

$\mathrm{f} 4 \mathrm{i}=\mathrm{n} 4 \mathrm{ij} *\left(2 * \mathrm{pi}^{\wedge} 2\right) * \sin (\mathrm{pi} * \mathrm{xeij}) * \sin \left(\mathrm{pi}^{*}\right.$ yeij)*(4+si+sj)/96;

$\mathrm{f} 5 \mathrm{i}=\mathrm{n} 5 \mathrm{ij} *\left(2 * \mathrm{pi}^{\wedge} 2\right) * \sin \left(\mathrm{pi}^{*} \mathrm{xeij}\right) * \sin \left(\mathrm{pi}^{*} \mathrm{yeij}\right) *(4+\mathrm{si}+\mathrm{sj}) / 96$

f6i=n6ij*(2*pi^2)*sin(pi*xeij)*sin(pi*yeij)*(4+si+sj)/96;

f7i $=n 7 i j^{*}\left(2 * \mathrm{pi}^{\wedge} 2\right) * \sin \left(\mathrm{pi}^{*}\right.$ xeij $) * \sin ($ pi*yeij $) *(4+\mathrm{si}+\mathrm{sj}) / 96$;

f8i=n8ij*(2*pi^2)*sin(pi*xeij)*sin(pi*yeij)*(4+si+sj)/96;

f9i=n9ij*(2* pi $\left.^{\wedge} 2\right) * \sin ($ pi*xeij)*sin $($ pi*yeij $) *(4+s i+s j) / 96$;

$\mathrm{f} 10 \mathrm{i}=\mathrm{n} 10 \mathrm{ij} *\left(2 * \mathrm{pi}^{\wedge} 2\right) * \sin \left(\mathrm{pi}^{*} \mathrm{xeij}\right) * \sin \left(\mathrm{pi}^{*}\right.$ yeij $) *(4+\mathrm{si}+\mathrm{sj}) / 96$;

f1 1i $=n 11 \mathrm{ij} *\left(2 * \mathrm{pi}^{\wedge} 2\right) * \sin \left(\mathrm{pi}^{*} \mathrm{xeij}\right) * \sin \left(\mathrm{pi}^{*}\right.$ yeij $) *(4+\mathrm{si}+\mathrm{sj}) / 96$;

$\mathrm{f} 12 \mathrm{i}=\mathrm{n} 12 \mathrm{ij} *\left(2 * \mathrm{pi}^{\wedge} 2\right) * \sin \left(\mathrm{pi}^{*} \mathrm{xeij}\right) * \sin ($ pi*yeij $) *(4+\mathrm{si}+\mathrm{sj}) / 96$;

$\mathrm{f}(1,1)=\mathrm{f}(1,1)+\mathrm{f} 1 \mathrm{i} * \mathrm{wi} * \mathrm{wj}$

$\mathrm{f}(2,1)=\mathrm{f}(2,1)+\mathrm{f} 2 \mathrm{i} * \mathrm{wi} * \mathrm{wj}$;

$\mathrm{f}(3,1)=\mathrm{f}(3,1)+\mathrm{f} 3 \mathrm{i} * \mathrm{wi} * \mathrm{wj}$;

$\mathrm{f}(4,1)=\mathrm{f}(4,1)+\mathrm{f} 4 \mathrm{i} * \mathrm{wi} * \mathrm{wj}$;

$\mathrm{f}(5,1)=\mathrm{f}(5,1)+\mathrm{f} 5 \mathrm{i} * \mathrm{wi} * \mathrm{wj}$;

$\mathrm{f}(6,1)=\mathrm{f}(6,1)+\mathrm{f} 6 i^{*} w i * w j ;$

$\mathrm{f}(7,1)=\mathrm{f}(7,1)+\mathrm{f} 7 \mathrm{i}^{*} \mathrm{wi} * \mathrm{wj}$

$\mathrm{f}(8,1)=\mathrm{f}(8,1)+\mathrm{f} 8 \mathrm{i} * \mathrm{wi}$ *wj;

$\mathrm{f}(9,1)=\mathrm{f}(9,1)+\mathrm{f9i}{ }^{*} \mathrm{wi}^{*} \mathrm{wj}$;

$\mathrm{f}(10,1)=\mathrm{f}(10,1)+\mathrm{f} 10 i^{*} w i * w j$;

$\mathrm{f}(11,1)=f(11,1)+f 11 i^{*} w i * w j ;$

$f(12,1)=f(12,1)+f 12 i * w i * w j$;

end

end

$\mathrm{f}=($ delabc $) * \mathrm{f} ;$

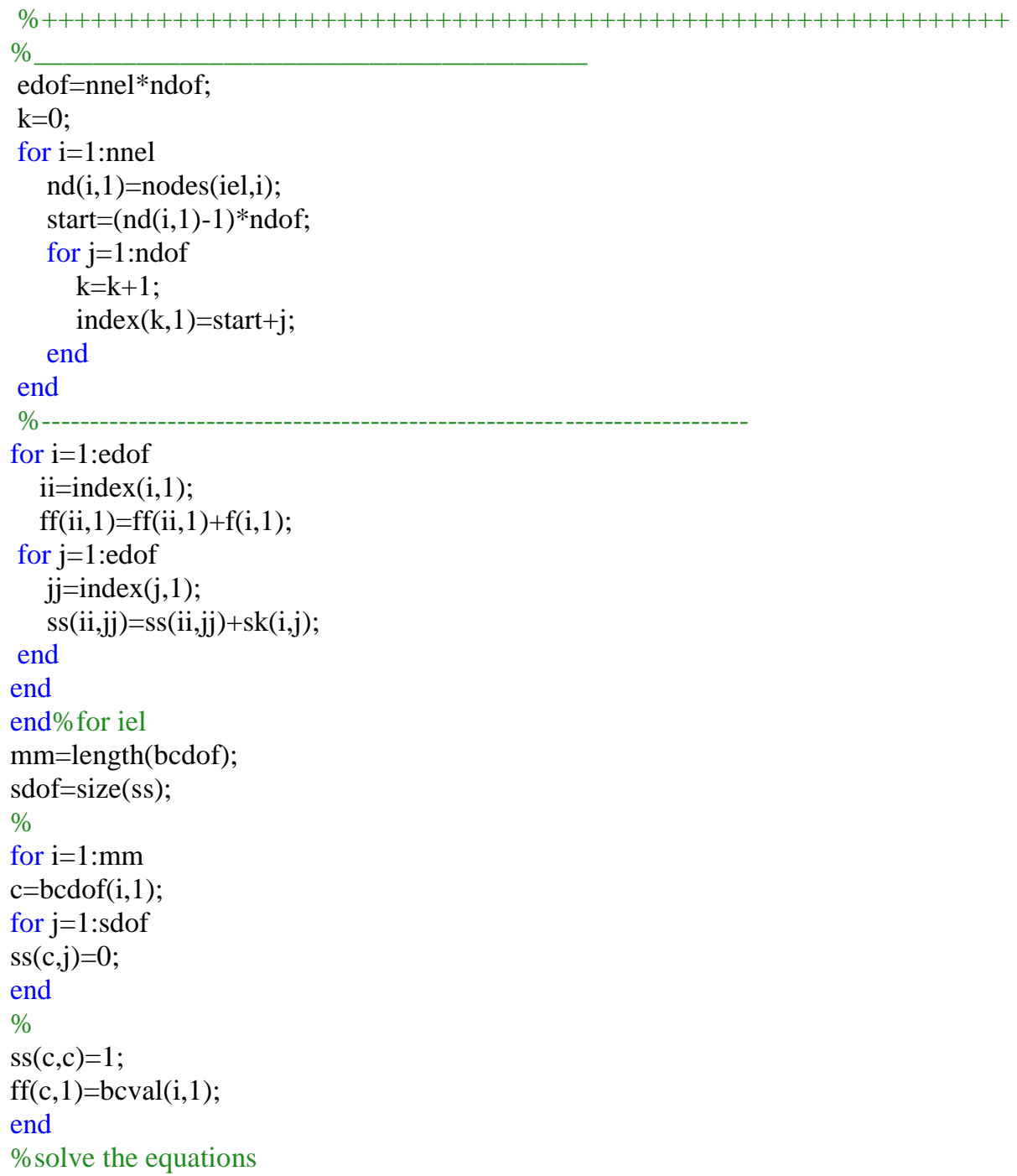


phi=ss $\backslash f f ;$

for $\mathrm{I}=1$ :nnode

$\mathrm{NN}(\mathrm{I}, 1)=\mathrm{I}$;

end

$\operatorname{disp}('$

disp('number of nodes, elements \& nodes per element')

[nnode nel nnel ndof]

$\operatorname{disp}('$

fem-computed values

anlytical(theoretical)-values

')

$\operatorname{disp}([\mathrm{NN}$ phi xi] $)$

$\operatorname{disp}('$

disp('number of nodes,elements \& nodes per element')

[nnode nel nnel ndof]

nodes

gcoord

$[\mathrm{x}, \mathrm{y}]=$ meshgrid $(0: 0.05: 1,0: 0.05: 1)$;

for $i=1: 21$

for $\mathrm{j}=1: 21$

for iel $=1:$ nel

$\mathrm{XX}=\mathrm{xx}($ iel, $1: 3)$

YY=yy(iel,1:3);

$\% \operatorname{disp}([\mathrm{X} Y])$

$\mathrm{xa}=\mathrm{XX}(1,1)$;

$\mathrm{xb}=\mathrm{XX}(1,2)$

$\mathrm{Xc}=\mathrm{XX}(1,3)$;

ya $=Y Y(1,1)$

$\mathrm{yb}=\mathrm{YY}(1,2)$;

$\mathrm{yc}=\mathrm{YY}(1,3)$

$\mathrm{aLPa}=\mathrm{xb} * \mathrm{yc}-\mathrm{xc} * \mathrm{yb}$

$\mathrm{aLPb}=\mathrm{xc} * \mathrm{ya}-\mathrm{xa} * \mathrm{yc}$;

bta $=y b-y c ; b t b=y c-y a ;$

$\mathrm{gma}=\mathrm{xc}-\mathrm{xb} ; \mathrm{gmb}=\mathrm{xa}-\mathrm{xc}$

delabc $=$ gmb $*$ bta-gma*btb;

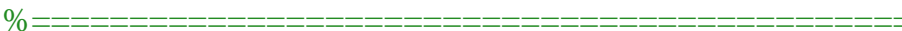

$\%$ node numbers of quadrilateral

nd1=nodes(iel,1);nd2=nodes(iel,2);nd3=nodes(iel,3);nd4=nodes(iel,4);

nd5=nodes(iel,5);nd6=nodes(iel,6);nd7=nodes(iel,7);nd8=nodes(iel,8);

nd9=nodes(iel,9);nd10=nodes(iel,10);nd11=nodes(iel,11);nd12=nodes(iel,12);

$\%$ coordinates of quadrilateral $(\mathrm{u}, \mathrm{v})$

$\mathrm{u}(1,1)=\operatorname{gcoord}(\mathrm{nd} 1,1) ; \mathrm{u}(2,1)=\operatorname{gcoord}(\mathrm{nd} 2,1) ; \mathrm{u}(3,1)=\operatorname{gcoord}(\mathrm{nd} 3,1) ; \mathrm{u}(4,1)=\operatorname{gcoord}(\mathrm{nd} 4,1) ;$

$\mathrm{v}(1,1)=\operatorname{gcoord}(\mathrm{nd} 1,2) ; \mathrm{v}(2,1)=\operatorname{gcoord}(\mathrm{nd} 2,2) ; \mathrm{v}(3,1)=\operatorname{gcoord}(\mathrm{nd} 3,2) ; \mathrm{v}(4,1)=\operatorname{gcoord}(\mathrm{nd} 4,2) ;$

$\%$ coordinates of the $\operatorname{grid}(\mathrm{x}, \mathrm{y})$

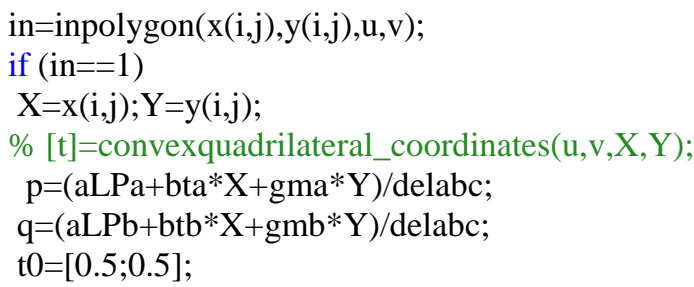

[t,iter $]=$ newtonmethod4spquadrileteral(t0,p,q,'parameqnspqd','paramdetJspqd','paraminvJspqd')

$\mathrm{r}=\mathrm{t}(1,1)$;

$\mathrm{s}=\mathrm{t}(2,1)$;

$\operatorname{shn} 1=\left((1-r) *(1-s) *\left(-10+9 *\left(\mathrm{r}^{\wedge} 2+\mathrm{s}^{\wedge} 2\right)\right)\right) / 32$;

$\operatorname{shn} 2=\left((1+\mathrm{r}) *(1-\mathrm{s}) *\left(-10+9 *\left(\mathrm{r}^{\wedge} 2+\mathrm{s}^{\wedge} 2\right)\right)\right) / 32$

$\operatorname{shn} 3=\left((1+\mathrm{r}) *(1+\mathrm{s}) *\left(-10+9 *\left(\mathrm{r}^{\wedge} 2+\mathrm{s}^{\wedge} 2\right)\right)\right) / 32$;

$\operatorname{shn} 4=\left((1-r) *(1+s) *\left(-10+9 *\left(r^{\wedge} 2+s^{\wedge} 2\right)\right)\right) / 32$

$\operatorname{shn} 5=(9 / 32) *\left((1-\mathrm{s}) *\left(1-\mathrm{r}^{\wedge} 2\right) *(1-3 * \mathrm{r})\right)$; 


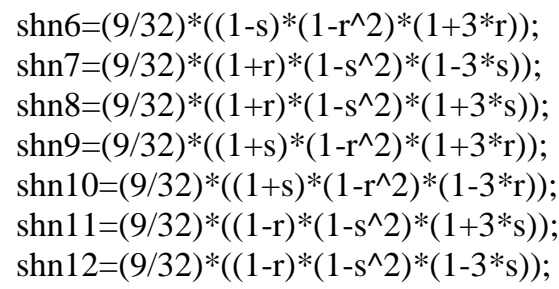

PHI $(\mathrm{i}, \mathrm{j})=\operatorname{shn} 1 * \operatorname{phi}(\mathrm{nd} 1,1)+\operatorname{shn} 2 * \operatorname{phi}(\mathrm{nd} 2,1)+\operatorname{shn} 3 * \operatorname{phi}(\mathrm{nd} 3,1)+\operatorname{shn} 4 * \operatorname{phi}(\mathrm{nd} 4,1)+\operatorname{shn} 5 * \operatorname{phi}(\mathrm{nd} 5,1)+\operatorname{shn} 6 * \operatorname{phi}(\mathrm{nd} 6,1)+\operatorname{shn} 7 * \operatorname{phi}(\mathrm{nd} 7$,

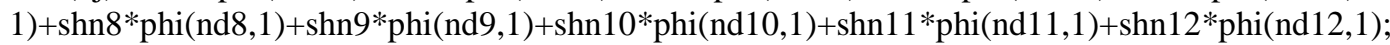

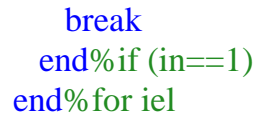


hold on

$[\mathrm{x}, \mathrm{y}]=$ meshgrid(0:.05:1,0:0.05:1)

$\mathrm{y}((\mathrm{y}>1 / 2) \&(\mathrm{y}<=1) \&(\mathrm{x}>1 / 2) \&(\mathrm{x}<=1) \&(\mathrm{x}+\mathrm{y}>3 / 2))=\mathrm{NaN}$;

contour(x,y,z,40,'g-')

$\%[\mathrm{c}, \mathrm{h}]=$ contour $\left(\mathrm{x}, \mathrm{y}, \mathrm{z}, \mathrm{g}_{-}{ }^{\prime}\right)$

xlabel('X-axis');

ylabel('Y-axis');

\%clabel(c,h);

axis square

title('contour level curves for exact solution: $\sin \left(\mathrm{pi}^{*} \mathrm{x}\right) * \sin \left(\mathrm{pi}{ }^{*} \mathrm{y}\right)$ ')

$\mathrm{mm}=0$;

for $\mathrm{i}=1: 21$

for $\mathrm{j}=1: 21$

$\mathrm{mm}=\mathrm{mm}+1$;

femsoln $(\mathrm{mm}, 1)=\mathrm{PHI}(\mathrm{i}, \mathrm{j})$;

$\operatorname{exactsoln}(\mathrm{mm}, 1)=\mathrm{z}(\mathrm{i}, \mathrm{j})$;

end

end

case 2

clc

hold off

clf

figure(1)

$\mathrm{X}=\left[\begin{array}{llll}0.0 & 1.0 & 1.0 & 0.0\end{array}\right]$;

$\mathrm{y}=\left[\begin{array}{llll}0.0 & 0.0 & 1.0 & 1.0\end{array}\right]$;

$\operatorname{patch}\left(\mathrm{x}, \mathrm{y},{ }^{\prime} \mathrm{w}\right)$

hold on

$[\mathrm{x}, \mathrm{y}]=$ meshgrid(0:.05:1,0:0.05:1)

contour(x,y,PHI,40,'r-')

$\%[\mathrm{c}, \mathrm{h}]=$ contour $\left(\mathrm{x}, \mathrm{y}, \mathrm{PHI}, \mathrm{r}: \mathrm{:}^{\prime}\right)$

xlabel('X-axis');

ylabel('Y-axis');

$\%$ clabel $(\mathrm{c}, \mathrm{h})$;

axis square

st1='Contour level curves for ';

st2='FEM and Exact solutions using ';

st3='Twelve Noded ';

st4='Special Quadrilateral';

st5=' Elements on a SQUARE'

title([st1,st2,st3,st4,st5])

sst1='(MESH HAS '

sst2 $=$ num 2 str(nnode)

sst3=' NODES'

sst4=' AND

sst5=num 2 str(nel)

sst6=' ELEMENTS)'

text(0.25,-.08,[sst1 sst2 sst3 sst4 sst5 sst6])

figure(2)

$\mathrm{x}=\left[\begin{array}{llll}0.0 & 1.0 & 1.0 & 0.0\end{array}\right]$;

$\mathrm{y}=\left[\begin{array}{llll}0.0 & 0.0 & 1.0 & 1.0\end{array}\right]$;

$\operatorname{patch}\left(\mathrm{x}, \mathrm{y},{ }^{\prime} \mathrm{w}\right)$

hold on

$[\mathrm{x}, \mathrm{y}]=$ meshgrid(0:.05:1,0:0.05:1)

contour(x,y,z,40,'g-')

$\%[\mathrm{c}, \mathrm{h}]=$ contour $\left(\mathrm{x}, \mathrm{y}, \mathrm{z}, \mathrm{g}-{ }^{\prime}\right)$

\%xlabel('X-axis');

\%ylabel('Y-axis');

$\%$ clabel(c,h);

axis square

\%text(.6,.98,'\{ ...(red)FEM and--(green)EXACT $\} ')$

\%legend(' FEM',' EXACT')

title('contour level curves for exact solution: $\sin (\mathrm{pi} * \mathrm{x}) * \sin \left(\mathrm{pi}{ }^{*} \mathrm{y}\right)$ ')

$\mathrm{mm}=0$;

for $\mathrm{i}=1: 21$ 


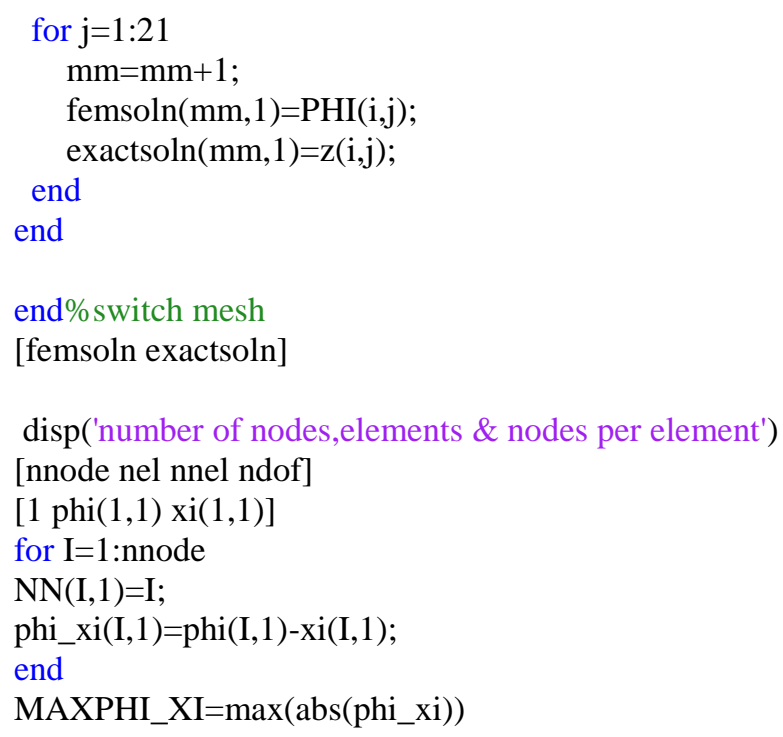

[18]D2PoissonEquationQ16MoinEx_MeshgridContourNew.m

function[]=D2PoissonEquationQ16MoinEx_MeshgridContourNew(n1,n2,n3,nmax,numtri,ndiv,mesh)

$\%$ ndiv $=2,4,6,8, \ldots \ldots$

$\%$

\%D2PoissonEquationQ16MoinEx_MeshgridContourNew([1;1;1;1;1;1;1],[2;3;4;5;6;7;8],[3;4;5;6;7;8;2],8,1,2,1)

\%D2PoissonEquationQ16MoinEx_MeshgridContourNew([1;1;1;1;1;1;1;1],[2;3;4;5;6;7;8;9],[3;4;5;6;7;8;9;2],9,1,2,2)

clc

clf,figure(1)

clf,figure(2)

syms coord

[coord,gcoord,nodes,nodetel,nnode,nel]=polygonal_domain_coordinates_3rd_orderLG(n1,n2,n3,nmax,numtri,ndiv,mesh) nnel=16;

ndof $=1$;

$\%$ nc $=(\text { ndiv } / 2)^{\wedge} 2$

$\%$ nnode $=($ ndiv +1$) *($ ndiv +2$) / 2+$ nc;

$\%$ nel $=3 *$ nc;

sdof $=$ nnode $*$ ndof;

$\mathrm{ff}=(\mathrm{zeros}(\mathrm{sdof}, 1)) ; \mathrm{ss}=(\mathrm{zeros}(\operatorname{sdof}, \mathrm{sdof}))$;

$\operatorname{disp}([$ nel nnode nnel ndof] $)$

format long $g$

for $i=1$ :nel

$\mathrm{N}(\mathrm{i}, 1)=\mathrm{i}$;

end

for $i=1$ :nel

$\mathrm{NN}(\mathrm{i}, 1)=\mathrm{i}$;

end

switch mesh

case 1

$\mathrm{nnn}=0$;

for $\mathrm{nn}=1$ :nnode

$\mathrm{xnn}=\mathrm{g} \operatorname{coord}(\mathrm{nn}, 1) ; \mathrm{ynn}=\mathrm{g} \operatorname{coord}(\mathrm{nn}, 2) ;$

if $(\mathrm{xnn}==0) \&((\mathrm{ynn}>=0) \&(\mathrm{ynn}<=1))$

nnn $=\mathrm{nnn}+1$;

$\operatorname{bcdof}(\mathrm{nnn}, 1)=\mathrm{nn}$;

$\operatorname{bcval}(n n n, 1)=0$;

end

end

\%boundary conditions-2

for $n n=1$ :nnode

$\mathrm{xnn}=\operatorname{gcoord}(\mathrm{nn}, 1) ; \mathrm{ynn}=\operatorname{gcoord}(\mathrm{nn}, 2)$;

if $(\mathrm{ynn}==0) \&((\mathrm{xnn}>=0) \&(\mathrm{xnn}<=1))$

$\mathrm{nnn}=\mathrm{nnn}+1$;

$\operatorname{bcdof}(\mathrm{nnn}, 1)=\mathrm{nn}$;

$\operatorname{bcval}(\mathrm{nnn}, 1)=0$;

end

end 


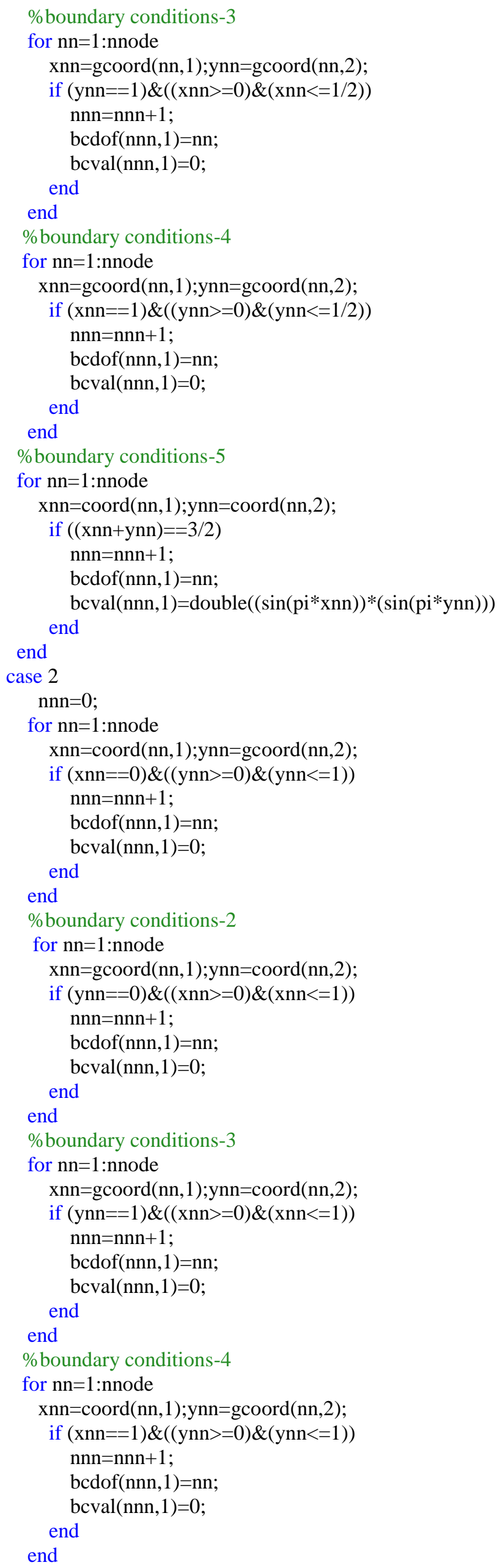


end

bcdof

$\mathrm{mm}=$ length(bcdof);

format long g

\%analytical solution

$\mathrm{xi}=(\operatorname{zeros}($ nnode, 1$))$

for $m=1$ :nnode

$\mathrm{xm}=(\operatorname{gcoord}(\mathrm{m}, 1)) ; \mathrm{ym}=(\operatorname{gcoord}(\mathrm{m}, 2)) ;$

$\mathrm{xi}(\mathrm{m}, 1)=\sin (\mathrm{pi} * \mathrm{xm}) * \sin (\mathrm{pi} * \mathrm{ym})$;

end

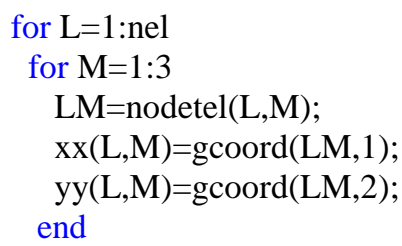


xe1=xe(1,1);xe2=xe(2,1);xe3=xe(3,1);xe4=xe(4,1);

ye1=ye $(1,1)$;ye $2=y e(2,1)$;ye $3=y e(3,1)$;ye4=ye $(4,1)$;

$\mathrm{f}(1: 16,1)=$ zeros $(16,1)$;

for $\mathrm{i}=1: \mathrm{ng}$

$\operatorname{si}=\operatorname{sp}(\mathrm{i}, 1) ; \mathrm{wi}=\mathrm{wt}(\mathrm{i}, 1)$;

h1i=-9* $(\operatorname{si}+1 / 3) *(\operatorname{si}-1 / 3) *(\operatorname{si}-1) / 16$;

$\mathrm{h} 2 \mathrm{i}=27 *(\mathrm{si}+1) *(\mathrm{si}-1 / 3) *(\mathrm{si}-1) / 16$;

$\mathrm{h} 3 \mathrm{i}=-27 *(\mathrm{si}+1) *(\mathrm{si}+1 / 3) *(\mathrm{si}-1) / 16$;

$\mathrm{h} 4 \mathrm{i}=9 *(\mathrm{si}+1) *(\mathrm{si}+1 / 3) *(\mathrm{si}-1 / 3) / 16$;

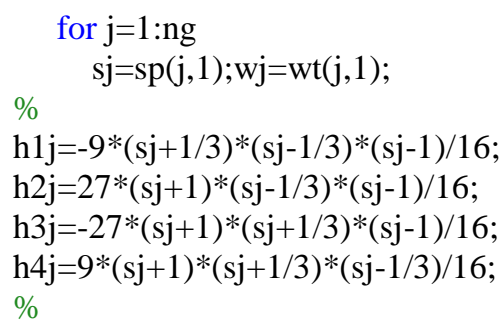




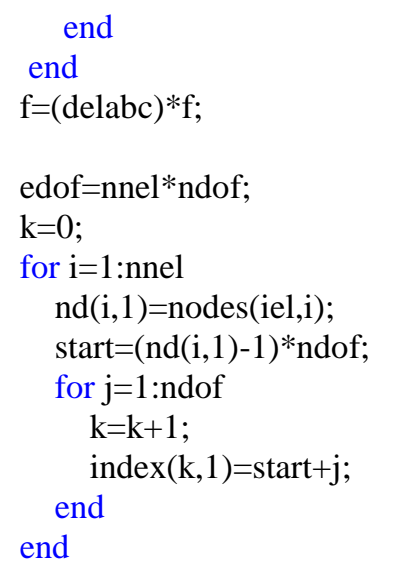

$\%$

for $i=1$ :edof

ii=index $(\mathrm{i}, 1)$;

ff(ii, 1$)=f f(i i, 1)+f(i, 1)$;

for $\mathrm{j}=1$ :edof

$\mathrm{jj}=\mathrm{index}(\mathrm{j}, 1)$;

ss(ii,jj) $=s s(i i, j j)+s k(i, j)$;

end

end

end $\%$ for iel

$\mathrm{mm}=$ length(bcdof);

sdof=size(ss);

$\%$

for $\mathrm{i}=1: \mathrm{mm}$

$c=$ bcdof $(i, 1)$;

for $\mathrm{j}=1$ :sdof

$\operatorname{ss}(\mathrm{c}, \mathrm{j})=0$;

end

$\%$

$\operatorname{ss}(\mathrm{c}, \mathrm{c})=1$;

ff $(c, 1)=\operatorname{bcval}(i, 1)$;

end

$\%$ solve the equations

phi=ss Iff;

for $\mathrm{I}=1$ :nnode

$\mathrm{NN}(\mathrm{I}, 1)=\mathrm{I}$;

end

$\operatorname{disp}('$

disp('number of nodes, elements \& nodes per element')

[nnode nel nnel ndof]

$\operatorname{disp}('$

$\operatorname{disp}\left(^{\prime}\right.$

$\operatorname{disp}([\mathrm{NN}$ phi xi] $)$

$\operatorname{disp}('$

disp('number of nodes,elements \& nodes per element')

[nnode nel nnel ndof]

nodes

gcoord

$[\mathrm{x}, \mathrm{y}]=$ meshgrid $(0: 0.05: 1,0: 0.05: 1)$;

for $i=1: 21$

for $\mathrm{j}=1: 21$

for iel=1:nel

$\%======$

$\mathrm{XX}=\mathrm{xx}(\mathrm{iel}, 1: 3)$;

YY=yy(iel,1:3);

$\% \operatorname{disp}([\mathrm{X} Y])$ 
$\mathrm{xa}=\mathrm{XX}(1,1)$;

$\mathrm{xb}=\mathrm{XX}(1,2)$;

$\mathrm{xc}=\mathrm{XX}(1,3)$;

ya $=Y Y(1,1)$;

$\mathrm{yb}=\mathrm{YY}(1,2)$;

yc=YY $(1,3)$;

$\mathrm{aLPa}=x b^{*} \mathrm{yc}-\mathrm{xc} * \mathrm{yb}$;

$\mathrm{aLPb}=\mathrm{xc} * \mathrm{ya}-\mathrm{xa} * \mathrm{yc}$

bta $=y b-y c ; b t b=y c-y a ;$

gma $=x c-x b ; g m b=x a-x c$;

delabc $=$ gmb $*$ bta-gma*btb;

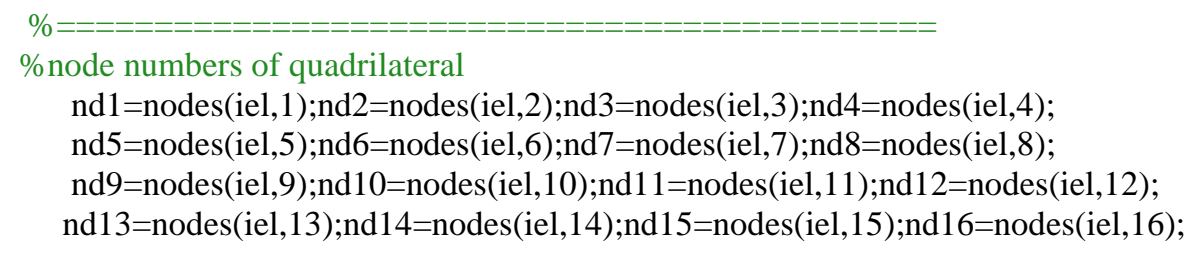

$\%$ coordinates of quadrilateral $(\mathrm{u}, \mathrm{v})$

$\mathrm{u}(1,1)=\operatorname{gcoord}(\mathrm{nd} 1,1) ; \mathrm{u}(2,1)=\operatorname{gcoord}(\mathrm{nd} 2,1) ; \mathrm{u}(3,1)=\operatorname{gcoord}(\mathrm{nd} 3,1) ; \mathrm{u}(4,1)=\operatorname{gcoord}(\mathrm{nd} 4,1)$;

$\mathrm{v}(1,1)=\operatorname{gcoord}(\mathrm{nd} 1,2) ; \mathrm{v}(2,1)=\operatorname{gcoord}(\mathrm{nd} 2,2) ; \mathrm{v}(3,1)=\operatorname{gcoord}(\mathrm{nd} 3,2) ; \mathrm{v}(4,1)=\operatorname{gcoord}(\mathrm{nd} 4,2)$;

$\%$ coordinates of the $\operatorname{grid}(\mathrm{x}, \mathrm{y})$

in=inpolygon $(x(i, j), y(i, j), u, v)$;

if (in==1)

$\mathrm{X}=\mathrm{x}(\mathrm{i}, \mathrm{j}) ; \mathrm{Y}=\mathrm{y}(\mathrm{i}, \mathrm{j})$;

$\%[\mathrm{t}]=$ convexquadrilateral_coordinates $(\mathrm{u}, \mathrm{v}, \mathrm{X}, \mathrm{Y})$;

$\mathrm{p}=(\mathrm{aLPa}+\mathrm{bta} * \mathrm{X}+\mathrm{gma} * \mathrm{Y}) /$ delabc;

$\mathrm{q}=(\mathrm{aLPb}+\mathrm{btb} * \mathrm{X}+\mathrm{gmb} * \mathrm{Y}) /$ delabc;

$\mathrm{t} 0=[0.5 ; 0.5]$;

$\mathrm{r}=\mathrm{t}(1,1)$;

$\mathrm{s}=\mathrm{t}(2,1)$;

$\mathrm{h} 1 \mathrm{r}=-9 *(\mathrm{r}+1 / 3) *(\mathrm{r}-1 / 3) *(\mathrm{r}-1) / 16$;

$\mathrm{h} 2 \mathrm{r}=27 *(\mathrm{r}+1) *(\mathrm{r}-1 / 3) *(\mathrm{r}-1) / 16$

$\mathrm{h} 3 \mathrm{r}=-27 *(\mathrm{r}+1) *(\mathrm{r}+1 / 3) *(\mathrm{r}-1) / 16$;

$\mathrm{h} 4 \mathrm{r}=9 *(\mathrm{r}+1) *(\mathrm{r}+1 / 3) *(\mathrm{r}-1 / 3) / 16$;

$\mathrm{h} 1 \mathrm{~s}=-9 *(\mathrm{~s}+1 / 3) *(\mathrm{~s}-1 / 3) *(\mathrm{~s}-1) / 16$;

$\mathrm{h} 2 \mathrm{~s}=27 *(\mathrm{~s}+1) *(\mathrm{~s}-1 / 3) *(\mathrm{~s}-1) / 16$;

$\mathrm{h} 3 \mathrm{~s}=-27 *(\mathrm{~s}+1) *(\mathrm{~s}+1 / 3) *(\mathrm{~s}-1) / 16$;

$\mathrm{h} 4 \mathrm{~s}=9 *(\mathrm{~s}+1) *(\mathrm{~s}+1 / 3) *(\mathrm{~s}-1 / 3) / 16$;

$\%$

$\operatorname{shn} 1=\mathrm{h} 1 \mathrm{r} * \mathrm{~h} 1 \mathrm{~s} ; \operatorname{shn} 5=\mathrm{h} 2 \mathrm{r} * \mathrm{~h} 1 \mathrm{~s} ; \operatorname{shn} 6=\mathrm{h} 3 \mathrm{r} * \mathrm{~h} 1 \mathrm{~s} ; \operatorname{shn} 2=\mathrm{h} 4 \mathrm{r} * \mathrm{~h} 1 \mathrm{~s} ;$

$\operatorname{shn} 12=h 1 \mathrm{r} * \mathrm{~h} 2 \mathrm{~s} ; \operatorname{shn} 13=\mathrm{h} 2 \mathrm{r} * \mathrm{~h} 2 \mathrm{~s} ; \operatorname{shn} 14=\mathrm{h} 3 \mathrm{r} * \mathrm{~h} 2 \mathrm{~s} ; \operatorname{shn} 7=\mathrm{h} 4 \mathrm{r} * \mathrm{~h} 2 \mathrm{~s} ;$

$\operatorname{shn} 11=h 1 r * h 3 s ; \operatorname{shn} 16=h 2 r * h 3 s ; \operatorname{shn} 15=h 3 r * h 3 s ; \operatorname{shn} 8=h 4 r * h 3 s ;$

shn4=h1r*h4s;shn10=h2r*h4s;shn9=h3r*h4s;shn3=h4r*h4s;

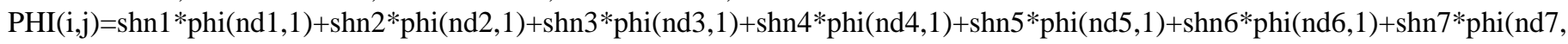

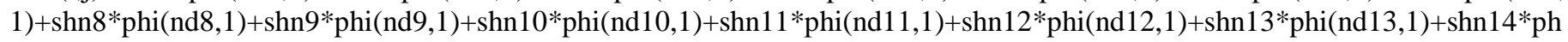
i(nd14,1)+shn15*phi(nd15,1)+shn16*phi(nd16,1);

break

end $\%$ if (in==1)

end $\%$ for iel

$\%$ THE PROGRAM EXECUTION JUMPS TO HERE if (in==1)

end $\%$ for $\mathrm{j}$

end $\%$ for $\mathrm{i}$

$\mathrm{z}=\sin \left(\mathrm{pi}^{*} \mathrm{x}\right) . * \sin \left(\mathrm{pi}^{*} \mathrm{y}\right)$;

for $i=1: 21$

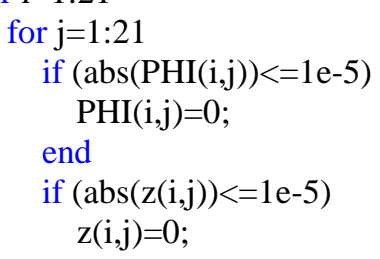


end

end

end

switch mesh

case 1

clc

hold off

clf

figure(1)

$\mathrm{x}=\left[\begin{array}{lllll}0.0 & 1.0 & 1.0 & 0.5 & 0.0\end{array}\right]$;

$\mathrm{y}=\left[\begin{array}{lllll}0.0 & 0.0 & 0.5 & 1.0 & 1.0\end{array}\right]$;

$\operatorname{patch}\left(\mathrm{x}, \mathrm{y},{ }^{\prime} \mathrm{w}\right)$

hold on

[x,y]=meshgrid(0:.05:1,0:0.05:1)

$\mathrm{y}((\mathrm{y}>1 / 2) \&(\mathrm{y}<=1) \&(\mathrm{x}>1 / 2) \&(\mathrm{x}<=1) \&(\mathrm{x}+\mathrm{y}>3 / 2))=\mathrm{NaN}$

contour(x,y,PHI,40,'r-')

$\%[\mathrm{c}, \mathrm{h}]=$ contour $(\mathrm{x}, \mathrm{y}, \mathrm{PHI})$

xlabel('X-axis');

ylabel('Y-axis');

\%clabel(c,h);

axis square

st1='Contour level curves for ';

st2='FEM solution of ';

st3=' Sixteen Noded ';

st4='Special Quadrilateral';

st5=' Elements'

title([st1,st2,st3,st4,st5])

sst $1=$ '(MESH HAS '

sst $2=$ num 2 str(nnode)

sst3=' NODES'

sst4=' AND

sst5=num $2 \operatorname{str}($ nel)

sst6=' ELEMENTS)'

text(0.25,-.08,[sst1 sst2 sst3 sst4 sst5 sst6])

figure(2)

$\mathrm{x}=\left[\begin{array}{llllll}0.0 & 1.0 & 1.0 & 0.5 & 0.0\end{array}\right]$;

$\mathrm{y}=\left[\begin{array}{lllll}0.0 & 0.0 & 0.5 & 1.0 & 1.0\end{array}\right]$;

$\operatorname{patch}\left(\mathrm{x}, \mathrm{y},{ }^{\prime} \mathrm{w}\right)$

hold on

$[\mathrm{x}, \mathrm{y}]=$ meshgrid(0:.05:1,0:0.05:1)

$\mathrm{y}((\mathrm{y}>1 / 2) \&(\mathrm{y}<=1) \&(\mathrm{x}>1 / 2) \&(\mathrm{x}<=1) \&(\mathrm{x}+\mathrm{y}>3 / 2))=\mathrm{NaN}$;

contour(x,y,z,40,'g-')

$\%[\mathrm{c}, \mathrm{h}]=$ contour $(\mathrm{x}, \mathrm{y}, \mathrm{z})$

xlabel('X-axis');

ylabel('Y-axis');

$\%$ clabel(c,h);

axis square

title('contour level curves for exact solution: $\sin \left(\mathrm{pi}^{*} \mathrm{x}\right) * \sin \left(\mathrm{pi}{ }^{*} \mathrm{y}\right)$ ')

$\mathrm{mm}=0$;

for $\mathrm{i}=1: 21$

for $\mathrm{j}=1: 21$

$\mathrm{mm}=\mathrm{mm}+1$;

femsoln $(m m, 1)=P H I(i, j)$;

exactsoln $(m m, 1)=z(i, j)$;

end

end

case 2

clc

hold off

clf

figure(1)

$\mathrm{x}=\left[\begin{array}{llll}0.0 & 1.0 & 1.0 & 0.0\end{array}\right]$;

$\mathrm{y}=\left[\begin{array}{llll}0.0 & 0.0 & 1.0 & 1.0\end{array}\right]$;

$\operatorname{patch}\left(\mathrm{x}, \mathrm{y}, \mathrm{w}^{\prime}\right)$ 
hold on

$[\mathrm{x}, \mathrm{y}]=\operatorname{meshgrid}(0: .05: 1,0: 0.05: 1)$

contour(x,y,PHI,40,'r-')

$\%[\mathrm{c}, \mathrm{h}]=\operatorname{contour}(\mathrm{x}, \mathrm{y}, \mathrm{PHI})$

xlabel('X-axis');

ylabel('Y-axis');

\%clabel(c,h);

axis square

st1='Contour level curves for ';

st2='FEM solution of ';

st3='Sixteen Noded ';

st4='Special Quadrilateral';

st5=' Elements'

title([st1,st2,st3,st4,st5])

sst1='(MESH HAS '

sst $2=$ num 2 str(nnode)

sst3=' NODES'

sst4=' AND

sst5=num2str(nel)

sst6=' ELEMENTS)'

text(0.25,-.08,[sst1 sst2 sst3 sst4 sst5 sst6])

figure(2)

$\mathrm{x}=\left[\begin{array}{llll}0.0 & 1.0 & 1.0 & 0.0\end{array}\right]$;

$\mathrm{y}=\left[\begin{array}{llll}0.0 & 0.0 & 1.0 & 1.0\end{array}\right]$;

$\operatorname{patch}\left(\mathrm{x}, \mathrm{y}, \mathrm{w}^{\prime}\right)$

hold on

$[\mathrm{x}, \mathrm{y}]=$ meshgrid(0:.05:1,0:0.05:1)

contour(x,y,z,40,'g-')

$\%[\mathrm{c}, \mathrm{h}]=$ contour $(\mathrm{x}, \mathrm{y}, \mathrm{z}) \%$

xlabel('X-axis');

ylabel('Y-axis');

$\%$ clabel(c,h);

axis square

title('contour level curves for exact solution: $\left.\sin \left(\mathrm{pi}^{*} \mathrm{x}\right) * \sin (\mathrm{pi} * \mathrm{y}){ }^{\prime}\right)$

$\mathrm{mm}=0$;

for $\mathrm{i}=1: 21$

for $\mathrm{j}=1: 21$

$\mathrm{mm}=\mathrm{mm}+1$;

femsoln $(\mathrm{mm}, 1)=\mathrm{PHI}(\mathrm{i}, \mathrm{j})$;

$\operatorname{exactsoln}(\mathrm{mm}, 1)=\mathrm{z}(\mathrm{i}, \mathrm{j})$;

end

end

end\%switch mesh

[femsoln exactsoln]

for $\mathrm{I}=1$ :nnode

$\mathrm{NN}(\mathrm{I}, 1)=\mathrm{I}$

phi_xi(I,1)=phi(I,1)-xi(I,1);

end

MAXPHI_XI=max(abs(phi_xi))

[NN phi]

for $\mathrm{I}=1$ :nel

$\mathrm{NNN}(\mathrm{I}, 1)=\mathrm{I}$;

end

[NNN nodes]

disp('number of nodes, elements \& nodes per element')

[nnode nel nnel ndof]

$[1 \operatorname{phi}(1,1) \times i(1,1)]$

\section{[19]newtonmethod4spquadrileteral.m}

function $[\mathrm{t}$, iter $]=$ newtonmethod 4 spquadrileteral $(\mathrm{t} 0, \mathrm{p}, \mathrm{q}, \mathrm{f}, \mathrm{det} \mathrm{J}, \mathrm{inv} \mathrm{J})$

$\%$ Newton-Raphson method applied to a

$\%$ system of linear equations $\mathrm{f}(\mathrm{x})=0$,

$\%$ given the jacobian function $\mathrm{J}$, with 
$\% \mathrm{~J}=\operatorname{del}(\mathrm{f} 1, \mathrm{f} 2, \ldots, \mathrm{fn}) / \operatorname{del}(\mathrm{x} 1, \mathrm{x} 2, \ldots, \mathrm{xn})$

$\% \mathrm{x}=[\mathrm{x} 1 ; \mathrm{x} 2 ; \ldots ; \mathrm{xn}], \mathrm{f}=[\mathrm{f} 1 ; \mathrm{f} 2 ; \ldots ; \mathrm{fn}]$

$\% \mathrm{x} 0$ is an initial guess of the solution

format short

$\mathrm{N}=100 ; \%$ define max. number of iterations

epsilon $=1 \mathrm{e}-10 ; \%$ define tolerance

maxval $=10000.0 ; \%$ define value for divergence

$\mathrm{tt}=\mathrm{t} 0 ; \%$ load initial guess

while $(\mathrm{N}>0)$

$\operatorname{det} J \mathrm{~J}=\mathrm{feval}(\operatorname{det} \mathrm{J}, \mathrm{tt})$;

if abs(detJJ)<epsilon

error('newtonm - Jacobian is singular - try new x0');

abort;

end;

tn = tt -feval(invJ,tt)*(feval(f,tt)-[p;q]);

if abs(feval(f,tn)-[p;q])<epsilon

$\mathrm{ftn}=$ feval(f,tn)-[p;q];

$\mathrm{t}=\mathrm{tn}$;

iter $=100-\mathrm{N}$;

return;

end;

if $\operatorname{abs}(f e v a l(f, t t)-[p ; q])>\operatorname{maxval}$

iter $=100-\mathrm{N}$;

$\operatorname{disp}([$ 'iterations = ',num2str(iter)]);

error('Solution diverges');

abort;

end;

$\mathrm{N}=\mathrm{N}-1$

$\mathrm{tt}=\mathrm{tn}$

end;\% while

error('No convergence after 100 iterations.');

abort;

$\%$ end function

\section{[20]parameqnspqd.m}

function $[\mathrm{f}]=$ parameqnspqd(tt)

$\mathrm{f}=[-((\operatorname{tt}(1,1)-1) *(\operatorname{tt}(2,1)+5)) / 24 ;-((\operatorname{tt}(1,1)+5) *(\operatorname{tt}(2,1)-1)) / 24]$;

[21]paramdetJspqd.m

function $[$ det $]=$ paramdetJspqd(tt)

$\operatorname{det} \mathrm{J}=\operatorname{tt}(1,1) / 96+\operatorname{tt}(2,1) / 96+1 / 24$;

[22]paraminvJspqd.m

function $[\mathrm{inv}]=$ paraminv Jspqd $(\mathrm{tt})$

invJ $=[-(\operatorname{tt}(1,1) / 24+5 / 24) /(\operatorname{tt}(2,1) / 96+\operatorname{tt}(1,1) / 96+1 / 24),(\operatorname{tt}(2,1) / 24-1 / 24) /(\operatorname{tt}(2,1) / 96+\operatorname{tt}(1,1) / 96+1 / 24) ; \ldots$

$(\operatorname{tt}(1,1) / 24-1 / 24) /(\operatorname{tt}(2,1) / 96+\operatorname{tt}(1,1) / 96+1 / 24),-(\operatorname{tt}(2,1) / 24+5 / 24) /(\operatorname{tt}(2,1) / 96+\operatorname{tt}(1,1) / 96+1 / 24)] ;$

[23]glsampleptsweights.m:this program is regarding Gauss Legendre sampling points and weight coefficients and it is available in references[27-33] 